\title{
MPLS-BASED MICRO-MOBILITY ARCHITECTURE FOR 5G VEHICULAR VISIBLE LIGHT COMMUNICATION NETWORKS
}

\author{
by \\ SAMEERA SIDDIQUI
}

Thesis submitted to the University of Ottawa in partial fulfillment of the requirements for the degree of

\section{Doctor of Philosophy}

\author{
Ottawa-Carleton Institute for Electrical and Computer Engineering \\ School of Electrical Engineering and Computer Science \\ University of Ottawa
}

(C) Sameera Siddiqui, Ottawa, Canada, 2020 


\begin{abstract}
Future vehicular networks in the emerging era of $5 \mathrm{G}$ will be characterized by ubiquitous connectivity across heterogeneous networks (HetNets) while maintaining their required Quality of Service (QoS). Visible light communication (VLC) and mmWave are both promising candidates for use in last mile communication networks. However, selection of appropriate technology for vehicular systems is dependent on their performance in outdoor networks. Due to cell densification inherent in 5G communication networks, selection of an appropriate micromobility scheme that ensures seamless connectivity with QoS provision is critical. This thesis focuses on the investigation of these issues.
\end{abstract}

VLC and mmWave are both evaluated for outdoor vehicular networks based on their propagation characteristics and attenuation due to adverse weather conditions. Based on comparative design analysis, we proposed the adoption of a dual-link, multi beam VVLC system for outdoor infrastructure vehicular communication networks. The design analysis was carried out using existing empirical and mathematical models. Compared to mmWave system, the proposed model was found a safer, power efficient and economically more viable solution.

Multiprotocol Label Switching (MPLS) based micro-mobility management has the advantage of fast and efficient packet forwarding. This thesis proposes a hierarchical IntServ based MPLS architecture to overcome the limitations found in existing MPLS based micro-mobility schemes in terms of latency and explicit QoS support necessary for 5G vehicular communication networks.

The survivability of communication networks is another critical issue. This thesis also proposes an improved fast re-routing scheme that decreases the path restoration delay compared to existing solutions proposed by IETF and found in the open literature.

Our proposed schemes are evaluated through both mathematical and simulative analysis. The models were developed and simulated using the OPNET simulator to validate the analytical results. The results were also compared with other existing schemes to demonstrate the performance benefits of the proposed schemes. 


\section{ACKNOWLEDGEMENTS}

Indeed, all praises and thanks belong to Allah S.W.T, the Master of the Universe, the most merciful and the most beneficent. Without His will, this work would have never materialized. I owe Him my existence and all of my achievements.

I would like to express my sincere gratitude to my supervisor, Dr. Dimitrios Makrakis for his valuable technical guidance, support and encouragement throughout this work and for making my $\mathrm{Ph} . \mathrm{D}$ journey a profound learning experience. His analysis and feedback on this work based on his extensive knowledge and expertise in the field of networking and communication systems motivated me in enhancing my researching capabilities and boosted my knowledge.

I would also like to thank students in the Broadband Wireless and Internet Lab for their help and advice during this research.

My deepest gratitude goes to my parents for instilling the love of education in me since a very early age. They taught me to take on the challenges of life with positive attitude. Many thanks to my brothers for being there for me. Special thanks to my friends for always encouraging me, they didn't let me give up. Also, to my kids, Omer, Ammaar and Emaad, who light up my days even in the darkest moments. Many thanks to my parents-in-laws for supporting and encouraging me throughout. Last but not least, I am really thankful to my husband, Farrukh, who has been a pillar of strength and encouragement for me. His consistent support, genuine advices and constant efforts to uplift me in the hardest times are forever appreciated and treasured. 


\section{TABLE OF CONTENTS}

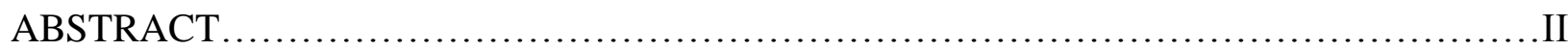

ACKNOWLEDGEMENTS .................................................................

TABLE OF CONTENTS ............................................................ IV

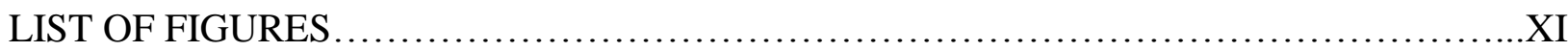

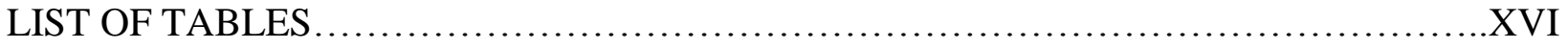

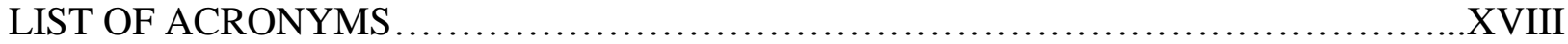

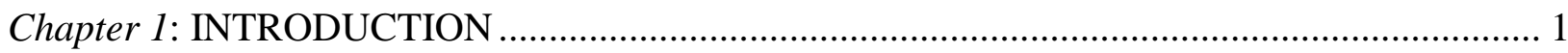

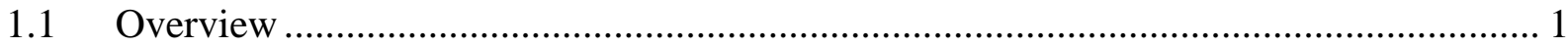

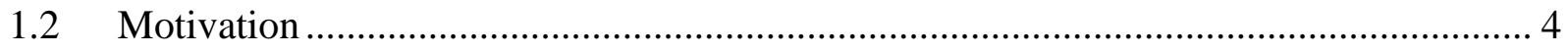

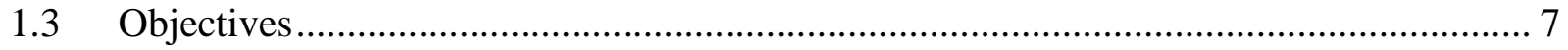

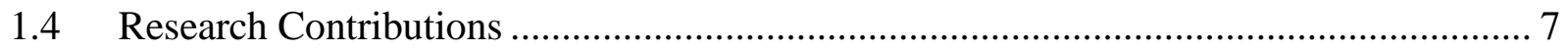

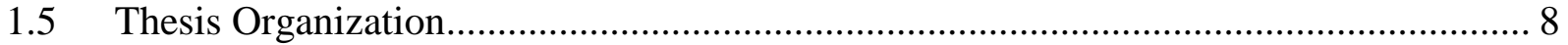

Chapter 2: LITERATURE REVIEW OF 5G VVLC NETWORKS ..................................... 10

2.1 Key Transformations in Future Vehicular Communication Networks ....................... 10

2.1.1 Autonomous Vehicles ................................................................................ 10

2.1.2 Road Safety and traffic management services ........................................... 13

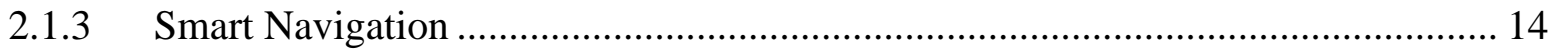

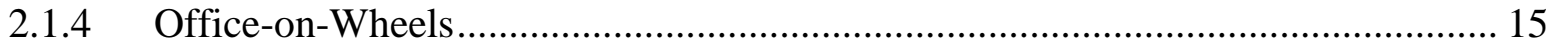

2.1.5 Transportation As A Service (TaaS) ........................................................ 15

2.1.6 Automatic Maintenance Notifications ...................................................... 16

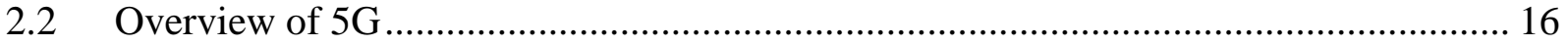

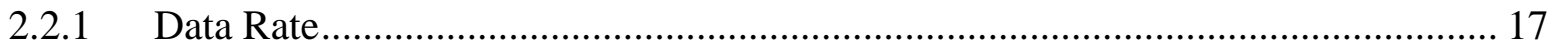

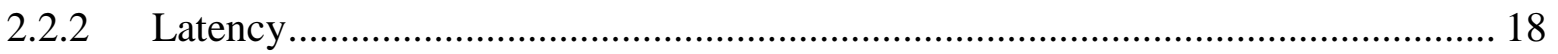

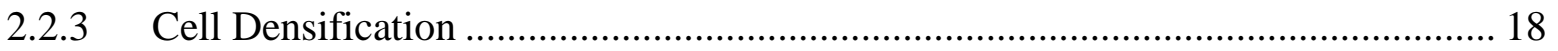


2.2.4 Huge Bandwidth \& mmWave Spectrum ........................................................ 19

2.2.5 Device Diversity and Density ………………................................................... 19

2.2.6 Massive MIMO \& 3D Beamforming .............................................................. 19

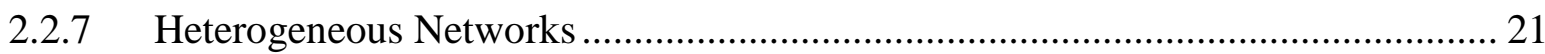

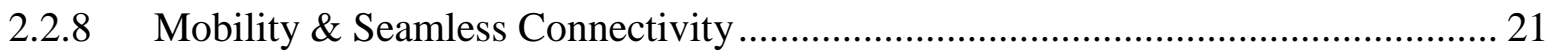

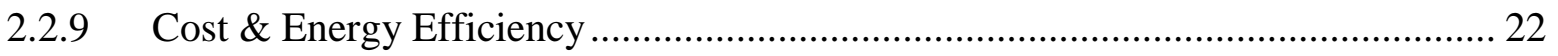

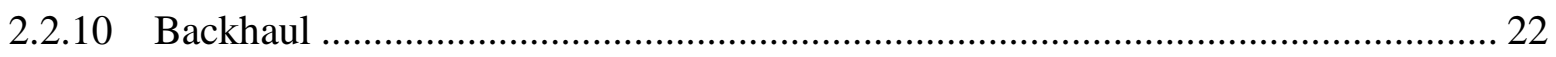

2.3 Link Between Future Vehicular Networks And 5G Objectives.................................... 23

2.3.1 5G For Vehicular Communication....................................................................... 24

2.3.1.1 Device-to-Device Communication ………………..................................... 24

2.3.1.2 Mobile Edge Computing (MEC) ……………............................................ 25

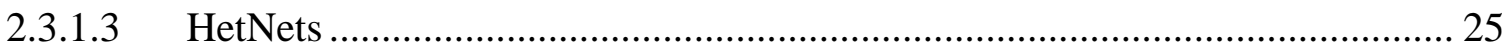

2.3.2 Technical Requirements For Vehicular Applications Mapped To 5G ................... 26

2.4 Limitations of Existing Communication Technologies ................................................. 29

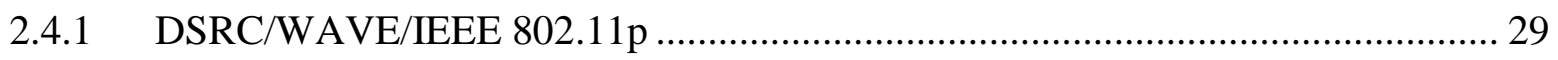

2.4.2 4G/LTE Infrastructure -based communication ………………………………..... 31

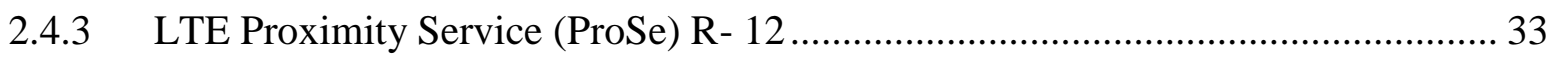

2.4.4 Existing Short Range Communication Technologies .............................................. 33

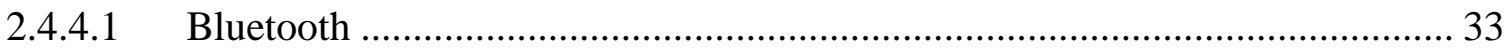

2.4.4.2 Ultra-Wideband ................................................................................ 34

2.4.4.3 ZigBee ……………………………………......................................... 34

2.5 New Technologies Proposed To Be Used in 5G ...................................................... 34

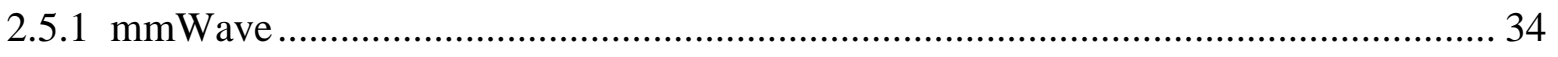

2.5.1.1 Range and Directional Communication ......................................................... 35

2.5.1.2 Atmospheric Absorption............................................................................ 35 


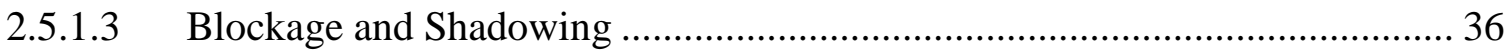

2.5.1.4 Heterogeneous Networking Issues ............................................................... 36

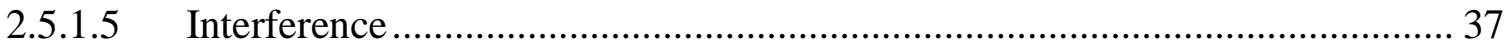

2.5.1.6 Multiple Access Mechanism ...................................................................... 37

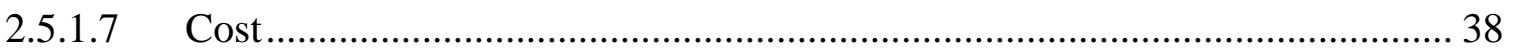

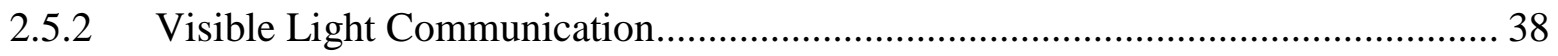

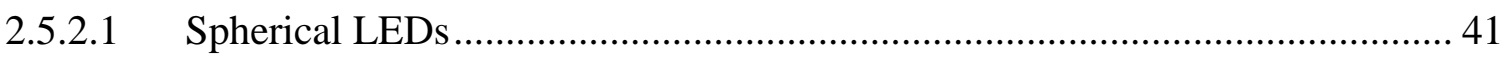

2.5.2.2 Separate Uplink and Downlink.................................................................... 41

2.5.2.3 Control and User Data Plane Separation ........................................................ 42

2.5.2.4 LOS Blocking ...................................................................................... 42

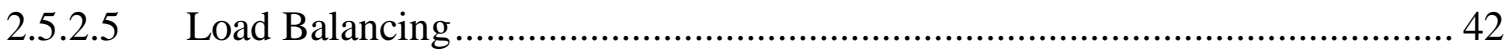

2.5.2.6 BackHaul ............................................................................................. 43

2.6 Comparative Commentary between VLC and mmWave .................................................. 44

2.7 Mobility Management for 5G Vehicular Networks ............................................................ 46

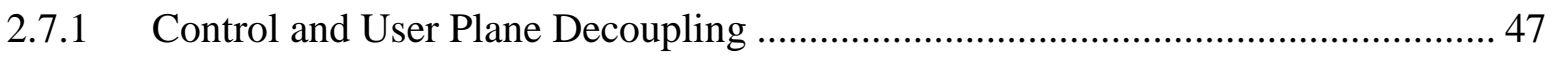

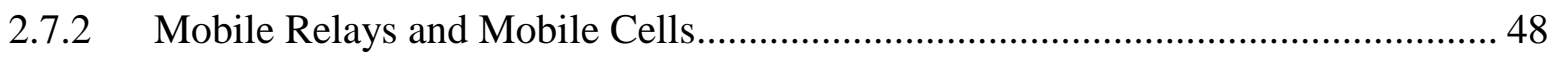

2.7.3 Geo-Aided Fast Handover .................................................................................... 49

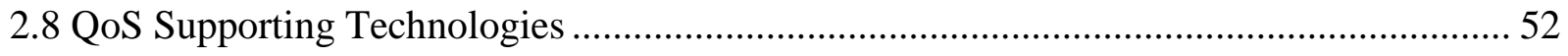

2.8.1 Multiprotocol Label Switching \& Traffic Engineering ............................................... 52

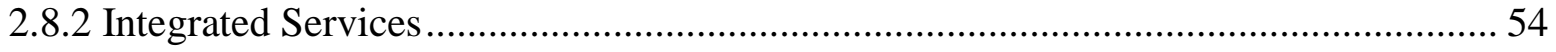

2.8.2.1 Guaranteed Service ………………………….................................................. 54

2.8.2.2 Controlled Load Service …………………………..................................... 55

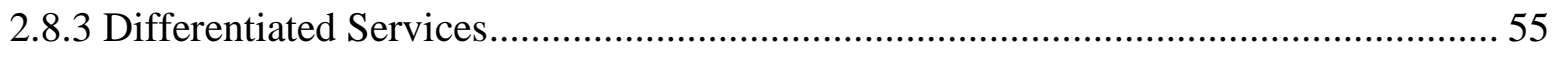

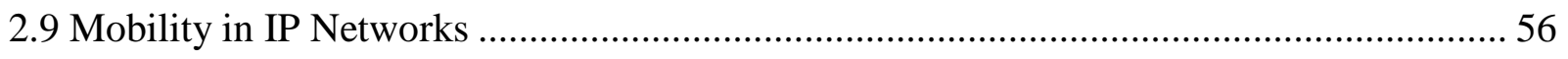

2.9.1 Mobile IP for Macro-mobility ………......................................................... 57 


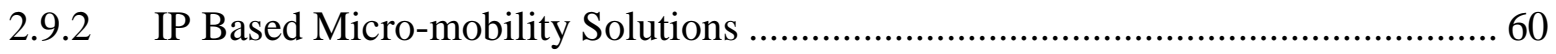

2.9.3 MPLS Based Micro-mobility Management........................................................... 61

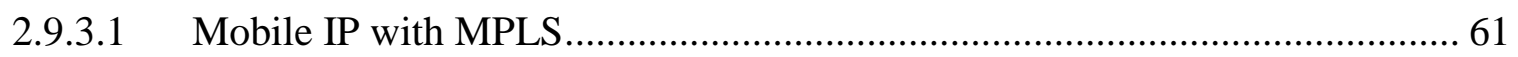

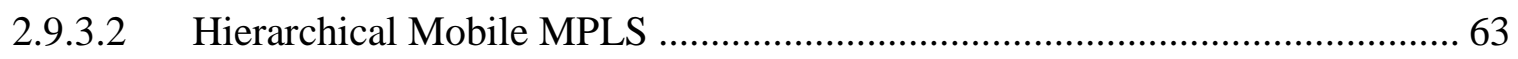

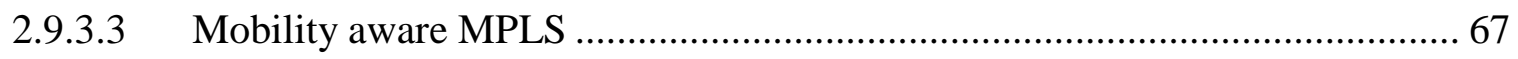

2.9.3.4 MPLS based HMIP ............................................................................ 73

2.9.3.5 Micro-cell Mobile MPLS ......................................................................... 77

2.9.3.6 Label-Edge Mobility Agent (LEMA) ……………………............................ 80

2.9.3.7 MPLS-based Micro- mobility Scheme ……………………………………...... 83

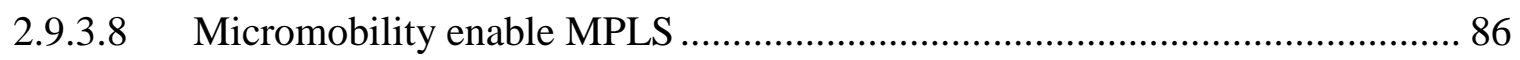

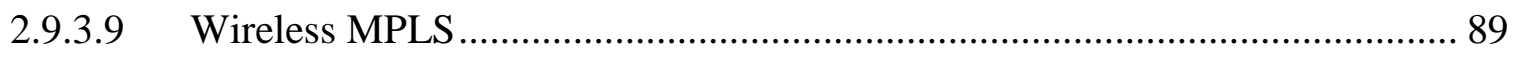

2.9.3.10 Virtual LSP (V-LSP) based QoS capable Micro Mobility Scheme ................ 92

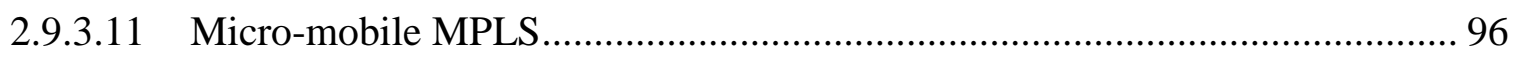

2.9.3.12 Mobile Traffic Engineering with QoS aware MPLS...................................... 101

2.9.3.13 Comparison Between MPLS-based Micro Mobility Schemes ....................... 105

Chapter 3: COMPARATIVE ANALYSIS BETWEEN VL AND MMWAVE FOR 5G

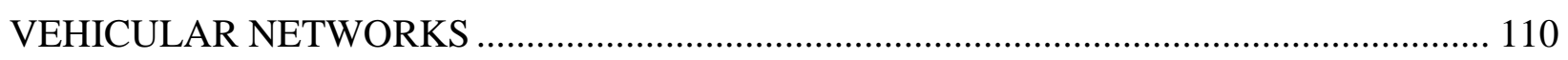

3.1 Visible Light Communication .................................................................................. 110

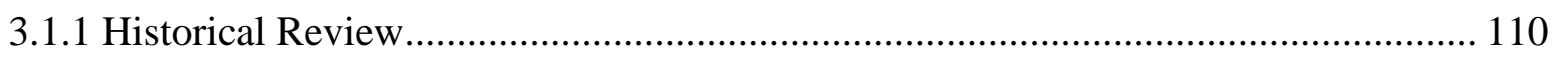

3.1.2 Channel Models For Vehicular Visible Light Communication ................................ 113

3.1.3 Visible Light Propagation Characteristics .............................................................. 115

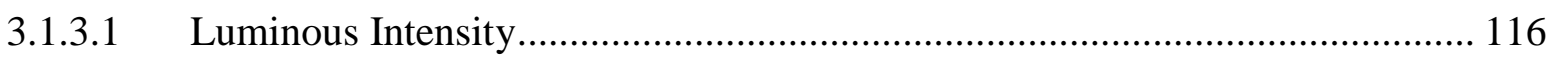

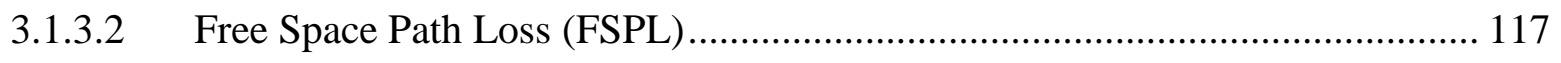

3.1.3.3 Specific Attenuation due to Adverse Weather ................................................... 119

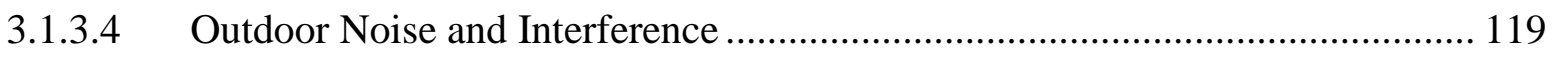




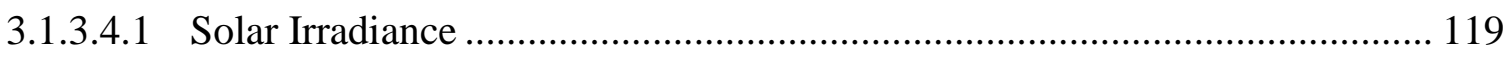

3.1.3.4.2 Daytime Sky Radiance ............................................................................... 120

3.1.3.4.3 Nighttime Sky Radiance................................................................................ 120

3.1.3.4.4 Interference due to Artificial Lighting Sources............................................. 121

3.1.3.5 Directivity of Propagation Links...................................................................... 121

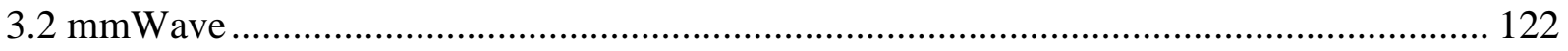

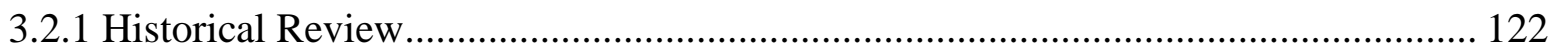

3.2.2 Channel Models For Outdoor mmWave Communication ........................................ 122

3.2.3 mmWave Propagation Characteristics ................................................................... 124

3.2.3.1 Free-Space Path Loss ................................................................................ 125

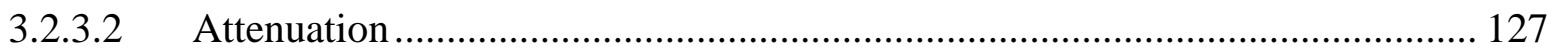

3.2.3.2.1 Atmospheric Attenuation ............................................................................ 127

3.2.3.2.2 Weather Specific Attenuation .................................................................. 128

3.2.3.2.3 Foliage .......................................................................................... 128

3.2.3.3 Propagation Mechanism ............................................................................... 130

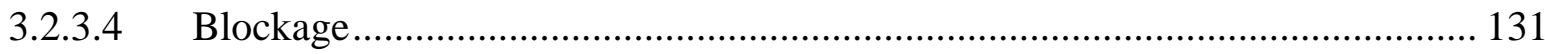

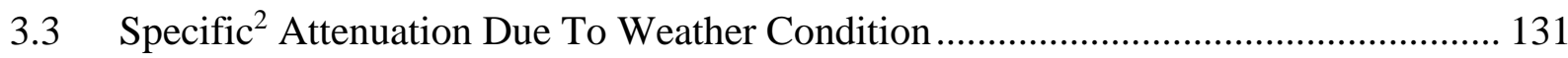

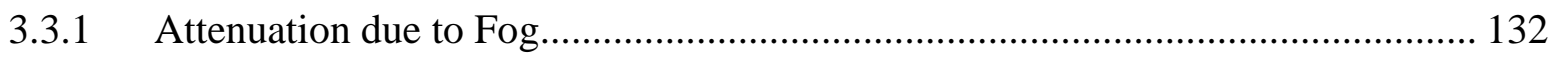

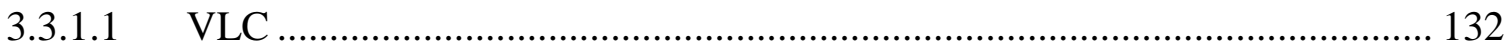

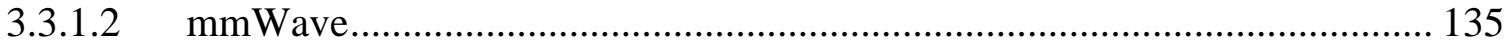

3.3.2 Attenuation due to Rain .............................................................................. 138

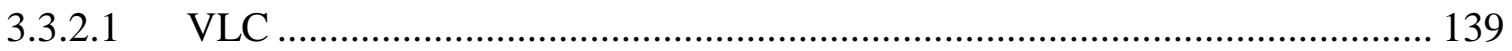

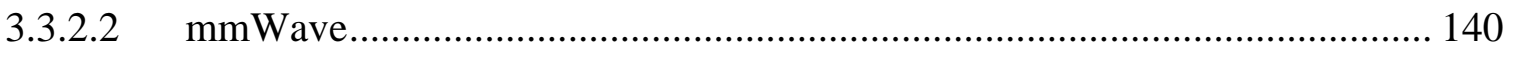

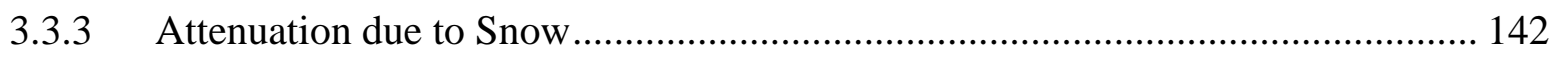

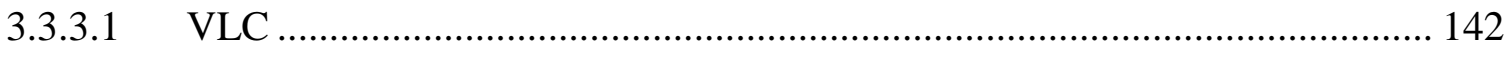


3.3.3.2 mmWave 145

3.4 Comparison between VLC and mmWave based on Attenuation due to Adverse Weather 148

3.5 Design Analysis for 5G vehicular Networks.................................................. 152

3.5.1 Dual-link VLC System ....................................................................... 152

3.5.1.1 Joint design of high rate and low rate dual link system............................ 158

3.5.1.2 Dual link system with weather attenuation.......................................... 164

3.5.1.3 Mapping of power transmission levels to traffic lights ............................. 168

3.5.2 Link Budget Analysis of Outdoor mmWave System ................................... 169

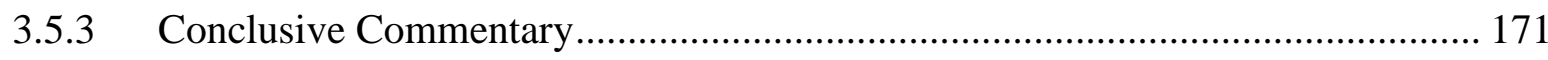

Chapter 4: HIERARCHICAL FORWARDING CHAIN BASED MPLS SCHEME FOR 5G

ULTRA DENSE VEHICULAR NETWORKS ........................................................... 175

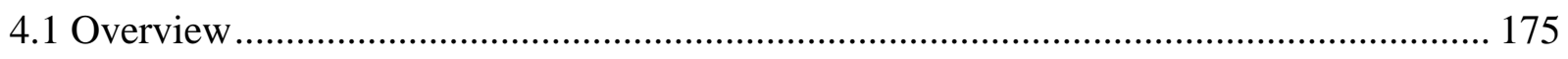

4.2 Architecture for Proposed Hierarchical Forwarding Chain based MPLS scheme for 5G

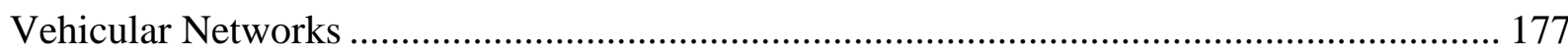

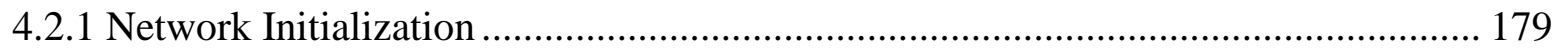

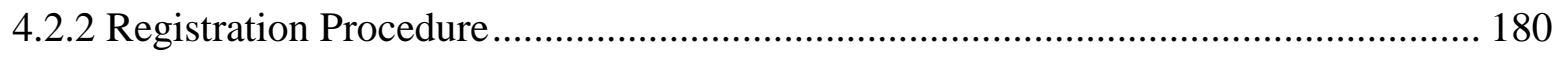

4.2.3 Mobility Management and Seamless Handoff.................................................. 181

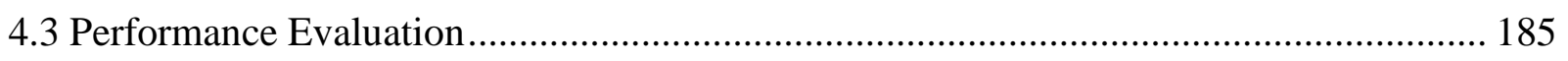

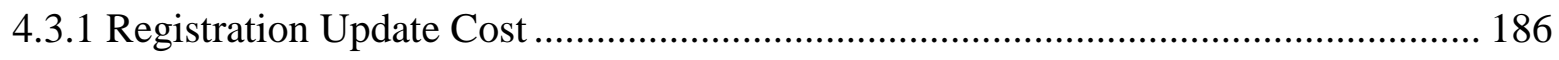

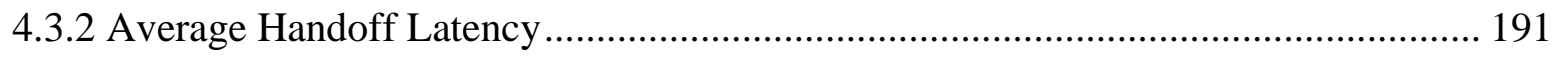

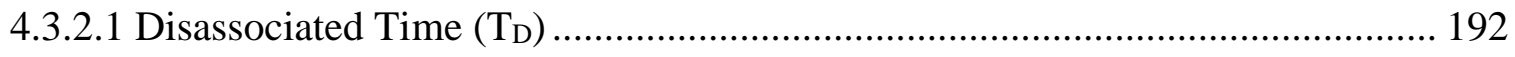

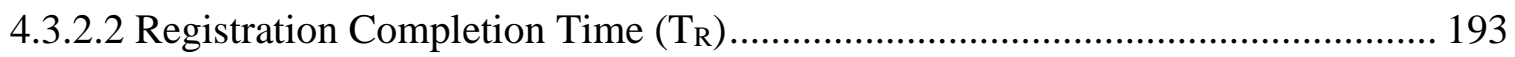

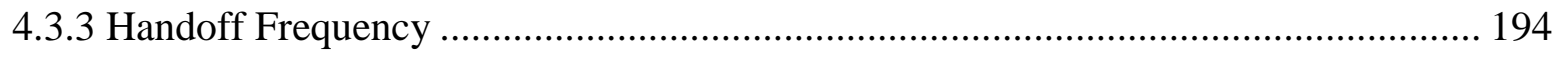

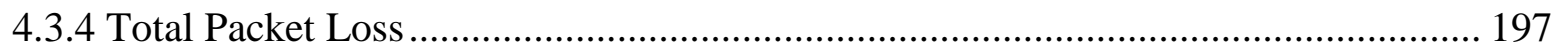

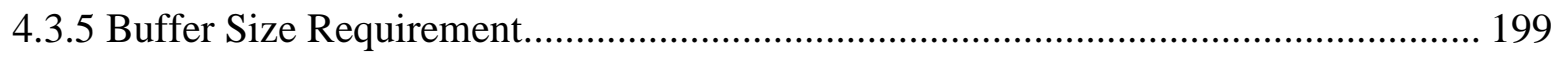




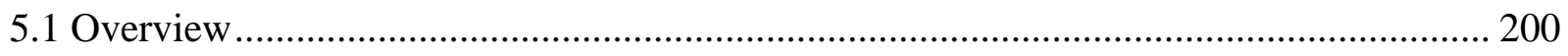

5.2 Existing MPLS Fast-Rerouting Schemes................................................................. 202

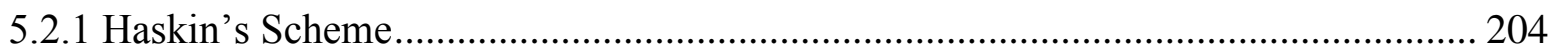

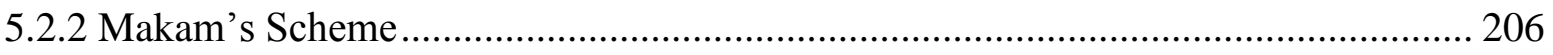

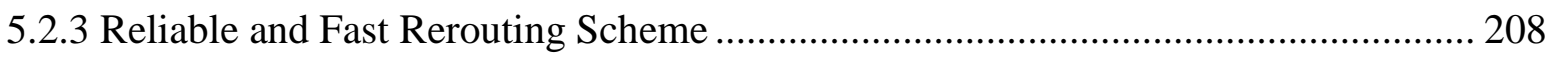

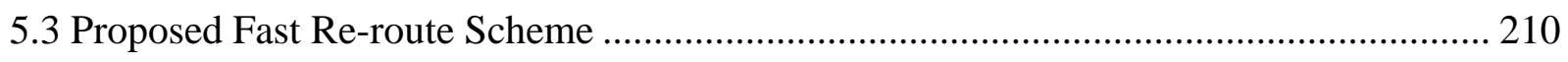

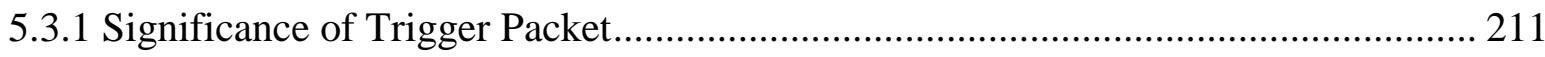

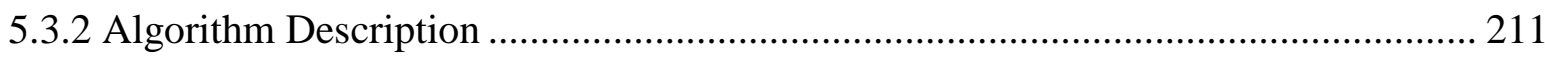

5.3.3 LFIB Table Management ................................................................................... 217

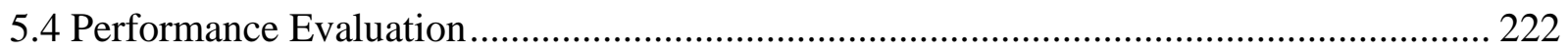

5.4.1 Path Restoration Time............................................................................................ 222

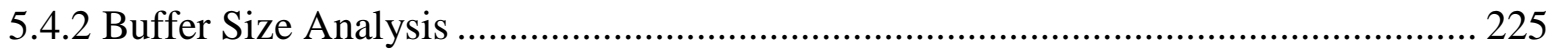

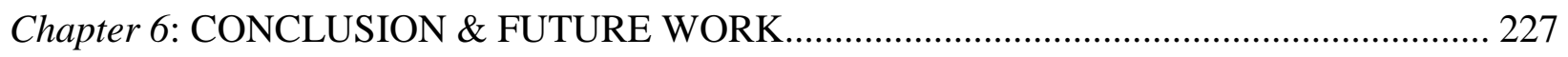

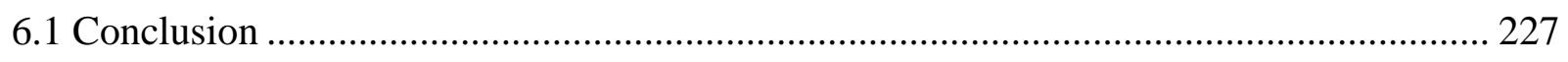

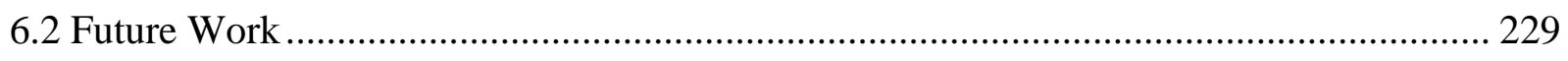

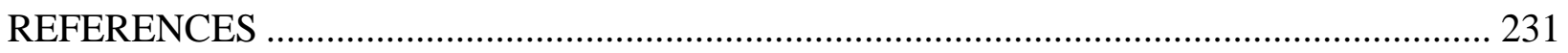




\section{LIST OF FIGURES}

Figure 1.1 5G Automotive Vision supporting heterogeneous technologies for vehicle-to-

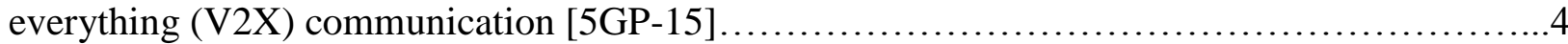

Figure 2.1 Six levels of vehicular automation according to SAE/VDA. [VDA-15]............12

Figure 2.2 Example see-through case for vehicular safety application. [5GP-15].............14

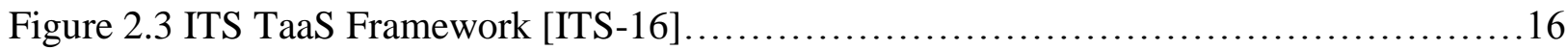

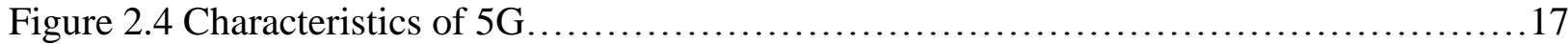

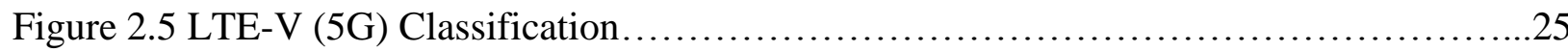

Figure $2.6 \mathrm{CRAN}$ architecture for $5 \mathrm{G}$ vehicular networks..............................47

Figure 2.7 SDN based architecture with MPLS in user plane (reproduced from [GAR-16]).....51

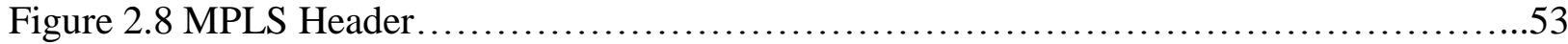

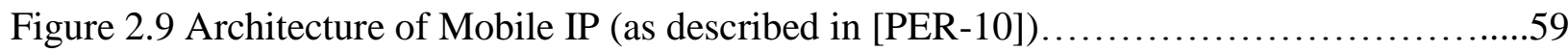

Figure 2.10 Timing flow of messages in Mobile IP .................................60

Figure 2.11 Architecture of MIP-MPLS (as described in [REN-01] .......................62

Figure 2.12 Timing diagram of registration/handoff message exchange in MIP-MPLS.........63

Figure 2.13 Architecture of HMPLS (reproduced from [YAN-01]) $\ldots \ldots \ldots \ldots \ldots \ldots \ldots \ldots \ldots \ldots . . .65$

Figure 2.14 Timing diagram of messages exchanged during handoff in HMPLS (reproduced

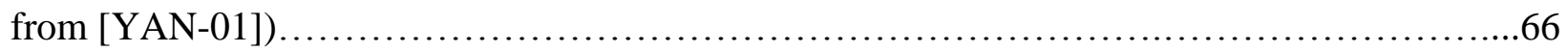

Figure 2.15 Architecture of MAMPLS-DiffServ with static LSPs (reproduced from [KIM-

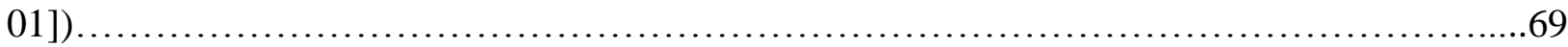

Figure 2.16 Handoff in MAMPLS-DiffServ with static LSPs..........................70

Figure 2.17 Architecture of MAMPLS-IntServ with dynamic LSPs (reproduced from [KIM-

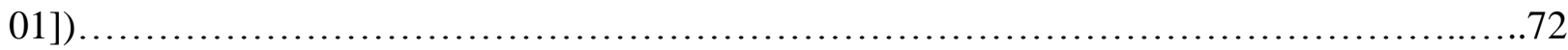

Figure 2.18 Handoff in MAMPLS-IntServ with dynamic LSPs........................73

Figure 2.19 Architecture of MPLS-HMIP (as described in [URN-01]) .....................75

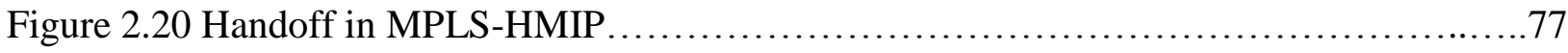

Figure 2.21 MM-MPLS architecture (reproduced from [YAN-02]) $\ldots \ldots \ldots \ldots \ldots \ldots \ldots \ldots \ldots \ldots$

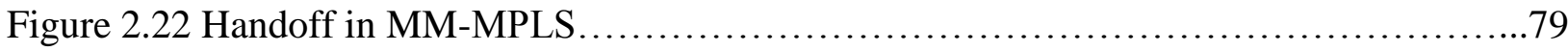

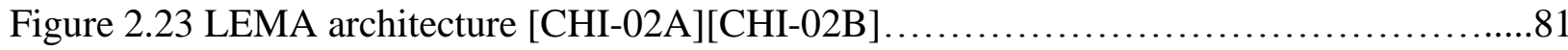

Figure 2.24 Handoff in LEMA [CHI-02A][CHI-02B] .................................. 82 
Figure 2.25 MB-MMS architecture (reproduced from [XIE-03]) ....................... 84

Figure 2.26 Handoff in presence of PS in MB-MMS .................................. 85

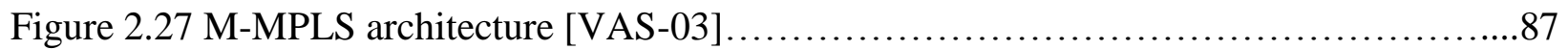

Figure 2.28 Message exchange for LSP set up in M-MPLS overlay model [VAS-03]...........88

Figure 2.29 Message exchange for registration in W-MPLS (reproduced from [SET-04]).......90

Figure 2.30 Message exchange during handoff in W-MPLS ............................91

Figure 2.31 Architecture of V-LSP MPLS scheme (reproduced from [DON-05])..............93

Figure 2.32 Message exchange during (A) V-LSP set up (B) handoff in V-LSP MPLS scheme (DON-05)

Figure 2.33 Message exchange during handoff in FH-MMMPLS showing pre-registration and

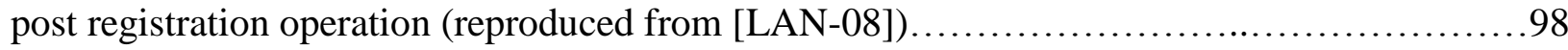

Figure 2.34 FC-MMMPLS architecture (reproduced from [LAN-08])...................... 100

Figure 2.35 Message exchange during handoff in FC-MMMPLS........................101

Figure 2.36 Architecture of Mobile Traffic Engineering with QoS aware MPLS scheme (reproduced from [GAN-08]). 103

Figure 2.37 Intra MFA handoff in Mobile Traffic Engineering with QoS aware MPLS scheme

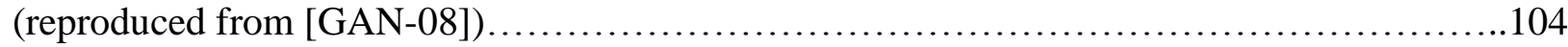

Figure 3.1 Outdoor Propagation Characteristics of Optical Signals........................116

Figure 3.2 Optical transmitter and receiver. Receiver has been reproduced from [KAH-97]....118

Figure 3.3 Directed LOS link (A) and Non-directed LOS link (B)......................121

Figure 3.4 Propagation Characteristics of outdoor mmWave Channel.....................125

Figure 3.5 Comparison of mmWave and LTE/4G FSPL............................. 127

Figure 3.6 Attenuation Due to Foliage at mmWave Frequencies........................ 129

Figure 3.7 Attenuation due to fog according to Kim and Kruse model at $\lambda=630 \mathrm{n} \ldots \ldots \ldots \ldots 134$

Figure 3.8 Attenuation due to dense, moderate and light fog at $75 \mathrm{GHz}$ and $300 \mathrm{GHz} \ldots \ldots \ldots 138$

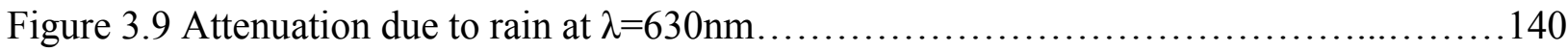

Figure 3.10 Attenuation Due to Rain at $60 \mathrm{GHz}$ and $150 \mathrm{GHz} \ldots \ldots \ldots \ldots \ldots \ldots \ldots \ldots \ldots \ldots \ldots \ldots$

Figure 3.11 Attenuation Due to Rain at mmWave Frequencies........................... 142

Figure 3.12 Attenuation due to Dry Snow for light, moderate and Heavy Snowfall at

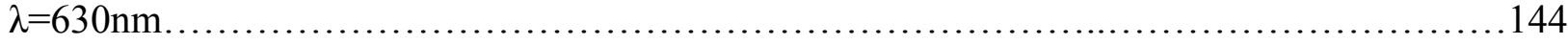


Figure 3.13 Attenuation due to Wet Snow for light, moderate and heavy snowfall at $\lambda=630 \mathrm{~nm}$. 145

Figure 3.14 Attenuation due to light, moderate and heavy snow at mmWave wavelengths....147

Figure 3.15 Attenuation due to light, moderate and heavy snow at $60 \mathrm{GHz}$ and $300 \mathrm{GHz} . . . .147$

Figure 3.16 Comparison between mmWave $(75 \& 300 \mathrm{GHz})$ and VL (Kim/Kruse model) for dense fog conditions. 148

Figure 3.17 Comparison between mmWave $(75 \& 300 \mathrm{GHz})$ and VL (Kim/Kruse model) for

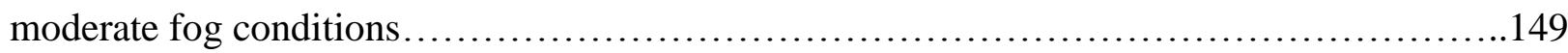

Figure 3.18 Comparison between mmWave $(60 \& 150 \mathrm{GHz})$ and VL for heavy rain..........150 Figure 3.19 Comparison between mmWave $(60 \& 150 \mathrm{GHz})$ and VL for moderate rain.......150 Figure 3.20 Comparison between mmWave $(60 \& 300 \mathrm{GHz})$ and VL (wet/dry) at heavy snowfall

Figure 3.21 Comparison between mmWave $(60 \& 300 \mathrm{GHz})$ and VL (wet/dry) at moderate snowfall

Figure 3.22 Transmitter and receiver configuration mapped to traffic light and vehicle.....

Figure 3.23 Transmitter power vs distance for low rate links 161

Figure 3.24 Data rate for high rate links vs power ratio 163

Figure 3.25 Distance vs transmitted power for various fog intensities. 165

Figure 3.26 Distance vs transmitted power for various rain intensities 165

Figure 3.27 Distance vs transmitted power for various intensities of wet snow.... 166

Figure 3.28 Distance vs transmitted power for various intensities of dry snow................166

Figure 4.1 Architecture of the Micro Mobile Hierarchical MPLS Access Network.............175

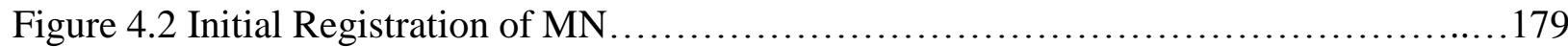

Figure 4.3 Timing Sequence for Intra MCL forwarding chain extension.

Figure 4.4 Timing Sequence for Inter MCL handoff after the forwarding chain threshold is reached....

Figure 4.5 Timing Sequence for Inter CL handoff after the forwarding chain threshold is reached.... 183 Figure 4.6 Analytical vs simulated results for registration cost of MN at different velocities...187 Figure 4.7 Comparison between registration cost of $\mathrm{MN}$ using different schemes in suburban areas. 
Figure 4.8 Comparison between registration cost of $\mathrm{MN}$ using different schemes in urban areas....

Figure 4.9 Comparison between registration cost of MN using different schemes on

highways 190

Figure 4.10 MN moving between two non-overlapping subnets.

Figure 4.11 Comparison between handoff latencies of different schemes.

193

Figure 4.12 Analytical vs simulative comparison between the frequency of handoff messages sent to $\mathrm{GW}$ in proposed architecture.

Figure 4.13 Comparison between frequency of handoff messages sent to GW using different schemes in suburban areas.

Figure 4.14 Comparison between frequency of handoff messages sent to GW using different schemes in urban areas.

Figure 4.15 Comparison between frequency of handoff messages sent to GW using different

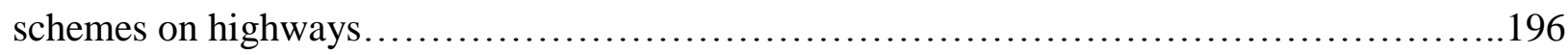

Figure 4.16 Total packet loss vs subnet resident time for various schemes....

Figure 5.1 Haskin's MPLS fast reroute scheme (A) Reverse LSP is created along protected LSP and back up LSP (B) Link failure is detected, traffic is reversed from aware-LSR (C) Traffic is directed on the back up LSP. .204

Figure 5.2 Makam's MPLS fast reroute scheme (A) Backup LSP is created along protected LSP (B) Link failure is detected, FIS message is generated by aware-LSR (C) Traffic is directed on the back up LSP. .206

Figure 5.3 Timing diagram for RFR scheme. .208

Figure 5.4 Simplified version of the proposed hierarchical MPLS based micro-mobility architecture.

Figure 5.5 State machine diagram of intermediate LSRs on the protected path. 213

Figure 5.6 State machine diagram of intermediate LSRs on the protected path. .213

Figure 5.7 Message timing diagram describing the proposed scheme 216

Figure 5.8 Protected LSP and backup LSP originating from CL1 .217

Figure 5.9 LFIB at CL1 (A) Before Fault Detection (B) After fault is detected and buffer is activated (C) Buffer is being emptied (D) Incoming packets are directly sent to back up LSP (E) Protected LSP is restored. 219 
Figure 5.10 Timing sequence at CL1 during fault detection and LSP redirection..............220 Figure 5.11 Total restoration delay compared to the number of LSRs in the protected path for the

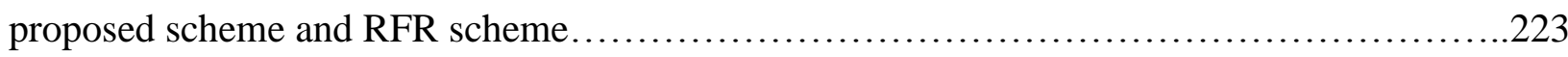
Figure 5.12 Buffer size required at PSL compared to the number of LSRs in the protected

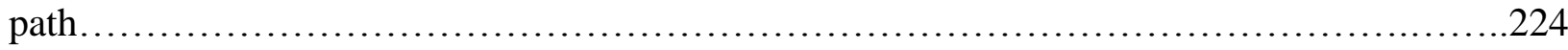




\section{LIST OF TABLES}

Table 2.1 Technical Requirements for Vehicular Applications...............................29

Table 2.2 METIS KPI's for Vehicular Communication [MET-14]..........................29

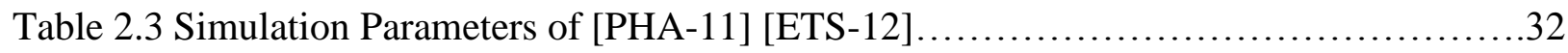

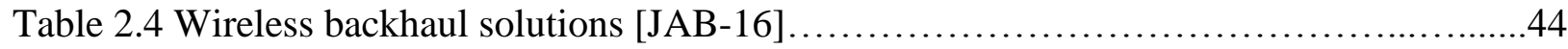

Table 2.5 Comparison between mmWave and VLC Communication.........................45

Table 2.6 Comparison between MPLS-based micro-mobility schemes......................105

Table 3.1 Channel models for VVLC systems presented in open literature...................115

Table 3.2 lrradiance Levels for Several Light Sources [KAR-88]........................ 120

Table 3.3 Classification of channel models for mmWave outdoor systems [HUA-19].........123

Table 3.4 Visibility Range Corresponding to Weather Conditions.............................133

Table 3.5 Specific Attenuation according to Kim and Kruse model $(\lambda=450 \mathrm{~nm}, 530 \mathrm{~nm} \&$

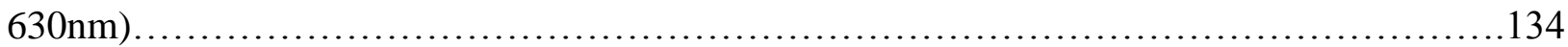

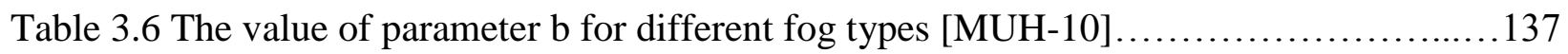

Table 3.7 Attenuation due to fog at mmWave Frequencies.............................. 137

Table 3.8 Specific Attenuation due to Rain at Visible Light Spectrum.......................139

Table 3.9 SpecificAttenuation due to Rain at mmWave Spectrum.........................141

Table 3.10 Values of parameter a and $b$ for wet and dry snow [ITU-07]....................143

Table 3.11 Visibility corresponding to snowfall rate according to WMO....................143

Table 3.12 Specific Attenuation due to snow (dB/km) for visible light spectrum..............144

Table 3.13 Specific Attenuation due to snow $(\mathrm{dB} / \mathrm{km})$ for $\mathrm{mmWave}$ frequencies..............146

Table 3.14 Values of $\mathrm{V}(\lambda)$ for various wavelengths as specified by CIE [ROB-09]...........155

Table 3.15 Parameters related to the dual link design analysis...........................159

Table 3.16 Values of various parameters related to the dual link design analysis...............163

Table 3.17 Intensities of $200 \mathrm{~mm}$ and $300 \mathrm{~mm}$ modules near the center of the lane

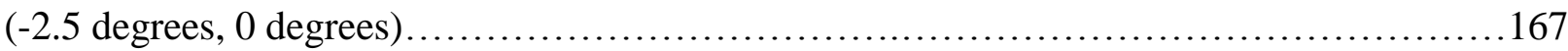

Table 3.18 Transmitted Power specifications for traffic light according to [TTA-12]..........168

Table 3.19 Link Budget Analysis for mmWave outdoor system........................170

Table 3.20: Comparative summary of mmWave and VLC for $5 \mathrm{G}$ outdoor system under proposed

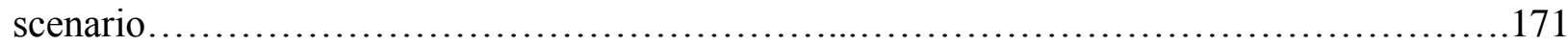

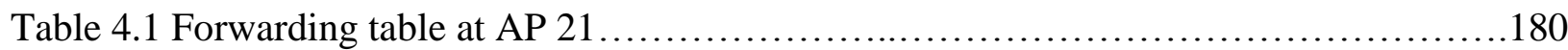


Table 4.2 Parameters used in the performance analysis of MPLS hierarchical architecture......185

Table 4.3 Values of the parameters used in the performance analysis of MPLS hierarchical

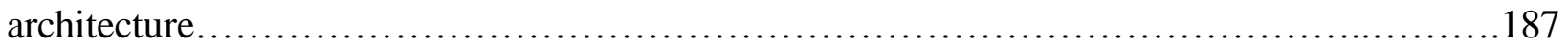

Table 5.1 Values of the parameters used for analysis..................................223 


\section{LIST OF ACRONYMS}

2D

3D

3G

$4 \mathrm{G}$

$5 \mathrm{G}$

ABS

$\mathrm{AC}$

ADAS

ADC

$\mathrm{AF}$

A-LER

AMSU

AP

AR

ARP

AV

BBU

$\mathrm{BE}$

BER

BS

BU

CAM

CAP

CBQ

CCA

$\mathrm{CCH}$

CFP

CL

$\mathrm{CN}$

COA
2 Dimension

3 Dimension

$3^{\text {rd }}$ Generation of Communication

$4^{\text {th }}$ Generation of Communication

$5^{\text {th }}$ Generation of Communication

Automated Braking System

Access Categories

Advance Driver Assistance System

Analog to Digital Converters

Assured Forwarding

Access Label Edge Router

Advanced Microwave Sounding Unit

Access Point

Access Router

Address Resolution Protocol

Autonomous Vehicle

Baseband Unit

Best Effort

Bit Error Rate

Base Stations

Binding Update

Cooperative Awareness Messages

Contention Access Period

Class Based Queuing

Clear Channel Assessment

Control Channel

Contention Free Period

Cell Leader

Corresponding Node

Care of Address 


\begin{tabular}{|c|c|}
\hline CoMP & Coordinated Multipoint Transmission Scheme \\
\hline $\operatorname{CoS}$ & Class of Service \\
\hline C-Plane & Control Plane \\
\hline CRAN & Cloud Radio Access Networks \\
\hline CR-LDP & Constraint based Routing Label Distribution Protocol \\
\hline CSI & Channel State Information \\
\hline CSMA/CA & Carrier Sense Multiple Access/Collision Avoidance \\
\hline $\mathrm{CW}$ & Contention Window \\
\hline CWDM/DWDM & Coarse/Dense Wavelength Division Multiplexing \\
\hline D2D & Device to Device \\
\hline DAPRA & Defense Advance Research Project \\
\hline DC & Direct Current \\
\hline DENM & Decentralized Environmental Notification Messages \\
\hline DHCP & Dynamic Host Configuration Protocol \\
\hline DiffServ & Differentiated Services \\
\hline D-Light & Data-Light \\
\hline DOT & Department of Transportation \\
\hline DSCP & Differentiated Services Code Point \\
\hline DSRC & Dedicated Short Range Communication \\
\hline DS-TE & Differentiated Services aware Traffic Engineering \\
\hline $\mathrm{ECN}$ & Explicit Congestion Notification \\
\hline EDCA & Enhanced Distributed Channel Access \\
\hline $\mathrm{EF}$ & Expedited Forwarding \\
\hline EGW & Edge Gateway Router \\
\hline EIRP & Equivalent Isotropically Radiated Power \\
\hline ERO & Explicit Route Object \\
\hline EXP & Experimental \\
\hline FA & Foreign Agent \\
\hline FC & Forwarding Chain \\
\hline FCC & Federal Communication Commission \\
\hline FD & Foreign Domain \\
\hline
\end{tabular}


FDA

FEC

FF

FIS

FOV

FRS

FSO

FSPL

GBDM

GFA

GPS

GTS

GW

HA

HAWAII

HetNets

HFA

HFNO

HMIPV6

IETF

IFA

IM/DD

IntServ

IP

IR

IrDA

ISI

ISP

ITE

ITS

IVC
Foreign Domain Agent

Forwarding Equivalence Class

Fixed Filter

Failure Indication Signal

Field Of View

Failure Recovery Signal

Free Space Optical

Free Space Path Loss

Geometry Based Deterministic Models

Gateway Foreign Agent

Global Positioning System

Guaranteed Time Slots

Gateway Router

Home Agent

Handoff-Aware Wireless Access Internet Infrastructure Heterogeneous Networks

Hierarchical Foreign Agent

Handoff Notification

Hierarchical Mobile IPv6

International Engineering Task Force

Intermediate Foreign Agent

Intensity Modulated/Direct Detection

Integrated Services

Internet Protocol

Infrared

Infrared Data Association

Inter Symbol Interference

Internet Service Provider

Institute Of Transportation Engineers

Intelligent Transportation System

Inter Vehicle Communication 
KPI

LCoA

LDC

LDP

LED

LEMA

LER

LERG

LFA

LFIB

LIDAR

$\mathrm{LiFi}$

LOS

LSC

LSP

LSR

LTE

MaaS

MAC

MAP

MCL

MEC

MFA

MFA

MFC

MIH

MIMO

MIP

MIPV4-RR

mmWave

$\mathrm{MN}$
Key Performance Indicator

Local Care of Address

Local Data Center

Label Distribution Protocol

Light Emitting Diode

Label Edge Mobility Agent

Label Edge Router

Label Edge Router Gateway

Lowest Foreign Agent

Label Forwarding Information Base

Light Detection and Ranging System

Light Fidelity

Line Of Sight

Local Service Center

Label Switching Path

Label Switching Router

Long Term Evolution

Mobility as a Service

Medium Access Control

Mobility Access Point

Micro-Cell Leader

Mobile Edge Computing

Master Forwarding Agent

Mobile Forwarding Agent

Master Forwarding Chain

Media Independent Handover

Multiple Input Multiple Output

Mobile IP

Mobile IPv4 Regional Registration

Millimeter Wave

Mobile Node 


\begin{tabular}{ll} 
MNDB & Mobile Node Data Base \\
MPLS & Multi-Protocol Label Switching \\
MPLS-TE & MPLS based Traffic Engineering \\
MR & Mobile Relay \\
MSF & Multiple Stream Forwarding \\
NLMP & Neighborhood Label Mapping Table \\
NLOS & Non Line Of Sight \\
OBU & On-Board Unit \\
OEM & Original Equipment Manufacturers \\
OF & Open Flow \\
OOK & On-Off Keying \\
OPNET & Optimized Network Engineering Tool \\
OSPF & Open Shortest Path First \\
PD & Photo diodes \\
PHB & Per Hop Behaviour \\
PLC & Power Line Communication \\
PLR & Packet Loss Rate \\
PS & Paging Server \\
PSL & Path Switching LSR \\
QoS & Quality of Service \\
RAR & Radio Access Routers \\
RAT & Radio Access Technology \\
RCoA & Regional Care of Address \\
RED & Random Early Detection \\
RF & Radio Frequency \\
RFA & Regional Foreign Agent \\
RIO & RED with In and Out \\
RONJA & Reasonable Optical Near Joint Access \\
RRO & Record Route Object \\
RRS & Round Robin Scheduler \\
RRU & Remodiohead Unit \\
\hline
\end{tabular}




\begin{tabular}{ll} 
RS-GBSM & Regular Shaped Geometry Based Stochastic Model \\
RSU & Road Side Unit \\
RSVP & Resource Reservation Protocol \\
RSVP-TE & RSVP-Traffic Engineering \\
RVR & Runway Visual Range \\
SCH & Service Channel \\
SDN & Software Defined Networking \\
SE & Shared Explicit Style \\
SID & Segment Identifier \\
SLA & Service Level Agreement \\
SNR & Signal to Noise Ratio \\
SSF & Single Stream Forwarding \\
SR & Segment Routing \\
TaaS & Transportation as a Service \\
TDD & Time Duplex Division \\
TE-LSP & Traffic Engineered Label Switching Path \\
ToS & Type of Service \\
TTL & Time To Live \\
U-Plane & User Plane \\
UWB & Ultra Wideband \\
V2I & Vehicle-to-Infrastructure \\
V2V & Vehicle-to-Vehicle \\
V2X & Vehicle-to-Everything \\
VANET & Vehicular Ad hoc Network \\
VL & Visible Light \\
VLC & Visible Light Communication \\
VLCC & Visible Light Communication Consortium \\
VLED & Visible Light Emitting Diode \\
V-LSP & Virtual Label Switching Path \\
VNI & Visual Network Index \\
VPN & Virtuate Networks \\
\hline
\end{tabular}


VRU

VVLC

WAVE

WF

WHO

WiFi

WiMax

WMO

WRED
Vulnerable Road User

Vehicular Visible Light Communication

Wireless Access in Vehicular Environment

Wildcard Filter Style

World Health Organization

Wireless Fidelity

Worldwide Interoperability for Microwave Access

World Metrological Organization

Weighted Random Early Detection 


\section{Chapter 1: INTRODUCTION}

\subsection{Overview}

Casualties due to traffic accidents have reached an alarming rate worldwide. According to the recent report published by World Health Organization (WHO), around 1.35 million people involved in road accidents lose their lives every year [WHO-18]. It is the number one cause of death among those aged 15-29 years. Intelligent Transportation System (ITS) was established in 1993 [ISO-17] with the vision of enhancing transportation safety for masses. ITS supports wide range of applications for collection, processing and sharing of information in order to ensure safety, ease congestion and improve traffic management while ensuring that the environmental impacts of these applications are minimized. To prevent the needless loss of lives, the vehicles are increasingly integrated with safety applications utilizing expensive sensors, radars, cameras and other state-of-the-art technologies that would enhance public safety and convenience.

With the progression of wireless technology in recent years, the focus of academia, industry and government shifted towards more intense use of wireless communication in the vehicular environment as, along with safety applications [VEG-13] [HAR-10], they can also support traffic management applications [ABB-11] and infotainment vehicular applications [HUE-12] which

enrich travel experience. Hence, wireless vehicular communication technology is developed with the goals to: 1) ensure traveller's safety, 2) be suitable for fast and easy deployment, 3) be of low cost, 4) provide large bandwidth capacity, 5) support reliable traffic management and comfort applications with required level of QoS and 6) support both vehicle-to-infrastructure (V2I) and vehicle-to-vehicle (V2V) communication. However, most existing wireless solutions only meet part of the desired goals.

The DSRC/WAVE [LI-12] [MOR-10] family of standards, developed and adopted under the auspices of the US Department of Transportation (DOT) emerged as the most comprehensive set of developed standards to support physical and link layer in vehicular ad hoc networks (VANET). The adopted standard for local network access is the IEEE 802.11p [STD-10], operating within the $5.85 \mathrm{GHZ}$ range. Each DSRC channel is $10 \mathrm{MHz}$ wide and supports 
transmission speeds between 6-27 Mbps. Unfortunately, DSRC applications was not able to penetrate market as expected primarily due to the high implementation cost associated with the hardware such as roadside antennas and on-board units (OBUs) as well as lack of applications that attracts consumer adoption of DSRC technology. In 2013, The Federal Communication Commission (FCC) put forward a proposal allowing unlicensed technologies to share the 5.9 GHz ITS band due to its vacuity with the release of NRPM (13-22) (ET-DOCKET 13-49). This step was expected to relieve the heavily congested RF spectrum at lower frequencies by $35 \%$ [FCC-13]. However, it may be the cause of severe implications, e.g. increase in interference, delay, packet drop rate, etc., especially after the decision of US Department of Transportation (DOT) in February 2014 [DOT-14] to proceed with the installation of V2V communication technology in newly manufactured cars and trucks. Initially it was expected that it will take more than two years for functional implementation of DSRC applications on the road with minimum market penetration rate of $10 \%$ even if all the new vehicles are equipped with DSRC transceivers [YU-13], yet after six years DSRC supported vehicular network fails to exist. Hence, earlier consumers opting for DSRC equipped vehicles would not have gained any functional benefits regardless of the hefty cost they paid.

Naturally, cellular and wireless mesh broadband networks (e.g. 3G [GOO-05], 4G LTE [LTE14], 4G/WiMax [WiM-10]) are also in the market of offering services to the vehicular user. The broadband nature of these networks enables delivery of broadband content to the vehicular population. However, these cellular solutions come with their own set of challenges. In the context of vehicular communication, 3G brought larger coverage area and instant access to data transfer but at the expense of higher cost. To combat the high cost, several architectures proposed to integrate $\mathrm{WiFi}$ with 3G [BAL-10] [HOU-11]. Another approach was to use complex algorithms for route optimization in the data download process but at the expense of increasing delay [CHE-13]. A Cluster-based solution [BEN-11] is an example of centralized approach to achieve routing improvement in $3 \mathrm{G}$ supported vehicular communication networks.

There are some serious reservations on the use of 4G LTE in high speed mobile environment since it relies on an expensive beamforming architecture for communication that requires perfect alignment between transmitter and receiver; a hard task to achieve with antennas on fast moving 
vehicles. Both WiMAX and LTE also suffers from inter symbol interference (ISI), It becomes more destructive at cell edge area [RAH-10][JIN-10][MAO-08] hence reducing the edge rate. To resolve these issues, researchers have proposed the use of coordinated multipoint transmission scheme (CoMP) for LTE, where multiple base stations transmit in a coordinated manner to improve the edge rate [GHO-10]. Although, CoMP rectify the edge rate issue to some extent, it does not improve the spectral efficiency. Moreover, majority of the solutions presented to improve edge rate throughput studied fixed or nomadic nodes scenarios [RAH-10][JIN10][MAO-08][MIT-08]. High speed vehicular environment adds further challenges such as scalability [MOR-17] and multipath issues [AHM-08] [LEU-05].

Academia and industry started concentrating on some alternative solutions, autonomous vehicles being one of them. Autonomous vehicles do not rely on inter vehicle communication (IVC) but utilize extensive number of sensors and actuators to navigate the road safely. The modern automotive era was developed as a result of various research funding programs supported by Defense Advance Research Project (DARPA), research agency of USA military [AUT-18]. In the private sector, GOOGLE was the one to initiate a self-driving car research project in 2009 followed closely by Uber, Tesla and several start-ups. Prototype self-driving cars appeared on urban roads in 2012. The technology has since improved considerably but is still far from mass deployment. A fully autonomous vehicle must fulfil the 3Ps: Perception (figuring out the driving environment), Prediction (what can/will happen) and Policy (taking the appropriate action). An autonomous vehicle utilizes a combination of sensors, cameras, radars and light detection and ranging system (LIDAR) - a radar like technique that uses pulses of invisible light to construct a 3D map of the driving environment. Significant hurdle in the adoption of autonomous vehicles is the cost due to use of massive number of expensive sensors [DRE-14] and LIDAR systems that currently cost tens of thousands of dollars. Apart from the cost, for public acceptance and adoption, the technology must also overcome two other major issues: judgement in case of ethical conflict and safety from cyber- attacks.

It can be extracted from the discussion that implementing an efficient vehicular network is not possible by utilizing any single technological solution. Currently research towards heterogeneous vehicular networking is gaining momentum. The onus of heterogeneous vehicular networking is 
not to concentrate on a single technology for vehicular communication but rather utilize multiple available technologies. Heterogeneous vehicular networks may be formed by either enabling vehicles to select and connect with the best available technology or by assigning vehicular applications to specific technologies.

This makes vehicular networks an inherent candidate for 5G. The emerging era of $5 \mathrm{G}$ is going to change the communication paradigm in many ways. $5 \mathrm{G}$ will be highly integrative and will be supporting various technologies so as to produce seamless user experience. It will provide 1000x

\section{G Automotive Vision}

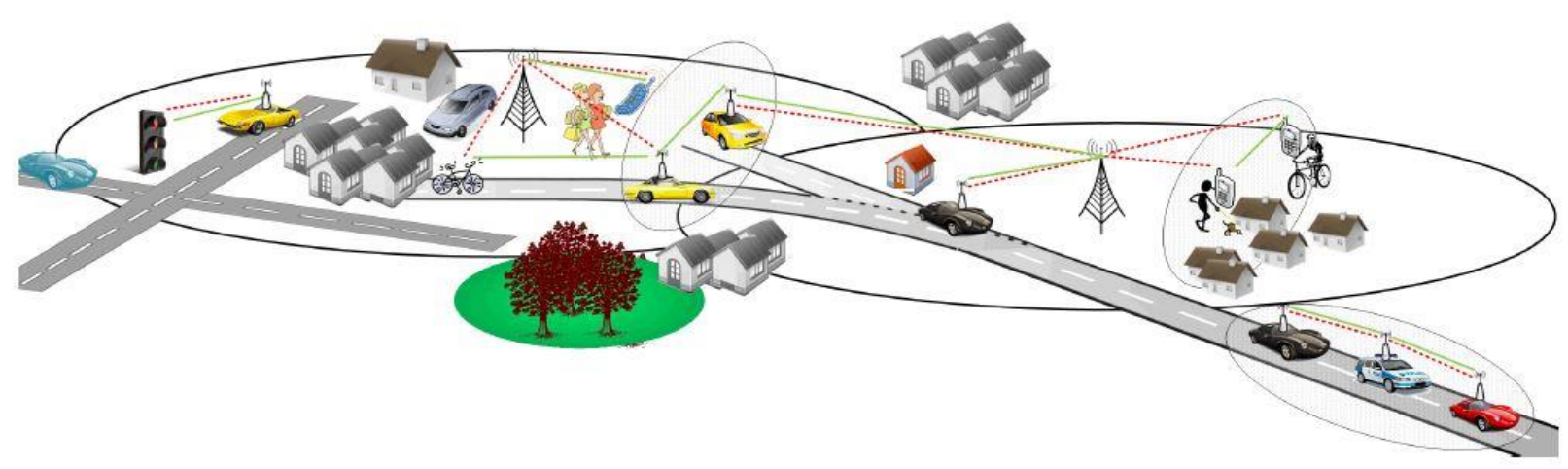

Figure 1.1: $5 G$ Automotive Vision supporting heterogeneous technologies for vehicle-to-everything (V2X) communication [5GP-15].

higher throughput than $4 \mathrm{G}$ with smaller latency (in some cases up to $1 \mathrm{~ms}$ ), and low energy consumption at a reasonable cost. Figure 1.1 reflects the perceived vehicular communication paradigm with the advent of 5G [5GP-15].

\subsection{Motivation}

Visible light communication (VLC) is a perfect candidate for $5 \mathrm{G}$ based heterogeneous vehicular network service. Not only it can be implemented as a supporting technology that could provide reliable high speed data transfer without interference to the existing technologies, but it could also provide communications in those areas where RF has limitations. VLC systems employ LEDs transmitting in the visible light spectrum as transmitters and photodiodes as receivers. Due 
to the energy and cost efficiency of LEDs over traditional lighting methods such as fluorescent and incandescent lamps, there has been a remarkable increase in the number of lighting fixtures using LEDs transmitting at visual spectrum. Use of VLC in vehicular communications is particularly easy, as most of the traffic lights, lamp posts and vehicle headlights and taillights are already equipped with Visible LEDs (VLEDs). Use of VLC in vehicular communication applications provides the following advantages:

Ease of deployment as LED based lighting infrastructure in public spaces, included roads already exists.

No dependency on GPS for vehicle tracking or synchronization ${ }^{1}$

Operates in licence free and unregulated spectrum

Cost efficient

Interference free

Does not pose health hazard.

It can achieve much higher data rate than RF, hence enabling it to support easily broadband applications.

Light does not penetrate through walls hence the network is more secure.

These advantages are in-line with the main drivers of 5G. e.g. cost-efficiency and increased capacity. To increase capacity in $5 \mathrm{G}$, one approach under consideration is to increase the densification of cells by reducing the cell size, hence increasing spectrum reuse. This quality is inherent in VLC systems where the cell size is limited by the lighting range. Ability to co-exist with other technologies without generating any interference to them is also in agreement with the targets of future highly integrative $5 \mathrm{G}$ networks.

It is important that the user gets seamless connectivity while roaming between different cells. Cell densification in $5 \mathrm{G}$ will arise many handoff challenges especially since $5 \mathrm{G}$ is expected to

\footnotetext{
1 "GPS satellite includes atomic clock that are synchronized to coordinated universal time (UTC). So for time synchronization, the GPS signal is received and processed by a local master clock and passed on to other devices, systems, or networks so their internal clocks are synchronized to UTC. It is the process of synchronization to GPS that can provide atomic clock accuracy without the need for a local atomic clock. GPS clock synchronization eliminates the need for manual clock setting to establish traceability to UTC standards so various events can be correlated even when they are time-stamped by different clocks" [SPE18]. Synchronization between vehicles is essential to support communication protocols for safety and non-safety applications. Vehicle tracking based on periodic signals send to GPS satellite enables vehicle recovery in case of vehicle theft.
} 
support large number of diverse sets of devices. Therefore, it is significant that investigation should be directed towards the establishment of a reliable quality-of-service (QoS) enabled architecture capable of supporting undisrupted connectivity of active connections, and do so regardless of their roaming velocity and frequency. This becomes very challenging in high mobility cases, where optimized solutions are required to minimize hand-off latency, reduce signaling overhead and maintain QoS.

Although several protocols that support micro-mobility in IP networks such as Mobile IPv4 regional registration (MIP-RR) [GUS-04], Hierarchical Mobile IPv6 (HMIPv6) [SOL-05], Cellular IP [VAL-99], and Handoff-Aware Wireless Access Internet Infrastructure (HAWAII) [RAM-02] exist, they are unsuitable for highly mobile vehicular environments as these architecture experience extensive handoff delay and lack scalability to support increasing number of data flows with increasing vehicular traffic. Moreover these schemes have limited capability of providing QoS support.

During the last two decades, with the increase in demand for scalable and low latency IP networks, the research interest shifted towards fusing the Multiprotocol Label Switching (MPLS) technology [IET-02] with IP mobility management protocols. Several MPLS based micro mobility schemes [CHI-02A], [CHI-02B], [GRI-01], [KIM-01], [REN-01], [SET-04], [URN-01], [VAS-03], [XIE-02], [XIE-03], [YAN-01], [YAN-02] have emerged with the intent of providing low latency handoff and rapid packet forwarding.

MPLS is a scalable and efficient layer 2.5 technology. In MPLS, each packet is assigned a label. The intermediate routers in the forwarding path have the capability to read, swap and forward the packet based on the label through pre-established label switching paths (LSPs). Packet forwarding through LSPs requires much less overhead than through conventional IP routing and reduces the packet forwarding time considerably. Additionally, MPLS offers QoS support by establishing QoS supporting traffic engineered LSPs (TE-LSPs). Guarantees on bandwidth provision and end-to-end delay can be ascertained by incorporating Integrated Services (IntServ) with MPLS. Alternatively, "soft” QoS support based on prioritization, e.g. as done with Differential Services (DiffServ) [BLA-98]. QoS support could be provided on the basis of 
individual flows or flow aggregates. Most importantly, the fast LSP redirection capability of MPLS can accelerate the handoff process considerably. Therefore, MPLS-based micro-mobility schemes are highly suitable for the intermittent mobile vehicular environment, where constantly changing network topology requires an efficient packet forwarding mechanism for ensuring continuous connectivity. However, existing MPLS based micro-mobility schemes are not suitable for vehicular networks as they fail to provide scalability and QoS support as explored in detail in Chapter 2 of this thesis.

Therefore, one of the focus areas of this thesis is to design, study and evaluate an MPLS based micro-mobility scheme capable of supporting effectively highly mobile vehicular users, be scalable in terms of the number of supported devices and number of data flows, provide seamless connectivity and support the QoS levels required by safety and comfort applications.

\subsection{Objectives}

The objective of this research work is to present a MPLS based architecture for seamless vehicular communication using free-space optical technology for $5 \mathrm{G}$ vehicular networks. This vehicular visible light communication network shall be able to transport urgent public safety messages with the required latency, provide quick delivery of travel facilitation information, and also enhance passenger experience by supporting broadband applications and voice communications.

\subsection{Research Contributions}

This thesis establishes the use of free-space optical communications as the main technology to support public safety applications along with provision of low cost broadband communication services in vehicular environment. The aim of the work is to provide designs and integrative architecture for the access and transport network. The contributions of this work are:

- Evaluated the performance of visible light (VL) and mmWave propagating through atmosphere and under different weather conditions such as fog, rain and snow. A detailed comparative design analysis between VL and mmWave small cell vehicular network is carried out as well, taking into consideration weather inflicted attenuation factors. 
- Designed a hierarchical MPLS architecture capable of supporting seamless handoff in vehicular networks, while maintaining QoS provisions to the data streams. Mathematical and simulative analysis of the MPLS registration cost and hand-off latency is carried out as well.

- Designed a fast link restauration scheme for LSP protection in MPLS that preserves the QoS provision to applications under link failures. Mathematical and simulation based analysis of the MPLS path protection scheme in terms of path restoration delay is carried out.

Although, the comparison of performance between VL and mmWave aids in identifying critical design and implementation issues, the main contribution of this work is the proposed hierarchical MPLS based architecture to support micro-mobility in 5G vehicular networks.

\subsection{Thesis Organization}

The rest of the thesis is organized as below:

Chapter 2 comprises of two parts. It begins with a discussion of the key transformations that the automotive industry is headed towards in the approaching era of 5G. This part highlights the link between goals of future vehicular communication networks and $5 \mathrm{G}$ objectives. The first part concludes with a comprehensive overview of proposed technologies for $5 \mathrm{G}$ and their suitability in the vehicular communication environment. The second part comprises of a thorough review of the existing literature utilizing MPLS architectures for seamless connectivity. The chapter concludes with a thorough discussion on the shortcomings of the existing schemes when mapped to a VVLC 5G network.

Chapter 3 concentrates on the performance analysis of VL and mmWave through atmosphere while discussing and reviewing the reported work utilizing these technologies for outdoor mobile communication. The chapter also presents comparative design analysis between outdoor mmWave and VLC small cell 5G systems.

Chapter 4 presents an MPLS based hierarchical micro-mobility schemes for fast handoff in 5G VVLC networks. 
Chapter 5 proposes a fast reroute scheme to be adopted in case of link failures. Analytical and simulative results are presented and discussed.

Chapter 6 provides a detailed conclusion of the work.

References are listed at the end. 


\section{Chapter 2: LITERATURE REVIEW OF 5G VVLC NETWORKS}

\subsection{Key Transformations in Future Vehicular Communication Networks}

Ubiquitous connectivity will be the prime focus of future vehicular networks. To fulfil this objective, future vehicles will use a comprehensive set of wireless technologies to exchange information among themselves (vehicle-to-vehicle (V2V)), the network infrastructure (vehicleto-infrastructure (V2I)), and even the pedestrians (vehicle-to-pedestrian (V2P)). Vehicle-toeverything (V2X) communication is a more comprehensive term that has been widely adopted by academia and industry to elaborate the extent of communication that would be required and carried out by vehicles of this emerging era. Automotive industry is already undergoing transformation, which will become massive transformation in the next few years. Below we are discussing some areas that researchers and industries are focusing and where we witness major innovations occurring with fast pace.

\subsubsection{Autonomous Vehicles}

In principle, autonomous vehicles (AV) are capable of sensing the environment and navigating roads without information exchange with neighbouring vehicles. However, a highly autonomous vehicle would require a plethora of sensors, radars, high end GPS and LIDARs in order to drive safely in a stand-alone configuration. The US Society of Automotive Engineers (SAE) and the German Association of Automotive Industry (VDA) have defined six levels of automation, ranging from zero automation (requiring full human control) to full automation (no driver involvement). Figure 2.1 shows the six levels of automation as presented in the SAE standard J3016 [SAE-14]. There are definite perks fully automated vehicles can offer, such as stress-free journey for drivers as they no longer need to monitor their surroundings, become "green mobility solution" as these cars will also increase efficiency and congestion by optimizing traffic flow, 
reduce $\mathrm{CO}_{2}$ emission by minimizing braking and reaccelerating while driving [LEO18].Researchers at MIT have developed a slot-based intersection model specifically for autonomous vehicles that would replace the traditional traffic light signal while doubling road capacity and significantly reducing traffic delays for various vehicle arrival rates [AND-17] [TAC-16] . Most importantly, they will increase the mobility of the disabled population. However, these functionalities can be considerably expanded with increased connectivity. V2V or V2I communication can inform drivers about hazardous driving conditions (e.g. presence of black ice), accidents or traffic congestions ahead. As a result, cars can switch lanes or change route beforehand to pass the hazard without driver's intervention. During adverse weather conditions, such as fog, snow, rain etc., when the reliability and accuracy of car sensors could be challenged, V2X communication can be extremely beneficial for safely navigating the car. Currently the onus of research is mostly at levels 3 and 4 as there are still legal, ethical, and social challenges that would have to be resolved before allowing high degree of responsibility on the automation component. Most recent developments in autonomous vehicles are at the test drive stage; Audi A7 was tested in 2015 from Silicon Valley to Las Vegas [ENG-15], Blackberry QNX Lincoln MKZ in 2017 in Kanata, Ottawa [CBC-17] and a recent release of Audi A8 [ENG17] that functions at level 3. Given the advancement and continued research in the field of AV, it is predicted that autonomous vehicle will increase total vehicle travel in the range of 3-9\% by 2035 as empty vehicles will travel to pick up and drop off passengers and non-drivers will also have access to vehicle travel as shared autonomous rides or autonomous taxis [TRO-16][LIT-18]. 


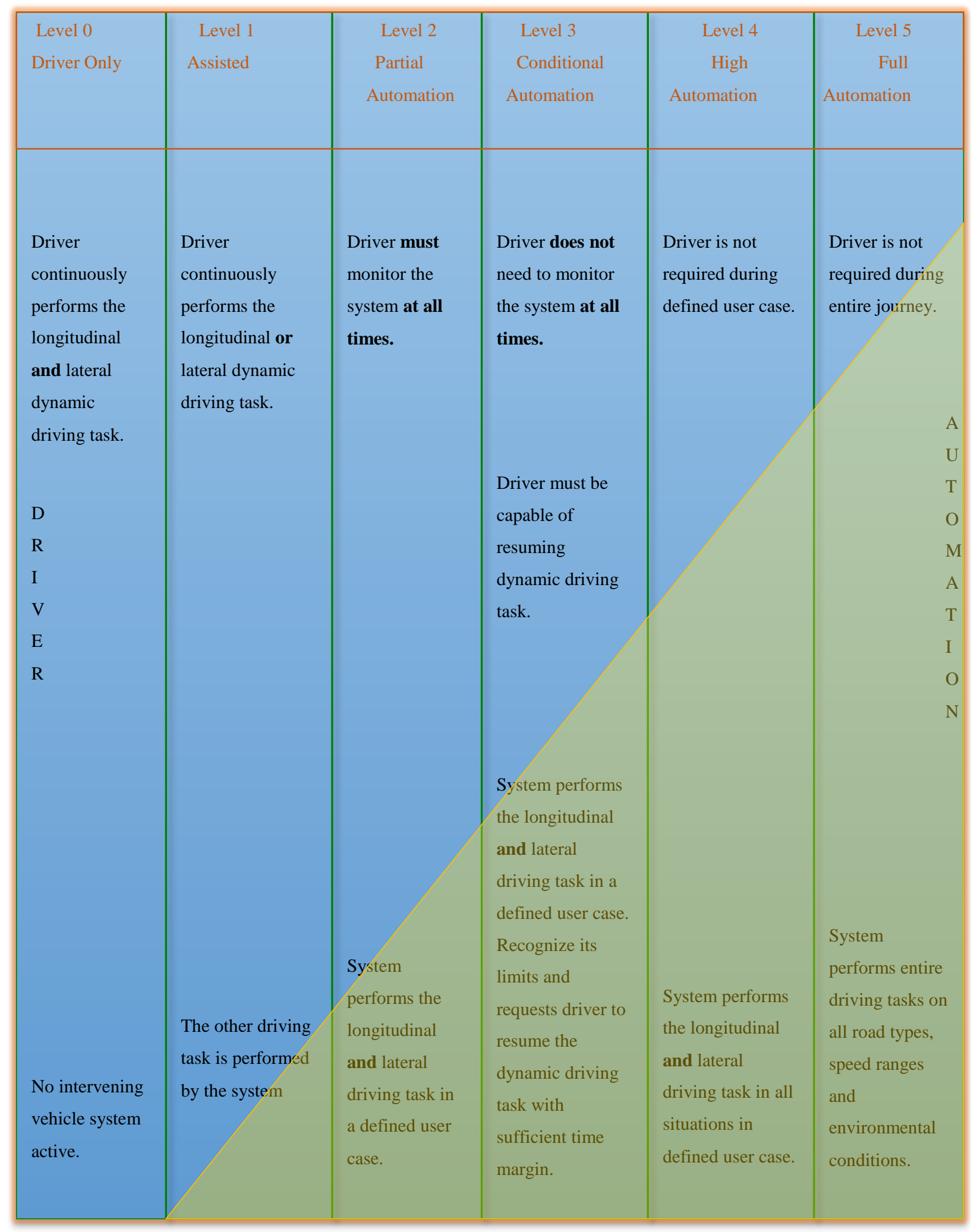

Figure 2.1: Six levels of vehicular automation according to SAE/VDA. [VDA-15] 


\subsubsection{Road Safety and traffic management services}

Increased V2I and V2V communication will improve road safety for drivers owning partially automated vehicles. Over the decades, various safety functions have been increasingly integrated in vehicles to improve personal safety such as cruise control, automated braking system (ABS), and various levels of Advance Driver Assistance System (ADAS) varying from vendor to vendor. These systems generally enhance safety by providing forward collision warning, lane departure warning, automated emergency braking and pedestrian detection. eCALL has been made mandatory in all Europe from April 1 ${ }^{\text {st }}, 2018$ [DIG-15]. eCALL enables a vehicle to automatically send location and information related to collision to emergency responders without the driver's intervention by utilizing available wireless technologies. Hence the vehicles increasingly need to exchange data in order to enhance reliability of the following safety features:

- Collision avoidance warning.

- Post/Pre crash warning.

- Emergency Vehicle Approaching.

- Road hazard warning.

- Electronic emergency brake.

- Stop sign /traffic light violation warning.

- Traffic congestion warning.

In order to increase the road safety for vehicles and pedestrians, support of various stringent latency applications such as bird's eye view, see-through vehicles and vulnerable road user (VRU) notifications by future vehicle networks is of paramount importance. These applications need to collect data from the on-board sensors of the neighboring cars and share it with all of the vehicles in the locality, in a manner that each vehicle gets the full view of impending intersections, obstacles, road blockages or sudden impediment that could directly affect them. These information messages can be periodically exchanged or can be event-driven. An example 
of a see-through application is shown in Figure 2.2 [5GP-15] where the vehicle behind a truck gets the full view of a pedestrian crossing located ahead, in front of the leading truck.

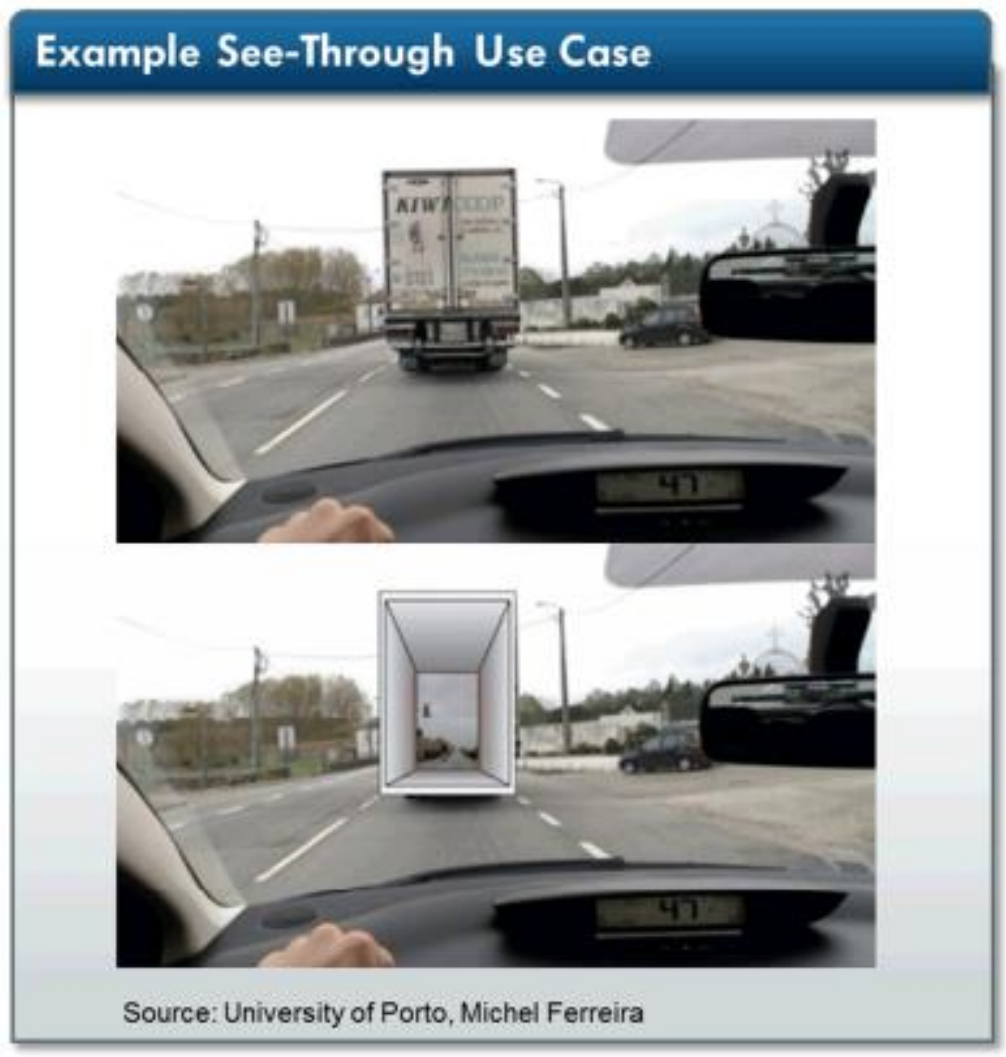

Figure 2.2 Example see-through case for vehicular safety application. [5GP-15]

\subsubsection{Smart Navigation}

Modern navigation systems use GPS and digital maps to provide real-time guidance to the driver and assistance to select the optimum route. Not only the stand-alone navigation systems are utilizing this feature, but newer vehicles are increasingly embedded with on-board navigation systems that also provide "point-of-interest" notifications. These notifications allow drivers easy access to transportation and personal services such as finding a gas station or a restaurant. Some of them can also present the most favourable options by providing quick comparisons e.g. gas prices as advertised by two neighboring gas stations. Needless to say, these navigation services require considerable volume of information to be exchanged between neighboring vehicles and network. With emerging techniques such as mobile edge computing (MEC), extensive 
information can be gathered, stored efficiently, processed, analyzed and disseminated closer to the user that will enhance the navigation services and improve the driver experience.

\subsubsection{Office-on-Wheels}

The concept of office-on-wheels is to utilize the commute time as work time. This concept allows flexible work time. In Europe, fleet of buses were launched as part of the office on wheels project in June 2017 [ANT-17]. This is a joint venture between BAAV (Professional Federation of Bus and Automobile Company, Belgium) and Smart Mobility Expertise Center, Antwerp. These office buses are equipped with fast internet, power outlets and USB connections.

This concept can be further extended to cars, where the interior of the car may be set up with the essentials of performing office work, enabling the drivers to use cars as a second office. Furthermore, partial or full automation will enable the driver to conduct group meetings and carry out individual and group tasks at different locations or while on the way. IDEO [STI-14], based in California, is working on designing automotive solutions with complete overhaul of traditional vehicles to support the key transformation of future work space.

\subsubsection{Transportation As A Service (TaaS)}

TaaS [HIE-16] [ASE-17][GOO-17] shifts the paradigm of mobility from owning a vehicle to consuming transportation as a service. The experimental debut of TaaS or alternatively called mobility as a service (MaaS) was in 2016 at Helsinki, Finland. The concept of TaaS is to offer the consumer different mobility solutions as monthly packages from commercial operators depending on service level agreements (SLA). These packages are tailored according to consumer needs and include all sort of transportation options; from public transportation to rental cars. Needless to say, TaaS requires access to real-time traffic information and a massive database containing information about all sorts of public transportation, including taxis and rental cars. This is possible by extensive information exchange between the centralized controller units, the traffic management system and the public transportation database. Figure 2.3 depicts the framework of TaaS [ITS-16] 


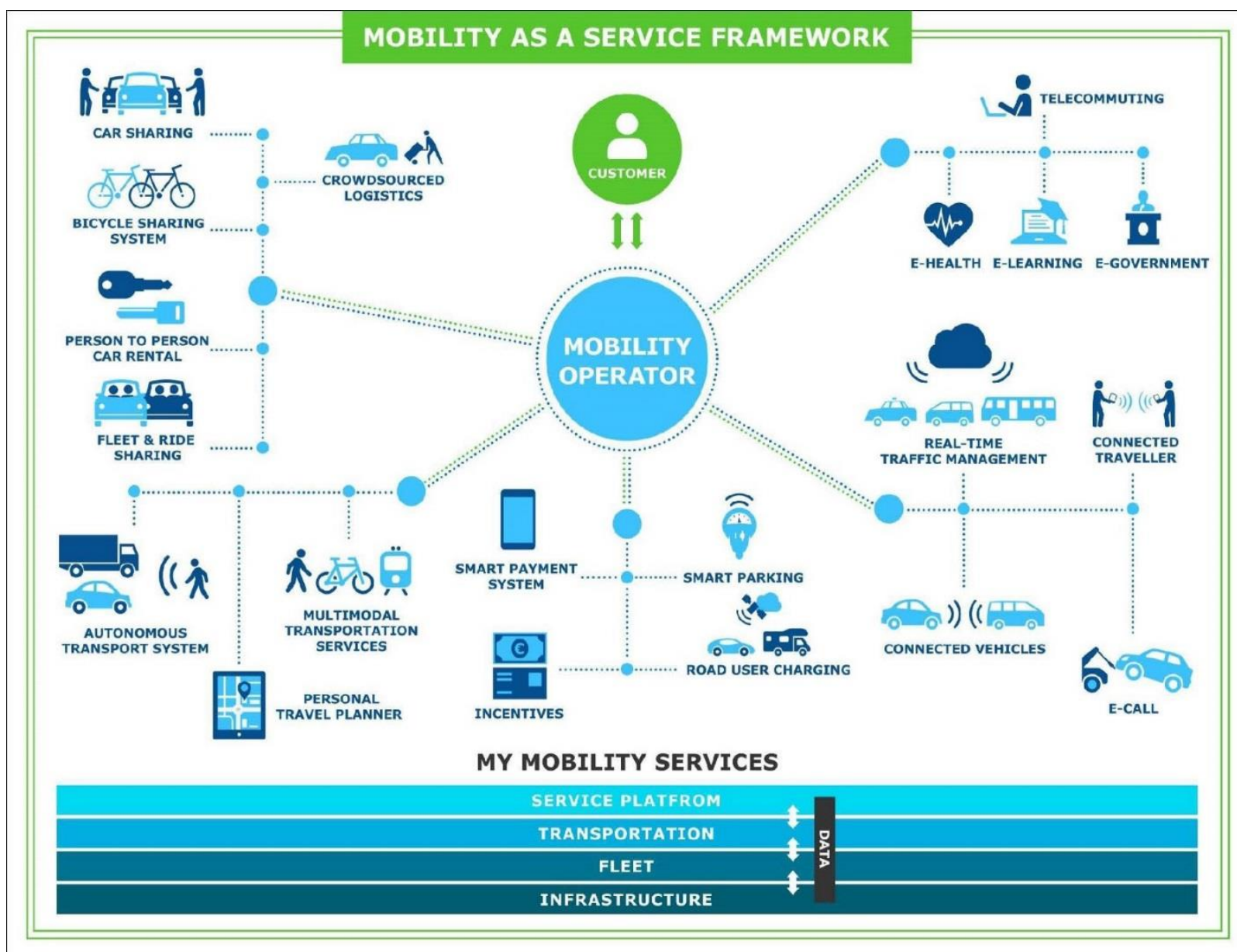

Figure 2.3 ITS TaaS Framework [ITS-16]

\subsubsection{Automatic Maintenance Notifications}

Future vehicles will be able to generate automatic maintenance notifications and disseminate to the drivers based on data collected by on-board sensors. This information is not only useful to the customer to initiate timely maintenance but will also be beneficial to the vendors and Original Equipment Manufacturers (OEMs) who will be able to offer service-based packages to the customers and collect performance statistics of their components and vehicles. Hence, the business model will extend far beyond sales of product, becoming an entire solution package, which will offer extended customer support by offering after sales maintenance and repair.

\subsection{Overview of $5 G$}

The next generation of wireless cellular technology, 5G, should be capable of supporting and serving the communication needs of billions of connected devices with optimized trade-offs 
between speed, latency, bandwidth, cost and energy-efficiency. Although 5G devices will become commercially available by 2020 , it will take several years for maturation of $5 \mathrm{G}$ devices and networks to accommodate the increasing reliance on mobile and internet-enabled devices. $5 \mathrm{G}$ devices are expected to become mainstream by 2030 . The main characteristics of $5 \mathrm{G}$ are presented in figure 2.4 .

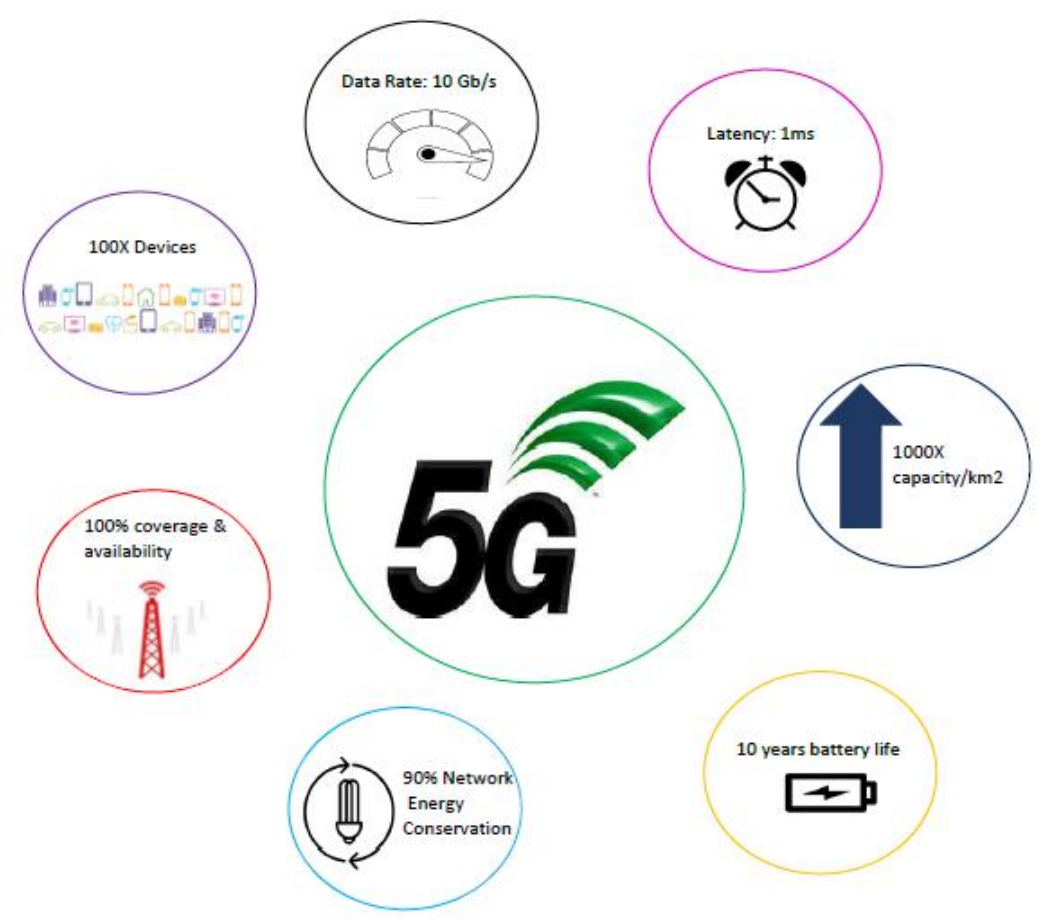

Figure 2.4 Characteristics of $5 G$

\subsubsection{Data Rate}

According to the recent visual network index (VNI) report released by CISCO [VNI-17], IP data traffic handled by wireless networks has increased massively. It is predicted that by 2021 annual global IP traffic will reach 278 exabytes. This is nearly 127 times increase in traffic from 2005. Moreover, it is predicted that traffic from wireless and mobile devices will account for more than $63 \%$ of the total IP traffic by 2021, with smart phones utilizing $33 \%$ of total IP data traffic; a $20 \%$ increase from 2016. The need to support this exponentially increasing data traffic is one of the main drivers behind 5G. It is expected that 5G systems will efficiently support extremely high data rates with lowest acceptable data rate at network's edge or edge rate between 500 Mbps to $1 \mathrm{Gbps}$, which is 100 times the rate supported by $4 \mathrm{G}$ systems, and peak rate in tens of 
Gbps. Area capacity, measured in bits per second per unit area, will increase by 1000 times in comparison to that offered by $4 \mathrm{G}$ systems [AND-14].

\subsubsection{Latency}

One of the most prominent advantages of $5 \mathrm{G}$ is low latency operation. $5 \mathrm{G}$ will support a roundtrip latency of around $1 \mathrm{~ms}$ [KAV-18] [HOR-18] for applications that require real-time feedback. 4G/LTE has latency in the order of about $50 \mathrm{~ms}$ with $1 \mathrm{~ms}$ sub frame or $0.5 \mathrm{~ms}$ slot that can accommodate 14 symbols [INN-10] [SAN-18]. Like 4G, 5G will also have slot-based channel access architecture. However, apart from allowing the same symbol configuration in same duration slots, $5 \mathrm{G}$ also introduces $500 \mu \mathrm{sec}, 250 \mu \mathrm{sec}$ and $125 \mu \mathrm{sec}$ "mini-slots" [SAN-18]. These mini-slots are able to carry up to seven symbols in the same time duration that carries only one symbol in 4G/LTE and 5G basic configuration. This will further reduce the latency and will be extremely helpful in applications that require frequent exchange of small amount of data such as sensors or keep alive messages during a broadband connection.

\subsubsection{Cell Densification}

Cellular wireless networks are composed of cell sites divided into sectors, with a base station in each sector. This base station is responsible for utilizing radio waves to transmit data to all the associated devices within the boundaries of that sector. Unlike 4G, which requires high power, large cell towers to radiate signals over long distances, $5 \mathrm{G}$ will introduce numerous small cells, implementing extreme densification, in order to increase capacity. The main advantage of cell densification is that it allows dense spectrum reuse and reduces the number of contention nodes for resource allocation at each base station. One main concern is that the increasing densification may affect the signal quality, as shorter distance between base stations generates the potential for causing higher interference to neighboring cells. However according to [DHI-12], the signal to interference ratio (SIR) remains constant irrespective of the cell size as long as the power-law path loss model stays intact. Nonetheless, extreme densification will result in substantial increase in the number of base stations, devices and antennas and the installation and maintenance of such system may be costly. 


\subsubsection{Huge Bandwidth \& mmWave Spectrum}

To meet the constantly and exponentially growing data traffic demand, 5G requires huge capacity. Since the most straightforward way to increase capacity is to increase the available bandwidth, use of higher frequency bands makes sense. mmWave is being considered as the new perspective spectrum for $5 \mathrm{G}$ technology. The mmWave spectrum extends from $30-300 \mathrm{GHz}$ where wavelengths are within the 1 to $10 \mathrm{~mm}$ range. Up until recently the mmWave spectrum was not considered for communication due to the higher free space pathloss when compared to the pathloss experienced at frequency bands used by current wireless technologies, e.g. cellular and WiFi [LIU-16][AYA-14]. While achieving almost ten times increase in operating frequency from $700 \mathrm{MHz}$ (LTE/4G) and 2.4-5GHz (WiFi) when moving to $30 \mathrm{GHz}$, use of mmWave also increases the free space pathloss by $20-30 \mathrm{~dB} / \mathrm{km}$. mmWave is also susceptible to adverse weather conditions such as rain, fog and snow. Heavy rainfall can further attenuate the mmWave signal by $20 \mathrm{~dB} / \mathrm{km}$. It also experiences strong pathloss when passing through solid objects such as foliage, human hands or bricks, and has low diffraction factor around obstacles. Moreover, mmWave beams are highly directional and would require very precise beam management solutions that encompass beamforming, beam alignment and beam failure recovery and realignment. Section 2.5 and 2.6 provide a more detailed discussion on mmWave technology.

\subsubsection{Device Diversity and Density}

5G will need to support efficiently vast variety and number of devices. 4G can support around two thousand connections per square kilometer [NOT-17]. However, this is insufficient for the upcoming era, where consumers would require internet connectivity for everything; from smart phones to dishwashers. Hence, $5 \mathrm{G}$ will need to support nearly millions of connections per square kilometer [HUA-15]. Moreover, devices will become increasingly diverse in terms of the types of traffic and their QoS requirements. Hence, it is crucial that 5G should provide support to both, high and low data rate users as defined by their specific communication requirements.

\subsubsection{Massive MIMO \& 3D Beamforming}

MIMO is a wireless communication technology that enhances the capacity of a radio link by utilizing multiple antennas at transmitter and receiver. It utilizes the signals generated by multipath propagation constructively to enhance channel capacity by simultaneously supporting 
data transfer on multiple spatial links over the same radio channel, achieve higher data rate using spatial multiplexing, or combat fading by exploiting diversity compared to single antenna systems [VIS-14]. A MIMO system characterizes each spatial channel between user device and antenna element created due to multiple paths, by its unique transfer function. The estimation of the transfer function is referred as channel state information (CSI). The CSI is arranged in a matrix and is used in the digital processing of transmitted and received data [MAS-17]. Massive MIMO corresponds to a large-scale antenna system and is under consideration for use in 5G systems with high number of antenna elements per base station. Compared to standard MIMO systems, that utilizes 2 to 4 antenna elements, massive MIMO has more complex architecture, however, the exact number of antenna elements depends on the vendor's and system's specifications. It generally varies from tens to hundreds antenna elements per base station. For example Ericsson's AIR 6468 utilizes 64 transmit and 64 receiver antennas [ERI-19], whereas ZTE and HUAWEI have demonstrated massive MIMO architecture utilizing 96-128 antenna elements per base station [AGE-20]. Massive MIMO is expected to enhance the spectral efficiency enormously, however the performance is limited by pilot contamination (pilot signal interference by adjacent base stations during training phase). Also the hardware complexity increases with the number of antenna elements [HOY-13] [RUS-13]. Beamforming technology enables directionally steering data streams to a particular user or sector using multiple transmitter antennas. Beamforming can be analog or digital. Digital beamforming can increase the system capacity as it allows spatial multiplexing, however it is an expensive option for massive MIMO as each antenna element require one RF chain. On the other hand, although analog beamforming does not require exclusive RF chain for each antenna element, supports diversity and improves BER, it compromises on spatial multiplexing. Hence, for massive MIMO hybrid beamforming architectures that provide economical multi user/ multi data streams support are emerging [KUT16]. While 2D beamforming adopted in 4G LTE cellular networks allows only horizontal steering of the data signal, 3D beamforming will enable the formation of directional radiation pattern in both, elevation and azimuth plane. In $5 \mathrm{G}$ networks, 3D beamforming will be adopted along massive MIMO architectures to enhance user support, as it offers prominent advantages over 2D beamforming systems, such as increased channel capacity and improved spectrum efficiency, as multiple vertical tilts result in more sectors thus enhancing frequency reuse, less interference between sectors and signals and better coverage [RAZ-14]. In 5G networks due to 
cell densification, each macro-cell employing mmWave wireless backhaul with massive MIMO and 3D beamforming would provide ubiquitous coverage for numerous underlying small cells. However, several challenges related to beamforming architecture, power consumption, scalability in terms of number of user devices, antenna correlation, mutual coupling, overhead reduction for CSI and cost must be addressed before massive MIMO can be adopted and deployed.

\subsubsection{Heterogeneous Networks}

$5 \mathrm{G}$ will witness increasingly heterogeneous networks. A key feature of $5 \mathrm{G}$ is the integrated support it should provide to 3G, 4G, WiFi and VLC devices, spread across many spectral bands. Hence, modeling, analyzing and optimizing base station association for each user will be a complex task. The optimal base station selection for each associated user would depend on many factors such as SINR, the instantaneous load at each base station and protocol constraint requirement such as use of the same BS for both uplink and downlink to facilitate resource allocation procedures [ROY-12] [QYE-13]. Simplified procedures such as "biasing" and "macro cell muting" can be adopted to accomplish this task [AND-14]. Biasing refers to load balancing between small cells and macro cell, whereas macro cell muting refers to temporarily shutting down transmission from macro cell in order to reduce interference in small cells [FOO-13] [BED-13] [DEB-14].

\subsubsection{Mobility \& Seamless Connectivity}

Due to increased densification and presence of heterogeneous networks, one of the central and crucial features of $5 \mathrm{G}$ will be to provide seamless connectivity with smooth handoffs in both moderate and highly mobile environments. Various handoff approaches have been studied by researchers to support efficient handoff in 5G networks. One approach is to select the next base station on the criteria of maximum achievable throughput instead of signal strength [GHO-17]. Energy efficient solutions also exist in the literature, where the ratio between data rate required by each node and total power consumed are decisive factors for base station selection [LIU-16] [ZHO-12] [MES-14]. In the mmWave spectrum, handoffs will become even more challenging, as transmitter and receiver beams must be perfectly aligned to carry out handoffs for communication. In that scenario, it is quite possible that an opportunistic approach is adopted, 
where users communicate with multiple coordinated base stations [KIM-14]. It is expected that mmWave based 5G systems will witness a high handoff rate in different mobility scenarios, as high as having handoff every $0.75 \mathrm{sec}$ [TAL-14].

\subsubsection{Cost \& Energy Efficiency}

It is important that $5 \mathrm{G}$ systems are deployed with reasonable cost and power consumption. Small cells should be cheaper and more power efficient than 4G macro cells. One major expense would be associated with backhaul connections and maintenance of large number of base stations and antenna systems. Due to the increased network density, access network will take the lion's share in terms of energy consumption. Various approaches that can be adopted to reduce energy consumptions are:

1) To intentionally opt in favour of "green devices" while deploying $5 \mathrm{G}$ access networks or use renewable energy sources for base stations, e.g. solar powered base stations.

2) To apply intelligent resource allocation even if it means compromising on lower data rate for achieving large energy saving, as long as data rate is at par with the application's requirement.

3) To have base stations and devices go through periodic sleep/wake cycles during idle and active communication periods [HAN-16].

\subsubsection{Backhaul}

Backhaul in cellular networks consists of communication links between base stations and the core network. These communication links can be wired (e.g. fiber, copper) or wireless such as microwave or free space optical (FSO). In 5G networks, due to extreme cell densification, massive amount of data will be transferred between core and edge networks and backhaul could be a huge bottleneck. It is not practical and sometimes impossible to use fiber based wired backhaul connections from each base station to the core network, as some terrain may have deployment limitations and other times cost could be a major factor. In this scenario, wireless backhaul solutions are most favourable options for 5G networks. However, the backhaul for 5G network will be a combination of available wireless technologies such as microwave (ptp,ptmp), FSO and mmWave and will be deployed based on various factors such as maximum uplink, downlink data rate, deployment terrain, latency, jitter and cost [JAB-16]. 


\subsection{Link Between Future Vehicular Networks And 5G Objectives}

At present, vehicles can exchange limited information with the infrastructure and among themselves. This information is regarding road conditions, accidents and point of interest. Realtime traffic information, in addition to digital maps, is available to the drivers in various urban locations. However, the coverage area of such information availability and exchange is limited. Also the latency in some cases is high, which makes the information useless such as in cases of temporary road blockage scenarios. It is therefore essential that existing communication standard such as DSRC/IEEE 802.11p / WAVE in North America and ITS-G5 in Europe are updated or complemented with new emerging technologies in order to enhance V2I and V2V communication. These enhancements should result in guaranteed delivery of data messages with lowest latency while maintaining seamless connectivity between highly mobile vehicular nodes within the communication range. The existing short-range adhoc based IEEE 802.11 V2I and V2V communication system does not provide delay guarantees and fails to provide QoS support for broadband data. As vehicles advance toward higher automation levels and deal with increasingly complex road situations, they will need to exchange collection of diverse information. Therefore there is a growing need for a complementary communication technology for the exchange of cooperative information between V2I and V2V, having higher bandwidth, low cost and improved reliability. The future requirements of vehicular networks are well beyond the capabilities of existing wireless and $4 \mathrm{G}$ cellular networks in terms of latency, availability and reliability. The goals of $5 \mathrm{G}$ are harmonized with the requirement of future vehicular networks. Ubiquitous network availability is crucial for widespread deployment of ITS services. Network coverage should be available along roads at all times; even in cases of low vehicular density. In absence of infrastructure, direct V2V or device-to-device (D2D) communication should be efficiently supported. Data has to be accessible by all devices at all times. $5 \mathrm{G}$ is the emerging access technology that provides a unifying platform for various heterogeneous networks and ensures essential support for ultra-reliable and low latency applications required by evolving vehicular networks. 


\subsubsection{G For Vehicular Communication}

$5 \mathrm{G}$ aims to support new and existing access technologies and air interfaces in order to carry out low latency and high capacity communication. In this section, we review some fundamental building blocks of $5 \mathrm{G}$ in vehicular context.

\subsubsection{Device-to-Device Communication}

Device-to-Device (D2D) communication is the exchange of information between two devices directly, without going through the cellular base station. D2D communication is one of the key building blocks of $5 \mathrm{G}$ as it enables devices to directly engage in data exchange, hence reducing latency, increasing throughput and reducing the traffic load applied on base stations. 3GPP LTEV Release 14 [3GP-17] outlines the enhancement over LTE-A to support V2X communication for $5 \mathrm{G}$. This release of LTE-V introduces two radio interface. One cellular interface called $\mathrm{U}_{\mathrm{u}}$ to support V2I information exchange. The second interface is called PC5 and supports V2V communication based on D2D. The earlier modes (1 \&2 ) for D2D communication that were announced in release 12 were able to save battery life in exchange of latency and hence deemed unsuitable for the vehicular environment, as low latency is extremely crucial in the context of vehicular communication. Modes $3 \& 4$, included in Release 14, are proposed specifically for vehicular D2D [MAS-17A]. In mode 3, the cellular base stations are responsible for device discovery and managing resources. However, in mode 4 the vehicles autonomously select and manage resources based on their requirements. Figure 2.5 presents the LTE -V 5G classification. A centralized adaptive resource allocation scheme for broadcasting safety messages using D2D broadcast for vehicular communication was found to perform better than fixed resource allocation schemes [GHO-17]. The performance improvement is in terms of the number of broadcast group members that were able to receive "all" broadcast packets, packet loss rate and efficient spectrum reuse while adaptively managing broadcast group load and reuse of spatial resources. Since the simulation scenarios were centralized, interference between D2D and cellular transmission was not considered. A distributed cell-less D2D vehicular communication architecture with selected vehicles acting as AP's (either pre-configured or co-operatively) was compared against only $\mathrm{V} 2 \mathrm{~V}$ communication for latency in terms of number of hops traversed [WAN-17]. It was found that the latency was considerably reduced when the vehicles are selected co-operatively compared to only vehicle based relaying or pre-selecting vehicle as 
moving AP. Naturally, the downside of such schemes is the overhead increase due to the messages exchanged between vehicles for selecting the AP.

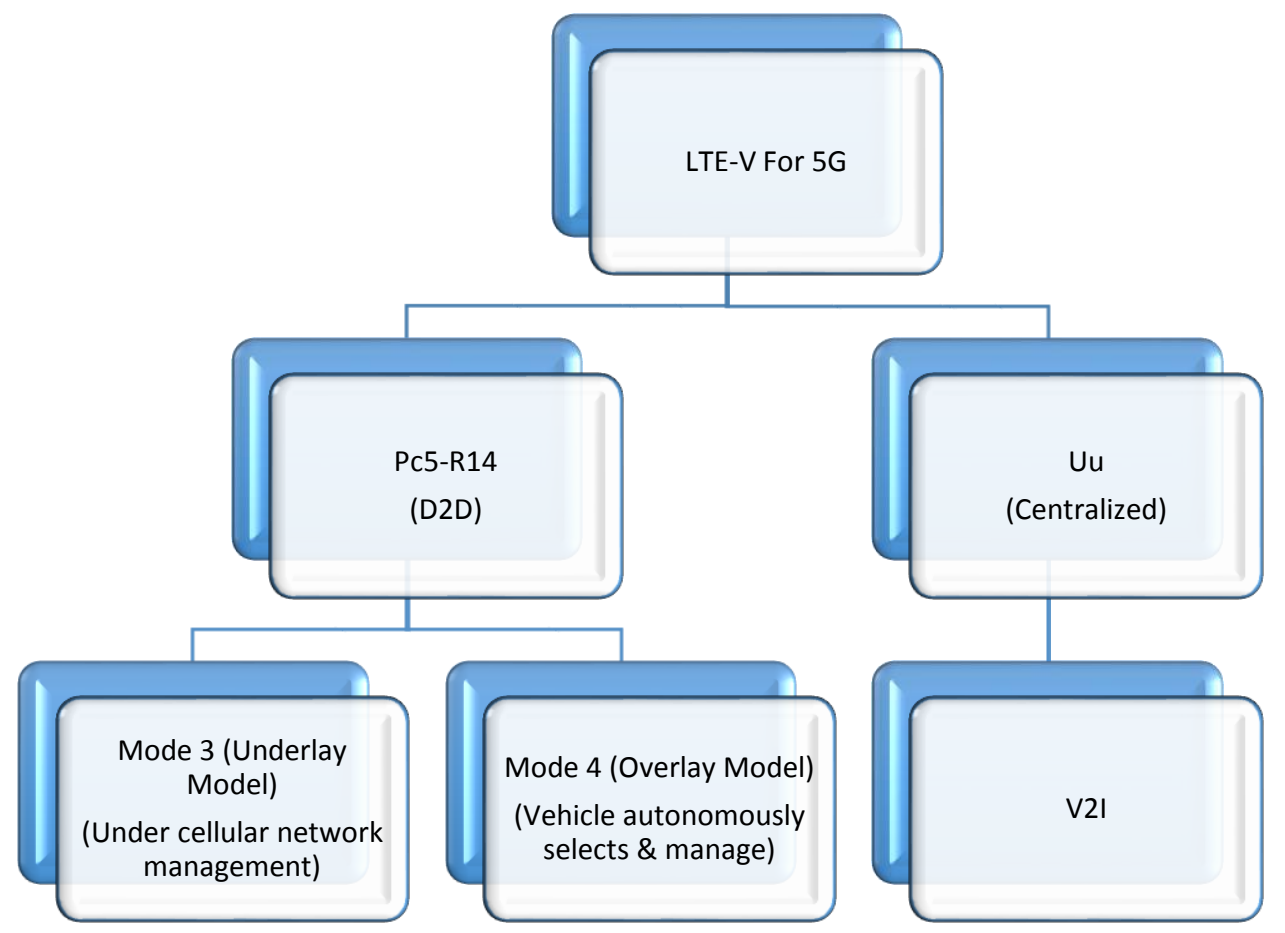

Figure 2.5 LTE-V (5G) Classification

\subsubsection{Mobile Edge Computing (MEC)}

One of the key advantages of $5 \mathrm{G}$ is mobile edge computing (MEC). MEC provides IT services such as data collection, aggregation and processing as well as cloud computation closer to the mobile devices. The purpose of providing these services closer to the mobile users is to reduce latency, ensure highly efficient network operation and ultimate personal experience. In vehicular context, safety information can be gathered, processed and disseminated locally with low latency. Even infotainment applications can be stored locally, near the mobile users, and be available on demand. A $5 \mathrm{G}$ enabled vehicular network with buffers on the base stations improves the delay for video streaming and thus enhances the entertainment experience for the passengers [QIA-18].

\subsubsection{HetNets}

$5 \mathrm{G}$ is going to provide integrated support to multiple access technologies. It will be able to support existing and new technologies. In vehicular communication, due to intermittent 
connectivity, frequent handoff is the main issue. HetNets are utilized in vehicular networks to efficiently navigate handoff issues. In general, two-tier network architecture is created where the small cells are responsible for data transfer only, whereas macro cells are responsible for resource reservation and handoff [MUM-15] [DON-17] [GE-16]. Another architecture applying spectrum sharing (for vehicular networks) between DSRC and mmWave is presented in [ZHO17].

\subsubsection{Technical Requirements For Vehicular Applications Mapped To 5G}

The technical requirements for the vehicular application belonging to safety and non-safety applications must be accomplished under different environmental circumstances. It will be easier to achieve $\mathrm{V} 2 \mathrm{~V}$ communication with the required latency on moderately crowded roads for $20 \mathrm{~m}$ distance between the transmitting and receiving vehicles, however it will be difficult achieving it for high vehicular density and longer distances. Following are the important communication parameters:

1. Latency: End-to-end latency is defined as the time elapsing between the time the data packet is generated to the time it is received at the destination node.

2. Maximum packet loss rate (PLR): Defines the reliability of the communication link by providing a limit on the maximum fraction of packets that may be lost while traversing through the link between sender and receiver vehicle.

3. Data rate: The minimum speed (in bits/sec) of data required to be exchanged between sender and receiver that allow the application to function correctly.

4. Cycle-time: The delay after which periodic information is repeated by the sender.

5. Transmission Mode: Whether the information is periodically exchanged by the vehicles or event-driven.

6. Communication Range: The maximum coverage area, in which data should be received by all vehicles.

7. Velocity: The speed at which vehicles are travelling.

8. Density: The concentration of vehicles in the area/road.

The table below lists technical requirements for major safety and non-safety applications, based on their ability to enhance public safety and reduce traffic accident, as published by National 
Highway Traffic Safety Administration (NHTSA) under US Department of Transportation (USDOT) [NHT-05]. Key performance indicators (KPI's) for futuristic vehicular applications (e.g. bird's eye view) are also included in the table.

\begin{tabular}{|c|c|c|c|}
\hline Applications & Description & Communication Range & Key Parameters \\
\hline $\begin{array}{l}\text { Traffic signal violation } \\
\text { warning. Stop sign } \\
\text { violation warning. }\end{array}$ & $\begin{array}{l}\mathrm{I} 2 \mathrm{~V} \text { issues warning if it is } \\
\text { predicted that the vehicle } \\
\text { will violate the signal, so } \\
\text { that the driver could } \\
\text { adhere to legal location } \\
\text { limits. }\end{array}$ & $250 \mathrm{~m}$ & $\begin{array}{l}\text { Latency }=100 \mathrm{~ms} \\
\text { Cycle-time }=10 \mathrm{~ms}\end{array}$ \\
\hline Automated overtake. & $\begin{array}{l}\text { V2V data exchange on } \\
\text { two-way road to } \\
\text { automatically overtake } \\
\text { the leading vehicles. }\end{array}$ & $\begin{array}{l}\text { Within } 30 \mathrm{~cm} \text { positioning } \\
\text { error }\end{array}$ & $\begin{array}{l}\text { Latency }=10 \mathrm{~ms} \\
\mathrm{PLR}=10^{-5}\end{array}$ \\
\hline $\begin{array}{l}\text { Left turn assistant. Stop } \\
\text { sign moment warning. }\end{array}$ & $\begin{array}{l}\text { On-board sensors and } \\
\text { I2V help vehicle to take } \\
\text { left turn on signals } \\
\text { without dedicated left } \\
\text { turn arrows and/or } \\
\text { vehicles departing after } \\
\text { stopping at a stop sign. }\end{array}$ & $300 \mathrm{~m}$ & $\begin{array}{l}\text { Latency }=100 \mathrm{~ms} \\
\text { Cycle-time }=10 \mathrm{~ms}\end{array}$ \\
\hline $\begin{array}{l}\text { Cooperative collision } \\
\text { avoidance. }\end{array}$ & $\begin{array}{l}\mathrm{V} 2 \mathrm{~V} \text { data exchange in } \\
\text { order for vehicles to } \\
\text { deviate from trajectory to } \\
\text { avoid collision } \\
\text { cooperatively. }\end{array}$ & N/A & $\begin{array}{l}\text { Latency }=10-100 \mathrm{~ms} \\
\text { PLR }=10^{-5}-10^{-3}\end{array}$ \\
\hline See-through vehicle. & $\begin{array}{l}\text { On-board sensors, V2I } \\
\text { and V2V }\end{array}$ & N/A & $\begin{array}{l}\text { Data rate }=10 \mathrm{Mbps} \\
\text { Latency }=50 \mathrm{~ms}\end{array}$ \\
\hline Bird's eye view. & $\begin{array}{l}\text { On-board sensors, V2I } \\
\text { and V2V }\end{array}$ & N/A & $\begin{array}{l}\text { Data rate }=40 \mathrm{Mbps} \\
\text { Latency }=50 \mathrm{~ms}\end{array}$ \\
\hline Platooning. & $\begin{array}{l}\text { Requires V2V } \\
\text { communication. } \\
\text { Combines vehicle data } \\
\text { with real-time position } \\
\text { and map information. }\end{array}$ & $100 \mathrm{~m}$ & $\begin{array}{l}\text { Latency }=10 \mathrm{~ms} \\
\text { PLR }=10^{-5} \\
\text { Cycle-time }=20 \mathrm{~ms}\end{array}$ \\
\hline $\begin{array}{l}\text { Automated vehicular } \\
\text { telematics }\end{array}$ & $\begin{array}{l}\text { Information released by } \\
\text { in-car sensors for } \\
\text { automated driving }\end{array}$ & N/A & Latency $=1 \mathrm{~ms}$ \\
\hline
\end{tabular}




\begin{tabular}{|c|c|c|c|}
\hline $\begin{array}{l}\text { Vulnerable road user } \\
\text { (VRU) discovery }\end{array}$ & $\begin{array}{l}\text { Discovery of pedestrian, } \\
\text { bicycle, elderly or } \\
\text { children in the driving } \\
\text { trajectory. }\end{array}$ & $\begin{array}{l}200 \mathrm{~m} \text { with maximum } 10 \\
\mathrm{~cm} \text { positioning error }\end{array}$ & Latency $=5 \mathrm{~ms}$ \\
\hline $\begin{array}{l}\text { Intersection collision } \\
\text { warning }\end{array}$ & $\begin{array}{l}\text { I2V data transfer to warn } \\
\text { the vehicle if a collision } \\
\text { at approaching } \\
\text { intersection is predicted. }\end{array}$ & $300 \mathrm{~m}$ & $\begin{array}{l}\text { Latency }=100 \mathrm{~ms} \\
\text { Cycle-time }=10 \mathrm{~ms}\end{array}$ \\
\hline Blind merge warning & $\begin{array}{l}\mathrm{V} 2 \mathrm{~V} \text { and } \mathrm{V} 2 \mathrm{I} \text { information } \\
\text { exchange to warn the } \\
\text { vehicle is merging with } \\
\text { blind spots. }\end{array}$ & $200 \mathrm{~m}$ & $\begin{array}{l}\text { Latency }=100 \mathrm{~ms} \\
\text { Cycle-time }=10 \mathrm{~ms}\end{array}$ \\
\hline $\begin{array}{l}\text { Emergency vehicle } \\
\text { approaching }\end{array}$ & $\begin{array}{l}\text { V2I and V2V to inform } \\
\text { vehicles to yield right-of- } \\
\text { way to approaching } \\
\text { emergency responders }\end{array}$ & $1000 \mathrm{~m}$ & Latency $=1 \mathrm{sec}$ \\
\hline Post-crash warning & $\begin{array}{l}\mathrm{V} 2 \mathrm{~V} \text { from disable vehicle } \\
\text { due to crash to oncoming } \\
\text { traffic }\end{array}$ & $300 \mathrm{~m}$ & $\begin{array}{l}\text { Latency }=0.5 \mathrm{sec} \\
\text { Cycle-time }=1 \mathrm{~s}\end{array}$ \\
\hline In-vehicle signage & $\begin{array}{l}\text { Information from I2V } \\
\text { about approaching road } \\
\text { conditions and released } \\
\text { from vehicle sensor } \\
\text { system to driver or central } \\
\text { controller in case of } \\
\text { automated driving }\end{array}$ & $200 \mathrm{~m}$ & Latency $=1 \mathrm{sec}$ \\
\hline $\begin{array}{l}\text { Cooperative forward } \\
\text { collision warning, } \\
\text { highway merge assistant } \\
\text { and blind spot warning }\end{array}$ & $\begin{array}{l}\mathrm{V} 2 \mathrm{~V} \text { and } \mathrm{I} 2 \mathrm{~V} \text { for } \\
\text { enabling safe vehicular } \\
\text { maneuvering }\end{array}$ & $150 \mathrm{~m}$ & $\begin{array}{l}\text { Latency }=100 \mathrm{~ms} \\
\text { Cycle-time }=10 \mathrm{~ms}\end{array}$ \\
\hline Visibility notification & $\begin{array}{l}\mathrm{V} 2 \mathrm{~V} \text { for deteriorating } \\
\text { weather conditions such } \\
\text { as rain, fog, snow that } \\
\text { affects visibility }\end{array}$ & $200 \mathrm{~m}$ & $\begin{array}{l}\text { Latency }=100 \mathrm{~ms} \\
\text { Cycle-time }=2 \mathrm{~s}\end{array}$ \\
\hline Pre-crash sensing & $\begin{array}{l}\text { V2V warning for } \\
\text { impending crash }\end{array}$ & $50 \mathrm{~m}$ & $\begin{array}{l}\text { Latency }=20 \mathrm{~ms} \\
\text { Cycle-time }=20 \mathrm{~ms}\end{array}$ \\
\hline
\end{tabular}


The mobile and wireless communication enablers for the twenty-twenty information society (METIS) has also released its own specification for vehicular communications [MET-14]. The METIS KPI's for vehicular communications would maintain or place further stringent requirements on existing parameters supported by legacy solutions such as DSRC. Table 2.2 lists the METIS KPI's.

\begin{tabular}{|c|c|c|c|c|c|c|}
\hline Scenario & $\begin{array}{l}\text { Velocity } \\
(\mathrm{km} / \mathrm{hr})\end{array}$ & $\begin{array}{l}\text { Communication Range } \\
\text { (m) }\end{array}$ & $\begin{array}{l}\text { User Density } \\
\text { (user } / \mathrm{km}^{2} \text { ) }\end{array}$ & Latency (ms) & $\begin{array}{l}\text { Traffic } \\
\text { Volume } \\
\left(\mathrm{Gbps} / \mathrm{km}^{2}\right)\end{array}$ & Reliability \\
\hline Urban Traffic & 60 & 300 & 1000 & 5 & 0.1 & $99.99 \%$ \\
\hline Rural Traffic & 120 & 500 & 100 & 5 & 0.01 & $99.99 \%$ \\
\hline Highway & 250 & 1000 & 100 & 5 & 0.01 & $99.99 \%$ \\
\hline VRU & $3-30$ & & $150-5000$ & 5 & & $99.99 \%$ \\
\hline
\end{tabular}

Table 2.2 METIS KPI's for Vehicular Communication [MET-14]

\subsection{Limitations of Existing Communication Technologies}

\subsubsection{DSRC/WAVE/IEEE 802.11p}

DSRC/WAVE/IEEE 802.11p technology is exclusively defined for the vehicular communication environment. DSRC enabled systems consist of on-board units (OBU's) and road side units (RSUs). The vehicles can directly communicate with each other (V2V) or through the RSU i.e. V2I. The DSRC band is divided into seven radio channels of $10 \mathrm{MHz}$ each, with one control channel $(\mathrm{CCH})$ and six service channels $(\mathrm{SCH})$. The multichannel WAVE architecture requires the DSRC on-board radios to keep switching between control channel to receive and transmit safety information and service channels to make use of available services. IEEE 802.11p standard utilizes the IEEE 802.11e enhanced distributed channel access (EDCA) medium access control (MAC) layer. EDCA use CSMA/CA as the transmission protocol and has four different access categories (ACs) in order to provide prioritization to diverse types of data communication. 
DSRC enables vehicle to exchange data between V2V and between V2I where road side units (RSUs) act as infrastructure. Conceptually, the AdHoc mode of IEEE 802.11p is suitable for the vehicular environment, as connection between peers can be established "on demand" and does not require a central controller except to carry out management and security functionalities. This improves latency however, apart from broadcasting safety messages, road side units (RSUs) are also gateways providing support for diverse internet-based applications such as gaming, realtime video, audio and video download and office-on-wheels applications. Fully utilizing the capabilities of DSRC, requires a wide spread deployment of RSUs which is not feasible economically since comparable cellular infrastructure already exist. Moreover, due to the terrain conditions, it may not be practical and/or possible to install RSUs everywhere.

One of the major drawbacks of IEEE 802.11p is the relatively fast performance degradation it experience with increase in network load. This is due to two reasons. Firstly, each vehicle in the network is required to periodically broadcast beacon crucial for executing safety applications. Naturally, the capacity of network is reached quickly as vehicle density within the area increases. Secondly, the CSMA/CA protocol is an inefficient protocol, providing utilization below 33\%. It requires vehicular applications to sense the medium and only transmit if the channel is sensed idle. In case of collision, the stations are required to back-off and retransmit after the back-off window. With increase in the number of stations contending for channel access, number of collision and hence the back-off duration and back-off frequency increases, which degrades the latency of the system. Hence, the maximum latency cannot be guaranteed, which makes the protocol unsuitable even for safety applications.

Another factor is unavailability of QoS support in IEEE 802.11p. Although, there are four different access categories, each with its own contention window and back-off period, the fairness of access cannot be guaranteed as even for the highest priority user, there are few contention window $(\mathrm{CW})$ sizes to select from, which means users reach fast the zero back-off at the same time. This becomes especially significant if safety message packets are generated during SCH intervals or guard band. As soon as CCH starts, all of the vehicles with safety messages will try to access the channel in order to broadcast their safety messages. This will result in high collision rate. Hence, the maximum throughput and latency cannot be guaranteed even for high priority applications. 
In addition to this problem, other sources of impairments are at play, placing especially safety applications into jeopardy. Such sources of impairment are the hidden node behavior, interchannel interference between channels etc. In highly congested vehicular networks, safety beacons may be received after their time to live (TTL) has expired and will be discarded. Such delays in frame delivery become common under increased traffic loading. Hence, CSMA/CA is a highly unstable protocol when there are multiple users and random traffic and fails to provide any guarantees in terms of throughput and delay.

\subsubsection{G/LTE Infrastructure -based communication}

The infrastructures or base stations in LTE 4G are called E-UTRAN node B (eNB). In cellular LTE infrastructure base communication, several challenges IEE 802.11p has are resolved. In cellular networks, uplink and downlink transmissions are scheduled such that interference is minimized. Also, it is easier to provide guaranteed QoS as resources can be centrally allocated and maintained by utilizing admission control. However, presence/use of only infrastructure based communication has its own implications. Firstly, each time vehicles want to exchange data, the data has to transverse at least through one uplink and one downlink connection. This increases latency and causes a bottleneck with increase in traffic as the cell approaches its maximum capacity. Also, it also creates a single point of failure (eNB in this case) for the entire communication network.

In vehicular environment, it is necessary for vehicles to periodically exchange short beacon messages that enable neighboring vehicles to estimate the position, velocity and direction of other vehicles. These messages are referred as Cooperative Awareness Messages (CAM) according to ETSI documents. This information is crucial to safety applications. However, since LTE was mainly developed to support broadband services, it is unsuitable for exchanging short messages resulting in underutilization of the channel resources [5GP-15]. Scalability is another issue as latency and number of collisions rise with the increase in the number of devices located within the cellular coverage area. Latency rises as the number of vehicles in the cell coverage area increase. This is true for both CAM and event-driven messages that are referred as Decentralized Environmental Notification Messages (DENM) in ETSI documents [ETS-10]. CAM are short heartbeat messages that are broadcasted in the immediate neighbourhood of the vehicle and give details regarding position, kinematics and basic status of the vehicle. DENM 
messages are distributed to the relevant area that would be effected by the event. It was analytically concluded in [VIN-12] that the unicast distribution of CAM messages through eNB to every vehicle in the cell heavily overloaded the network and infrastructure based LTE transmissions failed to support the delivery requirement of CAM messages. Another detailed simulation based study was conducted in [PHA-11] to conduct capacity analysis and to evaluate the feasibility of LTE infrastructure based networks in case of both CAM and DENM message dissemination. The findings of this study contributed in the proposed framework by ETSI [ARA13]. In this study, the CAM messages were filtered and were only unicasted through eNB to the selected vehicles. The main simulation parameter are presented in table 2.3.

\begin{tabular}{|c|c|}
\hline System band width & $\begin{array}{c}5 \mathrm{MHz} \text { DL UL }+5 \mathrm{MHz} \text { DL (FDD) } \\
7 \text { sites with } 3 \text { sectors } / \text { site } 21 \text { cells }\end{array}$ \\
\hline Network size & $\begin{array}{c}\text { Urban scenario: } 500 \mathrm{~m} \text { at } 2 \mathrm{GHz} \text {; antenna tilt: } 11^{\circ} \text { Rural } \\
\text { scenario: } 6 \mathrm{~km} \text { at } 800 \mathrm{MHz} \text {; antenna tilt: } 1^{\circ}\end{array}$ \\
\hline Inter-site-distance (ISD) and carrier frequency & $1 / 2(\mathrm{SIMO})$ \\
\hline Tx $/$ Rx antennas & $13,9 \mathrm{~m} / \mathrm{s}=50 \mathrm{~km} / \mathrm{h}(\mathrm{urban}) 22,2 \mathrm{~m} / \mathrm{s}=80 \mathrm{~km} / \mathrm{h}(\mathrm{rural})$ \\
\hline User speed & $120 \mathrm{bytes}$ \\
\hline CAM size & $120 \mathrm{bytes}$ \\
\hline DENM size & \\
\hline
\end{tabular}

Table 2.3: Simulation Parameters of [PHA-11] [ETS-12]

Even the filtered CAM dissemination significantly overload the LTE network in both rural and urban scenarios and a higher number of vehicles per cell were only supported if CAM rate was reduced to 2 packets per second. In urban scenario, the maximum number of cars per cell was 275 with 10 neighbours receiving CAM messages every 2 seconds for latency greater than $100 \mathrm{~ms}$. For rural scenario, the maximum number of cars supported for each cell reduced to 99. Although DENM messages temporarily overload the network, it was found that the capacity was limited to 140 devices in urban and 100 devices in rural scenarios with latency as high as $200 \mathrm{~ms}$. These studies were conducted under the assumption that LTE capacity is exclusively used for CAM/DENM messages and do not consider any background traffic that would require QoS support. Hence, these studies points to serious limitations that infrastructure based LTE networks may face in vehicular environment. 
Providing cellular coverage in underground vehicular environments such as tunnels is also important issue since safety applications should be supported to the utmost degree depending on the feasibility of communication environment.

\subsubsection{LTE Proximity Service (ProSe) R-12}

LTE ProSe release 12 addresses D2D communication. In D2D communication, data is directly exchanged between the devices located on the vehicles without traversing infrastructure. LTE R12 specifies two different modes of transmission:

- Mode 1: The transmission is scheduled through infrastructure/BS. This mode requires the availability of BS for scheduling and resource reservation purposes. The devices exchanging data are required to be registered with the BS.

- Mode 2: The data is directly exchanged between devices without any sort of intervention from BS. The devices are not required to be registered or be in the vicinity of any BS. Release 12 provisions low mobility without any support for QoS or multicast/broadcast options. Moreover, in mode 1 the request for scheduling initiated by the device may take longer due to the involvement of the BS thus affecting latency considerably. Handoffs are also not prioritized. Since latency, QoS and freedom of communication pattern i.e. unicast, multicast or broadcast will be essential for future vehicular networks, R-12 falls substantially short in terms of capability to support V2V communication.

There is high probability of collisions in mode 2 due to hidden node issues. The sub-frame structure supports only half-duplex transmission i.e. a node can either transmit or receive in one sub-frame. For short emergency and periodic messages there is no dynamic scheduling mechanism and hence the efficiency and latency of transmission are highly compromised.

\subsubsection{Existing Short Range Communication Technologies}

\subsubsection{Bluetooth}

Bluetooth technology supports data exchange over short distances in ISM band (from 2.4-2.485 $\mathrm{GHz}$ ). The in-vehicle specific applications of Bluetooth are facilitating many applications such as hands-free voice communications, linking DVD, MP3 players to speakers, remote start-up, 
garage door control, toll road payments. Bluetooth enforces a piconet structure which is the main disadvantage it faces for inter vehicular communication. Due to intermittent connectivity it is impossible to maintain piconet structure. Furthermore, piconet formation and the process for new nodes to join piconet faces considerable delay, which is not acceptable in VANETs. The range of communication is of the order of tens of meters, which is also another limitation.

\subsubsection{Ultra-Wideband}

Due to its low latency and high bandwidth occupancy, Ultra-wideband (UWB) is also a potential candidate for vehicular communication. Use of UWB has been examined and analyzed for use in underground communication, specifically for subway trains [MFO-14]. It is applicable for use in vehicular collision-detection systems and suspension systems that respond to road conditions [GHA-07]. But as UWB signals generated for those other applications could potentially interfere with communication signals, it imposes a technical problem that must be solved before it could be used in vehicular systems. Like all traditional RF technologies, UWB's radio coverage can also extend to uninvolved vehicles that may propagate unnecessary information flow.

\subsubsection{ZigBee}

ZigBee is based on the IEEE 802.15.4 standard and is suitable for wireless sensor network (WSN) with coverage range up to $20 \mathrm{~m}$. It supports low power and low data rate applications. ZigBee employs multihop, mesh architecture to assure robustness of the communication network and to extend the communication range. Although in vehicular context, ZigBee can be used at the intra vehicular monitoring system such as tire pressure monitoring, temperature control, remote keyless entry, ventilation and lighting control [RAH-14] [TSA-07], it is not suitable for inter vehicle communication due to low data rate and limited communication range.

\subsection{New Technologies Proposed To Be Used in 5G}

\subsection{1 mmWave}

The mmWave spectrum refers to wavelengths from $1-10 \mathrm{~mm}$, corresponding to frequencies in the range of 30-300 GHz. While the mmWave spectrum offers much wider bandwidth than the ones available to current cellular system, it was not considered for mobile communication till now due to unfavourable propagation environment. Signals at these frequencies experience high free space pathloss, atmospheric absorption, attenuation under poor weather conditions, especially rain, they are susceptible to high loses in the presence of obstacles obstruction and have to deal 
with strong phase noise. Even now, although it is considered as the main spectrum for $5 \mathrm{G}$ pico cell access network, back haul and front haul [KUO-17] due to its high bandwidth availability, several significant issues remain that need to be addressed before use of this frequency range in $5 \mathrm{G}$ is materialized.

mmWave technology is in line with the goal of $5 \mathrm{G}$ to increase channel capacity by cell densification. Due to its propagation limitations, the size of a mmWave cell is limited to around $200 \mathrm{~m}$. However, mmWave technology requires highly directional transmission with beamforming for efficient mobile communication. A mmWave transmission system will consist of a number of mmWave base stations deployed randomly at a distance of about few hundred meters from each other, having directional antenna arrays. All of the user devices are expected to be equipped with a number of miniaturized antennas, fabricated at different angles of coverage, to overcome the possibility of human body's obstruction, as is the case when holding a cell phone [RAN-14]. The design and adoption of the mmWave system will need significant changes. Some major challenges are discussed below.

\subsubsection{Range and Directional Communication}

The free-space pathloss between the transmitter and receiver antenna grows with $\mathrm{fc}^{2}$. Thus moving up in frequency from 3 to $30 \mathrm{GHz}$ increases the pathloss by a factor of $20 \mathrm{~dB}$ [AND-14]. To compensate for this loss, the directionality of the antenna needs to be increased. However use of very directional antennas highly mobile environment is quite challenging, impacting among others the initial synchronization of the devices with the cellular system and the handoff delay and rate. Both BSs and mobile devices are required to scan over a number of channels before the signals can be detected and devices are authorized to join the cellular network. This directionality may increase the handoff delay substantially as the devices transit from one base station to another. Moreover, both the device and the base station antennas need to be very well aligned.

\subsubsection{Atmospheric Absorption}

mmWaves have high atmospheric absorption. They are absorbed by the gases in the atmosphere especially oxygen at $60 \mathrm{GHZ}$, water vapor at $24 \mathrm{GHZ}$ and $128 \mathrm{GHz}$ and experience high pathloss 
over long distances. The pathloss becomes significant in the presence of adverse weather conditions such as rain, fog, or snow; especially heavy rainfall that can further attenuate the signal up to $20 \mathrm{~dB} / \mathrm{km}$. Therefore, the transmission coverage area is limited to few hundred meters. However, since one of the aspects of $5 \mathrm{G}$ is cell densification, especially in urban scenarios where cell area is envisioned to be limited to $200 \mathrm{~m}$, the atmospheric absorption experienced by mmWave is inconsequential. The cell isolation due to atmospheric absorption enables and reinforces efficient spectrum reuse by distant BSs. However, mmWave BSs would require large and highly directional antenna array to perform precise beamforming in order to successfully accomplish narrow-beam communication without experiencing interference between neighboring devices/cells.

\subsubsection{Blockage and Shadowing}

mmWave signals are highly susceptible to blockage and shadowing. The channel is dependent on the presence of an unobstructed line-of-sight (LOS) and the link quality can quickly degrade to complete outage and intermittent channel quality. This high probability of blockage can also result in indistinct cell boundaries as it is quite possible that devices become linked with a distant BS bypassing the physically closest one due to the shadowing affect. Simple building materials such as bricks can attenuate the signal by $80 \mathrm{~dB}$ [ZHA-13] and a human hand in the LOS can cause a $25-35 \mathrm{~dB}$ loss [SLU-12].

\subsubsection{Heterogeneous Networking Issues}

mmWave systems cannot be stand-alone due to their nature. In order to provide reliable and ubiquitous coverage it is important to provide a cellular back up system possibly operating at microwave frequencies. There are several ways HetNets can support a mmWave system. One way is to separate data and control planes with the mmWave BS only employed for data exchange whereas control information such as handoff and resource allocation messages been handled by microwave base stations [ISH-12][QLI-13]. This way even the issue of intermittent connectivity becomes resolved to some extent as the lost data can be retrieved through retransmissions managed by macro base stations operating at microwave frequencies. Another approach is for the mobile user to simultaneously connect with multiple BSs. This is called carrier aggregation technique [3GP-10][YUA-10].However, this require complex CoMP 
architecture and requires transmission scheduling and path switching support. Another issue with mmWave arises due to the huge bandwidth. Since the mmWave spectrum is quite vast, it is highly likely that multiple operators will share licence for specific spectrum bands in mmWave spectrum, thus interoperability support regarding handoffs and interference between cells is crucial.

\subsubsection{Interference}

Due to the small radii of mmWave cell and high directionality required for communication, the cells are inherently immune to inter BS interference. However, in vehicular context, D2D transmission will most probably take place simultaneously. This will introduce interference issues as D2D transmission can be accomplished in both overlay and underlay models. In the underlay model, the BS is responsible for scheduling and resource allocation for D2D transmission hence interference can be handled. However the overlay model enables D2D transmission directly without the intervention of cellular BS. In this scenario, it is highly likely that D2D transmission will cause interference to another concurrent V2I transmission.

\subsubsection{Multiple Access Mechanism}

Currently mmWave frequencies are used for point-to-point communication between satellite sensors such as advanced microwave sounding unit (AMSU) on NASA satellite [RSS-17]. Presently, AMSUs are installed on eight satellites: NOAA-15 to NOAA-19, Metop-A, Metop-B and NASA AQUA. "Listen before talk" is generally employed as multiple access technique for communication in the lower radio spectrum. However, due to highly directional transmission requirement of mmWave, it is expected that inter device interference will not exist or in some cases selected areas will require channel sensing before transmission. For example, it is possible that due to background noise multiple user select same beam or beams close to each other for data transmission. This would increase the probability of interference between devices. To overcome this issue, compressive sensing (CS) algorithms may be used to reconstruct the received signal. CS is a signal processing technique that reconstruct the received signal from limited number of samples of the original signal. However, this technique requires channel state information. Hence clear channel assessment (CCA) procedures need to be revisited for mmWave communication. New and efficient multiple access mechanisms need to be deployed 
for handling multiple simultaneous communications taking into consideration the extensive spectrum reuse and efficient utilization of channel capacity; critical factors for achieving high throughput communication using mmWave.

\subsubsection{Cost}

mmWave systems require novel transmitter architecture equipped with multiple miniature antennas. This becomes especially critical in smart phones that would require mass scale adoption of new forms of handset to keep the cost reasonable. Power consumption will also be significant due to presence of multiple miniature antennas in the handset, each requiring advanced RF circuitry. Moreover, the BSs have to be deployed at high density since the communication range is very limited. The deployment as well as maintenance cost would be a significant factor. In addition, the power consumption of A/D conversion process increases linearly in terms of sampling rate and exponentially by the number of bits/sample [RAP11][MUR-13] making higher quantization at wide bandwidth and use of large number of antennas extremely power draining to devices.

\subsubsection{Visible Light Communication}

Visible light communication (VLC) refers to the data transfer in visible spectrum that ranges from $400 \mathrm{THz}(780 \mathrm{~nm})$ to $800 \mathrm{THz}(375 \mathrm{~nm})$ using LEDs as transmitter and photo diodes as receivers. Due to their fast switching capabilities and longer lifetime with lower energy dissipation, LEDs are the perfect candidate for visible light data transfer. In VLC systems, LEDs are used simultaneously for illumination and communication. It is predicted that by 2030 LEDs will have captured $99 \%$ of the lighting fixture market [HOL-14].

5G systems will use cell densification technique to increase channel capacity and spectrum reuse. Due to its dependency on LOS for data communication, VLC inherently agrees with small attocell deployment configuration, thus enabling spectrum reuse with minimum interference between two neighboring VLC attocells. However, even this interference could be completely nullified if APs transmit using different colors in neighboring cells. VLC is widely proposed for adoption in indoor configuration between users with lighting infrastructure serving as access points (AP). In outdoor environment, due to the probability of signal degradation in adverse 
weather conditions such as rain, fog or snow, and interference from solar radiation and ambient lighting sources, VLC is considered to be used jointly with back-up wireless radio technology. It should be noted that since $5 \mathrm{G}$ will provide unifying platform for different communication technologies, attenuation of light rays due to weather is not a factor that would affect widespread adoption of VLC technology for outdoor communications.

The IEEE task group for Personal Area Network (PAN) published the IEEE 802.15.7 Physical and MAC layer standard for visible light communication in 2011. The standard supports indoor communication at speeds up to $96 \mathrm{Mb} / \mathrm{s}$ and outdoor communication at $266 \mathrm{kbps}$ [STD-11]. The standard was revised in 2018 to extend the operating wavelengths from 190nm to $10000 \mathrm{~nm}$ and was renamed as short range optical wireless communication standard [STD-18]. The IEEE 802.15.7 MAC superframes are bounded by beacons. The superframe is divided into active and inactive periods. Data communication is supported along illumination during active periods whereas only illumination takes place during inactive periods. The active period is divided into beacon, contention access period (CAP) and contention free period (CFP). There are four different random access techniques that can be used for data transfer: slotted and unslotted random access without channel slotted CSMA/CA and unslotted CSMA/CA. During the CAP duration, the user sends short data messages or request for guaranteed time slots (GTS) using one of the four protocols. The central coordinator assigns the GTS upon request and availability. This scheme has several drawbacks for vehicular networks. CSMA/CA suffers from inherent disadvantage of not providing delay and delivery guarantees, which are extremely crucial for safety applications. Fast moving and small attocell based mobile environment would require multiple handoffs and the protocol does not provide any handoff support. Moreover, the protocol does not provide any QoS support as the GTS are also assigned on first come first serve principle.

Mostly VLC research concentrates on point-to-point or unidirectional channel instead of fully duplex communication links, as developing such system is not an easy task. The use of same visible band in both directions would result in self-interference at transceivers. There are three different ways to address the issue of crosstalk arising due to self-interference. The first approach is to install photo diodes and LEDs physically separated from each other. The second approach is 
to use different color bands for uplink and downlink transmission and the third approach is to use time duplex division (TDD) where the same channel is used for both uplink and downlink but not simultaneously [BUR-14].

In vehicular systems, it is possible to introduce reasonable distance between transmitter and receiver so that there is no interference between transmitter and receiver. e.g. vehicle headlights can act as transmitters whereas photodiodes mounted on the top of vehicle will act as receivers. Also use of different wavelengths (colors) is a viable solution. However, Outdoor VLC networks suffer from some substantial drawbacks that make them inappropriate as a stand-alone technology. The performance of outdoor vehicular visible light communication (VVLC) networks deteriorates considerably with bad weather conditions. Rain, snow, and fog may degrade network's performance substantially. Decrease in visibility reduces throughput. Although VLC networks do not cause electromagnetic interference and are immune to it, they are sensitive to the visible solar and man-made light interference. Solar light and ambient light generated by lighting sources such as halogen, fluorescent or incandescent lamps and LED lamps are the main source of interference in VLC systems. Their presence in the field of view (FOV) of the receiver can severely affect the network's functioning.

The basic link types for VVLC systems are line-of sight (LOS) and non-line-of-sight (NLOS) direct and diffused links. For high data rate communication, direct LOS link is required. In this case the transmitter is pointed to the receiver and forms an unobstructed LOS path, passing through its FOV. The diffused links make use of the reflected light rays to establish connectivity between transmitter and receiver and achieve comparatively lower data rates. Usually any VLC system will make use of both the direct and diffused links for communication. The high speed direct LOS link is susceptible to "shadowing" effect which means that any obstruction in the link path will result in complete blockage and the system throughput would fall back to the lower data rate or in some cases to zero. This is unacceptable in a highly mobile VVLC environment where vehicles need to communicate with each other and with infrastructure for safety purposes, and failure to do so might result in serious accident. These are the dominant factors that favors the deployment of VVLC as a hybrid technology where in case of bad weather conditions or 
obstruction in the FOV, a backup RF/OF link can be employed to maintain the performance of the network.

The underground or tunnel network does not face such challenges as it is shielded from weather variations. However, it can be extended to form a complete VVLC network, which is viable underground and over ground. In this case, it is important to evaluate the suitability of hybrid architecture and analyze if it is beneficial for throughput. Various recommendations to resolve fundamental issues are discussed.

\subsubsection{Spherical LEDs}

One of the rudimentary requirements of VLC system is the establishment of a non-obstructed LOS link between the transmitter and the receiver. This can be achieved by directly pointing the receiver to the transmitter. However, in outdoor environment, this is not easy. Due to the high speed mobile environment, it is not practical to expect maintaining the alignment between transmitter and receiver. Spherical LEDs may solve the issue of maintaining LOS for longer duration and do so at different relative angles between the 2 vehicles. In the design of spherical LEDs a node is covered with multiple transmitters and receivers [NAK-09]. This is achieved as the spherical node provides angular diversity in three dimensions and hence creates and maintains LOS links at different orientations between transmitter and receiver, as long as there is no obstruction. However, this solution also has its own drawback as more energy is consumed to provide 3D coverage. Similar to RF adaptive beamforming architecture, VLC spherical nodes can also be installed with auto-configurable circuits that detect the direction of communication and enhance the LOS energy in that direction while lowering the LED power in other directions [YUK-09].

\subsubsection{Separate Uplink and Downlink}

Using VLC for both uplink and downlink communication can be a source of serious interlink interference. To overcome this issue, one approach is to apply a TDMA based approach, providing half-duplex transmission. Another approach is to employ the HetNets and use an alternate access technology for uplink communication while utilizing VLC for downlink. Blue 
tooth, Infra-red (IR), ultraviolet (UV) and WiFi are all options for providing low to medium rate full duplex communication links.

\subsubsection{Control and User Data Plane Separation}

The presence of multiple radio access technologies (RATs) can also be used to overcome the issue of multiple and frequent handoffs faced by VVLC networks. VVLC networks are highly intermittent and due to the VLC attocell coverage area, it is quite likely that they need to perform handoff every few seconds. If the VLC cell is also providing QoS based resource allocation and reservations, these handoffs will become riskier as the network has to maintain the QoS requested by the devices. By using multiple RATs, control and data plane can be segregated. In that case, the data is transferred by using high capacity VLC links while maintaining the low latency and high data rate requirement while handoff and resource reservation becomes the responsibility of control plane supported by different radio technology e.g. use of umbrella RAT for control messaging [ZHA-15] supported by media independent handovers (MIH) as first presented in IEEE 802.21 standard [IEE-09].

\subsubsection{LOS Blocking}

LOS blocking is the most severe problem in VLC as it may potentially result in network failure. It is recommended that the lighted area is provided back-up from another angle by utilizing multiple lighting sources. However, in case of losing the link with an AP, immediately switching to another AP is not a viable solution as it may result in ping-pong effect. It is better to use a predictive approach in order to minimize the handoff signaling load while taking into consideration the latency needs of the applications [NGU-13]. In such approach, the delay tolerant applications should keep their association with the current VLC AP if there is a high probability that the link will be re-established successfully. In contrast, users using real-time or safety critical application may need to immediately switch to neighboring VLC AP or alternate RAT in order to maintain the flow of data with required QoS.

\subsubsection{Load Balancing}

Uplinks may experience heavy multimedia traffic in a mobile environment as the demand grows. In this scenario, there is a mounting need for a sophisticated load balancing and traffic-reshaping 
framework for uplink and downlink communication in order to make a smart choice between the available uplink and downlink scenarios.

\subsubsection{BackHaul}

Providing a cost-effective and fast backhaul solution is very important for a VLC system. Initially, power line communication (PLC) was opted as the backhaul choice for VLC systems. However, opting for PLC had its own challenges as PLC may limit the data rate due to presence of noise and dispersive nature of powerline. An alternate was the use of fiber-based backhaul solutions as they can provide high data rate backhaul for VLC systems. However, based on the size of attocells, connecting each VLC AP with the optical fiber backbone appeared an expensive and impractical solution. With the advent of G.fast [ITU-14] and XG-Fast [COO-15] that are able to provide up to $10 \mathrm{~Gb} / \mathrm{s}$ data over a pair of copper cable for distances up to $150 \mathrm{~m}$, it is now possible to adopt various fiber-based solutions such as fiber to the home (FTTH), fiber to the frontage (FTTF) and fiber to the distribution point (FTTdp) in combination to provide an affordable solution for high speed VLC networks. An alternate approach to the wired backhaul solutions is to opt for wireless backhaul. The emerging era of $5 \mathrm{G}$ promises to provide a unified platform to support diverse access technologies. Several wireless backhaul solutions have been proposed based on the latency, QoS requirements of the application, jitter, and distance covered. In addition to traditional microwave links, current proposals also suggests use of free space optical utilizing lasers beams, mmWave, satellite and sub $6 \mathrm{GHz}$ transmission as potential backhaul solutions. Table 2.4 [JAB-16] lists proposed wireless backhaul solutions for 5G networks.

\begin{tabular}{|l|l|l|l|l|l|}
\hline Technology & Options & $\begin{array}{l}\text { Upstream } \\
\text { Throughput }\end{array}$ & $\begin{array}{l}\text { Downstream } \\
\text { Throughput }\end{array}$ & Latency/Jitter & Distance \\
\hline Microwave PtP & PtP & $1 \mathrm{Gbps}$ & $1 \mathrm{Gbps}$ & $<1 \mathrm{msec} / \mathrm{hop}$ & $2-4 \mathrm{Km}$ \\
\hline $\begin{array}{l}\text { Microwave } \\
\text { PtmP }\end{array}$ & PtmP & $1 \mathrm{Gbps}$ & $1 \mathrm{Gbps}$ & $<1 \mathrm{msec} / \mathrm{hop}$ & $2-4 \mathrm{Km}$ \\
\hline Satellite & LOS & $15 \mathrm{Mbps}$ & $50 \mathrm{Mbps}$ & $\begin{array}{l}30 \mathrm{msec} \text { one } \\
\text { way latency, 5- }\end{array}$ & $\sim$ ubiquitous \\
\hline $\begin{array}{l}\text { mmWave } \\
60 \mathrm{GHz}\end{array}$ & LOS & $1 \mathrm{Gbps}$ & $1 \mathrm{Gbps}$ & $200 \mu \mathrm{msec}$ jitter & $1 \mathrm{Km}$ \\
\hline $\begin{array}{l}\mathrm{mmWave} 70- \\
80 \mathrm{GHz}\end{array}$ & LOS & $10 \mathrm{Gbps}$ & $10 \mathrm{Gbps}$ & $65-350 \mu \mathrm{sec}$ & $3 \mathrm{Km}$ \\
\hline
\end{tabular}




\begin{tabular}{|l|l|l|l|l|l|}
\hline $\begin{array}{l}\text { Sub 6GHz 800 } \\
\text { MHz-6GHz }\end{array}$ & NLOS & 170 Mbps & 170 Mbps & $\begin{array}{l}5 \text { msec single } \\
\text { hop 1 way }\end{array}$ & $\begin{array}{l}1.5-2.5 \mathrm{Km} \\
\text { urban, 10 Km } \\
\text { rural }\end{array}$ \\
\hline $\begin{array}{l}\text { Sub 6 GHz } \\
2.4,3.5,5 \mathrm{GHz}\end{array}$ & NLOS & $150-450 \mathrm{Mbps}$ & $150-450 \mathrm{Mbps}$ & $2-20 \mathrm{msec}$ & $250 \mathrm{~m}$ \\
\hline FSO & LOS & $10 \mathrm{Gbps}$ & $10 \mathrm{Gbps}$ & low & $1-3 \mathrm{Km}$ \\
\hline
\end{tabular}

Table 2.4 Wireless backhaul solutions [JAB-16]

Since VVLC networks are expected to support timely transfer of critical safety data, use of microwave technology, mmWave and FSO employing lasers are all possible options for backhaul implementation.

\subsection{Comparative Commentary between VLC and mmWave}

VLC possess several advantages that are crucial for 5G such as high capacity, high data rate, high spectral efficiency, low battery consumption, high energy efficiency and low latency. It is due to these benefits that VLC has emerged as an alternative technology to mmWave in shortrange communication for the approaching $5 \mathrm{G}$ era. VLC can be employed in both indoor and outdoor environments. It can be successfully used for localization techniques, D2D communication, V2I by utilizing traffic signals and lamp posts as infrastructures and vehicle head and taillights. Most importantly, it is easily usable in electromagnetic-sensitive environment such as hospitals, underground mines, airplanes and even underwater communication. VLC exhibit similar advantages and disadvantages as mmWave such as availability of huge spectrum, ability to support data rate at rates of tens of Gbps, easy implementation of spectrum reuse, highly secure and interference-free transmission while on the other hand suffers from shadowing, link blockages, and attenuation in adverse weather conditions such as snow, rain or fog. Similar to mmWave beam alignment, VLC also requires LOS alignment for communication and can be used for high rate data transfer for short distances.

However, in some aspects VLC has a definite edge over mmWave communication. First and foremost VLC is much more cost efficient as it employs LED for transmitter and photodiodes as receivers. Lighting infrastructure is increasingly adopting LEDs due to the low cost and energy 
efficient characteristics of the technology. Hence the infrastructure for both indoor and outdoor VLC communication already exists and can be placed in service at a minimal additional cost and practically no use of additional power, whereas a mmWave communication system would require novel transceiver and base station architectures both to be equipped with steerable, highly directional arrays of antennas. This would translate to very high deployment and maintenance cost. Additionally, VLC spectrum is richer and licence-free compared to mmWave spectrum. It has the bandwidth capacity to support communication requirement for devices even beyond $5 \mathrm{G}$. VLC can also co-exist with other radio technology with no interference whereas mmWave cells require narrow beamforming architecture for interference-free transmission. However, if the cell density in mmWave cellular networks increases beyond $120 \mathrm{BSs} / \mathrm{km}^{2}$, then a BS can experience interference from neighboring BSs [REB-16]. Moreover, it is expected that the mmWave spectrum will be shared among different operators due to the availability of around $270 \mathrm{GHz}$ bandwidth i.e. 30-300 GHz. The spectral bands can be exclusively assigned to the operators or shared through a common pool. In case of common pool, same bandwidth will be used by different operators hence resulting in co-channel interference [REB-17]. Comparison between mmWave and VLC is shown in table 2.5.

\begin{tabular}{|l|l|l|}
\hline & \multicolumn{2}{|c|}{ mmWave } \\
\hline Spectrum & $30-300 \mathrm{GHz}$ & $385-790 \mathrm{THz}$ \\
\hline License & Partly free & Free [HAA-19] \\
\hline Safety & Medium & High \\
\hline Cost & High & Low \\
\hline Power Consumption & High & Low \\
\hline Range & $100-200 \mathrm{~m}$ & $\sim 50 \mathrm{~m}$ \\
\hline Susceptibility to Shadowing & Significant & Significant \\
\hline Requires beamforming & Yes & No \\
\hline Interference & Yes for cell density beyond 120 BSs/km ${ }^{2}$ & Solar in outdoor communication \\
\hline Co-exist with other radio technologies & Yes & Yes \\
\hline LOS required & Yes & Yes \\
\hline Stand-alone technology & No & No \\
\hline
\end{tabular}

Table 2.5 Comparison between mmWave and VLC Communication 


\subsection{Mobility Management for 5G Vehicular Networks}

5G mobility management solutions are expected to cater a vast range of speediness from extremely high mobility, like airplanes and bullet trains, to exceptionally low mobility like pedestrians. In this context, the deployment and operational environment plays a pivotal role in devising the optimum solution according to the mobility requirement [FAN-16]. 5G vehicular networks will have very profound requirement of reliable and seamless connectivity. The fundamental challenges in designing mobility management solution in high speed vehicular networks are: (1) frequent handoffs due to ultra-cell densification, (2) maintaining QoS support while roaming through different small cells and (3) minimizing signalling overheads with quick decisive operations for example, optimum cell selection. A number of new architectures and techniques are proposed for $5 \mathrm{G}$ networks to support the latency and bandwidth requirement in highly mobile environment. Some of these architectures such as cloud radio access networks (CRANs)[CMR-11] improves the deployment environment and transmission efficiency by supporting the transmission of high speed signals over longer distances by utilizing large scale centralized architecture. CRANs are composed of centralized base band unit (BBU) also known as BBU pool where individual units are kept together with interconnections between them, remote radio head (RRU) networks that provide radio connectivity between access points and users, and high speed front haul to provide connectivity between BBU pool and RRUs. The front haul can be implemented according to Common Public Radio Interface (CPRI) standard utilizing optical fiber supporting Coarse/Dense Wavelength Division Multiplexing (CWDM/DWDM) technology, cellular communication or mmWave. Any base band unit (BBU) in the BBU pool can interact with another unit with very low latency and high bandwidth due to their physical layer interconnections. CRANs can be used for $5 \mathrm{G}$ high speed network deployment. Multiple RRUs can be connected to one BBU to reduce handoff frequency and packet loss during handoffs. CRAN platforms can also dynamically allocating resources to various BBUs providing connectivity to several RRUs by taking network load and topology into consideration. For example, when vehicles are moving along the highway exchanging information through RRUs connected to different BBUs located in a centralized BBU pool. However, resource allocation to ensure highest QoS through a common BBU pool comes with its own challenges. Various publications exists in open literature that are focused on the trade-offs between resource allocation and energy/power consumption as well as operational cost and front haul capacity 
limitations in CRANs. Figure 2.6 shows CRAN architectural configuration in context of 5G vehicular network. CRANs supports real time network virtualization functionalities using open platforms where hardware and software can be provided by separate vendors. This is a major migration from traditional BBUs that were implemented using vendor specific customized and integrated hardware/software combination. The real time virtualization enables on-demand resource allocation to BBUs according to the network load and configuration.

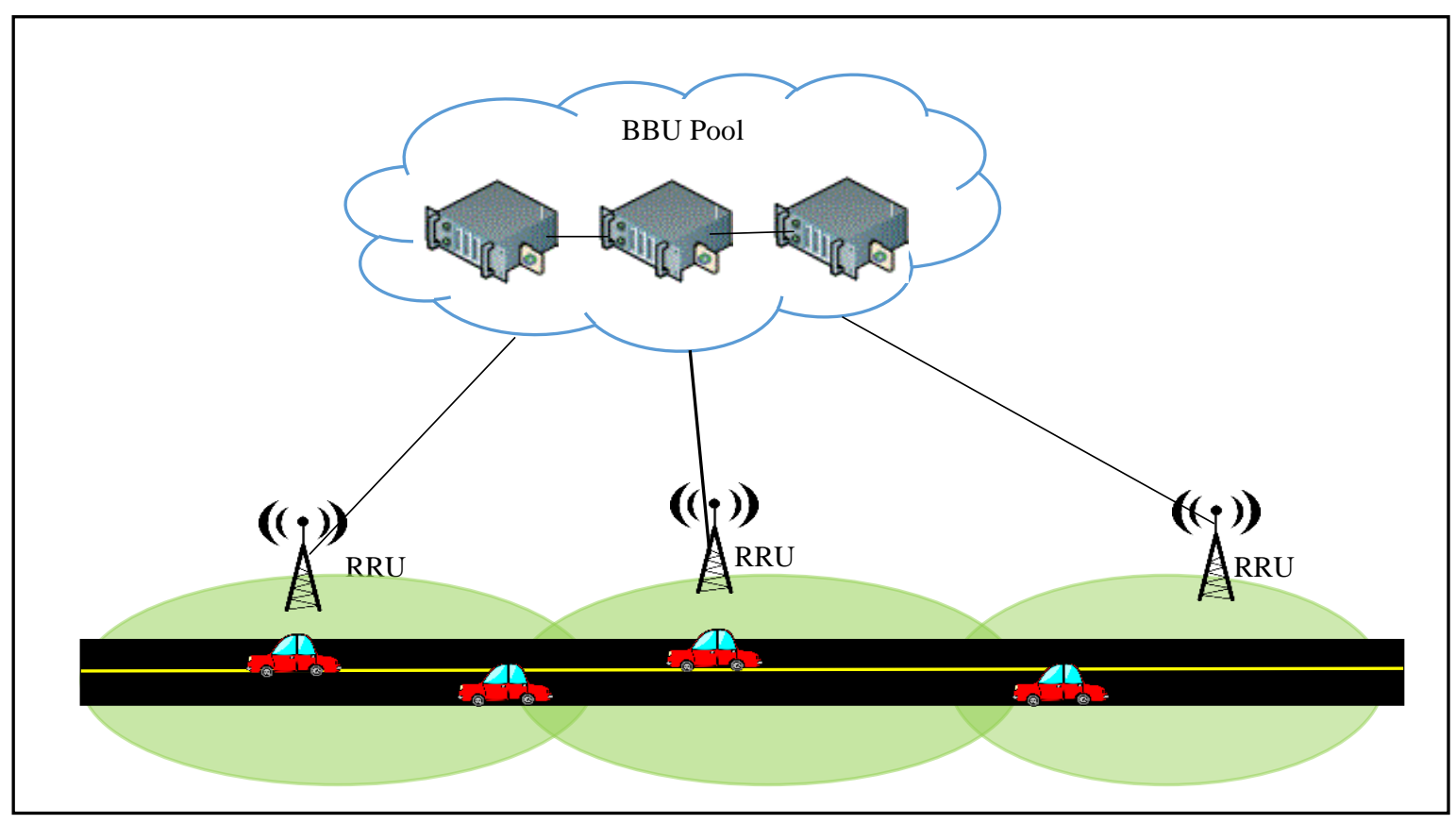

Figure 2.6 CRAN architecture for $5 G$ vehicular networks

Various emerging techniques such as control and user plane (C/U-plane) decoupling [LIN-16], where packet forwarding is assigned to the user plane (U-plane) and all of the mobility management functionalities are transferred to control plane, use of mobile relays [3GP-14] and mobile cells to make handoff transparent to the devices, and geo-aided fast handover where handoff decisions are made on geographical location in conjunction with CRANs can be adopted to provide an effective and efficient mobility management solution for $5 \mathrm{G}$ high mobility users. These prominent mobility management techniques are discussed below.

\subsubsection{Control and User Plane Decoupling}

The decoupling of control plane (C-plane) and user plane (U-plane) is strongly proposed for the future 5G network architecture. In this architecture, U-plane is utilized for user centric 
communication that is exchange of data packets, while the control signalling intended for network resource provision, access mechanisms and link maintenance is transmitted on the Cplane. If we consider a highly dense small cell vehicular network, vehicles would require handoff every few seconds while travelling, possibly resulting in frequent session interruptions, as the users would be crossing the cell boundaries frequently. With decoupled C/U plane, it is possible that the wider coverage area, served by a macro base station, is responsible for control and handoff signalling while the small cells are responsible only for data transmission. This decoupling of C-plane and U-plane can enhance the mobility management since handoff signalling load is shifted from small cells to a more stable macro base station. [WAN-17A] proposes two different network architectures with $\mathrm{C} / \mathrm{U}$ decoupling in centralized and distributed fashion to provide mobility management solution for ultra dense and high speed data traffic. Both architectures were compared in terms of handoff latency, handoff signalling load, packet delivery rate and general signalling load with centralized scheme performing better than the distributed configuration. In the centralized configuration, the U-plane consisting of APs and Cplane consisting of macro BS are connected to the local access server. The local access server consists of two modules: local service center (LSC) and local data center (LDC). The local service center is responsible for handoff initiation, new AP selection, handoff signalling and back haul maintenance whereas data forwarding to the internet is handled by LDC which concentrates the traffic from SC APs and forward it to the core network. In the distributed configuration, the APs along with LSC carry out both C-U and U-P functionalities.

\subsubsection{Mobile Relays and Mobile Cells}

Mobile relays (MRs) [3GP-14] are considered a solution to streamline handoffs in one hop architecture where each mobile node connects to the base station directly and each session needs to be handed off separately when the cell boundaries are crossed. These mobile relays are mounted on top of public buses, high speed trains and cars and stores collective channel state information (CSI) feedback from user devices within the vehicle [STE-12][WLI-12]. In this configuration, only the MR perform handoff with the stationary BS while maintaining connection to the user devices. Hence making the handoff transparent to the user devices. Mobile cells extends the concept of mobile relays by integrating it with small cell technology. 
It introduces the concept of moving network where each small cell is capable of changing its connection to the core network by changing its location. Similar to mobile relays, the mobile cells are deployed on buses, trains and cars and serves as an AP to offer connectivity to the users within the vehicle [CHE-14]. Mobile cells also reduce the handoff signalling overhead in the network. However, frequent movement will result in extensive registration signalling load.

\subsubsection{Geo-Aided Fast Handover}

In vehicular networks, the direction and speed of the vehicles are predictable. In such cases, the geographical information can be effectively utilized to initiate handoff. In such schemes, multiple radio units or APs are centrally controlled by a single baseband unit (BBU) in CloudRAN architecture. GPS receivers or sensors can be used to trace the vehicle's trajectory and the BBU selects and switches the AP based on the geographical information. However, these scheme have a single point of failure in the form of BBU and needs to include back up mechanism otherwise, in case of failure of central BBU, the packet loss may increase significantly and hence QoS can be highly effected.

In general, a number of challenges related to modelling, designing and performance evaluation of high mobility vehicular environment must be overcome in $5 \mathrm{G}$ networks in order to offer a fitting mobility management solution. We argue that MPLS based packet forwarding, handoff procedures and QoS provisions can be easily extended and merged with 5G network architecture and techniques such as $\mathrm{C} / \mathrm{U}$-plane decoupling, extending small cell range by merging multiple radio units coverage area, and hierarchical network configuration to devise a micro-mobility solution capable of supporting high speed 5G vehicular networks. As an example, we can consider software defined networking (SDN) in the context of future 5G networks. SDN is already pursued as an attractive solution to provide $\mathrm{C} / \mathrm{U}$ plane segregation in $5 \mathrm{G}$ networks. SDN utilizes a central controller to carry out C-plane functionalities. Packet forwarding in SDN is not done by looking up destination address in IP header but is rather focused on data flows that are identified by collectively considering several fields in the packet headers. The SDN controller using OpenFlow (OF) protocol defines the rules that are used to forward per flow packets based on five tuple (source address, destination address, source port, destination port, and protocol). The SDN controller can dynamically change the forwarding rules on each packet flow by 
updating the forwarding tables at SDN switches. To extend SDN to wireless networks, base stations have to be equipped with OpenFlow switches with OpenFlow protocol running on them in order to communicate with the central controller. MPLS is looked up as key SDN enabler [SZA-15]. The current version of OpenFlow protocol v1.5 [OPF-15] is capable of handling MPLS labels. [GAR-16] presented 5G architecture for mobile networks in which the U-plane is connected to the SDN controller through a regional router (RR). Each base station in the U-plane is connected to the regional router through OF switches. The packet forwarding in this access domain is done by enabling MPLS on OF switches and RR. Figure 2.7 shows the proposed architecture. Similarly, [DCS-18] presents a simulative study where MPLS is implemented across the SDN-enabled core networks. Hence, it can be recognised that the MPLS model is complementary to fundamental features of future 5G network. In a nutshell, MPLS model supports:

- Separation of control signalling and data forwarding.

- Separation of service from transport.

- Minimizing the formation and maintenance of long routing tables on each router.

- Fast and efficient packet forwarding by using labels on packet headers and signalling to the forwarding paths.

In the following sections, existing MPLS schemes are surveyed in context of QoS provisions and low handoff latencies that are key requirements of future $5 \mathrm{G}$ vehicular networks. 


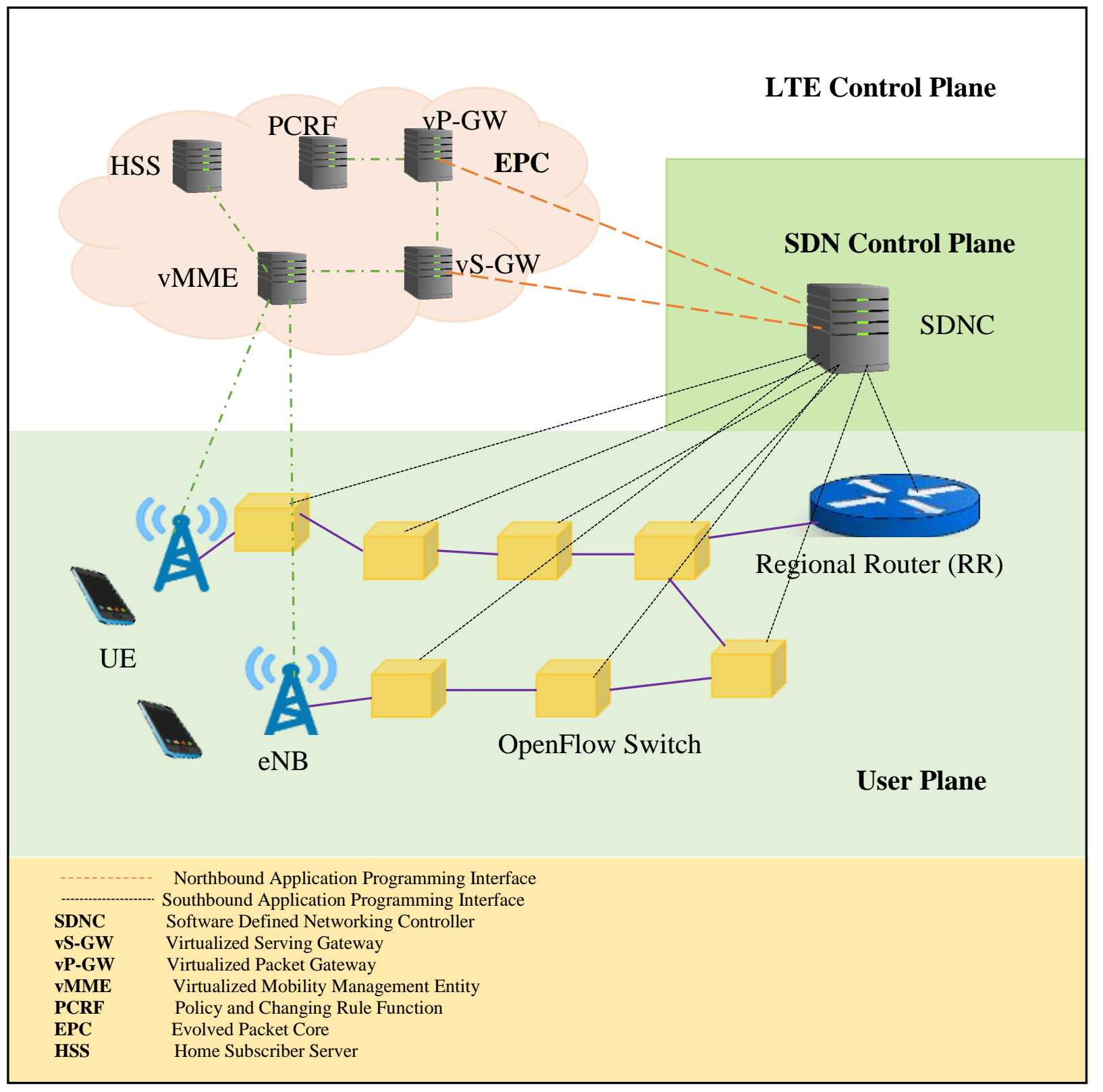

Figure 2.7 SDN based architecture with MPLS in user plane (reproduced from [GAR-16]) 


\subsection{QoS Supporting Technologies}

The International Engineering Task Force (IETF) has developed several standards to meet the ever-growing demand of supporting QoS over Internet. MPLS, Integrated Services (IntServ) and Differentiated Service (DiffServ) being the most distinguished architectures. These solutions were initially defined for only wired backbone networks. However, with the constant increase in mobile data traffic, upcoming era of connected smart cities and enhanced requirement for reliable and fast network for specific and critical services such as telemedicine, these architectures are progressively extended to support the access network as well. In the next sections, a comprehensive literature review is provided to overview various QoS solutions that are devised by academia and industry by merging and extending MPLS [VIS-01], IntServ [CLA94] and DiffServ [DAV-98] architecture to provide seamless support to edge network.

\subsubsection{Multiprotocol Label Switching \& Traffic Engineering}

Multiprotocol Label Switching (MPLS) [VIS-01] is a vital Internet based technology that facilitates packet forwarding in an IP network by implementing fast label look-up based packet switching. It offers a range of IP-based services such as virtual private networks (VPNs), class of service (CoS) based forwarding and traffic engineering. MPLS protocol resides between TCP/IP layer 2 and 3 and is often addressed as layer 2.5 protocol. It can operate over any layer 2 network, e.g. ATM, frame relay, IEEE 802 networks, etc. When an IP packet enters an MPLS enabled network, it obtains an MPLS header, which is inserted between the link layer's header and the Internet layer's header. Figure 2.8 shows the MPLS shim header. The 4-byte header contains a 20-bit label field, a 3-bit field for experimental use, 1-bit stack field, and 8-bit time to live (TTL) field. The label identifies the forwarding equivalence class (FEC) to which that packet is assigned and has only local significance inside that MPLS domain. A set of packets belonging to the same FEC are forwarded over the same path. The 3-bit EXP field is designated for experimental purposes and has been used in conjunction with the DiffServ [DAV-98] architecture to enable QoS support in MPLS networks. The stack field indicates the order of the label in MPLS label stacking, when more than one label header is attached to a single IP packet. This stack bit is set to 1 if the label is at the bottom of the stack and is set to 0 otherwise. TTL field in the MPLS label is similar to the TTL field in IP headers as it is copied from the IP TTL field. 
There are two types of routers in MPLS domain: label edge routers (LERs) and label switching routers (LSR). The LER acts as both ingress and egress routers. Each MPLS-enabled router maintains a local packet forwarding database called label forwarding information base (LFIB). Each entry in the LFIB consists of an incoming label, an outgoing label, an incoming port number and an outgoing port number. An ingress (LER) performs packet classification, assigns an initial MPLS label to each packet and sends it out to the appropriate output port. An egress LER accepts the incoming MPLS packets, strips off the MPLS label and forwards the packet using IP routing.

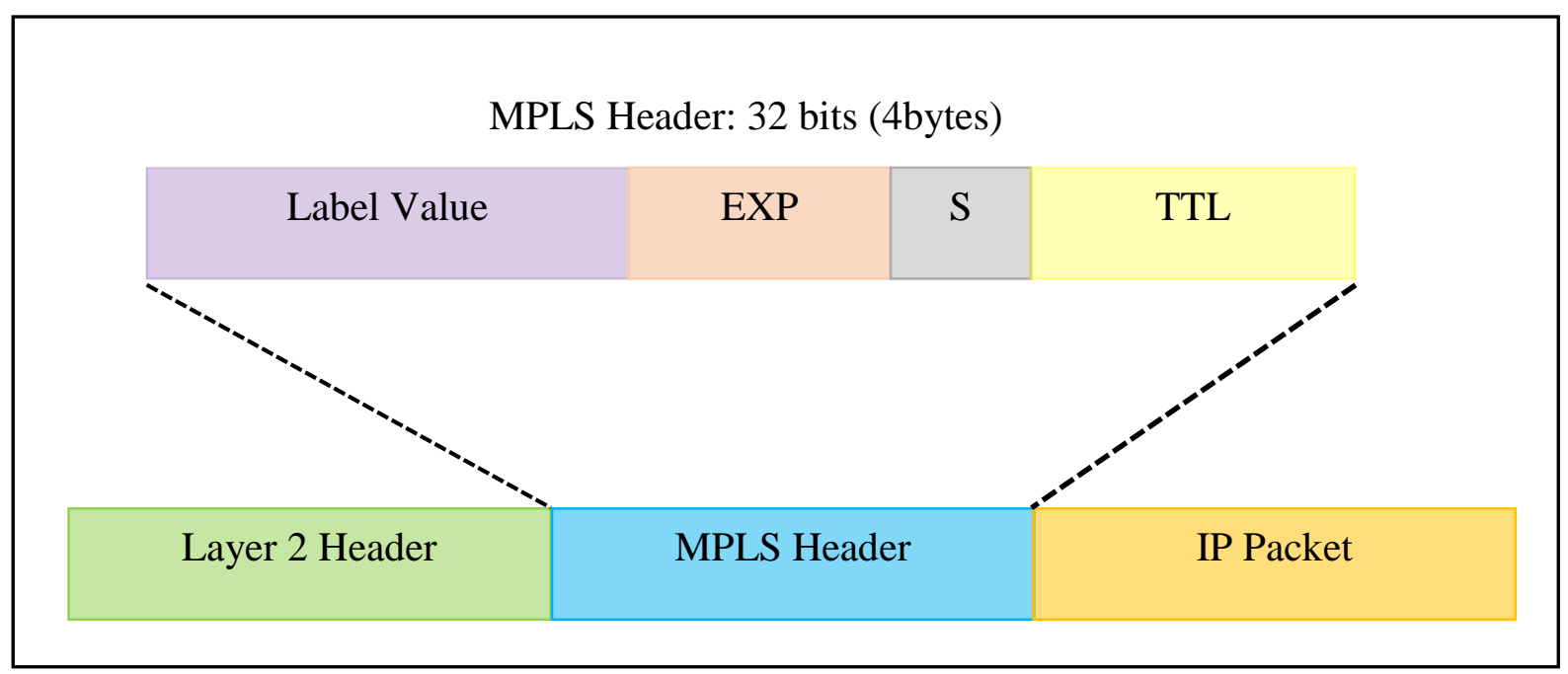

Figure 2.8 MPLS Header

The LSRs are intermediate routers that use the incoming label and the input port number to retrieve the outgoing label and the outgoing interface from the LSR's LFIB, swap the incoming label with the outgoing label, and direct the packet to the selected outgoing interface for transmission to the next hop. The packet routing paths between the ingress and the egress LSRs are called the label switching paths (LSPs).The LSPs are setup by utilizing a number of path discovering, resource reservation and label distribution protocols such as OSPF [MOY-98], RSVP [ZHA-97], and LDP [MIN-07]. These LSPs are used for label distribution and packet forwarding. Traffic Engineering encompasses scientific measurements, modelling, distribution and control of traffic flow through a service provider's network while optimizing the performance of the network. MPLS based Traffic Engineering (MPLS-TE) [CET-11] employs 
constrain-based routing in order to fully utilize available network resources and achieve optimized traffic performance. Traditional IP routing techniques are based on the optimization of one scalar metric, for example, OSPF [MOY-98] calculates the route with least hops between source and destination. Constrain based routing is not based on one scalar metric but adheres to a set of constrains while calculating the optimum route. Hence it is possible to efficiently utilize the available resources such as bandwidth allocation in conjunction with the shortest number of hops. Since in MPLS the packet forwarding is done entirely based on labels, explicit paths can be created between source and destination using exclusive signalling techniques such as RSVPextension or CR-LDP that includes bandwidth allocation and instructions about packet forwarding through specific hops/routers.

\subsubsection{Integrated Services}

Integrated Service (IntServ) architecture was put forward by IETF [CLA-94] to support real-time applications by providing service guarantees. These guarantees are achieved by explicit resource reservation for each flow at each router hop between sender and receiver. IntServ model utilizes a soft state signalling mechanism, RSVP for per flow resource reservation at each router in the path. In RSVP, the sender initiates resource reservation by originating a PATH message. This PATH message includes the traffic characteristics also known as TSPecs. When the packet reaches the destination, the receiver performs resource reservation and responds with RESV message. Both PATH and RESV messages are routed through the network as IP packets. The intermediate routers that forward the RESV message back to the sender also perform resource allocation. If any router does not have the asked resources, it sends a notification back to the sender. Once the RESV message reaches the sender, a resource allocated route has been established. These resource reservation are periodically refreshed by PATH messages. IntServ models supports three service classes: Best Effort, Guaranteed Service and Controlled Load Service.

\subsubsection{Guaranteed Service}

Guaranteed Service [SHE-97] traffic class requires stringent end-to end delay and specific bandwidth allocation guarantees. In perspective of Guaranteed Service, each flow is described using a token bucket. Based on this description, the network can estimate the maximum delay 
each flow will experience utilizing token bucket measurement parameters such as bucket rate, bucket depth and peak rate. The total delay that a packet experiences while traversing a network is composed of three parts: transmission delay, propagation delay and queuing delay. The transmission delay and propagation delay are fixed. Guaranteed Service controls the maximum queuing delay. This service class ensures that traffic flows that adhere to the specified traffic parameters are delivered within the agreed upon delay bounds and are not discarded due to queue overflow.

\subsubsection{Controlled Load Service}

Controlled Load [WRO-97] Service supports applications that require performance equivalent to traditional best effort traffic class under lightly loaded network condition. The token bucket model is also used for controlled load services, however, the difference from guaranteed services is that controlled load services do not provide any delay bounds. The traffic flows that do not adhere to the specified traffic parameters are forwarded as best effort.

The major disadvantage of IntServ architecture is its poor scalability as the resources are reserved per flow at each hop. The fact that RSVP is a soft state signalling mechanism only adds to the scalability issue as it further burdens the network with excessive signalling load.

\subsubsection{Differentiated Services}

Differentiated Services (DiffServ) [DAV-98] architecture was proposed to overcome the scaling issues inherent in IntServ. In DiffServ, real-time applications are supported by aggregating various traffic flows into classified traffic classes. Thus, there is no need for each router in the network to store per flow state or participate in per flow signaling. Hence DiffServ offers scalable solution to QoS critical applications by performing coarse level of traffic classification. To map IP packets into different traffic classes, an alternative header field, called the DS field, is defined in the DiffServ architecture. The DS field supersedes the definitions of IPV4 Type of Service (ToS) octet and IPV6 traffic class octet. In the DS field, 6 bits are used to identify differentiated service code point (DSCP) that will define the per hop behaviour (PHB) of the IP packet at each node. The remaining 2 bits are used for explicit congestion notification (ECN). 
The network can be visualised as divided into multiple domains. Each network domain is under an Internet Service Provider (ISP). In order to support DiffServ, ISPs offer service level agreements (SLA) to the users. SLA are formal contracts that list the details about the agreed upon aspects of services such as maximum and minimum delay, jitter, and throughput according to traffic classes. The edge routers at each domain intercepts the incoming traffic in that domain and are responsible for classification and conditioning (marking, policing and shaping) of the incoming traffic according to the respective SLAs.

The fundamental difference between DiffSer and IntServ lies in two areas. Firstly, DiffServ architecture is class-based instead of flow-based. This makes DiffServ more scalable than IntServ since the amount of information stored at each network node is proportional to the number of classes rather than to the number of flows. Although, the number of classes are limited due to the limited size of DS field. Maximum 64 different traffic classes can be defined with 6 bit DSCP field. Secondly, only the edge routers of the ISP domain are responsible for admission control, classification, marking, policing and shaping of incoming traffic. Once the marked packets enters the DiffServ domain, core routers only perform packet forwarding based on PHB assigned to the corresponding traffic class. PHB specifies the forwarding behaviour at each network node by identifying the bounds of delay, jitter and throughput as well as packet dropping probability. PHB also provides the framework for network node to allocate resources such as buffer space and bandwidth according to different traffic classes. IETF has standardized three different PHBs: Default, Assured Forwarding [HEI-99] and Expedited Forwarding [DAV02].

\subsection{Mobility in IP Networks}

Mobility management is an essential constituent of IP networks. With the upcoming era of 5G and Heterogeneous Networks (HetNets), it is necessary that user mobility between various subnets is supported seamlessly independent of the underlying technology. Traditionally, mobility in IP networks can be classified into two categories: Macro-mobility that is concerned with mobility management of user between two distant wide area wireless networks, and micromobility that deals with mobility management locally when user changes the point of attachment within a network domain. 


\subsubsection{Mobile IP for Macro-mobility}

Mobile IP [PER-10] is an IETF standard protocol to manage user mobility. It allows the mobile user to roam between networks while keeping permanent IP address and maintaining ongoing connections. Although, Mobile IP is suitable for macro-mobility, it is inefficient in micro-mobile environment. With high speed mobile users, it is essential that the registration latency and handoff delays are minimized. However, Mobile IP is not optimized for handling frequent changes in point of attachment for a number of users within a short time frame.

Mobile IP describes four different entities: mobile node (MN) that is the roaming user, corresponding node $(\mathrm{CN})$ that is the peer node communicating or trying to establish communication with MN, home agent (HA) that is the router which is the point of contact of the $\mathrm{MN}$ in its home domain and foreign agent (FA) that is the router which is the point of attachment of $\mathrm{MN}$ in the visiting foreign domain. However, presence of FA in the foreign domain is optional as Mobile IP allows a MN to communicate even in the absence of FA.

A MN has a permanent IP address, also called home address, originating from its registration to the HA in its home network. When the MN moves to a foreign domain, it obtains care-of-address (COA) from the foreign network. There are two different types of COA's that can be acquired: the FA's COA, which is the IP address of the FA or the co-located COA, which is a local IP address and is acquired by the MN probably by using Dynamic Host Configuration Protocol (DHCP). The co-located COA is used by the MN if FAs are not deployed in the visiting domain. In case FAs are present in the foreign network, the MN receives agent advertisement message from the FA in the visiting domain. The $\mathrm{MN}$ then sends a registration request message to the $\mathrm{HA}$ using the FA's COA. The HA, upon receiving the registration request, creates a binding entry for the MN with this COA. The HA then sends the registration response to the MN through FA by using this $\mathrm{COA}$. When a $\mathrm{CN}$ initiates communication with the $\mathrm{MN}$, it uses the permanent address of the MN. These packets are intercepted by the HA. The HA checks its binding table and upon finding the binding entry for MN, encapsulates each IP packet within another IP packet with the destination address set as COA of the MN. This packet encapsulation is known as IP-in-IP tunneling. These packets are then forwarded from the HA to the FA, which is the end point of the tunnel and de-encapsulates the IP packets. In the absence of FA in the visiting domain, the 
packets are tunneled to the $\mathrm{MN}$ that performs de-encapsulation. In the reverse direction, $\mathrm{MN}$ sends the packets directly to the $\mathrm{CN}$ without going through the HA. When the MN moves into another subnet, it initiates the handoff process by obtaining new COA and sending another registration request to the HA with the updated COA. Mobile IP does not guarantee that all the packets will be delivered during the handoff process as the registration latency may significantly delay the redirection of packet to the new residing position of MN. Route Optimization (RO) enhancement in Mobile IPV6 has further developed the mobility support of base Mobile IP by allowing the MN to send binding update to the $\mathrm{CN}$. This enables the $\mathrm{CN}$ to send IP packets directly to the MN hence eliminating triangular routing [PER-11A]. Figure 2.9 shows Mobile IP architecture along the message exchange sequence. Figure 2.10 shows the timing flow of messages in Mobile IP between various nodes. 


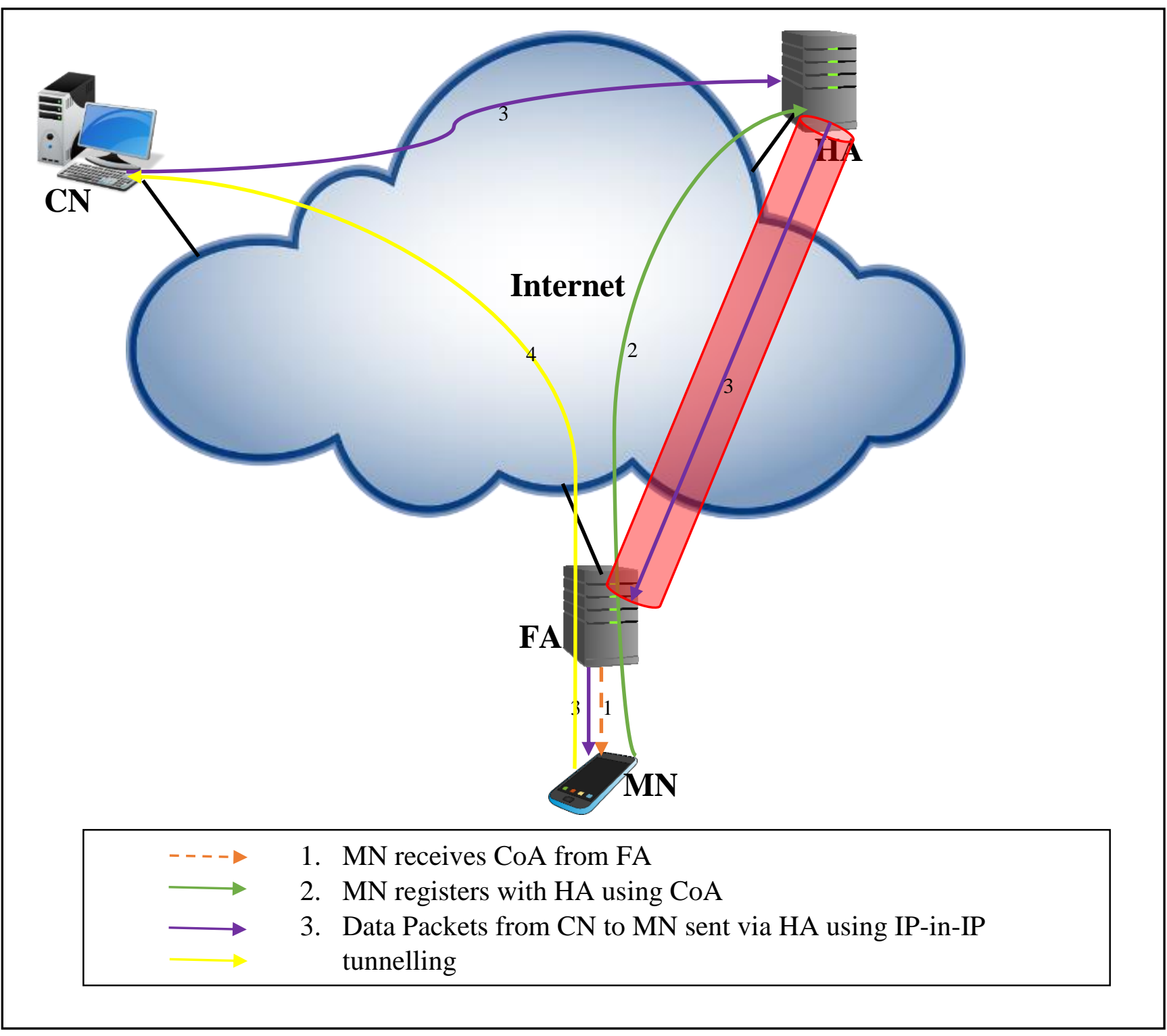

Figure 2.9 Architecture of Mobile IP (as described in [PER-10]) 


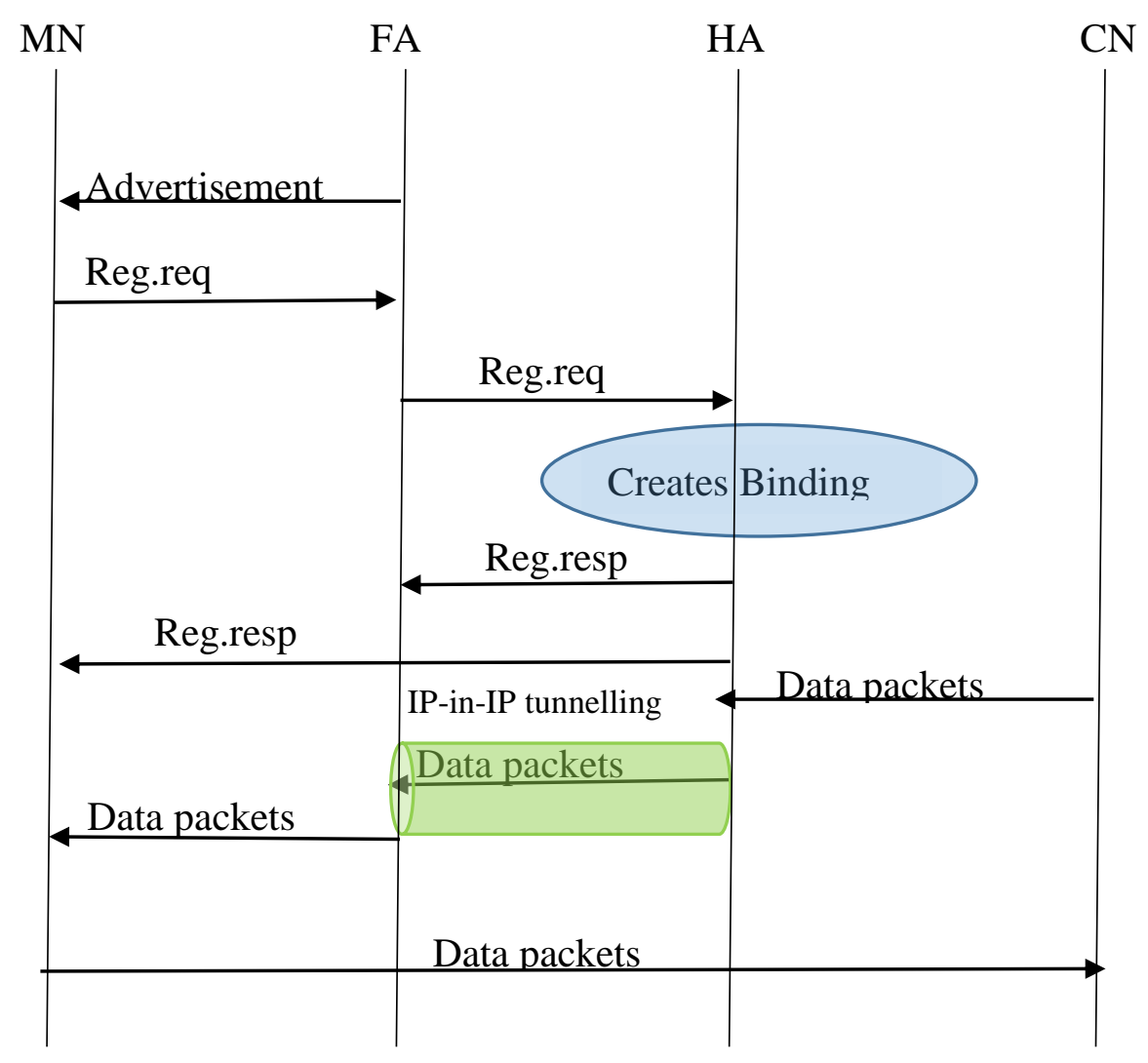

Figure 2.10 Timing flow of messages in Mobile IP

\subsubsection{IP Based Micro-mobility Solutions}

Mobile IP has serious limitations if extended to support micro-mobility. Mobile IP requires that an MN performs a new registration every time it visits a new foreign domain (FD). This may incur unreasonably long registration delay if the FA and HA are at distant locations from each other. Moreover, the signalling load in the network is increased considerably. In an environment with frequent handoffs due to high speed mobility of users and small cell sizes, there is a need for specific protocols that provides mobility management with minimum handoff latencies and low signaling load while maintaining seamless connectivity and fulfilling the QoS requirement of the IP traffic. Various IP micro-mobility protocols have been proposed in the literature (e.g. Cellular IP [CAM-00], HAWAII [RAM-02], and Hierarchical Mobile IP [MAL-00]). Since this work concentrates on MPLS based micro-mobility schemes, IP based micro-mobility solutions are not reviewed in detail in this thesis. 


\subsubsection{MPLS Based Micro-mobility Management}

There has been increasing interest in applying MPLS in wireless access networks. Compared with other micro-mobility schemes, MPLS based micro-mobility schemes have several advantages. It provides the capability to incorporate constraint-based routing and the support of traffic engineering and QoS in wireless access networks. The second is the ease of deployment. Within the wireless access network, only few selected nodes, e.g. gateways, selected hierarchical leaders (routers locally responsible for handovers between subnets), need to be aware of the mobility of the node. Several schemes have been proposed in the literature that use MPLS based micro-mobility solutions for fast handoff and QoS provision. This section provides the literature review of these MPLS based micro-mobility schemes.

\subsubsection{Mobile IP with MPLS}

One of the first schemes in open literature that discusses the integration of Mobile IP with MPLS (MIP-MPLS) was reported in [REN-01]. This is essentially a macro-mobility scheme in which two LSP segments are created: one between $\mathrm{CN}$ to HA and the next between HA to FA. This work assumes that the LSP between CN and HA has already been established. Once the MN arrives in a foreign domain, it acquires $\mathrm{COA}$ from the $\mathrm{FA}$ and sends a registration request to the FA. The FA upon receiving the registration request records MN's address and the incoming interface as the outgoing interface for the $\mathrm{MN}$ in its routing table. The request is then forwarded by FA to HA using traditional IP routing. When the HA receives the registration request, it establishes and LSP between itself and FA by using LDP protocol. Once the LSP is established, the registration response is sent by the HA to the FA. When the FA receives the registration 


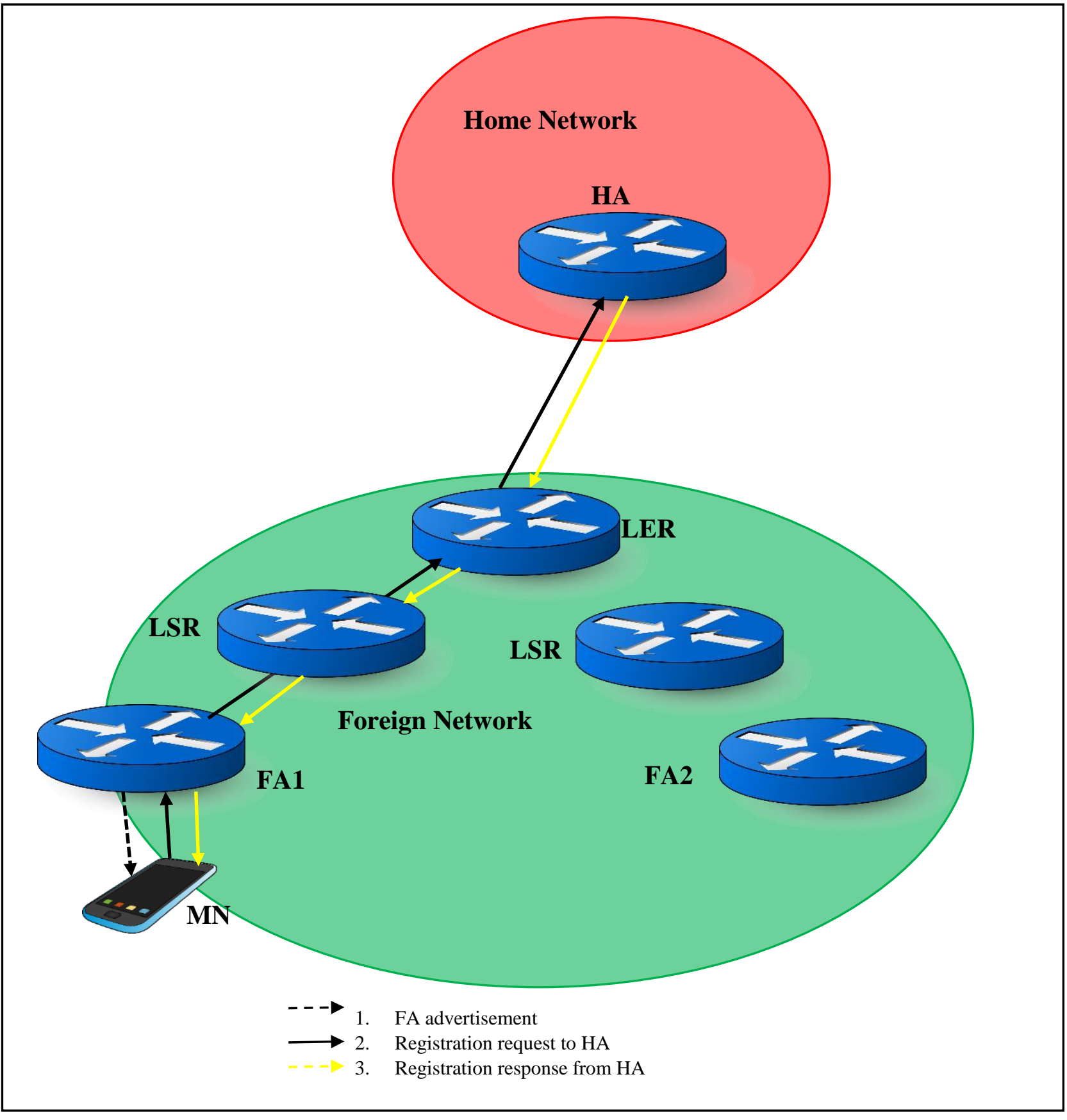

Figure 2.11 Architecture of MIP-MPLS (as described in [REN-01]

response from the HA, it updates its label forwarding information base (LFIB) and forwards the registration response to $\mathrm{MN}$ using IP routing. When the $\mathrm{CN}$ wants to send packets to the $\mathrm{MN}$, they are first sent to the HA using the LSP between CN and HA and are then forwarded to the MN by the HA using the LSP between the HA and the FA. When the MN moves under a new 
FA, the registration process with HA is carried out again and a new LSP is created between the HA and the new FA. Figure 2.11 and 2.12 presents the MIP-MPLS architecture and the timing diagram of message exchange during handoff. Although, this scheme eradicated IP-in-IP tunnelling of the data packets inherent in MIP, it has several drawbacks. One significant drawback is that since the path is re-established from HA to FA after each handoff, this scheme is unsuitable for micro-mobility. Secondly, since the size of forwarding table at HA is directly dependent on the number of roaming MNs, scalability issues may arise.

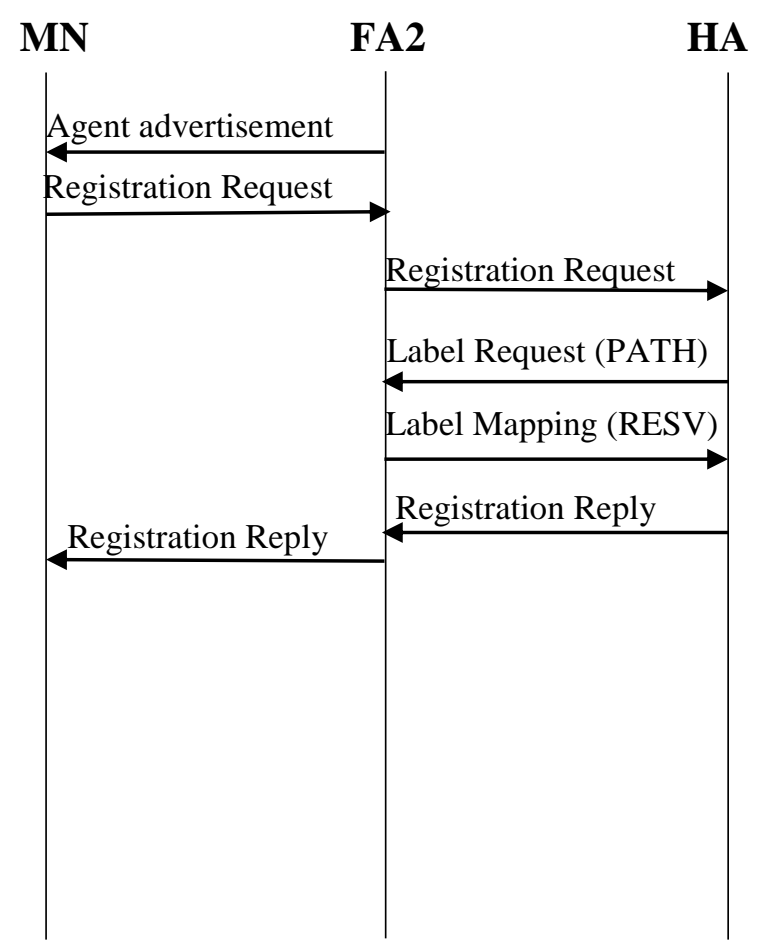

Figure 2.12 Timing diagram of registration/handoff message exchange in MIP-MPLS

\subsubsection{Hierarchical Mobile MPLS}

Hierarchical Mobile MPLS (HMPLS) [YAN-01] is one of the earliest proposals that introduces MPLS based micro-mobility architecture. In this scheme, a Foreign Domain Agent (FDA) is introduced into each MPLS domain to support intra domain mobility. As the name indicates, this is a hierarchical scheme, where FDA is at the top level of hierarchy and foreign agents (FA) at the lowest level of hierarchy serves as the point of attachment for the MN. HMPLS assumes that the LSP between HA and CN has been already established. 
Once the MN discovers that it is in a foreign domain, it acquires a local COA from the FA and sends a registration request message to the FDA through the FA. FDA after updating the binding cache for $\mathrm{MN}$, forwards the registration request to the HA. Upon receiving the registration request message from FDA, the HA sends a label request to the FDA using LDP with FDA's address as the FEC. The FDA responds by sending a label mapping message back to HA. It also sends a label request message to the FA with the FA's address as FEC. When HA receives the label mapping messages, the LSP between HA and FDA is established. Similarly, with the arrival of label mapping message originated from the FA and reaching FDA, the LSP between FDA and FA is established. Finally, the registration response from HA is forwarded through these two LSPs to the FA.

When the $\mathrm{CN}$ send data packets to MS, they are intercepted by HA and are forwarded to FDA through LSPs using label swapping. FDA strips off the packets label and sends the packets to the IP layer. By checking its binding cache, the FDA recognizes the FA which is serving MN. The packets are encapsulated in MPLS headers with FEC set to FA's address. These packets are then forwarded though the already established LSP from FDA to FA. The FA strip off the packet labels and forward them to the MN using IP routing. Figure 2.13 shows the architecture of HMPLS along with message exchange sequence during registration. 


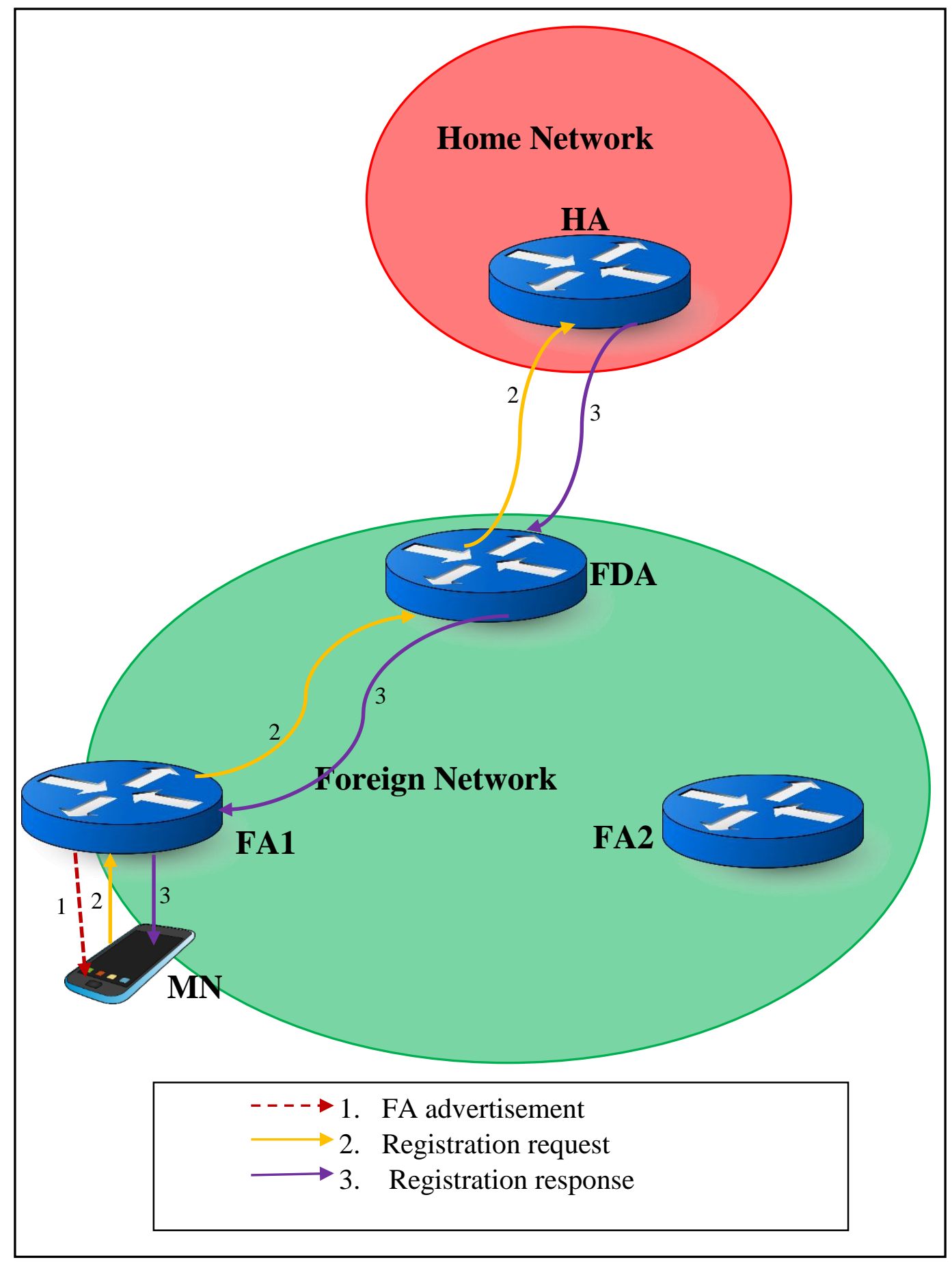

Figure 2.13 Architecture of HMPLS (reproduced from [YAN-01])

Handoff is initiated when the MN moves under a new FA, the registration request is forwarded to the FDA through the new FA. Upon receiving the registration request, the FDA updates its binding cache for the MN and a new LSP is established between FDA and the new FA, leaving 
the LSP between HA and FDA intact. Hence handoff is accomplished locally. Also, this work introduces the concept of active and passive LSPs where, passive LSPs are set up from the FDA to the neighboring subnet that the mobile node is expected to visit. These LSPs become active once the update message is received from the MN. The MN may also send a binding update announcing the new FA's address as COA to the old FA. This enables the old FA to cache the binding entry for MN's new location. In case outdated cache entry is used by the FDA to forward packets for MN, they will wrongfully arrive at old FA. Based on the binding entry for MN, the old FA will create an LSP between itself and new FA serving MN. The packet will then be forwarded to the MN through this LSP. The timing diagram of exchanged messages during handoff in HMPLS scheme is shown in figure 2.14.

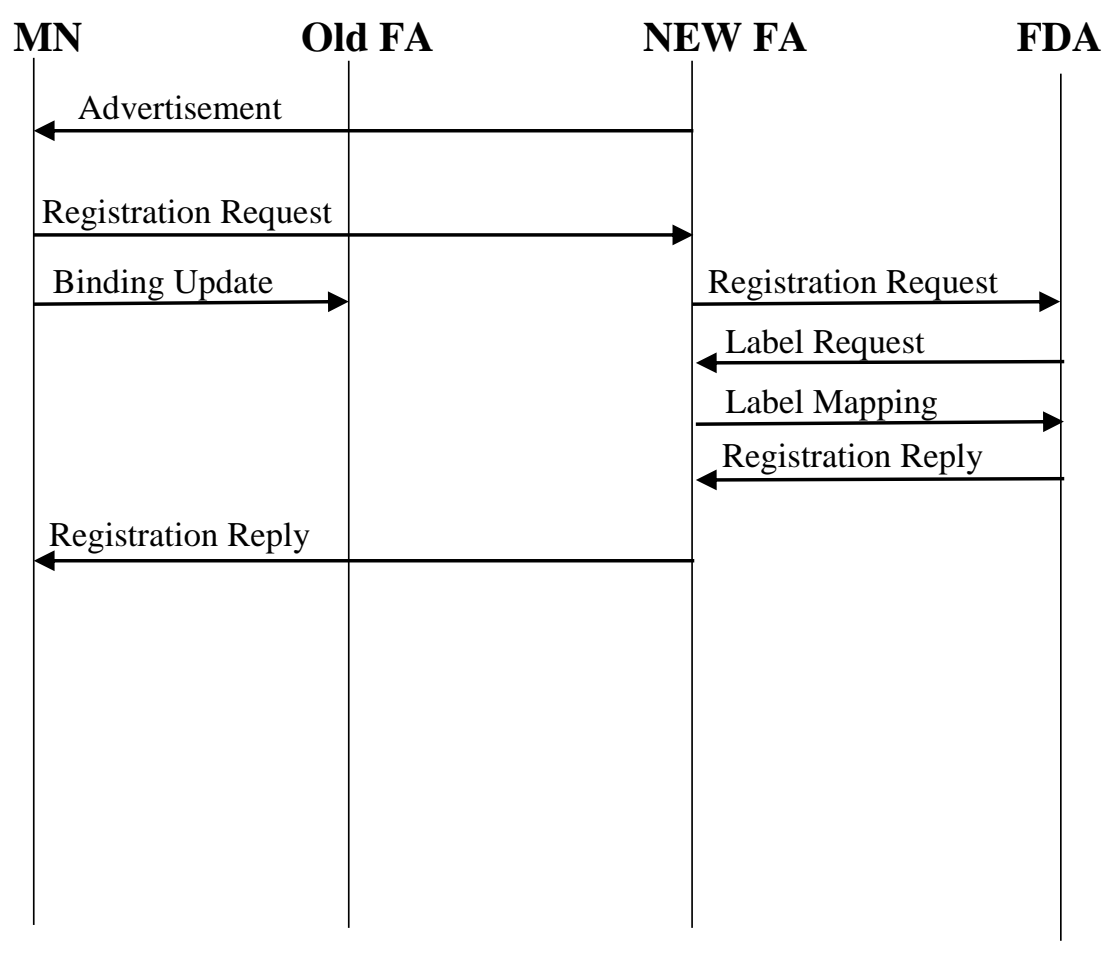

Figure 2.14 Timing diagram of messages exchanged during handoff in HMPLS (reproduced from $[Y A N-01])$

Although, HMPLS scheme supports scalability as the LSPs established between HA and FDA and FDA to FAs can be used by all of the MN under these entities, it fails to provide QoS support as the bandwidth is shared between the MNs. Moreover, only FDA can redirect the LSP 
in case of handoff and depending upon the number of intermediate routers, this delay may become significant. This delay can be reduced if cross over routers can perform LSP redirection.

\subsubsection{Mobility aware MPLS}

The objective of Mobility aware MPLS (MAMPLS) [KIM-01] is to establish a micro-mobility model that integrates micro-mobility management with MPLS, specifically taking advantage of MPLS characteristics that provides IP QoS support. This scheme presents two different LSP configurations: static and dynamic. The static LSPs establishment technique supports DiffServ QoS architectures through MPLS by utilizing the EXP field in MPLS shim header to create EXP-> PHB mappings. Based on EXP value, FECs are mapped to two different LSPs preconfigured according to Assured Forwarding (AF) PHB and Expedited Forwarding (EF) PHB, while the dynamic LSP establishment technique enables QoS support by merging MPLS and per-hop resource reservation using RSVP-TE signalling mechanism according to IntServ architecture.

Mobility Aware MPLS also adopts a hierarchical approach. The network is divided into access domains with a gateway router (GW) residing at the top level of each domain that provides connectivity between the access domain and the internet. Base stations (BS) are at the lowest level of hierarchy serving as point of attachment for the MN. MA-MPLS addresses mobility management within the access domain. When the MN moves between MPLS access domains, it can use either Mobile IP or LSPs if the domains are connected through MPLS mesh.

When the MN initially arrives in the Mobility Aware MPLS access domain, an LSP is created between the HA and GW by adopting similar procedure as in HMPLS. In the DiffServ LSP establishment technique, LSPs are pre-established between the GW and the BS. Since DiffServ model has two main QoS PHB, Assured Forwarding (AF) and Expedited Forwarding (EF), four LSPs (two in the uplink direction and two in the downlink direction) are established. Two more LSPs are created for signalling in uplink and downlink direction. Hence, between the BS and GW there are six pre-established LSPs. Once a MN wakes up in the access domain, it sends a registration request to the $\mathrm{BS}$ with strongest signal. The base station forwards this registration request to the GW using the pre-established LSP for signalling in uplink direction. Upon receiving the registration request, the GW creates a binding entry in its LFIB table associating MN's IP address and the interface from which the registration request was received. The GW 
keeps the MN's IP address in the FEC column associated with the common downlink signalling LSP of the BS. The CN sends marked DSCP packets to the MN. When these packets arrive at the $\mathrm{GW}$, it looks at the DSCP field, assigns an appropriate label and updates the LFIB table by placing the MN's IP address in the FEC field of the pre-established LSP according to the PHB. These packets are then forwarded through the corresponding LSP to the MN. When the MN has packets to send, the connecting BS performs similar LSP mapping operation. In case of handoff, the new BS forwards the registration request to the GW and performs LSP transfer according to the PHB for all of the packets from MN. The GW also performs the LSP transfer in downlink direction. Figure 2.15 and 2.16 shows the DiffServ-MAMPLS architecture and the exchange of messages during handoff. 


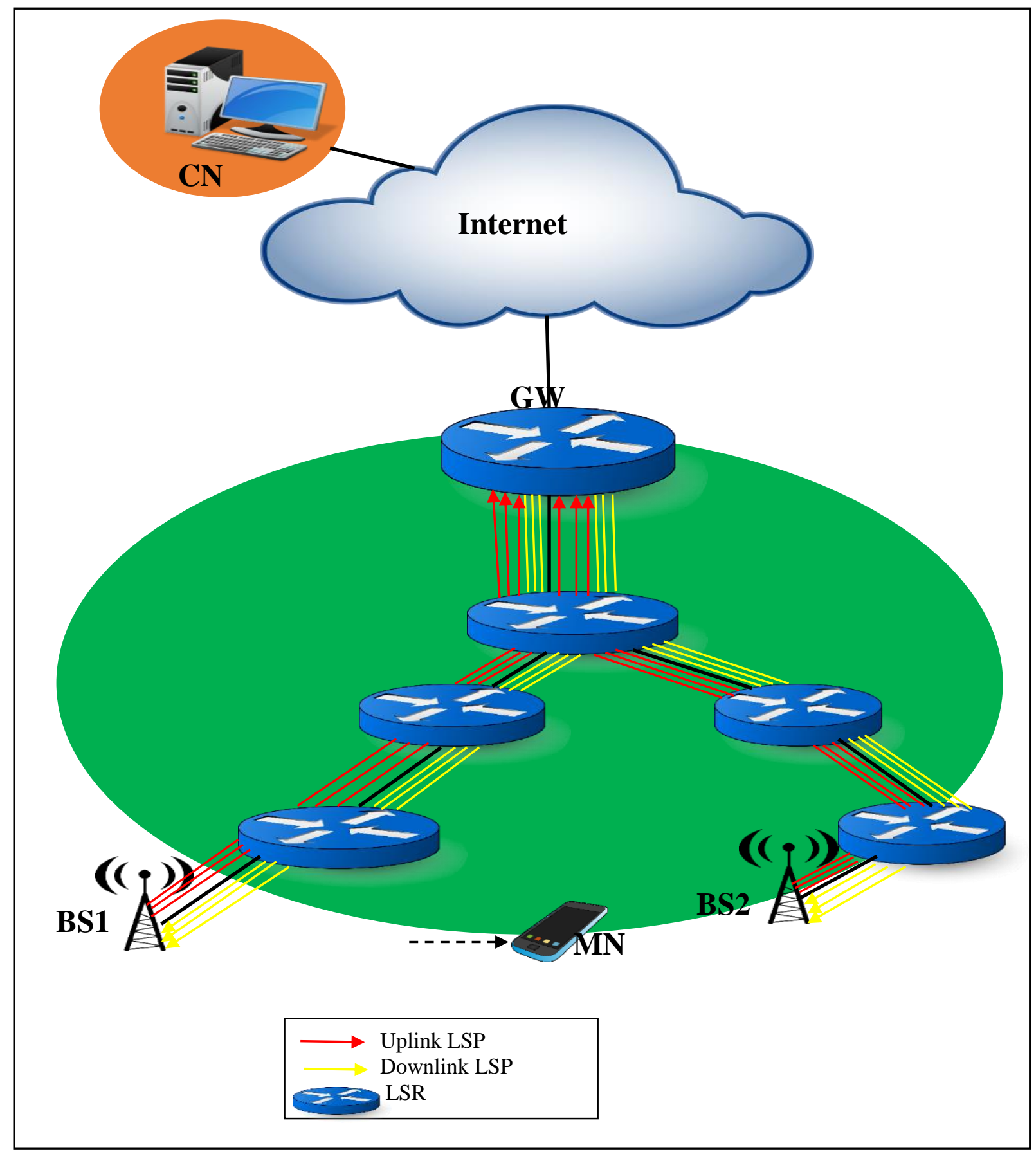

Figure 2.15 Architecture of MAMPLS-DiffServ with static LSPS (reproduced from [KIM-01]) 


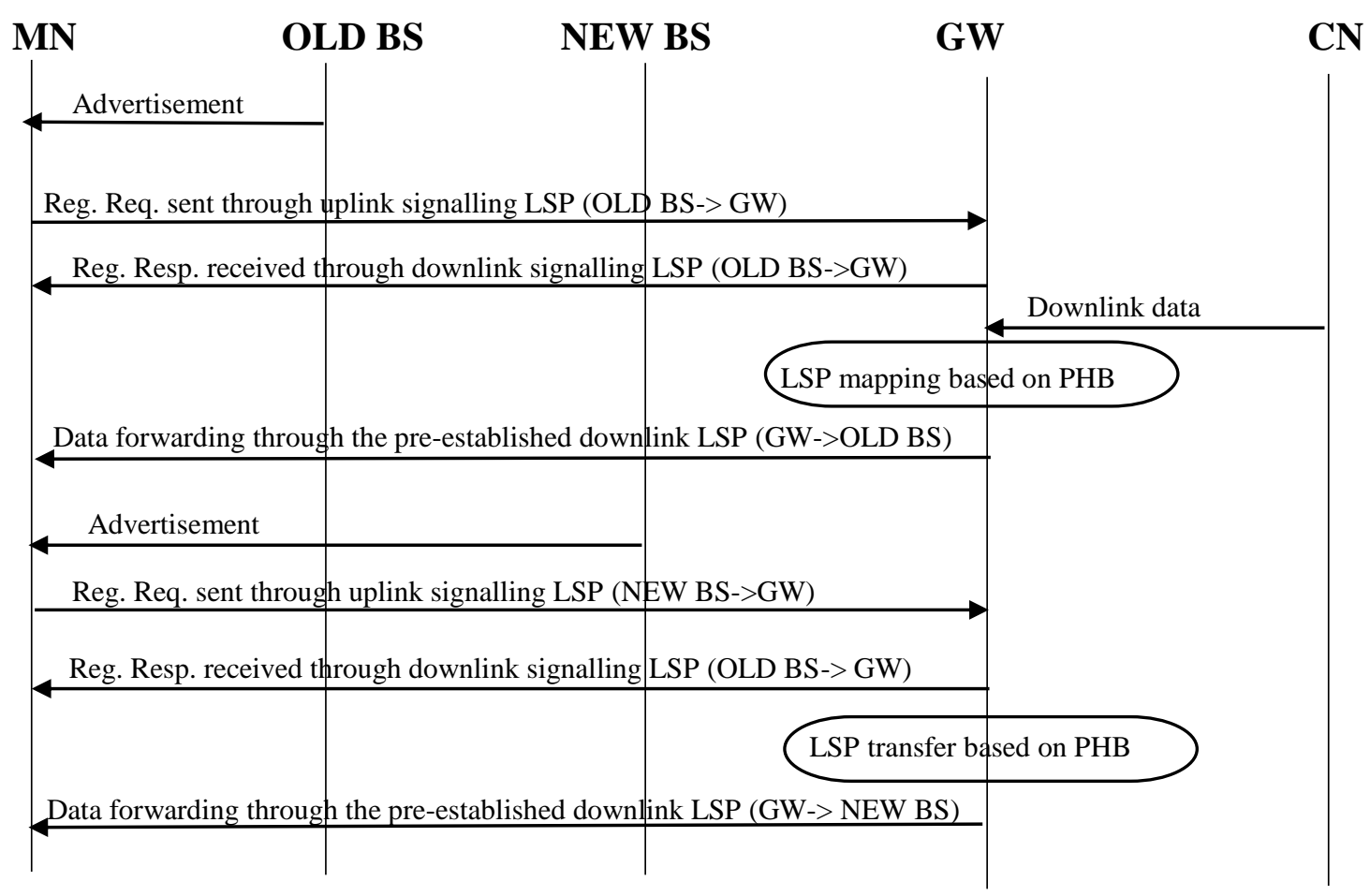

Figure 2.16 Handoff in MAMPLS-DiffServ with static LSPS

For supporting IntServ, a dynamic LSP scheme is introduced. In dynamic LSP scheme, LSPs are created between BS and GW based on per-flow using RSVP signalling mechanism. Two preestablished LSPs exist from GW to the BS for uplink and downlink signalling. When the CN wishes to communicate with the MN, it sends a PATH message. This message is routed to the GW through MN's HA. The GW uses the downlink signalling LSP to forward the PATH message to the MN. Upon reception of PATH message, MN responds back with RESV message through the uplink signalling LSP. For creating flow to label bindings, the scheme introduces the use of LABEL OBJECT. This LABEL OBJECT is carried inside the RESV message. When an LSR wants to reserve resources, it creates a label to be used as incoming label at the input port and sends it upstream inside the LABEL OBJECT in RESV message. Each intermediate node en route to GW performs similar flow to label binding for the MN by creating a LABEL OBJECT and forwarding it to the next hop in RESV message. When the GW receives the RESV message, it uses the LABEL OBJECT in the RESV message to create outgoing label binding with the interface and forwards RESV to the CN. This establishes the downlink LSP corresponding to the 
specific flow. When a packet with appropriate mappings for this specific flow arrives at the GW, the GW first classifies the incoming packet by accessing information in IP and transport header. It then applies appropriate traffic policing and traffic shaping QoS procedures according to the flow specific reservations. Each LSR on the way to MN, forwards the packet according to the QoS requirements specific to the flow by using the label to flow mapping stored in their forwarding tables. Handoff is initiated by the MN by sending a RESV message to the new BS. The new BS forwards the RESV message upstream containing new label in LABEL OBJECT to the GW. Each intermediate router check its routing table to find binding information for the MN. The cross over router (CR) upon finding the binding information, updates its routing table and stops forwarding the RESV message to the next hop. This way traffic is redirected from the CR. Figure 2.17 and figure 2.18 shows the IntServ architecture using dynamic LSPs and the timing diagram for signal exchange during handoff.

There are some major limitations in this scheme. The first issue is in the static LSP technique supporting DiffServ model. In this scheme, the GW updates the routing table by placing MN's IP address in the FEC of the corresponding LSP. This made LSP exclusive to that MN and it can no longer support aggregated traffic which is the purpose of DiffServ architecture. Secondly, in both static and dynamic schemes, the proposed protocol employs common LSPs to route signalling traffic. However, packet forwarding at LSRs through LSPs usually involves scanning the forwarding tables, identifying the mapping, and label swapping according to the corresponding forwarding table entry. Signalling mechanism RSVP, which is utilized in dynamic scheme conveys significant resource reservation information and should be inspected at IP layer instead of being sent through common signalling LSP. Since LSRs cannot support RSVP readily. Although, the scheme uses LABEL OBJECT to map between flow specs, interfaces and labels, it is not elaborated how the labels are used for applying traffic policing/shaping at intermediate LSRs. It is not even clarified how the LSRs in common signalling LSP distinguishes between control messages for example, between a registration request (that should be simply forwarded to $\mathrm{GW}$ ) and a PATH/RESV request (which should be forwarded to the next LSR only after reserving resources and appending a label to the incoming/outgoing interface). Moreover, since the PATH and RESV messages are forwarded along pre-established common LSPs, all of the reservations in both direction are done only on those LSRs along the static LSP. This introduces 
serious scalability issues with increasing number of MNs under a base station in the network. If the CRs are common in various LSPs, they may cause significant bottleneck for traffic as well.

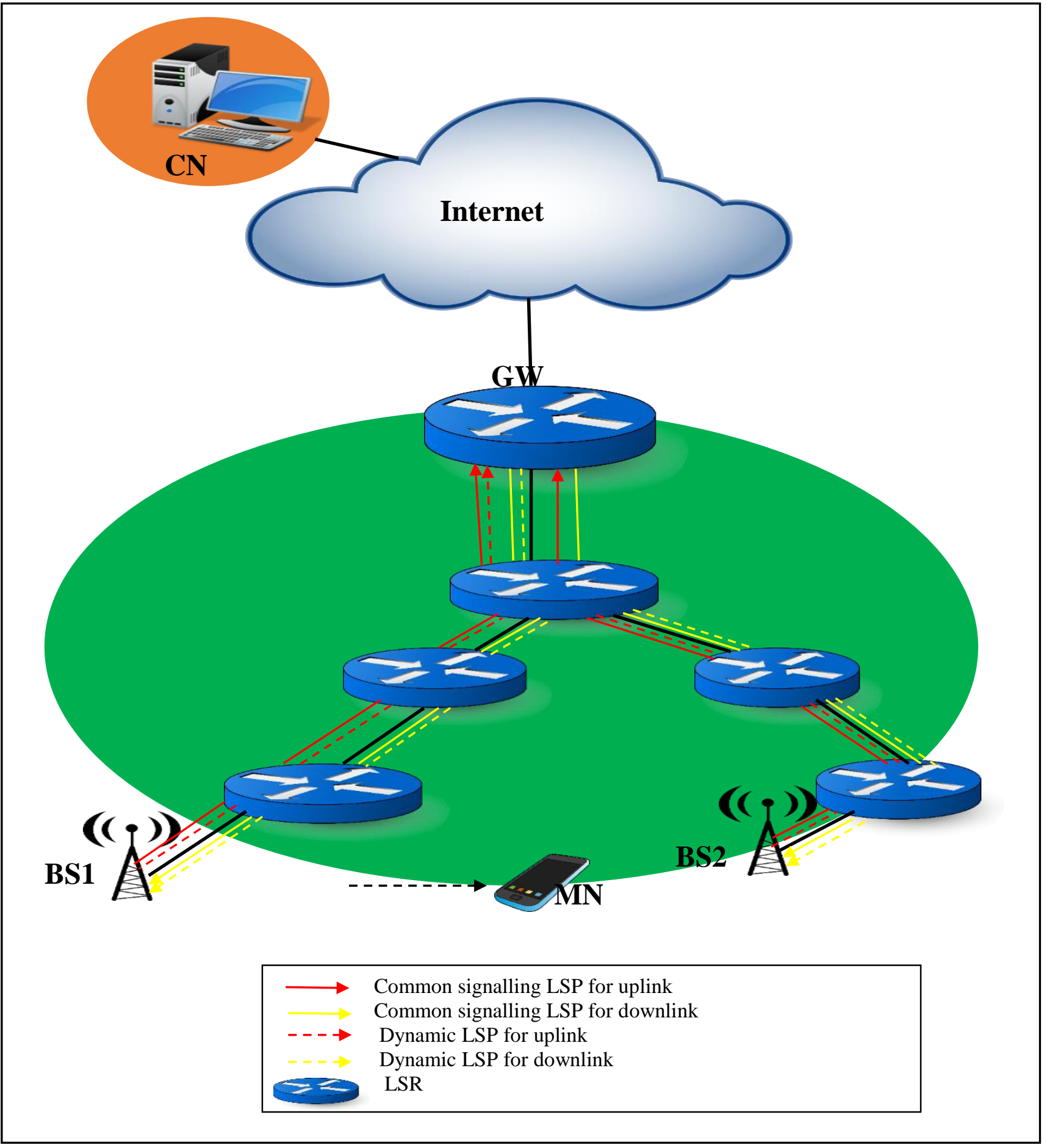

Figure 2.17 Architecture of MAMPLS-IntServ with dynamic LSPs (reproduced from [KIM-01]) 


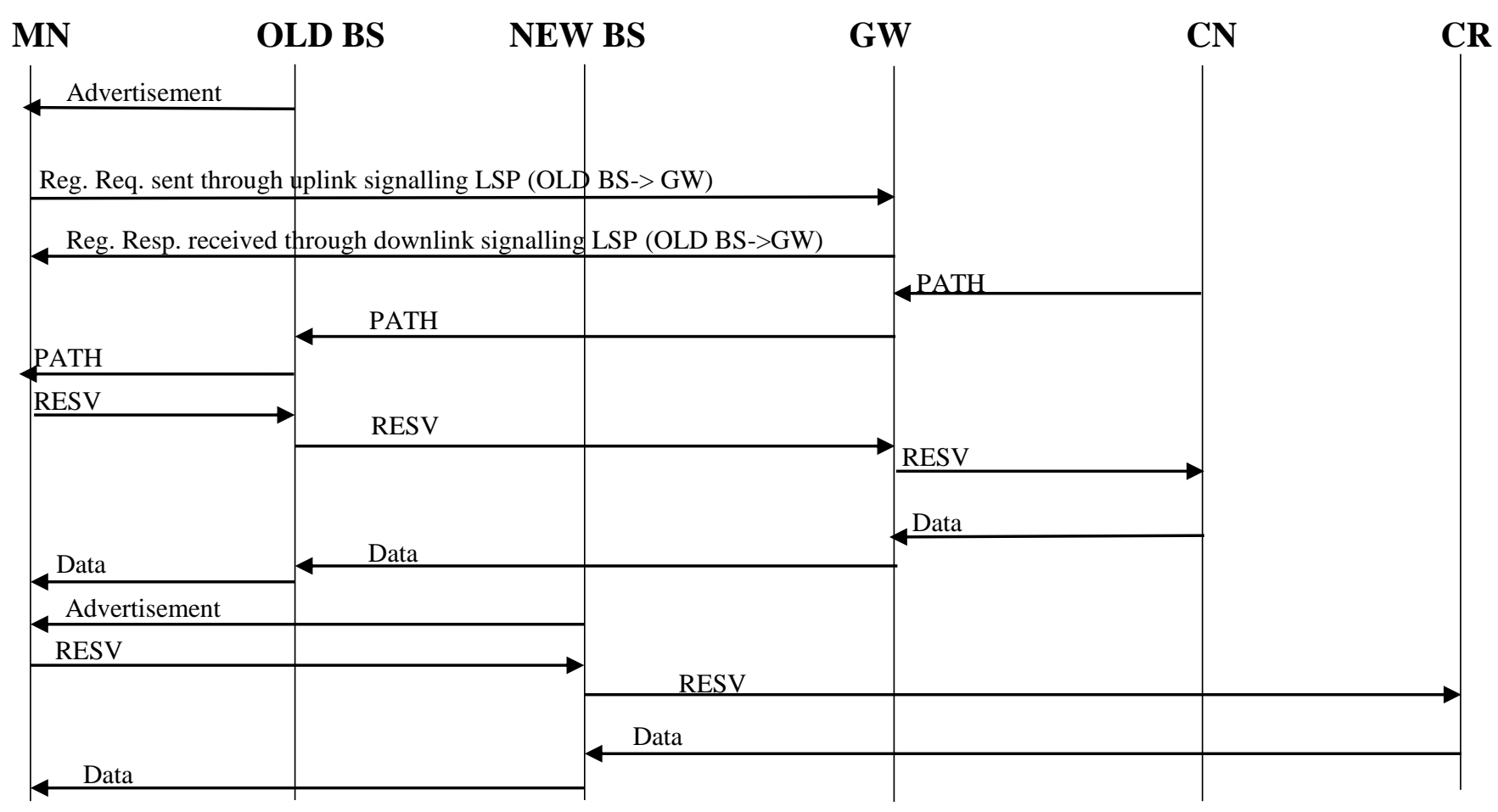

Figure 2.18 Handoff in MAMPLS-IntServ with dynamic LSPS

\subsubsection{MPLS based HMIP}

MPLS-based Hierarchical mobile IP (MPLS-HMIP) [URN-01] integrates MPLS with

Hierarchical MIP. The aim of this scheme is to eliminate the MPLS-IP-MPLS conversion during packet forwarding at each LER. It also proposes LSP re-routing instead of LSP re-establishment during handoff. This scheme introduces tree like structure where FAs are arranged in hierarchical order. Gateway Foreign Agent (GFA) sits at the top of the hierarchy. Regional Foreign Agent (RFA) is in the middle of the tree structure connecting multiple FA that resides at the bottom of the tree providing connectivity to the MNs. There are multiple LSPs between these nodes that only perform packet forwarding by label swapping. The protocol assumes only TCP traffic to avoid multiple LSP set up and release that would happen repeatedly if UDP traffic is also considered since one LSP is created for each MN under a FA initiating from $\mathrm{CN}$ passing through CN->HA->GFA->RFA->FA->MN path . When MN initially arrives in a foreign domain, it receives the agent advertisement message from FA. This advertisement contains a lists of addresses of FAs in hierarchical order beginning with advertising FA and ending with GFA. The 
MN sends a registration request using GFA's address as the COA. When the FA receives the request, it caches MN's IP address in its visitor's table and forwards the request to the next FA that is RFA. When RFA receives the registration request, it inserts its own address in the registration request, add MN's address in its visitor's table and forward the request to the GFA. The GFA also add MN's address in its visitor table and forwards the request to MN's HA. When the HA receives the request, it creates the binding between GFA's address and MN's address and forwards the response to GFA. This response is received by the MN after passing through GFA, RFA and FA.

Figure 2.19 shows the architecture of this scheme along the registration message exchange sequence. It is assumed that the LSP between CN and HA has already been established. When the packets arrive for the $\mathrm{MN}$ from $\mathrm{CN}$ to the $\mathrm{HA}$, it sends a label request message to establish an LSP between GFA and HA. As this label request propagates down the hierarchical tree, two FEC entries are made in each FA's LFIB. The first entry corresponds to the IP address of the next downstream FA. The next entry corresponds to the IP address of the MN as FEC with same output port and same label mapping as the next downstream FA. Once the label response message is passed in upstream direction, the incoming port and label mapping are also completed and LSP is established. It should be noted that the scenario of two FEC entries would happen only once when the first MN would start communication. Henceforth, one entry per MN would be required with the ports and label matching to the next FA. Once the LSP is established, the packets can be forwarded from $\mathrm{CN}$ to $\mathrm{MN}$ only through label swapping eliminating the IP layer conversion at each FA node since MN's IP address would be used as FEC for LSP matching. Hence this scheme merges four LSP segments CN->HA, HA->GFA, GFA->RFA and RFA-> FA to create a single LSP. 


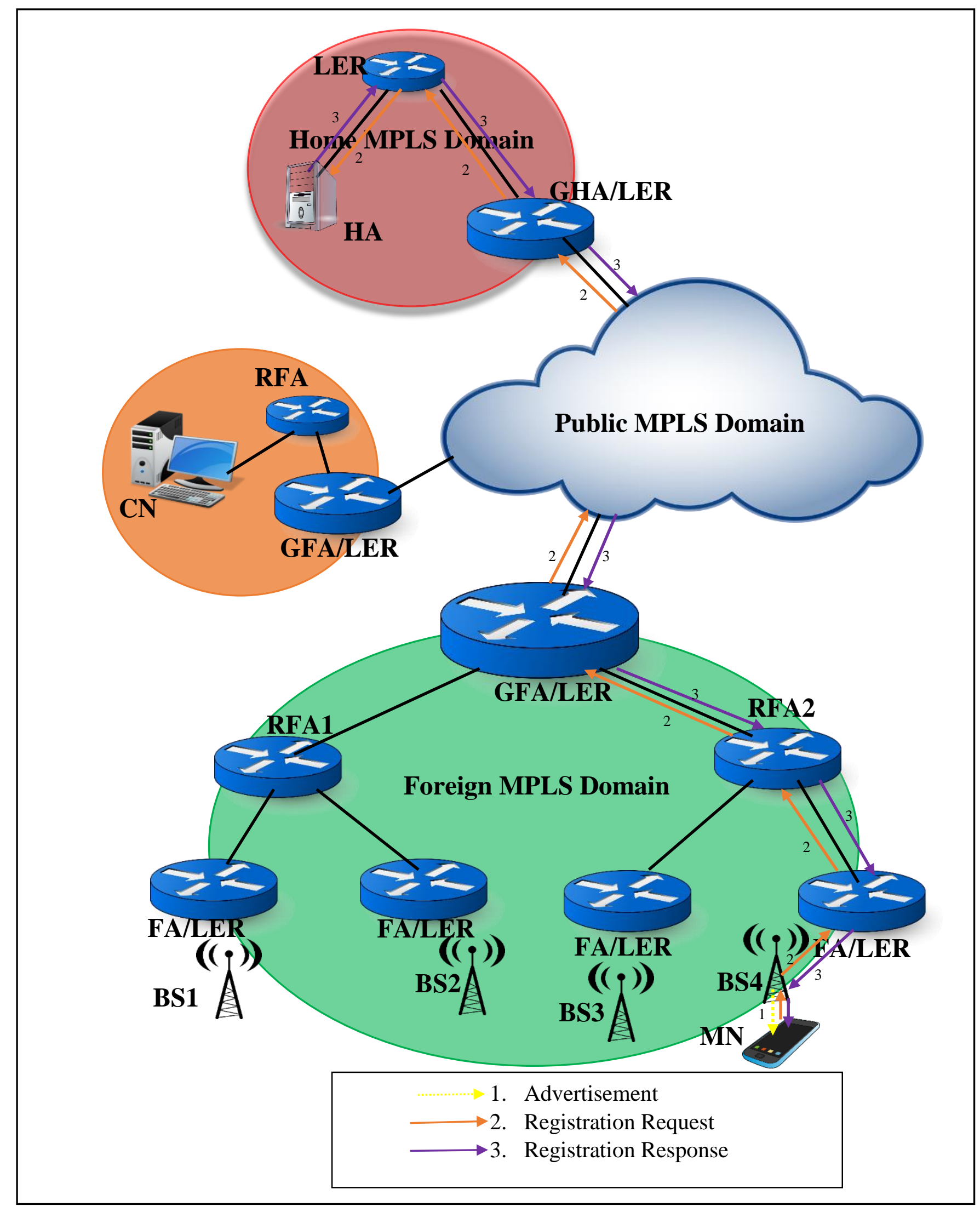

Figure 2.19 Architecture of MPLS-HMIP (as described in [URN-01]) 
During handoff, the scheme proposes LSP redirection technique. When the MN receives an agent advertisement message from the new FA, it sends the registration request through old BS and old FA. The old FA upon receiving the registration request forwards the request to new FA. The new FA sends the request upstream to the GFA. This request is intercepted by RFA, which is the common upstream FA between old and new edge FAs. The RFA sends label request to the new edge FA. Upon receiving the label mapping, the RFA updates its LFIB by changing the output port and label of MN's FEC entry. Hence the LSP is redirected from RFA. The RFA then sends a handoff command to the MN through old FA. Upon receiving the handoff command, the MN performs handoff and switches to new FA. Figure 2.20 shows the timing sequence of the message exchange during handoff. This scheme faces serious scalability issues as an exclusive LSP is required for each MN under a FA. Moreover, the GFA has to maintain separate label mapping for each $\mathrm{MN}$ in its domain which would increase the size of forwarding table considerably. Combining LSPs from HA to GFA by assigning same incoming label may not be used for multiple MNs as it would result in LSP disjoint at GFA. This point can be further clarified by an example. Suppose that GFA has LFIB entries of two different MN's as FECs with the same incoming label but different outgoing ports/labels as the MNs are under different RFAs. Upon receiving the packets with the same incoming label GFA would not be able to decide what outgoing label should be appended. Hence, GFA will have to strip off the MPLS label, send the packet to the IP layer to search for the MN's IP address before it can be encapsulated again and sent out. This is against the LSP integration technique which is the essence of this proposed scheme. 


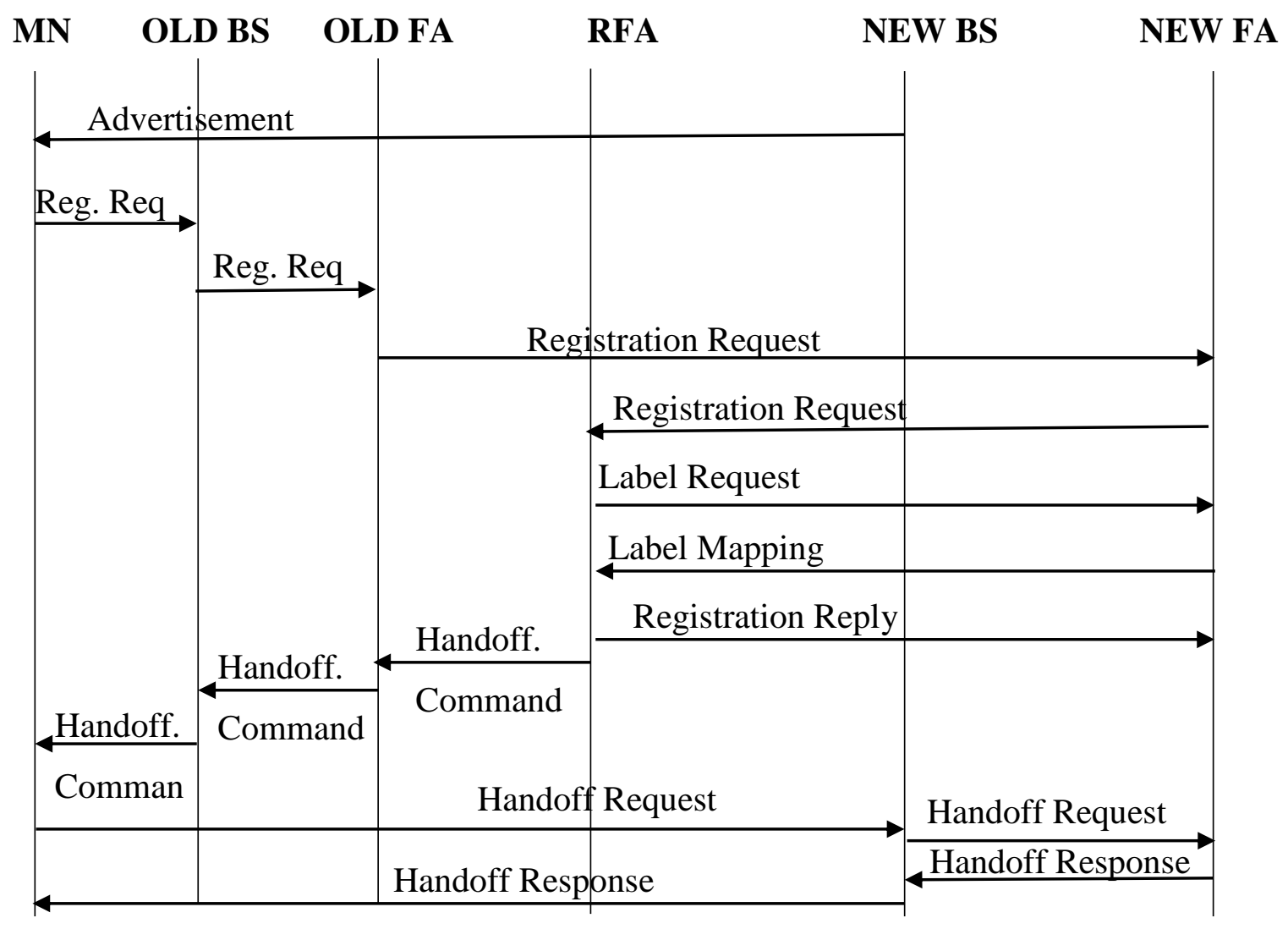

Figure 2.20 Handoff in MPLS-HMIP

\subsubsection{Micro-cell Mobile MPLS}

Micro-cell Mobile MPLS (MM-MPLS) [YAN-02] is an improvement of the HMPLS scheme in order to reduce the handoff delay. In HMPLS scheme, the LSP redirection was always done from the FDA. MM-MPLS has enhanced the architecture by introducing intermediate router LSR between FA and FDA that are capable of recognizing if the mobile node requesting new path establishment is already served through them. These cross over routers make the switch to the new location without tearing down the remaining path. This has the benefit of further reducing the delay and jitter during a handoff period. Figure 2.21 shows the architecture of MM-MPLS. 


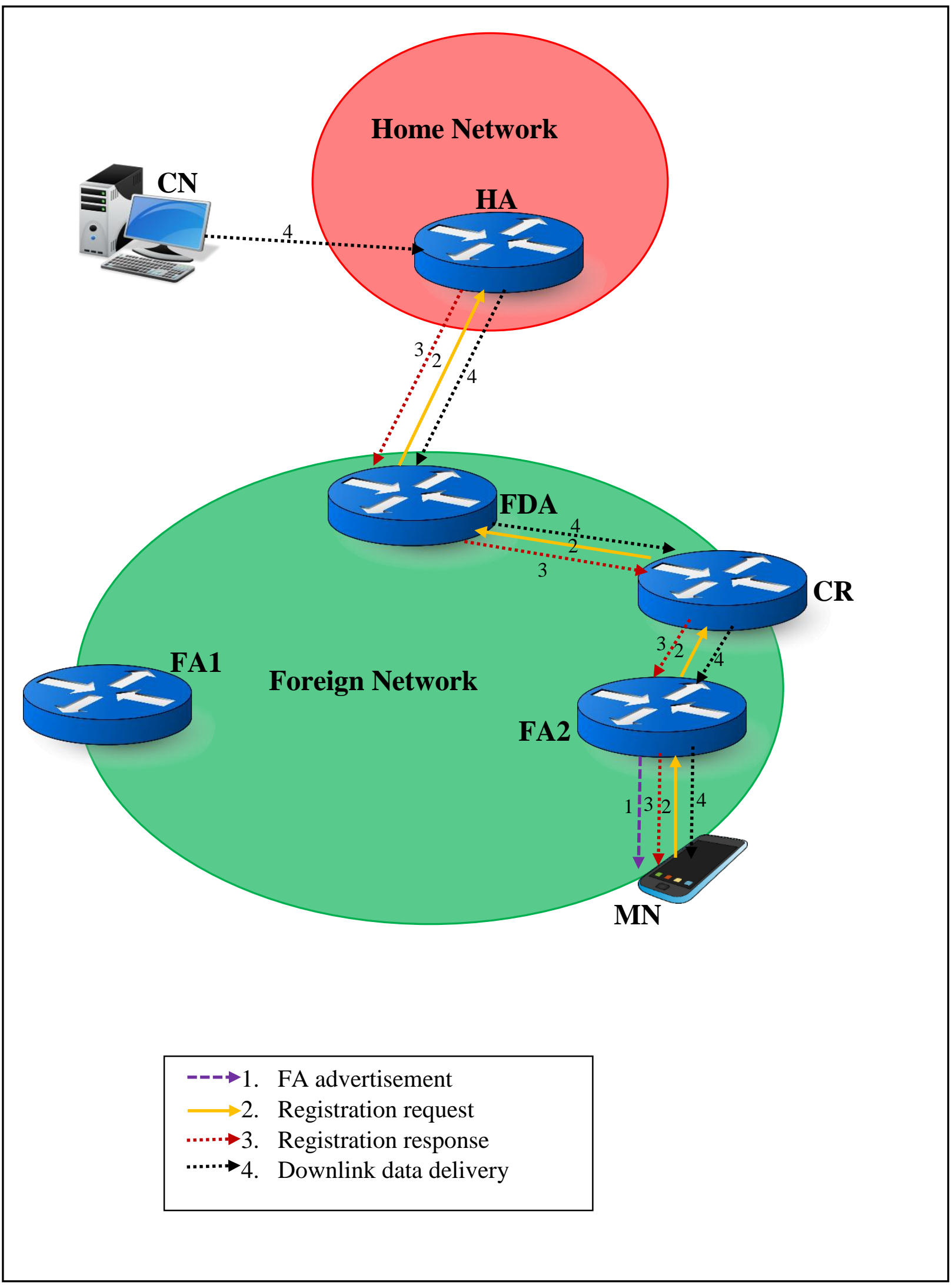

Figure 2.21 MM-MPLS architecture (reproduced from [YAN-02]) 
When a MN power-ups under a FA in MM-MPLS domain for the first time, an LSP is created between the FDA and FA using RSVP-TE protocol and MN's home address as the FEC. When this $\mathrm{MN}$ moves under a new FA, it sends a registration request towards the FDA. The intermediate LSR scan their LFIB to check if they have a forwarding entry with the MN's home address as FEC. When this registration message reaches a cross over router, it establishes a new redirected LSP to the MN's new location using MN's home address as the FEC. Since the LSP between the FDA and old FA was created using the RSVP-TE protocol, it is periodically refreshed by the FDA using PATH messages. When the CR intercepts the next PATH message from the FDA to the old FA, it responds by generating a RESV message containing a new EXPLICITROUTE OBJECT (ERO) [AWD-01]. This ERO updates the FDA regarding the new LSP for the MN from CR to the new FA. Hence, all the following PATH messages are redirected to the new FA. Although, MM-MPLS has shorter handoff delay compared to HMPLS scheme, it also faces scalability issues. The size of the forwarding table at the FDA increases with the number of visiting MN's. Also the intermediate routers are supposed to maintain per-flow forwarding table for each mobile MN. Figure 2.22 shows the message exchange during handoff in MM-MPLS.

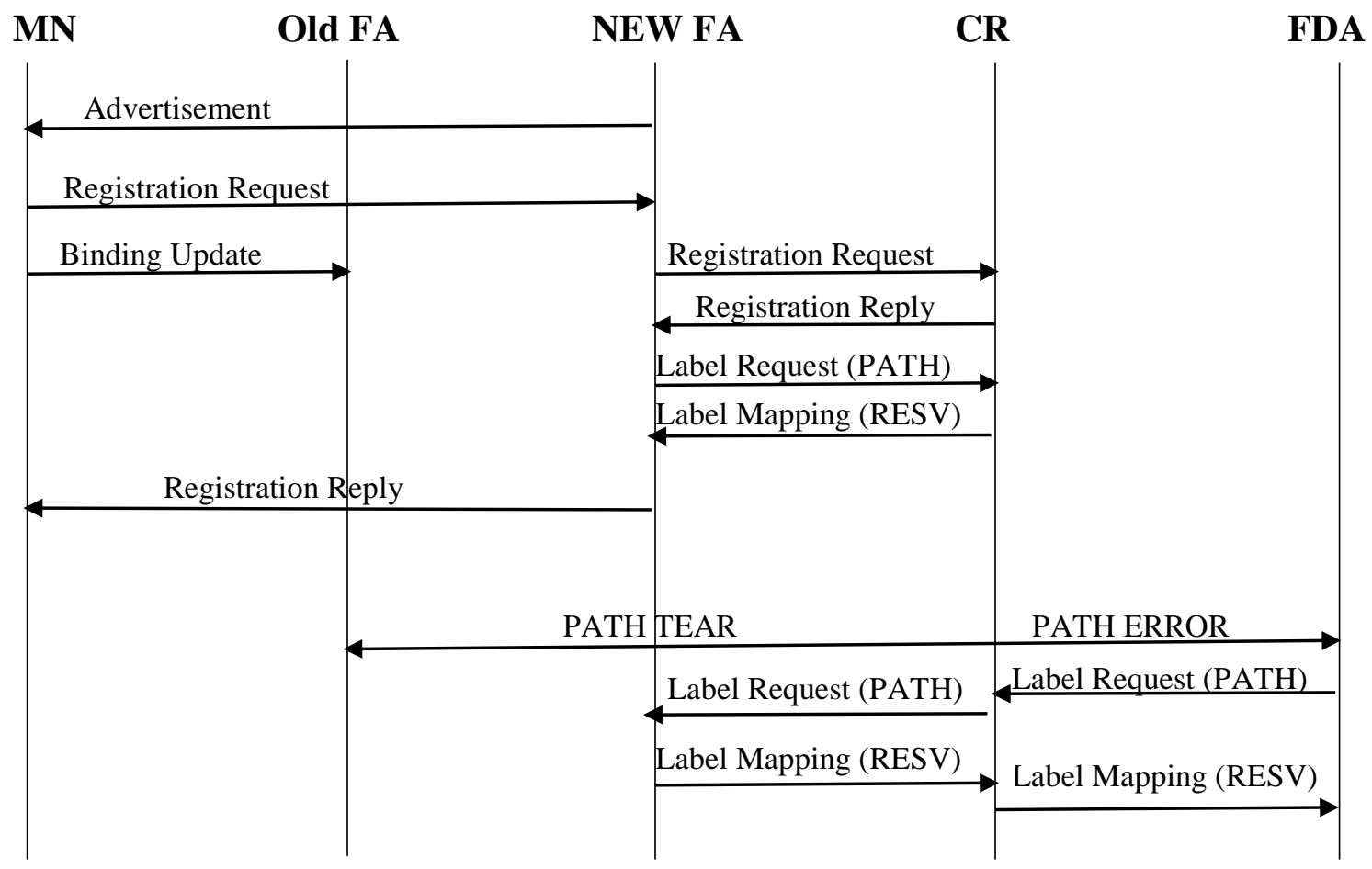

Figure 2.22 Handoff in MM-MPLS 


\subsubsection{Label-Edge Mobility Agent (LEMA)}

In [CHI-02A] [CHI-02B], an enhanced mobility management entity called the label edge mobility agent (LEMA) is introduced. LEMA presents a complete signaling framework for intradomain mobility in a traffic engineered network. The scheme has been shown to be scalable. Figure 2.23 shows the LEMA architecture.

The main contribution of this work is the introduction of LEMAs in the access network. It is mandatory to add LEMA functionality in ARs, in addition to selected LER. LEMAs are capable of performing three functions: 1) Map the IP address of the MN to FECs, 2) create a new mapping for the IP address to FECs in response to registration messages and 3) update the existing mapping of the IP address of the $\mathrm{MN}$ in response to redirect messages. It is not mandatory for LEMA to reside at the edge of MPLS access domain. A main drawback of this work is that all access routers should be modified to add LEMA functionality. LEMA nodes are connected though LSPs and may include several physical links.

LEMA has a hierarchical architecture. Each access router selects a chain of reachable LEMA router connected to its hierarchy and advertise them periodically. When a MN arrives in the LEMA domain, the highest level LEMA performs the registration with MN's HA using Mobile IP. Once the MN is registered, and receives the advertisement message from the access LEMA, it chooses to attach with a LEMA sub-chain from the advertised chain. MN uses the address of the top level LEMA in the selected sub-chain as its COA. The LEMAs selected by the MN to be in its chain maintain tables for the MN by mapping LSPs with the MN's home address. When the MN moves under a neighbouring LEMA access router, it searches the advertised chain by the new access router to find a common LEMA from the previous chain. In case a common LEMA is found, the MN includes this LEMA in the new chosen sub-chain and sends a re-direct message to the common LEMA. In case a common LEMA is not found, a new chain is selected by the MN. In this case, as the highest level LEMA is also changed, Mobile IP is employed to carry out registration with MN's HA. Figure 2.24 shows the signalling message exchange during handoff in LEMA. 


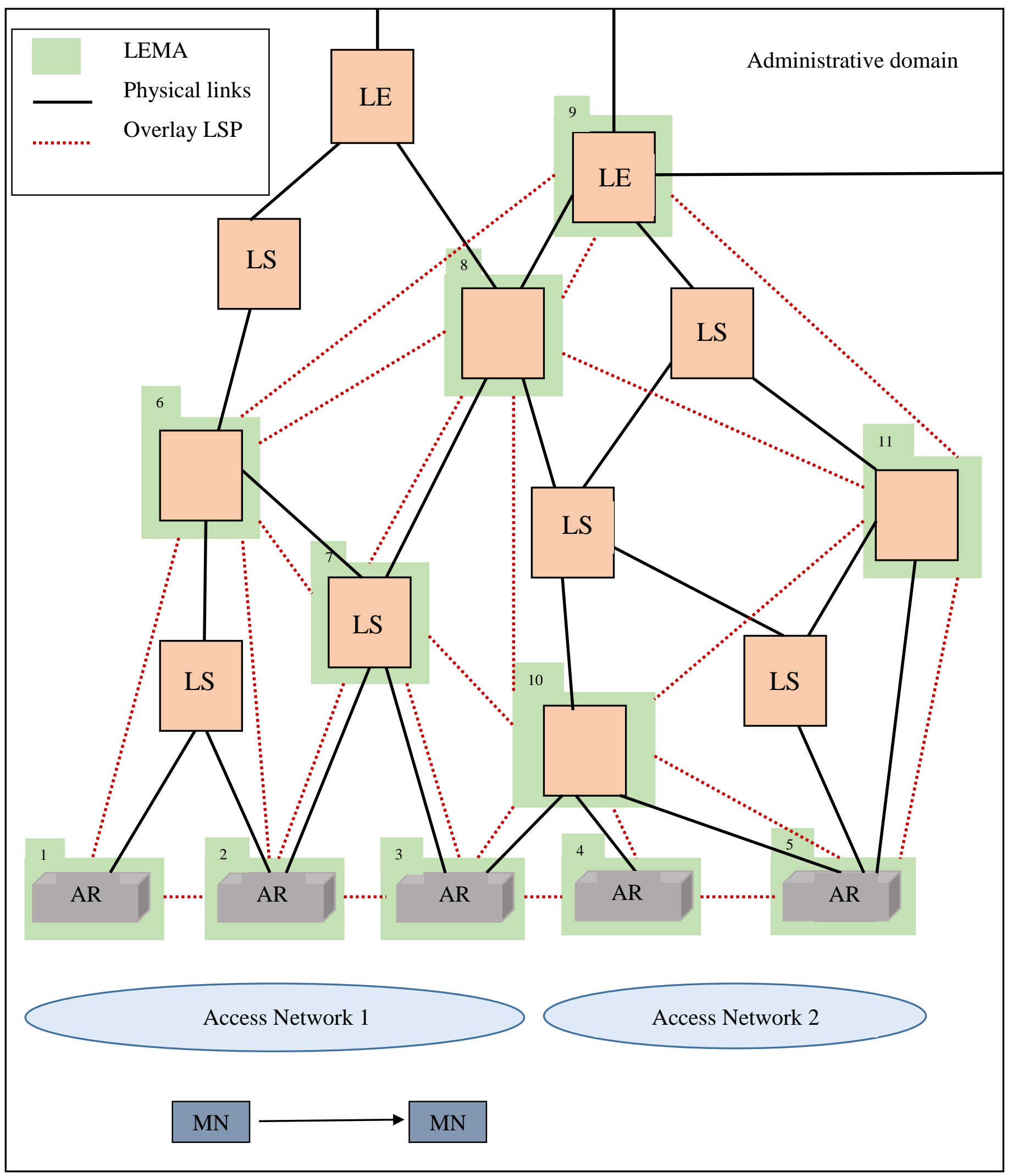

Figure 2.23 LEMA architecture [CHI-O2A][CHI-O2B] 


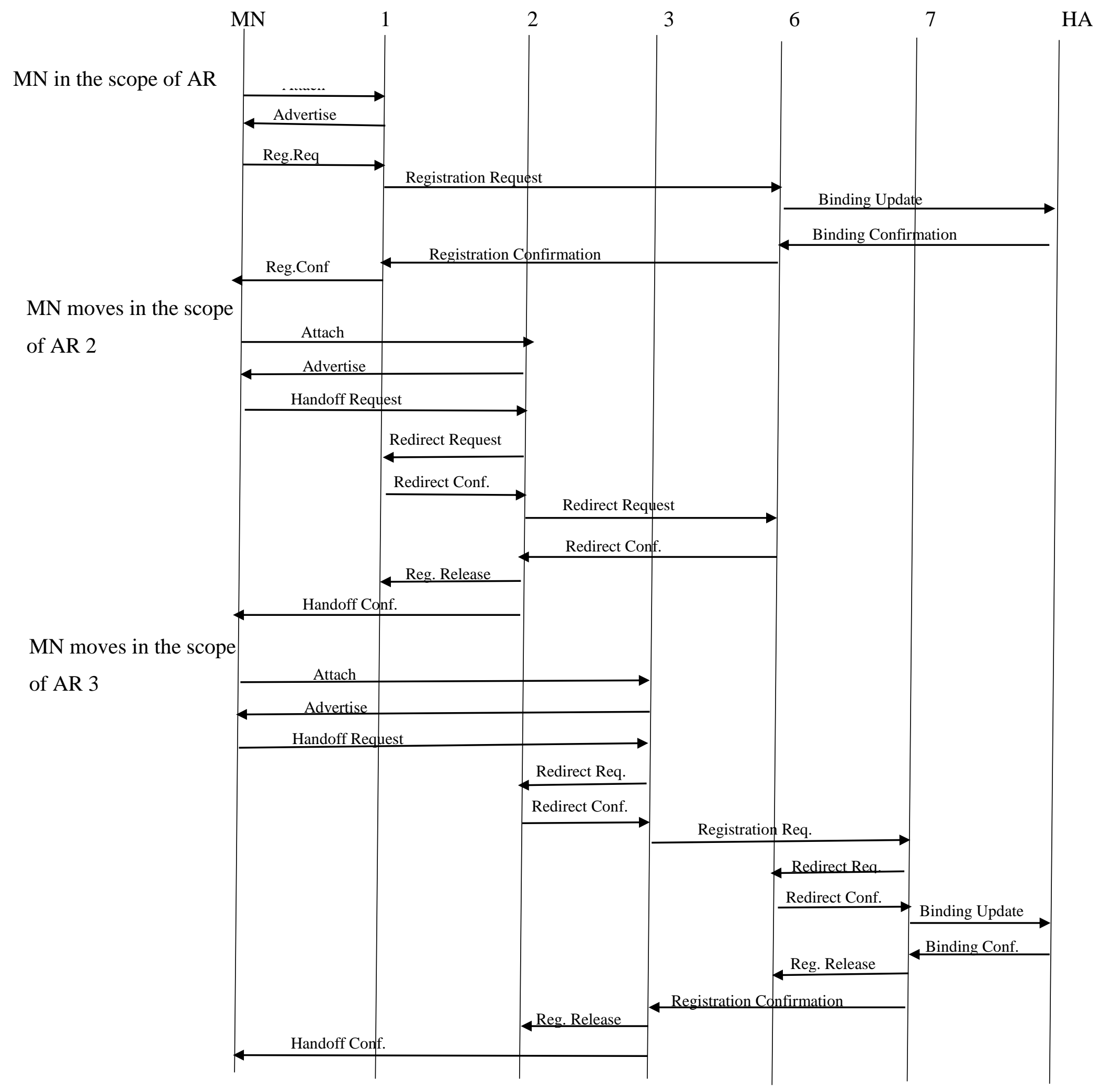

Figure 2.24 Handoff in LEMA [CHI-O2A][CHI-O2B] 
LEMA allows the MN to select its own LEMA chain. However, the algorithms that are used by the MNs for selecting the LEMA chain are complex. This results in increased cost for the implementation of LEMA architecture. Also, the selected chain by the MN may not be the shortest as it is hard to determine from the advertised message by the access LEMA router. If MN selects a sub-chain which is too short, it may trigger repeated Mobile IP home registrations thus generating excessive signalling load. Moreover, all MN have to be modified to support the LEMA architecture. This is a major drawback since it requires development and adoption of new protocols by the MNs that have incorporated procedures to communicate with the LEMA access router. Also, there is no information regarding the mechanism to refresh the location information, paging support or packet loss reduction specific to LEMA architecture.

\subsubsection{MPLS-based Micro- mobility Scheme}

MPLS-based micro-mobility scheme (MB-MMS) [XIE-03] presents a hierarchical architecture for micro-mobility management that includes LSP set up and packet forwarding details, handoff processing, and also paging. In order to prevent packet loss during handoff, two packet recovery mechanisms are also proposed.

The access network is divided into domains with the GW router residing at the top of the network and BSs at the bottom providing connectivity to the MNs. When the MNs first arrives or wakes up in the access network, it performs MIP registration with the GW. This work assumes that pre-configured LSP exist between $\mathrm{CN}$ to $\mathrm{HA}, \mathrm{HA}$ to GW and GW to BS. Upon receiving the registration request, the GW creates the FEC mapping of MN's IP address to the LSP between GW and BS. This work introduces the use of segmented flag "S" as an added field in the label forwarding information base (LFIB) table. This field if set indicates that another segment of the LSP exists and hence the LFIB should be searched again to forward the packet. If a packet destined for MN arrives from $\mathrm{CN}$ to $\mathrm{HA}$, it will look at the segmented field of the LFIB, upon detecting $S=1$, the label will be stripped off from the packets to read the destination address of the packet. HA will find the next segment of the LSP by using MN's destination address (IP address of the GW) to search LFIB again. Then the packet will be forwarded to GW. Upon receiving the packet, GW will perform identical operation i.e. stripping of the label, searching LFIB and re-encapsulation of the packet with another label before forwarding it to the BS serving MN. The BS will finally strip off the label and send the packet to the MN. During 
handoff, the new BS also sends a handoff notification (HFNO) message to the old BS. When the old $\mathrm{BS}$ receives HFNO message, it adds an LFIB entry for MN pointing to the LSP between itself and the new BS and set the segment flag in the LSP entry from GW to itself. The old BS also sets a timer and keeps forwarding the packets destined to the MN on the LSP segment between itself and new BS until the timer expires. Figure 2.25 shows the MB-MMS architecture.

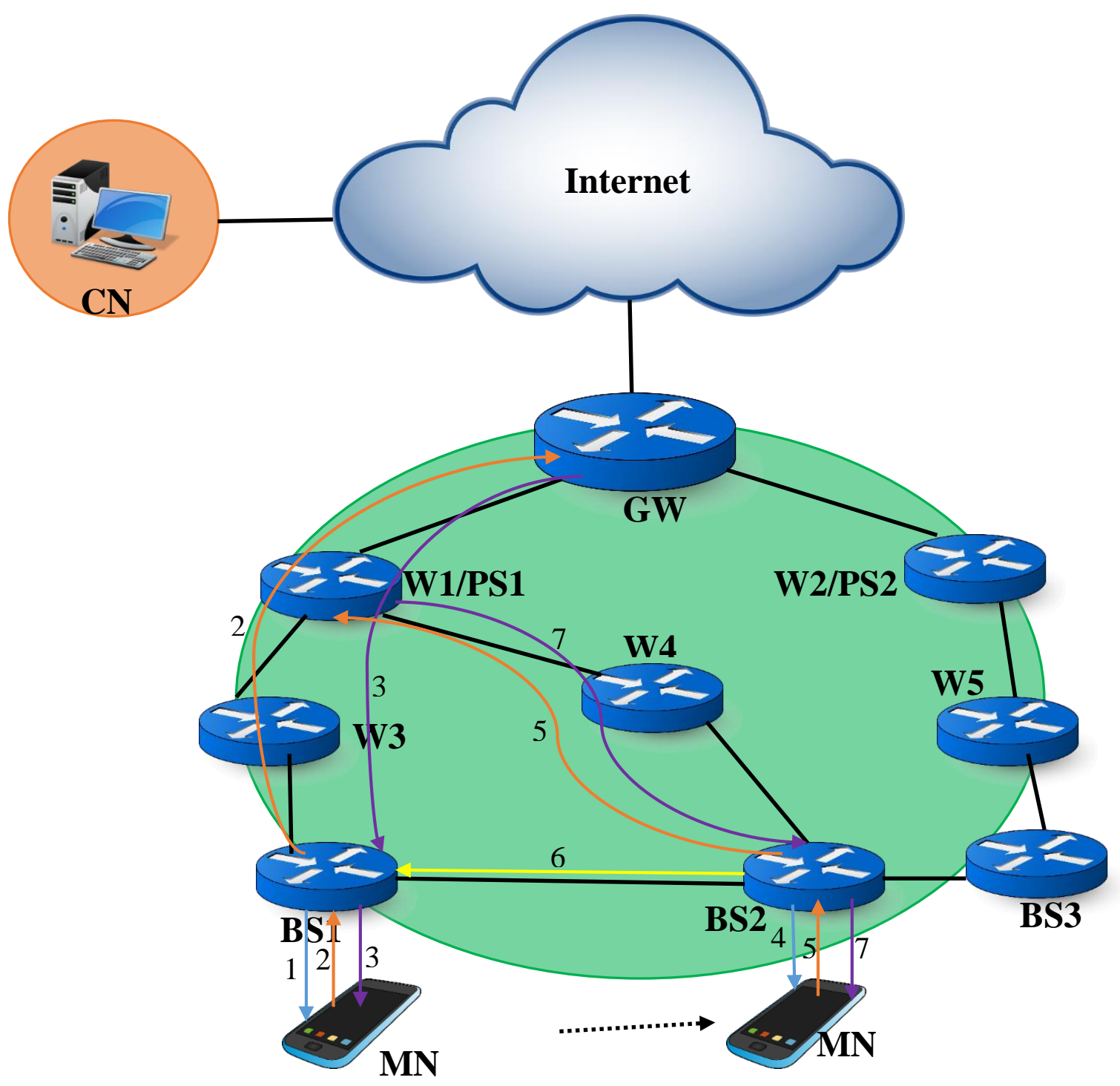

$\longrightarrow$ 1. $\quad$ MN receives advertisment from BS1.

2. MN send RREQ to GW through PS1.

3. MN receives RREP from GW through PS1.

4. $\mathrm{MN}$ receives advertisement from BS2.

5. MN sends RREQ to GW through BS2.

6. BS2 sends handoff notification to BS1.

7. MN receives RREP from PS1 through BS2. 
To reduce handoff latency, this architecture also introduces an intermediate LSR called paging server (PS) between GW and MN. In the presence of PSs, the GW only maintains the information about the location of the $\mathrm{MN}$ in the dominion of paging server. The handoff requests are sent to GW by the MN not upon crossing BS boundaries but on crossing PS boundaries. When the mobile node is in the idle state, the PS stores all the downlink data packets and sends a paging request to the associated BSs through multicast. The BSs forward the paging request through layer 2. The MN responds to the paging request and the BS sends the response message to the paging server. The paging server creates an entry in its LFIB corresponding to $\mathrm{MN}$ and forwards the data packets. Figure 2.26 shows the MB-MMS architecture with signal flow for handoff in the presence of paging server.

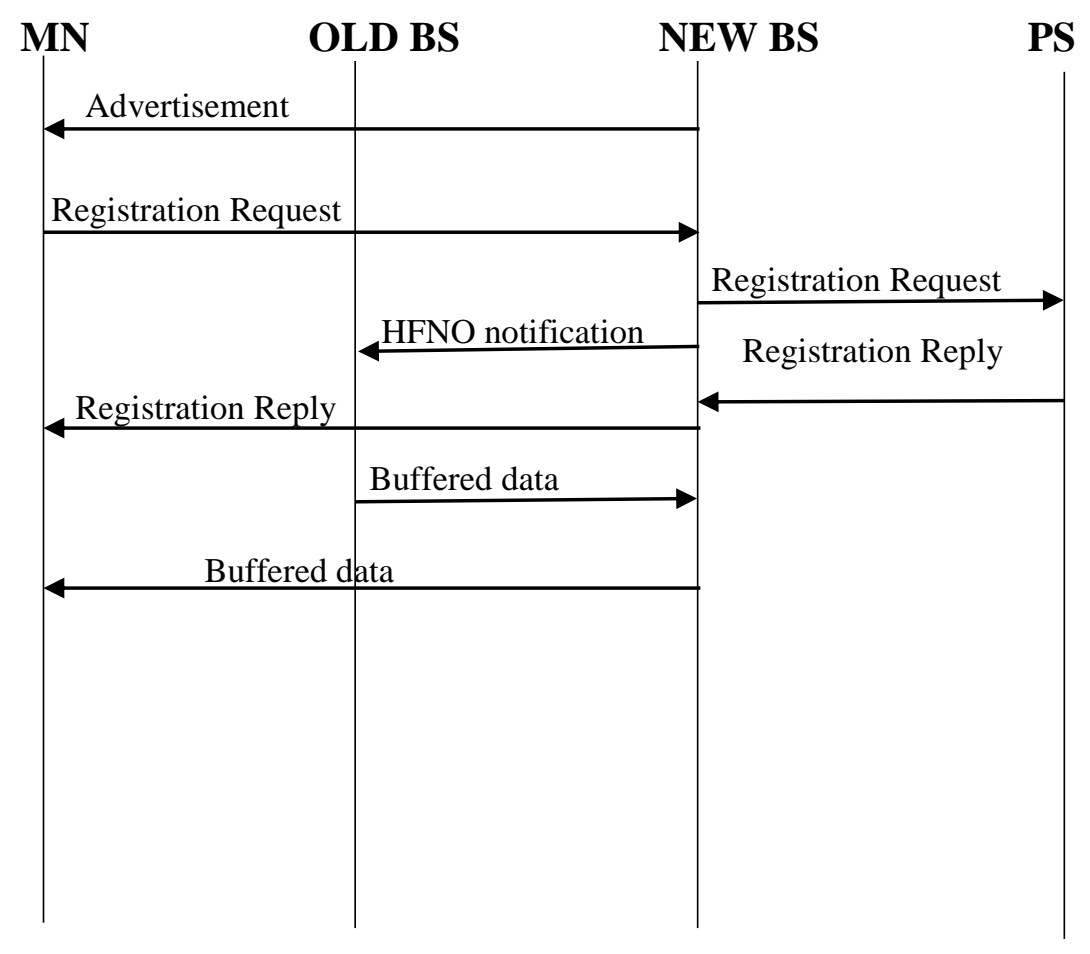

Figure 2.26 Handoff in presence of PS in $M B-M M S$

This scheme also introduces two different packet buffering mechanisms during handoff: Buffer time based packet recovery scheme and MAC layer assisted packet recovery scheme. In buffer time based packet recovery scheme, each packet is time stamped and then stored in the buffer at the old BS. Once the handoff is accomplished, the old AP decides to forward or discard the packet based on buffering time (the maximum duration allowed for packet to fulfill latency 
requirements). In MAC -layer assisted packet recovery scheme, all outgoing packets are buffered at the old AP. Hence all of the packets that are not acknowledged are forwarded from old BS to new BS once the handoff is completed. Simulation results prove that MAC- layer assisted packet recovery scheme is more beneficial in the micro-mobile environment.

This scheme has two drawbacks. The first drawback is common to all paging schemes. Although, the paging schemes offers energy consumption to the MNs by allowing them not to send registration messages in idle state and also lowers the signalling load by sending the handoff requests to the GW only in case the boundaries of PSs are crossed, it hinders the QoS support for real-time applications by providing no delay bounds since the packets have to be stored at the PS and will be forwarded after multiple message exchange between the MN, BS and PS. Secondly, the GW has an entry for each visiting MN in its forwarding table. Hence, the scheme also faces scalability issues in terms of maintaining large forwarding tables at GW.

\subsubsection{Micromobility enable MPLS}

The Micromobility-based MPLS (M-MPLS) [VAS-03] scheme merges MPLS with hierarchical mobile IPV6 (HMIPV6) and defines two different models: overlay model and integrated model. HMIPV6 [MAL-00] is based on IPV6 and introduces an entity called Mobility Anchor Point (MAP). The fundamental objective of HMIPV6 is that the MN uses the MAP's IP address as CoA. Like other gateway based domain approaches, the handoffs inside the domain are handled by MAPs and no changes corresponding to MN's location is communicated to the HA or CN. Hence, keeping the signalling load light and handoff latency low. M-MPLS access networks consists of LSRs, LERs that are called radio access routers (RAR) and one or more gateway routers called edge gateways (EGW). The RARs connects MNs to the M-MPLS access domain through BSs. The M-MPLS architecture is shown in figure 2.27 


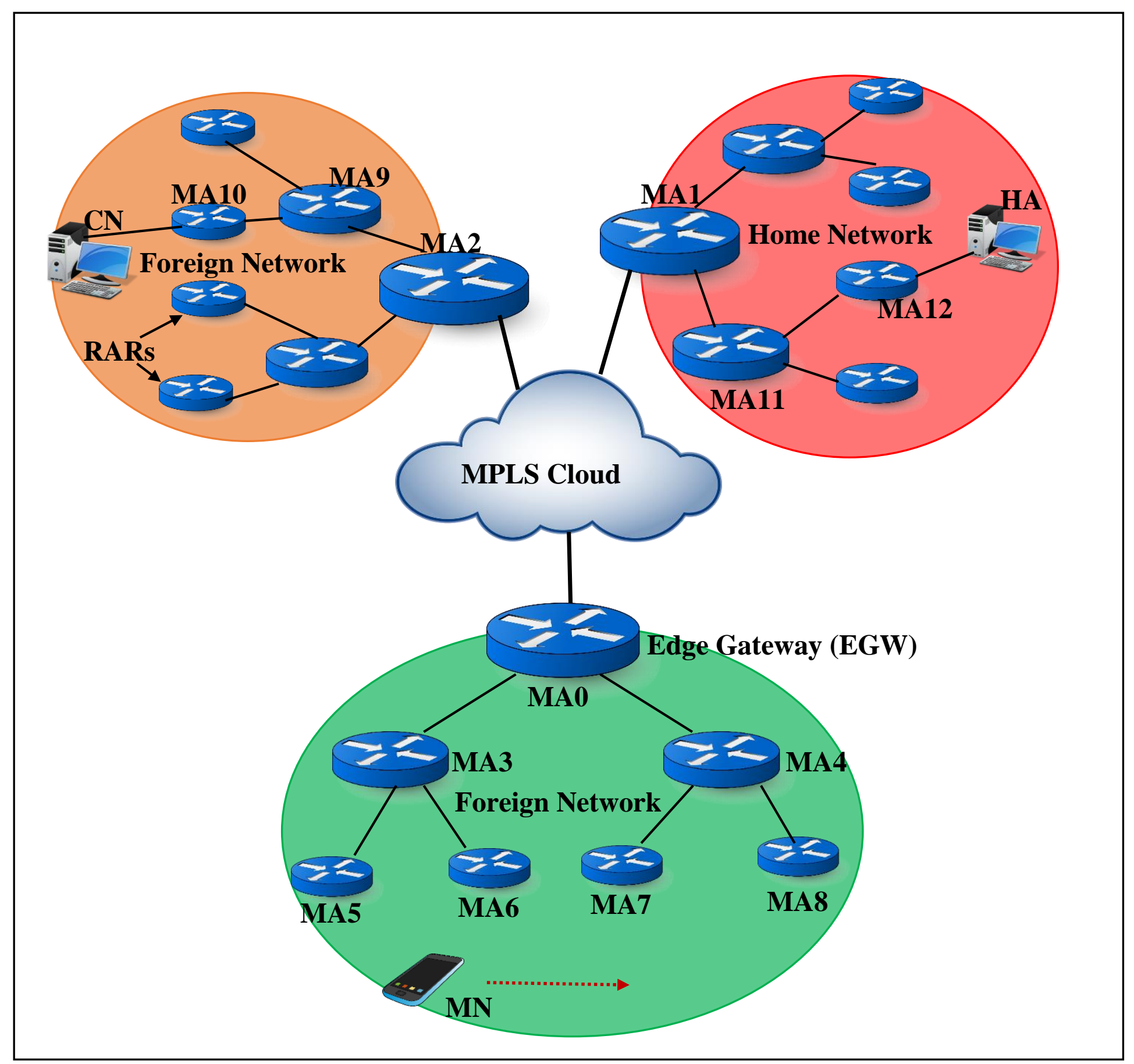

Figure 2.27 M-MPLS architecture [VAS-03]

In the overlay model, the MN first performs registration with the HA using the MAP's address as the COA. When the $\mathrm{CN}$ initiates communication, the packets are forwarded through HA to MN using three different LSPs: first between CN to HA, second between HA to MAP of MN's visiting domain and third between MAP to the RAR which is serving as point of attachment for the $\mathrm{MN}$. The $\mathrm{MN}$ upon receiving packets from the $\mathrm{CN}$ though $\mathrm{HA}$ updates the $\mathrm{CN}$ with its binding information so the $\mathrm{CN}$ can send future packets directly addressed to MN's CoA. Once 
the MN moves to the new RAR, MAP handles traffic redirection by creating a new LSP from MAP to the new RAR. Figure 2.28 shows the timing diagram of message exchange for the overlay model.

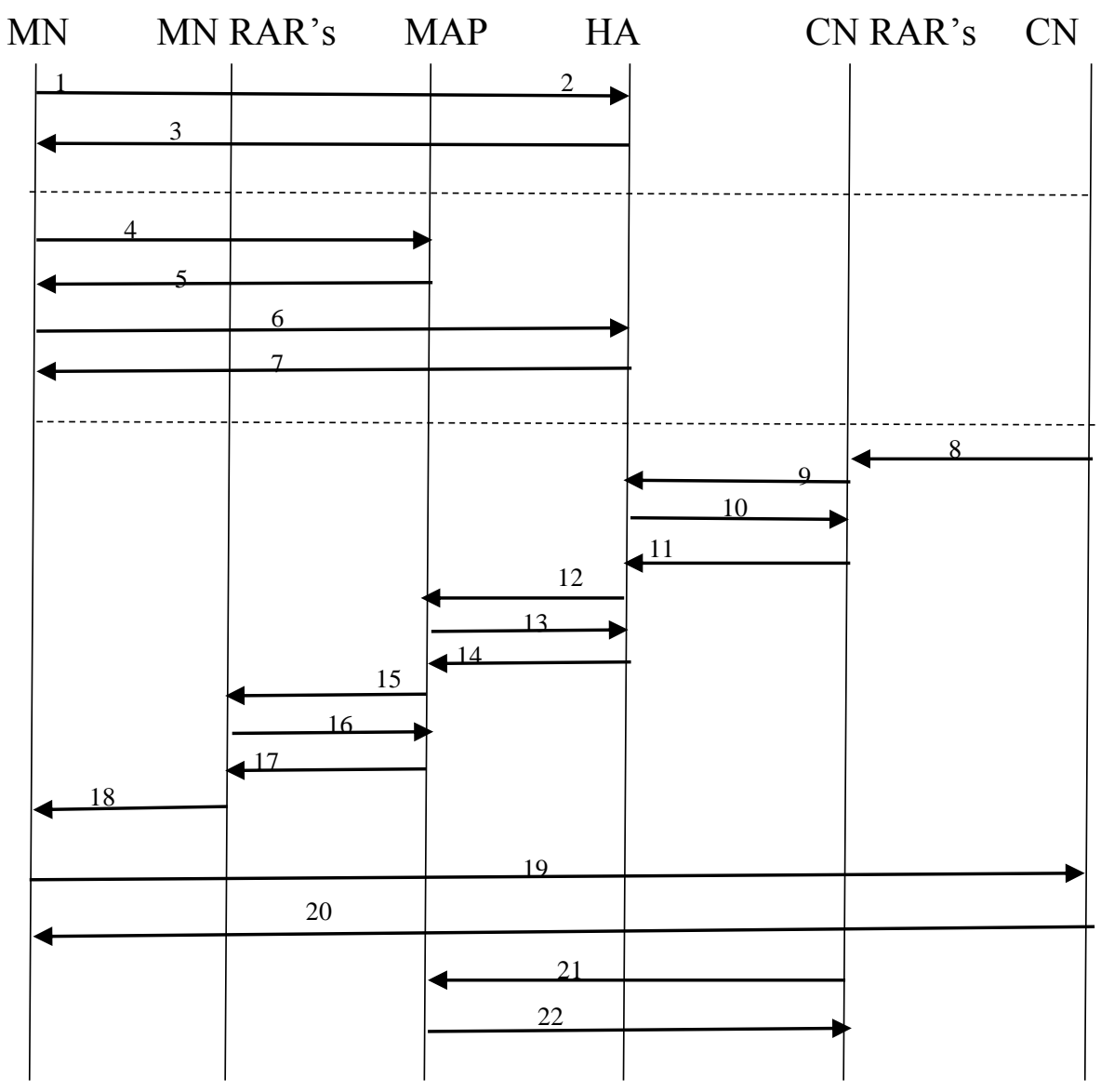

1. RCoA and LCoA discovery

2. HA discovery request

3. HA discovery reply

--Registration Procedure--

4. MAP registration $B U$

5. MAP Reg.BU Ack

6. HA Registration $\mathrm{BU}$

7. HA Reg. BU Ack

--MN is registered with MAP and HA--

8. Data packets

9. Label Request (PATH)

10. Label Mapping (RESV)

--LSP now setup between CN's RAR and HA--

11. Packets labelled and put on LSP

12. Label Request (PATH)

13. Label Mapping (RESV)

--LSP now setup between HA and MAP--

14. Packets labelled and put on LSP

15. Label Request (PATH)

16. Label Mapping (RESV)

--LSP now setup between MAP and MN's RAR

17. Packets labelled and put on LSP

18. Label popped and IP packets delivered.

19. Binding Update

20. Binding Ack

21. Label Request

22. Label Mapping

--LSP now setup between CN and MAP--

Figure 2.28 Message exchange for LSP set up in M-MPLS overlay model [VAS-03]

In the integrated approach, the packet forwarding process is improved by using "LFIB ptr field". This is a new field in LFIB and points to the next segment of the LSP. For example, when the MN changes its regional care of address (RCoA). It registers its new RCoA to the HA. Now HA has two entries corresponding to the MN in its LFIB. The pointer in the LFIB ptr field point to the recent entry and hence the packets are forwarded on the correct LSP.

The shortcoming of this approach lies in the fact that LFIB ptr field has to stay updated. As the pointer points to a row in the LFIB table, if the row is deleted due to timer expiry, the pointer will directs the packet to the wrong LSP. Another limitation is that the LFIB ptr cannot be used 
at the MAP's LFIB. Consider the scenario where multiple MNs are communicating between the same HA and MAP. When the HA forwards the packets using MAP's RCoA as the FEC for MN. The MAP cannot distinguish to which LSP it should forward the packets as they do not have an outgoing label. Therefore, the packets will have to be sent to the IP layer, in order to read the destination address. Another MPLS label with the output port corresponding to the correct LSP will have to be created and appended to the packets in order to send them to the MN. Hence, this scheme has serious deficiency and does not eliminate the IP layer transition as originally claimed.

\subsubsection{Wireless MPLS}

Wireless MPLS (W-MPLS) [SET-04] presents an MPLS-based administrative domain architecture. It address location management and handoff management procedures. A gateway router RG sits at the highest level of hierarchy and acts an ingress/egress router for the MPLS traffic. This work assumes that LSPs are pre-established between all MPLS routers using RSVPTE. In this architecture, the MN are connected through BS to Access LERs (A-LER). A-LERs are the first IP capable entity providing IP connectivity to the MNs. Each A-LER has an additional table for packet forwarding to the MNs that are attached locally to that A-LER. This data base is called Mobile Node Data Base (MNDB). When the MN first arrives in the W-MPLS domain, it obtains a CoA from the W-MPLS domain. This CoA will not change as long as mobile nodes moves within the domain. A-LER also creates a specific entry linking MN's CoA to its MAC address in the MNDB. When the $\mathrm{MN}$ sends a registration request to the $\mathrm{RG}$, it is forwarded through the pre-establish LSP between A-LER and RG. RG upon receiving the message creates an LSP entry with MN's COA as the FEC. The outgoing port and outgoing label for this entry is identical to the static LSP that exist between the RG and the A-LER. Figure 2.29 shows the registration process in the W-MPLS domain. W-MPLS assumes that CN is aware of the MN's CoA and sends them directly to the RG. The RG scans the forwarding table and forwards the packets along the static LSP. When the A-LER receives the packets, it extracts destination address and scan its MNDB to find the IP address. In case, the IP address is not found, it uses traditional IP routing to forward the packet. Otherwise, if the IP address is found, it uses the corresponding MAC address to forward the packet. 


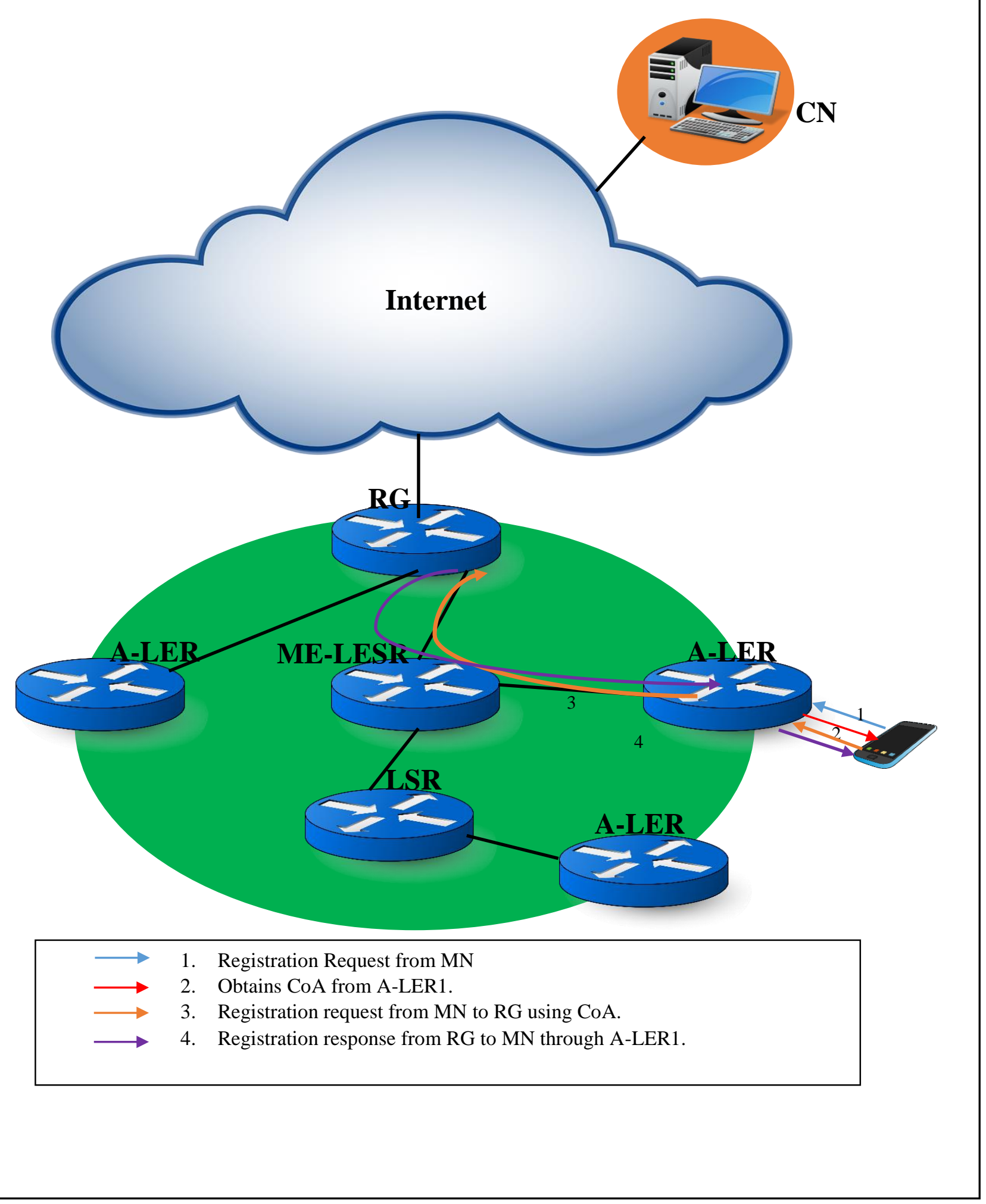

Figure 2.29 Message exchange for registration in W-MPLS (reproduced from [SET-04])

In case of handoff, $\mathrm{MN}$ sends another registration request to the RG through the new A-LER. Then the RG update its forwarding table by changing the outgoing port and outgoing label for 
MN's FEC to match the static LSP between RG and the new A-LER. W-MPLS also employs the idea of cross over routers called as Mobility Enhanced LSRs (ME-LSR). Similar to traditional handoff techniques employing CR, ME-LSR search its forwarding table to find the CoA of MN upon receiving the registration request. If the entry is found, ME-LSR changes the outgoing port and outgoing label to match the static LSP for the new A-LER. Otherwise, if the entry is not found, handoff is carried out by RG. Figure 2.30 presents the timing diagram of the messages exchanged during handoff.

The limitations of this scheme is that it assumes that CNs are aware of the MN's CoA, however the procedure followed for keeping the CNs aware of MN's location is not discussed. Another major limitation of this work lies in the claim of redirection of LSPs from ME-LSRs. It was assumed in this work that the LSPs are statically defined between RG and A-LERs. In this scenario, the ME--LSRs have no local binding cache of MN's FEC and hence static LSP cannot be redirected to perform handoff.

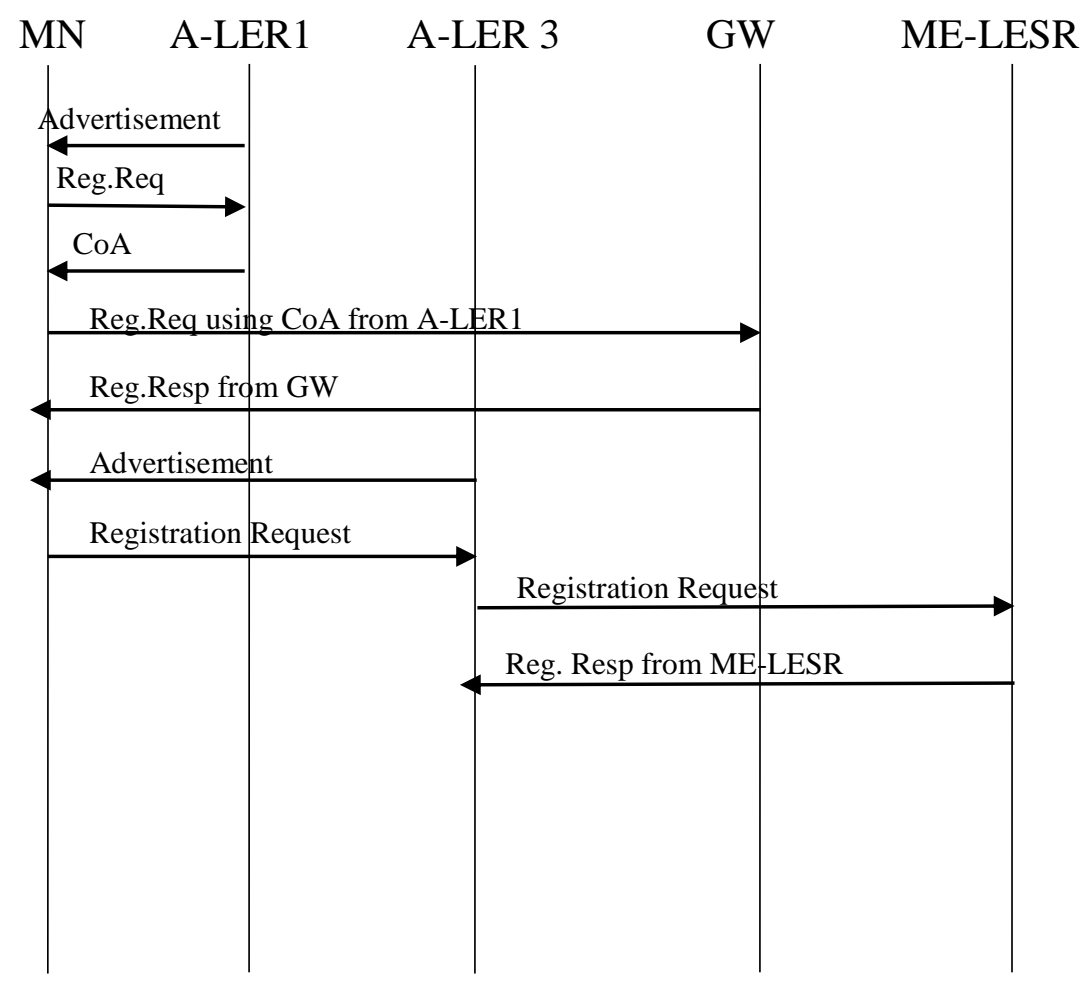

Figure 2.30 Message exchange during handoff in W-MPLS 


\subsubsection{Virtual LSP (V-LSP) based QoS capable Micro Mobility Scheme}

A virtual LSP based QoS capable MPLS based micro-mobility scheme (V-MPLS) was presented in [DON-05]. This scheme integrated HMIPV6 and MPLS and utilizes DiffServ aware MPLS Traffic Engineering (DS-TE) to provide QoS support. Moreover, it eradicates the MPLS-IPMPLS layer conversions at the LSRs that are carried out at the meeting point of two LSPs by introducing virtual LSP. These virtual LSPs are built over existing LSPs and improves packet forwarding.

The architecture is similar to HMIPV6 [MAL-00] architecture. Three MPLS domains are considered to be attached though MPLS cloud. One is the home domain of the MN, the other is the home domain of $\mathrm{CN}$ and the third is the visiting domain of the MN. Each MPLS domain has a Mobility Anchor Point (MAP) which acts as the local HA and can be located anywhere in the domain. LERs serves as the point of attachment for the MNs. When an MN arrives in the domain, it performs registration with LER to get local care of address (LCoA) and MAP to acquire regional care of address (RCoA). This RCoA is used to create binding entry of the MN with HA and CN. The MN also send the binding of its LCoA to the MAP so the path between MAP and LER can be established. Packets from MAP reaches the LER through label switching. At the LER, the label is removed and the packets are forwarded to MN using IP routing. Figure 2.31 shows the architecture of this scheme. 


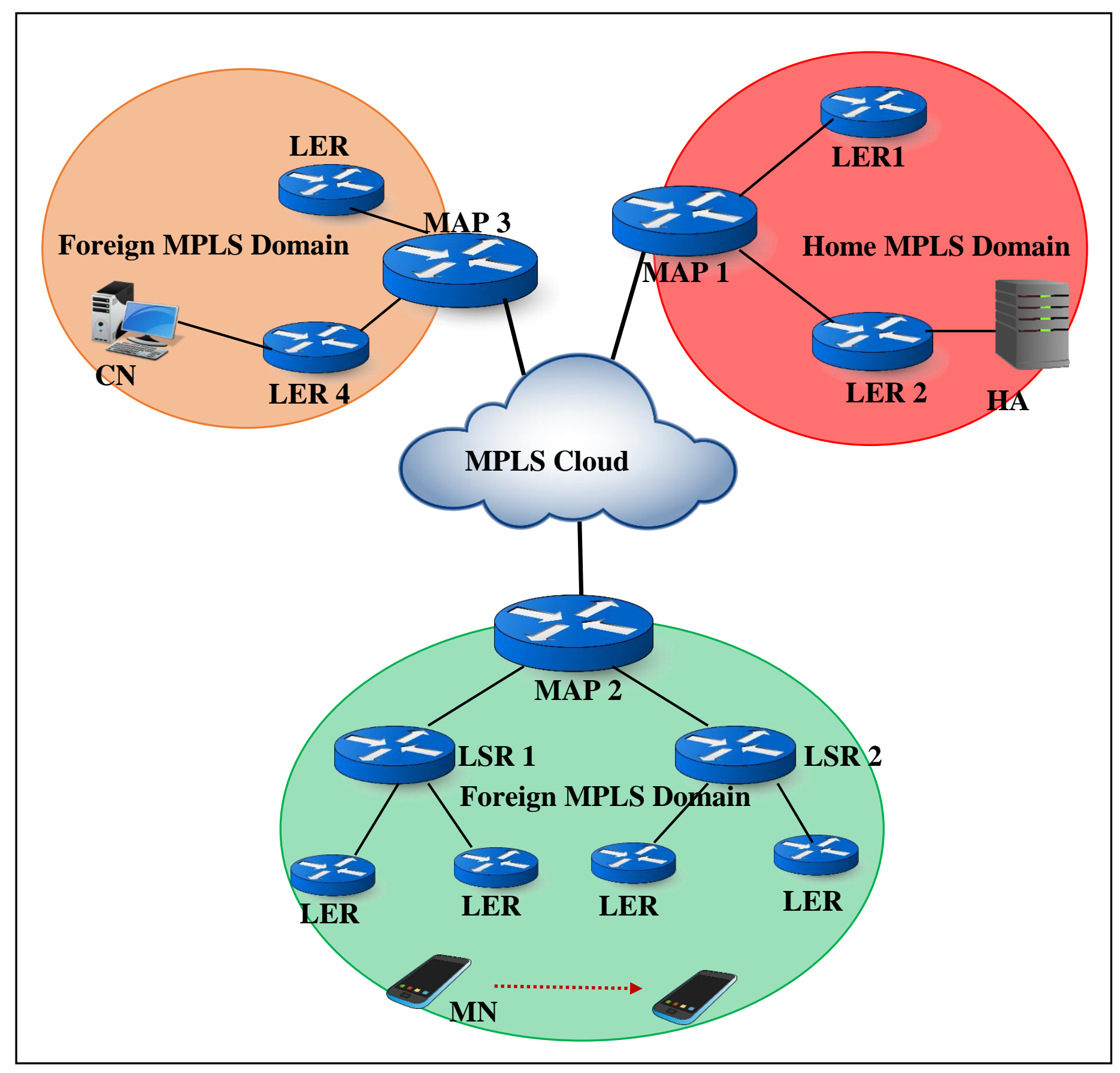

Figure 2.31 Architecture of V-LSP MPLS scheme (reproduced from [DON-05])

This scheme adopts DiffServ combined with MPLS for QoS provision. It assumes that LSPs are pre-established between MAP and LERs using RSVP-TE. Multiple MNs are able to share these LSPs, hence these LSPs are transferring aggregated data traffic between the MAP and the LER. This approach eliminates the requirement of storing per-flow information at each LSR and reduces the signaling traffic. To further improve the packet forwarding process by eliminating 
the transition from MPLS-IP-MPLS software modules at LSR, this work introduces virtual LSPs (V-LSP). These V-LSPs are created on top of existing LSPs using RSVP-TE and make use of the label stacking property of the MPLS header. When a CN initiates a conversation with the MN, the packets are first sent to the HA. The HA forwards the packets to the MAP using preestablished LSP. When MN receives the packets through the MAP $\rightarrow$ LER LSP, it sends the binding info to the $\mathrm{CN}$ directly. When the $\mathrm{CN}$ receives the binding information, the LER of the $\mathrm{CN}$ initiates the virtual path set up by sending a virtual label request to the MAP of the MN. The MAP forwards the request to the LER and receives the label response. The MAP then responds back to the LER of the CN by sending it a label. The forwarding tables at the MAP, LER of the MN and the LER of the CN are updated with two new fields called "Next Entry Ptr" and "Next FEC". Both perform the same function. Next entry pointer is preferred for use as using the Next FEC would result in researching the entire forwarding table again to find the match whereas, Next Entry Ptr field directly points to the number of next FEC entry in the forwarding table. The LER of the CN first scans the forwarding table using the MN as FEC, it then checks the "Next Entry Ptr" field and encapsulates the packets one more time using the MAP as the FEC. Hence, the packets at the $\mathrm{CN}$ are encapsulated twice, the outer label uses the MAP as the FEC whereas the inner label uses the MN as the FEC. These steps are repeated when the packets are received at MAP. Finally when the packets are received at MN's LER, both labels are stripped off and the packets are sent through IP routing.

During handoff, if the MN attaches to a LER under a new MAP, then the entire VLSP is reestablished between new MAP, HA and the CN. If however, the new LER and the old LER are under the same MAP, partial V-LSP update is performed by re-routing the V-LSP from MAP to the new LER. Figure 2.32 shows the timing sequence of messages exchanged during initial VLSP set up and during handoff when the new LER and old LER are under the same MAP. 


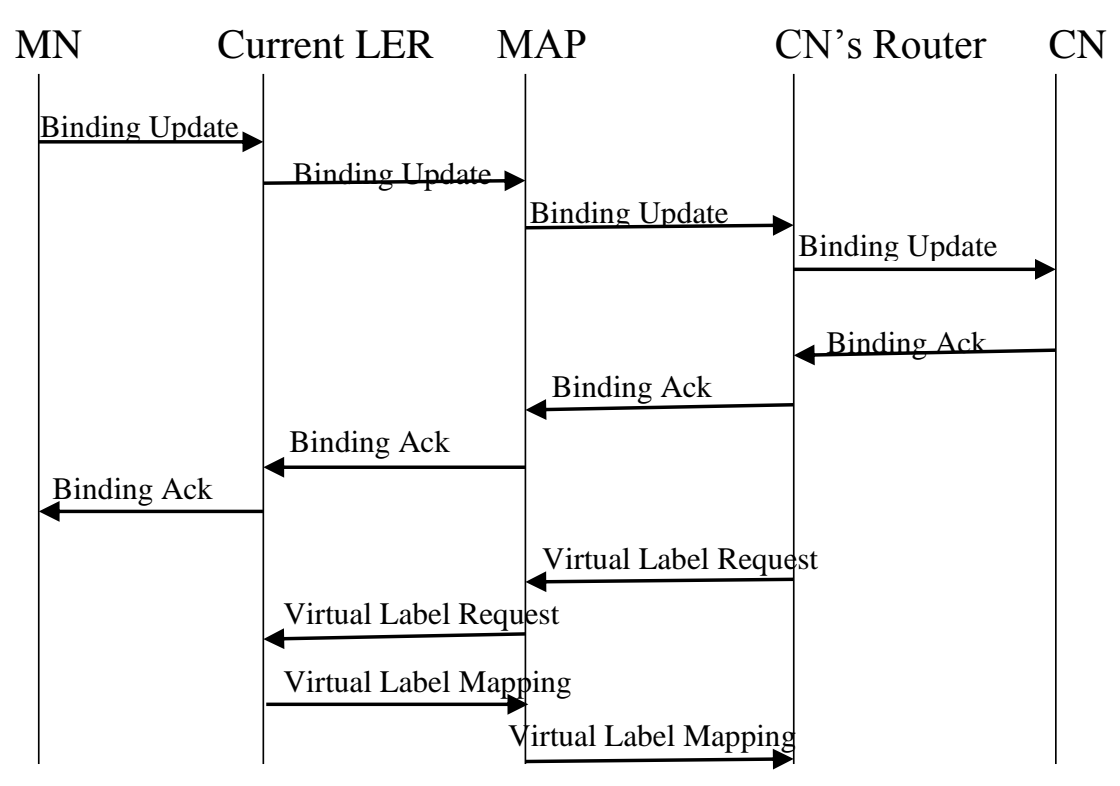

(A)

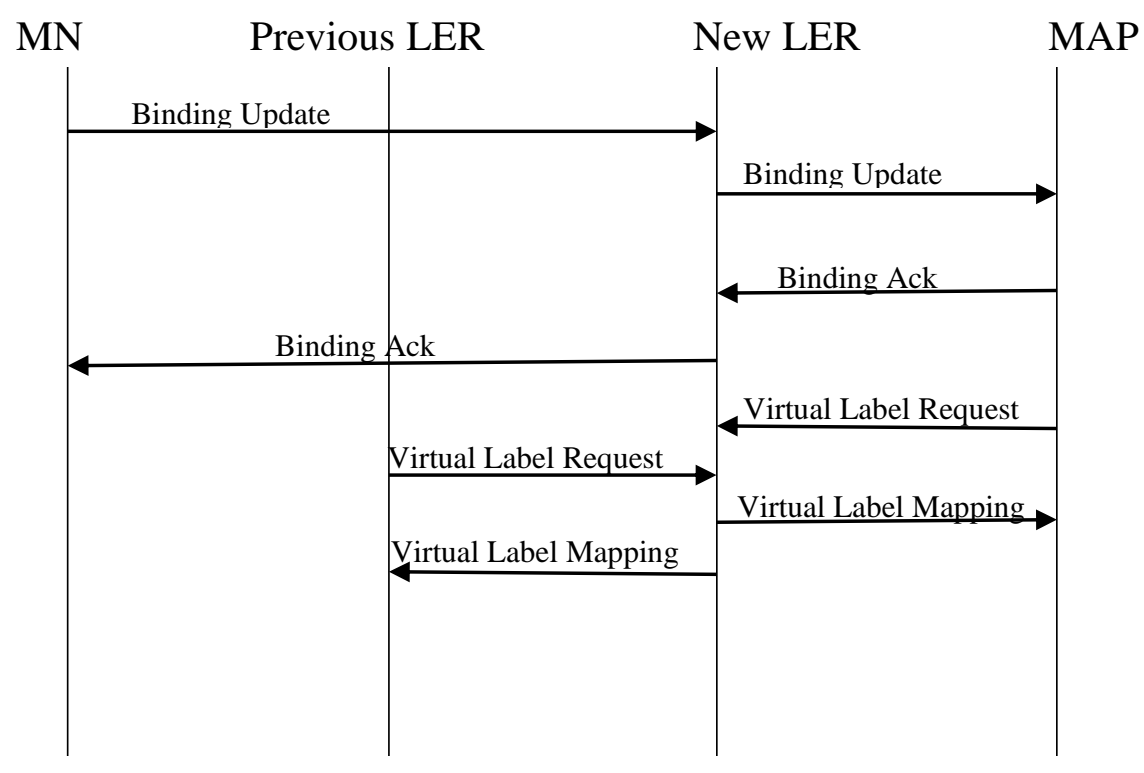

(B)

Figure 2.32 Message exchange during (A) V-LSP set up (B) handoff in V-LSP MPLS scheme $(D O N-05)$

This scheme faces few challenges. When V-LSPs are created, the forwarding tables are updated using Next Entry Ptr, however, if the entry is deleted or changed for example, due to timer 
expiry, the Next Entry Ptr will point to the wrong LSP. Hence, a table management procedure is required. Secondly, the scheme does not discuss any packet buffering mechanism that is carried out to store in-flight packets during handoff. V-LSP are created from CN's LER to the MN's LER, which requires the forwarding tables to have one entry corresponding to each MN. This means that the forwarding table will increase in size considerably if the number of MNs between these LERs increase.

\subsubsection{Micro-mobile MPLS}

The Micro-Mobile MPLS [LAN-08] scheme comprises of three mechanisms that enhances mobility management and reduce handoff latency. The Micro-Mobile MPLS domain is hierarchical with one gateway node (LERG), several LSRs and FA that are the MPLS-enabled edge routers for $\mathrm{MN}$. MN is connected to the edge routers through access point (AP). The FAs are the first IP capable entities as seen from MN. This work assumes that FAs maintain a neighbor mapping table that contain entries mapping the IP addresses of the neighboring APs to their MAC addresses. Upon activation the MN performs Mobile IP registration with the LERG. Henceforth, the description of three hand-off schemes are provided. The first scheme, called fast handoff (FH-Micro Mobile MPLS), anticipates the LSP procedure setup with an adjacent subnet that an MN is likely to visit. This mechanism is proposed to reduce service disruption by using the L2 functionalities. Once the MN reaches the boundary area of two neighboring cells, it receives the $\mathrm{L} 2$ beacon from the new AP that is also under a new FA. MN immediately initiates the handoff procedure by sending a handoff initiation message to the old FA. This message contains the MAC address of the new AP.

When the old FA receives the handoff initiation message, it performs three functions. Firstly, it scans the neighborhood mapping table to find the IP address of the new FA and sends it to the LERG so that the passive LSP set-up may begin according to the QoS requirement of the MN's on-going sessions between LERG and new FA. Secondly, it forwards the IP address of the new FA to the MN so that it may begin Mobile IP registration through the new AP immediately, without waiting for address advertisement from the new FA. Thirdly, it starts buffering the inflight packet for the MN. Once the MN moves under the AP under new FA, it sends a registration request to the new FA using the IP address of new FA as the RCoA. This activates 
the passive LSP already established between the new FA and LERG. The MN receives registration reply from LERG. LERG also sends a message to the old FA to forward the in-flight packets for the MN. These packets are forwarded from the old FA to the new FA through static MPLS tunnel. Figure 2.33 shows the handoff procedure in FH-Micro Mobile MPLS. 


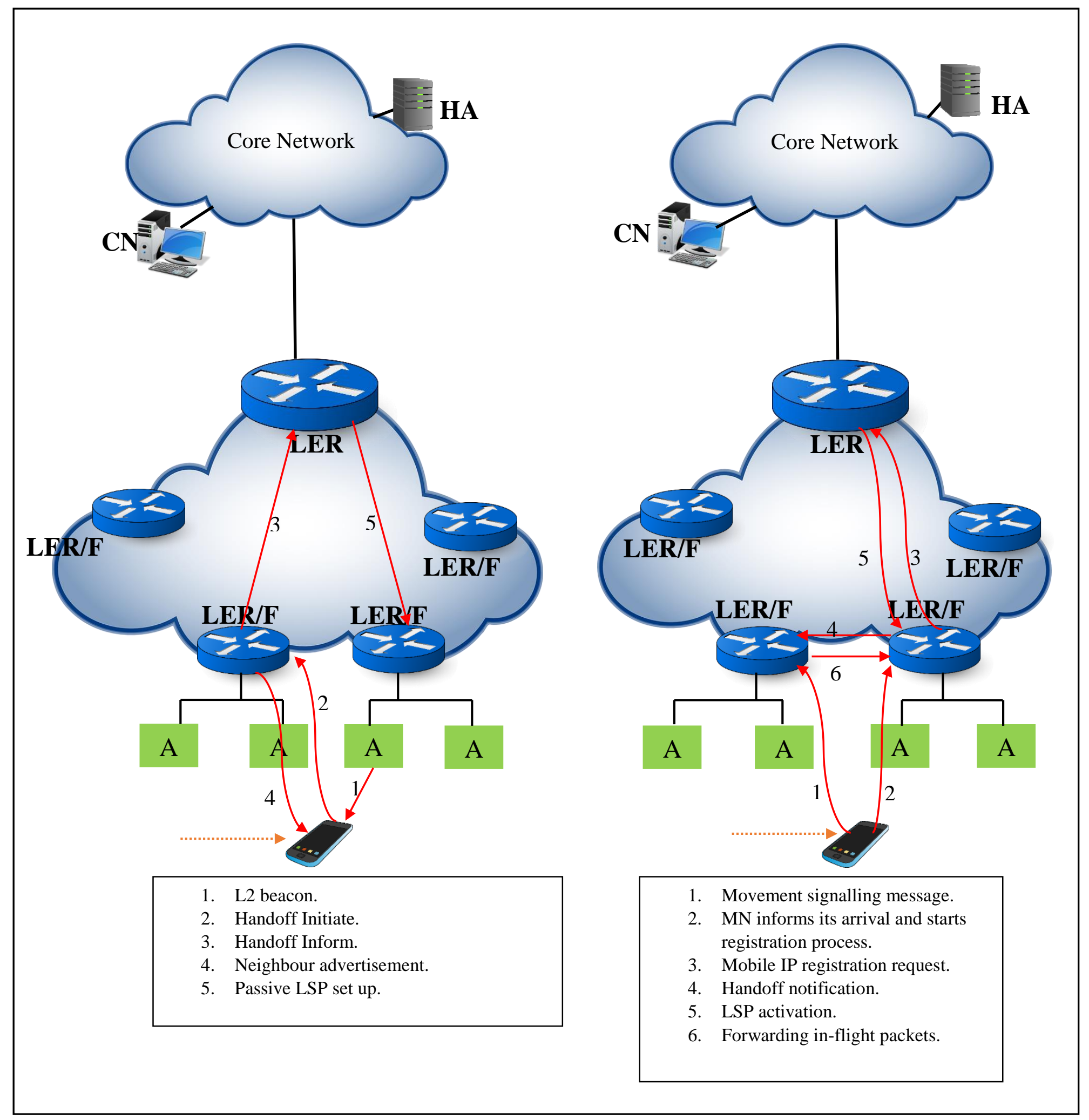

Figure 2.33 Message exchange during handoff in FH-MMMPLS showing pre-registration and post registration operation (reproduced from [LAN-08]) 
In the second scheme called FC-Micro Mobile MPLS, a simple forwarding chain (FC) concept with loop removal is introduced to track efficiently the host mobility within a domain. In this approach, each time the MN moves to a new subnet it registers with the old foreign agent (FA) instead of the gateway. The old FA sends a label request to the new FA and the new FA responds with the label mapping. This way, instead of setting up a new LSP, the existing LSP is extended as MN moves from one subnet to another till threshold is reached after which the registration is performed with LERG. The forwarding chain can reduce registration updates cost (i.e., volume of signaling messages exchanged during handoff operations) and provide low handoff latency and QoS support due to MPLS capabilities. Figure 2.34 and 2.35 shows the architecture and exchange of timing messages during handoff.

In the third mechanism called M (Master) FC-Micro Mobile MPLS, a new attribute, called residing area is defined for each $\mathrm{MN}$ to achieve further reduction of the signaling costs. This mechanism is a two-dimensional variant of the previous scheme. In MFC scheme, when the MN arrives in the MM-MPLS domain, the first FA with which it performs the registration becomes master FA (MFA) or anchored FA. Henceforth, after each movement which can be under any FA considering a 2-D mobility model, the new FA calculates the shortest path from itself to the MFA. The forwarding chain is then established between the MFA and the new FA. It should also be noted that in FC scheme, the path is always extended by one-hop but in MFC scheme it may be extended by (M-1) hops with M being the threshold of the master forwarding chain. 


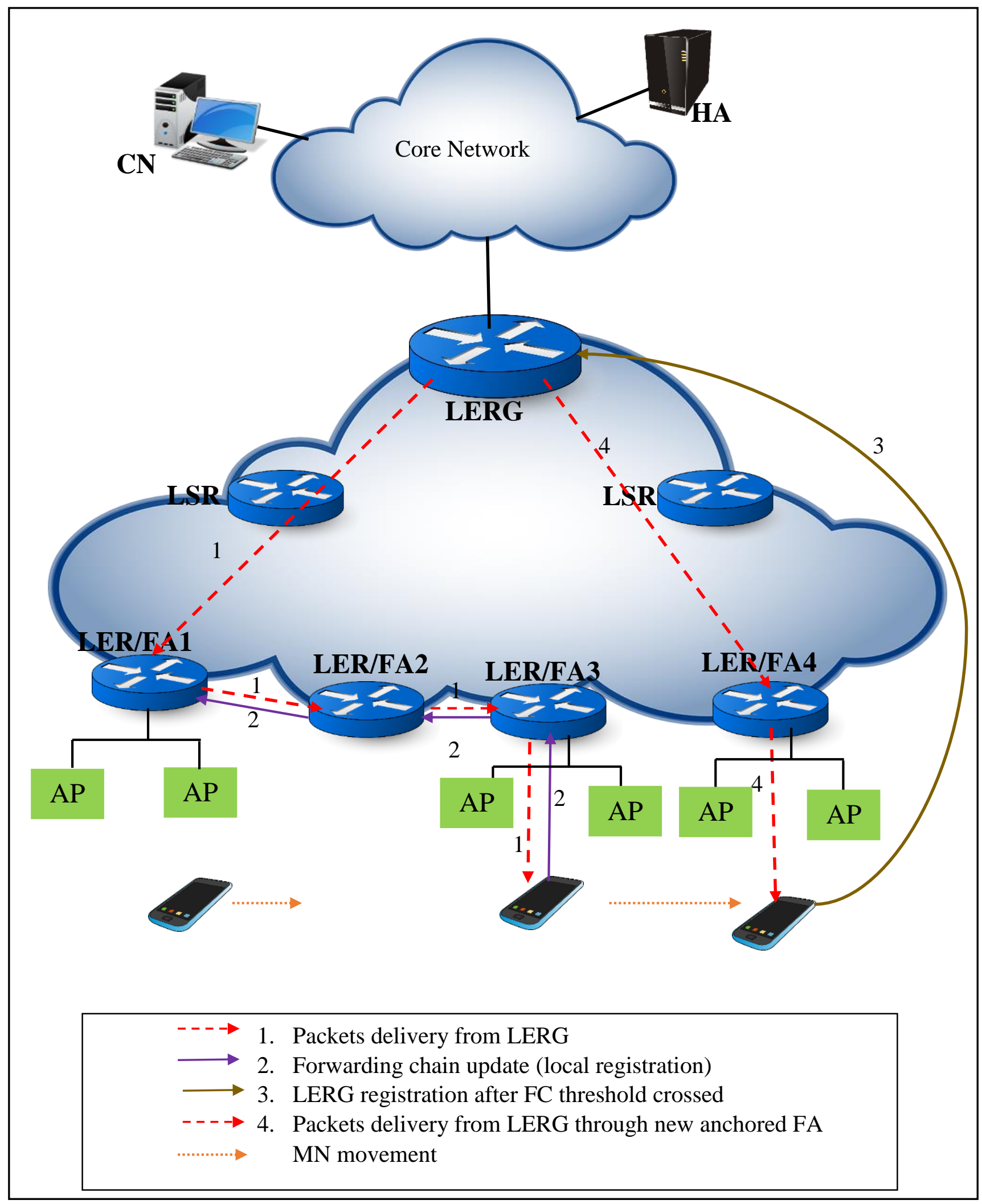

Figure 2.34 FC-MMMPLS architecture (reproduced from [LAN-08]) 


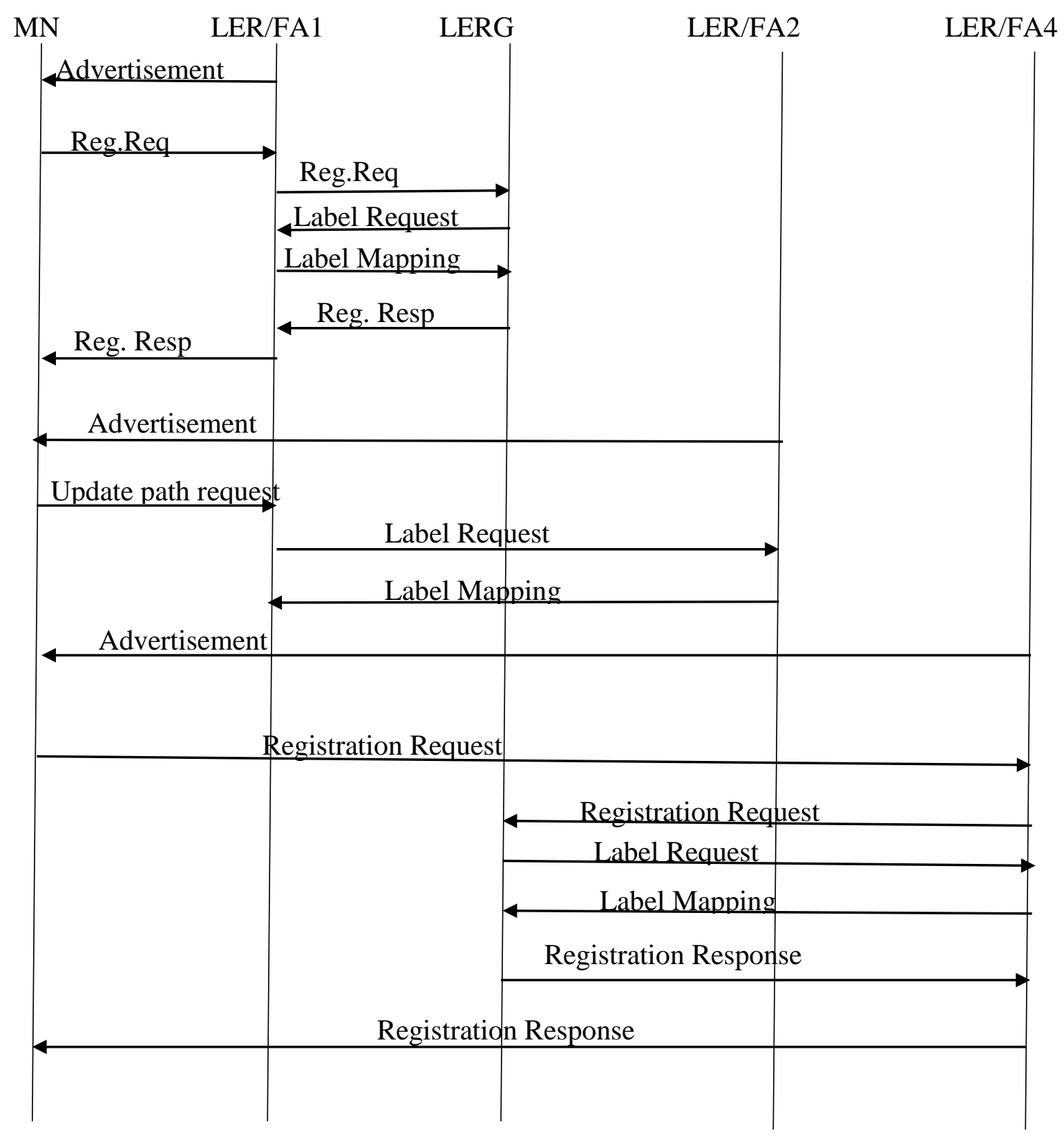

Figure 2.35 Message exchange during handoff in FC-MMMPLS

\subsubsection{Mobile Traffic Engineering with QoS aware MPLS}

Mobile Traffic Engineering with QoS aware MPLS to provide QoS in wireless domain is presented in [GAN-08]. The wireless access domain is hierarchical with gateway foreign agents (GFA) at the boundary of wireless domain and core network and mobile foreign agents (MFA) that are point of contact for MNs. Each MFA is connected to several APs and the APs provide connectivity to the MNs. The GFA's and MFA's are connected through pre-established DiffServ 
Traffic Engineered (DS-TE) tunnels that are created using DS-TE. DS-TE is an extension of RSVP-TE and provide QoS functionalities. It should be noted that multiple GFA's are present in the wireless domain and MFA's are connected to more than one GFA. The architecture is shown in figure 2.36 .

The tunnels are set up by MFA. First, the MFA sets up the uplink DS-TE tunnels with designated bandwidth for each QoS class to the GFA. Then the MFA sends the registration request to the GFA using a modified MIP registration request message. The modified message has a type, length and value extension. In the value extension field, the number of QoS applications based on DSCP and the requested bandwidth for each application is listed. GFA creates the downlink DS-TE tunnels and sends the modified MIP registration response to the MFA. This registration response also includes the CoA of the GFA. Once the tunnels are established, the MFA starts sending the agent advertisement messages, these messages contains the CoA of all connected GFAs.

When the MN moves into the wireless domain, it receives the agent advertisement message through the MFA and follows by sending the registration request to the MFA using the address of the first GFA in the list as CoA. The request is forwarded to the HA through GFA. When the GFA receives the reply from the HA, it adds the entry of the MN in its FEC table and link the entry to the corresponding DS-TE tunnel. It then uses the established DS-TE tunnel to send the response to MFA. When this response arrives at the MFA, it also adds an entry linking the FEC of the MN to the DS-TE tunnel and forwards the response to the MN. These DS-TE tunnels carry aggregate traffic for each QoS class from multiple MNs connected to the same MFA.

Two types of handoffs are discussed in this scheme. Intra-MFA and Inter MFA. In Intra-MFA, when the connection to the current $\mathrm{AP}$ is lost, $\mathrm{MN}$ scans for a new AP and perform layer 2 registration. It then waits for the MFA agent advertisement message or sends the mobile solicitation message. MFA updates it Address Resolution Protocol (ARP) table and no further process happens. 


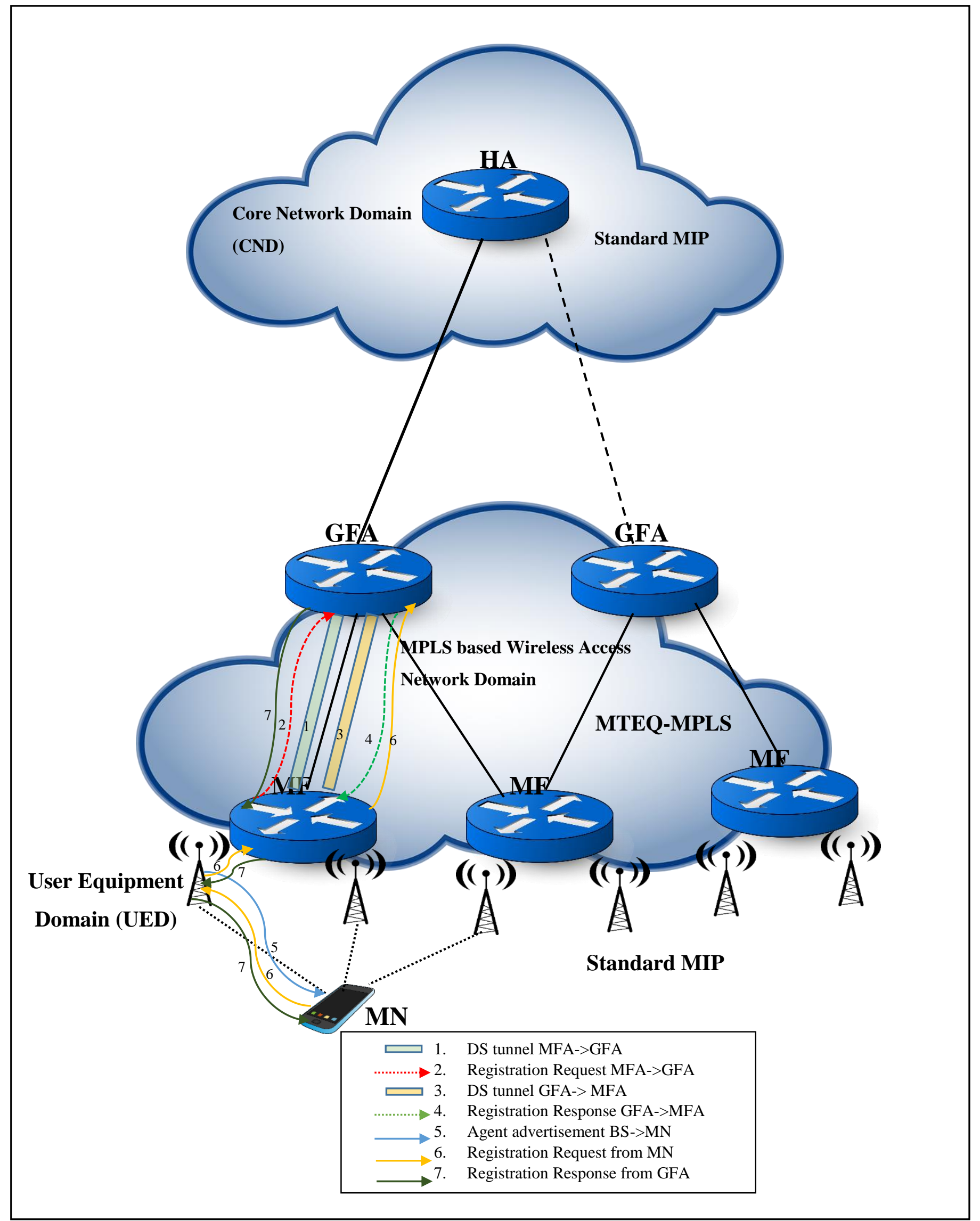

Figure 2.36 Architecture of Mobile Traffic Engineering with QoS aware MPLS scheme 
In case of Inter MFA registration, the entire registration process is repeated upto GFA or HA depending upon if the new MFA is connected to the same GFA or different GFA. To further reduce handoff latency, pre-registration procedure is also proposed. In this scheme, it is assumed that a monitoring component is installed in the $\mathrm{MN}$, which keeps evaluating the strength of the radio link between the MN and AP. Once the signal strength drops below a certain threshold, it triggers the handoff mechanism. The MN scans the neighboring APs and send a list of the perspective MFA's to the old MFA via proxy solicitation message. The old MFA selects a new MFA and sends the CoA to the MN. The MN initiates the registration procedure with the new MFA via the MFA. Once the registration process is complete and the GFA becomes updated, the MN disconnects from the old MFA without further signalling. This work essentially presents hard handoff and does not present any packet buffering or packet recovery schemes hence QoS can be significantly effected in this scheme. Figure 2.37 shows the intra MFA handoff operation.

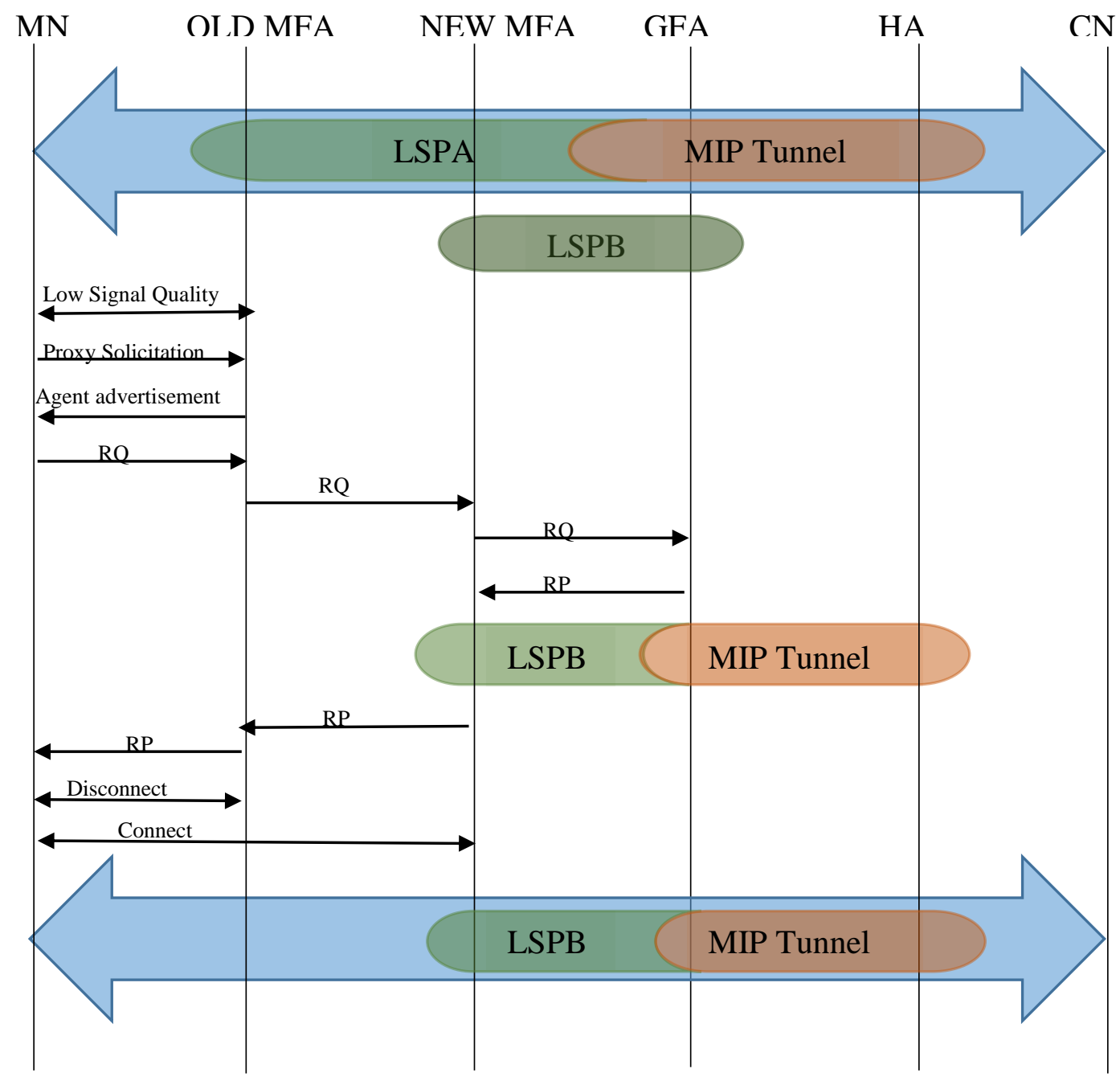

Figure 2.37 Intra MFA handoff in Mobile Traffic Engineering with QoS aware MPLS scheme (reproduced from [GAN-08]) 


\subsubsection{Comparison Between MPLS-based Micro Mobility Schemes}

\begin{tabular}{|c|c|c|c|c|c|c|c|c|c|c|c|c|c|c|}
\hline & $\begin{array}{l}\text { MIP- } \\
\text { MPLS }\end{array}$ & HMPLS & MAMPLS & $\begin{array}{l}\text { MPLS- } \\
\text { HMIP }\end{array}$ & $\begin{array}{l}\text { MM- } \\
\text { MPLS }\end{array}$ & LEMA & $\begin{array}{l}\text { MB- } \\
\text { MM } \\
\text { S }\end{array}$ & $\begin{array}{l}\text { M- } \\
\text { MPLS }\end{array}$ & $\begin{array}{l}\text { W- } \\
\text { MPLS }\end{array}$ & $\begin{array}{l}\text { V- } \\
\text { MPLS }\end{array}$ & $\begin{array}{l}\text { FH-MM- } \\
\text { MPLS }\end{array}$ & $\begin{array}{l}\text { FC- } \\
\text { MM_MPL } \\
\text { S }\end{array}$ & $\begin{array}{l}\text { MFC- } \\
\text { MM- } \\
\text { MPLS }\end{array}$ & $\begin{array}{l}\text { MTE- } \\
\text { MPLS }\end{array}$ \\
\hline $\begin{array}{l}\text { LSP redirecting } \\
\text { nodes }\end{array}$ & $\mathrm{HA}$ & FDA & $\mathrm{CR}$ & RFA & $\mathrm{CR}$ & LEMA & PS & MAP & $\begin{array}{l}\text { ME- } \\
\text { LSR }\end{array}$ & MAP & LERG & FA & MFA & GFA \\
\hline $\begin{array}{l}\text { Passive } \\
\text { LSPs/Static } \\
\text { LSPs }\end{array}$ & $\mathrm{N} / \mathrm{A}$ & Yes & Yes & No & No & Yes & No & No & No & No & Yes & No & No & Yes \\
\hline $\begin{array}{l}\text { Signalling } \\
\text { Protocol (LSP) }\end{array}$ & LDP & LDP & RSVP & LDP & $\begin{array}{l}\text { RSVP- } \\
\text { TE }\end{array}$ & $\begin{array}{l}\text { Not } \\
\text { Specified }\end{array}$ & LDP & LDP & $\begin{array}{l}\text { RSVP- } \\
\text { TE }\end{array}$ & $\begin{array}{l}\text { RSVP- } \\
\text { TE }\end{array}$ & $\begin{array}{l}\text { Not } \\
\text { Specifie } \\
\text { d }\end{array}$ & $\begin{array}{l}\text { Not } \\
\text { Specified }\end{array}$ & $\begin{array}{l}\text { Not } \\
\text { Specified }\end{array}$ & DS-TE \\
\hline $\begin{array}{l}\text { Mobility } \\
\text { managing nodes }\end{array}$ & $\begin{array}{l}\mathrm{CN}, \mathrm{HA}, \mathrm{F} \\
\mathrm{A}\end{array}$ & FDA,FA & $\begin{array}{l}\text { GW,CR,BS, } \\
\text { MN }\end{array}$ & $\begin{array}{l}\text { GFA,RF } \\
\text { A,FA }\end{array}$ & $\begin{array}{l}\mathrm{FDA}, \\
\text { FA,CR }\end{array}$ & $\begin{array}{l}\text { MN, } \\
\text { LEMA } \\
\text { LSR, } \\
\text { LEMA } \\
\text { AR }\end{array}$ & $\begin{array}{l}\text { GW } \\
\text { PS, } \\
\text { BS. }\end{array}$ & $\begin{array}{l}\text { EGW, } \\
\text { MAP, } \\
\text { RAR }\end{array}$ & $\begin{array}{l}\text { RG, } \\
\text { A-LER, } \\
\text { ME- } \\
\text { LSR }\end{array}$ & $\begin{array}{l}\text { MAP, } \\
\text { LER }\end{array}$ & $\begin{array}{l}\text { LERG, } \\
\text { FA }\end{array}$ & LERG, FA & $\begin{array}{l}\text { LERG, } \\
\text { MFA, FA. }\end{array}$ & $\begin{array}{l}\text { GFA, } \\
\text { MFA }\end{array}$ \\
\hline $\begin{array}{l}\text { LSP between } \\
\text { neighbouring } \\
\text { FAs }\end{array}$ & No & No & No & No & No & No & Yes & Yes & Yes & Yes & Yes & Yes & No & No \\
\hline $\begin{array}{l}\text { Packet Buffering } \\
\text { during handoff }\end{array}$ & No & No & No & $\begin{array}{l}\text { Nol } \\
\text { Buffer } \\
\text { size } \\
\text { evaluatio } \\
n\end{array}$ & No & No & Yes & No & No & No & Yes & Yes & Yes & No \\
\hline $\begin{array}{l}\text { Explicit QoS } \\
\text { Support }\end{array}$ & No & No & $\begin{array}{l}\text { DiffServl } \\
\text { IntServ }\end{array}$ & No & No & No & No & No & No & $\begin{array}{l}\text { DiffSer } \\
v\end{array}$ & No & No & No & DiffServ \\
\hline Signaling Load * & $\mathrm{VH}$ & $\mathrm{H}$ & $M$ & $M$ & $M$ & $M$ & $M$ & $\mathrm{H}$ & $\mathrm{H}$ & $\mathrm{H}$ & $M$ & $\mathrm{~L}$ & $\mathrm{~L}$ & $\mathrm{H}$ \\
\hline
\end{tabular}

${ }^{*} \mathrm{VH}=$ Very High, $\mathrm{H}=$ High, M=Moderate, L=Light

* Signaling load is considered VH when LSP redirection is done from HA, $\mathrm{H}$ when redirection is from Domain Root Router, $M$ when redirection is from CR, and $L$ when redirection is from another $F A$.

Table 2.6 Comparison between MPLS-based micro-mobility schemes

Table 2.6 provides a comparison between the reviewed schemes. The items the schemes are compared on are listed in the leftmost column of table 2.6. The LSP redirection function is included in most schemes however, some schemes require redirection from the domain root router, which tends to generate high handoff latency. The HMPLS, MAMPLS, LEMA, and FHMM-MPLS schemes either employ a pre-established static LSP or dynamically established LSP during handoff to further reduce handoff latency. In terms of signaling protocols, LDP, RSVP, RSVP-TE and DS-TE have all been used as possible choices. Most schemes have utilized an additional CR as mobility management router in addition to FA and gateway router. This results in fast LSP redirection during handoff at the expense of increased complexity of the proposed architecture. LEMA especially can be looked up as one example as it requires all of the nodes to be LEMA enhanced nodes. MB-MMS, M-MPLS, W-MPLS, V-MPLS, FH-MM-MPLS and FC- 
MM-MPLS schemes allow LSP creation between neighbouring FAs during/after handoff. This enables forwarding of en-route packets to MN through the new FA thus resulting in lower packet loss during handoff. Most schemes do not include buffering mechanisms in their architecture. The outcome from adopting such approach is increased packet loss, which might degrade QoS during hand-off process. For example, in MTE-MPLS, initially DS-TE tunnels are created for providing QoS support, however, when a MN moves between MFAs, hard handoff is employed and QoS is affected considerably. FC-MM-MPLS and MFC-MM-MPLS schemes support lowest signalling load during handoff as the LSPs are redirected from neighbouring FAs.

In the context of $5 \mathrm{G}$ vehicular networks, fast and efficient handoff with low signalling load and minimum packet loss is significant. Continuous QoS support is also critical as 5G vehicular networks would be exchanging data for safety critical applications, which are becoming especially significant applications with the emergence of autonomous vehicles. The presence of a network where vehicles are interdependent for safety of masses is highly desirable as it will reduce the dependency of vehicles entirely on stand-alone sensors and would result in reduction of vehicle prices.

Hence, considering the presented capabilities of MPLS to support QoS effectively in a practical and very reliable manner, we need to devise an MPLS based scheme that integrates the benefits of existing schemes and offers a comprehensive solution for fast and efficient handoff with low signaling load and seamless QoS provision. Below we discuss some of the main challenging issues that need to be addressed before high speed micro-mobile MPLS vehicular networks can be widely deployed.

\section{Fast Packet Forwarding}

Packet forwarding in the existing schemes are accomplished based on two different models: Overlay and Integrated. In the overlay model, the packets are forwarded through multiple conversions between MPLS-IP-MPLS layers. In this approach, the LSP from CN to MN consists of various disjoint segments of LSPs that terminate at special routers capable of performing the MPLS based micro-mobility management. When a packet arrives at such router, e.g. Gateway router of an MPLS domain, through a pre-established LSP, the gateway router strips off the MPLS label, sends the packet to the IP layer to read its destination address binding, reencapsulates it in another MPLS header based on LFIB entry of appropriate label and port and forwards it towards the current location of MN. The drawback of this approach is consumption 
of time and computational resources. On the other hand, in the integrated approach, the disjoint segments of LSPs are merged at the combining nodes (usually mobility aware routers) to create a single LSP to the current location of MN. This is accomplished by creating one exclusive entry in the forwarding table for each $\mathrm{MN}$ at the combining nodes and using a pointer or next FEC entry in the forwarding table to send the packets to the current location of the MN. The integrated approach eradicates the MPLS-IP-MPLS conversion, simplifies processing at each node and enables faster, more efficient packet forwarding. However, this approach results in having to maintain a larger forwarding table at each node. This is due to the fact that an exclusive LSP is established for each MN. Considering the number of MNs in each MPLS domain, adopting this approach may result in serious scalability issues as the number of MNs in the MPLS domain increases.

\section{Registration Load}

Low registration load is important in MPLS domain supporting vehicular networks. Due to highly mobile environment, vehicles will cross the subnet boundaries at a much higher rate than traditional low to moderate speed users. Registration load is directly related to the number of handoffs during a session, the size of the registration message and the number of hops traversed by the message to carry out the handoff. When the registration is carried out by the MNs, the LSPs are redirected to forward the data packets to the current location of the MNs. In some of the schemes such as HMPLS, FH-MM-MPLS and MTE-MPLS, the LSPs are redirected from the root domain router. Obviously, considering the high number of handoffs that would be required by the vehicular networks, adoption of these schemes would result in extensive registration messages processing and introduces delay at each node. Hence, these schemes are highly unsuitable for vehicular networks. Some schemes utilize an intermediate or CR to carry out the registration. In these schemes, such as MAMPLS, MPLS-HMIP, MM-MPLS, and MB-MMS, the registration message sent from the $\mathrm{MN}$ to the domain gateway router is intercepted by the $\mathrm{CR}$. The CR searches its forwarding table to find the binding entry of the MN and updates the next hop and next label entry the forwarding table to match the current location of the MN. Hence the message is not forwarded all the way to the root of the domain. Although, this results in less registration message processing in the MPLS domain, it may still introduce considerable processing delay in the domain, depending on the location of the CR. If the CR is located only one or two hops away from the domain gateway, then there will still be considerable registration 
load on the MPLS network. A more appropriate approach is the use of forwarding chain mechanism, as presented in FC-MM-MPLS and MFC-MM-MPLS schemes. In these schemes, a forwarding chain is established and extended for each subnet movement of the MN up to a certain pre-determined threshold in terms of number of hops. Once the threshold is reached the handoff is carried out from the domain gateway. The advantage of this scheme is that the registration load in the MPLS network is reduced significantly, as the message traverses between one to a maximum (M-1) hops, where $\mathrm{M}$ is the threshold of the forwarding chain.

\section{Handoff Latency}

Minimum handoff latency is important in the $5 \mathrm{G}$ vehicular networks. Safety applications, directly related to autonomous vehicles control, would have the latency requirement of less than $1 \mathrm{~ms}$. Handoff latency is directly proportional to the processing delay incurred by each node to process registration message and the number of hops that the message traverse to carry out reregistration. Hence, the schemes that redirect LSPs from domain gateway router will introduce longer handoff delay whereas, forwarding chain based MPLS schemes would have noticeably lower handoff latency. It should also be considered that handoff latency also includes the time that the MNs spends without connectivity to any subnet, hence the speed of the vehicle and coverage area of the cells are also significant.

\section{Seamless QoS Provision} Seamless QoS provision is of vital importance for future $5 \mathrm{G}$ vehicular networks. Since future vehicular networks are envisioned to be fully automated and would require minimum human interaction, it is important that safety critical information exchange is prioritized over other data exchange. MPLS can provide QoS support by establishing traffic engineered LSPs. RSVP-TE signalling protocol is commonly adopted to establish TE-LSPs. Packet forwarding on these TELSPs can be flow-based according to IntServ model or class-based as in DiffServ architecture. In DiffServ architecture, IP networks utilize the 6 bit DSCP field to classify the IP packets and assign the per hop behaviour and drop precedence at each network node. In MPLS domain, two different types of LSPs, E-LSP and L-LSPs can be used to enable class based forwarding according to the DiffServ architecture. In E-LSPs, the 3 bit EXP field in MPLS header is used to indicate PHB. Traffic belonging to different FECs, for example EF and AF1, may be forwarded on the same LSP (same label) but be stored in different queues within each LSR. In L-LSP, PHB is extracted from the label value. Each traffic class is forwarded on separate LSP and is stored in different queues at LSRs. In L-LSPS, packet drop precedence is extracted from the EXP field. As 
IntServ based MPLS architecture provides latency, delivery and bandwidth guarantees, it is more suitable than the MPLS DiffServ scheme for 5G vehicular networks. This benefit is obtained at the expense of sizable forwarding tables that need to be created and maintained at each MPLS router.

\section{Scalability}

Both MPLS packet forwarding schemes i.e. integrated and overlay approach faces scalability issues. In the integrated model (as adopted in ME-MMS, V-MPLS and M-MPLS schemes) the individual LSPs are linked together at the "LSP meeting nodes" usually, gateway or crossover routers. This is done by introducing additional fields in the forwarding tables and using pointers to indicate the next LSP segment that is required for forwarding packets to the current location of MNs. Obviously this results in fast and efficient packet forwarding at the expense of large forwarding tables. This introduces serious scalability issues as the number of MNs in the MPLS domain increases. On the other hand, the overlay approach (as adopted in HMPLS, W-MPLS and FH-MM-MPLS) requires MPLS-IP-MPLS conversion at each connecting node to direct the packet to the current location of MN. The benefit of this approach is that the data traffic can be aggregated and forwarded between connecting nodes without the need of maintaining large forwarding tables since exclusive LSPs are not required for MNs. However, this approach require considerable processing at the LSP connecting nodes. The conversion between software modules and hence number of processing operations increases with the number of MNs in the MPLS domain as well and introduces extensive processing scalability issues. Depending on the processing delay at each node, the overlay approach may also not be suitable to fulfill the QoS requirements of the network. 


\section{Chapter 3: COMPARATIVE ANALYSIS BETWEEN VL AND MMWAVE FOR 5G VEHICULAR NETWORKS}

\subsection{Visible Light Communication}

\subsubsection{Historical Review}

Visible light communication (VLC) refers to the data transfer in visible spectrum that ranges from $400 \mathrm{THz}(780 \mathrm{~nm})$ to $800 \mathrm{THz}(375 \mathrm{~nm})$ using ordinary light sources such as fluorescent lamps [JAC-98] and light emitting diodes (LEDs) as transmitter and photo diodes as receivers. Due to their fast switching capabilities and longer lifetime with lower energy dissipation, LEDs are the perfect candidate for visible light data transfer. Although research in VLC using traditional lighting infrastructure has gained momentum during the last 15 years, the perception of using visible light for communication is quiet ancient. The first referenced use of visible light for communication employing appropriate model dates back to 1880 with the invention of Photophone by Dr. Alexander Graham Bell. In modern times, the formation of infrared data association (IrDA) in 1993 [IrD-93] played a key role in research advancement utilizing the infrared part of the electromagnetic spectrum for secure wireless optical communication. However, infrared can be used only for very short range and is suitable for low to medium speed transmission in indoor environment. Although recent advancement in technology with the adoption of Giga-IR standards [GIG-14] enables high data rate communication using IR, it is recognized that high data rate transmission increases the associated visual safety risk with amplified emission energy and invisibility, thus, making VLC a safer choice.

With cheap and energy efficient LEDs replacing the conventional light sources such as incandescent and fluorescent lamps and the RF spectrum becoming heavily congested, researchers have shifted their focus towards VLC as an alternative to achieve high rate data communication. The recent work employing visible light for communication dates back to early 2000. In December 2001, a user controlled project Reasonable Optical Near Joint Access 
(RONJA) [RON-01] developed in Czech Republic became the first device to transmit in free space at $10 \mathrm{Mb} / \mathrm{s}$ over a distance of $1.4 \mathrm{Kms}$ providing optical point-to-point data link using red light. However, RONJA suffers from severe performance degradation with adverse weather conditions such as fog or snow. The vast majority of the work on visual light free space optical communications at the early years was performed in Japan. In November 2003, the Visible Light Communication Consortium (VLCC) [VLC-03] was established, with the majority of its members coming from the Japanese academia and industry. In 2004, at CEATEC Japan, the VLCC displayed four different exhibits showcasing visible light communication [CEA-04]. These exhibits displayed sound and light collaboration, traffic signal communication system, Illumination ID, and VLC applied to cell phone display. VLC was also used on trial basis in parts of Kansai airport, Japan, in 2005 as the means of sending flight information to cell phones. In 2007, the VLCC proposed two visible light communication standards to the Japan Electronics and Information Technology Industries Association (JEITA). The two proposed standards were accepted by JEITA (CP-1221 and CP 1222) [JEI-07] as the standards for "visible light communication system" and "visible light ID system". Since 2008, research in the area grew, and several large projects dealing with the subject were initiated, generating new interesting results all over the world. Work performed under the OMEGA [OME-11] project, funded by European Union, demonstrated the potential to transmit data indoors using VLC at $100 \mathrm{Mb} / \mathrm{s}$ and higher. The Cooperative Research Center on Optical Wireless Applications (COWA) [COW-10] was established by the collaboration of National Science Foundation (NSF), Pennsylvania State University (PSU), Georgia Institute of Technology (GT) and industry. This center is conducting research for promoting VLC in navigation and positioning systems, health care, and other commercial and hazardous environments, where RF cannot be used. The center for Ubiquitous Communication by Light (UC-Light) [UCL-10] at University of California is conducting active research in VLC systems, networks, and ASIC designs. In 2009, the VLCC released their first physical layer specification standard adopting and expanding the infrared Data Association (IrDA) physical layer standard to reduce the deployment cost [BUS-09]. In 2011, at TED Global, VLC was given the label "Li-Fi" [HAA-11] by Prof. Harold Hass of University of Edinburgh and the Li-Fi consortium [LiF-11] was shortly formed afterwards to promote research in the visible spectrum with a vision of achieving data transfer at the rate of $10+\mathrm{Gb} / \mathrm{s}$. University of Edinburgh also initiated a D-Light (Data-Light) project [POV-11] that has developed modulation 
techniques to achieve over $100 \mathrm{Mb} / \mathrm{s}$ [HHI-13] data rate using off the shelf LEDs. The IEEE task group for Personal Area Network (PAN) published the IEEE 802.15.7 Physical and MAC layer standard for visible light communication in 2011. The standard supports indoor communication at speeds up to $96 \mathrm{Mb} / \mathrm{s}$ and outdoor communication at $266 \mathrm{kbps}$ [STD-11]. Various researchers have reported VLC systems achieving data rates from $500 \mathrm{Mb} / \mathrm{s}$ [VUC-10], [LAN-13] to 800 $\mathrm{Mb} / \mathrm{s}$ (with offline processing) [PAR-12] in indoor environment. In 2013, there were two major developments in VLC communication. Firstly, Fraunhoufer Heinrich Hertz Institute reported having tested components enabling data transfer using LED's at $3 \mathrm{~Gb} / \mathrm{s}$ in laboratory environment [HHI-13a]. Secondly, the researchers of Ultra-parallel visible light communication project (UP-VLC) [UPV-15] which is a joint venture of universities of Oxford, Cambridge, Edinburgh, St Andrews and Strathclyde, and funded by the Engineering and Physical Sciences Research Council reported that they reached $10 \mathrm{~Gb} / \mathrm{s}$ [VIN-13] transmission speed. In November 2013, National Instruments (NI) and University of Edinburgh announced their collaboration for the development of a massive MIMO indoor VLC prototype using reconfigurable FPGAs, software defined radio bundles of NI and interchangeable I/O adapter modules that will operate beyond the rates of commercial RF wireless systems, as a step towards the $5 \mathrm{G}$ wireless communications [NAT-13]. At TED GLOBAL held in July 2011, there was live demonstration of high definition video being transmitted from LED lamps. By 2010, commercial VLC products started appearing in the market such as VLC transceivers by LVX Minnesota in December 2010 [LVX-10] and Axrtek's MOMO transceivers in 2014, that are capable of uplink and downlink transmission at $300 \mathrm{MB} / \mathrm{s}$ at 25 feet [AXR-14]. During 2010-2017, VLC has found its ground in food retail [PHI-15], toys [GIU-12] and even banking [GUO-15].

As RF spectrum becomes overcrowded, the presence of a high bandwidth, license-free alternative operating in currently vacant spectrum is attractive. The capability of using existing lighting infrastructure compared to RF network that requires installation of expensive RF equipment makes VLC a very cost-effective solution. Vehicular communication has emerged as an attractive domain for use of VLC technology as alternative of RF based communications, in addition to those cases where use of RF has limitations due to safety, security and/or implementation issues such as aviation [QUI-13] [PER-11], underwater information exchange [DAS-12], or where RF power is deemed hazardous environment, as is the case for hospitals 
[SON-14], toys [COR-14] and mines [ITU-14]. Also, localization using optical as compared to RF becomes considerably more accurate [PRI-12] [COS-11]

\subsubsection{Channel Models For Vehicular Visible Light Communication}

The goal of channel modeling is to predict the behavior of the propagation environment through which the communication links will traverse. Hence accurate channel modeling is essential for the development of consistent, robust and competent communication system.

VVLC channel modeling is a challenging task as the existing radio communication channel models for vehicular systems cannot be adopted to represent the behavior of optical wireless channels. This is because the optical wireless channel exhibits characteristics fundamentally different from radio propagation channels. For example, multipath fading is considered a significant reason for signal distortion in radio communications however, in optical wireless communication as the dimensions of photodetectors are significantly higher in magnitude than the size of optical wavelengths, they provide enough spatial diversity to nullify the effects of multipath fading. In addition, the effect of Doppler shift is insignificant in VLC systems [ELG09]. On the other hand, VLC signals are effected by multipath dispersion, which results in inter symbol interference (ISI).

The wireless optical channel models can be widely characterized as being deterministic or stochastic. The channel models that are most commonly adopted in literature for VVLC communication are geometry based deterministic models (GBDM). Deterministic channel models are established by taking into account specific detailed description of the propagation environment (e.g. presence of buildings, neighboring vehicles, etc.), channel scenario (e.g. LOS, NLOS), and the position and orientation of the transmitters and receivers. These details are significant in obtaining site-specific channel impulse response. GBDM considering the LOS channel for V2I communication is reported in [WAD-05] [PAN-02] [ARA-08] [IWA-08]. Further, ray tracing based deterministic channel modeling for V2V and V2I communication in VVLC systems has also been reported in literature [LEE-12] [LEE-12A]. Ray tracing models are generated through specific ray tracing software, which models the channel with trivial specification regarding the environment such as geometry, furnishing and reflectivity of the surfaces in the immediate vicinity of the vehicles. Generating ray tracing models is time- 
consuming procedure as ray tracing models tracks all rays travelling from source, generating and computing the reflected and transmitted component for each obstacle in its path and this pattern is individually followed for each receiving point. As evident from the procedure, these models are highly scenario specific and are hard to generalize.

Another geometry based model considering the LOS and NLOS component that comprises of the first reflection from the asphalt road surface is presented in [LUO-15]. This work considers tungsten halogen headlamp beam radiation pattern instead of Lambertian radiation pattern which is commonly adopted for VVLC system. Further, the authors extended the work to develop a 2x2 multiple input multiple output (MIMO) system for car-to-car communication. A taillight model developed specifically for LOS communication using off-the-shelf scooter taillights as transmitters for V2V scenario is presented in [VIR-13]. The presented channel model estimates the optical channel behaviour more accurately than existing work in terms of received optical power and packet reception rate. However, this model can be used in a very specific application scenario, which is communication between two scooters. [KIN-18] presents a regular shaped geometry based stochastic model (RS-GBSM) for VVLC channels that considers both LOS and NLOS components. The reflections off the moving neighbouring vehicles in the vicinity of transmitter and receiver and the stationary roadside surroundings are all considered in the developed channel model. As GBSMs are derived from "predefined stochastic distribution of effective scatterers by applying the fundamental laws of wave propagation" [YIN-16], it is more flexible and can be easily generalized compared to GBDM that are site-specific. However, they are also considered less accurate than the GBDM. Table 3.1 summarizes the channel models presented for VVLC systems.

\begin{tabular}{|c|c|c|c|c|c|}
\hline Reference & Modelling Approach & Environment & Scenario & $\begin{array}{c}\text { Evaluated Channel } \\
\text { Characteristics }\end{array}$ & $\begin{array}{c}\text { Operating } \\
\text { Wavelength }\end{array}$ \\
\hline [WAD-05] & GBDM & LOS & I2V & $\begin{array}{c}\text { Transmission } \\
\text { Speed }\end{array}$ & $\begin{array}{c}\lambda=650 \mathrm{~nm}, \\
540 \mathrm{~nm}, \\
\end{array}$ \\
& & & & & $580 \mathrm{~nm}$. \\
\hline [PAN-02] & GBDM & LOS & I2V & H(f), BER, SNR & $\lambda=650 \mathrm{~nm}$ \\
\hline [ARA-08] & GBDM & LOS & I2V & BER with data & $\lambda=650 \mathrm{~nm}$, \\
& & & & priority & $540 \mathrm{~nm}$, \\
& & & & & $580 \mathrm{~nm}$ \\
\hline
\end{tabular}




\begin{tabular}{|c|c|c|c|c|c|}
\hline [IWA-08] & GBDM & LOS & I2V & BER & $\begin{array}{c}\lambda=650 \mathrm{~nm}, \\
540 \mathrm{~nm}, \\
580 \mathrm{~nm}\end{array}$ \\
\hline [LEE-12] & Ray-tracing & LOS, NLOS & I2V, V2V & $\mathrm{h}(\mathrm{t})$ & $\begin{array}{c}\lambda=380- \\
780 \mathrm{~nm}\end{array}$ \\
\hline [LEE-12A] & Ray-tracing & LOS, NLOS & I2V, V2V & Delay profile & $\begin{array}{c}\lambda=380- \\
780 \mathrm{~nm}\end{array}$ \\
\hline [LUO-15] & First Reflection & LOS, NLOS & V2V & BER, & $\begin{array}{c}\lambda=380- \\
\text { Communication } \\
\end{array}$ \\
& & & & range & $780 \mathrm{~nm}$ \\
\hline [VIR-13] & Taillight Model & LOS & V2V & H(f), BER, PRR & $\lambda=650 \mathrm{~nm}$ \\
\hline [KIN-18] & RS-GBSM & LOS, NLOS & V2V & H(0), h(t), DRMS & $\lambda=380-$ \\
& & & & & $780 \mathrm{~nm}$ \\
\hline
\end{tabular}

Table 3.1 Channel models for VVLC systems presented in open literature

VVLC systems are still in the early stages of analysis, modelling, and evaluation, hence open literature still lacks in-depth survey of challenges that the real life VVLC systems will encounter such as impact of adverse weather condition and multipath interference.

\subsubsection{Visible Light Propagation Characteristics}

Atmosphere is the propagation channel for visible light signals. While traversing the atmosphere, visible light waves lose energy due to scattering, reflection, refraction and other propagation phenomenon in addition to free space loss. Visible light possess unique properties such as no interference to RF frequencies, sensitivity to blockage and multipath induced signal distortion in diffused VLC links which makes modelling of VLC channels much different than those for RF communication. Although, use of visible light technology for indoor communication has been extensively reported, in outdoor environment it has been less pursued. The main reasons for this is due to the fact that the dual use of light emitting diodes (LED's) is not always required in the outdoor VLC environment e.g. during the daytime, sun is already illuminating the environment and lamp posts and lighting poles are not required for illumination purposes. Secondly, the level of noise and interference due to solar radiations and artificial light sources are considerably higher in outdoor VLC compared to indoor VLC channels. However, with upcoming era of 5G devouring essential requirement of high data rate communication within small cell radius and absence of a reliable communication technology specifically for V2V and V2I communication, 
VLC has emerged as an attractive technology for outdoor communication. Various results have been reported in literature confirming reception of high power signals in medium to short range outdoor channel [CAI-16] [CAI-17] [KUM-12] [LIU-12]. Some major propagation characteristics of the outdoor high speed channel are presented in figure 3.1 and briefly described thereafter.

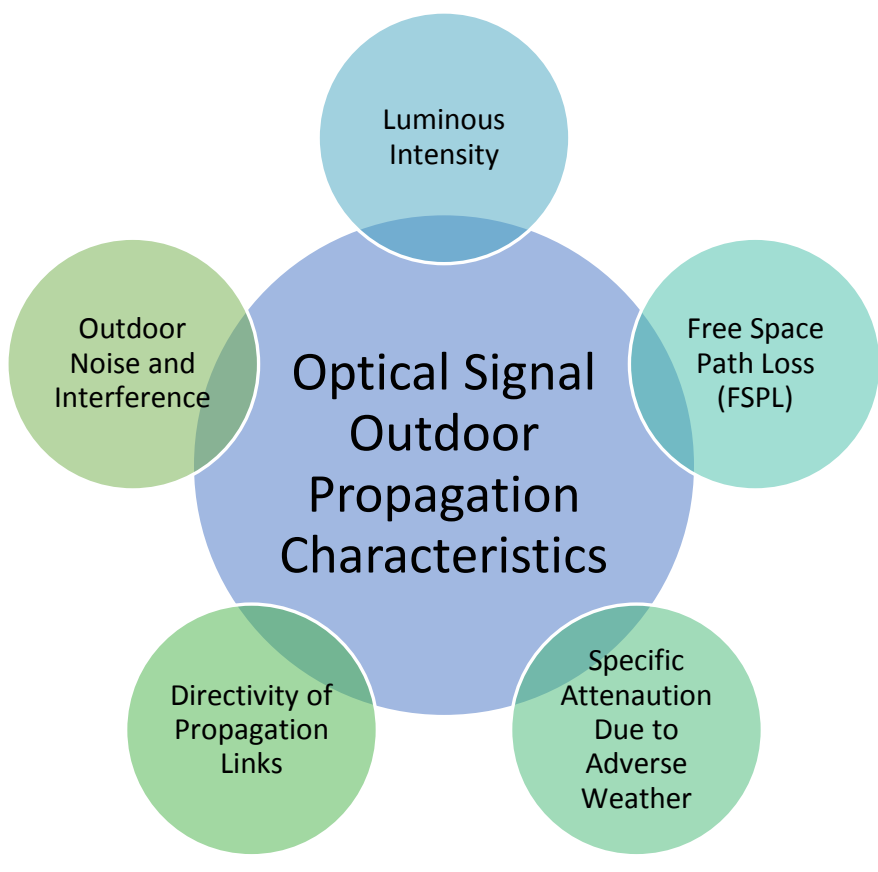

Figure 3.1: Outdoor Propagation Characteristics of Optical Signals

\subsubsection{Luminous Intensity}

Lighting characteristics of LEDs are defined by photometric and radiometric quantities. The photometric quantity provides information about the perceived brightness of the LEDs, whereas, the radiometric quantity provides measurement about absolute brightness of the LEDs. The photopic spectral luminous efficiency curve $V(\lambda)$ provides the spectral response of the human eye to various wavelengths of light and is used to provide conversion between photometric and radiometric terms [THO-18]. Luminous intensity in photometric terms is defined as the luminous flux per solid ${ }^{1}$ angle unit. It corresponds to the brightness of a LED as perceived by the human eye and is measured in candelas (cd). The corresponding terminology in radiometric terms is

1. "A plane angle, $\theta$, made up of the lines from two points meeting at a vertex, is defined by the arc length of a circle. The unit of plane angle is the radian, with $2 \pi$ radians in a full circle. A solid angle, $\omega$, made up of all the lines from a closed curve meeting at a vertex, is defined by the surface area of a sphere subtended by the lines and by the radius of that sphere. The dimensionless unit of solid angle is the steradian, with $4 \pi$ steradians in a full sphere". A. V. Arecchi, T. Messadi, and R. J. Koshel, Field Guide to Illumination, SPIE Press, Bellingham, WA (2007). Simply, plane angles are 2 dimensional whereas solid angles are 3 dimensional. 
radiant intensity and is measured in Watts per steradian ${ }^{1}$. Luminous intensity is given by following expression:

$$
I=\frac{d \emptyset}{d \Omega}
$$

Where $I$ is the luminous intensity, $\varnothing$ is the luminous flux, and $\Omega$ is the solid angle.

\subsubsection{Free Space Path Loss (FSPL)}

Free space path loss is defined as the difference between transmitted and received power while the optical signal is propagating through free space in line-of-sight (LOS). Some important terminologies that are used in this section are defined as:

- LED Half Power Angle: LED half power angle is defined as the measured angle with respect to LED's emission centerline at which the intensity of LED emission has fallen to $50 \%$ of its originating intensity.

- Field of View (FOV): Field of view is defines as a solid angle through which light may enter the receiver's photosensitive area.

- Angle of incidence: "The angle between the light ray incident on a surface and the line perpendicular to the surface at the point of incidence.’[MER-19]

- Angle of irradiance: The angle between the light emitted by the transmitter in a particular direction towards a surface area and the line perpendicular to transmitter is called angle of irradiance.

- Optical Filter: Optical filters are devices that transmit particularly chosen wavelengths of light while blocking other wavelengths.

- Optical Concentrator: Optical concentrators converge the incident light rays on the receiver's aperture to the photodetector.

Figure 3.2 shows optical transmitter and receiver with defined terminologies. 


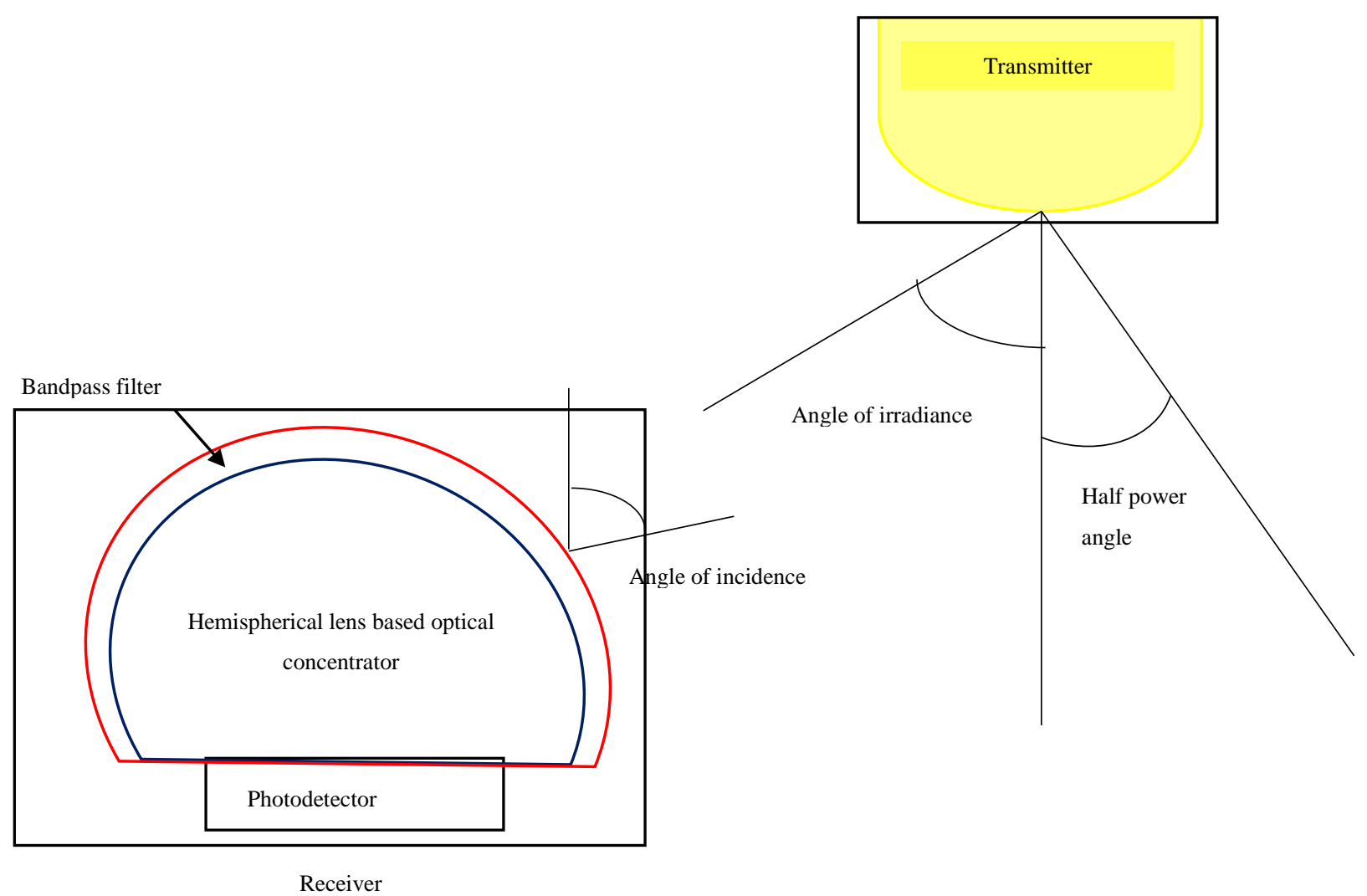

Figure 3.2: Optical transmitter and receiver. Receiver has been reproduced from [KAH-97]

The LEDs are directional light sources and they come in different shapes and sizes and various radiation patterns. Here we assume LEDs with uniform radiation pattern (Lambertian sources) for analysis. These LEDs are follow Lambert's cosine law power. Hence, the emission from LED transmitters $\mathrm{R}_{\mathrm{e}}$ can be modeled using a generalized Lambertian radiant intensity:

$$
R_{e}(\varnothing)=\frac{m+1}{2 \pi} P_{t} \cos ^{m}(\varnothing)
$$

Where $\mathrm{P}_{\mathrm{t}}$ is the transmission optical power and the order $\mathrm{m}$ is related to LED half angle, $\phi_{1 / 2}$ by

$$
m=-\frac{\ln 2}{\ln \cos \phi_{\frac{1}{2}}}
$$

Correspondingly, the pathloss is given by: 


$$
P l=\left\{\begin{array}{rr}
\frac{(m+1) A}{2 \pi d^{2}} \cos ^{m}(\varnothing) T s(\Psi) g(\Psi) \cos (\Psi) ; & 0<\Psi<\Psi_{c} \\
0 & \Psi>\Psi_{c}
\end{array}\right\}
$$

Where, $\mathrm{A}$ is the physical photosensitive area of the detector photodiode, $\emptyset$ is the angle of irradiance, $\boldsymbol{\Psi}$ is the angle of incidence, $\operatorname{Ts}(\boldsymbol{\Psi})$ is gain of optical filter used at transmitting LED, $\mathrm{g}(\boldsymbol{\Psi})$ is the optical concentrator gain in the receiver and $\boldsymbol{\Psi}_{\mathrm{c}}$ is the receiver's field of view (FOV).

The gain from an idealized non imaging concentrator having an internal refractive index $n$ can be calculated by the following expression:

$$
g(\Psi)=\left\{\begin{array}{ll}
\frac{n^{2}}{\sin ^{2} \Psi_{c}} ; & 0<\Psi<\Psi_{c} \\
0 & \Psi>\Psi_{C}
\end{array}\right\}
$$

\subsubsection{Specific Attenuation due to Adverse Weather}

Visible light signals are highly attenuated due to adverse weather such as fog, rain and snow. While going through foggy patches, the signals are attenuated due to scattering as the molecule sizes are comparable to visible wavelength. Dry snow attenuates visible signal extensively as it completely blocks the visible link. The attenuation due to adverse weather conditions are discussed in detail in section 3.3.

\subsubsection{Outdoor Noise and Interference}

\subsection{Solar Irradiance}

Solar radiation is the most significant interference source during day time. Depending on the FOV of photo detector and sun's position in the sky, the photo detector can easily become saturated [LIU-10]. It is essential that the transmitted signal is isolated from the background solar radiation. However, the photodetector captures only the DC component of solar radiations, which can be eliminated by utilizing a band pass filter, leaving behind the shot noise component that cannot be alleviated. This shot noise component must be accounted for while designing a VLC system. The irradiance due to solar radiation is given by:

$$
I_{S U N}=W(\lambda) \Delta \lambda
$$

Where $\mathrm{W}(\lambda)$ is the spectral radiance of the sun at wavelength $\lambda$ and $\Delta \lambda$ is the bandwidth of the optical BPF at the receiver. Here it is assumed that the bandwidth of the optical filter is narrow so it can be approximated as having flat spectral density in the areas of passing through 
wavelengths, and $\lambda$ is central frequency of the optical filter's spectral response. The variance of the background shot noise is given by:

$$
\sigma_{B g}^{2}=2 q B P_{b g} R\left(I_{S K Y}+I_{S U N}\right)
$$

Where $\mathrm{B}$ is the bit rate of the signal, $\mathrm{R}$ is the responsivity of the detector and $\mathrm{P}_{\mathrm{bg}}$ is the noise power incident on the photodiode. This optical noise power is directly proportional to the transmittance and spectral width of the optical bandpass filter.

\subsection{Daytime Sky Radiance}

The day time sky radiance is caused by the scattering of solar light. This scattered energy will be dependent on the frequency, angle of the sun and the scattering mechanism. For example, the spectral radiance of day time sky within $30^{\circ}$ inclination and $180^{\circ}$ inclination of the sun is produced mainly by large particles or Mie scattering [KAR-88]. Mie scattering results in the spectral radiance values that are inversely proportional to wavelength and humans perceive the sky in those areas as being "white". On the other hand, at around $90^{\circ}$ from the sun, the spectral radiance is due to small particles that are scattered according to Rayleigh model which is proportional to the wavelength by the fourth-order inverse power law [KAR-88]. This enhances the shorter wavelength of solar spectrum, and human eye perceives the sky as being "blue" in those areas. Table 3.2 lists the radiance level at different light conditions.

\begin{tabular}{|l|l|}
\hline Light Conditions & Irradiance Levels $\left(\mathrm{W} / \mathrm{m}^{2}\right)$ \\
\hline Solar irradiation at sea level on a clear day & $10^{3}$ \\
\hline Solar irradiation at sea level on a lightly overcast day & $10^{2}$ \\
\hline Solar irradiation at sea level on a heavily overcast day & 10 \\
\hline Well-lighted interior & 1 \\
\hline Lunar irradiation, full moon, sea level, horizontal surface & $10^{-3}$ \\
\hline First magnitude star's irradiance on surface normal to rays & $10^{-8}$ \\
\hline
\end{tabular}

Table 3.2 Irradiance Levels for Several Light Sources [KAR-88]

\subsection{Nighttime Sky Radiance}

Nigh time sky radiance is caused by zodiacal light, diffused galactic radiation, scattered skylight, and luminance due to moon and stars. These are the most prominent sources of optical noise. 
Compared to solar radiance, the noise induced due to night time sky radiance is pretty low with difference between the solar radiance and lunar radiance being around $60 \mathrm{~dB}$ [KAR-88].

\subsection{Interference due to Artificial Lighting Sources}

Another source of interference in VLC systems is due to the presence of artificial light sources such as halogen, fluorescent lamps and neon sign boards. The optical power received at the photodetector due to artificial light sources introduces a noticeable shot noise component that must be compensated for in order to maintain and enhance system performance. If the artificial lights are located near the VLC transmitter, they also interfere with the transmitted signal by producing a periodic and deterministic optical signal [MOR-97] [KAH-95]. Intervention models have been developed to estimate the interference effecting VLC links due to the presence of artificial light source [MOR-97].

\subsubsection{Directivity of Propagation Links}

The outdoor VLC vehicular channel model is composed of directed and non-directed line-ofsight (LOS) communication links. In directed LOS links, the transmitter and receiver are directly pointing to each other. In non-directed LOS links, the alignment between transmitter and

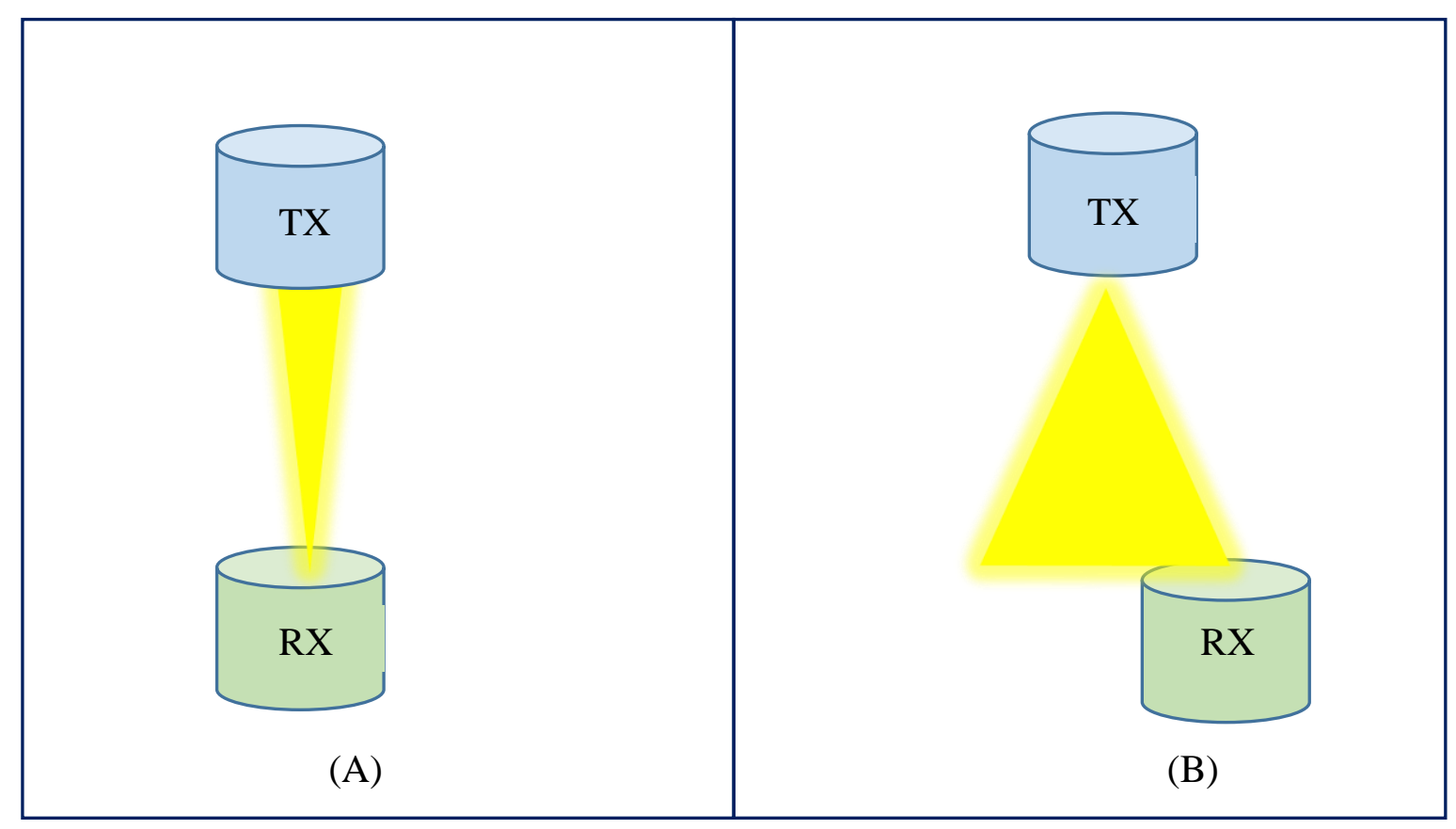

Figure 3.3 Directed LOS link (A) and Non-directed LOS link (B) 
receiver is not necessary, however, the receiver's FOV lies within the radiation field of transmitter. Figure 3.3 exhibits the link types in the outdoor vehicular VLC networks.

\section{$3.2 \mathrm{mmWave}$}

\subsubsection{Historical Review}

The first experimentations on mmWave signals dates back to 1895. In 1895, J.C.Bose utilized $5 \mathrm{~mm}$ wavelength corresponding to $60 \mathrm{GHz}$ frequency to remotely ring a bell and explode gun powder at a distance of $23 \mathrm{~m}$. mmWave spectrum was investigated by researchers as the frequency of interest for radar technology during World War II. However, until 1960s, mmWave technology was mainly used for experimental purposes both by academic and government laboratories. In 1960, mmWave was adopted in radar astronomy applications and later, around 1970s, was pursued by military for short-range communication. By 1980, commercial integrated circuits capable of operating at mmWave started to appear in the market thus opening doors to potential development of mmWave commercial products. The first consumer based product operating at mmWave frequency of $77 \mathrm{GHz}$ was for automotive collision avoidance radar, manufactured around 1990.

Federal Communication Commission (FCC) released the 59-64 GHZ mmWave band for unlicensed wireless communications. This boasted commercial manufacture of radar equipment and also for broadband communication. Later in 2003, FCC released 70-80-90 GHz bands for licenced operations. mmWave has been increasingly proposed as one of the new technologies in the upcoming $5 \mathrm{G}$ era due to the available bandwidth that could potentially provide relief to the congested sub 5GHz radio spectrum. Most recently, FCC has placed forward the first allotment of mmWave spectrum in the range of $27.500-28.350 \mathrm{GHz}$, divided into two $425 \mathrm{MHz}$ blocks, with a total of around 3000 licenses for auction. This will be followed by the auction of three more mmWave frequency bands at 37GH, 39GHz and 47GHz, scheduled for December 2019.

\subsubsection{Channel Models For Outdoor mmWave Communication}

Accurate channel modeling becomes significantly essential at mmWave frequencies as the propagation behaviour of the communication channels operating at these frequency range are 
noticeably different compared to those of sub-5GHz band channels. Radio waves at this frequency experience higher path loss, higher penetration loss, and attenuation due to foliage and human blockage. Various research institutes and standardization organizations have proposed channel models for mmWave communication systems. These channel models were developed by adopting and enhancing the key parameters obtained through measurement campaigns from existing channel models taking into consideration the carrier frequency, propagation environment and the limitation of the existing channel models [HEM-18]. The main limitation of the existing channel models were the absence of specific parameters and scenarios that would be crucial in designing mmWave systems such as effects of spatial characteristics of the communication links, huge bandwidth, higher Doppler spread, use of directional and large scale antennas and 3-D beamforming. Proposed mmWave outdoor communication channel models can be classified as deterministic, stochastics and quasi-deterministic. Table 3.3 specifies the classification of proposed mmWave standardized models as listed in [HUA-19].

\begin{tabular}{|l|l|l|l|l|}
\hline Modeling Approach & Classification & Standardized Models & Scenario & Features \\
\hline Ray tracing & Deterministic & IEEE 802.11ad & Indoor/Outdoor & $\begin{array}{l}\text { Site specific and high } \\
\text { complexity }\end{array}$ \\
\hline Map-based & Deterministic & METIS [JAM-15] & Indoor/Outdoor & $\begin{array}{l}\text { Support massive } \\
\text { MIMO and } \\
\text { beamforming }\end{array}$ \\
\hline Q-D & Quasi deterministic & $\begin{array}{l}\text { MiWEBA and IEEE } \\
802.11 \text { ay }\end{array}$ & Outdoor & $\begin{array}{l}\text { Support non- } \\
\text { stationary } \\
\text { environments }\end{array}$ \\
\hline GBSM & Stochastic & $\begin{array}{l}\text { NYU WIRELESS, } \\
\text { 3GPP TR 38.901, } \\
\text { METIS, and } \\
\text { mmMAGIC }\end{array}$ & Indoor/Outdoor & $\begin{array}{l}\text { Characterize 3-D and } \\
\text { non-stationary } \\
\text { properties }\end{array}$ \\
\hline
\end{tabular}

Table 3.3: Classification of channel models for mmWave outdoor systems [HUA-19].

IEEE 802.11ad [MAL-10] adopts the ray tracing modeling approach to provide a deterministic characterization of the mmWave indoor/outdoor channels at $60 \mathrm{GHz}$. In ray tracing models, the geometrical modeling of the propagation environment and diffraction theory are used in conjunction to calculate the diffraction paths as well as the delay, amplitude and arrival and 
departure angle of each ray traversing the channel between transmitter and receiver. This method is highly site-specific and complex. METIS has proposed a map-based channel model. Although, this model is also based on the ray-tracing approach, it utilizes a simple 3-D geometric modeling of the propagation environment. In these models, the first step is map generation by specifying the transmitter, receiver and scattering objects in the communication path. The pathways are then defined specifying the angle of arrival and departure of each radio wave. The channel impulse response takes into consideration the shadowing loss, and effects of reflection, diffraction and scattering. This model provides accurate and realistic path loss modeling in case of V2V communication. A new modeling approach combining the deterministic and stochastic approach, called quasi-deterministic channel modeling, is proposed for mmWave systems. This approach is adopted by both MiWEBA [MAL-14] [MAL-14A] and IEEE 802.11ay [MAL-16] for modeling outdoor communication channel at $60 \mathrm{GHz}$ [WAN-18]. The benefit of using this hybrid approach is that it does not require site-specific parameters as in ray tracing models and is more accurate than general stochastic models. The most commonly adopted stochastic model is the geometry based stochastic model (GBSM). NYU WIRELESS [RAP-13], 3GPP TR 38.901[ETS-17], METIS [JAM-15], and mmMAGIC [MAG-17] are based on GBSM. These models takes into consideration antenna parameters, large-scale parameters and small scale parameters. The channel coefficients are obtained through extensive channel measurements.

\subsection{3 mmWave Propagation Characteristics}

The quest for higher bandwidth and availability of vacant semi licenced spectrum are the main drivers behind the emergence of mmWave frequency band as spectrum of interest for $5 \mathrm{G}$ networks. The feasibility of mmWave spectrum for robust and reliable high data rate communication has been extensively evaluated by both academia [AKD-14] [AYA-14] [ZHA10] [DAN-10] and industry [GIG-10] [PI-11] [PI-12] [BLE-13]. However, the propagation characteristics of mmWave bands are quite different from those below $5 \mathrm{GHz}$ radio bands where 3G/4G/WiFi communication networks are established. Compared to classical lower frequencies, mmWave frequencies are much more susceptible to atmospheric effects, foliage blockage, human shadowing and cannot penetrate or propagate around obstructions. All these factors become more significant for outdoor vehicular networks and have to be carefully considered and included in the channel modeling process for outdoor mmWave communication systems. The 
major propagation characteristics that are significant for high speed outdoor channel modeling are presented in figure 3.4 and briefly described thereafter

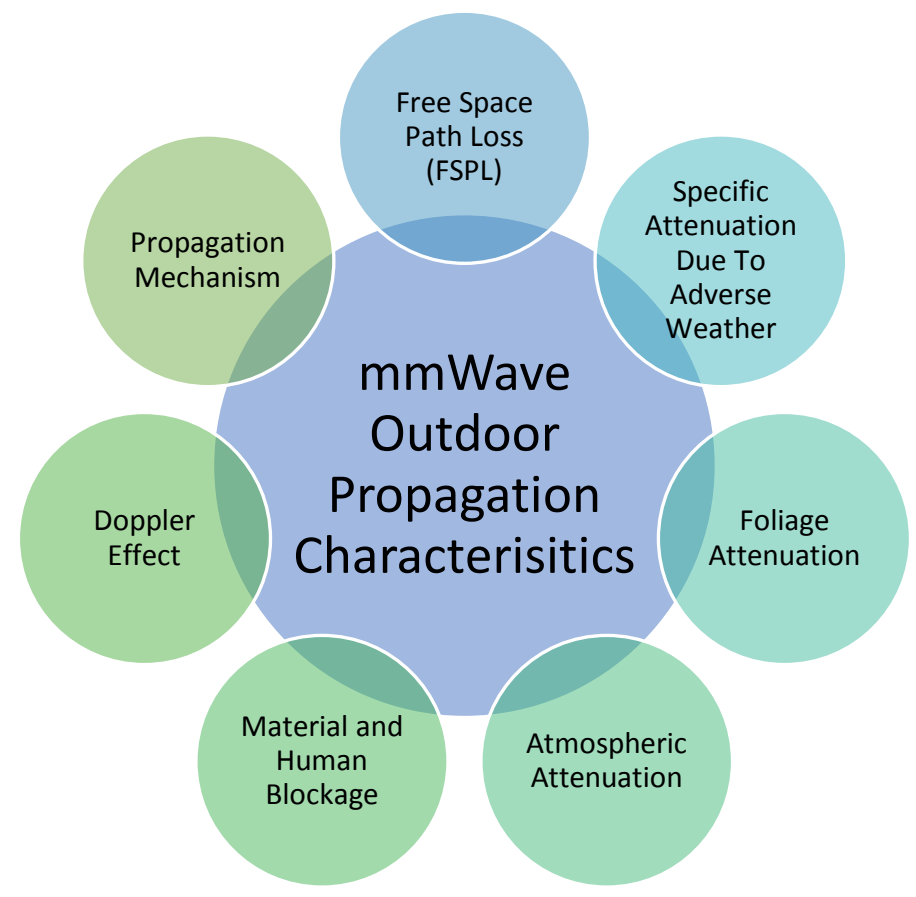

Figure 3.4 Propagation Characteristics of outdoor mmWave Channel

\subsubsection{Free-Space Path Loss}

Free space path loss is defined as the loss in the strength of transmitted signal in line of sight (LOS) propagation through free space without any obstructions. The free space path loss considering isotropic transmitter and receiver antennas that have uniform radiation pattern in all directions, can be derived through Friss transmission equation that relates received power to the amount of incident transmitted power on the receiver antenna's effective aperture [FRI-46].

$$
P_{r}=A_{e f f} P_{o}
$$

Here,

$P_{r}$ is the received power, $A_{e f f}$ is the effective aperture of the receiver antenna and $P_{o}$ is the "power flow per unit area at the incident field of the receiver antenna" [FRI-46]. The power flow per unit area at a distance $d$ from the transmitter antenna is given as

$$
P_{o}=\frac{P_{t}}{4 \pi d^{2}}
$$


Substituting equation 3.2.2 in 3.2.1 yields,

$$
P_{r}=A_{e f f} \frac{P_{t}}{4 \pi d^{2}}
$$

For isotropic antennas,

$$
A_{e f f}=\frac{\lambda^{2}}{4 \pi}
$$

Substituting 3.9 in 3.8 yields,

$$
\frac{P_{r}}{P_{t}}=\left(\frac{\lambda}{4 \pi d}\right)^{2}
$$

Which is known as the Friss's law of free space propagation loss [FRI-46] [HEM-18].

The free space path loss (FSPL) between isotropic transmitting and receiving antennas at a distance $\mathrm{d}$ in kilometers $(\mathrm{km})$ and operating at $\mathrm{GHz}$ frequencies is given as [HEM-18] [WAL88]:

$$
F S P L(d B)=92.45+20 \log f(G H z)+20 \log d(\mathrm{~km})
$$

It is evident from equation 3.11 that free space path loss is dependent on both the distance between transmitting and receiving antennas and the operating frequency. Hence the free space path loss at mmWave frequencies will be higher than $1.9 \mathrm{GHz}$ and $2.6 \mathrm{GHz}$ which are the commonly used frequency bands for 4G LTE service provision in Canada by Rogers, Telus and Bell [BAD-16]. Figure 3.5 compares the FSPL at various mmWave frequencies with 4G/LTE frequencies. 


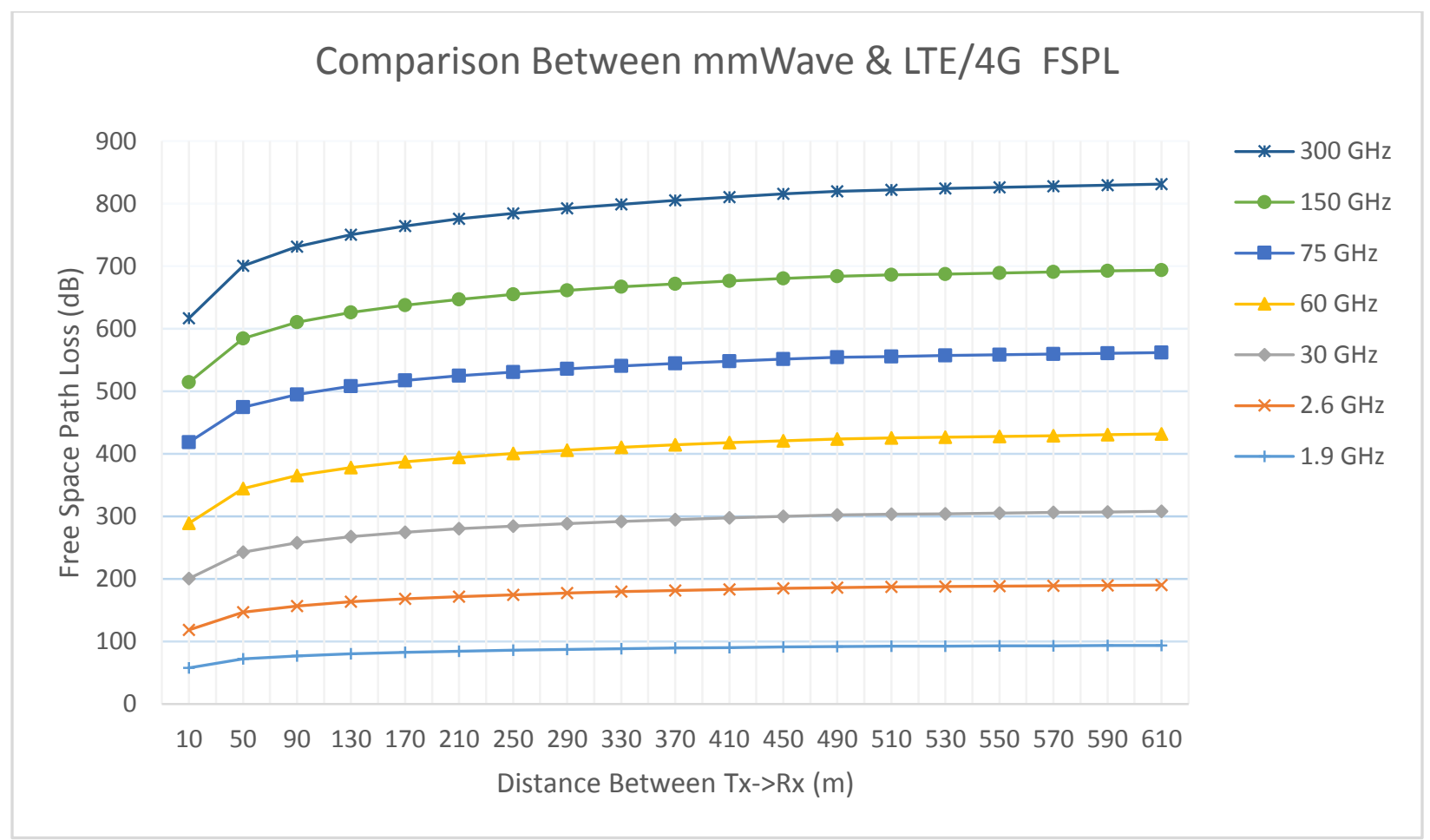

Figure 3.5 Comparison of mmWave and LTE/4G FSPL

\subsubsection{Attenuation}

\subsection{Atmospheric Attenuation}

Earth's atmosphere is composed of many gases. The two major gases that cause significant attenuation of mmWave are oxygen and water vapor [KHA-11] [PI-11] [PIE-12] [RAN-14]. This attenuation is dependent on the gaseous pressure, temperature, density and the carrier frequency. Oxygen molecules exhibit magnetic dipole moment and water molecules exhibit electric dipole moment. Due to these dipole moments, mmWave signals losses part of radio energy while passing through the atmosphere as it is absorbed while causing enhanced vibration of the oxygen and water vapor molecules. The strength of the vibrations depends on the relation between the molecules' resonance frequency and the carrier frequency. Needless to say, that the absorption of energy and corresponding vibration is most significant when the carrier frequency is the same with the resonance frequency of the gas molecules [GIA-99]. According to ITU-R [ITU-16], the specific attenuation due to atmosphere is given by:

$$
\gamma=\gamma_{o}+\gamma_{H_{2} o}=0.1820 f\left(N^{\prime \prime}\left(\mathrm{f}_{\mathrm{o}}\right)+\mathrm{N}^{\prime \prime}\left(f_{\mathrm{H}_{2} \mathrm{o}}\right)\right)
$$


where, $\gamma_{\mathrm{o}}$ and $\gamma_{\mathrm{H}_{2} \mathrm{O}}$ are specific attenuations due to oxygen and water vapor, $f$ is the frequency in $\mathrm{GHz}, N^{\prime \prime}\left(\mathrm{f}_{\mathrm{o}}\right)$ and $\mathrm{N}\left(f_{\mathrm{H}_{2} \mathrm{O}}\right)$ are the imaginary component of the frequency dependent complex refractivity.

In the mmWave range, the attenuation due to oxygen was found to be maximum at $60 \mathrm{GHz}(15$ $\mathrm{dB} / \mathrm{km})$ and $119 \mathrm{GHz}(1.4 \mathrm{~dB} / \mathrm{km})$, whereas the maximum attenuation due to water vapor was found to be at $183 \mathrm{GHz}(28.35 \mathrm{~dB} / \mathrm{km})$ and $323 \mathrm{GHz}(38.6 \mathrm{~dB} / \mathrm{km})$ [HEM-18] [MAR-05] [ITU16]. However, the impact of this attenuation is considerably reduced when mmWave expected cell sizes are taken into consideration. It is expected that the maximum cell coverage area in mmWave communication will be around $200 \mathrm{~m}$, which would result in having maximum attenuation due to oxygen at $60 \mathrm{GHz}$ close to $3 \mathrm{~dB}$. Similarly maximum attenuation due to water vapor will be reduced to $5.6 \mathrm{~dB}$ at $183 \mathrm{GHz}$ and $7.72 \mathrm{~dB}$ at $323 \mathrm{GHz}$ correspondingly.

\subsection{Weather Specific Attenuation}

In addition to atmospheric attenuation, outdoor mmWave signals also undergo attenuation due to adverse weather conditions such as fog, rain and snow. Rain is the most significant cause of attenuation in mmWave range as the wavelength of mmWave radio signals is between $1 \mathrm{~mm}$ $10 \mathrm{~mm}$. Since the size of rain drops is also few $\mathrm{mm}$, moderate to heavy rain can cause complete blockage of mmWave signals resulting in significant attenuation of radio signals at mmWave frequencies compared to longer wavelength. A detailed analysis of mmWave attenuation due to specific weather conditions has been presented in section 3.3.

\subsection{Foliage}

mmWave frequencies are highly susceptible to attenuation due to foliage. Depending on the density and depth of vegetation, the specific attenuation at various frequency bands of mmWave varies from few $\mathrm{dB}$ to few dozens of $\mathrm{dB}$ for example, a single tree will cause less foliage attenuation than a line of trees and a line of trees will cause less attenuation than a forest [HEM18]. The prediction models for foliage attenuation can be classified based on the propagating ray geometry as either horizontal path, where the elevation angle between transmitter and receiver antennas through treeline is less than $3^{0}$ and the path depth can be short (through 1-2 trees) or long (line of trees but not forest) or as a slant path, where the elevation angle is around $10^{0}$ and 
the path length is short (through 1-2 trees only) [MEN-10]. The traditional empirical model used for estimating foliage loss at radio frequencies is the exponential decay model. In its generalized form, it is as follows:

$$
F L_{d B}=A f^{b} D_{f}^{c}
$$

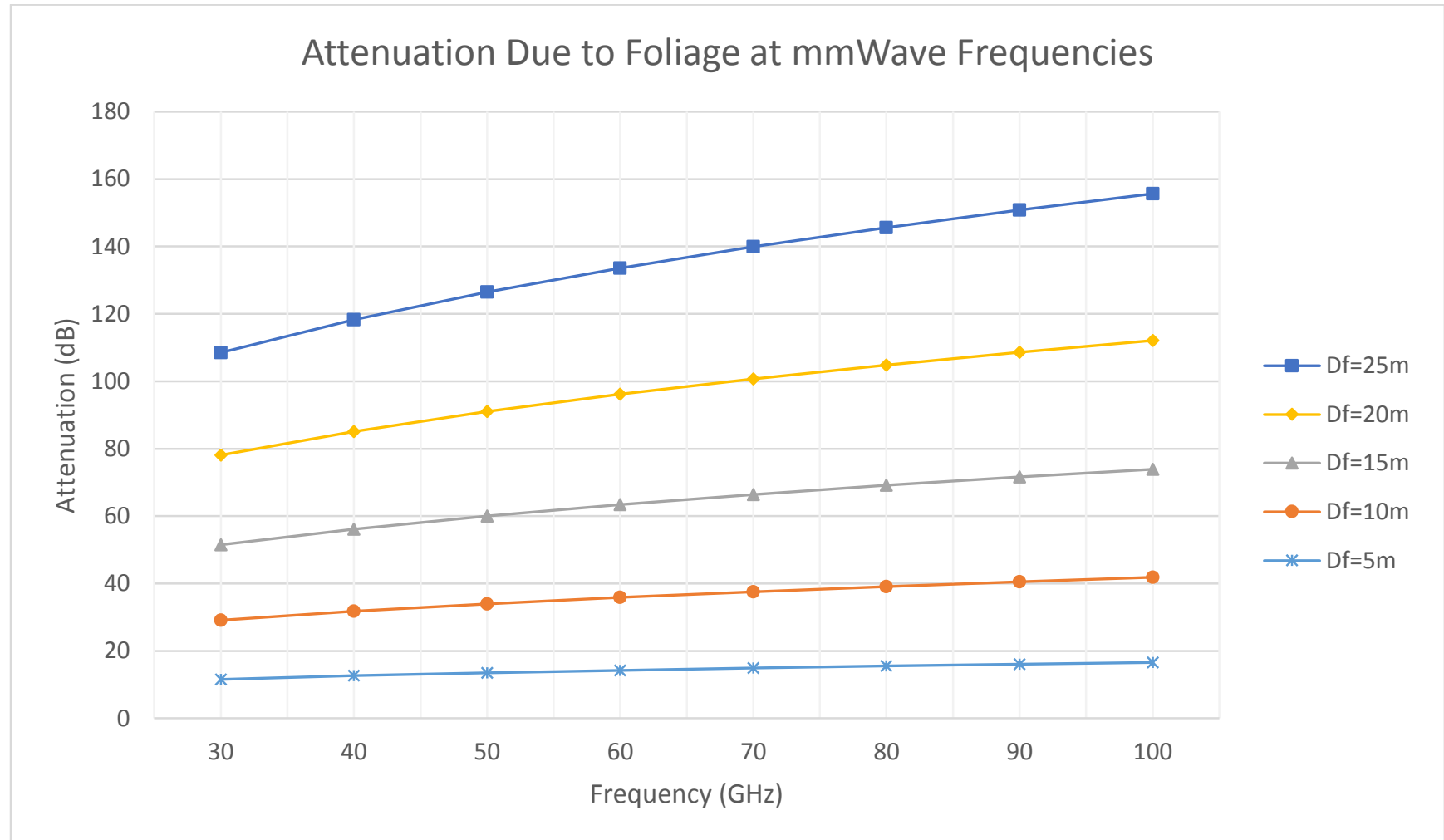

Figure 3.6 Attenuation Due to Foliage at mmWave Frequencies

Where, $F L$ is the loss due to foliage, $f$ is the frequency in $\mathrm{MHz}, D_{f}$ is the foliage depth in meters and $A, b, c$ are empirical parameters that are dependent on the model used, foliage type and the propagation mechanism. Various modifications have been proposed in literature over the traditional model, suggesting different values for empirical parameters, with the Weissberger model [WEI-82] being one of the first reported and ITU-R (CCIR Rpt 236-2) model being the most commonly adopted [MAR-05] [PI-11][KHA-11]. A detailed review of several other models for determining foliage loss is presented in [MEN-10]. Based on the empirical values adopted in ITU-R model, eq.3.13 can be written as:

$$
F L_{d B}=0.2 f^{0.3} D_{f}^{0.6}
$$

The above relationship can be used to estimate foliage loss for $D<400 \mathrm{~m}$ and $f$ up to $100 \mathrm{GHz}$. Foliage attenuation at various mmWave frequencies is plotted in figure 3.6. It can be deduced 
from the figure that increase in the depth of foliage highly attenuates the mmWave signal even at lower frequencies.

\subsubsection{Propagation Mechanism}

Radio waves are affected by diverse propagation mechanisms such as reflection, refraction, diffraction and scattering while traversing the atmosphere. Depending highly on the carrier frequency and propagation environment, radio waves undergo attenuation and distortion to various extent due to these mechanisms. Due to the short wavelength, i.e. between 1-10mm, mmWave communication will take place at frequencies far above the sub $5 \mathrm{GHz}$ band and hence will exhibit extremely different propagation characteristics compared to those exhibited by signals in the classical radio band. In the context of outdoor vehicular environment, line-of-sight (LOS) signal propagation would be the main mode of communication for mmWave. In the absence of a directed LOS path between transmitter and receiver, the signal may still reach the receiver via reflections or scattering from buildings, cars and other objects in proximity of the receiver. The short wavelength of mmWave results in low diffraction as the effects of diffraction decreases rapidly with the decrease in wavelength and only has significance if the size of the object in the signal path is exceptionally small. Typically, diffraction has negligible effect on the mmWave signal as compared to the signals of lower bands.

Reflection is the key propagation mechanism in non-line-of-sight (NLOS) communication. Reflection can be defined as specular, where the incident signal is reflected in a single direction thus maximizing the signal energy and creating a beneficial multipath component, or diffused where the incident signal is scattered in various directions resulting in spread signal power and attenuated specular component. The degree of reflectivity depends upon the roughness of the reflecting surface [WEI-14]. In outdoor propagation environment, the roughness of exterior building material may considerably attenuate the mmWave signal through diffused reflection.

Cell size is also extremely significant in deciding the dominant propagation mechanism in mmWave. It has been found that in the case of $100 \mathrm{~m}$ radius from the mmWave base station, $57.5 \%$ of the area was catered through LOS links , $22.2 \%$ of the area relied on reflected beams as main mode of interaction, $14.5 \%$ of the area maintained connectivity through diffracted beams and $5.8 \%$ area had no detectable beams. These percentages changed noticeably as the cell radius 
was increased from $100 \mathrm{~m}$ to $200 \mathrm{~m}$ with LOS links-based coverage area reduced to $24.4 \%$ and coverage due to reflected links reduced to $14.9 \%$ coverage area by diffracted beams increased to $38.7 \%$ forcing around $22 \%$ area within the coverage radius to complete signal outage [ZHA$15 \mathrm{~A}]$.

\subsubsection{Blockage}

The mmWave signals are also sensitive to material and human blockage [LU-12][WU-15][JAM15][XU-02]. It has been found that presence of one person in the mmWave signal path attenuates the received signal by $20 \mathrm{~dB}$ [GAR-10] increasing it to $50 \mathrm{~dB}$ for 2-3 people in the LOS [LU-12] hence, reducing the effective throughput considerably. However, it has also been debated that attenuation due to human blockage is not dependent on the number of people between transmitting and receiving antennas but rather on antenna configurations (gain, half power bandwidth and elevation) [COL-04] [MAC-16]. Another analysis of outdoor the mmWave channel concluded that the transmitter height relative to the receiver's, receiver's dimension (length and width), human density and distance between transmitter and receiver are allparamount in computing the channel blockage probability [GAP-16].

\subsection{Specific $^{2}$ Attenuation Due To Weather Condition}

Huge bandwidth availability at both mmWave and optical spectrum motivates their adoption for support of high data rate in 5G networks. However, reliability and availability of links are crucial for any communication network. The performance of both mmWave and optical signals is highly dependent on environmental factors such as fog, rain and snow. In terms of optical signal, the presence of heavy fog, rain or snow reduces visibility. Visibility is characterized by runway visual range (RVR) which is the distance a parallel luminous beam covers through the atmosphere until its intensity is decreased by $2 \%-5 \%$ of the original luminous flux [KAU-17] [KAR-11] [GHA-10]. This reduction in illumination is due to absorption thus directly effects the quality of the received optical signal and maximum coverage area of the optical cell. The mmWave traversing through adverse weather conditions loses significant energy, as shorter wavelengths are more prone to scattering through ice, water and oxygen particles, than classical lower frequencies. This section discusses and compares the attenuation optical and mmWave signals experience in fog, rain and snow. 


\subsubsection{Attenuation due to Fog}

Fog is formed due to the condensation of water vapor in the air. This condensation happens when the difference between the temperature of surrounding area and dew point is less than $2.5^{\circ} \mathrm{C}$ and results in the formation of tiny water droplets $(1-20 \mu \mathrm{m})$ [KAR-11]. Fog can be classified into many different types. Advection fog and radiation fog are considered two major categories of fog [GHA-10] [NAB-04].

- ADVECTION FOG: Advection fog is formed due to the movement of warm air over cold surface, such as ice or cool water. The lower layer of warm air that comes into contact with the cold surface reaches dew point quickly resulting in the formation of advection fog.

- RADIATION FOG: Radiation fog is formed when the land cools after sunset. The release of thermal energy enables the ground to lose heat fast, cooling the moist air above it to the dew point. Radiation fog is highly localized and dense.

\subsubsection{VLC}

Fog attenuates optical signal significantly due to scattering as the particle size is comparable to the optical wavelength. Mie scattering theory can be used to predict the attenuation the optical signal endures due to fog. However, this prediction procedure requires complex computation based on detailed specification of fog parameters. Hence, to estimate fog attenuation experienced by optical signal an alternative approach has emerged, which is the use of an empirical formula based on visibility range and a simplified Mie scattering model. The specific attenuation due to fog is given by the following empirical formula for Mie scattering [KAU-17] [KAU-12] [GHA10] [ITU-07]:

$$
\beta_{f o g}(\lambda)=\frac{3.91}{V}\left(\frac{\lambda}{550}\right)^{-p}
$$

Where, $\beta_{f o g}(\lambda)$ is the wavelength dependent specific attenuation due to fog, $\lambda$ is the wavelength in $\mathrm{nm}, V$ is the visibility range in kilometers $(\mathrm{km})$ and $p$ is the size distribution coefficient of scattering. The value of $p$ is given by the Kim or Kruse models [NAD-08] [KAU-17].

2. Specific Attenuation: To characterize the attenuation of optical signal propagating through a medium, a term called "specific attenuation" is used which means attenuation per unit length expressed in $\mathrm{dB} / \mathrm{km}$ 
According to Kim model;

$$
p=\left\{\begin{array}{lr}
1.6 & V>50 \\
1.3 & 6<V<50 \\
0.16 V+0.34 & 1<V<6 \\
V-0.5 & 0.5<V<1 \\
0 & V<0.5
\end{array}\right\}
$$

According to Kruse model;

$$
p=\left\{\begin{array}{lr}
1.6 & V>50 \\
1.3 & 6<V<50 \\
0.585 V^{1 / 3} & V<6
\end{array}\right\}
$$

Visibility ranges corresponding to different weather conditions are specified in table 3.4 [KAU17] [KIM-01A] [ITU-12].

\begin{tabular}{|c|c|}
\hline Weather Condition & Visibility Range $(\mathrm{Km})$ \\
\hline Thick Fog & 0.2 \\
\hline Moderate Fog & 0.5 \\
\hline Light Fog & $0.77-1$ \\
\hline Thin Fog \ Heavy Rain $(25 \mathrm{~mm} / \mathrm{hr})$ & $1.9-2$ \\
\hline Haze $\backslash$ Medium Rain $(12.5 \mathrm{~mm} / \mathrm{hr})$ & $2.8-4$ \\
\hline Light Haze $\backslash$ Light Rain $(2.5 \mathrm{~mm} / \mathrm{hr})$ & $5.9-10$ \\
\hline Clear\ Light Drizzle $(0.25 \mathrm{~mm} / \mathrm{hr})$ & $18-20$ \\
\hline Very Clear & $23-50$ \\
\hline
\end{tabular}

Table 3.4 Visibility Range Corresponding to Weather Conditions 
Based on the above empirical formula, the specific attenuation coefficients for the $450 \mathrm{~nm}$, $530 \mathrm{~nm}, 580 \mathrm{~nm}$ and $630 \mathrm{~nm}$ wavelengths were computed for thick, moderate and light fog conditions. These wavelengths are chosen as they correspond to blue, green, and red colors that are the primary colors utilized in vehicular environment. Table 3.5 lists the specific attenuation due to fog at the mentioned wavelength in the visible spectrum.

\begin{tabular}{|c|c|c|c|c|c|c|c|c|c|}
\hline Fog Density & Visibility (Km) & $p=$ Kim & $p=$ Kruse & \multicolumn{2}{|c|}{$\begin{array}{c}\text { Attenuation(db/km) } \\
\lambda=450 \mathrm{~nm}(\mathrm{blue})\end{array}$} & \multicolumn{3}{|c|}{$\lambda=530 \mathrm{~nm}(\mathrm{Green})$} & $\lambda=630 \mathrm{~nm}(\mathrm{Red})$ \\
\hline Heavy & 0.2 & 0 & 0.3439 & 19.55 & 20.94 & 19.55 & 19.8 & 19.55 & 18.65 \\
\hline Moderate & 0.5 & 0 & 0.464 & 7.82 & 8.58 & 7.82 & 7.9 & 7.82 & 7.35 \\
\hline Light & $0.77-1$ & 0.5 & 0.585 & 4.8 & 4.88 & 4.42 & 4.43 & 4.05 & 4.02 \\
\hline Thin & $1.9-2$ & 0.644 & 0.7353 & 2.22 & 2.26 & 2.02 & 2.00 & 1.79 & 1.77 \\
\hline
\end{tabular}

Table 3.5 Specific Attenuation according to Kim and Kruse model $(\lambda=450 \mathrm{~nm}, 530 \mathrm{~nm} \& 630 \mathrm{~nm})$

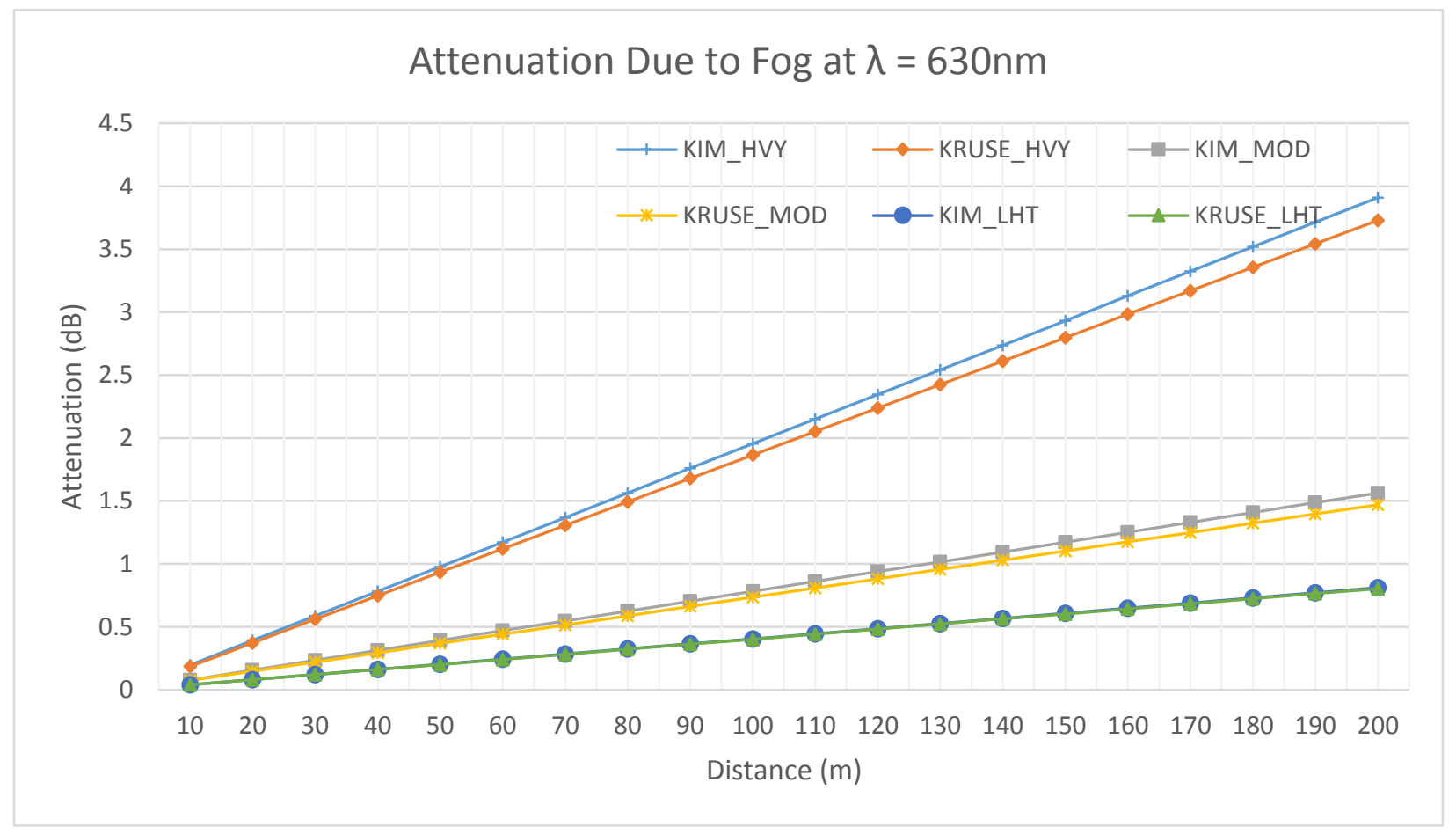

Figure 3.7 Attenuation due to fog according to Kim and Kruse model at $\lambda=630 \mathrm{~nm}$

Based on table 3.5, the specific attenuation for various fog densities are plotted in figure 3.7 for up to $200 \mathrm{~m}$ distance between transmitter and receiver. It can be seen that the specific attenuation at heavy fog is around $3.8 \mathrm{~dB} / \mathrm{km}$ and decrease to $1.5 \mathrm{~dB} / \mathrm{km}$ for moderate fog conditions. However, VLC cells are perceived to be femto-cells, in which case cell area will be on the order 
of $10 \mathrm{~s}$ of meters and the specific attenuation will be around $1-1.5 \mathrm{~dB} / \mathrm{km}$ even in dense fog scenarios.

\subsubsection{2 mmWave}

At $\mathrm{GHz}$ frequencies, the attenuation due to fog is a complex function of the particle size distribution, density, index of refraction, liquid water content and wavelength [ALT-84]. The attenuation induced by fog on the electromagnetic waves is directly proportional to the frequency of radio wave, hence mmWave propagating through foggy weather will experience significantly more attenuation than lower frequency. At frequency less than $10 \mathrm{GHz}$, attenuation due to fog becomes insignificant [KAU-12]. The specific attenuation due to fog up to the carrier frequency of $200 \mathrm{GHz}$ can be expressed through the following empirical relation developed according to Rayleigh absorption approximation of Mie scattering theory [CHE-04] [GAL-06] [ITU-17]:

$$
\alpha_{f o g}=K M
$$

Where $\alpha_{f o g}$ is the specific attenuation due to fog $(\mathrm{dB} / \mathrm{km}), \mathrm{M}$ is the liquid water content in the fog $\left(\mathrm{g} / \mathrm{m}^{3}\right)$ and $\mathrm{K}$ is the specific attenuation coefficient $(\mathrm{dB} / \mathrm{km}) /\left(\mathrm{g} / \mathrm{m}^{3}\right)$. The value of $\mathrm{K}$ is determined through a mathematical model based on Rayleigh scattering [ITU-17]. This mathematical model uses a double-Debye representation of the dielectric permittivity $\epsilon(f)$ of the water. Dielectric permittivity ${ }^{3}$ consists of real and imaginary parts, $\epsilon(f)=\epsilon^{\prime}(f)+\epsilon^{\prime \prime}(f)$.

$$
K=\frac{0.819 f}{\epsilon^{\prime \prime}\left(1+\eta^{2}\right)}
$$

Where $f$ is the frequency in $\mathrm{GHz}$,

$$
\eta=\frac{2+\epsilon^{\prime}}{\epsilon^{\prime \prime}}
$$

The complex dielectric permittivity of water is given by:

$$
\begin{gathered}
\epsilon^{\prime \prime}(f)=\frac{f\left(\epsilon_{0}-\epsilon_{1}\right)}{f_{p}\left[1+\left({ }^{f} / f_{p}\right)^{2}\right]}+\frac{f\left(\epsilon_{1}-\epsilon_{2}\right)}{f_{s}\left[1+\left({ }^{f} / f_{s}\right)^{2}\right]} \\
\epsilon^{\prime}(f)=\frac{\left(\epsilon_{0}-\epsilon_{1}\right)}{\left[1+\left(f^{f} / f_{p}\right)^{2}\right]}+\frac{\left(\epsilon_{1}-\epsilon_{2}\right)}{\left[1+\left(f^{f} / f_{s}\right)^{2}\right]}+\epsilon_{2}
\end{gathered}
$$

3. The real component is "the" permittivity (which is a "constant" in the static limit and decreases with increasing frequency), the imaginary component is called dielectric loss. The loss component (which may also have contributions from the ohmic conductivity) is in phase with the field. 
Where,

$$
\epsilon_{0}=77.6+103.3(\theta-1)
$$

Where,

$$
\begin{gathered}
\epsilon_{1}=0.0671 \epsilon_{0} \\
\epsilon_{2}=3.51 \\
\Theta=300 / \mathrm{T}
\end{gathered}
$$

Here $\mathrm{T}$ is the temperature in Kelvins (K).

The principle and secondary relaxation frequencies in $\mathrm{GHz}$ are as:

$$
\begin{gathered}
f_{p}=20.20-146(\theta-1)+316(\theta-1)^{2} \\
f_{s}=39.8 f_{p}
\end{gathered}
$$

Relaxation frequency is defined as "the frequency at which the dielectric loss factor $(\in$ ") reaches a maximum, for a dielectric material that has no static conductivity and that is subjected to an alternating electromagnetic field" [OXF-19]. For a given temperature, the maximum dielectric loss factor peaks at two frequencies in double Debye model that corresponds to primary and secondary relaxation frequencies. The variation in the fog density due to the presence of water vapor causes a considerable variability in the visibility range. Numerous researchers have aimed at defining relationships between associated visibility range reduction, the liquid water density in the fog clouds and its associated extinction coefficient. Extinction coefficient is also called attenuation coefficient and reflects the degree of attenuation the light will experience while passing through a medium (in this case fog clouds). The general empirical formula relating the liquid water content of fog and the visibility range is as follows [MUH-10] [NAD-08] [KAU12]:

$$
V=b M^{-2 / 3}
$$

Here $\mathrm{V}$ is the visibility range $(\mathrm{km}), \mathrm{M}$ is the liquid water content of fog $\left(\mathrm{g} / \mathrm{m}^{3}\right)$ and $b$ is a specific parameter and its value changes depending on the fog type as indicated in table 3.6.

\begin{tabular}{|c|c|}
\hline Fog Type & $b$ \\
\hline Dense Haze & 0.013 \\
\hline Continental Fog (Dry \& Cold) & 0.034 \\
\hline Maritime Fog (Wet \& Warm) & 0.060 \\
\hline Dense Haze and Selective Fog & 0.017 \\
\hline Stable \& Evolving Fog & 0.024 \\
\hline
\end{tabular}


Table 3.6 The value of parameter $b$ for different fog types [MUH-10]

Considering the density of fog, liquid water content in thin fog is about $0.001-0.005 \mathrm{~g} / \mathrm{m}^{3}$, in moderately dense fog is typically about $0.05 \mathrm{~g} / \mathrm{m}^{3}$ and $0.5-1 \mathrm{~g} / \mathrm{m}^{3}$ for thick fog [ITU-17][MUH10]. Table 3.7 lists the attenuation due to fog at $\mathrm{mmWave}$ frequencies. Figure 3.8 plots the specific attenuation corresponding to distance between transmitter and receiver for up to $200 \mathrm{~m}$.

\begin{tabular}{|c|c|c|c|c|}
\hline$\lambda$ & $f$ & $\begin{array}{l}\text { Attenuation }(\mathrm{dB} / \mathrm{km}) \text { in } \\
\text { dense fog; } M=1 \mathrm{~g} / \mathrm{m}^{3}\end{array}$ & $\begin{array}{l}\text { Attenuation }(\mathrm{dB} / \mathrm{km}) \text { in } \\
\text { moderate fog; } \mathrm{M}=0.05 \mathrm{~g} / \mathrm{m} 3\end{array}$ & $\begin{array}{l}\text { Attenuation }(\mathrm{dB} / \mathrm{km}) \text { in thin } \\
\text { fog } ; M=0.001 \mathrm{~g} / \mathrm{m} 3\end{array}$ \\
\hline $1 \mathrm{~mm}$ & 300 & 15.5 & 0.775 & 0.0155 \\
\hline $2 \mathrm{~mm}$ & 150 & 13.71 & 0.68 & 0.0137 \\
\hline $3 \mathrm{~mm}$ & 100 & 9.823 & 0.49 & 0.0098 \\
\hline $4 \mathrm{~mm}$ & 75 & 2.8125 & 0.14 & 0.0028 \\
\hline $5 \mathrm{~mm}$ & 60 & 1.90 & 0.095 & 0.0019 \\
\hline $6 \mathrm{~mm}$ & 50 & 1.39 & 0.0695 & 0.00139 \\
\hline $7 \mathrm{~mm}$ & 43 & 1.047 & 0.0523 & 0.00104 \\
\hline $8 \mathrm{~mm}$ & 37.5 & 0.812 & 0.0406 & 0.000812 \\
\hline $9 \mathrm{~mm}$ & 33 & 0.633 & 0.03165 & 0.000633 \\
\hline $10 \mathrm{~mm}$ & 30 & 0.527 & 0.02635 & 0.000527 \\
\hline
\end{tabular}

Table 3.7: Attenuation due to fog at mmWave Frequencies 


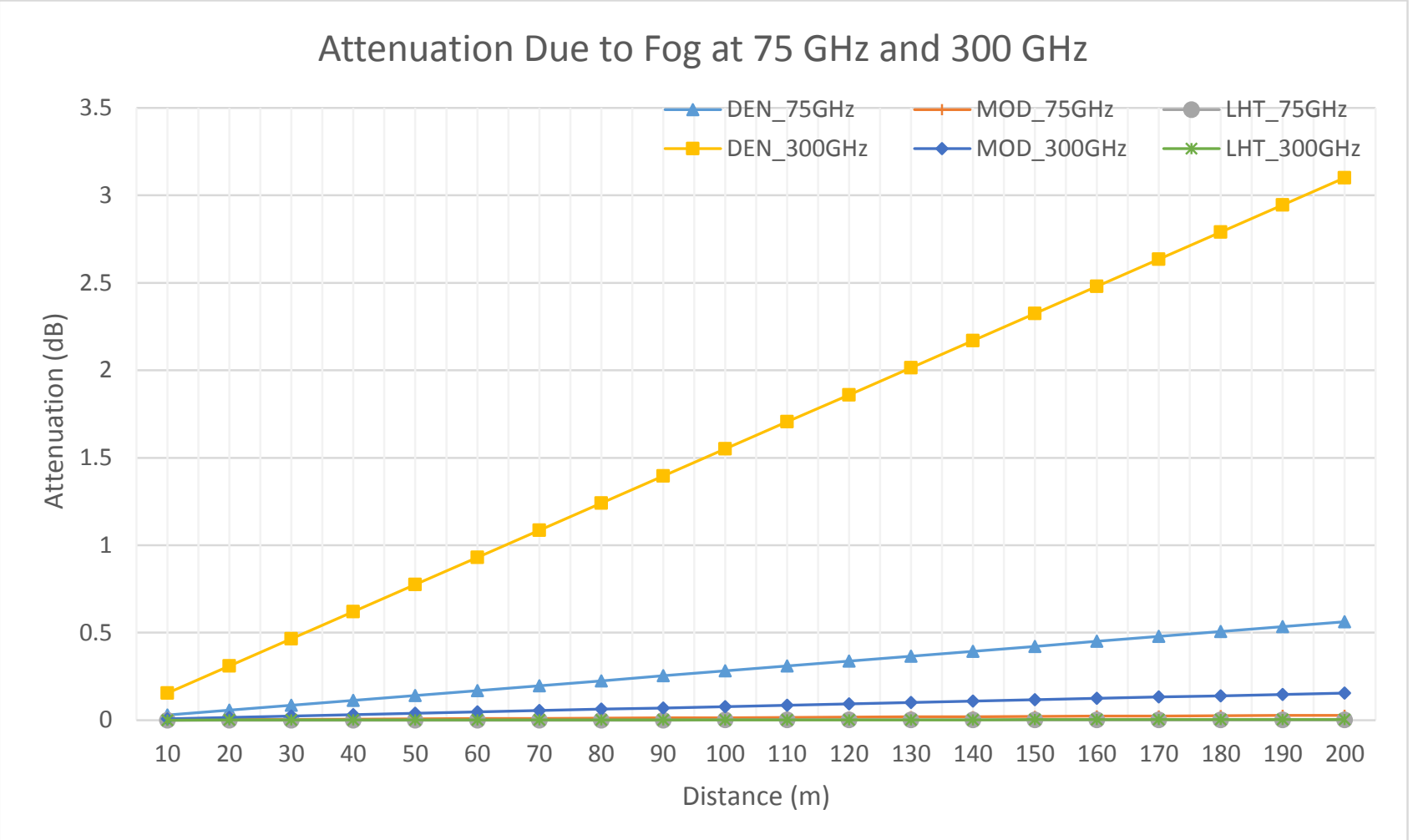

Figure 3.8 Attenuation due to dense, moderate and light fog at $75 \mathrm{GHz}$ and $300 \mathrm{GHz}$

It can be deduced from the figure that specific attenuation due to fog increases with decreasing wavelength. At $300 \mathrm{GHz}$, the specific attenuation for dense fog is around $3 \mathrm{~dB}$. However, at lower frequencies such as $75 \mathrm{GHz}$, the attenuation due to fog is negligible.

\subsubsection{Attenuation due to Rain}

Rain is composed of water droplets varying in size from $10 \mu \mathrm{m}$ to $10 \mathrm{~mm}$ [KAR-11]. Attenuation due to rain of telecommunications signals depends on rain droplet size, density of rain droplets, and the area covered by the rain, commonly known as rain cell between the transmitter and receiver [AKI-04]. Depending on the cell size, it is possible that a signal traveling through the atmosphere will experience attenuation due to rain even if rain fall is not happening in the closer vicinity of transmitter or receiver but rather in some area between the communication links. In general, mmWave signal experiences significant attenuation due to rain as the wavelength is comparable to the size of rain droplets. 


\subsubsection{VLC}

Rain drops cause scattering of optical signals independent of wavelength but dependent on rainfall rate. Attenuation due to rain is more significant in $\mathrm{GHz}$ range whereas, attenuation due to snow is more significant at optical frequencies. The specific attenuation due to rain increases linearly with rain fall rate and is given as follow [ITU-07] [ISH-16]:

$$
\beta_{\text {Rain }}=k R^{\alpha}
$$

Where, $\beta_{\text {Rain }}$ is the specific attenuation due to rain, $\mathrm{R}$ is the rain fall rate ( $\left.\mathrm{mm} / \mathrm{hr}\right), k$ and $\alpha$ are the parameters dependent on rain characteristics. The most commonly used values for these parameter in literature are [ITU-07] [KAU-17]:

$$
\begin{gathered}
k=1.076, \text { and } \\
\alpha=0.67
\end{gathered}
$$

Alternatively, rain attenuation for visible light links can be approximated by following the empirical formula below [KAU-17]:

$$
\gamma_{\text {Rain }}=\frac{2.8}{V}
$$

Here, $\gamma_{\text {Rain }}\left(\frac{d B}{k m}\right)$ is the specific attenuation due to rain and $V(\mathrm{~km})$ is the visibility range

\begin{tabular}{|c|c|c|c|}
\hline Rain Fall Type & Visibility $(\mathrm{Km})$ & $\begin{array}{c}\text { Attenuation }(\mathrm{dB} / \mathrm{Km})= \\
2.8 / \mathrm{V}\end{array}$ & $\begin{array}{c}\text { Attenuation } \\
(\mathrm{db} / \mathrm{km})=1.076 \mathrm{R}^{0.67}, \mathrm{R}=\text { rain } \\
\text { rate }\end{array}$ \\
\hline Heavy: $25 \mathrm{~mm} / \mathrm{hr}$ & $1.9-2$ & 1.4 & 9.2 \\
\hline Moderate $; 12.5 \mathrm{~mm} / \mathrm{hr}$ & $2.8-4$ & 0.93 & 5.84 \\
\hline Light: $2.5 \mathrm{~mm} / \mathrm{hr}$ & $5.9-10$ & 0.28 & 1.98 \\
\hline Drizzle: $0.25 \mathrm{~mm} / \mathrm{hr}$ & $18-20$ & 0.147 & 0.425 \\
\hline
\end{tabular}
according to rain fall rates as indicated in table 3.4. Table 3.8 lists the specific attenuation due to rain at different wavelengths of visible light spectrum calculated through rain rate based and empirical formula. Specific attenuation due to rain is plotted in figure 3.9.

Table 3.8 Specific Attenuation due to Rain at Visible Light Spectrum 
It can be concluded from the above graph that rain does not affect VLC links as the maximum attenuation is around $1.8 \mathrm{~dB}$ for heavy rainfall.

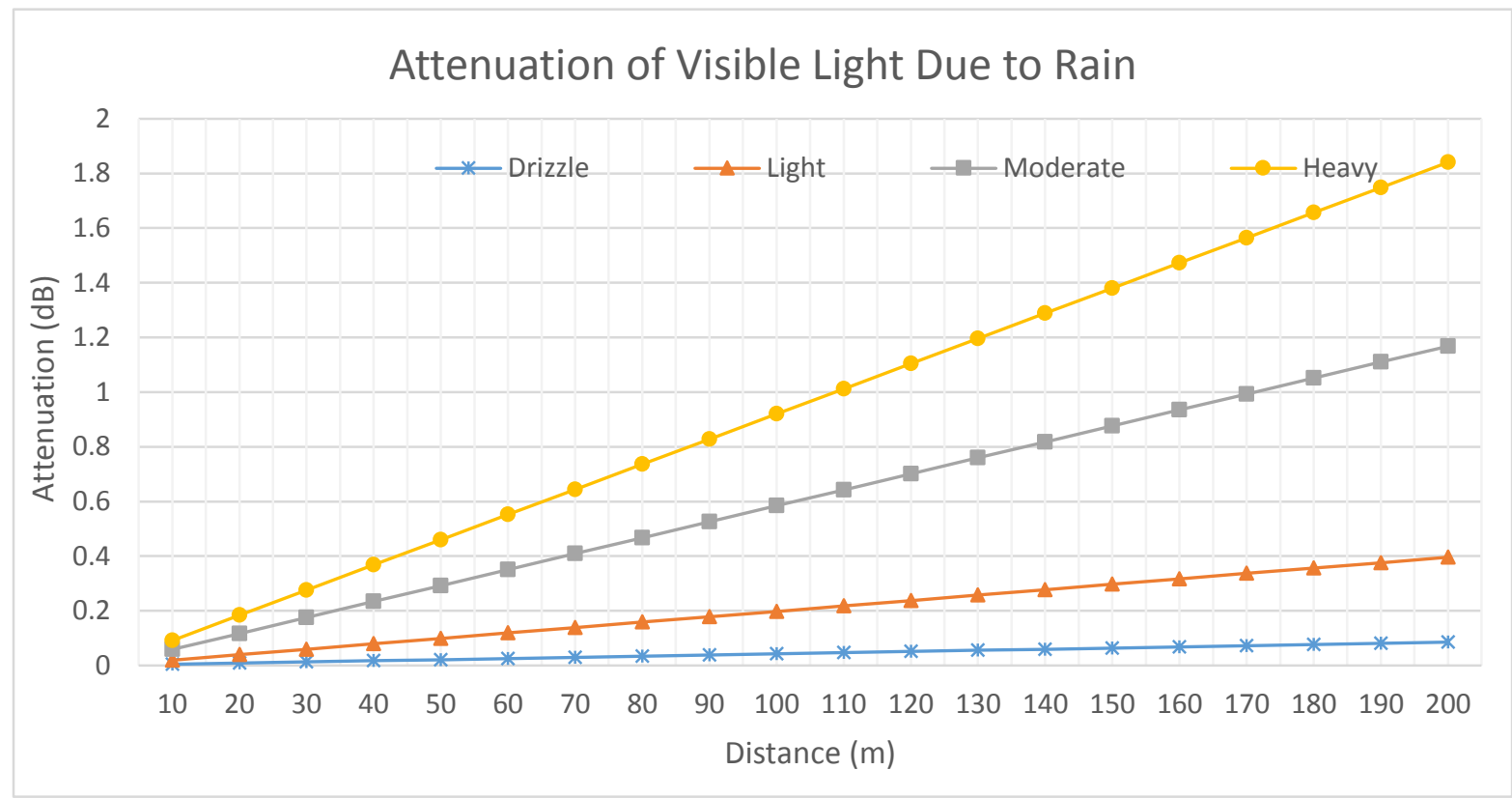

Figure 3.9 Attenuation due to rain at $\lambda=630 \mathrm{~nm}$

\subsubsection{2 mmWave}

The attenuation due to rain at $\mathrm{GHz}$ frequencies cannot be overlooked. The specific attenuation due to rain at mmWave frequencies is obtained from the power-law relationship:

$$
\alpha_{\text {Rain }}=k R^{a}
$$

where, $\alpha_{\text {Rain }}(\mathrm{dB} / \mathrm{km})$ is the specific attenuation due to rain, $R(\mathrm{~mm} / \mathrm{hr})$ is the rain fall rate, and values for the coefficients $k$ and $a$ are determined as functions of frequency, $f(\mathrm{GHz})$, in the range from 1 to $1000 \mathrm{GHz}$ and can be found in ITU-R documentation [ITU-05]. Table 3.9 lists the value of specific attenuation due to rain at various mmWave frequencies.

\begin{tabular}{|c|c|c|c|c|c|c|}
\hline$\lambda$ & $f$ & $\mathrm{k}$ & $\mathrm{a}$ & $\begin{array}{c}\text { Heavy Rain: } \\
25 \mathrm{~mm} / \mathrm{hr}\end{array}$ & $\begin{array}{c}\text { Moderate Rain } \\
: 12.5 \mathrm{~mm} / \mathrm{hr}\end{array}$ & $\begin{array}{c}\text { Light Rain: 2.5 } \\
\mathrm{mm} / \mathrm{hr}\end{array}$ \\
\hline $1 \mathrm{~mm}$ & 300 & 1.628 & 0.6296 & 12.35 & 7.984 & 2.89 \\
\hline $2 \mathrm{~mm}$ & 150 & 1.5823 & 0.649 & 12.78 & 8.15 & 2.86 \\
\hline $3 \mathrm{~mm}$ & 100 & 1.3671 & 0.681 & 12.24 & 7.63 & 2.55 \\
\hline $4 \mathrm{~mm}$ & 75 & 1.1048 & 0.722 & 11.28 & 6.84 & 2.14 \\
\hline
\end{tabular}




\begin{tabular}{|c|c|c|c|c|c|c|}
\hline $5 \mathrm{~mm}$ & 60 & 0.8606 & 0.765 & 10.09 & 5.94 & 1.734 \\
\hline $6 \mathrm{~mm}$ & 50 & 0.66 & 0.808 & 8.89 & 5.07 & 1.383 \\
\hline $7 \mathrm{~mm}$ & 43 & 0.508 & 0.847 & 7.76 & 4.31 & 1.103 \\
\hline $8 \mathrm{~mm}$ & 37.5 & 0.378 & 0.889 & 6.61 & 3.569 & 0.853 \\
\hline $9 \mathrm{~mm}$ & 33 & 0.29 & 0.92 & 5.60 & 2.96 & 0.673 \\
\hline $10 \mathrm{~mm}$ & 30 & 0.24 & 0.94 & 4.94 & 2.57 & 0.567 \\
\hline
\end{tabular}

Table 3.9 Specific Attenuation due to Rain at mmWave Spectrum

Figures 3.10 and 3.11 show the specific attenuation corresponding to the rain cell size and carrier frequency.

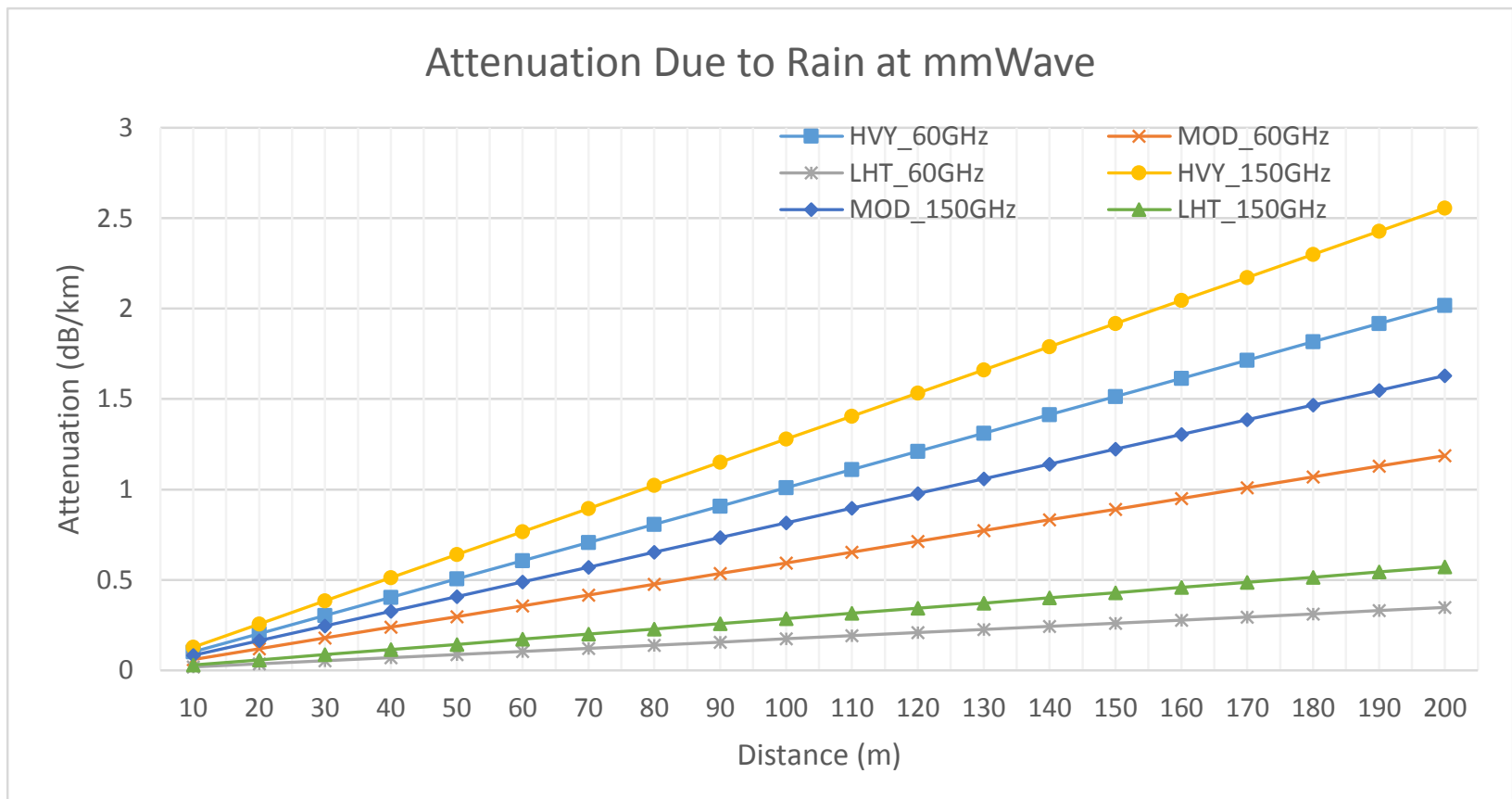

Figure 3.10 Attenuation Due to Rain at $60 \mathrm{GHz}$ and $150 \mathrm{GHz}$ 


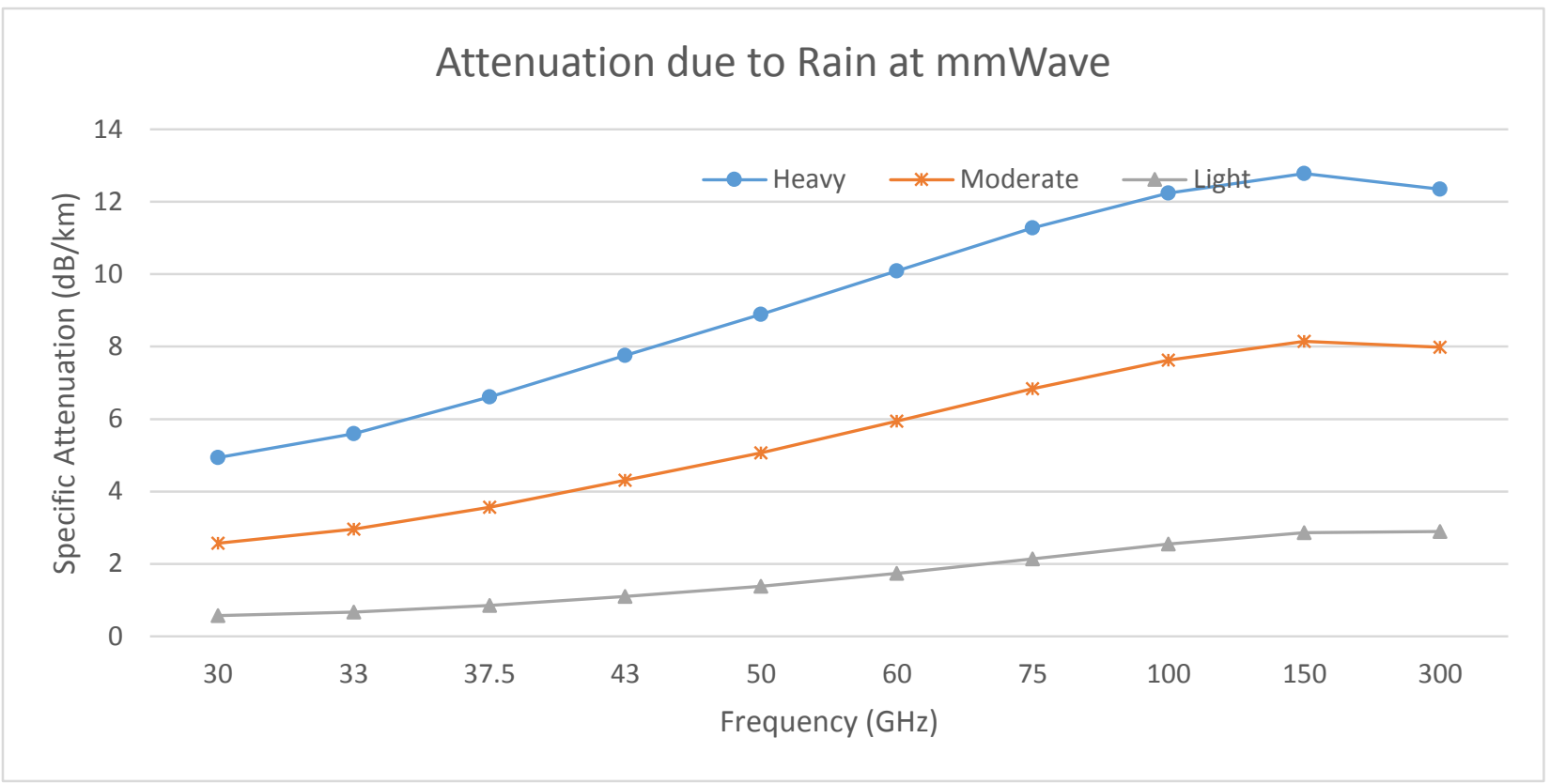

Figure 3.11 Attenuation Due to Rain at mmWave Frequencies

The highest attenuation is evident at the carrier frequency of $150 \mathrm{GHz}$ where the attenuation is almost $13 \mathrm{~dB} / \mathrm{km}$.

\subsubsection{Attenuation due to Snow}

Snow is a major source of attenuation for both mmWave signals and optical signals.

Communication links established between transmitter and receiver located across snowy regions may experience high bit error rate or in extreme cases, complete link failures, depending on the size of snow flakes, snow fall density and the position of ice crystals and snowflakes that are restricting the signal path. As snow particles are greater in size than rain drops, the attenuation due to snow is also larger than that due to rain of same intensity.

\subsubsection{VLC}

The attenuation at visible light frequencies due to snow depends upon the size of snow flake and the rate of snowfall. As the size of snow flake is larger than rain drops and fog particles, they cause major attenuation at nm wavelength [AKI-08]. In extreme cases snow flake size of up to $20 \mathrm{~mm}$ has been reported resulting in total blockage of visible light link and hence severely effecting the Quality of Service (QoS) of the communication network [YUT-06] [SON-98]. The attenuation due to snow is classified into wet snow attenuation and dry snow attenuation. The 
specific attenuation of visible light links due to snow in $\mathrm{dB} / \mathrm{km}$ is given by following relation [ITU-07] [KAU-17] [NAD-09]:

$$
\beta_{\text {snow }}=a S^{b}
$$

Where, $\beta_{\text {snow }}$ is the specific attenuation due to snow $(\mathrm{dB} / \mathrm{km}), S$ is the snowfall rate $(\mathrm{mm} / \mathrm{hr}), a$ and $b$ are wavelength dependent parameters and take different numerical values for wet and dry snow as indicated in table 3.10.

\begin{tabular}{|c|c|c|}
\hline Parameter & Wet Snow & Dry Snow \\
\hline$a$ & $0.000102 \lambda+3.79$ & $0.0000542 \lambda+5.50$ \\
\hline$b$ & 0.72 & 1.38 \\
\hline
\end{tabular}

Table 3.10 Values of parameter $a$ and $b$ for wet and dry snow [ITU-07]

An alternative approach to estimate attenuation due to snow based on visibility range is given by the following empirical formula [KAU-17]:

$$
\gamma_{\text {Snow }}=\frac{58}{V}
$$

Visibility range is dependent on snowfall levels. According to the report published by Aerodrome Meteorological Observation and Forecast Study Group (AMOFSG), the snowfall thresholds to distinguish between light, moderate and heavy snow fall and corresponding visibility range by World Metrological Organization (WMO) is as indicated in table 3.11 [AMO08]

\begin{tabular}{|c|c|c|}
\hline Snow Fall Intensity & Snow Fall Rate $(\mathrm{mm} / \mathrm{hr})$ & Visibility Range $(\mathrm{km})$ \\
\hline Light & $0-1$ & $>1$ \\
\hline Moderate & $1-5$ & $0.4<\mathrm{V}<1$ \\
\hline Heavy & $>5$ & $<0.4$ \\
\hline
\end{tabular}

Table 3.11 Visibility corresponding to snowfall rate according to WMO Table 3.12 presents the specific attenuation due to snow experienced by blue, red and green colors of visible light using both wavelength dependent relationship and empirical formula. 


\begin{tabular}{|c|c|c|c|c|c|c|c|c|}
\hline Snow Rate & $\begin{array}{l}\text { Visibility } \\
(\mathrm{Km})\end{array}$ & $\begin{array}{c}\text { Empirical } \\
\text { attenuation }(\mathrm{dB} / \mathrm{km}) \\
=58 / \mathrm{V}\end{array}$ & \multicolumn{3}{|c|}{ Attenuation $(\mathrm{db} / \mathrm{Km})=\mathrm{aS}^{\mathrm{b}}$} & & & \\
\hline & & & \multicolumn{3}{|c|}{$\begin{aligned} \text { Dry Snow }: a & =5.42 * 10^{-} \\
{ }^{5} \lambda+5.4958776, b & =1.38, S=\text { Rate }\end{aligned}$} & \multicolumn{3}{|c|}{$\begin{array}{c}\text { Wet Snow: }: a=1.023 * \\
10^{-4} \lambda+3.7855466, b=0.72 \\
, S=\text { Rate }\end{array}$} \\
\hline & & & $\lambda=450 \mathrm{~nm}$ & $530 \mathrm{~nm}$ & $630 \mathrm{~nm}$ & $450 \mathrm{~nm}$ & $530 \mathrm{~nm}$ & $630 \mathrm{~nm}$ \\
\hline High : $5-10 \mathrm{~mm} / \mathrm{hr}$ & $0.2-0.4$ & 193 & 80.94601 & 81.06959 & 81.08906 & 15.554 & 15.58 & 15.62 \\
\hline Moderate: $1-5 \mathrm{~mm} / \mathrm{hr}$ & $0.4-1$ & 58 & 14.36751 & 14.3788 & 14.3929 & 6.311 & 6.324 & 6.341 \\
\hline Light: $0-1 \mathrm{~mm} / \mathrm{hr}$ & $1-10$ & 5.8 & 2.12 & 2.122 & 2.124 & 2.326 & 2.331 & 2.337 \\
\hline
\end{tabular}

Table 3.12 Specific Attenuation due to snow (dB/km) for visible light spectrum.

Figure 3.12 and 3.13 plots the specific attenuation due to dry and wet snow at VL spectrum based on table 3.10 for distance between transmitter and receiver up to $200 \mathrm{~m}$.

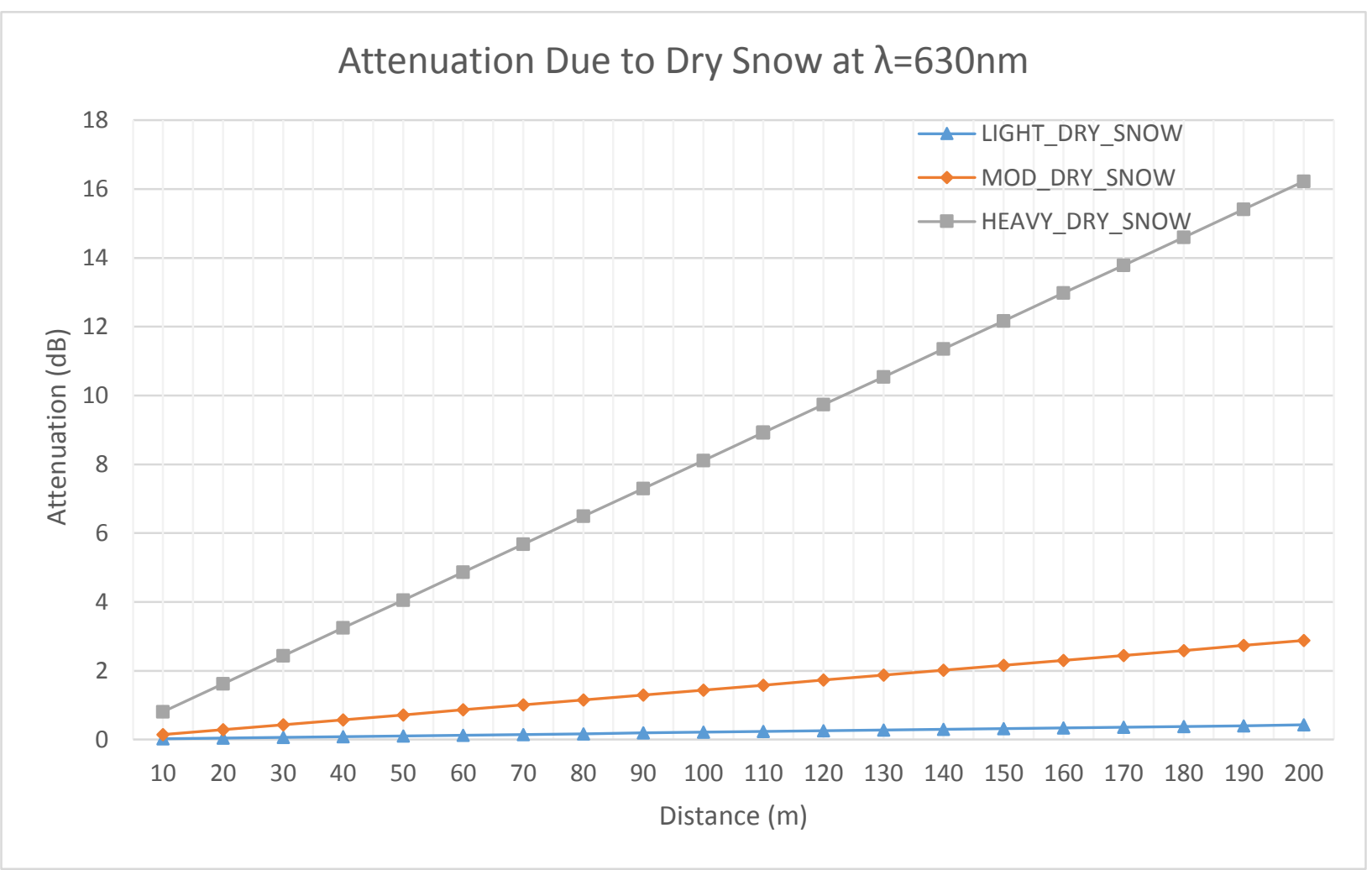

Figure 3.12 Attenuation due to Dry Snow for light, moderate and Heavy Snowfall at $\lambda=630 \mathrm{~nm}$ 


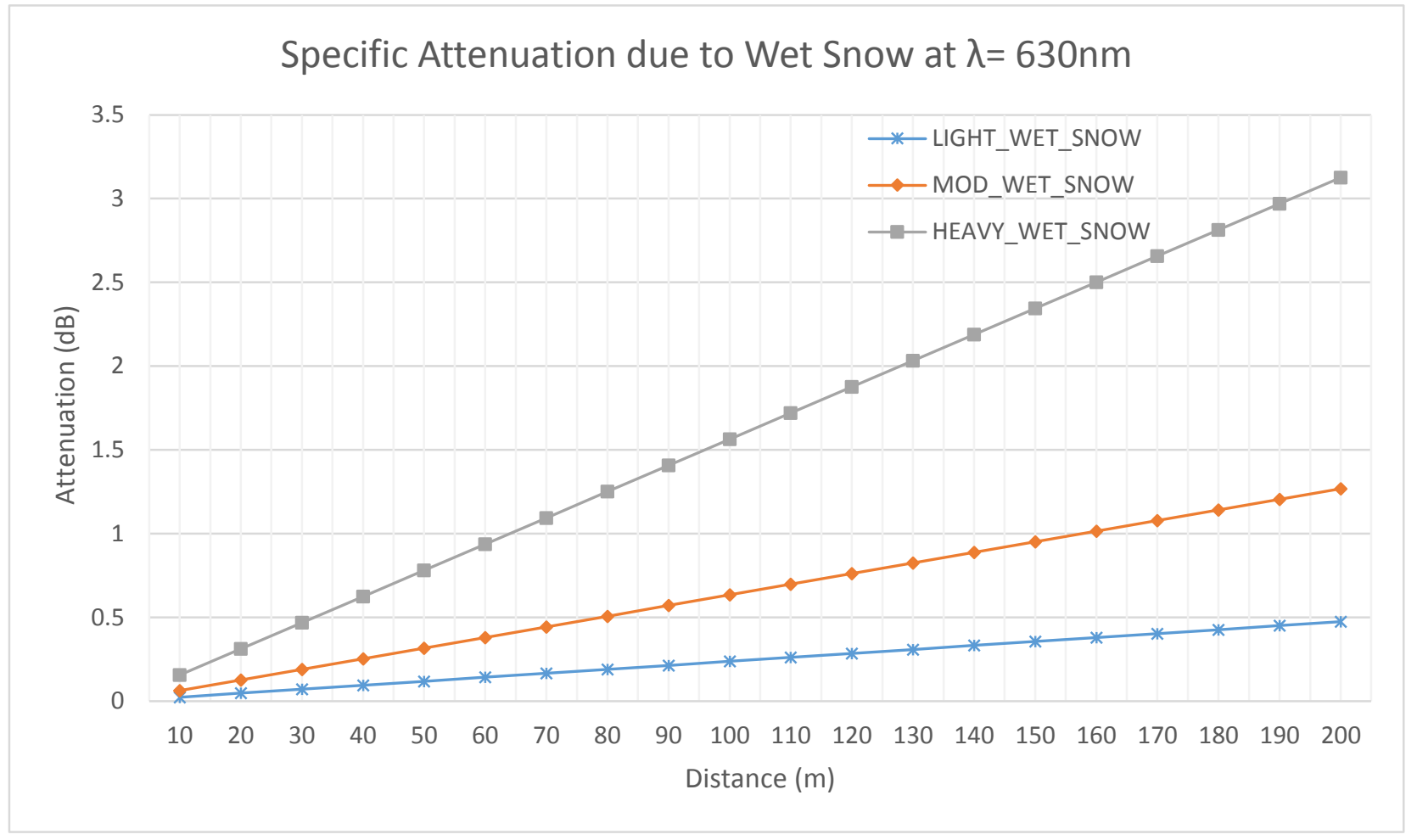

Figure 3.13 Attenuation due to Wet Snow for light, moderate and heavy snowfall at $\lambda=630 \mathrm{~nm}$

The attenuation due to dry snow at VL spectrum is much more pronounced than due to wet snow with maximum attenuation of upto $16 \mathrm{~dB}$ at $200 \mathrm{~m}$ for heavy snowfall. However, since VLC will take place at femto cell size range of upto $60 \mathrm{~m}$, the attenuation due to dry snow reduces to $5 \mathrm{~dB}$ while the attenuation due to wet snow at heavy snowfall becomes almost negligible.

\subsubsection{2 mmWave}

mmWaves signals are attenuated as a result of scattering when colliding with snowflakes.

Snowflakes are created by combination of ice, water and air molecules in different fractions. The knowledge of dielectric properties of the composite molecules is essential to the estimation of the extent of scattering, shorter electromagnetic wavelengths will undergo while travelling through the atmosphere. However, due to complex composition of snow flakes, it is not an easy task. The shape, density, water content and mixing rate of snow particles depends considerably on the 
exposed environmental factors. Therefore, the effective dielectric function can only be obtained by approximations and assumptions [NAD-09]. Hence, at mmWave frequencies, the attenuation due to snow is difficult to determine. Wet snow results in attenuation that is comparable and sometimes surpasses the attenuation due to rain of same intensity by 6 times [OOM-71]. The snow attenuation becomes increasingly significant at shorter wavelength as it was demonstrated that dry snow at $0.96 \mathrm{~mm}$ exceeds the attenuation due to rain by $40 \%$ [OGU-83]. The specific snow attenuation in the GHz range is given as follows [NAD-09] [OGU-83]:

$$
\alpha_{\text {Snow }}(\lambda)=0.00349 \frac{S^{1.6}}{\lambda^{4}}+0.00224 \frac{S}{\lambda}
$$

Where, $\alpha_{\text {Snow }}$ is the specific attenuation due to snow $(\mathrm{dB} / \mathrm{km}), S$ is the snow fall rate and $\lambda$ is the wavelength. Table 3.13 lists the specific attenuation due to snow at mmWave frequencies.

\begin{tabular}{|c|c|c|c|c|}
\hline$\lambda$ & $\mathrm{f}$ & $\begin{array}{c}\text { Heavy Snow: 5- } \\
10 \mathrm{~mm} / \mathrm{hr}\end{array}$ & $\begin{array}{c}\text { Moderate Snow :1- } \\
5 \mathrm{~mm} / \mathrm{hr}\end{array}$ & $\begin{array}{c}\text { Light Snow : 0-1 } \\
\mathrm{mm} / \mathrm{hr}\end{array}$ \\
\hline $1 \mathrm{~mm}$ & 300 & 22.38 & 11.19 & 2.25 \\
\hline $2 \mathrm{~mm}$ & 150 & 11.19 & 5.595 & 1.125 \\
\hline $3 \mathrm{~mm}$ & 100 & 7.47 & 3.73 & 0.75 \\
\hline $4 \mathrm{~mm}$ & 75 & 5.61 & 2.79 & 0.5625 \\
\hline $5 \mathrm{~mm}$ & 60 & 4.494 & 2.238 & 0.45 \\
\hline $6 \mathrm{~mm}$ & 50 & 3.75 & 1.865 & 0.375 \\
\hline $7 \mathrm{~mm}$ & 43 & 3.23 & 1.6039 & 0.3225 \\
\hline $8 \mathrm{~mm}$ & 37.5 & 2.79 & 1.39 & 0.281 \\
\hline $9 \mathrm{~mm}$ & 33 & 2.49 & 1.23 & 0.2475 \\
\hline $10 \mathrm{~mm}$ & 30 & 2.2755 & 1.11 & 0.225 \\
\hline
\end{tabular}

Table 3.13 Specific Attenuation due to snow $(\mathrm{dB} / \mathrm{km})$ for mmWave frequencies.

Figure 3.14 shows the specific attenuation at light, moderate and heavy snowfall corresponding to wavelength. It can be seen that the specific attenuation due to heavy snowfall will attenuate the $300 \mathrm{GHz}$ mmWave signal by $23 \mathrm{~dB} / \mathrm{km}$. Figure 3.15 provides the specific attenuation versus the distance between transmitter and receiver. For a maximum distance of $200 \mathrm{~m}$, attenuation at $300 \mathrm{GHz}$ will be $4.5 \mathrm{dBs}$. 


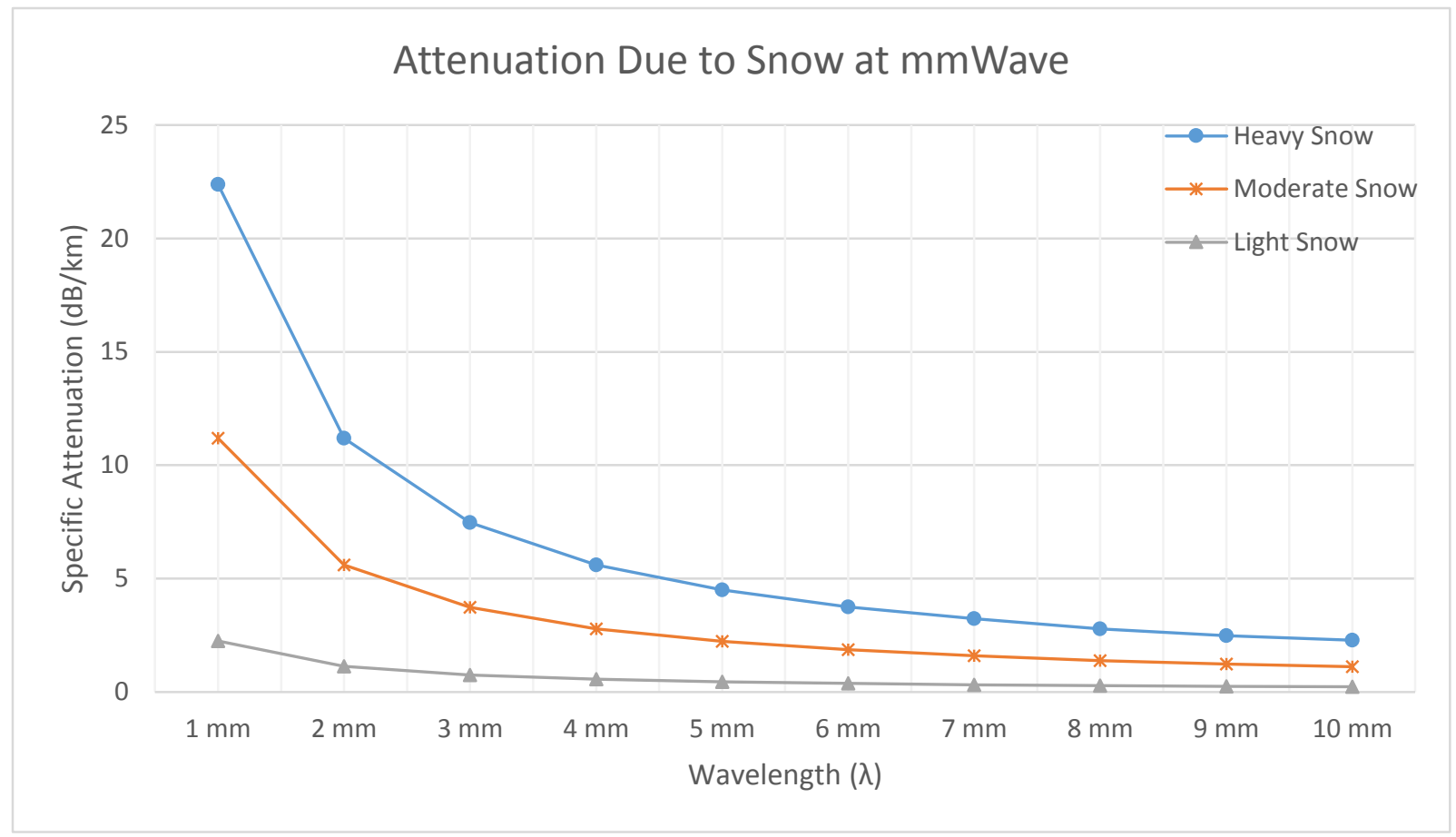

Figure 3.14 Attenuation due to light, moderate and heavy snow at mmWave wavelengths

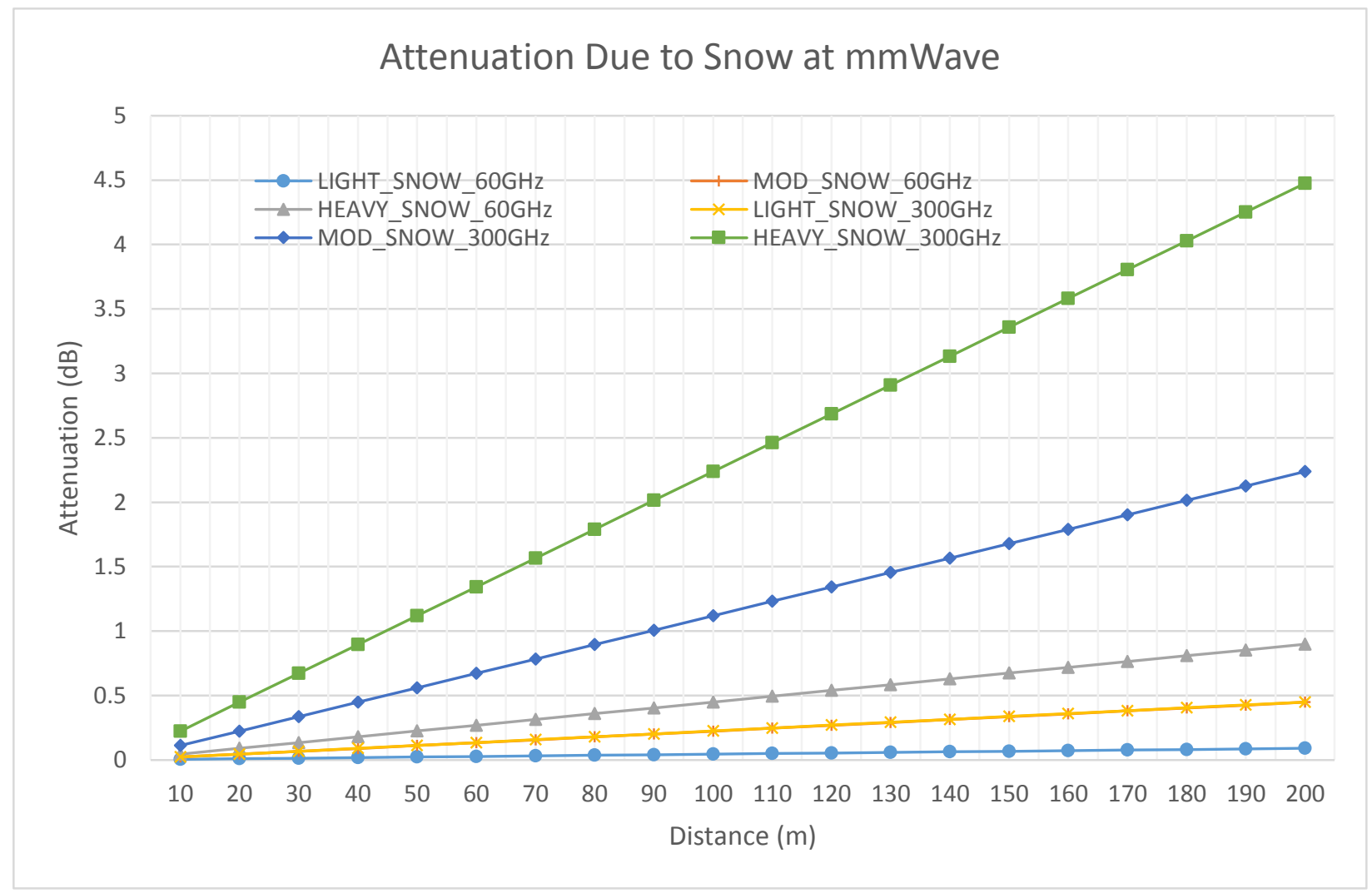

Figure 3.15 Attenuation due to light, moderate and heavy snow at $60 \mathrm{GHz}$ and $300 \mathrm{GHz}$ 


\subsection{Comparison between VLC and mmWave based on Attenuation due to Adverse Weather}

This section provides comparison between mmWave and VLC based on the specific attenuation calculated in the previous section. Figure 3.16 and 3.17 provides comparison between signal attenuation under dense fog and moderate fog conditions.

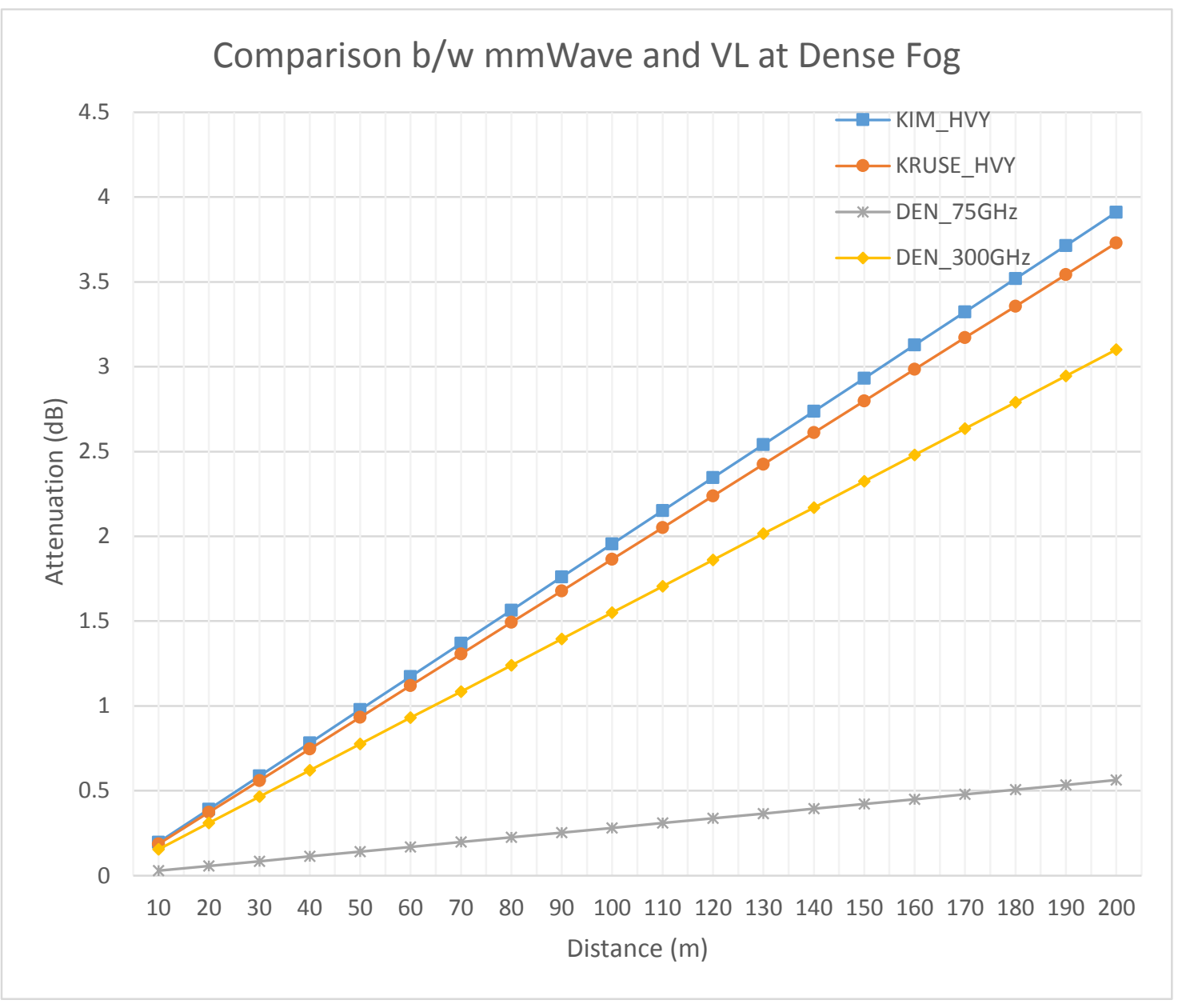

Figure 3.16 Comparison between mmWave $(75 \& 300 \mathrm{GHz})$ and VL (Kim/Kruse model) for dense fog conditions 


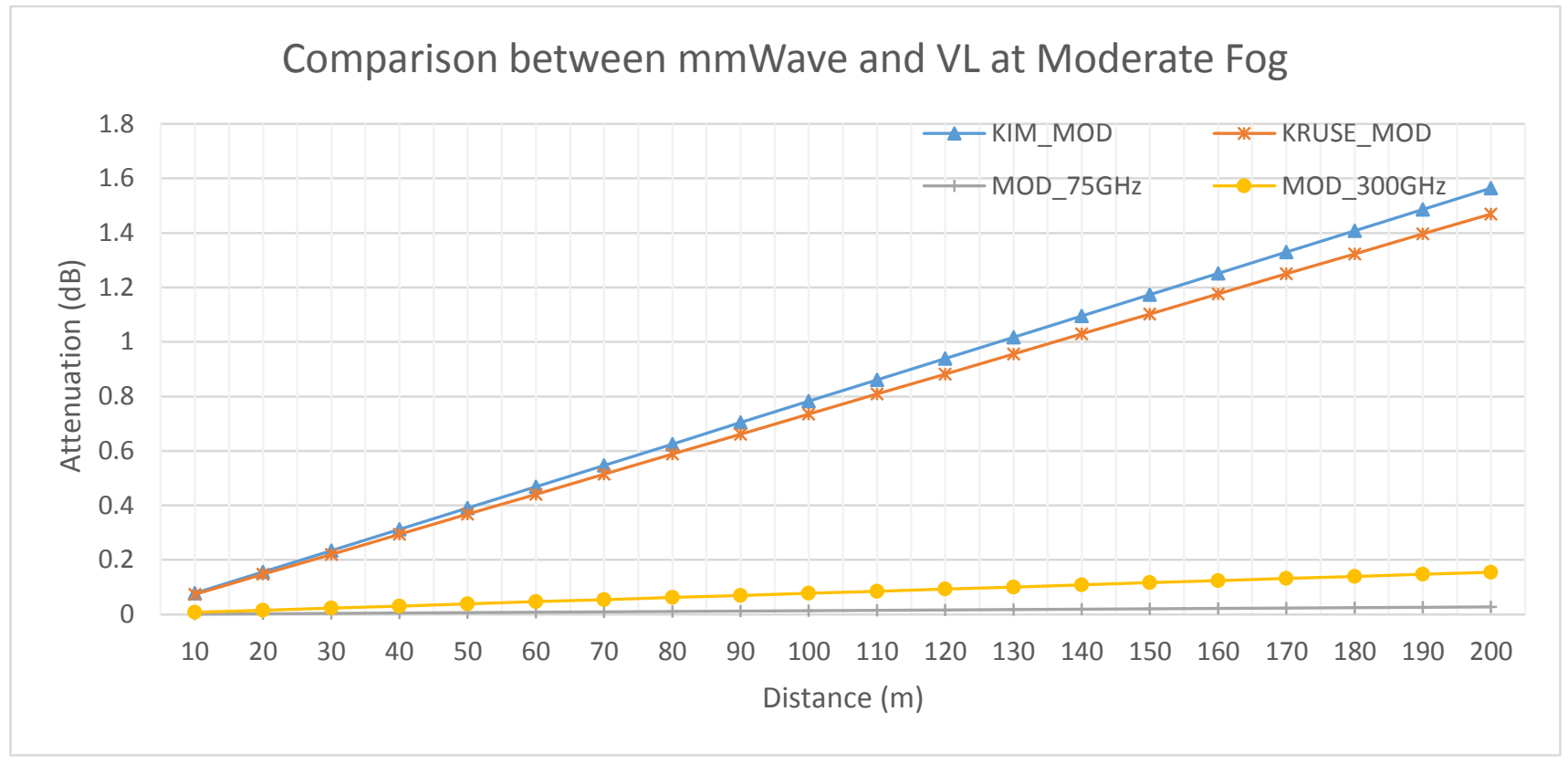

Figure 3.17 Comparison between mmWave (75 \& $300 \mathrm{GHz})$ and VL (Kim/Kruse model) for moderate fog conditions

It can be deduced from the above figures that fog effects VL waves more than mmWave frequencies for both dense and moderate foggy weather. Kim and Kruse models follow each other closely for short distance, in this case upto 200m, however, the specific attenuation of VL is around $1 \mathrm{~dB}$ more than mmave in both scenarios which is not very significant. 


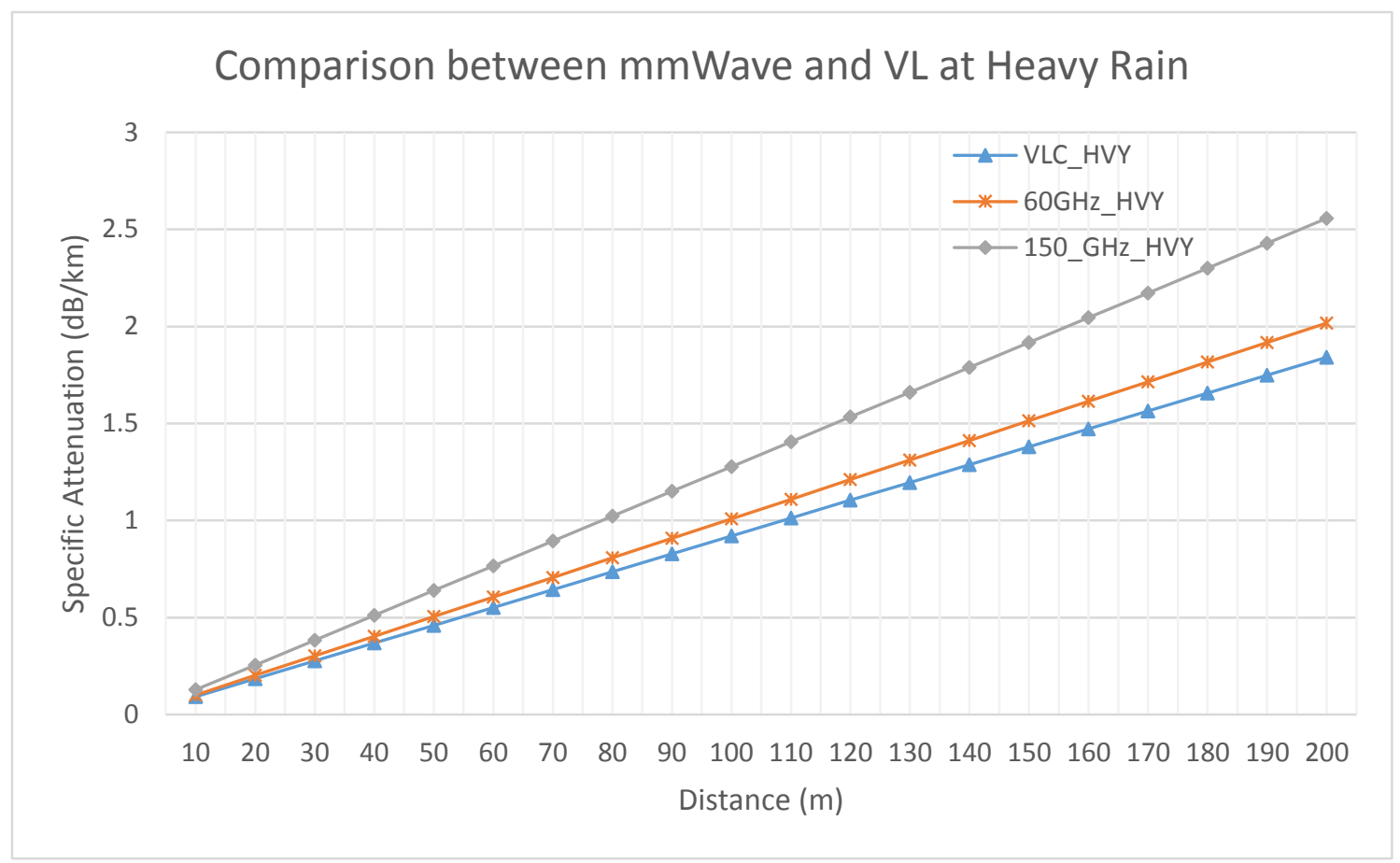

Figure 3.18 Comparison between mmWave $(60 \& 150 \mathrm{GHz})$ and VLfor heavy rain

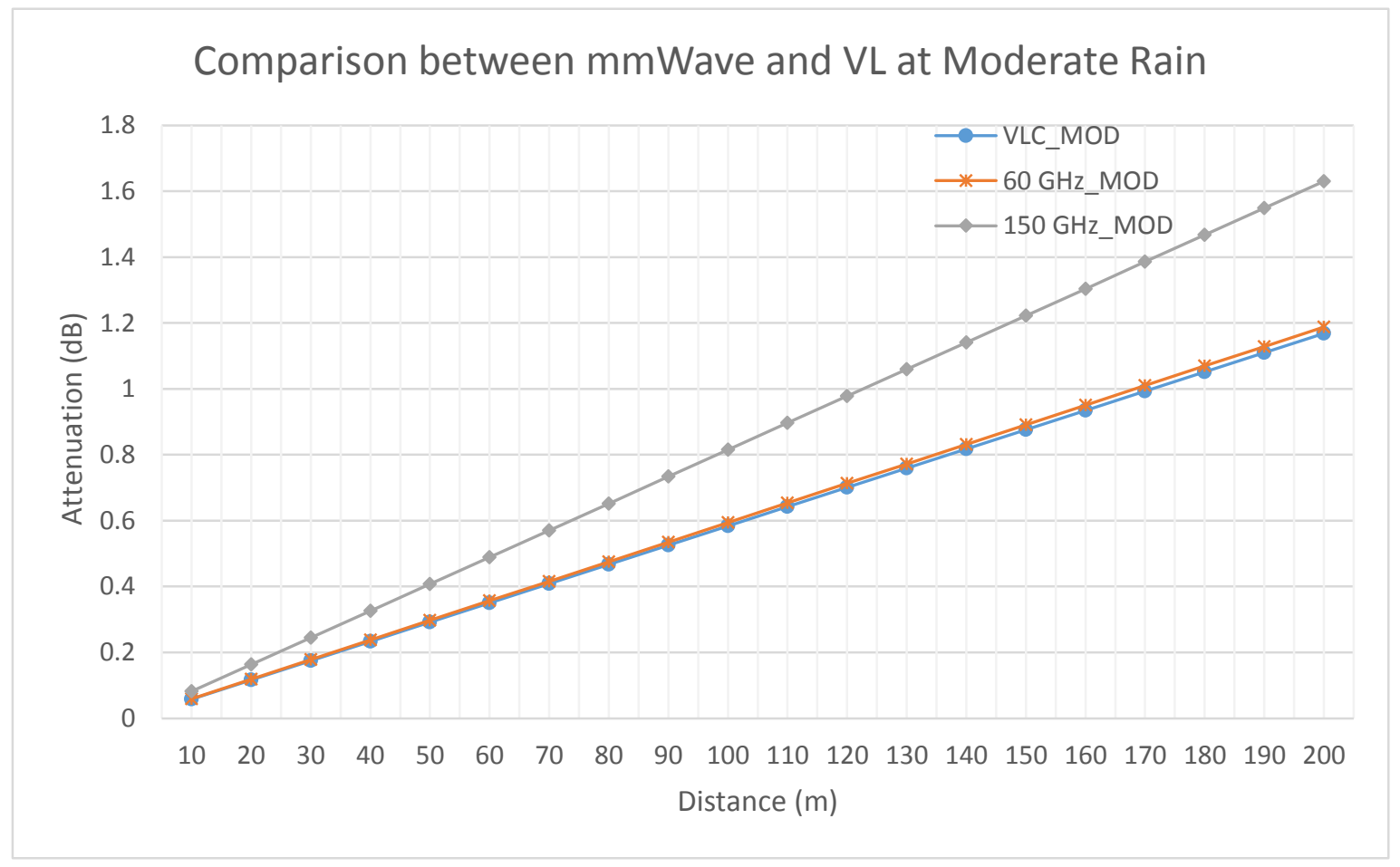

Figure 3.19 Comparison between mmWave $(60 \& 150 \mathrm{GHz})$ and VL for moderate rain 
For both heavy and moderate rain fall, VL signals are less attenuated than mmWave signals, however, the attenuation at cell size of up to $200 \mathrm{~m}$ still witness a maximum deviation of around $2 \mathrm{~dB}$ from clear weather.

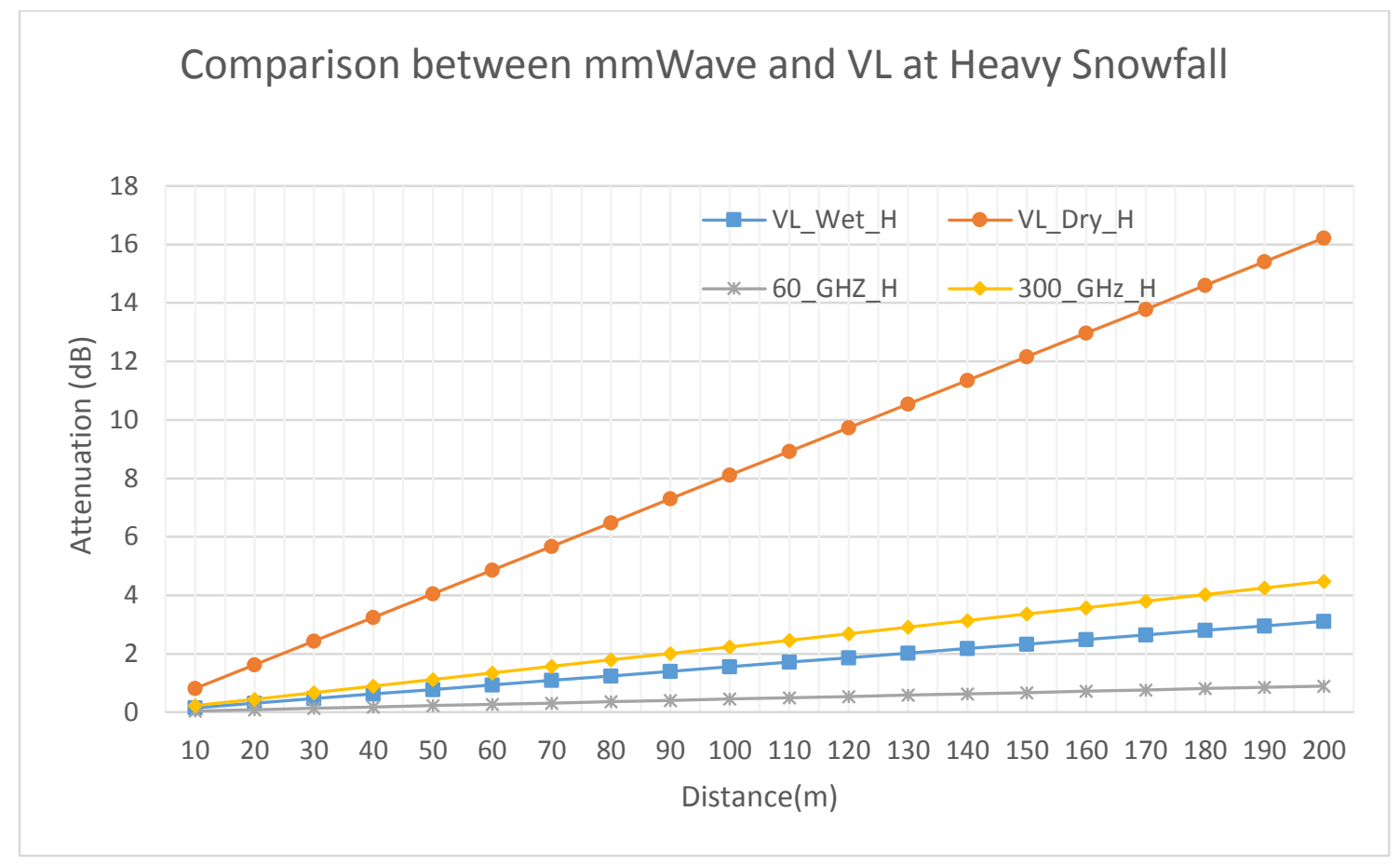

Figure 3.20 Comparison between mmWave $(60 \& 300 \mathrm{GHz})$ and VL (wet/dry) at heavy snowfall

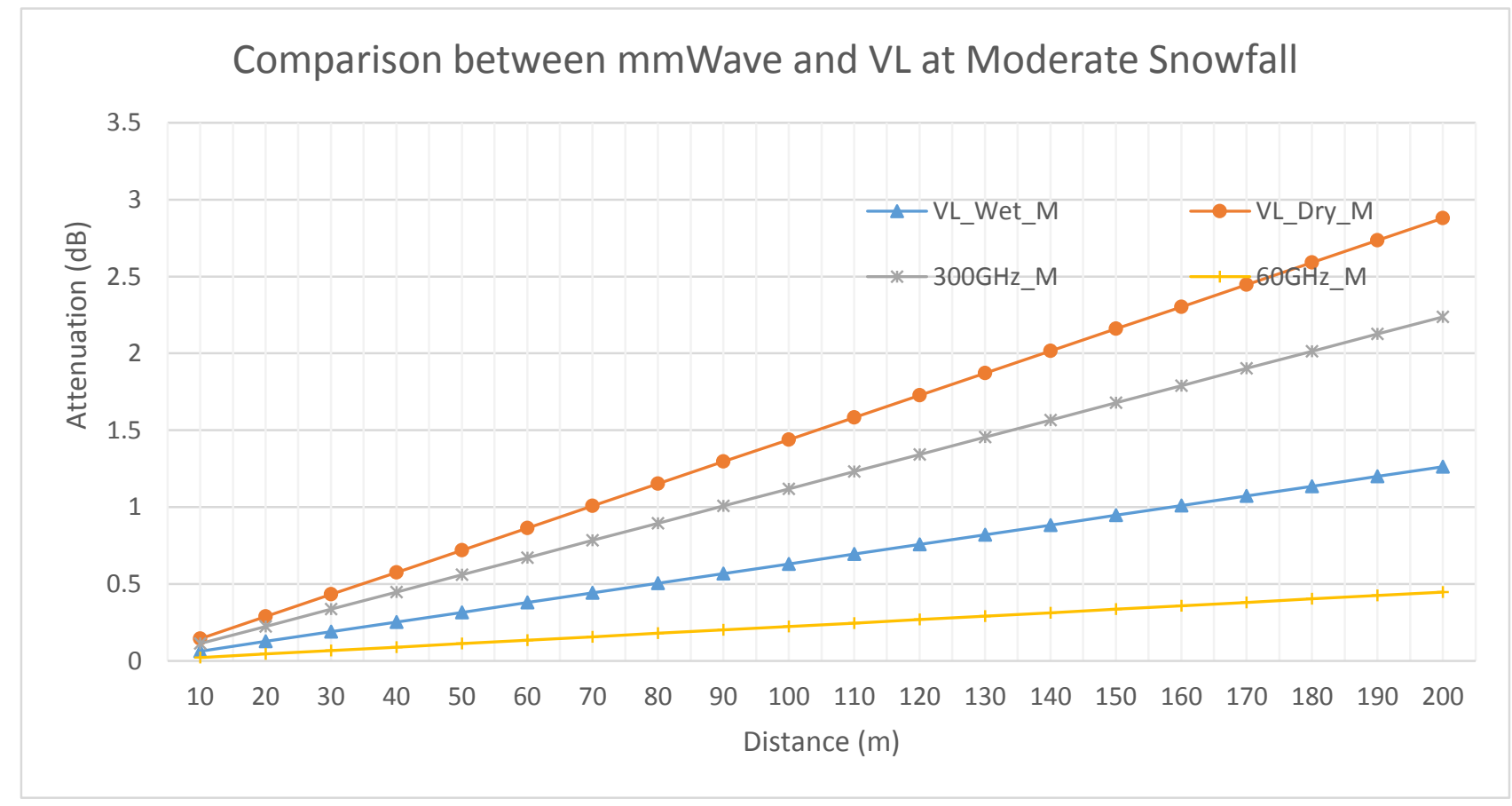

Figure 3.21 Comparison between mmWave $(60 \& 300 \mathrm{GHz})$ and VL (wet/dry) at moderate snowfall 
Although the attenuation of VL waves for moderate snowfall is comparable to mmWave signals, the attenuation due to dry snow at heavy snow fall is almost $12 \mathrm{~dB}$ more than mmWave signals at $200 \mathrm{~m}$.

It can be concluded from above figures that both mmWave signal and VL signals undergo similar attenuation under adverse weather conditions. Rain and snow are major attenuating factors for mmWave whereas fog and snow cause noticeable attenuation at VL. The only significant difference in attenuation going up by around $12 \mathrm{dBs}$ for VL compared to mmWave is for a scenario of heavy snowfall. While this value cannot be ignored, it should be recalled that VL technology is most suitable for femto cell deployment where the maximum cell size will be in the order of $10 \mathrm{~s}$ of meter. Hence reducing the difference between mmWave and VL specific attenuation due to snow to around 4-5 dBs. The next section provides the comparison between the performance analysis for both mmWave and VL outdoor channels by taking into consideration not only the weather specific attenuation but also the power transmission levels and feasibility of implementation of both systems for $5 \mathrm{G}$ vehicular networks.

\subsection{Design Analysis for 5G vehicular Networks}

\subsubsection{Dual-link VLC System}

For 5G vehicular networks, this work proposes the use of dual-link VLC system. The dual-link system comprises of two different types of optical links: wide beam links to maintain connectivity and transfer of sensor data and narrow beams, to support high data rate communication. Although, dual link LED communication systems has been proposed before for optical wireless communication [SHE-14], this work furthers the concept by specifically discussing the design analysis for outdoor VLC systems taking into consideration signal attenuation due to solar interference and weather attenuation. The power transmission levels obtained for targeted bit rate are mapped to the acceptable power transmission levels of traffic signals. Hence this discussion of a multi-beam dual-link VVLC system provides the mapping between power transmission levels for targeted bit rate and its translation to commercial fixtures and components.

The link model considered is for LOS communication between traffic lights/lamp posts and vehicle (I2V). The scenario considers that there is no power constraint thus we have strong SNR. 
The assumption is based on the fact that the street lights and traffic lights are the ones acting as infrastructure access points, and vehicle has enough power to support transmission of strong optical signal as well. In such case, the channel appears as an ON-OFF channel; if there is a blockage of the LoS the performance becomes very poor, since whatever energy is received through reflections cannot support high transmission rates reliably. The system is not designed to be able to function with diffusive energy. The FOV of the used lenses on transmitting LEDs and photo-detectors is assumed narrow. This reduces the power of transmitted and received diffusive radiation, as well as the spread of propagation delay of received rays. Reflections affect the impulse response of the channel by spreading it. This spread generated significant inter-symbol interference (ISI) that deteriorates performance. However because we are considering very small cells and focused light transmission (e.g. light pattern generated by light posts), the reflections are not expected to have significant time differential from the LoS, thus spreading should not be excessive. The system is based on intensity modulation/direct detection (IM/DD). Figure 3.22 shows the LED transmitter configuration for the proposed dual link VVLC system. The system consists of one wide beam link and two narrow beam links. $\phi_{\frac{1}{2}}$ is the half power beamwidth angle and $\varphi$ is the angle of alignment error for the transmitter. If we assume $\varphi=0$, it means that transmitter is directly pointed to the receiver. The range of the transmitter is defined as $2 \theta$, where $\Theta$ is the maximum pointing offset angle allowed between the axis of the transmitter and distance $d$ between transmitter and receiver that would still maintain connectivity. The FOV of the receiver is defined by the optical concentrator half power angle $\Psi_{c}$ and angle of alignment error of the receiver $\psi$. Assumption of $\psi=0$ indicates that receiver is directly pointed at transmitter. 


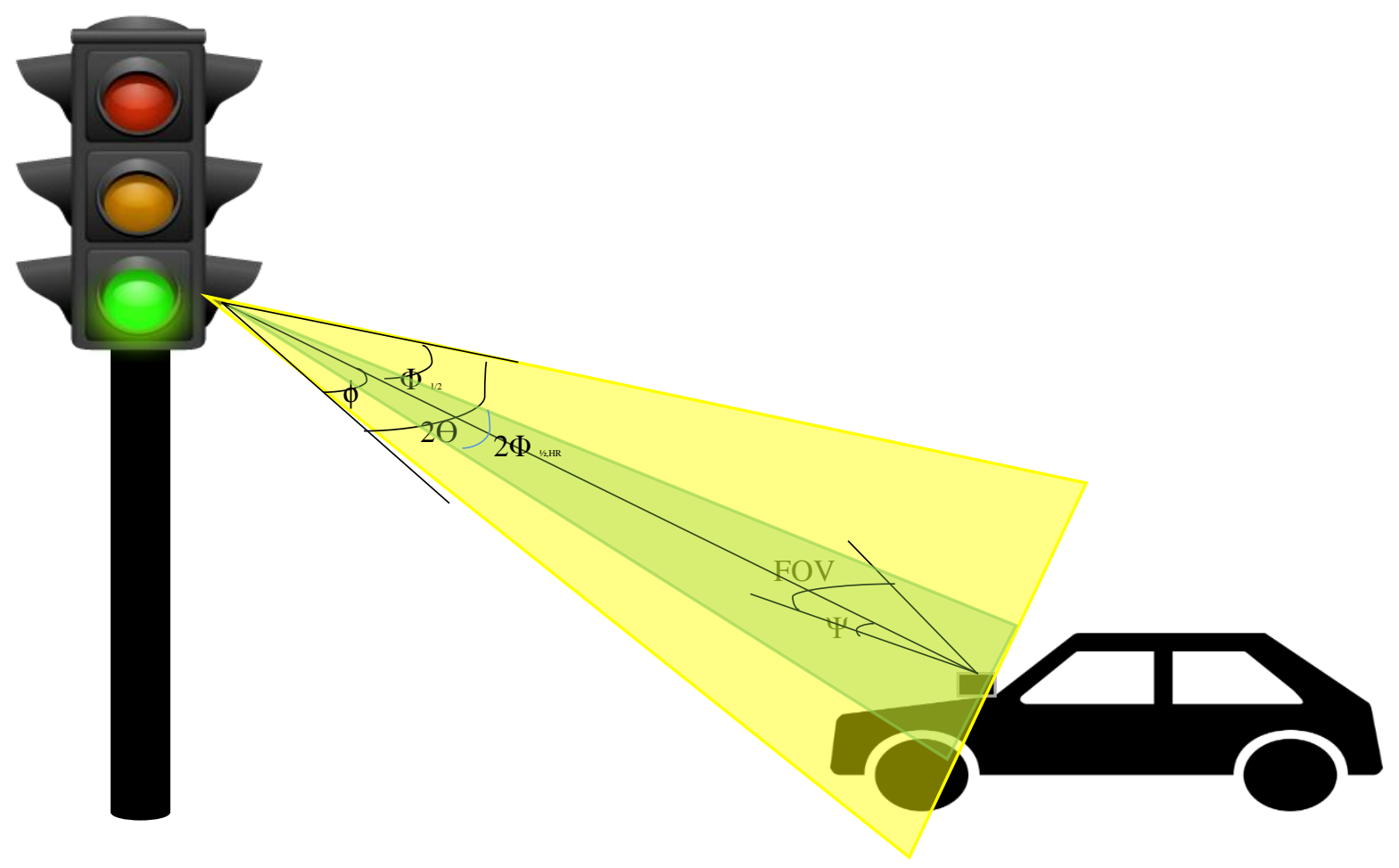

Figure 3.22 Transmitter and receiver configuration mapped to traffic light and vehicle

It is not possible to use a single LED for generating enough luminous intensity supporting both illumination and communication. Hence, multiple LEDs are arranged as an array in the traffic light. These arrays can be configured in square, triangular or circular arrangement. The total transmission power of these arrays can be calculated by linearly adding the power from each LED. For each LED, its power can be calculated by integrating the spectral response $\mathrm{St}(\lambda)$ over the wavelength of the visible light $\lambda$ as:

$$
P_{t}=\int_{\lambda_{L}}^{\lambda_{H}} S_{t}(\lambda) d \lambda
$$

where $\lambda_{\mathrm{L}}$ and $\lambda_{\mathrm{H}}$ are the minimum and the maximum wavelengths of the visible light, respectively. The absolute spectral response $\mathrm{St}(\lambda)$ is calculated from the normalized spectral distribution $\operatorname{St}(\lambda)$ as:

$$
S_{t}(\lambda)=c S_{t}^{\prime}
$$

where $\mathrm{St}_{\mathrm{t}}{ }^{\prime}(\lambda)$ is generally available in the LED datasheet and $\mathrm{c}$ is a scaling factor. The total luminous flux $F_{t}$, which is expressed as the measurement of the brightness of the light source as perceived by human eyes is also an important factor available in the data sheets. $F_{t}$ is a function of St $(\lambda)$ expressed as follows: 


$$
F_{t}=683 \int_{380 \mathrm{~nm}}^{780 \mathrm{~nm}} S_{t}(\lambda) V(\lambda) d \lambda
$$

where $380 \mathrm{~nm}$ to $780 \mathrm{~nm}$ is the wavelength range of visible light, and $\mathrm{V}(\lambda)$ is a spectral luminous efficiency function which is the measurement of human visual responses to several wavelengths of light. It is defined as the ratio between the radiant flux at a specified wavelength $\lambda_{s}$ to another wavelength $\lambda$ under similar photometric condition. Values of $V(\lambda)$ are pre-defined by International Commission on Illumination (CIE) for various wavelengths of light in the visible region and are given in table 3.14 [ROB-09].

\begin{tabular}{|l|l|l|l|}
\hline Wavelength $(\mathrm{nm})$ & $\begin{array}{l}\text { Spectral luminous efficiency } \\
(V(\lambda))\end{array}$ & Wavelength $(\mathrm{nm})$ & $\begin{array}{l}\text { Spectral luminous efficiency } \\
(V(\lambda))\end{array}$ \\
\hline 380 & 0.00004 & 580 & 0.870 \\
\hline 390 & 0.00012 & 590 & 0.757 \\
\hline 400 & 0.0004 & 600 & 0.361 \\
\hline 410 & 0.0012 & 610 & 0.503 \\
\hline 420 & 0.0040 & 620 & 0.381 \\
\hline 430 & 0.0116 & 630 & 0.265 \\
\hline 440 & 0.023 & 640 & 0.175 \\
\hline 450 & 0.038 & 650 & 0.107 \\
\hline 460 & 0.060 & 660 & 0.061 \\
\hline 470 & 0.091 & 670 & 0.032 \\
\hline 480 & 0.139 & 680 & 0.017 \\
\hline 490 & 0.208 & 690 & 0.0082 \\
\hline 500 & 0.323 & 700 & 0.0041 \\
\hline 510 & 0.503 & 710 & 0.0021 \\
\hline 520 & 0.710 & 720 & 0.00105 \\
\hline 530 & 0.862 & 730 & 0.00052 \\
\hline 540 & 0.954 & 740 & 0.00025 \\
\hline 550 & 0.995 & 750 & 0.00012 \\
\hline 560 & 0.995 & 760 & 0.00007 \\
\hline 570 & 0.952 & 770 & 0.00003 \\
\hline & & & \\
\hline
\end{tabular}

Table 3.14 Values of $V(\lambda)$ for various wavelengths as specified by CIE [ROB-09].

Alternatively, using gaussian curve fitting for $V(\lambda)$

$$
V(\lambda) \cong 1.019 e^{-285.4(\lambda-0.559)^{2}}, \quad \lambda: \text { in } \mu m
$$

The scaling factor $\mathrm{c}$, can be derived as:

$$
c=\frac{F_{t}}{683 \int_{380 n m}^{780 n m} s_{t}^{\prime}(\lambda) V(\lambda) d \lambda}
$$


$\mathrm{P}_{\mathrm{t}}$ can be found using these equations.

Assuming that transmitter is composed of LEDs that follow Lambertian radiation pattern, the irradiance at a distance $d$ between transmitter and receiver and as function of $\varphi$, where $\varphi$ corresponds to the angle of transmitter misalignment or pointing error is defined as [SHE-14]

$$
I_{s}(d, \varphi)=\mathrm{P}_{t} \frac{m+1}{2 \pi d^{2}} \cos ^{m}(\varphi)
$$

Here, $\mathrm{P}_{t}$ is the average transmitted optical power in watts and $m$ is the parameter defining half power emission beamwidth. The half-power beamwidth angle $\Phi_{1 / 2}$ is related to $m$ by the expression:

$$
m=-(\ln 2) / \ln \left[\cos \left(\Phi_{\frac{1}{2}}\right)\right]
$$

The received optical power at the photodiode at the distance $d$ is given as [SHE-14]:

$$
P_{R}=I_{S} A_{e f f}
$$

Where, $A_{\text {eff }}$ corresponds to the total effective area that gathers incident signal at the receiver. Assuming that the receiver also employs an optical concentrator $\mathrm{A}_{\mathrm{eff}}$ is defined as:

$$
A_{e f f}=g(\psi) T s(\psi) A \cos (\psi)(3.40)
$$

Here, A is the active area of receiver, Ts is related to optical filter and is defined as the optical power that is allowed to enter the filter. It should be noted that TS may correspond to the average filter transmission over a range of wavelengths if the transmission spectrum is wide or an average of filter transmission over a range of angles if light strikes the receiver at different incident angles. Specifically, in this scenario $\operatorname{Ts}(\psi)$ corresponds to the fraction of optical power that is allowed to pass through the optical filter at an incident angle $(\psi) . g(\psi)$ is the gain of optical concentrator. Assuming an ideal optical concentrator, the gain is calculated by the following expression:

$$
g(\psi)=\left\{\begin{array}{ll}
n^{2} / \sin ^{2}\left(\Psi_{c}\right), & \text { if }|\psi| \leq \Psi_{c} \\
0, & \text { if }|\psi|>\Psi_{c}
\end{array}\right\}
$$

Here, $\mathrm{n}$ is the refractive index of the concentrator and $\Psi_{c}$ is the half-angle field of view of the concentrator.

The incident received power generates the photocurrent, which is given by the following expression:

$$
I_{R}=R P_{R}
$$

Where, $R$ is the responsivity of the photodiode and is expressed in amperes/watts $(A / W)$. 
In outdoor VLC systems, the most prominent noise source is the spectral radiation of sunlight, skylight and ambient light sources. The received optical power from these sources introduces dc photocurrent which is the most dominant noise called as "shot noise". It is necessary to employ an optical bandpass filter to reduce the ambient noise power incident on the receiver's photodiodes. For this analysis, we assume a narrow band pass filter such as an ideal "boxcar" with effective spectral bandwidth of $\Delta \lambda$ and filter transmittance $T_{n}$ within the passband. It is also assumed that the background noise has a constant power spectral density within the passband, i.e. it is considered "white noise". The spectral irradiance of the noise sources are given as $p_{n}(\mathrm{~W}$ $\left./ \mathrm{nm}-\mathrm{cm}^{2}\right)$. Spectral irradiance is defined in terms of incident noise power per unit area of photodiode and per unit wavelength. It should be noted that the value of $p_{n}$ is considered independent of wavelength within the filter passband as considered in [KAH-97]. As we are assuming white noise and an ideal optical concentrator of refractive index $n$, the ambient power received by the photodiodes is given by the following expression [BAR-94][PHO-95]:

$$
P_{b g}=p_{n} \Delta \lambda T_{n} A n^{2}
$$

The shot noise caused by the ambient light sources are modeled as additive white Gaussian noise (AWGN). The variance of the shot noise is given by:

$$
\sigma_{S H O T}^{2}=2 q R P_{b g} B
$$

Here, B (bits/s) is the bit rate of the signal. Another source of noise is known as thermal noise or Johnson-Nyquist noise. This is the noise caused by the thermal fluctuations of electrons in a receiver circuit of equivalent resistance $\mathrm{R}_{\mathrm{L}}$ at Temperature $\mathrm{T}_{\mathrm{e}}$. Assuming silicon $\mathrm{p}$-i photodiode, the thermal noise variance is given by:

$$
\sigma_{T H}^{2}=\frac{4 K T_{e} B}{R_{L}}
$$

Where, $\mathrm{K}$ is Boltzman's constant $(\mathrm{K}=1.381 \mathrm{E}-23), q$ is the electronic charge $(\mathrm{q}=1.6 \mathrm{E}-19 \mathrm{C})$, and $T e$ is the temperature (290K). Another noise is quantum noise. This is defined as the shot noise due to the statistical nature of the optical detection process and is caused by the dark current, $I_{D}$. Its value is very small, having variance:

$$
\sigma_{Q t m}^{2}=2 q B R I_{D}
$$

The total noise variance is given by:

$$
\sigma^{2}=\sigma_{S H O T}^{2}+\sigma_{Q t m}^{2}+\sigma_{T H}^{2}
$$


Using this noise model, the signal-to-noise ratio (SNR) at the receiver is expressed as:

$$
S N R=\frac{I_{R}{ }^{2}}{\sigma^{2}}=\frac{\left(R P_{R}\right)^{2}}{\sigma^{2}}
$$

Considering On-off keying modulation (OOK), the BER can be found as:

$$
B E R=Q(\sqrt{S N R})
$$

Where, $\mathrm{Q}$ is the probability of standard normal distribution, defined as:

$$
Q=\frac{1}{\sqrt{2 \pi}} \int_{x}^{\infty} e^{\frac{-y^{2}}{2} d y}
$$

It is possible to find the achievable highest bit rate in terms of transmitted power, noise levels, range and targeted BER for the proposed link model using equation (3.44) to equation (3.49).

$$
\begin{aligned}
& S N R=\frac{\left(R P_{R}\right)^{2}}{\sigma^{2}}=\frac{R^{2} P_{R}^{2}}{\sigma_{S H O T}^{2}+\sigma_{Q t m}^{2}+\sigma_{T H}^{2}} \\
& S N R=\frac{R^{2} P_{R}^{2}}{\left(2 q R P_{b g}+\frac{4 K T_{e}}{R_{L}}+2 q R I_{D}\right) B} \\
& B=\frac{R^{2} P_{R}^{2}}{\left(2 q R P_{b g}+\frac{4 K T_{e}}{R_{L}}+2 q R I_{D}\right)\left[Q^{-1}(B E R)\right]^{2}}
\end{aligned}
$$

Similarly, the maximum range of communication for a given BER, data rate and average transmitted power can be found by substituting equations (3.37), (3.39) and (3.40) into equation (3.53)

$$
\begin{gathered}
B=\frac{R^{2}\left(\mathrm{P}_{t}(\mathrm{~m}+1) \cos ^{m}(\varphi) g(\psi) T s(\psi) A \cos (\psi)\right)^{2}}{\left(2 \pi d^{2}\right)^{2}\left(2 q R P_{b g}+\frac{4 K T_{e}}{R_{L}}+2 q R I_{D}\right)\left[Q^{-1}(B E R)\right]^{2}} \\
d=\left(\frac{1}{\left(2 q R P_{b g}+\frac{4 K T e}{R_{L}}+2 q R I_{D}\right) B}\right)^{\frac{1}{4}}\left(\frac{R\left(\mathrm{P}_{t}(\mathrm{~m}+1) \cos ^{m}(\varphi) g(\psi) T s(\psi) A \cos (\psi)\right)}{2 \pi Q^{-1}(B E R)}\right)^{\frac{1}{2}}
\end{gathered}
$$

\subsubsection{Joint design of high rate and low rate dual link system}

The joint design of high rate and low rate system requires that the operational range for both links are same. Here, we are using narrow beams for more focused high rate data transfer, and 
wide beams for maintaining connectivity i.e., exchange of control and positioning information and low rate periodic or/and emergency sensor data. Henceforth, the wide beam links will be referred to as the low rate links and narrow beams will be referred to as high rate links.

Correspondingly, subscript HR and LR will be used to identify the parameters related to high rate and low rate links respectively. Table 3.15 lists all of the parameters that are used in this design analysis.

\begin{tabular}{|c|c|c|}
\hline Parameters & Description & Type of link \\
\hline $\mathrm{B}_{\mathrm{LR}}$ & Bit rate & Low rate \\
\hline $\mathrm{B}_{\mathrm{HR}}$ & Bit rate & High rate \\
\hline$P_{L R}$ & Transmitted power & Low rate \\
\hline$P_{\text {HR }}$ & Transmitted power & High rate \\
\hline Ts,LR & Filter transmittance & Low rate \\
\hline Ts,HR & Filter transmittance & High rate \\
\hline$\Delta \lambda_{L R}$ & Bandwidth of optical filter & Low rate \\
\hline$\Delta \lambda_{H R}$ & Bandwidth of optical filter & High rate \\
\hline$\varphi, \mathrm{LR}$ & Transmitter misalignment angle & Low rate \\
\hline$\varphi, \mathrm{HR}$ & Transmitter misalignment angle & High rate \\
\hline$\psi, \mathrm{LR}$ & Receiver pointing angle & Low rate \\
\hline$\psi, \mathrm{HR}$ & Receiver pointing angle & High rate \\
\hline$\Psi_{c, L R}$ & Optical concentrator angle & Low rate \\
\hline$\Psi_{c, H R}$ & Optical concentrator angle & High rate \\
\hline$m, \mathrm{LR}$ & Parameter associated with half power beamwidth & Low rate \\
\hline$m, \mathrm{HR}$ & Parameter associated with half power beamwidth & High rate \\
\hline$A_{\mathrm{LR}}$ & Physical area of the detector & Low rate \\
\hline$A_{\mathrm{HR}}$ & Physical area of the detector & High rate \\
\hline$\Phi_{1 / 2, \mathrm{LR}}$ & Half power angle of transmitter beamwidth & Low rate \\
\hline$\Phi_{1 / 2, \mathrm{HR}}$ & Half power angle of transmitter beamwidth & High rate \\
\hline
\end{tabular}

\section{Table 3.15 Parameters related to the dual link design analysis}

It is important that there is no interference between the high rate links and low rate links. It is assumed that both links use different wavelengths or light "color" for communication, hence eliminating the interference. In a highly mobile environment, it is not possible to maintain constant alignment between transmitter and receiver, therefore for this analysis, a range of different pointing angles from the transmitter axis is considered for low rate links. The transmitter range is defined by $2 \Theta$, where $\Theta$ is the maximum pointing error or misalignment allowed between transmitter and receiver that would still maintain connectivity and we assume 
that $\varphi_{\mathrm{LR}}=\theta$, for $\left(-\Theta<\varphi_{\mathrm{LR}}<\theta\right)$, a minimum bit rate $\mathrm{B}_{\mathrm{LR}}$ is maintained. If we change the pointing angle $\varphi_{\mathrm{LR}}$ within this range $|\Theta|$, the distance $d$ at which this bit rate is achievable also changes. The analysis considers different pointing angles to replicate real world scenarios rather than using $\varphi_{\mathrm{LR}}=0$ for an ideal case with perfect transmitter pointing. The low rate half angle beamwidth can be obtained by using $\varphi_{\mathrm{LR}}=\Theta$ and differentiating equation (3.55) with respect to $m$ [SHE-14].

$$
\begin{aligned}
m_{L R} & =-1-\frac{1}{\ln [\cos (\theta)]} \\
\Phi_{1 / 2, L R} & =\cos ^{-1}\left[\exp \left(-\frac{\ln 2}{m_{L R}}\right)\right]
\end{aligned}
$$

The distance $\mathrm{d}$ for minimum bit rate $\mathrm{B}_{\mathrm{LR}}$ can be found by substituting the values of $m_{L R}$ and $\varphi_{\mathrm{LR}}$ $=\Theta$ in equation (3.55).

Equation (3.54) and equation (3.55) can be used to calculate realistic range and data rates for the dual link VVLC system. Figure 3.23 plot provides the achievable distance as a function of transmitted power for low rate links. 


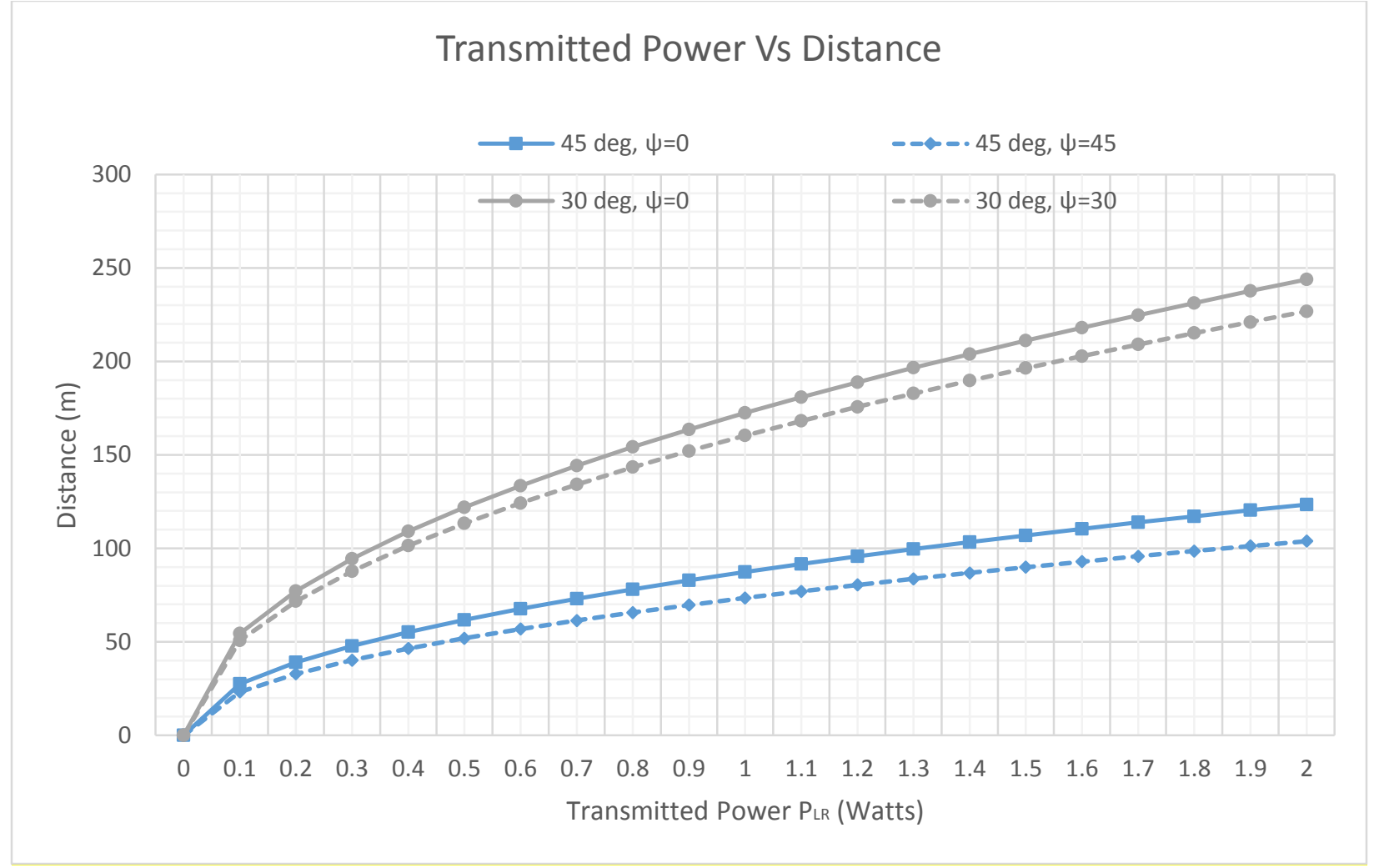

Figure 3.23 Transmitter power vs distance for low rate links

Figure 3.23 depicts the transmitted power required for two different values of transmitter pointing angle $\varphi_{\mathrm{LR}}=\Theta=45^{\circ}$ and $30^{\circ}$ and for the case of perfect receiver alignment $\psi=0$ and $\psi=\Theta$. It can be deduced from the above figure that for the case of perfect receiver alignment i.e. $\psi=0$ and transmitter pointing angle of $\varphi_{\mathrm{LR}}=45^{0}$ minimum bit rate (taken as $10 \mathrm{kbps}$ ) can be achieved at a distance of $50 \mathrm{~m}$ by transmitting 0.4 Watts. Generally, the value of $\mathrm{d}$ is dependent on many factors such as $\psi, \mathrm{R}, \mathrm{P}$ and data rate $\mathrm{B}$. Specifically in terms of data rate, higher data rates are achievable at short distances, whereas lower data rates are achievable at longer distances. However, it should be noted that the value of $\Phi_{1 / 2, L R}$ is directly dependent on

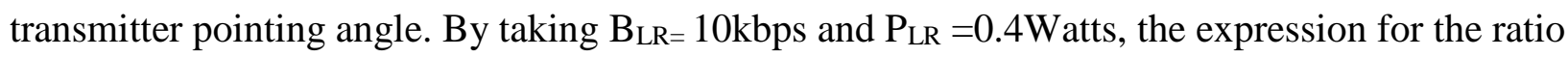
between high rate and low rate achievable was derived from equation (3.54). Since the shot noise due to sunlight and skylight is much greater than quantum noise and thermal noise, the total noise variance for deriving this expression is taken as $\sigma^{2}=\sigma_{S H O T}^{2}$. 


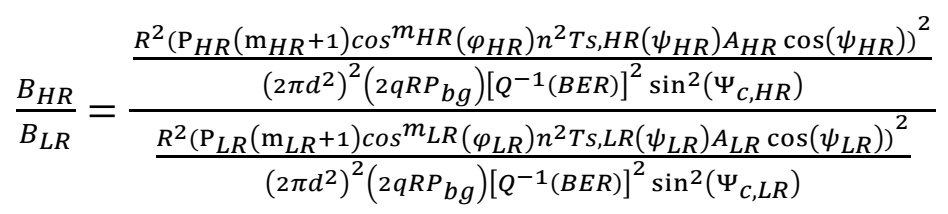

Simplifying,

$$
\begin{aligned}
& \frac{B_{H R}}{B_{L R}}= \\
& \left(\frac{T_{s, H R}\left(\psi_{H R}\right)}{T_{S, L R}\left(\psi_{L R}\right)}\right)^{2}\left(\frac{\mathrm{P}_{H R}}{\mathrm{P}_{L R}}\right)^{2}\left(\frac{\mathrm{m}_{H R}+1}{\mathrm{~m}_{L R}+1}\right)^{2}\left(\frac{A_{H R}}{A_{L R}}\right)^{2}\left[\frac{\cos ^{m_{H R}\left(\varphi_{H R}\right)}}{\cos m_{L R}\left(\varphi_{H R}\right)}\right]^{2}\left[\frac{\cos \left(\psi_{H R}\right)}{\cos \left(\psi_{L R}\right)}\right]^{2}\left(\frac{\Delta \lambda_{L R}}{\Delta \lambda_{H R}}\right)\left[\frac{\sin ^{2}\left(\Psi_{c, L R}\right)}{\sin ^{2}\left(\Psi_{c, H R}\right)}\right]^{2}
\end{aligned}
$$

Figure 3.24 plots the relationship between the high bit rate achievable to the ratio of high and low transmitted power for different half power angles of high rate links. These curves are plotted for transmitter pointing angle $\varphi_{\mathrm{LR}}=45^{\circ}$ and $\varphi_{\mathrm{LR}}=30^{\circ}$ and half power angle of high rate links $\Phi_{1 / 2, H R}=10^{0}$ and $\Phi_{1 / 2, H R}=20^{\circ}$. Table 3.16 lists the values of parameters that are used in this analysis. In these calculations, it is assumed that the receiver is composed of narrowband colored glass filter with passband width $\Delta \lambda$ with transmittance $\mathrm{T}_{\mathrm{s}}=\mathrm{T}_{\mathrm{n}}=0.8$. Silicon $\mathrm{p}$-i photodiode of responsivity 0.6A/W [HAM-19]. The optical concentrator is also assumed to be made of glass (hence the refractive index $n=1.5$ ).

\begin{tabular}{|c|c|}
\hline Parameters & Value \\
\hline$A_{H R}$ & $1 \mathrm{~mm}^{2}$ \\
\hline$A_{L R}$ & $1 \mathrm{~cm}^{2}$ \\
\hline$\Psi_{C, H R}$ & $5^{0}$ \\
\hline$\Psi_{C, L R}$ & $45^{0}$ \\
\hline$p_{n}$ & $5.8 \mu \mathrm{W} / \mathrm{nm} . \mathrm{cm}^{2}[\mathrm{KAH}-97][\mathrm{SHE}-14]$ \\
\hline $\mathrm{R}$ & $0.6 \mathrm{~A} / \mathrm{W}$ \\
\hline$\Delta \lambda_{L R}$ & $100 \mathrm{~nm}$ \\
\hline$\Delta \lambda_{H R}$ & $30 \mathrm{~nm}$ \\
\hline $\mathrm{n}$ & 1.5 \\
\hline$T_{S, H R}, T_{s, L R}, T_{n}$ & 0.8 \\
\hline $\mathrm{BER}_{\mathrm{BER}}$ & $10^{-6}$ \\
\hline $\mathrm{B}_{\mathrm{LR}}$ & $10 \mathrm{kbps}$ \\
\hline
\end{tabular}

Table 3.16 Values of various parameters related to the dual link design analysis 


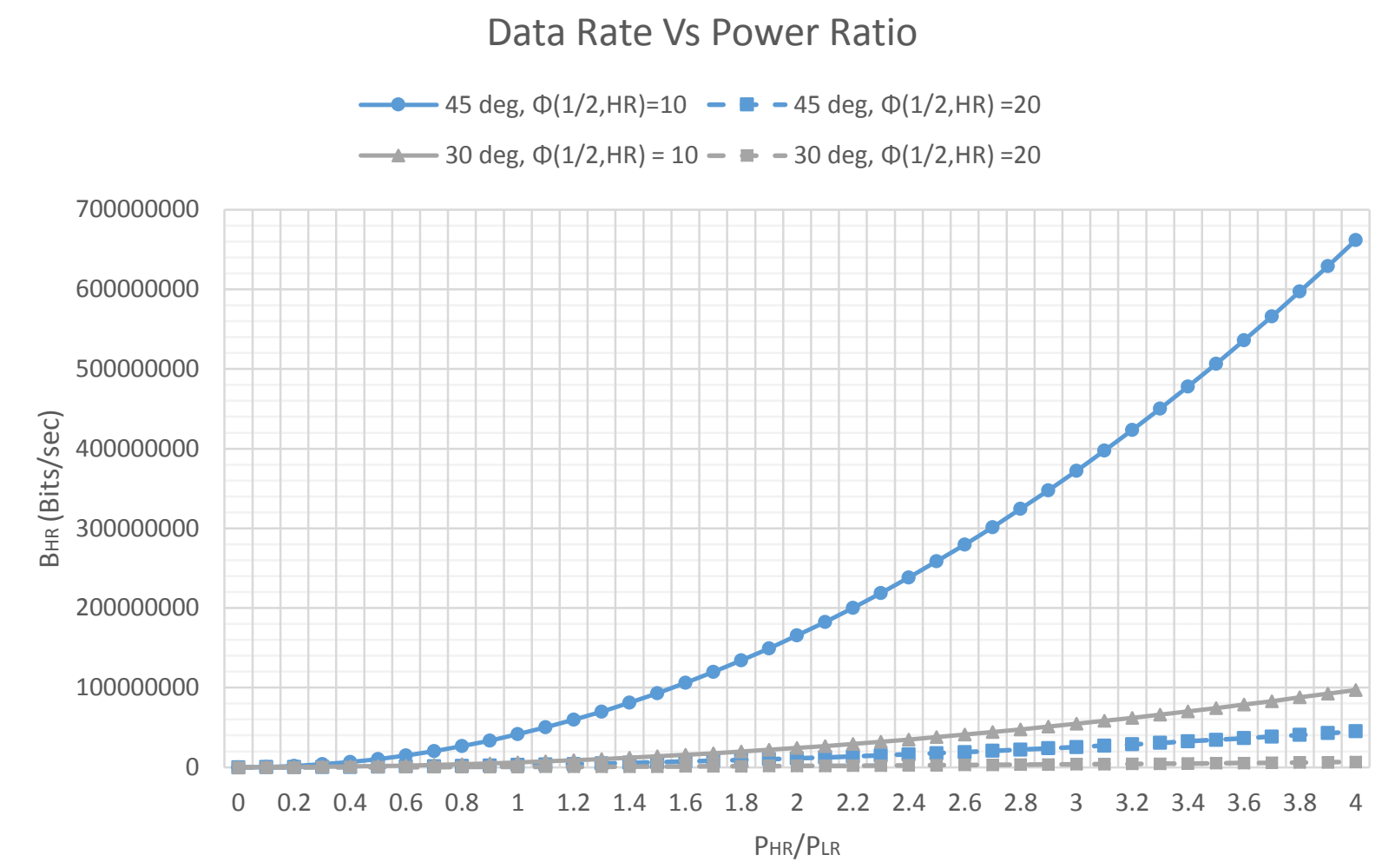

Figure 3.24 Data rate for high rate links vs power ratio

\begin{tabular}{|c|l|}
\hline & \\
\hline$A_{H R}$ & \\
\hline$A_{L R}$ & \\
\hline$\Psi_{c, H R}$ & \\
\hline$\Psi_{c, L R}$ & \\
\hline$p_{n}$ & \\
\hline & \\
\hline$\Delta \lambda_{L R}$ & \\
\hline$\Delta \lambda_{H R}$ & \\
\hline & \\
\hline$T_{S, H R}, T_{S, L R}, T_{n}$ & \\
\hline & \\
\hline
\end{tabular}


For further analysis, we take the values of low rate transmitter pointing angle as $45^{\circ}$ and half power angle of high rate links as $10^{\circ}$. It can be seen that in order to maintain a high link data rate of $500 \mathrm{Mb} / \mathrm{s}$ the ratio between $\mathrm{P}_{\mathrm{HR}} / \mathrm{P}_{\mathrm{LR}}$ should be equal to 3.4. Using the value of $\mathrm{P}_{\mathrm{LR}}$ obtained from figure 3.23 .

$$
\begin{gathered}
\frac{P_{H R}}{P_{L R}}=3.4 \\
P_{H R}=3.4 * 0.4=1.4 \mathrm{~W}
\end{gathered}
$$

\subsubsection{Dual link system with weather attenuation}

In this section, the effect of attenuation due to weather is analyzed for the dual link system. Recall that in section 3.3, effects of attenuation due to rain, fog and snow was investigated. In VLC, the illumination intensity is compromised due to unfavourable weather conditions. This decrease in illumination intensity affects the quality of the received optical signal and maximum coverage area of the optical cell. In order to keep the coverage area of the cell and achievable data rates within the cell same, it is desirable to increase the transmission power to overcome the loss of optical signal due to weather. An additional term $A_{t t}$ is introduced in the analysis to evaluate the attenuation due to weather dependent factors. Hence, equation (3.39) is modified as:

$$
P_{R}=I_{S} A_{e f f} A_{t t}
$$

Equation 3.54 and 3.55 are modified as:

$$
\begin{gathered}
B=\frac{R^{2}\left(\mathrm{P}_{t}(\mathrm{~m}+1) \cos ^{m}(\varphi) A_{e f f} A_{t t}\right)^{2}}{\left(2 \pi d^{2}\right)^{2}\left(2 q R P_{b g}+\frac{4 K T_{e}}{R_{L}}+2 q R I_{D}\right)\left[Q^{-1}(B E R)\right]^{2}} \\
d=\left(\frac{1}{\left(2 q R P_{b g}+\frac{4 K T_{e}}{R_{L}}+2 q R I_{D}\right) B}\right)^{\frac{1}{4}}\left(\frac{R\left(\mathrm{P}_{t}(\mathrm{~m}+1) \cos ^{m}(\varphi) g(\psi) T s(\psi) A \cos (\psi) A_{t t}\right)}{2 \pi Q^{-1}(B E R)}\right)^{\frac{1}{2}}
\end{gathered}
$$

Here, $A_{t t}$ is the specific coefficient for atmospheric attenuation and is given by the following equations for fog, snow and rain:

$$
\begin{gathered}
A_{t t, f o g}=e^{-\alpha f o g} \\
\alpha_{f o g}=\frac{3.912}{V}\left(\frac{\lambda}{\lambda_{0}}\right)^{-p}
\end{gathered}
$$


Where, $A_{t t, f o g}$ is the wavelength dependent specific attenuation due to fog, $\lambda$ is the wavelength in $\mathrm{nm}, V$ is the visibility range in kilometers $(\mathrm{km})$ and $p$ is the size distribution coefficient of scattering.

$$
A_{t, \text { rain }}=k R^{\alpha}
$$

Where, $A_{t t, \text { rain }}$ is the specific attenuation due to rain, $\mathrm{R}$ is the rain fall rate ( $\left.\mathrm{mm} / \mathrm{hr}\right), k$ and $\alpha$ are the parameters dependent on rain characteristics.

And,

$$
A_{t t, \text { snow }}=a S^{b}
$$

Where, $A_{t t \text {,snow }}$ is the specific attenuation due to snow $(\mathrm{dB} / \mathrm{km}), S$ is the snowfall rate $(\mathrm{mm} / \mathrm{hr})$, $a$ and $b$ are wavelength dependent parameters and take different numerical values for wet and dry snow. Figure 3.25-figure 3.28 shows the achievable distance for various intensities of fog, rain and snow compared to clear weather.

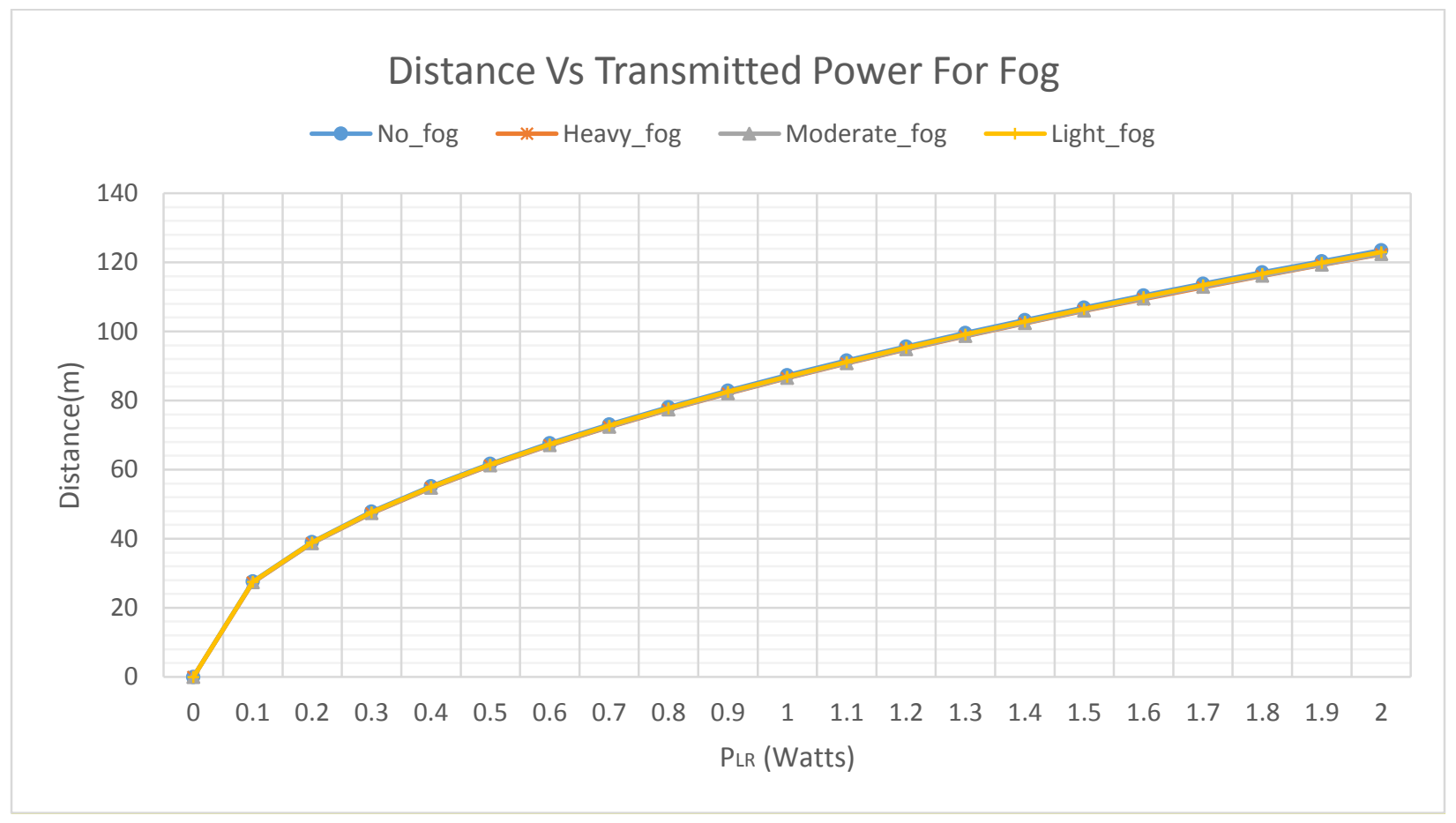

Figure 3.25 Distance vs transmitted power for various fog intensities 


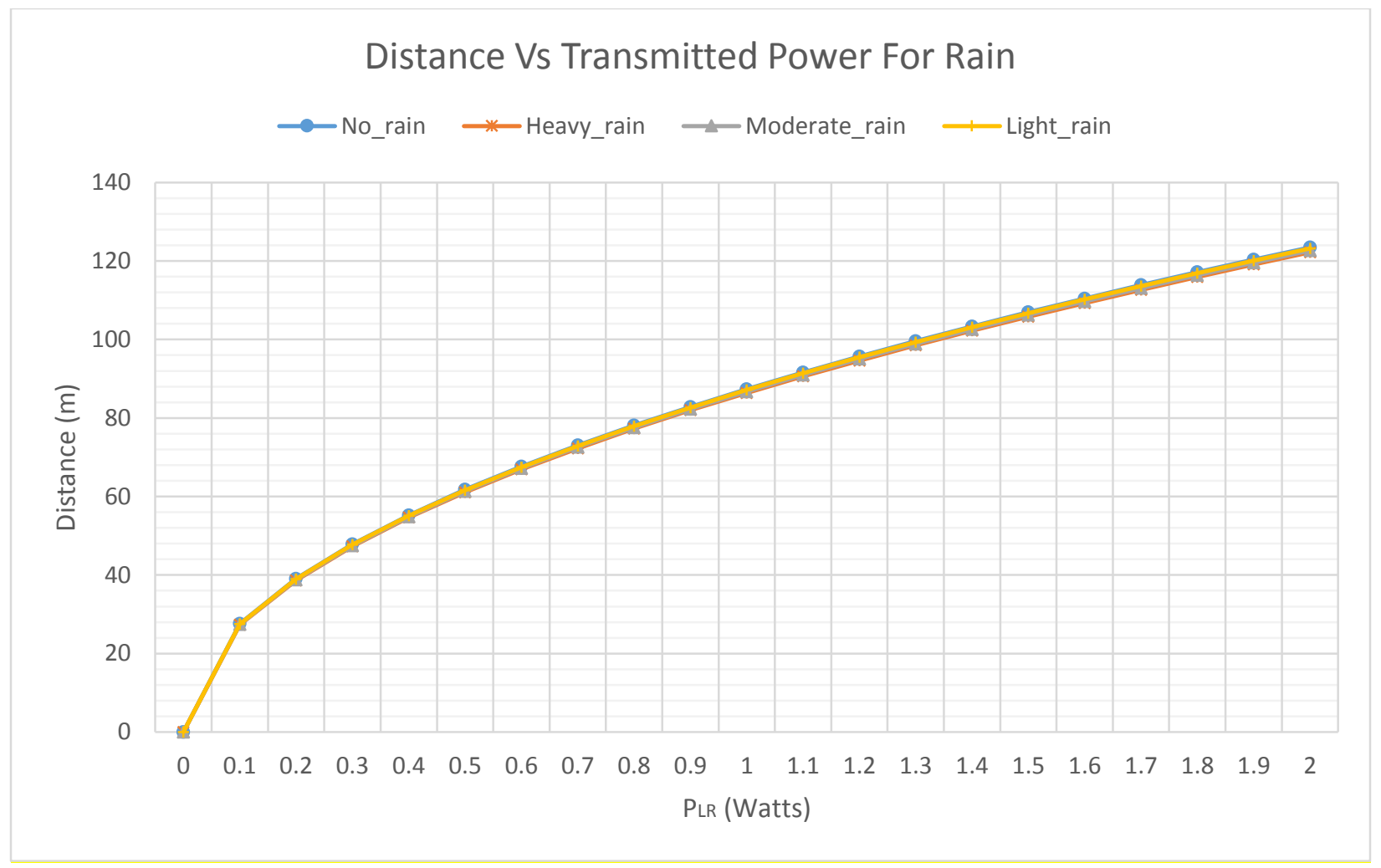

Figure 3.26 Distance vs transmitted power for various rain intensities 


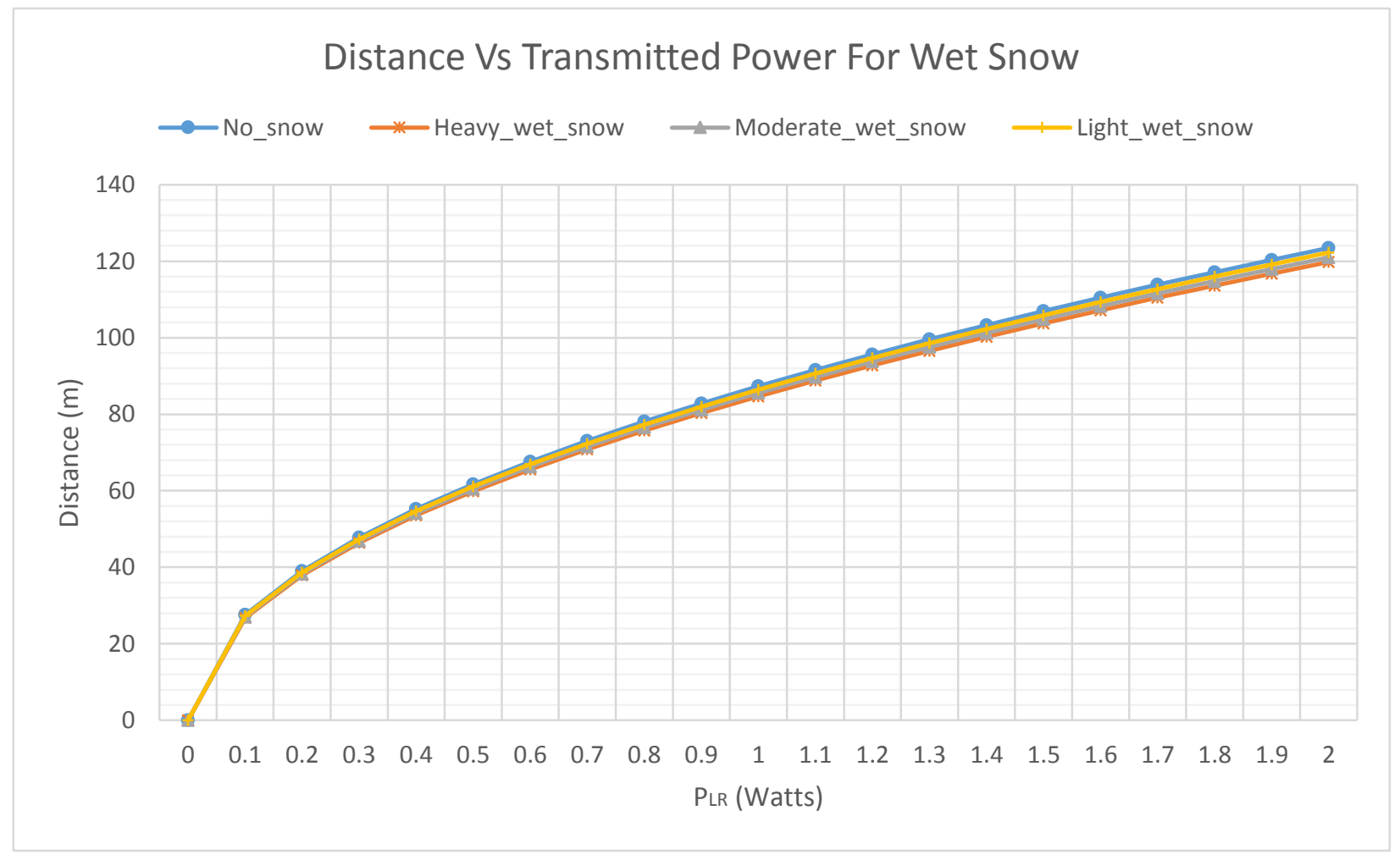

Figure 3.27 Distance vs transmitted power for various intensities of wet snow

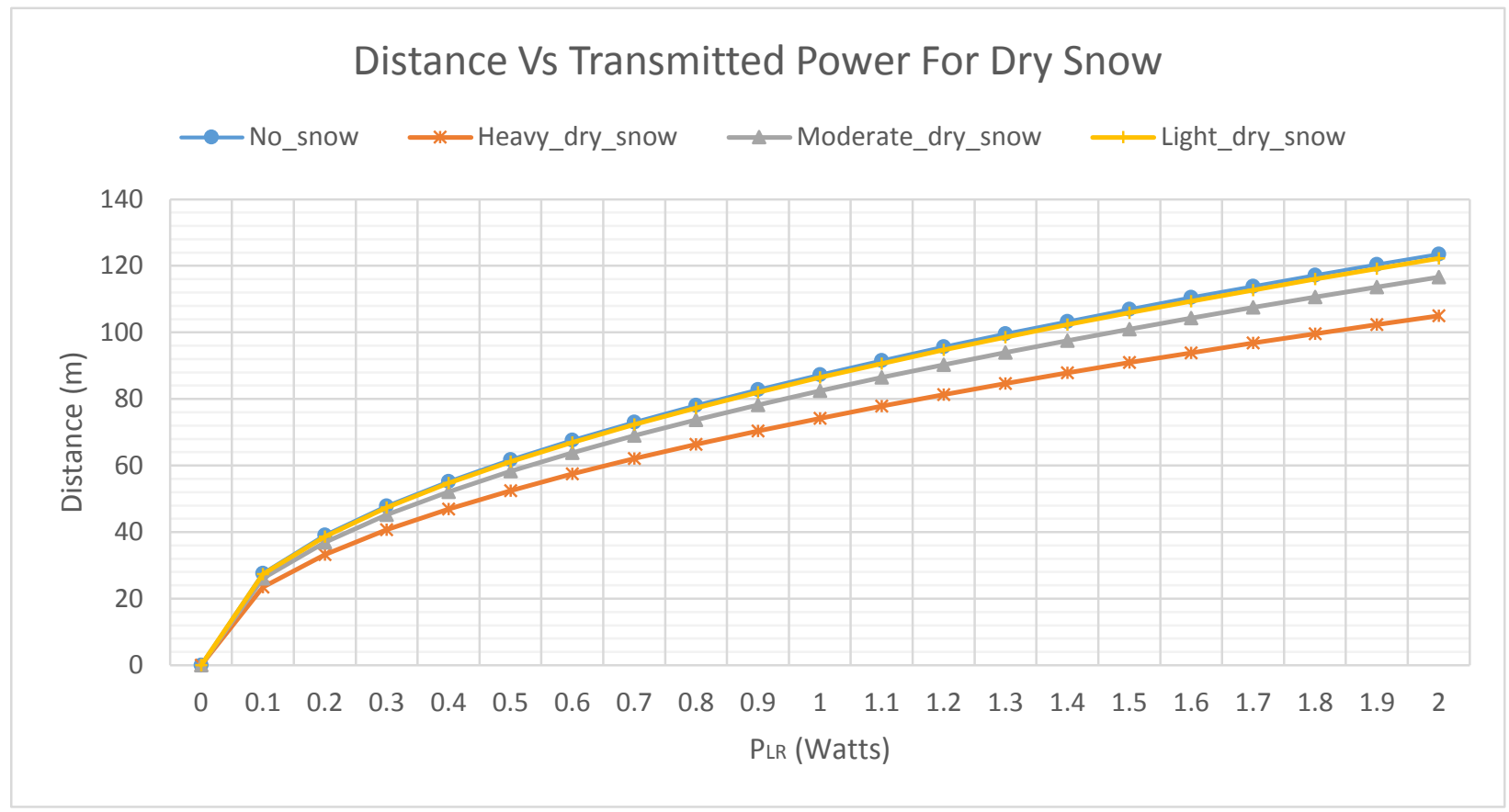

Figure 3.28 Distance vs transmitted power for various intensities of dry snow 
It can be concluded from the above graphs that transmitted power does not change much for the cases of fog, rain or wet snow. This is as expected. Recall that in section 3.3, it was evaluated that the loss or attenuation for distances up to $120 \mathrm{~m}$ was between 1-2.5 dB for different fog densities. The loss for even heavy rain was up to $1 \mathrm{~dB}$ and was around $2 \mathrm{dBs}$ for various intensities of wet snow. Hence, not much difference in power levels of transmitter LEDs are required. For the case of dry snow, the loss was much more significant, i.e around $10 \mathrm{~dB}$ for $120 \mathrm{~m}$. This is also evident in the above figure, which shows that for the same transmitted power, the maximum achievable distance in case of heavy dry snow reduces to $100 \mathrm{~m}$. This means that the transmission power will have to be boasted to maintain the same cell coverage area at required data rate in heavy dry snow. However, since we consider ultra small cells of around $50 \mathrm{~m}$ radius coverage area, the required boast in power increases from $0.4 \mathrm{~W}$ atts in clear weather to $0.56 \mathrm{~W}$ atts in heavy dry snow scenario i.e around $160 \mathrm{mWatts}$. Although, dependent on LED specification (forwarding voltage and drawn current), in general this is equivalent to using 1-2 more LEDs.

\subsubsection{Mapping of power transmission levels to traffic lights}

The institute of transportation engineers' (ITE) specification for LED circular traffic signals was published in 2005 under the name Vehicle Traffic Control Signal Heads Light Emitting Diode Circular Signal Supplement (VTCSH-LED)- Part 2 [BEH-05]. Manufacturers are required to adhere to the design guidelines and specifications provided in the standard in order to mark their modules "ITE compliant". VTSCH-LED provides mandatory specifications for LED vehicular traffic light system in terms of luminous intensity, electrical and physical requirements. There are two standard sizes for LED traffic lights: $200 \mathrm{~mm}$ and $300 \mathrm{~mm}$. In terms of luminous intensity, the intensity ratio between red, yellow and green (R:Y:G) for both $200 \mathrm{~mm}$ and $300 \mathrm{~mm}$ modules is $1: 2.5: 1.3$. Table 3.17 lists the intensity specification for $200 \mathrm{~mm}$ and $300 \mathrm{~mm}$ modules.

\begin{tabular}{|c|c|c|}
\hline Color & $200 \mathrm{~mm}$ & $300 \mathrm{~mm}$ \\
\hline Red & $165 \mathrm{~cd}$ & $365 \mathrm{~cd}$ \\
\hline Yellow & $410 \mathrm{~cd}$ & $910 \mathrm{~cd}$ \\
\hline Green & $215 \mathrm{~cd}$ & $475 \mathrm{~cd}$ \\
\hline
\end{tabular}

Table 3.17 Intensities of $200 \mathrm{~mm}$ and $300 \mathrm{~mm}$ modules near the center of the lane (-2.5 degrees, 0 degrees) 
The Canadian nominal and maximum wattage specifications for ITE compliant traffic light modules according to Toronto Transportation Authority [TTA-12] is presented in table 3.18.

\begin{tabular}{|c|c|c|c|c|}
\hline Color & Nominal.Wattage $(200 \mathrm{~mm})$ & $\begin{array}{c}\text { Max. } \\
\text { Wattage }(200 \mathrm{~mm})\end{array}$ & Nominal.Wattage(300mm) & $\begin{array}{c}\text { Maximum Wattage } \\
(300 \mathrm{~mm})\end{array}$ \\
\hline Red & 13 & 17 & 8 & 11 \\
\hline Yellow & 16 & 25 & 13 & 22 \\
\hline Green & 12 & 15 & 12 & 15 \\
\hline
\end{tabular}

Table 3.18 Transmitted Power specifications for traffic light according to [TTA-12]

Conventionally, LED based traffic lights consists of hundreds of LEDs arranged in an array configuration. It can be seen from above table and the required power calculations in previous section that for the proposed dual-link system with one narrow beam link and two high beam link, the total power required would be around 3.2 -3.4 watts. Hence, commercially available traffic light modules can easily support VLC within the acceptable transmitter power specification. It should be noted that in real life scenarios, LEDs installed in traffic lights, street lamp posts, vehicle headlights, taillights and additional fixtures (such as vehicle's roof) will all be used in conjunction to carry out VVLC.

\subsubsection{Link Budget Analysis of Outdoor mmWave System}

The standard Friss transmission equation [FRI-46] for mmWave gives the received power as function of distance $d$ as:

$$
P_{R X}=P_{T X} G_{T X} G_{R X}\left(\frac{\lambda}{4 \pi d}\right)^{2} \text { Att }
$$

where, $P_{R X}$ is the received power, $P_{T X}$ is the transmitted power, $G_{R X}$ and $G_{T X}$ are the gains of receiver and transmitter antenna, $\lambda$ is the wavelength and Att is the attenuation factor due to atmospheric absorption and other weather conditions. It should be noted that atmospheric loss due to oxygen absorption can be as high as $16 \mathrm{~dB} / \mathrm{km}$.

For link budget estimation in $\mathrm{dB}$, we use the following equation:

$$
P_{d B m(d)}^{R X}=E I R P_{d B m}-P_{L}(d)-O(d)-R(d)+G_{d B i}^{R X}
$$

Where, $P_{L}(d)$ is the free space path loss at the distance $d, O(d)$ is the attenuation due to oxygen absorption in the atmosphere, $G_{d B i}^{R X}$ is the gain on receiver antenna, $\mathrm{R}(\mathrm{d})$ is the rain attenuation and is replaced by $F(d)$ in case of fog and $S(d)$ in case of snow. 


$$
E I R P_{d B m}=G_{d b i}^{T X}+P_{d B m}^{T X}
$$

Although, various path loss models have been proposed in open literature, we adopt the one presented in [KIM-17] for outdoor mmWave systems.

The free-space path loss $\mathrm{P}_{\mathrm{L}}(\mathrm{d})$ is given for two different scenarios:

$$
P_{L}(d)=92.4+20 \log _{10} f_{G H z}+10 n \log d_{K m}
$$

For vehicle to vehicle (V2V) and,

$$
P_{L}(d)=82.02+10 n \log _{10}\left(\frac{d}{d_{o}}\right)
$$

For street canyon model or vehicle to infrastructure (device to base station). Here $\mathrm{d}_{0}$ is the reference distance in meters, and $n$ is the path loss coefficient and is equal to 2 for $\mathrm{V} 2 \mathrm{~V}$ model or 2.36 in street canyon model.

Since, we are considering V2I model, the value of free space path loss over a distance of 200m is calculated to be $119.82 \mathrm{dBm}$. Table 3.19 list the typical link budget for the scenario of $500 \mathrm{Mb} / \mathrm{s}$ transmission rate at $1.5 \mathrm{GHz}$ channel bandwidth and $60 \mathrm{GHz}$ operating frequency. Assuming QPSK modulation, the required SNR for an un-coded BER of around $10^{-7}$ is $13 \mathrm{~dB}$ [KUM-15]. The minimum received power is given as following [MAD-14]:

$$
P_{d B m}^{R X}(\min )=\left(\frac{E_{b}}{N_{o}}\right)_{d B}+\mathrm{R}_{d B}-174+N_{F}
$$

Where, $\mathrm{R}$ is the target bit rate, $-174 \mathrm{dBm} / \mathrm{Hz}$ is the thermal noise at $290 \mathrm{~K}$ and $\mathrm{N}_{\mathrm{F}}$ is the receiver noise figure. The equivalent isotropically radiated power (EIRP) value calculated from equation (3.71) is smaller than the maximum EIRP identified for the BS $(\sim 78 \mathrm{dBm})$ and slightly higher than the maximum EIRP identified for the $\mathrm{MN}(\sim 43 \mathrm{dBm})$ in the mmWave $5 \mathrm{G}$ system design guide by FCC [KUM-15][HUO-17]. This means that for the transmission from $\mathrm{MN}$ to BS, receiver gain will have to be increased in order to maintain the throughput.

\begin{tabular}{|c|c|}
\hline Link Budget Variables & 25.8 \\
\hline Transmit power (dBm) & 20 \\
\hline Transmit antenna gain (dBi) & 60 \\
\hline Carrier frequency (GHz) & 200 \\
\hline Distance (m) & 119.82 \\
\hline Free space propagation loss (dB) & 10 \\
\hline
\end{tabular}




\begin{tabular}{|c|c|}
\hline Receive antenna gain $(\mathrm{dB})$ & 20 \\
\hline Received power $(\mathrm{dBm})$ & -64.02 \\
\hline Bandwidth $(\mathrm{GHz})$ & 1.5 \\
\hline Thermal noise PSD $(\mathrm{dBm} / \mathrm{Hz})$ & -174 \\
\hline Noise figure & 10 \\
\hline Thermal noise $(\mathrm{dBm})$ & -77.02 \\
\hline SNR (dB) & 13 \\
\hline Implementation loss (dB) & 5 \\
\hline Data Rate (Mbps) & 500 \\
\hline
\end{tabular}

Table 3.19 Link Budget Analysis for mmWave outdoor system.

One of the significant challenge for meeting the proposed link budget requirements is to be able to overcome the propagation and attenuation losses by providing adequate beamforming gain. Beamforming with directed narrow beams providing 360 degree coverage in mmWave systems can boast system throughput and enables spatial reuse with low interference probability. However, this requires careful consideration for antenna selection and configuration decisions as base station and mobile users have different requirements. Base stations are generally divided into sectors. In order to provide complete coverage, multiples antennas with multiple elements per sector with efficient beam steering is to be used. The elevation and azimuthal tilt angle should be selected such as maximum power is delivered into the targeted direction with minimum leakage to the neighboring sectors.

\subsubsection{Conclusive Commentary}

This chapter provides an overview of the propagation behaviour of both VL and mmWave in outdoor environments through analytical models. The analysis also included weather dependent signal attenuation factors. Table 3.20 provides comparative summary of the two technologies in context of $5 \mathrm{G}$ vehicular networks under proposed scenario.

\begin{tabular}{|l|l|l|}
\hline & VL Communication & mm \\
\hline Case study & $\begin{array}{l}\text { Cell area of } 200 \mathrm{~m} \text { covered by } 4 \text { pico- } \\
\text { cells covering } 50 \mathrm{~m} \text { each. Dual beam } \\
\text { links with wide-beam supporting low } \\
\text { data rate and narrow beam supporting } \\
\text { high data rates. Each mini-cell is }\end{array}$ & $\begin{array}{l}\text { Cell area of 200m with one base station. } \\
\text { Base station is sectored with multiple } \\
\text { antennas covering each sector. } \\
\text { Each antenna has multiple antenna } \\
\text { elements and beam-steering for } \\
\text { complete coverage. }\end{array}$ \\
\hline
\end{tabular}




\begin{tabular}{|l|l|l|}
\hline & $\begin{array}{l}\text { covered by one low rate and two high } \\
\text { rate links. }\end{array}$ & \\
\hline Max. attenuation due to fog $(\mathrm{dB})$ & 3.9 & 3.1 \\
\hline Max. attenuation due to rain $(\mathrm{dB})$ & 1.84 & 2.5 \\
\hline Max. attenuation due to snow $(\mathrm{dB})$ & 16 & 4.5 \\
\hline Max data rate & $500 \mathrm{Mbps}$ & $500 \mathrm{Mbps}$ \\
\hline Transmission links & LOS & LOS \\
\hline Sensitivity to link misalignment & Moderate & High \\
\hline Required Transmission Power & 3.1 Watts for each pico-cell (12.4 Watts & 40 Watts \\
\hline $\begin{array}{l}\text { Is transmission power within } \\
\text { standardized range? }\end{array}$ & Yes $200 \mathrm{~m})$ & Yes for downlink, slightly higher for \\
\hline Installation and maintenance cost & Low to Moderate & uplink. \\
\hline
\end{tabular}

Table 3.20: Comparative summary of mmWave and VLC for $5 G$ outdoor system under proposed scenario

It can be concluded that for small cell sizes that would be inherent in $5 \mathrm{G}$ networks, both mmWave and VL show similar attenuation profiles and appears promising for practical implementation of LOS outdoor systems. However, small cell implementation of mmWave would be more challenging as there exists a gap between the existing mmWave products and promised commercial mmWave network [ZHA-16]. Successful implementation of mmWave requires advanced antenna designs and configurations such as phased array, horn antenna array, patch antenna array and modular antenna array. Each of these configurations further requires complex beamforming, tracking and beam steering capabilities. Additionally, each antenna array is composed of multiple antenna element that should be supported by RF circuitry such as analog to digital converters (ADC) and phase shifters. Use of directional antennas are strongly recommended to improve data rate, since it reduces Doppler spread and delay spread, but it increases the beam alignment sensitivity of the system. Although, several analytical and simulation evaluations have affirmed the viability of mmWave communication, extensive measurement campaigns covering different environmental scenarios are still missing in open literature. In a ray tracing based simulation model for outdoor mmWave networks, proposed in [LAR-13], it was found that increasing the density of base stations from $50 / \mathrm{km}^{2}$ to $133 / \mathrm{km}^{2}$, resulted in 56 times improvement (from $25 \mathrm{Mbps}$ to $1400 \mathrm{Mbps}$ ) in the cell edge rates. This further endorses the importance of density in mmWave networks for enhancing throughput. 
However, this also translates to increased cost of implementation and maintenance of mmWave networks. Another important factor to consider is the generation of mmWave signals. Generating mmWave signals in electrical domain is challenging due to the bandwidth limitations faced by existing electronic components. Hence techniques for generation of mmWave signal in optical domain are dominantly present in the open literature. [KIM-05] utilizes a dual mode lasers for generating mmWave. In this work, dual lasers with different wavelengths are locked by injection phase locking to generate a $25 \mathrm{GHz}$ mmWave carrier signal. [LI-05][FUK-01] adopts optical phase locking to generate mmWave signals. Direct intensity modulation uses mmWave carrier signals to directly modulate high-speed lasers. [HAR-03] utilizes distributed feedback laser (DFB) to directly modulate OFDM signal. [LEC-09] presented direct modulation of a mode-locked Fabry-Pérot laser with carrier frequency of $54.8 \mathrm{GHz}$. However, this approach is limited by the modulation bandwidth of lasers. Instead of direct modulation, external modulation has also been widely reported. In this approach, lasers operate in continuous wave $(\mathrm{CW})$ mode, whereas external modulators such as Mach-Zehnder modulator (MZM) or electro absorption modulator (EAM) are used for signal modulation. "By biasing the MZM at half-wave voltage of MZM, the optical carrier at centre wavelength is suppressed. The beat of upper and lower 1st side-modes will generate the required mm-wave signal with frequency twice to that of the mmwave signal applied to MZM [SIN-12]". [JIA-06] [MOH-08][LIN-09] report optical carrier suppression (OCS) external modulation over DWDM signals. Another approach is upconversion of Intermediate Frequency (IF) band to generate mmWave. In this technique, the IF band signal is optically instead of RF modulated and is sent over optical fiber. The conversion of IF to RF takes place in the BS utilizing mmWave carrier modulator. The process of electrical frequency conversion from IF to mmWave involves frequency mixers and mmWave local oscillator, which adds to the cost of BS [SMI-98][KIT-99] [PAR-07][LEC-09]. In addition optical heterodyning [LI-10] [GEO-95], non-linear techniques such as four-wave mixing [SHI10] [WAN-06] and stimulated Brillion scattering [LI-13][SCH-06] are also employed for the generation of mmWave. [MAR-14] employs coherent heterodyning by using optical frequency comb that is generated by using gain switching distributed feedback laser. However, each of these techniques have limitations and requires a plethora of lasers, photodiodes, filters, amplifiers, multiplexers, de-multiplexers and polarisation controllers. Hence, we are confident that all these technologies will be expensive compared to VL systems. 
VL systems on the other hand can be implemented partly with existing infrastructure such as traffic lights, lamp posts and vehicle headlights and taillights. Although, a completely operational implementation of VL systems would require additional LED transmitters and PDs, it has a benefit of reduced implementation cost as a single LED costs around 22-40 cents. 


\section{Chapter 4: HIERARCHICAL FORWARDING CHAIN BASED MPLS SCHEME FOR 5G}

\section{ULTRA DENSE VEHICULAR NETWORKS}

\subsection{Overview}

From the review of the existing versions of hierarchical MPLS based micro-mobility architecture presented in Chapter4, we conclude that the existing hierarchical models are not appropriate for VVLC networks. As part of future ultra dense 5G networks with multiple small cells and a highspeed vehicular environment where inherently the illumination area is very small, existing hierarchical models do not suffice. Adopting them as-is for the 5G VVLC network would again require extensive handoff messages and notifications being sent to GW. Additionally, the existing MPLS based schemes have limited capabilities to support QoS. Existing schemes such as V-MPLS and MTE-MPLS provide DiffServ based QoS support. However, since DiffServ models are class-based and have a pre-configured PHB, it cannot provide delay and delivery guarantees. The ability to provide delay and delivery guarantees is critical in $5 \mathrm{G}$ vehicular networks. $5 \mathrm{G}$ vehicular networks rely on exchange of precise and timely data between vehicles and infrastructure to support safety critical applications. In this scenario, IntServ based MPLS models are better fit. IntServ architecture is flow based and it is possible to configure LSPs with latency and bandwidth guarantees. Hence, a forwarding chain based hierarchical MPLS scheme with IntServ based QoS model is proposed for 5G VVLC networks. The proposed hierarchical MPLS based micro-mobile architecture is shown in figure 4.1. 


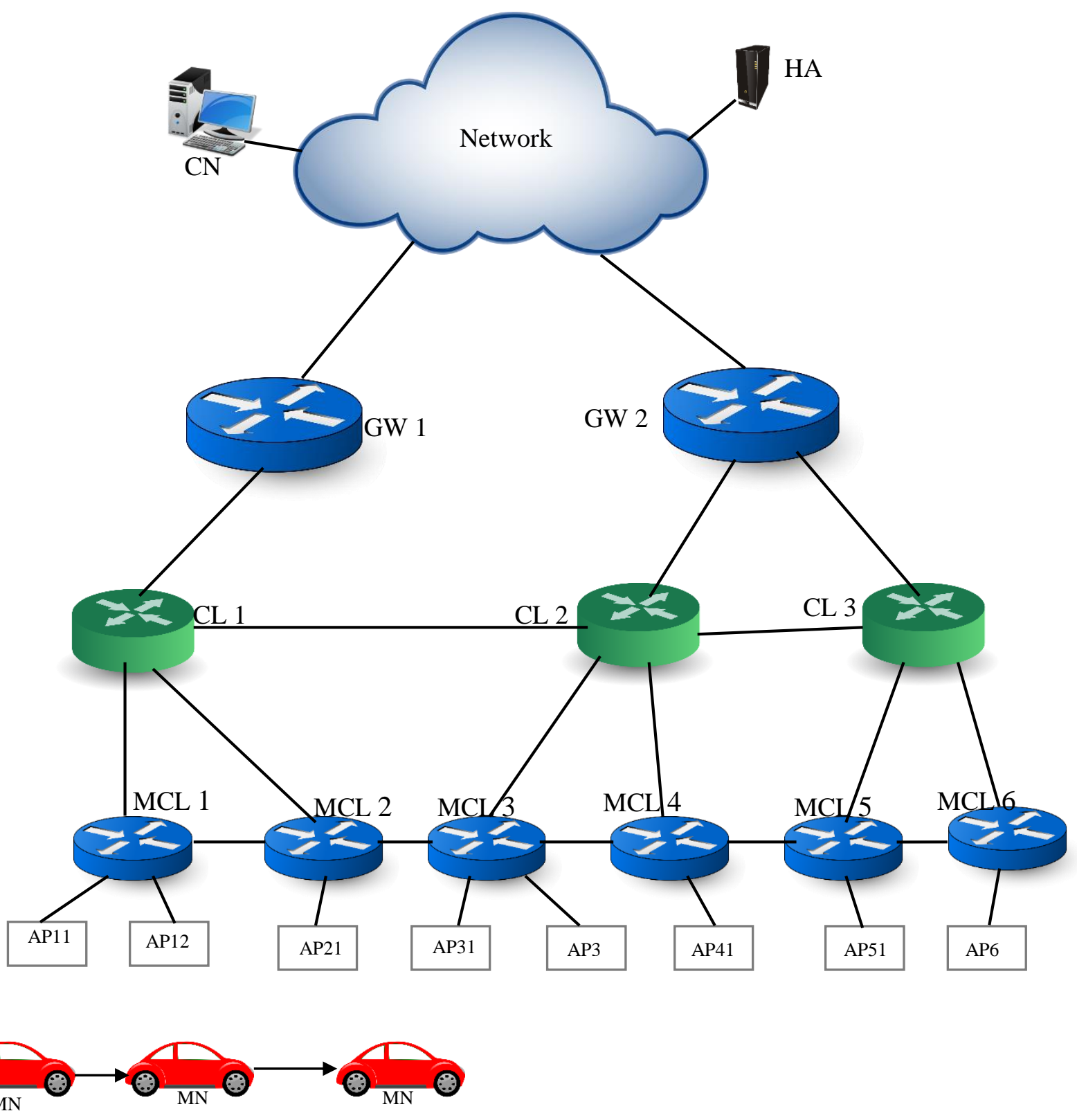

Figure 4.1 Architecture of the Micro Mobile Hierarchical MPLS Access Network

We will describe the Hierarchical FC based MPLS architecture in this section. The ultimate aim for the design of this architecture is to minimize the service disruption during subnet change, reduce registration cost (in terms of message length and link usage) and frequency of registration as well as maintain seamless connectivity during handoff. 


\subsection{Architecture for Proposed Hierarchical Forwarding Chain based MPLS scheme for 5G Vehicular Networks}

Our proposed architecture adopts hierarchical MPLS domain-based approach with preestablished LSPs that are based on the IntServ model and hence can provide data delivery and latency guarantees. The architecture is based on three-level hierarchy. At the top of each domain reside MPLS capable routers acing as the gateways $(\mathrm{GW})$. These are also the ingress/egress label edge routers (LER) for that domain. An MPLS access network exists between the GWs and the APs that serves as point of attachment for the mobile nodes. Each subnet is composed of multiple APs that are connected to one Micro-cell Leader. The Micro-cell Leader acts as a subnet-level point of attachment and provides a local care of address (LCoA) for the registered MNs in that subnet. The functionality of Micro-cell Leader is similar to the FA. At the intermediate level are the Cell Leaders (CL). CL are crossover label switching routers (LSR) capable of performing handoff locally between neighboring MCL and forward the packets from $\mathrm{GW}$ to the $\mathrm{MN}$ through appropriate CL-> AP connection. Every CL is connected to several MCLs and MCLs are connected to multiple APs.

It should be noted that each CL is connected to at least one $\mathrm{GW}$, with some CLs connected to multiple GWs. The GWs, CLs and MCLs are all connected via pre-established traffic engineered (TE-LSP) tunnels. These TE-LSP tunnels are similar in concept as forwarding adjacencies in GMPLS. The TE-LSP tunnel acts as a bigger LSP, which has the capability of carrying multiple aggregated flows inside. The LABEL REQUEST, LABEL, SESSION ATTRIBUTES, RECORD_ROUTE and EXPLICIT_ROUTE_OBJECT extensions to RSVP signalling mechanism provides flow-based QoS support by propagating bindings between flows and labels. The values of the QoS traffic parameters TLV (type, length, value) are extracted from the FLOWSPEC field of the RESV message. The TE-LSP tunnel must support the combined traffic parameters of the aggregated flows. The flow reservations can be of three different types: Fixed filter style (FF), Wildcard filter style (WF) and Shared explicit style (SE).

A brief definition of these extensions and types of reservations are provided below: 
- $\quad$ LABEL REQUEST: Label request object is created by the first MPLS-enabled router on the LSP tunnel path and forwarded in the PATH message. The LABEL REQUEST message specifies that a label binding for this LSP tunnel path is requested.

- $\quad$ LABEL: Upon receiving the label request object in the PATH message, intermediate and receiver nodes respond by appending a LABEL object in the RESV message. The RESV message is sent back upstream to the sender node, traversing the path taken by the PATH message. Each node that receives the LABEL object in the RESV message uses that label value to send outgoing packets on that LSP tunnel.

- EXPLICIT ROUTE OBJECT: Explicit route object (ERO) is inserted in the PATH message by the sender node if the sender node is aware of a route that meets the QoS requirements of a specific session or utilizes the network resources efficiently. ERO object explicitly identifies the router nodes through which the path should be established.

- RECORD ROUTE OBJECT: Record route object (RRO) is also created by the sender node. The benefit of using RRO is that the sender becomes aware of the route traversed by the PATH message. This is useful for loop detection.

- SESSION ATTRIBUTE: The session attribute object is created by the sender node for session identification and specifications such as setup and hold priorities.

- FIXED FILTER STYLE: In fixed filter reservation style a separate reservation is created for each sender. The total bandwidth reserved on the link is the sum of individually reserved bandwidths. As the reservations are separate from each other, an exclusive label is assigned to each sender.

- WILDCARD FILTER STYLE: In wildcard filter style reservation, a single reservation is used for multiple senders of the same session to the same receiver node. The total link reservation remains the same. The same label value is used by all senders of the same 
session. This reservation style should be utilized for the applications in which senders are using the link periodically and not continuously.

- $\quad$ SHARED EXPLICIT STYLE: In shared explicit style reservation, the receiver explicitly defines the senders included in a reservation. For all of the listed senders, there is a single reservation. The labels can be assigned per sender, generating separate LSPs on the same link, or a common label for multipoint to point LSPs. However, the common label can only be assigned when ERO is not used or have identical values for each sender.

For this work, we assumed the shared explicit style of reservation.

\subsubsection{Network Initialization}

The architecture uses pre-established TE tunnels for packet forwarding between GW, CLs, and MCLs. At the start-up, each MCL is pre-configured with the GW's address, the list of neighboring MCLs, the list of APs that are connected to it and the IP address of CL that is in precedence in the hierarchical chain. Similarly, each CL is pre-configured with the list of neighboring CLs, the list of MCLs that will be under its succession and the GW's address. The control plane can dynamically update these lists by utilizing periodic updates from link state protocols during network operation. The TE LSP tunnels' establishment is triggered by the downstream nodes. These LSPs are assumed to be established using RSVP-TE or CR-LDP registration messages with QoS extensions as discussed in earlier section. The TE LSPs are established between connecting router nodes i.e. GWs-> CLs and CLs->MCLs. Depending upon available resources, these tunnels may be bi-directional [DUB-03] or two unidirectional TELSPs in the reverse direction between the connecting nodes providing path for packet forwarding in both directions. The bi-directional LSPs have the benefit of being more efficient compared to unidirectional LSPs, as they require the same number of messages for setup and maintenance. "Implementation experience shows that the memory requirement for a bi-directional LSP when compared to two unidirectional LSPs is lower as fewer states need to be maintained for one bidirectional LSP when compared to two unidirectional LSPs" [DUB-03]. Unidirectional LSPs, on the other hand can be beneficial when connectivity between sender and receiver nodes in both directions is required and there are not enough resources available on the same path in both upstream and downstream directions. 


\subsubsection{Registration Procedure}

Once the MN powers up in a subnet domain, it receives the subnet identification advertisement from the AP. This advertisement contains the IP address of the Micro-cell Leader and the GW. The MN sends a registration request to the Micro-cell Leader. In this scheme, the MN also buffers the IP addresses of the visited subnets. The Micro-cell Leader adds the MN's address in the MIP visitor list and forwards the request to the $\mathrm{GW}$. Upon receiving the registration request from the MN, the GW also adds the MN's address in the MIP visitor list and forwards the request to the $\mathrm{HA}$ of the mobile node. Once the $\mathrm{GW}$ receives a registration response from the home agent, it creates an entry for the MN at the FEC table and links the entry to the corresponding TE tunnel towards the CL. The GW afterwards, only maintains the location information of that $\mathrm{MH}$ in the granularity of the CL. The registration request is sent again to GW only if the MN is performing handoff between two CLs. The initial registration and LSP set up process is presented in figure 4.2. Similarly, the MCL and the CL also create a FEC entry for the $\mathrm{MN}$ in their forwarding tables and links the entry to the appropriate TE tunnels. The MCL finally forwards the registration response to the MN through AP. This MCL provides the LCoA for the MN and is called anchored MCL of the MN, whereas GW provides the GCoA for the MN. All of

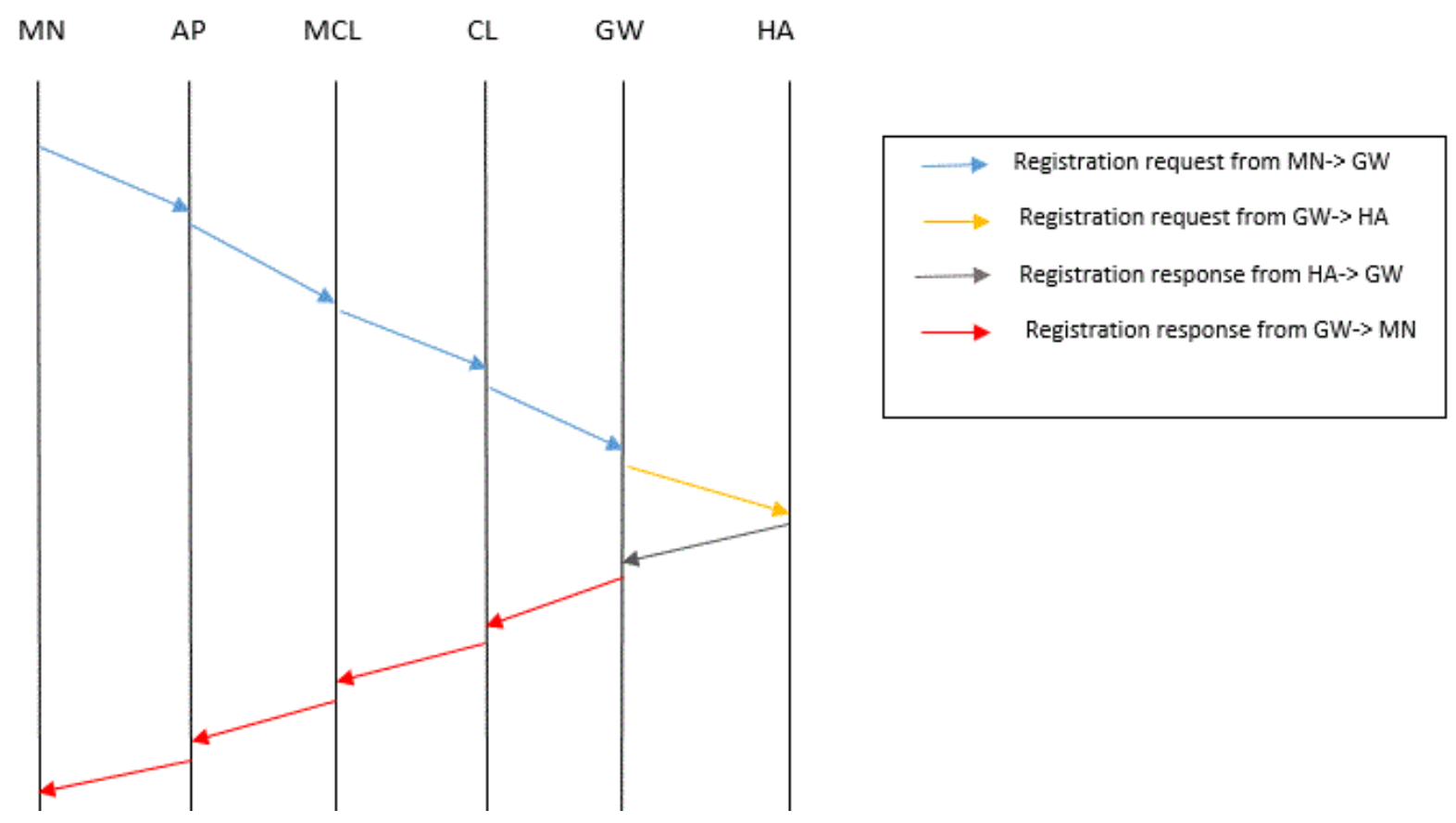

Figure 4.2 Initial Registration of MN. 
the MN that are connected to the same MCL uses the same tunnel for packet forwarding based on SE reservation style.

\subsubsection{Mobility Management and Seamless Handoff}

The APs are MPLS enabled switches working at layer 2.5. These APs are capable of inserting shim headers on the data packets but do not participate in TE-LSP creation. The LSPs are created between GW(s) and MCLs.

At the time of network initialization, each MCL broadcasts a neighborhood label-mapping table (NLMP) to its associated APs. This table contains the MAC IDs of the neighboring subnets (MCLs) and the label mapping corresponding to the pre-established TE tunnel. The APs are named as AP $a b$ where $a$ identifies the subnet and $b$ identifies the AP sequence. Based on the neighborhood-mapping table, the APs compile their own forwarding tables. Due to use of preestablished LSPs between subnets, the forwarding table has one more entry corresponding to next label that has to be used for creating the MPLS label stack. Consider table 4.1, which corresponds to forwarding table at AP21.

\begin{tabular}{|l|l|l|l|l|}
\hline MAC ID & Outgoing Interface & Outgoing Label & Operation & Next label \\
\hline MCL2 & 4 & E & Push & - \\
\hline MCL1 & 3 & F & Push/Set Stack bit & E \\
\hline
\end{tabular}

Table 4.1 Forwarding table at AP 21.

Now consider a MN, which is already registered with MCL1, initiating uplink communication with a $\mathrm{CN}$ in another domain. The work assumes constant data arrival rate both from $\mathrm{MN}$ to $\mathrm{CN}$ and from $\mathrm{CN}$ to $\mathrm{MN}$ as the TE tunnels are based on the IntServ model, the packets entering the MPLS domain would be transmitted according to the token bucket algorithm, resulting in a constant rate in non-saturated traffic conditions. The MN will send packets directly to the $\mathrm{CN}$ using its CoA. When the packets from the MN arrives at AP1n, the AP inserts a shim header with appropriate label. As long as MN is corresponding through AP 11-AP1n that are associated with MCL1, the forwarding operation is pretty straightforward. AP receives the packet, encapsulates with a label and forwards it to the outgoing port. When the packet is received at 
MCL1, MCL1 swaps the label and forwards it towards GW through CL. Whenever the MN arrives under a new AP associated with MCL1, the AP sends an UPDATE_PATH_REQ message to MCL1 so that MCL1 can update the tables and direct the downlink packets to the current AP. The APs periodically send the geographical co-ordinates of the subnet MCL1, through layer 2 beacons to the $\mathrm{MN}$ in their coverage area.

When the MN reaches the boundary of MCL1, it sends an ACT_BUF message to MCL1 through the last AP of the subnet. Upon receiving the message, MCL1 starts buffering the downlink packets destined for the MN. At the same time, MN starts buffering the uplink packets. The required buffer size is evaluated based on the velocity of vehicles, the data arrival rate and the distance between the APs and is given by the following expression:

$$
B=\left[d / V+2\left(s l_{M N->A P}+s l_{A P->M C L}\right)\right] R
$$

Where,

$\mathrm{B}$ is the size of buffered data

$\mathrm{R}$ is the data arrival rate (bps),

$\mathrm{d}$ is the distance between APs ( $\mathrm{d}=0$ for overlapping cells), $\mathrm{V}$ is the velocity of $\mathrm{MN}$, $\mathrm{sl}$ MN->AP and sl AP->MCL are the signalling latencies between mobile node and AP and AP and MCL respectively.

Now when the MN moves under AP21 which is directly associated with MCL2, MN detects the new subnet through network advertisement message. MN sends an UPDATE_PATH_REQ message to the new MCL2 with the CoA of MCL1 to establish a forwarding chain from MCL1 to MCL2. MCL2 sends an UPDATE_PATH_REQ message to MCL1 with MN's address. This message also indicates to MCL1 to forward the buffered packets for MN to MCL2. When MCL1 receives the UPDATE_PATH_REQUEST, it updates the LFIB table by linking MN's FEC to pre-established TE LSP between MCL1 and MCL2 and sends back the UPDATE_PATH_RESP along with buffered packets. When MCL2 receives the UPDATE_PATH_RESP from MCL1, it forwards the UPDATE_PATH_RESP to MN along with buffered packets received from MCL1. MCL2 also broadcast the MN's MAC address and the anchored MCL for the MN to the APs directly associated to MCL2 so that they can append the appropriate label stack to the uplink packets originating from $\mathrm{MN}$. The timing sequence is indicated in figure 4.3. 


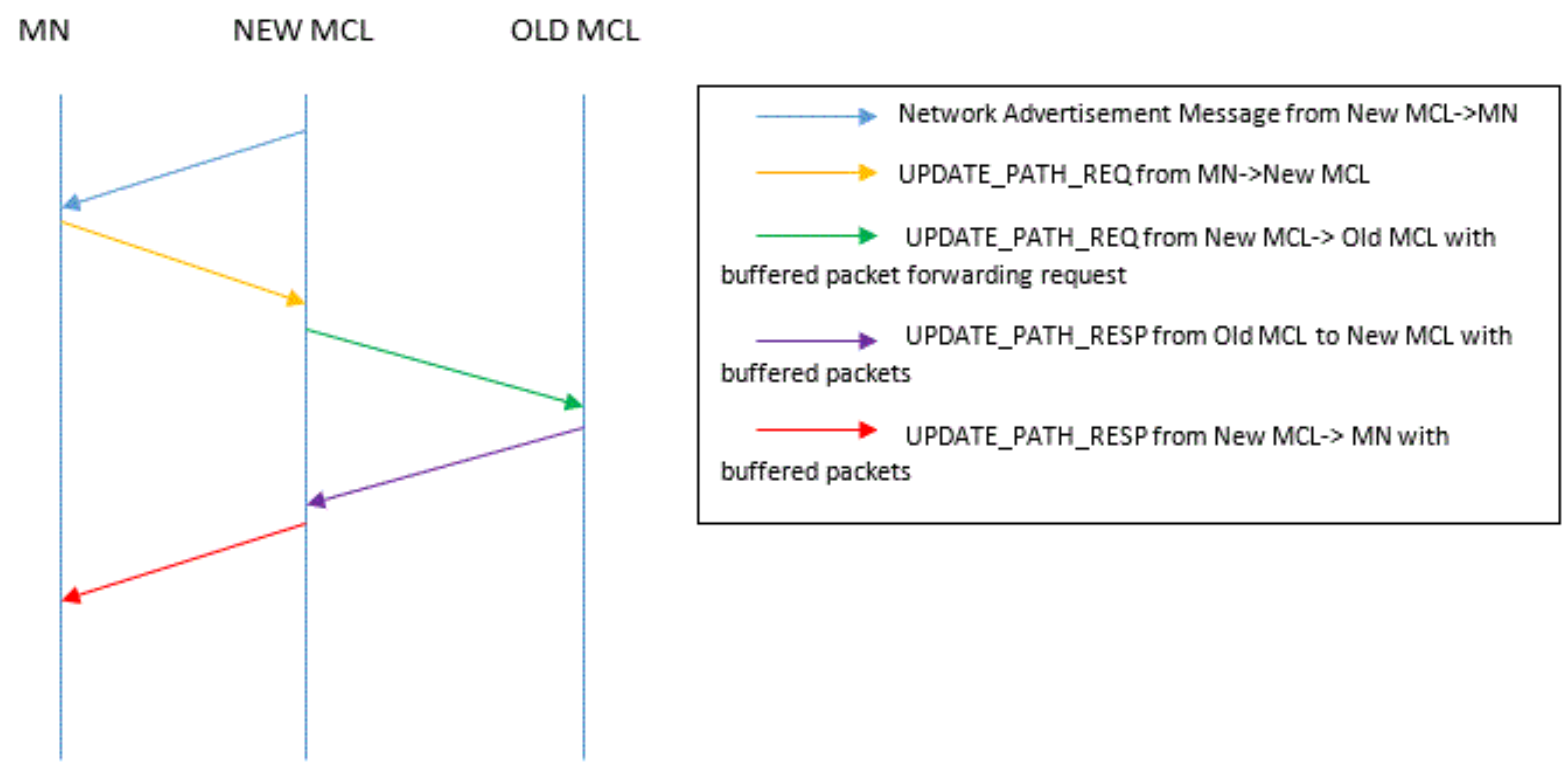

Figure 4.3 Timing Sequence for Intra MCL forwarding chain extension

When AP21 receives the packet from MN, it looks up in the forwarding table. It pushes two stacked labels on the packets and set the stack bit to 0 . The top label $\mathrm{E}$ corresponds to the link from AP21 to MCL2 and the label beneath F points to the already establish hierarchical tunnel between MCL2 ->MCL1. When MCL2 receives the MPLS packet from AP it will pop off the top label and forwards it to the MCL1 tunnel. Once the packet reaches MCL1, it is forwarded through CL1 to GW like packets from other MN being received from APs that are directly associated with MCL1. This tunnel-based forwarding continues until the latency-based maximum hop threshold $\mathrm{T}$, for $\mathrm{MN}$ is reached. After which, the $\mathrm{MN}$ carries out new registration with $\mathrm{MCL}(T+1)$ by sending a registration request instead of UPDATE_PATH_REQ. The MN will receive a registration response from CL or GW depending upon if the MCL's are under same or different CLs. Figure $4.4 \&$ figure 4.5 shows the timing sequence for these scenarios. 
MN OLDMCL NEW MCL CL
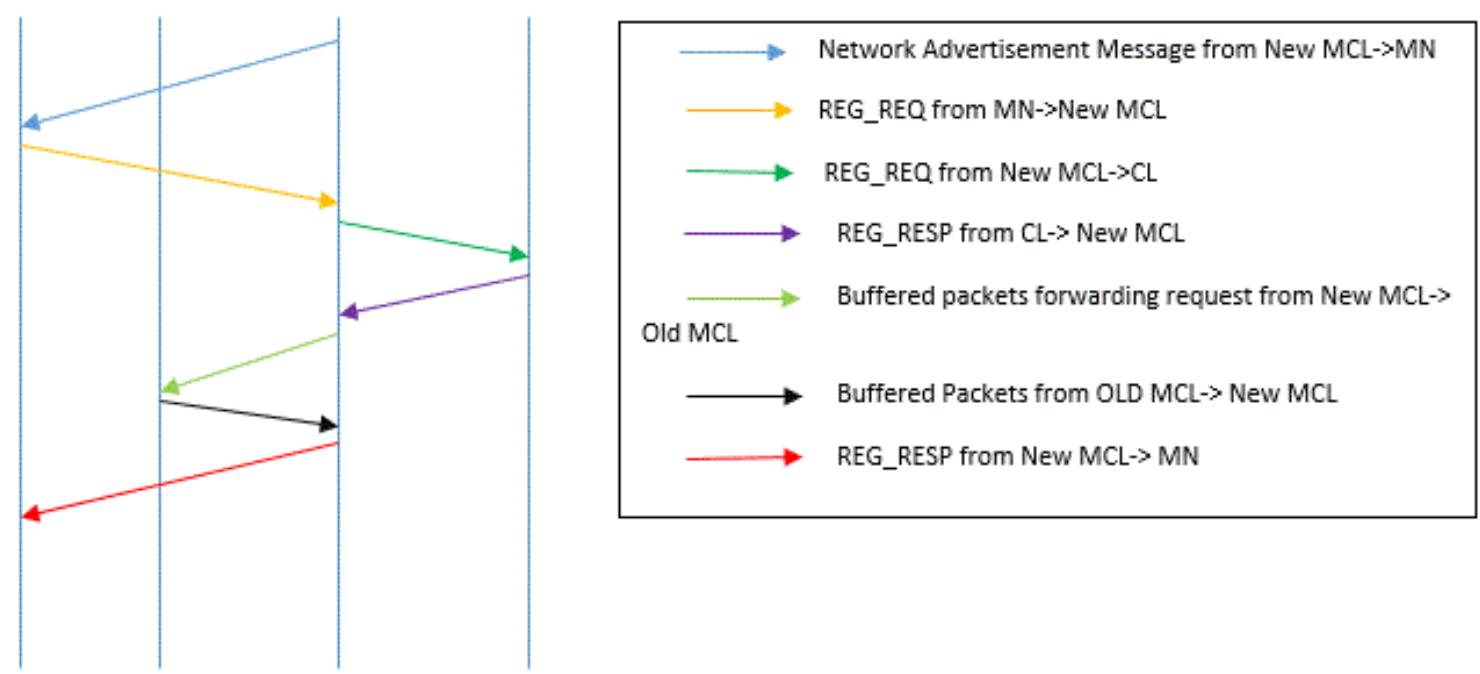

Figure 4.4 Timing Sequence for Inter MCL handoff after the forwarding chain threshold is reached

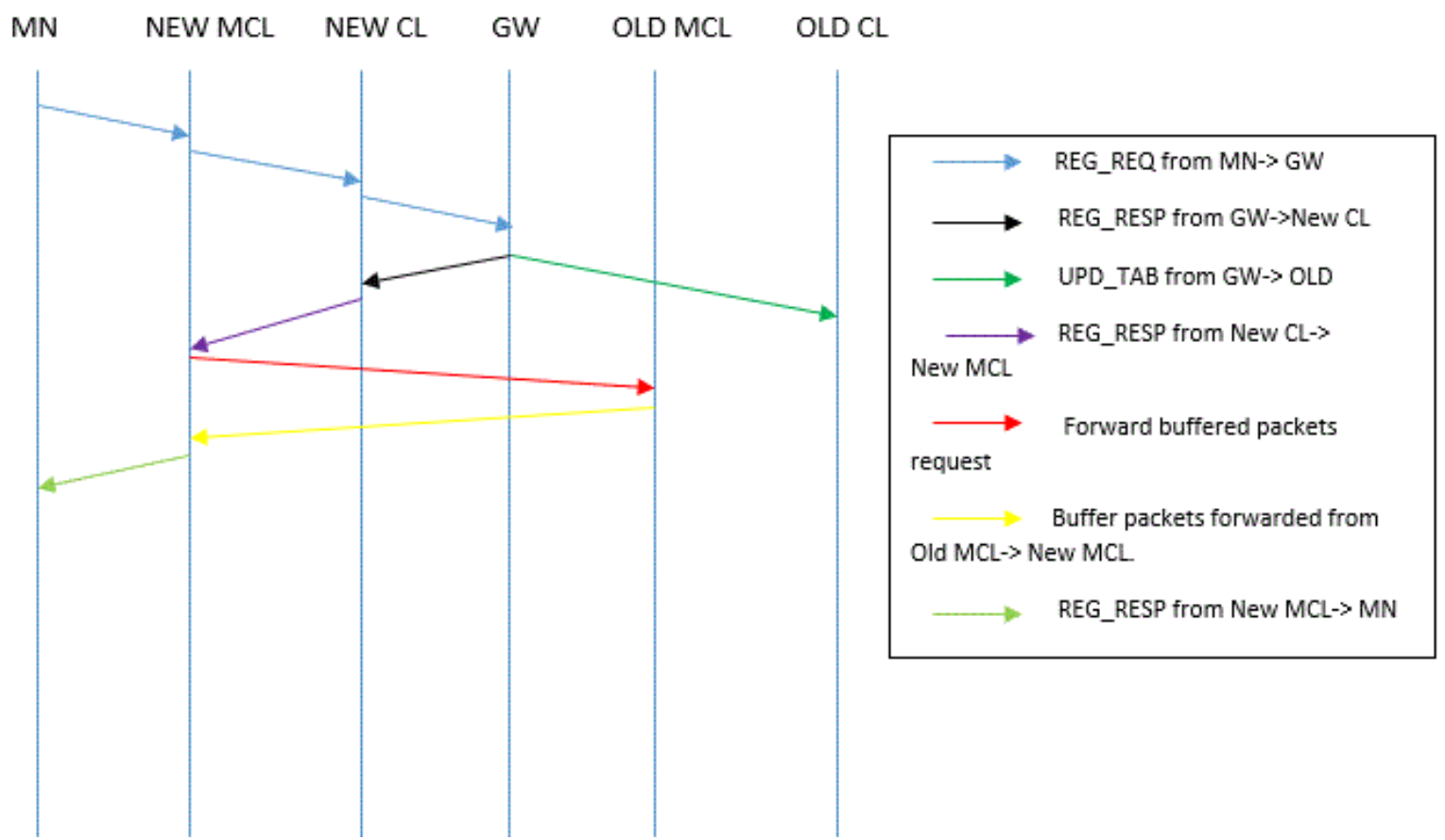

Figure 4.5 Timing Sequence for Inter CL handoff after the forwarding chain threshold is reached

If the $\mathrm{CN}$ initiates communication with the $\mathrm{MN}$, it initially sends packets destined for $\mathrm{MN}$ to the HA of the MN from where they are forwarded to the MN through GW. The MN sends its 
binding information to the $\mathrm{CN}$ and the communication is directly established. We assume that the $\mathrm{MN}$ is under MCL1, the packets are forwarded through GW-> CL1-> MCL1-> AP ->MN path. Now, when the MN moves under MCL2, then it sends an UPDATE_PATH_REQ to MCL1. Similar to uplink, a forwarding chain is established between the current MCL and anchored MCL of the MN. Each time the MN moves, this chain extends by one hop until the threshold is reached at which point the $\mathrm{MN}$ performs another registration and get anchored to a new MCL.

\subsection{Performance Evaluation}

This section evaluates the performance of the proposed scheme in terms of registration cost, frequency of registration updates and average handoff delay. The proposed scheme is evaluated for V2I communication model. The model involves a single car crossing subnets at various speeds, implementing various scenarios, e.g. driving in urban, suburban or highway. Although the scenario considers a single car, every user is assumed to have dedicated resource reservation hence presence of multi-users will not affect the network's performance. However, if network's capacity is reached and resources are fully reserved, then multi-user presence would generate performance degradation. Sessions may be dropped or rejected by the network, depending on admission control policies. This would result in degraded QoS support. This scenario is not addressed in this work. The proposed scheme is also compared with Mobile IP, MPLS-MIP, HMPLS, FH-Micro Mobile MPLS and MFC-Micro Mobile MPLS. Since the scheme is evaluated in vehicular environment, MN's mobility pattern is assumed to follow restricted unidirectional trajectory. In such linear models, each subnet is bounded by two neighbouring subnets and a MN in a subnet $x$ can only move to one of the neighboring subnets. It is assumed that the MN moves across a subnet with uniform speed. The parameters that are used in the analysis are listed in Table 4.2

\begin{tabular}{|l|l|l|}
\hline \multicolumn{1}{|c|}{ Parameters } & \multicolumn{1}{c|}{ Description } & Units \\
\hline$T_{S}$ & Average session time & $\mathrm{sec}$ \\
\hline$V$ & Velocity of the vehicle & $\mathrm{km} / \mathrm{hr}$ \\
\hline$D_{M C L}$ & Diameter of the Micro-cell Leader (Subnet) & $\mathrm{m}$ \\
\hline$D_{C L}$ & Diameter of the Cell Leader & $\mathrm{m}$ \\
\hline$T_{M C L}$ & Average MCL resident time (i.e. $\left.\mathrm{T}_{\mathrm{MCL}}=\mathrm{D}_{\mathrm{MCL}} / \mathrm{V}\right)$ & $\mathrm{sec}$ \\
\hline$T_{C L}$ & Average CL resident time (i.e. $\left.\mathrm{T}_{\mathrm{CL}}=\mathrm{D}_{\mathrm{CL}} / \mathrm{V}\right)$ & $\mathrm{sec}$ \\
\hline$F_{t h}$ & Forwarding chain threshold in terms of number of hops & - \\
\hline
\end{tabular}




\begin{tabular}{|c|c|c|}
\hline$S_{u}$ & Average size of signalling message for registration update & bytes \\
\hline$S_{l}$ & $\begin{array}{l}\text { Average size of signalling message for label mapping for LSP set } \\
\text { up }\end{array}$ & bytes \\
\hline$N_{M}$ & $\begin{array}{l}\text { Average number of movements across subnets during a session (i.e. } \\
\mathrm{N}_{\mathrm{M}}=\mathrm{T}_{\mathrm{S}} / \mathrm{T}_{\mathrm{MCL}} \text { ) }\end{array}$ & - \\
\hline$N_{R}$ & $\begin{array}{l}\text { Average number of renewal of the forwarding chain (i.e. } \mathrm{N}_{R}= \\
\mathrm{N}_{M} / \mathrm{F}_{\text {th }} \text { ) }\end{array}$ & - \\
\hline$N_{R C L}$ & $\begin{array}{l}\text { Average number of renewal of the forwarding chain sent to CL (i.e. } \\
\left.\mathrm{N}_{\mathrm{RCL}}=\mathrm{T}_{\mathrm{s}} /\left(\mathrm{T}_{\mathrm{MCL}} * \mathrm{~F}_{\mathrm{th}}\right)\right)\end{array}$ & - \\
\hline$N_{R G W}$ & $\begin{array}{l}\text { Average number of renewal of the forwarding chain sent to } \mathrm{GW} \\
\left.\text { (i.e. } \mathrm{N}_{\mathrm{RGW}}=\mathrm{T}_{\mathrm{S}} /\left(\mathrm{T}_{\mathrm{CL}} * \mathrm{~F}_{\mathrm{th}}\right)\right)\end{array}$ & - \\
\hline$H_{i-j}$ & $\begin{array}{l}\text { Average number of hops between any two entities } i \text { and } j \text { in the } \\
\text { network }\end{array}$ & \\
\hline$B_{w}$ & Bandwidth of the wired link & $\mathrm{Mb} / \mathrm{s}$ \\
\hline$B_{w l}$ & Bandwidth of the wireless link & $\mathrm{Mb} / \mathrm{s}$ \\
\hline$P_{w}$ & Propagation delay of the wired link & $\mathrm{sec}$ \\
\hline$P_{w l}$ & Propagation delay of the wireless link & $\mathrm{sec}$ \\
\hline
\end{tabular}

Table 4.2 Parameters used in the performance analysis of MPLS hierarchical architecture

\subsubsection{Registration Update Cost}

The registration update cost corresponds to the traffic load in the network due to exchange of signalling messages whenever a MN moves between subnets. It is denoted by $R_{C}$ and is specified by the relationship (hop x signalling message size). In Mobile IPV6, whenever MN moves to a new subnet, it has to perform registration update with new CoA to the HA. In MPLS-MIP scheme, every time the MN moves to a new subnet, it sends a registration update message to the HA. The HA will then initiate the LSP set up procedure. Hence, both signalling update message and LSP set up messages are exchanged. In H-MPLS scheme, the registration update messages and LSP set up messages are exchanged between the MPLS domain GW and MN. In both MPLS aware micro-mobility and MTE-QoS-MPLS schemes, only the registration update is sent to GW since the traffic is switched between pre-established DS-TE tunnels therefore, no new LSP set up is required. In FH-Micro-Mobile MPLS scheme, whenever the MN moves to a new subnet, the registration update with the $\mathrm{GW}$ is performed. In this scheme, since passive reservations are already made with the neighbouring subnet and hence LSP is already created, there is no need to exchanges LSP set up messages. However, a handoff notification message is exchange between 
the FA of the neighbouring subnets so that the in-flight downlink packets destined for the MN can be forwarded to it. In MMPLS scheme, the LSP is redirected from the GW for each subnet change of MN. Moreover, a signalling update is also send to the old FA so that the packets destined for MN can be forwarded from old FA to new FA. The three variants of the forwarding chain based MPLS schemes, MFC Micro-Mobile MPLS presented by the same group, involve the local registration and LSP set up or extension from a Master FA to the current FA of the MN. In the first variant, each time the $\mathrm{MN}$ arrives at a new subnet, it calculates the cost of sending an update to the GW or MFA. If the difference between the signalling costs is less than a predetermined threshold, then the registration is carried out with MFA, otherwise to the GW. In the second variant of the scheme, the MN performs registration update with the MFA until the predetermined threshold is reached. The shortest path to the MFA from the current FA in terms of number of hops is calculated by MN after every subnet change. In the third variant, the LSP between the old FA and the new subnet is extended every time MN moves to a new subnet. All these variants involve both registration update message and label mapping message exchange between the MFA and the current FA. We have the following relationships for the registration cost analysis:

$$
\begin{gathered}
R_{C}\left(\text { Mobile IPV6) }=2 S_{u} H_{M N-H A} N_{M}\right. \\
R_{C}(M P L S-M I P)=2\left[\left(S_{u} H_{M N-H A}\right)+\left(S_{l} H_{F A-H A}\right)\right] N_{M} \\
R_{C}(H M P L S)=2\left[\left(S_{u} H_{M N-G W}\right)+\left(S_{l} H_{F A-G W}\right)\right] N_{M} \\
R_{C}(M P L S-\text { Aware } M M / \mathrm{MTE}-\mathrm{Q} \mathrm{NS}-\mathrm{MPLS})=2 S_{u} H_{M N-G W} N_{M} \\
R_{C}(M M P L S)=2\left[\left(S_{u} H_{M N-G W}\right)+\left(S_{l} H_{F A-G W}\right)+\left(S_{u} H_{F A-F A}\right)\right] N_{M} \\
R_{C}(F H-M M P L S)=2 S_{u}\left(H_{M N-G W}+H_{F A-F A}\right) N_{M} \\
R_{C}(F C-M M P L S)=2\left[S_{u}\left(H_{M N-F A}+H_{F A-F A}\right)+S_{l} H_{F A-F A}\right] N_{M}+2 N_{R}\left[S_{u} H_{M N-G W}+\right. \\
\left.S_{l} H_{F A-G W}\right] \\
R_{C}(M F C-M M P L S)=2\left[S_{u}\left(H_{M N-F A}+H_{F A-M F A}\right)+S_{l} * H_{F A-M F A}\right] N_{M}+2 N_{R}\left[S_{u} H_{M N-G W}+\right. \\
\left.S_{l} H_{F A-G W}\right] \\
R_{C}(\text { Proposed })=2 S_{u}\left(H_{M N-M C L}+H_{M C L-M C L}\right) N_{M}+2 N_{R C L} S_{u} H_{M C L-C L}+2 N_{R G W} S_{u} H_{C L-G W}
\end{gathered}
$$

The values that were assigned to various parameters for analytical and simulative purposes are listed in table 4.3. 


\begin{tabular}{|l|l|}
\hline Parameters & Values \\
\hline Number of hops between MCL and CL & 5 \\
\hline Number of hops between CL and GW & 5 \\
\hline Threshold of MCL Chain & 4 \\
\hline Threshold of CL Chain & 4 \\
\hline Coverage area of MCL & $2 \mathrm{Km}$ \\
\hline Coverage area of CL & $20 \mathrm{Km}$ \\
\hline Latency of wired links & $5 \mu \mathrm{s} / \mathrm{Km}$ \\
\hline Latency of wireless link & $3.3 \mu \mathrm{s} / \mathrm{km}$ \\
\hline Bandwidth of wireless link & $500 \mathrm{Mbps}$ \\
\hline $\mathrm{P}_{\mathrm{c}}$ & $1 \mu \mathrm{s}$ \\
\hline
\end{tabular}

Table 4.3 Values of the parameters used in the performance analysis of MPLS hierarchical architecture

Figure 4.6 shows the registration cost in terms of session length to subnet area ratio. This parameter is obtained by dividing the duration of session initiated by the $\mathrm{MN}$ to the area of subnet. Depending upon the velocity of vehicles, different number of subnets will be crossed by the $\mathrm{MN}$ for a given session. Fig 4.7-4.9 shows the registration cost comparison between different schemes at different velocities.

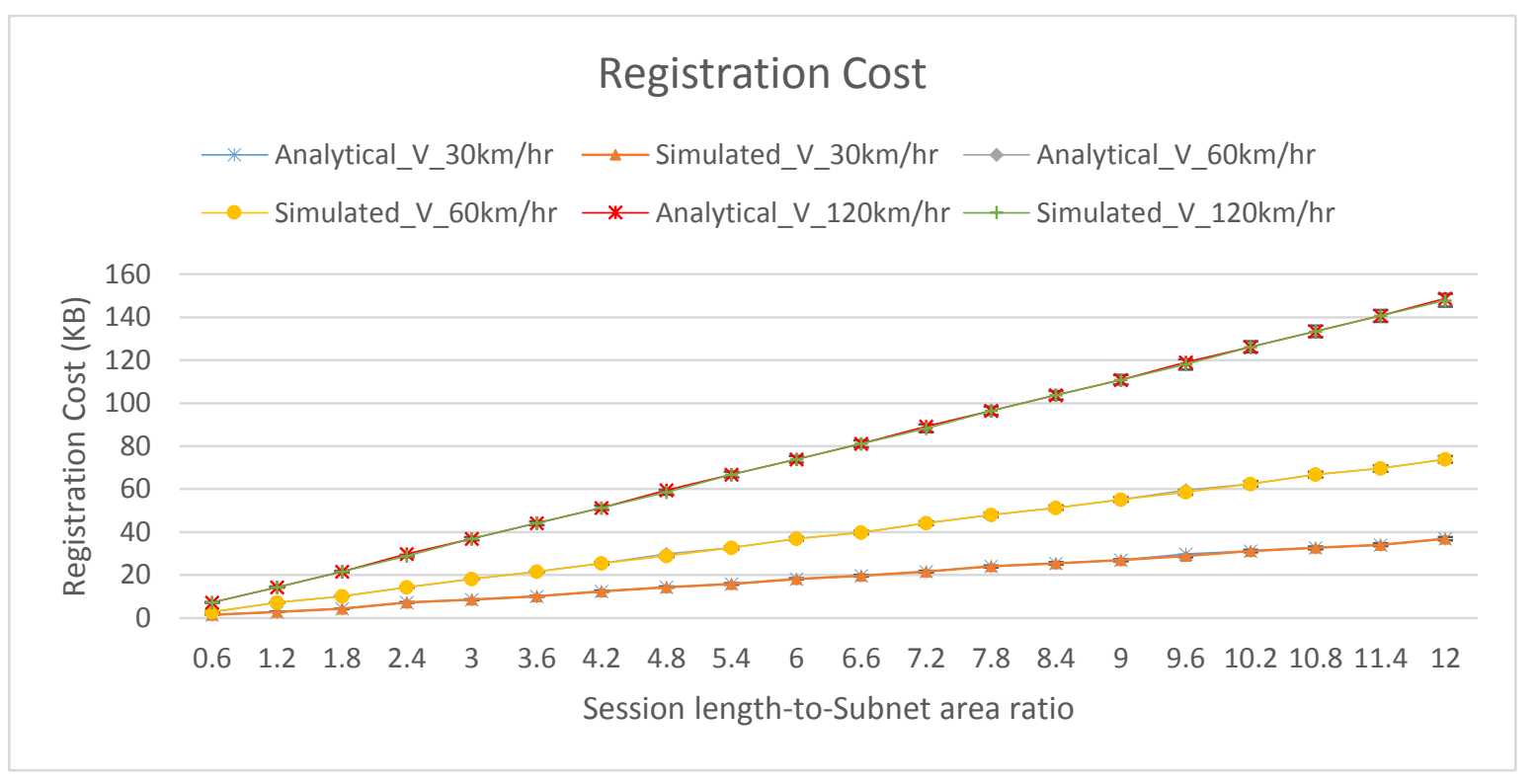

Figure 4.6 Analytical vs simulated results for registration cost of $M N$ at different velocities 


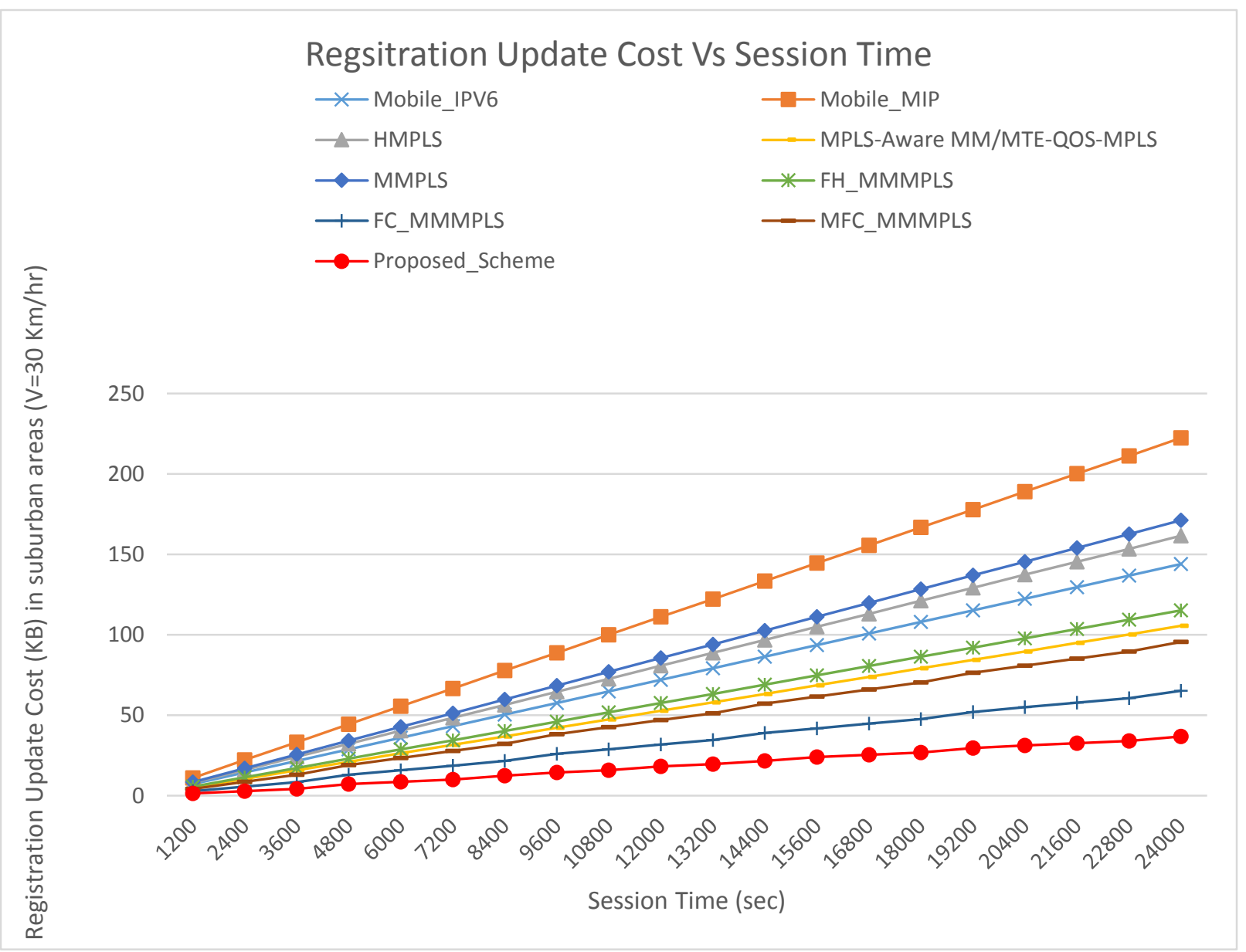

Figure 4.7 Comparison between registration cost of MN using different schemes in suburban areas 


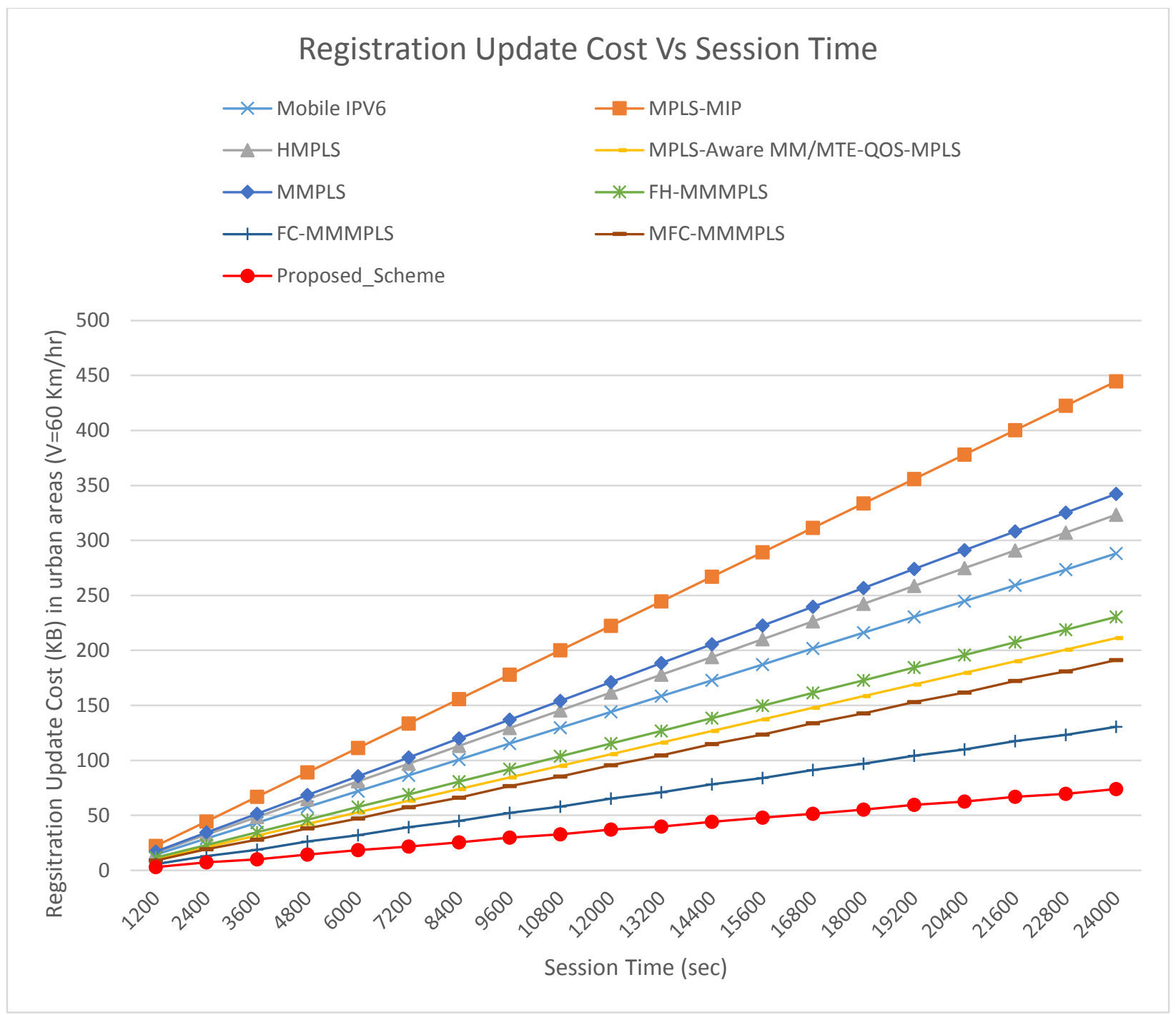

Figure 4.8 Comparison between registration cost of $M N$ using different schemes in urban areas 


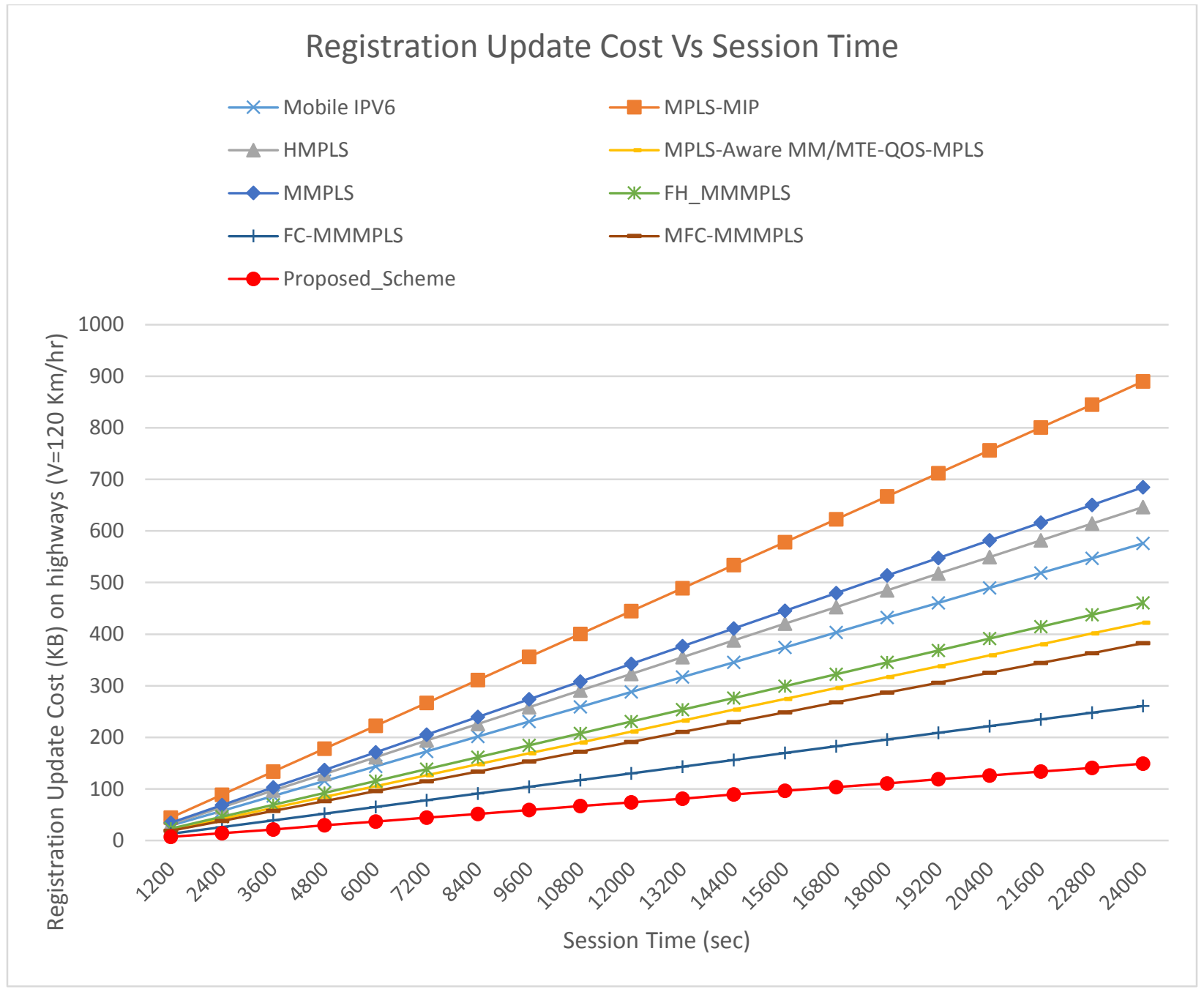

Figure 4.9 Comparison between registration cost of MN using different schemes on highways

\subsubsection{Average Handoff Latency}

Whenever a message is transmitted to/from MN, it will traverse through wireless and wired links. Therefore, while estimating the handoff latency, it is critical that the transmission and propagation delay through these links are also considered. Moreover, the processing delay that may include routing table look up, route cost processing and label table lookup should also be considered.

Let $t\left(s, H_{i-j}\right)$ denote the time that a message of size $s$ will take to traverse the link from $i$ to $j$ through wired and wireless links. $t\left(s, H_{i-j}\right)$ can be expressed as:

$$
t\left(s, H_{i-j}\right)=\left(S / B_{w l}+L_{w l}\right)+H_{i-j}\left(S / B_{w}+L_{w}\right)+P_{C}
$$


where $P c=$ Processing delay at each node

The handoff latency is expressed as the sum of the disassociated time $\left(\mathrm{T}_{\mathrm{D}}\right)$ and registration completion time $\left(T_{R}\right)$.

\subsubsection{Disassociated Time $\left(T_{D}\right)$}

The disassociated time $\left(\mathrm{T}_{\mathrm{D}}\right)$ is defined as the average time a $\mathrm{MN}$ spends without connection to any LER during handoff. This duration starts when the MN moves out from under one subnet and ends when the MN arrives under another subnet and discovers the subnet change. The subnet change is discovered by the MN upon reception of subnet advertisement message through the AP. In the worst case, this is equal to 3 times the super frame period, $T_{\text {sf. }}$ Hence $T_{D}$ is expressed as the sum of $T_{l}$ and $T_{s f}$. To find $T_{l}$, consider figure 4.10 ,

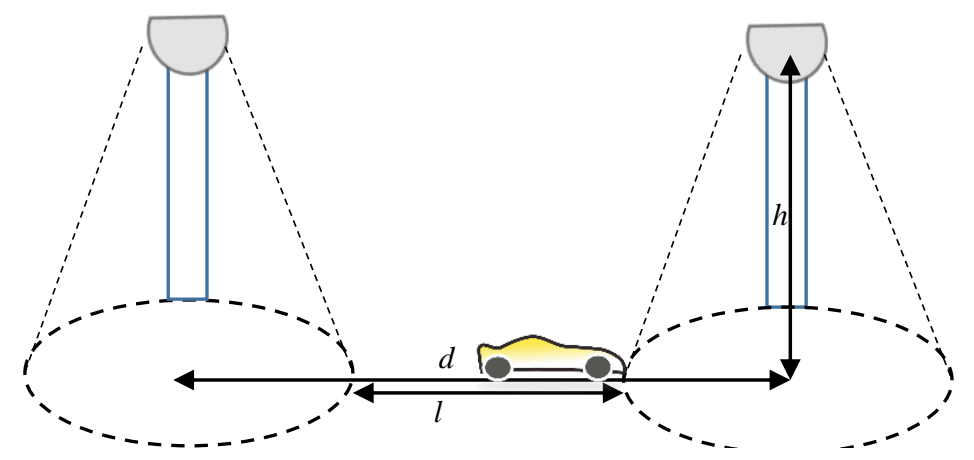

Figure 4.10 MN moving between two non-overlapping subnets.

In the above figure, it is assumed that the two light sources belong to two different subnets and there is no overlapping area between them (the case of overlapping cells would be discussed as later in derivation). The height of the light source from the ground is $h$, the distance between the two light sources is denoted by $d$ and $l$ is the non-overlapping area between the light sources. Then from [ZHU-14]:

$$
l=d-2 \tan \frac{\pi}{3} h
$$

Then the time taken by the MN to cover the non-overlapping area between the two light sources is given by:

$$
T_{l}=\frac{l}{V}
$$


where, $V$ is the vehicular velocity.

Hence, the handoff time can be expressed as follows:

$$
\begin{gathered}
T_{h}=T_{D}+T_{R} \\
T_{h}=T_{l}+T_{s f}+T_{R}
\end{gathered}
$$

In case of overlapping between the coverage area of light sources, $\mathrm{TD}=0$ and $\mathrm{Th}=\mathrm{Tr}$.

\subsubsection{Registration Completion Time $\left(T_{R}\right)$}

This is the time taken by the MN to complete the registration update. According to each scheme, $T_{R}$ is as follows:

$$
\begin{aligned}
& T_{R}\left(\text { Mobile IPV6) }=2 t\left(S_{u} H_{M N-H A}\right)\right. \\
& T_{R}(M I P-M P L S)=2 t\left[\left(S_{u} H_{M N-H A}\right)+\left(S_{l} H_{F A-H A}\right)\right] \\
& T_{R}(H M P L S)=2 t\left[\left(S_{u} H_{M N-G W}\right)+\left(S_{l} H_{F A-G W}\right)\right] \\
& T_{R}(M A M P L S / M T E-Q o S-M P L S)=2 t\left[S_{u} H_{M N-G W}\right] \\
& T_{R}\left(M B \_M M S\right)=2 t\left[\left(S_{u} H_{M N-G W}\right)+\left(S_{l} H_{F A-G W}\right)+\left(S_{u} H_{F A-F A}\right)\right] \\
& T_{R}(F H-M M M P L S)=2 t\left[S_{u}\left(H_{M N-G W}+H_{F A-F A}\right)\right] \\
& T_{R}(F C-M M M P L S)=2 t\left[S_{u}\left(H_{M N-F A}+H_{F A-F A}\right)+S_{l}\left(H_{F A-F A}\right)\right]+2 t\left[S_{u} H_{M N-G W}+\right. \\
& \left.S_{l} H_{F A-G W}\right] N_{R} \\
& T_{R}(M F C-M M M P L S)=2 t\left[S_{u}\left(H_{M N-F A}+H_{F A-M F A}\right)+S_{l} H_{F A-M F A}\right]+ \\
& 2 t\left[S_{u} H_{M N-G W}+S_{l} H_{F A-G W}\right] N_{R} \\
& T_{R}(P r o p o s e d)=2 t S_{u}\left(H_{M N-M C L}+H_{M C L-M C L}\right)+S_{u}\left(H_{M N \_M C L}\right)+ \\
& 2 t S_{u} H_{M C L-C L} \frac{F_{t h M C L}}{N_{m}}+2 t S_{u} H_{C L-G W} \frac{F_{t h C L}}{N_{m}}
\end{aligned}
$$

Assuming TD $=0$ or overlapping area, average handoff latency between different schemes is plotted in figure 4.11 


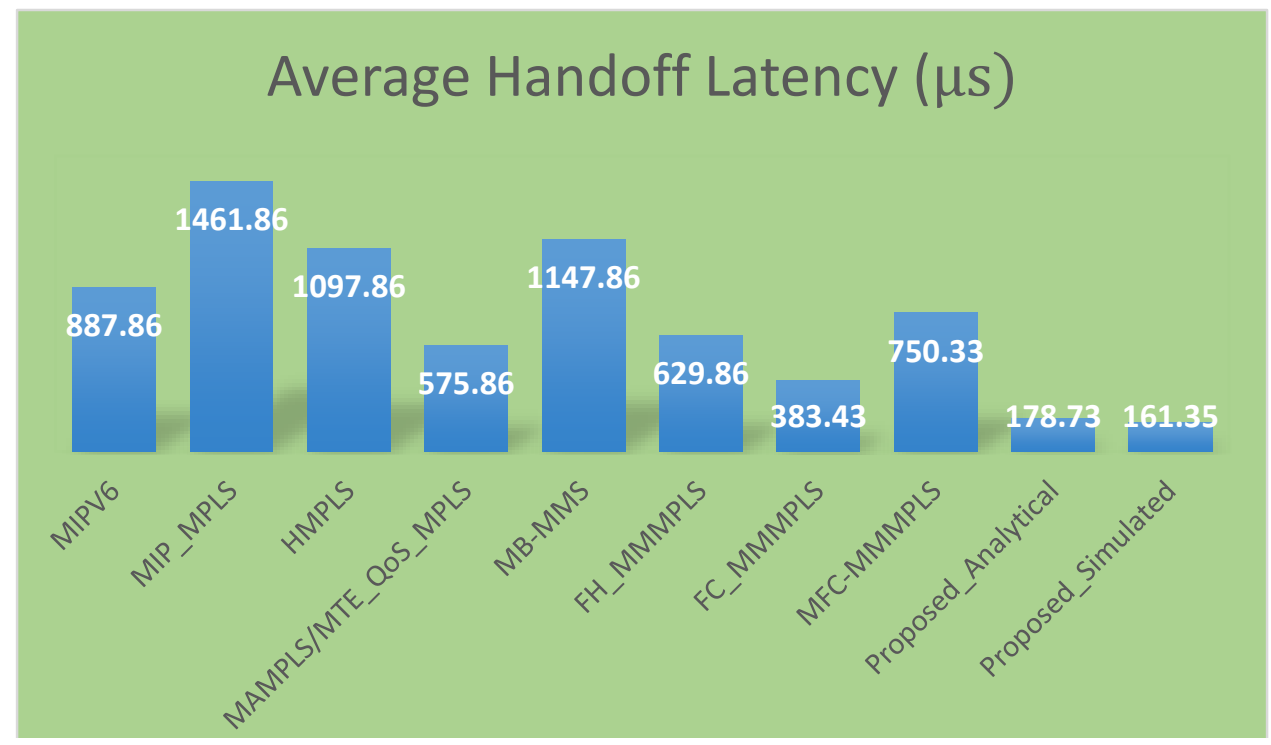

Figure 4.11 Comparison between handoff latencies of different schemes

\subsubsection{Handoff Frequency}

The handoff frequency is defined as the number of times the registration update is sent to the HA or root of the domain i.e. GW during a session so that the LSP can be re -configured to direct the packets to the MN.

HOF (Mobile IPV6/MPLS-MIP/HMPLS/FH-MMPLS/MMPLS/MPLS-AwareMM/MTE-QoS-

$$
\begin{aligned}
& M P L S)=N_{M} \\
& H O F(F C-M M P L S / M F C-M M P L S)=N_{R} \\
& H O F(\text { Proposed })=N_{R G W}
\end{aligned}
$$

Figure 4.12- 4.15 plots the handoff frequency comparisons between different schemes. 


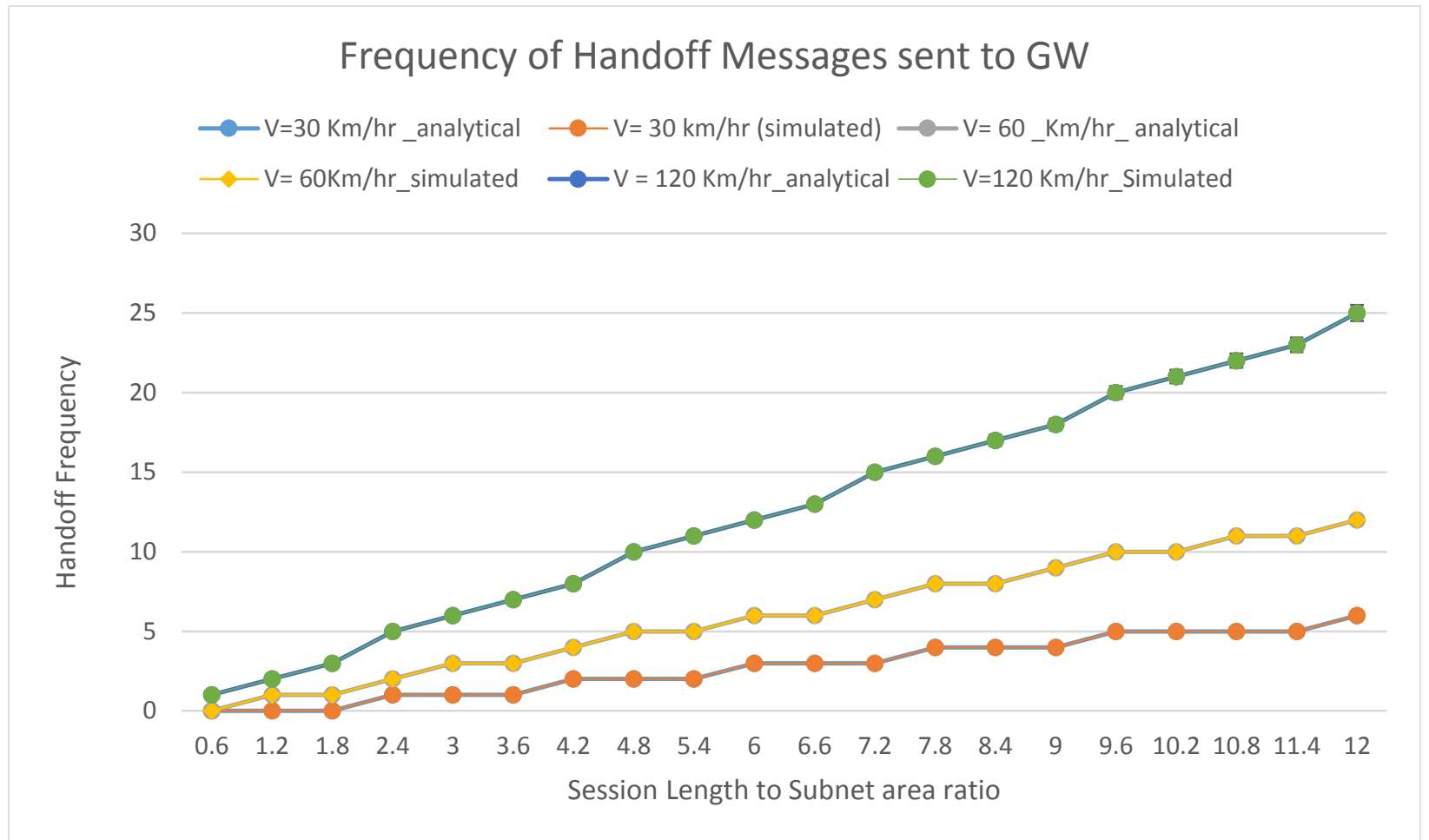

Figure 4.12 Analytical vs simulative comparison between the frequency of handoff messages sent to $G W$ in proposed architecture

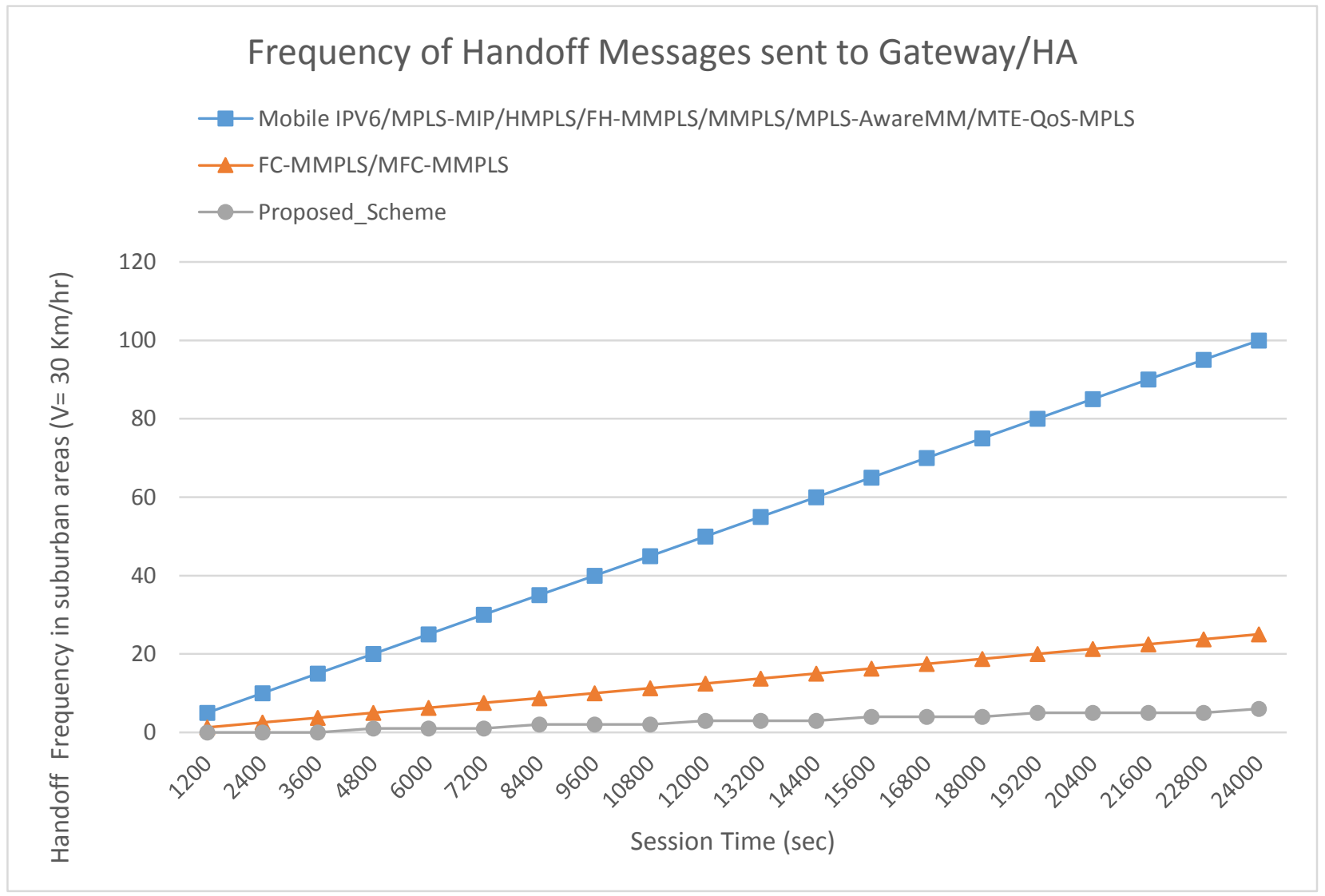

Figure 4.13 Comparison between frequency of handoff messages sent to $G W$ using different schemes in suburban areas 


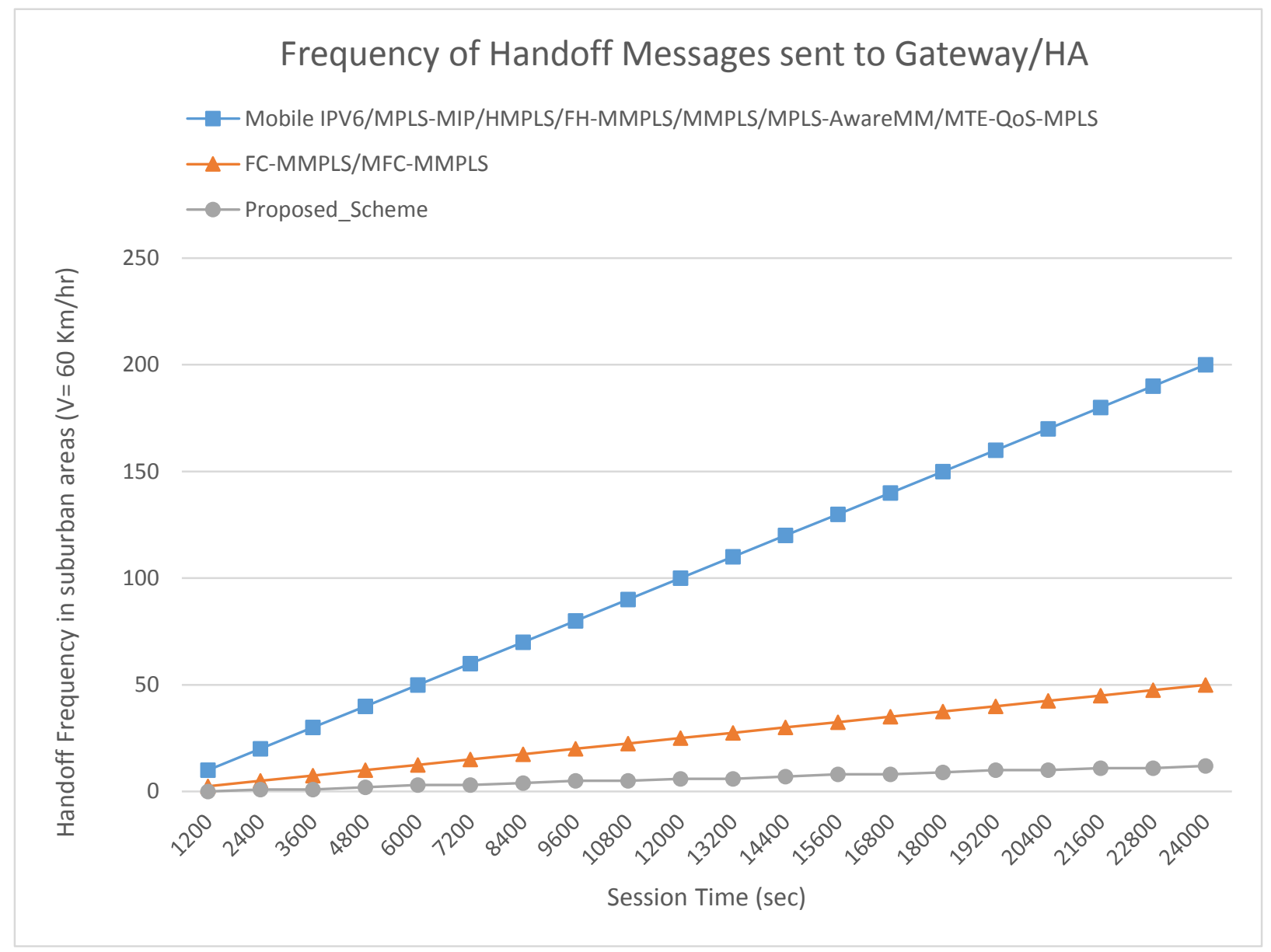

Figure 4.14 Comparison between frequency of handoff messages sent to GW using different schemes in urban areas 


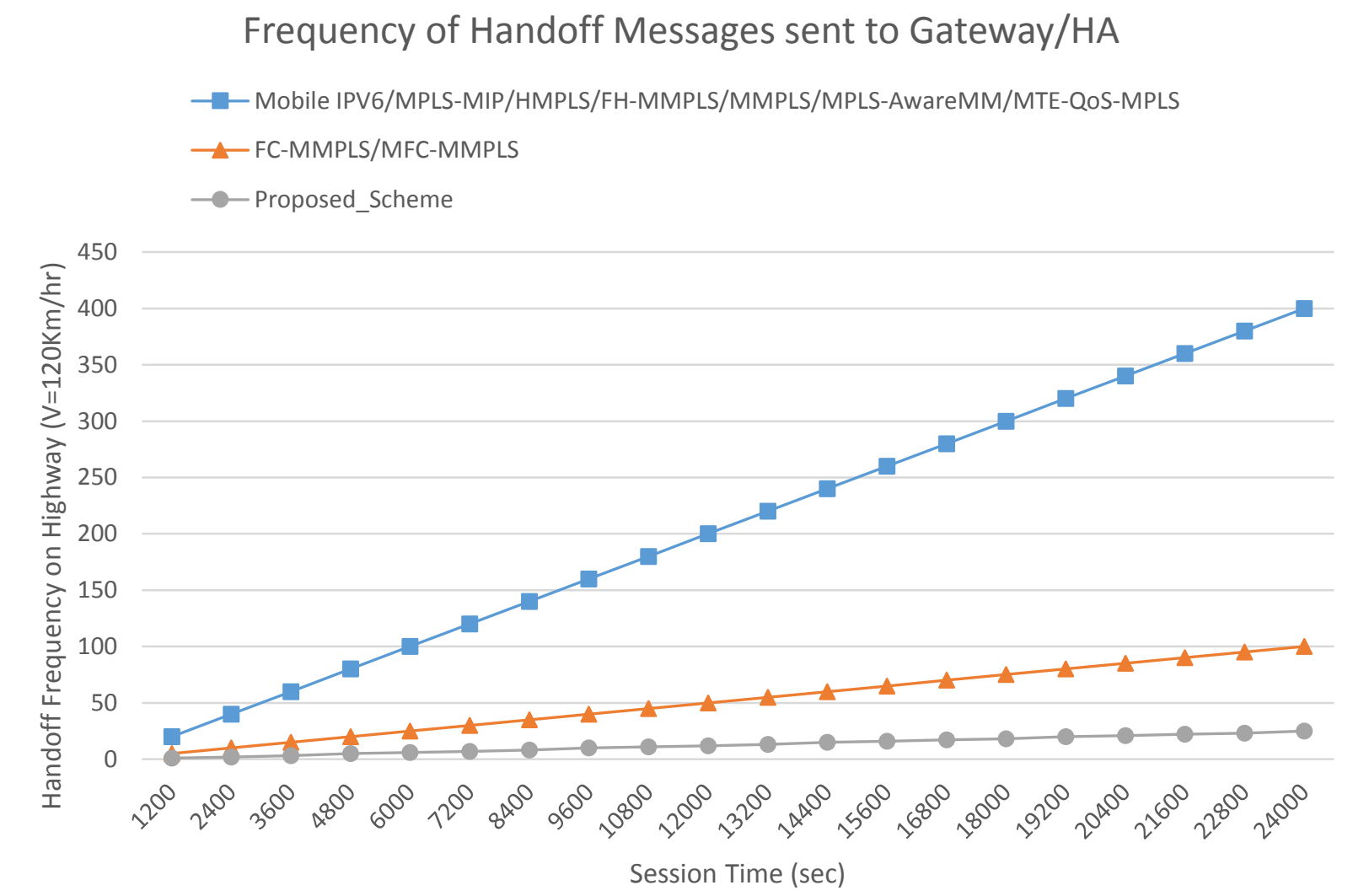

Figure 4.15 Comparison between frequency of handoff messages sent to GW using different schemes on highways

\subsubsection{Total Packet Loss}

The total packet loss (Pkt_loss) is defined as the sum of all packets lost during each handoff that takes place during the duration of the session. In Mobile IPV6, once the MN is attached to the new subnet, it sends a request to the previous access router or old FA to buffer and forward inflight packets to the new access router or new FA. The same mechanism is adopted in MMPLS scheme. In HMPLS, MPLS-MIP, MPLS-Aware-MM and MTE-QoS schemes, all of the in-flight packets are lost during handoff due to the absence of buffering mechanism. In FH-MMPLS, FCMMPLS, and MFC-MMPLS scheme the MN requests packet buffering to the old FA before it moves hence in-flight packets are lost only until the signalling message sent by MN reaches the old FA and buffering mechanism is initiated. In the following relationships $\lambda$ is the constant packet arrival rate. As the proposed scheme initiates the buffering mechanism before the MN 
changes subnet, there is no packet loss in this scheme under normal traffic flow. The expressions for the packet loss in other schemes are derived as follows:

$$
\begin{aligned}
& P k t_{\text {loss }}(M I P V 6 / M B M M S)=\left(T_{D}+t S_{u}\left(H_{M N-F A}+H_{F A-F A}\right)\right) * \lambda * N_{M} \\
& P k t_{\text {loss }}\left(M I P \_M P L S\right)=T_{h}(M P L S-M I P) * \lambda * N_{M} \\
& P k t_{\text {loss }}(H M P L S)=T_{h}(H M P L S) * \lambda * N_{M} \\
& P k t_{\text {loss }}(M A M P L S)=T_{h}\left(M P L S_{-} A w a r e_{-} M M\right) * \lambda * N_{M} \\
& P k t_{\text {loss }}(M T E-Q o S-M M P L S)=T_{h}(M T E-Q o S-M M P L S) * \lambda * N_{M} \\
& P k t_{l o s s}(F H-M M M P L S / F C-M M M P L S / M F C-M M M P L S)=t S_{u}\left(H_{M N-F A}\right) * \lambda * \\
& N_{M}
\end{aligned}
$$

Figure 4.16 plots the packet loss comaprison between different schemes based on the time spent by $\mathrm{MN}$ in subnet.

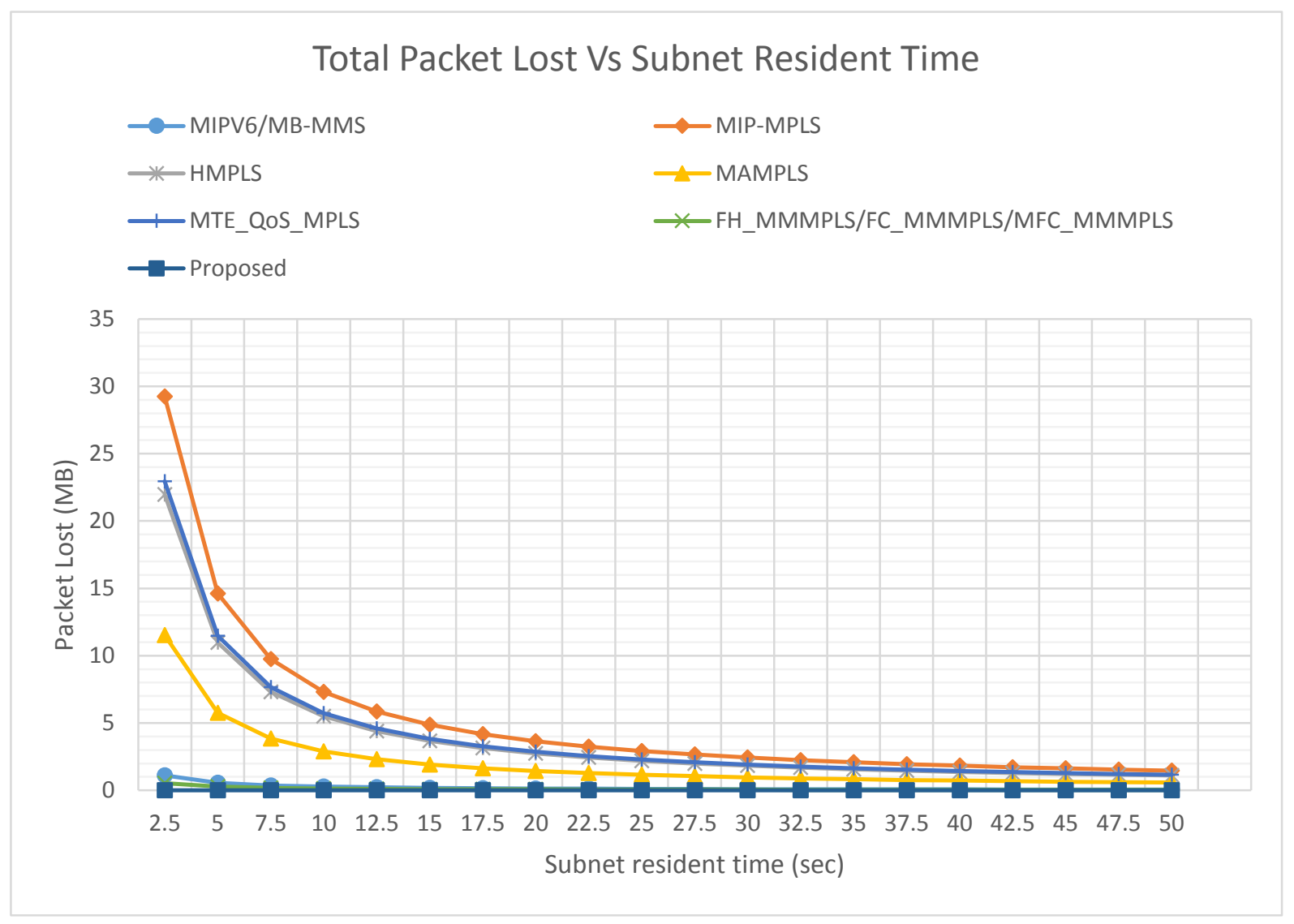

Figure 4.16 Total packet loss vs subnet resident time for various schemes 


\subsubsection{Buffer Size Requirement}

The buffer to store the in-flight packets in our proposed scheme is located at MCL (which is equivalent to FA in other schemes). The buffering of in-flight downlink packets starts from the time the signalling message sent from the MN is received at the old MCL and is discontinued once the registration update is received through the new MCL. The buffer size required is listed below:

$$
\text { Buffer_Size (Proposed) }=\left[T_{D}+t S_{u}\left(2 H_{M N-M C L}+H_{M C L-M C L}\right)\right] \lambda
$$

Assuming overlapping between neighbouring cells, at $\lambda=50 \mathrm{Mbps}$, it is concluded that the Buffer of size $1 \mathrm{~Gb}$ is sufficient to support over $18000 \mathrm{MNs}$ under one MCL.

From the above figures, it can be concluded that the proposed hierarchical architecture offers a noticeable improvement in registration cost, handoff latency, handoff frequency and packet loss rate compared to other existing schemes. For instance, the proposed scheme decreases the registration update cost for a $\mathrm{MN}$ at session time of $12000 \mathrm{sec}$ between $43 \%-82 \%$ in highway scenario compared to existing schemes. The number of handoff messages sent to gateways is also reduced between $74 \%-93 \%$ for the same session duration and same scenario. The average handoff latency of the proposed schem is $161.35 \mu \mathrm{sec}$. This is $88 \%$ lower compared to MIPMPLS scheme and 57\% lower than FC-MMMPLS scheme. The propsoed scheme offers zero packet loss during handoff because of the already established static TE-tunnels. Hence, we deduce that the proposed architecture is most suitable for the $5 \mathrm{G}$ vehicular environement due to its distinguised performance comapred to the existing schemes. 


\section{Chapter 5: MPLS BASED FAST REROUTING}

\subsection{Overview}

Multiprotocol label switching (MPLS) technology simplifies data forwarding by introducing high speed packet switching instead of hop-by-hop routing inherent in IP networks. In addition to high-speed data forwarding capability, MPLS also supports constraint based routing resulting in establishing Traffic Engineered LSPs (TE-LSPs), provision of QoS, and establishment of Virtual Private Networks (VPNs). MPLS utilizes short labels to forward the packets on preconfigured LSPs instead of longest prefix matching, adopted in conventional IP routing. This results in fast and efficient packet forwarding. However, MPLS has a connection-oriented architecture, which means that the entire LSP has to be established before packet forwarding can be initiated. This also means that for any link failure occurring in the MPLS network, reestablishment of LSP is needed. The new LSP has to be configured from the failure node or any other node (in case the failure node is not capable of establishing a new LSP). Hence, the ability of establishing an alternate LSP and fast traffic rerouting in case of link or node failure is quite significant for MPLS networks. In the case of MPLS architecture that is based on static LSPs such as that proposed in Chapter 4, fast rerouting becomes even more significant and challenging. Link failures can affect services and applications that require hard QoS guarantees. The delay in establishing the alternate LSP may result in session interruption. On the other hand, it is possible that the alternate LSP is too long or congested and hence the applications would experience degraded QoS as the latency, bandwidth and delivery constraints are not met. Hence, it is critical that alternate LSPs are established taking into consideration the QoS constraints of the applications and are configured in such a way that QoS guarantees of the traffic is fulfilled even in case of link failure.

MPLS path recovery and traffic restoration models can be categorized as centralized or distributed.

- Centralized Recovery Models: In centralized recovery models, a central management entity is responsible for fault detection, fault notification to the LSRs and computation of 
alternate LSP based on available resources in the network. The advantage of this model is that the central entity can configure the alternate path with optimal resource utilization but path restoration delay is longer due to the communication delay between the centralized entity and LSRs as well as the processing delay at the centralized node.

- Distributed Recovery: In distributed recovery model, each node in the network is able to reconfigure the path when node and/or link failure occurs. The advantage of this approach is shorter path restoration time. However, since the nodes do not have the "complete picture" of the network, the reconfigured path may not be efficient, as it may have more hops than required or include congested nodes.

The path restoration schemes in both, centralized or distributed models can be based on dynamic rerouting or fast re-routing (use of pre-computed paths).

- Dynamic rerouting scheme: These schemes_calculates the alternate path after the failure has occur. The path restoration delay is larger than fast re-routing schemes as more messages needs to be exchanged between LSRs for dynamic path calculation.

- Fast rerouting/protection switching: These schemes use pre-computed alternate routes to forward traffic after failure detection. Paths are quickly switched, however they may not be based on optimum resource utilization as the link utilizations may have changed since the paths were initially computed.

These path restoration schemes can use any of two modes for path re-routing: local repair or global repair.

- Global Repair: In global repair mode, the entire path between ingress and egress nodes is re-configured.

- Local Repair: In local repair mode, the traffic is redirected from the node closest to the failed link. The alternate path may include active nodes and links that were also part of failed LSP.

Local protection can be accomplished by using either one of the following two techniques: 
- One to one backup (1:1): In this technique, each protected LSP has a dedicated backup LSP.

- Tunnel Backup: In this technique, a backup tunnel is created that is shared by multiple protected LSPs. For this technique, the label stacking property of MPLS is utilized. In this scenario, a number of LSPs sharing the same bandwidth and delay constraints can be rerouted collectively over the same backup tunnel.

The issue with 1:1 backup technique is scalability. Depending upon the number of paths/links that require protection in the network, the number of backup LSPs can increase considerably. This is in addition to the signalling and routing load that is needed for supporting these extra LSPs. Another drawback is that it is highly possible that these backup LSPs are configured as dedicated links, which means under normal network operations, these LSPs do not carry any traffic. This results in huge bandwidth wastage.

On the other hand, using tunnel backup requires multiple LSPs to share the tunnel capacity, hence required QoS by individual protected LSPs may be compromised.

To minimize delay in restoration, distributed recovery schemes with fast rerouting based on preestablished LSPs with local repair are considered for proposed architecture. As initially the LSPs are established based on QoS requirements, it is assumed that back up LSP tunnel will also be established such that application QoS is not effected. This is accomplished by establishing integrated service MPLS model with dedicated bandwidth and delay guarantees.

\subsection{Existing MPLS Fast-Rerouting Schemes}

The benefit of fast re-routing scheme is shorter delay in path restoration as these schemes utilize pre-established back up LSPs. Before describing the existing fast re-routing schemes proposed by IETF and presented in open literature, we provide below some important terminologies and procedures: 
- $\quad$ Protected Path/Link/LSP: Protected path or link is the working path for data traffic under normal operation i.e. before the occurrence of fault. This is the link that requires protection or backup.

- $\quad$ Path switching LSR (PSL): An LSR that is the transmitter of both the protected path traffic and its corresponding backup path traffic. The PSL is responsible for switching the traffic from the protected path to the backup path. The PSL is the origin of the backup traffic path, but may or may not be the origin of the protected traffic path (that is the protected path may be transiting the PSL) [OWE-01].

- $\quad$ Backup Path/Link/LSP: This is the path or link on which traffic is redirected after the fault is detected by PSL.

- $\quad$ Path Merge LSR (PML): An LSR that receives both protected path traffic and its corresponding backup path traffic, and either merges their traffic into a single outgoing path, or, it is itself the destination, passes the traffic on to the higher layer protocols. The PML is the destination of the backup path but may or may not be the destination of the protected path [OWE-01].

- $\quad$ FIS (Fault Indication Signal): A signal that indicates that a fault along a path has occurred. It is relayed by each intermediate LSR to its upstream or downstream neighbor, until it reaches an LSR that is set up to perform MPLS recovery i.e. PSL [OWE-01].

- $\quad$ FRS (Fault Recovery Signal): A signal that indicates that a fault along a path has been repaired. Like the FIS, it is relayed by each intermediate LSR to its upstream or downstream neighbor, until it reaches PSL that performs a switchback from the backup path to the protected path for which the FIS was initially received [OWE-01].

- $\quad$ Reverse Path/LSP: The path in the reverse direction of protected/working path on which data packets will be sent back to the upstream node in case of link failure.

- $\quad$ Aware-LSR: The LSR that identifies the faulty link and generates FIS message. 
- Procedure for prior establishment of alternate paths: We assume that alternate paths are established, utilizing the same signaling mechanism used for setting up protection paths at the time of LSP establishment. This is achieved by using the RSVP-TE extensions. For enabling fast reroute and path protection, two new objects FAST_REROUTE and DETOUR are defined. Extensions are made to existing SESSION_ATTRIBUTES and RECORD_ROUTE_OBJECT in order to provide explicit node and bandwidth protection. An EXPLICIT_ROUTE_PROTECTION field is also added in RSVP_TE. These RSVPTE extensions are explained in detail in RFC-4090 \& IETF draft on MPLS path protection. (Note: CR_LDP protocol also have these extensions. So any of the protocols can be used for establishing back-up/alternate LSPs).

- Procedure for fault detection: It is assumed that faulty links are detected by the loss of liveness messages. Liveness messages are defined as generic messages that are exchanged between two neighbouring LSRs to check the integrity of the link in forward and backward direction. RSVP "hello" messages can serve as liveness messages. A LSR detects a faulty link when liveness messages are not acknowledged. The LSR keep sending the liveness messages until a pre-defined threshold is reached. After which the LSR identifies the link as faulty.

Currently, there are two IETF drafts proposing different schemes for MPLS fast-reroute:

\subsubsection{Haskin's Scheme}

Haskin's MPLS based fast re-route scheme is described in IETF draft [HAS-00]. This scheme proposes to establish a reverse LSP for each protected LSP in addition to the backup LSP. When the link failure happens, the LSR that identifies the failure (also called aware-LSR [GON-03]) start sending the packets back on the reverse LSP. Once the first packet on the reverse LSP reaches the PSL node of the protected path, the node starts directing the incoming packets directly on the backup LSP as well as packets coming through the reverse path. Although the scheme uses pre-established LSP and supports fast reroute, there are several issues. The first one is the delay experienced by the packets on the protected LSP that are redirected from the awareLSR. These packets experience twice the propagation delay. Secondly, the incoming packets at the PSL are mixed with the returning packets, hence packets arriving at the destination node will 
be out of order. Another issue that may arise in this scheme is based on the fault detection mechanism, if some packets are in transit on the faulty link before failure is detected, those packets will be lost. Figure 5.1 shows the MPLS fast re-route mechanism according to Haskin's scheme.

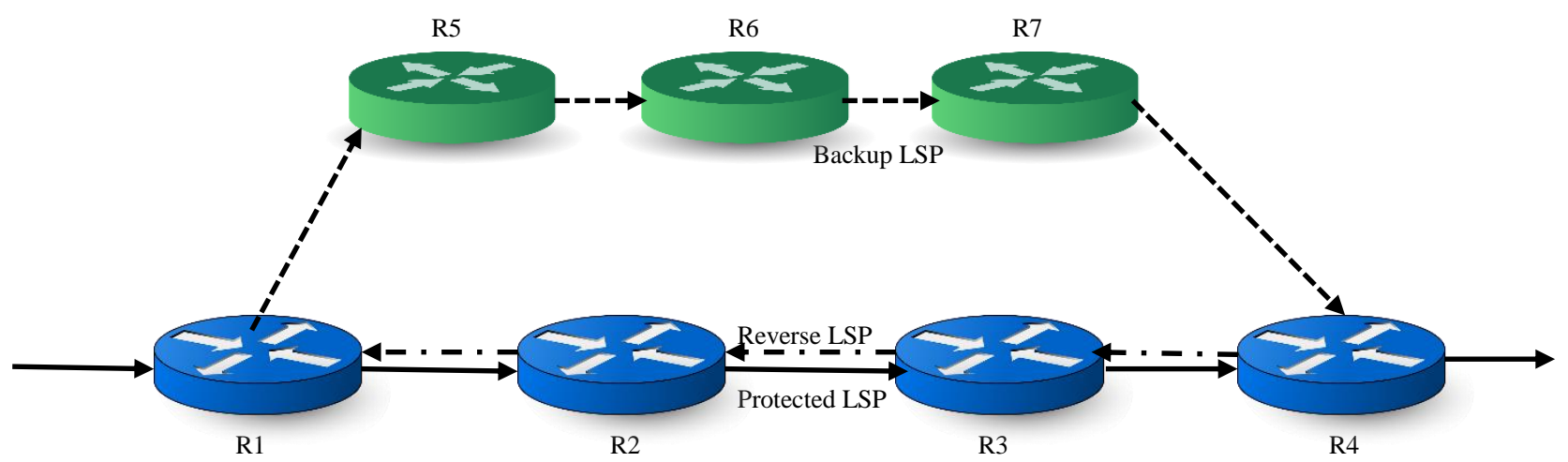

(A)
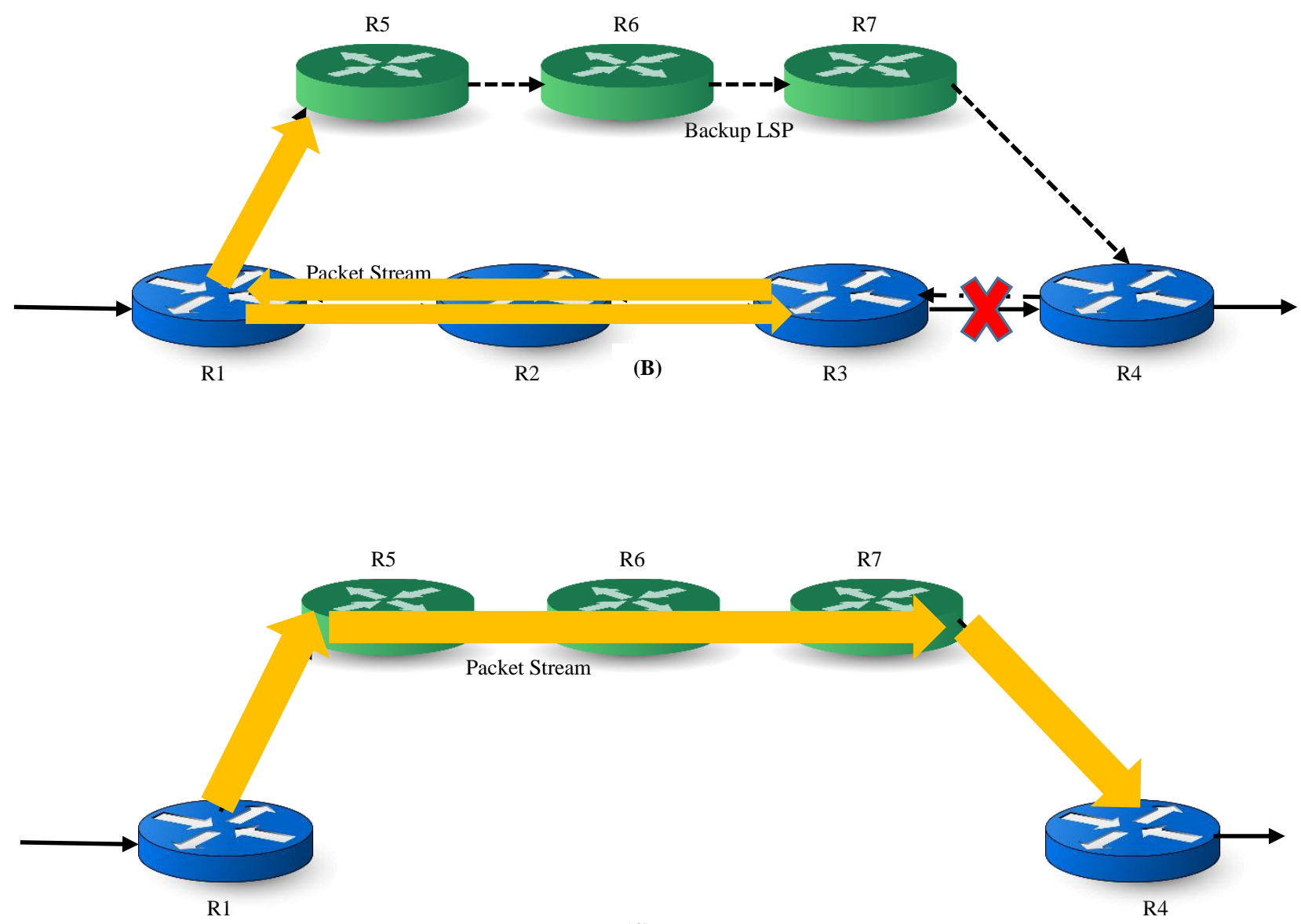

(C)

Figure 5.1 Haskin's MPLS fast reroute scheme (A) Reverse LSP is created along protected LSP and back up LSP (B) Link failure is detected, traffic is reversed from aware-LSR $(C)$ Traffic is directed on the back up LSP. 


\subsubsection{Makam's Scheme}

Makam's scheme is described in IETF draft [OWE-01]. Makam's scheme proposes using a FIS message to notify LSRs on the protected path about link failure instead of the reverse LSP. The FIS originates from the aware-LSR and is passed onto the PSL. Once the PSL receives FIS, it switches the link to the pre-established path and incoming packets are forwarded to the backup path. In this scheme, although order of incoming packets is preserved, but inflight packets on the protected path are lost until the FIS signal is received at the PSL. Figure 5.2 shows the Makam's proposed fast re-route scheme. 


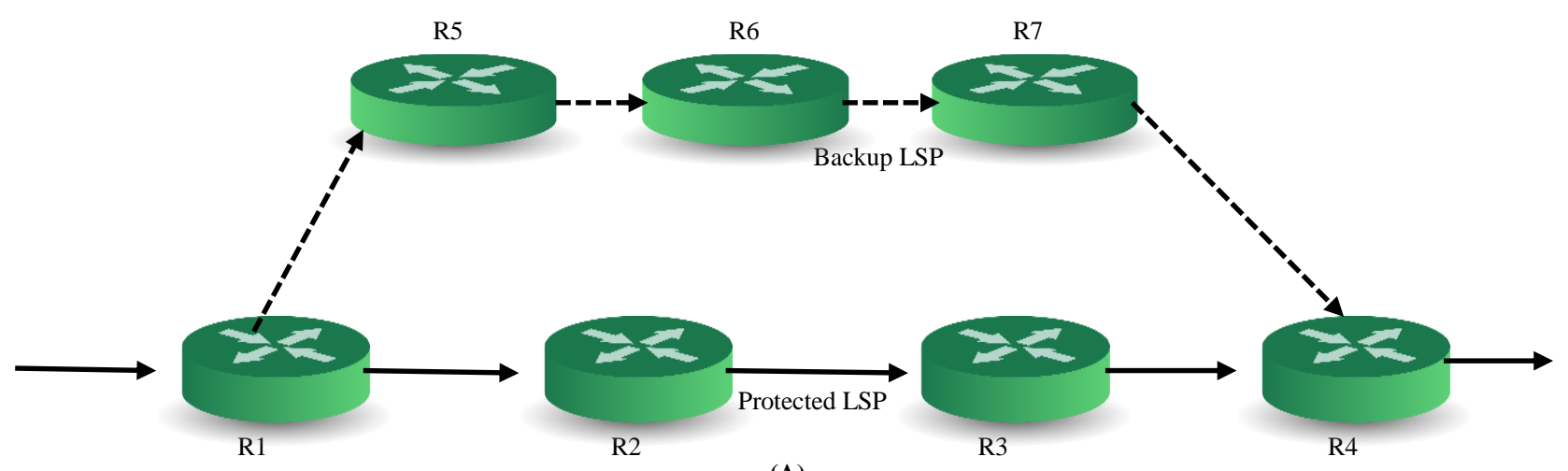

(A)

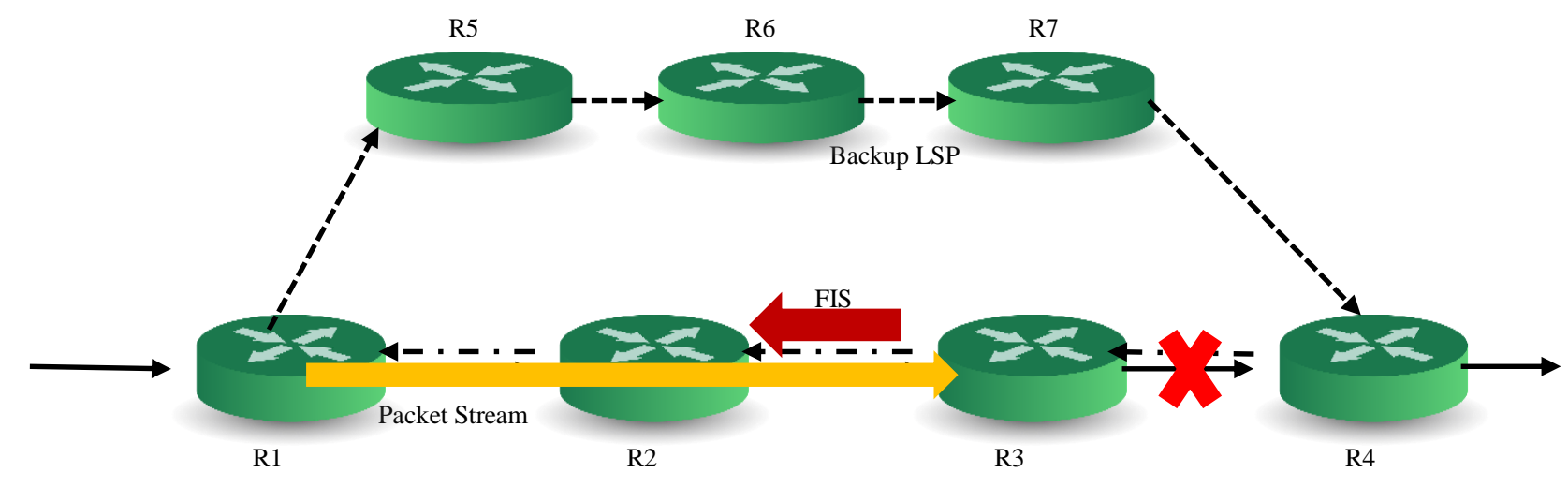

(B)

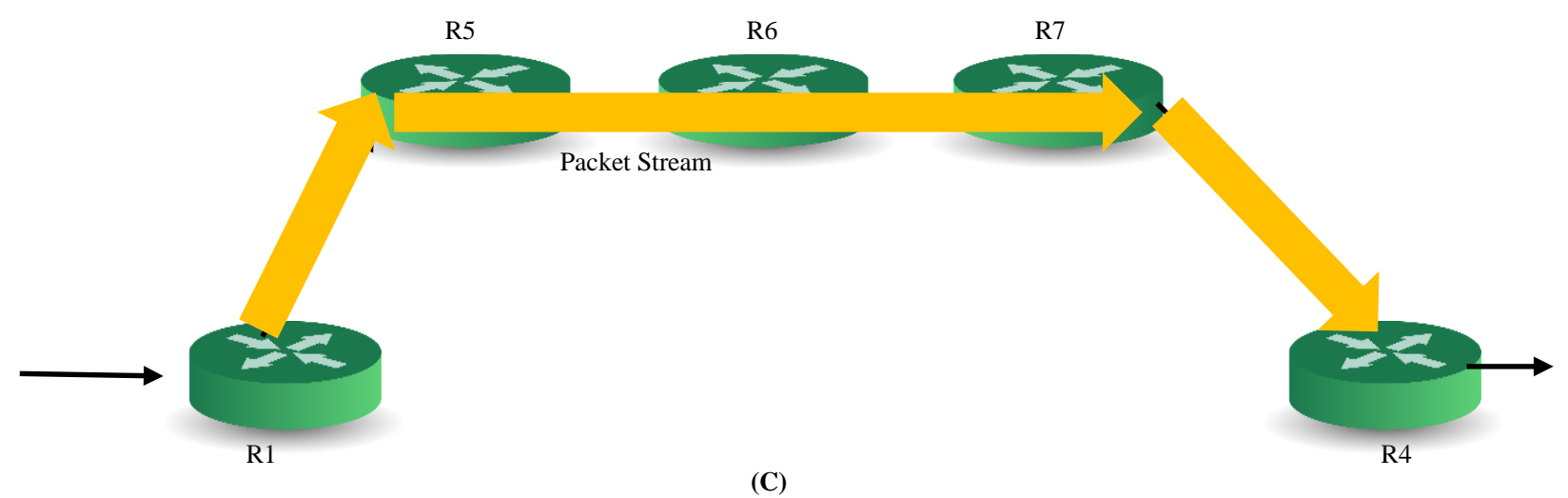

Figure 5.2 Makam's MPLS fast reroute scheme (A) Backup LSP is created along protected LSP (B) Link failure is detected, FIS message is generated by aware-LSR (C) Traffic is directed on the back up LSP. 


\subsubsection{Reliable and Fast Rerouting Scheme}

Another scheme called reliable and fast re-routing (RFR) is presented in [GON-03]. This scheme is based on Haskin's proposal and suggests modifications to overcome the limitations of Haskin's scheme. This scheme address three shortcomings of Haskin's scheme: 1) packet reordering; 2) restoration time delay; 3) packet loss.

This scheme proposes three additional fields in label forwarding information base (LFIB) at each LSR: Alternate_detect, Buffer_start and Buffer_empty. To address the issue of packet reordering and minimizing restoration time delay, RFR scheme proposes that the aware-LSR that detects the link failure, sends back the packet on the reverse path to the upstream LSR node. When the next upstream LSR node receives the first packet on the reverse path, it changes the LFIB entry to Alternate-detect, indicating that there is some issue with the downstream link. The next incoming packet for the protected link is "tagged" and forwarded regularly to the downstream path. This tagging materialized by using and "setting" one of the three "EXP" bits. Once the tagged packet is forwarded downstream, the node changes the LFIB entry from "Alternate_detect" to "Buffer_start" and starts buffering all packets intended for downstream forwarding on the protected path. This buffering continues until the tagged packet is received back on the reverse path. Once the tagged packet is received, there will be any no more packets that will be forwarded to that specific reverse link. The node changes the LFIB entry to "Buffer_empty" and will start sending the packets to the next upstream node using the reverse path. In the meantime, each node on the path to PSL node has started buffering the downstream packets upon receiving the first packet on the reverse LSP. This procedure continues until the tagged packet reaches the PSL node. This process preserves the "order of packet sequencing". Note that only one node, the one that is first in the upstream direction of the aware-LSR, tags the packet, and the same packet acts as a trigger for emptying the buffer at each node. Moreover, to eradicate packet loss, the scheme proposes that each LSR employs a small buffer, which continuously keeps a copy of the packet that is being forwarded on the downstream LSP. The benefit of this approach is that in case of link failure, the packets that are already on the link at the time of failure will not be lost. Instead, they will be sent on the reverse path from the buffer. The maximum size of this buffer was estimated to be equal to twice the number of packets that can occupy the given link of protected path. This value was selected so as to protect against both link and node failures. It was 
assumed that each node is directly connected to one upstream and one downstream node. Hence, in case of link failure, only the packets on that link would be lost, but in case of node failure, the packets on both upstream link and downstream link would be lost. Therefore, buffer size of twice the link capacity was chosen for complete recovery of all of the packets. This work also assumed that in case of link failure the intermediate nodes will discard the buffered packets and they will be forwarded from the aware-LSR node. Figure 5.3 shows the timing diagram for the RFR scheme.

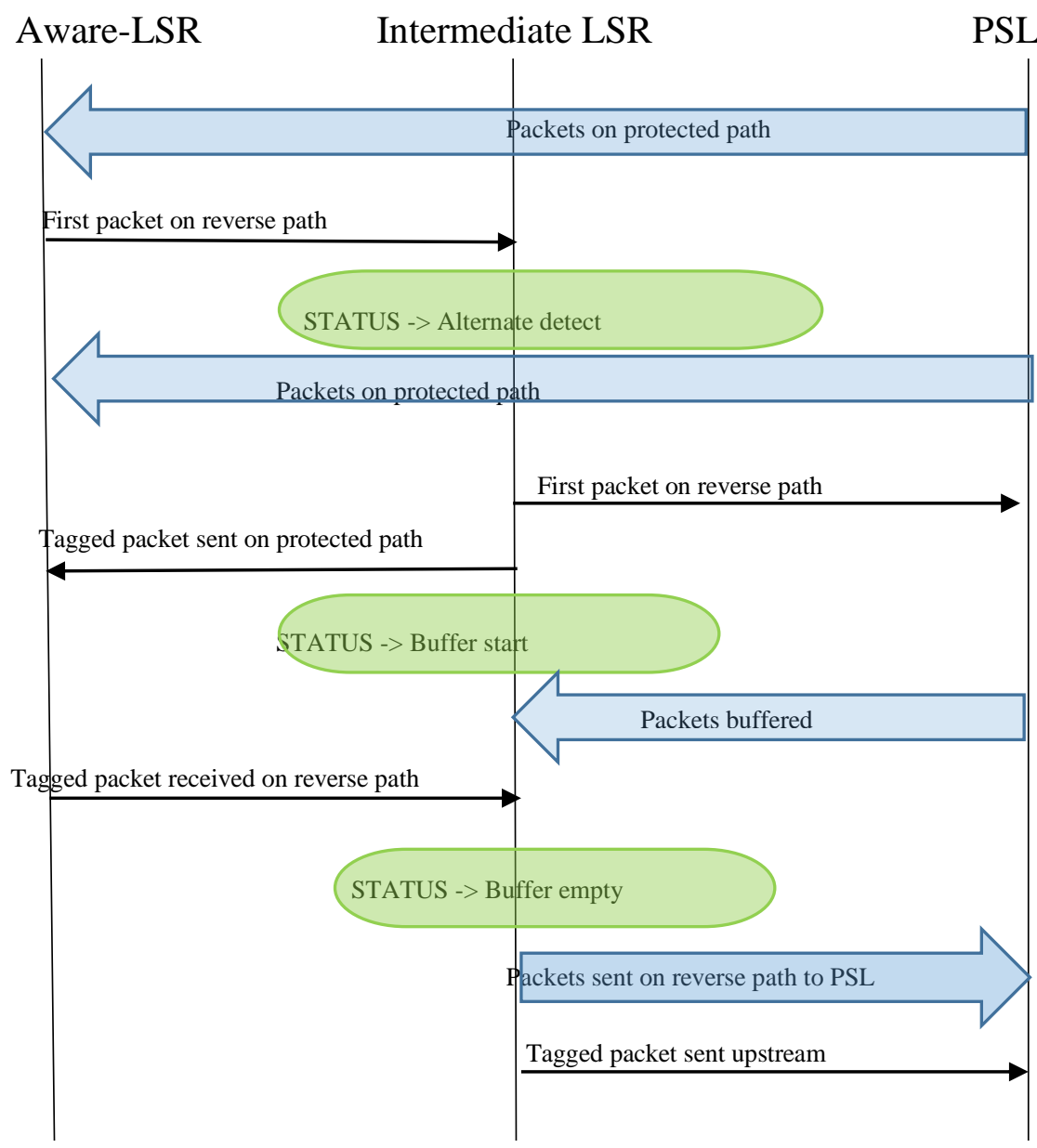

Figure 5.3 Timing diagram for RFR scheme

There are several serious shortcomings in this scheme. In this scheme one of the EXP bits are used to tag the packets. This makes the scheme unsuitable in MPLS networks supporting DiffServ based QoS model, since EXP bits are used to map the class of service and PHB on the packets. The scheme also proposes that the LSR that has tagged the packet will take two 
different actions while forwarding it on the reverse path. In the case, buffer is full, the tagged packet is forwarded "as is" on the reverse path after the buffer is emptied to indicate to the next node that no more packets are expected on this link. However, if the buffer is empty, the tagged packet is forwarded as a normal packet after re-setting the EXP bit to 0 . The purpose of this operation is unclear and raises serious questions on the validity of the scheme. Recall that the purpose of the tagged packet was to act as a trigger to initiate buffer emptying operation at each LSR on the way to PSL node. If the tagged packet is forwarded as normal packet with unset bit, the upstream LSR will think it is a normal packet on the reverse path, hence it will keep on buffering the incoming packets and will not initiate sending the already buffered packets back upstream on the reverse path. For the second part of the scheme, which suggests buffering the packets at each node doesn't address the situation when the link has not failed. How and when will the packets be discarded? Even in case of link failure all but aware LSR will discard the packets, this translates to additional processing complexity on the scheme.

\subsection{Proposed Fast Re-route Scheme}

The proposed scheme is called FIS based fast re-route (FFR) and is based on a combination of Makam's and Haskin's schemes. FIS message is used to notify the LSRs on the protected LSP about link failure, similar to Makam's scheme. However, reverse LSPs are also pre-configured to send data packets in backward direction similar to Haskin's scheme. The proposed scheme also utilize local buffers at LSRs to store data packets once the failure notification is received. The benefit of using short FIS message for fault notification is its quick transmission time. Recall that the PSL node keeps sending data packets on the protected path until it receives fault notification. Based on the number of hops between aware-LSR and PSL node, the delay in receiving the fault notification message would translate to more packets sent on the protected path. This means more packets will be in the system and it will take longer (collectively) to empty the buffers for the intermediate routers, hence increasing the path restoration delay.

Secondly, by utilizing local repair with special trigger packets and buffering at LSRs, the packet disorder and packet loss can be eradicated. This scheme address the issues in Haskin's scheme and RFR scheme. 


\subsubsection{Significance of Trigger Packet}

In the proposed scheme, the intermediate LSRs, upon receiving the FIS messages generated by aware-LSR sends a special "trigger" packet to the next downstream node. This is the last packet that is forwarded by this LSR on the protected path and serves as the indicator for end of packet stream on the protected path. The LSR then starts buffering all of the packets received on the protected path, intended for the next downstream node. This buffering continues until the trigger packet is received back by the LSR from which it originated on the reverse path. Upon receiving the trigger packet back, the LSR starts emptying its buffer and sends the buffered packets on the reverse path towards PSL. Using the "trigger" packet overcomes the unnecessary packet forwarding present in Haskin's scheme, where the packets are sent on the protected path by the LSRs even after they became aware that the link is faulty. This increases the path restoration delay that may become critical, depending on the number of hops between PSL and aware-LSR on the protected LSP.

\subsubsection{Algorithm Description}

Recall that the proposed architecture is the hierarchical MPLS network architecture, a simplified version of which is shown in figure 5.4. 


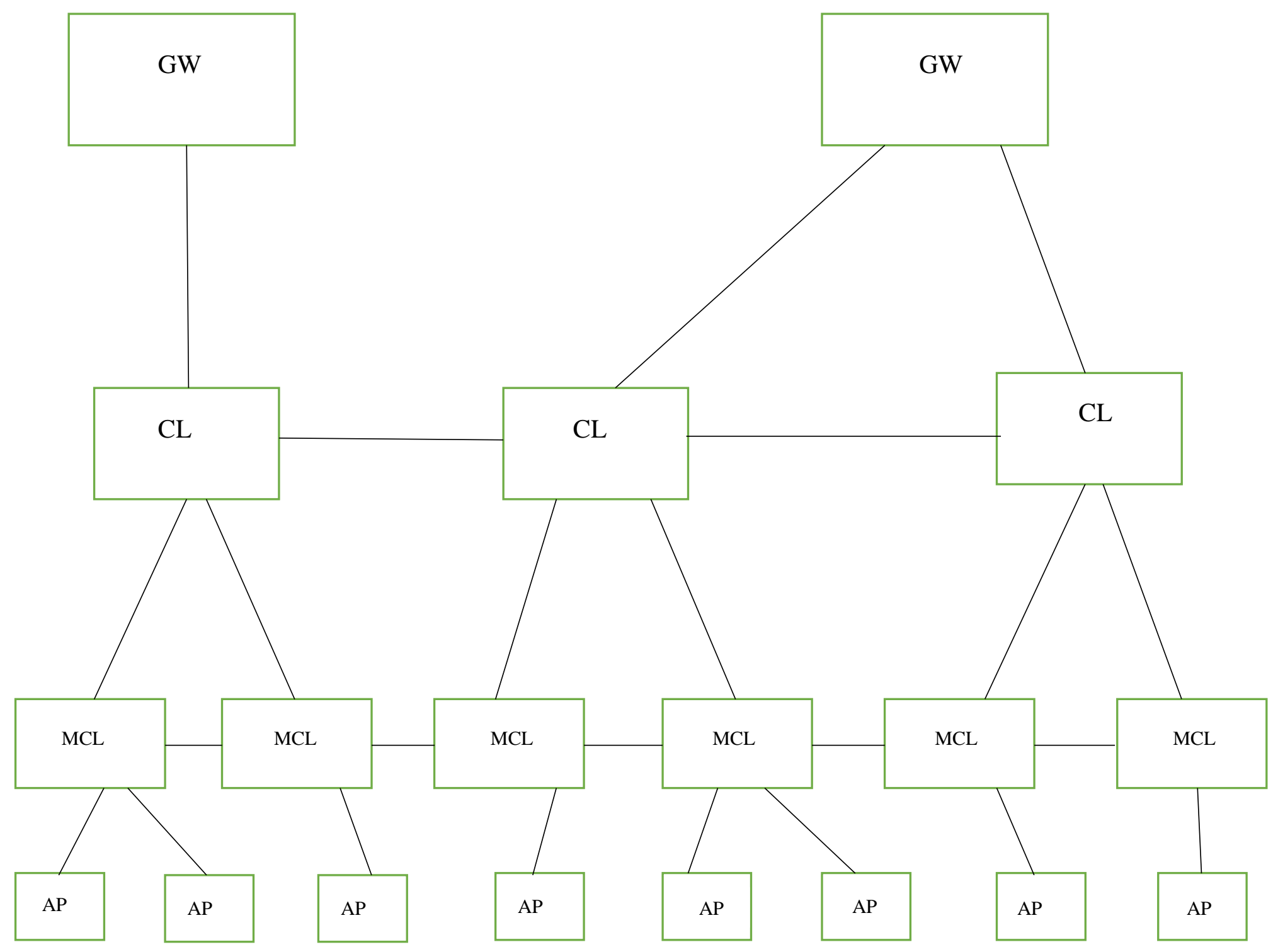

Figure 5.4 Simplified version of the proposed hierarchical MPLS based micro-mobility architecture

The hierarchical description of architecture is provided previously. Here we identify the PSL nodes that are MCLs and CLs. The black lines identify "links" between MCLs, CLs and GWs. Each link acts as an LSP tunnel carrying multiple LSPs with several LSR between the PSL and PML nodes. Each LSP is also protected through the use of backup tunnel. It is assumed that the primary tunnels and back up tunnels are established by using bandwidth guarantees. In this scenario, each LSP passing through the LSP tunnel on a link is established exclusively or may be aggregated as described in IntServ with MPLS documentation [TOM-01]. 
Figure 5.5 and 5.6 presents the state machine diagram of the proposed algorithm (FFR). The state machine diagrams are followed by a detailed description of the algorithm. The proposed algorithm introduces a new field called "Link Status" in the LFIB. The field can take four different states on intermediate LSRs on the protected path: ACTIVE, FAILED, BUFF_ACT and BUF_EMP. In PSL nodes, there is an additional state of BK_ACT.

- $\quad$ ACTIVE: This state corresponds to regular forwarding operation. In this state, the protected link is operational and packets are forwarded on the protected path after label SWAP/POP/PUSH operation.

- FAILED: This state corresponds to the detection of faulty link. The LSR(s) switches to this state after receiving the FIS message.

- BUFF_ACT: In this state, all of the incoming packets intended for protected LSP are buffered. The LSR(s) switched from FAILED state to BUFF_ACT state after sending the trigger packet on the protected LSP.

- BUFF EMP: In this state, the buffered packets are sent back on the reverse LSP to the next upstream LSR towards the PSL.

- BK_ACT: This state is exclusive to PSL node. In this state, the backup path is activated and all of the incoming packets are directly forwarded on the backup LSP. 


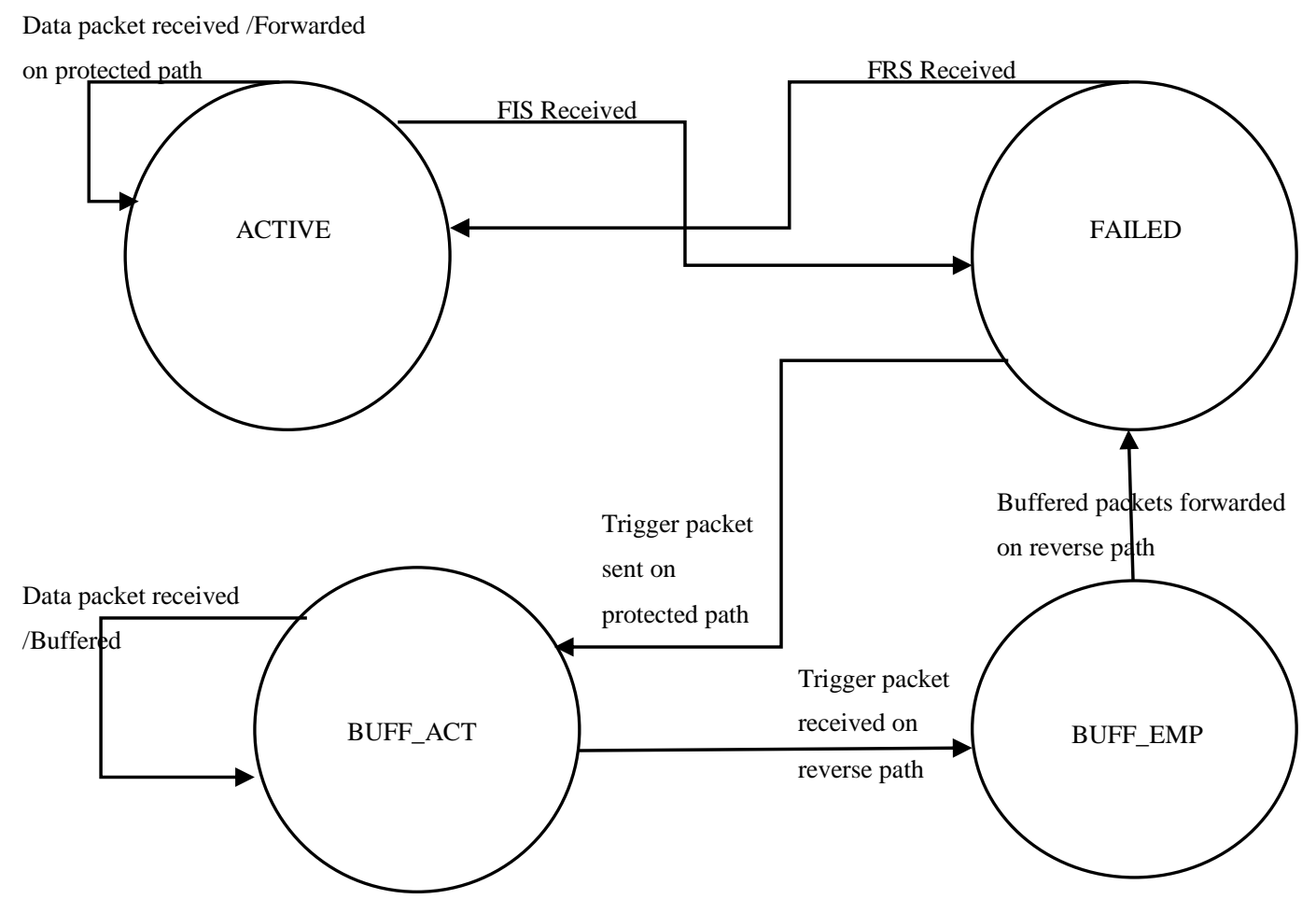

Figure 5.5 State machine diagram of intermediate LSRs on the protected path

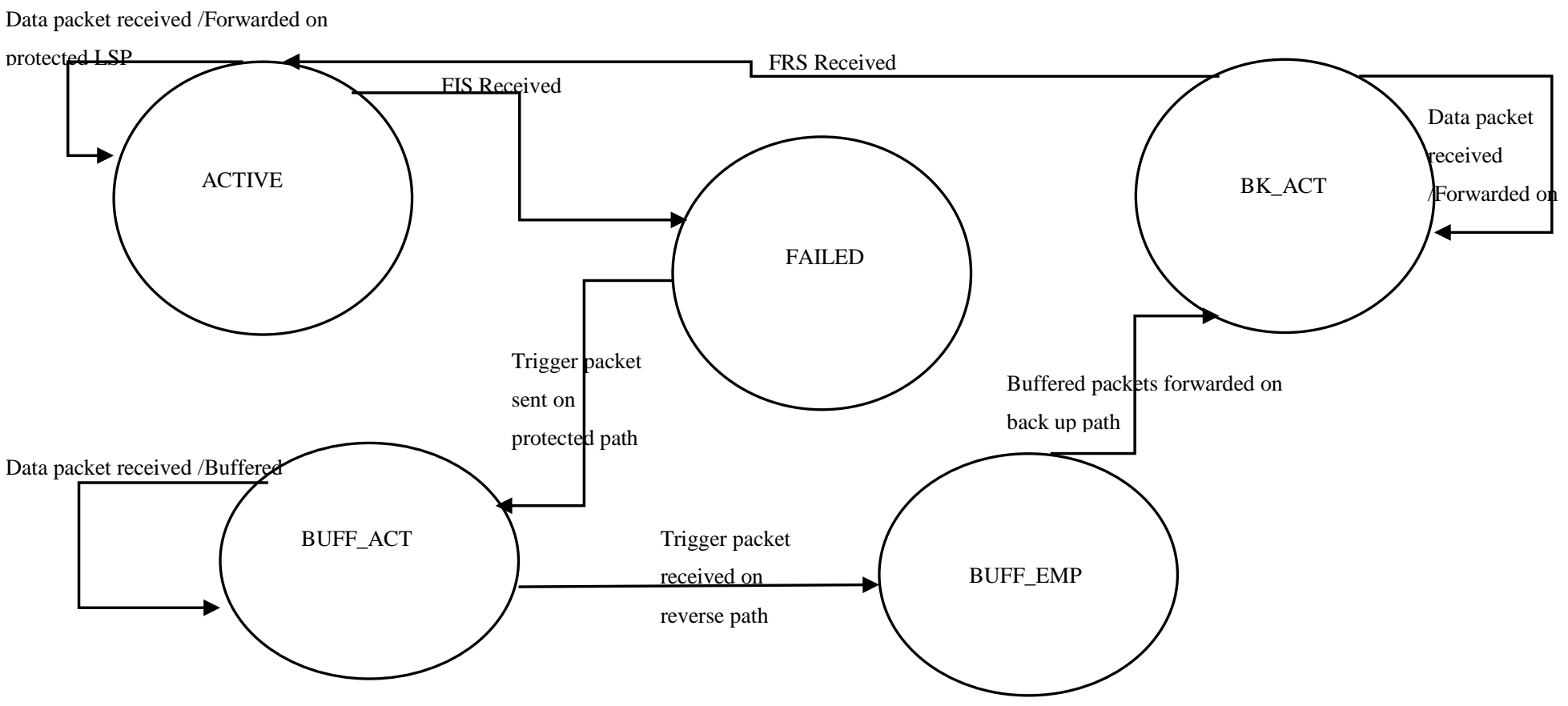

Figure 5.6 State machine diagram of PSL on the protected path 
For our scheme, we also propose the use of additional fields "BKUP_LABEL" and "BKUP_INTERFACE" on PSL nodes. The values of the BKUP_LABEL and

BKUP_INTERFACE were predetermined at the time of LSP creation. Incoming packets at each router are examined based on the incoming label, incoming interface and outgoing LINK STATUS field. LINK STATUS field before failure is ACTIVE. The LSRs check the incoming fields (interface and label) in LFIB and perform appropriate operation (PUSH/POP/SWAP) and places the packets in the queue towards the outgoing interface. It should be noted that each LSR has two buffers: 1) Failure buffer that would be utilized in the case an FIS message is received and 2) Protection buffer, that stores a copy of each packet forwarded on the upstream link for a pre-determined time period, which is equivalent to the threshold of lost liveliness messages. For example, if the liveliness messages are generated every millisecond and three unacknowledged liveliness messages will result in issuance of FIS message then the buffer size is based on $3 \mathrm{~ms}$ *peak aggregated data rate on the protected LSP. It should also be pointed out that this buffer may not necessarily store each packet on the link but can be exclusive to "selected LSPs" on the link. The selected LSPs are those that require guaranteed data delivery such as safety related critical packets. If there is no link failure, then the liveliness message will be acknowledged and the packets will be discarded after this duration.

On the other hand, if a LSR detects link failure, it will generate FIS message towards the PSL, change its "LINK STATUS" field to "FAILED", and forwards all the packets destined for that output link to the reverse path. The forwarded packets include the packets that are saved in the protection buffer. When the first upstream LSR receives the FIS message, it also sets the "LINK STATUS" field to "FAILED", and forwards the FIS message upstream. This LSR then generates and forwards a special packet called "trigger packet" downstream on the protected path. This is the last packet that is forwarded by this LSR on the protected path and serves as the indicator for end of packet stream on the protected path. The LSR also changes its LINK_STATUS field state to "BUFF_ACT" and starts buffering the incoming packets for the protected link locally in its failure buffer. Once all packets have been forwarded on the reverse LSP by the aware-LSR, the trigger packet is also received by the first upstream LSR on the reverse path. Upon receiving the trigger packet, the LSR knows that no more data packets will be forwarded from the aware LSR on the reverse path. The LSR then discards its trigger packet, changes its LINK_STATUS field 
to "BUFF_EMP" and forwards all buffered packets on the reverse path towards PSL. All intermediate LSRs on the protected LSP perform similar operation until the FIS signal reaches PSL. When PSL receives the FIS message, its "LINK STATUS" field is changed from "ACTIVE" to "FAILED". This will enable the "BKUP_LABEL" and "BKUP_INTERFACE" fields at PSL. Moreover, similar to other LSRs on the protected path, the PSL will also send a "trigger" packet downstream on the protected path after changing the LINK_STATUS field to "BUFF_ACT". It should be noted that the packets received on the reverse path are forwarded by the PSL node on the back up path using "BKUP_LABEL" and "BKUP_INTERFACE" field and only the incoming packets will be buffered. Once the trigger packet is received back by the PSL node on the reverse path, it will start forwarding its buffered packets on the backup path. Once the buffer is emptied, the LINK_STATUS field will change to "BK_ACT", the incoming packets will be affixed with the labels identifying the backup LSP and will be forwarded to the output interface of backup tunnel. This scheme preserves the order of the packet stream and also overcomes the unnecessary forwarding of packets inherent in HASKIN' scheme by employing failure buffers at each LSR. Moreover, protection buffering ensures that the QoS data delivery guarantees are met for selected flows. Figure 5.7 shows the timing diagram for message exchange in the proposed algorithm. 


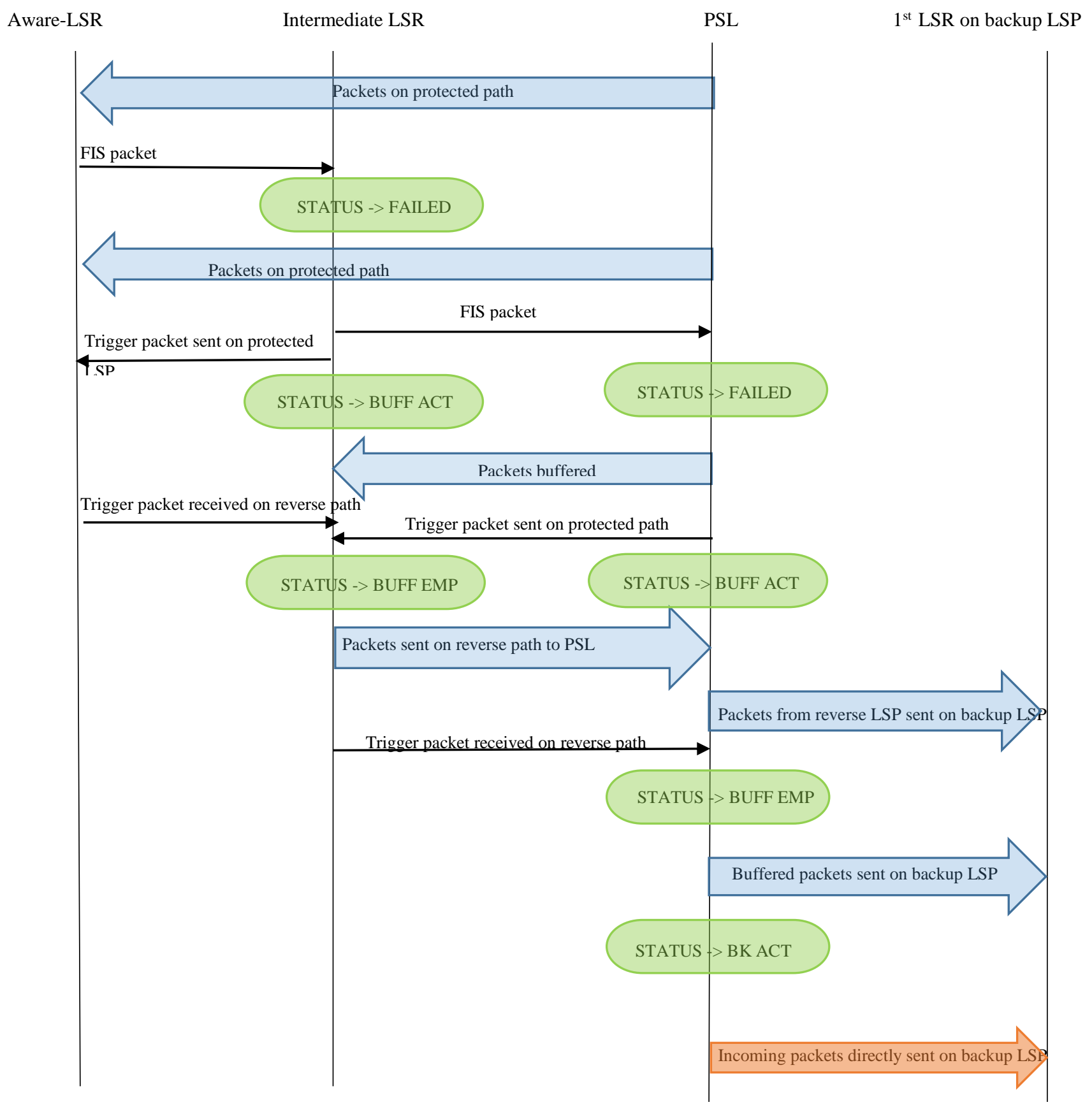

Figure 5.7 Message timing diagram describing the proposed scheme

\subsubsection{LFIB Table Management}

To understand LFIB table management, consider figure 5.8 as an example, which shows the links between CL1, CL2 and two gateways. 

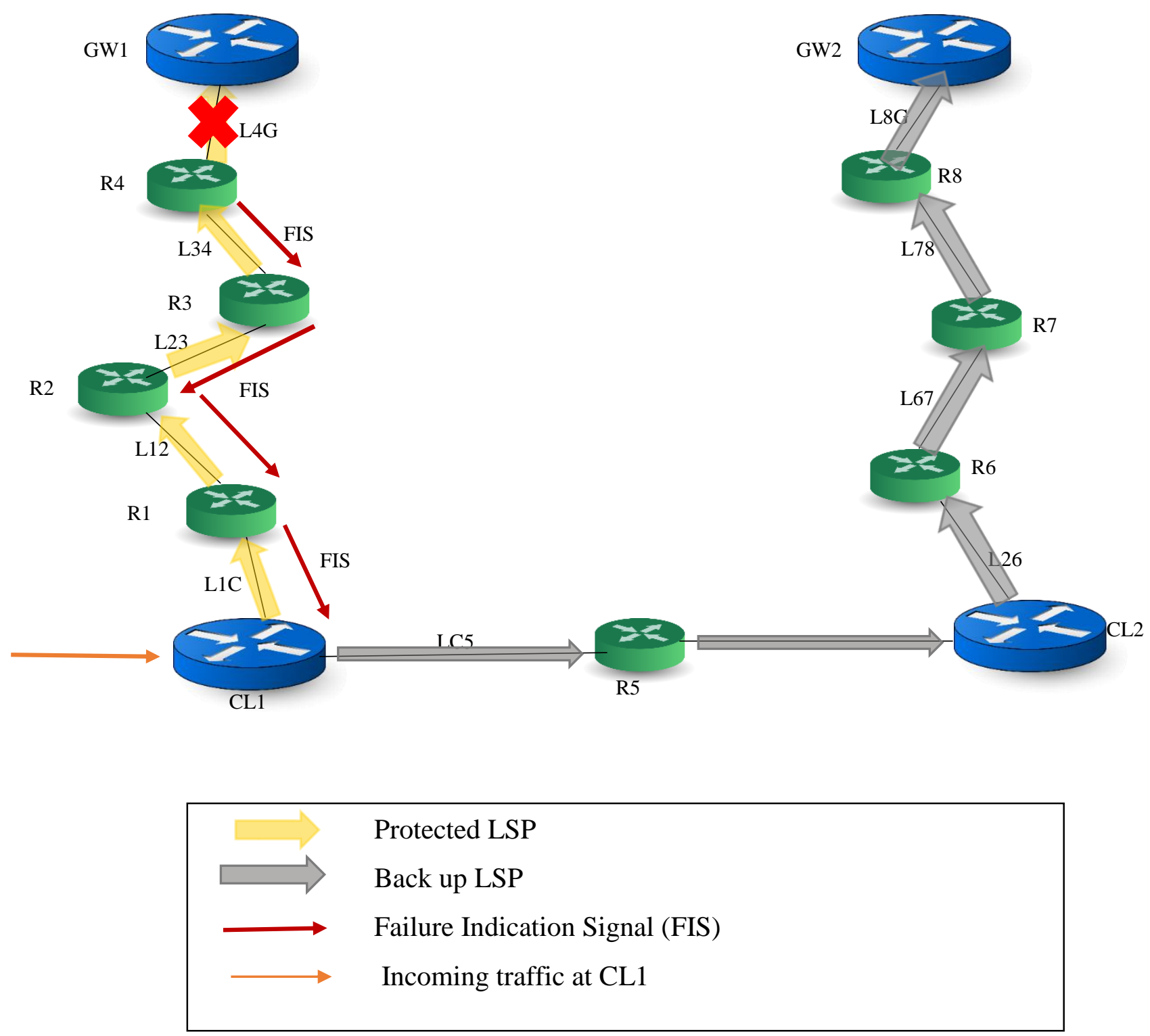

Figure 5.8 Protected LSP and backup LSP originating from CL1

Here the uplink traffic from the hierarchical network directed towards GW1 is forwarded from CL1. For simplification, we assume that the protected LSP shown is carrying only one type of traffic CT1, (that requires bandwidth, delivery and delay guarantees) and the backup LSP is also supporting CT1 traffic. Router R1 to R5 are LSPs on the protected path and router R5 to R8 are the LSR on the backup LSP. The links on the protected path are L1C, L12, L23, L34 and L4G, whereas LC5, L26, L67, L78and L8G are the links between the LSR on the backup path. I1C and IC5 are the output interface for protected and backup path at CL1. Suppose, that the uplink 
packets are forwarded on protected LSP from CL1, these packets are forwarded regularly upto link L34. LSR R4 detects link failure of the link L4G, it changes the LINK STATUS field in its LFIB to FAILED, forwards the stored packets to the protected buffer and incoming packets on the reverse link, generates and propagates FIS in the downlink direction towards CL1, which is the PSL node. The first downstream LSR node, R3 in this case, upon receiving the FIS signal, changes the LFIB LINK STATUS entry to FAILED, and forwards the FIS message to R2. It will then generate and forward a trigger packet on the protected path, changes LINK STATUS to BUFF_ACT and starts buffering the incoming packets from R2. When the trigger packet is received back on the reverse path, R3 changes the LINK STATUS to BUFF_EMP and forwards all buffered packets on the reverse path towards R2 and discards the trigger packet. Each LSR on the path to CL1, upon receiving the FIS signal, performs similar steps. When CL1 receives the FIS signal, it additionally starts sending the packets received through the reverse path on the back up path using the values in the fields BK_UP LABEL and BKUP_INTERFACE. Figure 5.9 shows the CL1 LFIB entries before, during and after the backup LSP is activated.

\begin{tabular}{|l|l|l|l|l|l|l|l|l|}
\hline FEC & $\begin{array}{l}\text { IN- } \\
\text { LABEL }\end{array}$ & $\begin{array}{l}\text { IN- } \\
\text { INTERFACE }\end{array}$ & $\begin{array}{l}\text { OUT- } \\
\text { LABEL }\end{array}$ & $\begin{array}{l}\text { OUT- } \\
\text { INTERFACE }\end{array}$ & $\begin{array}{l}\text { LINK } \\
\text { STATUS }\end{array}$ & $\begin{array}{l}\text { BK_UP } \\
\text { LABEL }\end{array}$ & $\begin{array}{l}\text { BK_UP } \\
\text { INTERFACE }\end{array}$ & ACTION \\
\hline 1 & 5 & 6 & 7 & I1C & ACTIVE & 9 & IC5 & SWAP \\
\hline
\end{tabular}

(A)

\begin{tabular}{|c|c|c|c|c|c|c|c|c|}
\hline FEC & $\begin{array}{l}\text { IN- } \\
\text { LABEL }\end{array}$ & $\begin{array}{l}\text { IN- } \\
\text { INTERFACE }\end{array}$ & $\begin{array}{l}\text { OUT- } \\
\text { LABEL }\end{array}$ & $\begin{array}{l}\text { OUT- } \\
\text { INTERFACE }\end{array}$ & $\begin{array}{l}\text { LINK } \\
\text { STATUS }\end{array}$ & $\begin{array}{l}\text { BK_UP } \\
\text { LABEL }\end{array}$ & $\begin{array}{l}\text { BK_UP } \\
\text { INTERFACE }\end{array}$ & ACTION \\
\hline 1 & 5 & 6 & 7 & $\mathrm{I} 1 \mathrm{C}$ & BUFF_ACT & 9 & IC5 & $\begin{array}{l}\text { Buffering } \\
\text { incoming } \\
\text { packets }\end{array}$ \\
\hline
\end{tabular}

(B)

\begin{tabular}{|c|c|c|c|c|c|c|c|c|}
\hline FEC & $\begin{array}{l}\text { IN- } \\
\text { LABEL }\end{array}$ & $\begin{array}{l}\text { IN- } \\
\text { INTERFACE }\end{array}$ & $\begin{array}{l}\text { OUT- } \\
\text { LABEL }\end{array}$ & $\begin{array}{l}\text { OUT- } \\
\text { INTERFACE }\end{array}$ & $\begin{array}{l}\text { LINK } \\
\text { STATUS }\end{array}$ & $\begin{array}{l}\text { BK_UP } \\
\text { LABEL }\end{array}$ & $\begin{array}{l}\text { BK_UP } \\
\text { INTERFACE }\end{array}$ & ACTION \\
\hline 1 & 5 & 6 & 7 & I1C & BUFF_EMP & 9 & IC5 & $\begin{array}{l}\text { Send } \\
\text { buffered } \\
\text { packets } \\
\text { on the } \\
\text { backup } \\
\text { LSP }\end{array}$ \\
\hline
\end{tabular}

\begin{tabular}{|l|l|l|l|l|l|l|l|l|}
\hline FEC & IN- & IN- & OUT- & OUT- & LINK & BK_UP & BK_UP & ACTION \\
& LABEL & INTERFACE & LABEL & INTERFACE & STATUS & LABEL & INTERFACE & \\
\hline
\end{tabular}




\begin{tabular}{|l|l|l|l|l|l|l|l|l|}
\hline 1 & 5 & 6 & 7 & I1C & BK-ACT & 9 & IC5 & $\begin{array}{l}\text { Send } \\
\text { incoming } \\
\text { packets } \\
\text { on the } \\
\text { backup } \\
\text { LSP }\end{array}$ \\
\hline
\end{tabular}

(D)

\begin{tabular}{|l|l|l|l|l|l|l|l|l|}
\hline FEC & $\begin{array}{l}\text { IN- } \\
\text { LABEL }\end{array}$ & $\begin{array}{l}\text { IN- } \\
\text { INTERFACE }\end{array}$ & $\begin{array}{l}\text { OUT- } \\
\text { LABEL }\end{array}$ & $\begin{array}{l}\text { OUT- } \\
\text { INTERFACE }\end{array}$ & $\begin{array}{l}\text { LINK } \\
\text { STATUS }\end{array}$ & $\begin{array}{l}\text { BK_UP } \\
\text { LABEL }\end{array}$ & $\begin{array}{l}\text { BK_UP } \\
\text { INTERFACE }\end{array}$ & ACTION \\
\hline 1 & 5 & 6 & 7 & I1C & ACTIVE & 9 & IC5 & SWAP \\
\hline
\end{tabular}

(E)

Figure 5.9 LFIB at CL1 (A) Before Fault Detection (B) After fault is detected and buffer is activated (C) Buffer is being emptied (D) Incoming packets are directly sent to back up LSP (E) Protected LSP is restored

Once all of the packets from the buffer are sent out on the backup link, the LINK STATUS field is changed to BK_ACT and the incoming packets at the CL1 are directly sent out on the backup path. Once the link is restored, the LSR (in this case R4) generates FRS signal directed towards CL1. Each router on the way from R4 to CL1 changes its LINK STATUS entry to ACTIVE and finally the signal is received by CL1. CL1 changes LINK STATUS to ACTIVE and starts forwarding the uplink packets on the protected LSP again. Figure 5.10 shows the timing sequence of buffering and packet forwarding mechanism at CL1. 

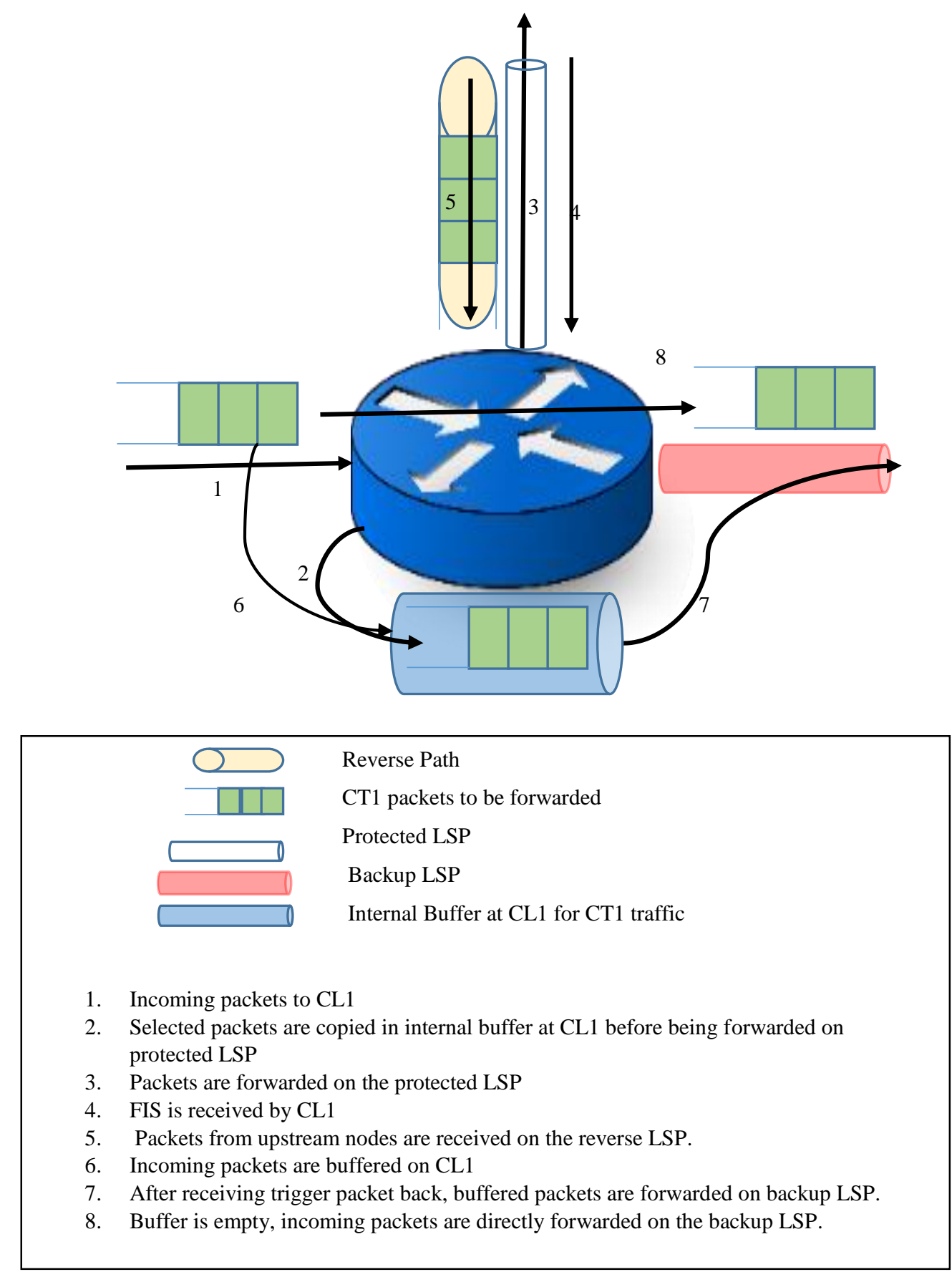

Figure 5.10 Timing sequence at CL1 during fault detection and LSP redirection

Compared to Haskin's scheme, this scheme significantly reduces the packet delay on the failed link. To understand this, please be reminded that in Haskin's scheme the packets have to traverse the path length between PSL and aware LER twice since they kept on propagating on the failed protected link until the PSL receives the first packet on the reverse path and becomes aware of 
the link failure. As the number of hops between the PSL and aware LER increases, this delay can become significantly large, which would result in degraded QoS during path restoration.

Compared to Makam's scheme, the proposed scheme eradicates packet loss and is more suitable for applications that require hard QoS delivery guarantees.

\subsection{Performance Evaluation}

The proposed scheme was evaluated for total restoration delay and the utilized minimum buffer size. The terminologies used in the derivation of mathematical relations are as follows:

$\mathrm{T}_{\mathrm{TR}}=$ Transmission delay on the link

$\mathrm{T}_{\mathrm{PR}}=$ Propagation delay on the link

$\mathrm{d}=$ distance between two adjacent LSRs= link length

$\mathrm{B}_{\mathrm{wl}}=$ Available LSP bandwidth for the protected link

$\mathrm{P}_{\mathrm{CM}}=$ Combined rate of the aggregated traffic on the protected LSP

$\mathrm{S}_{\mathrm{D}}=$ Data Packet Size

$\mathrm{S}_{\mathrm{C}}=\mathrm{FIS}$ and trigger packet size

$\mathrm{T}_{\mathrm{RT}}=$ Complete restoration time

$\mathrm{T}_{\mathrm{FD}}=$ Fault detection time

$\mathrm{T}_{\mathrm{SW}}=$ Switching time

$\mathrm{N}=$ Number of LSRs in protected path

\subsubsection{Path Restoration Time}

The complete restoration time is the aggregation of fault detection time and switching time. Here fault detection time is defined as the total time it takes the FIS message generated by the awareLSR node to reach the next downstream node and the round trip delay of the trigger packet generated by the next downstream node.

Hence,

$$
T_{F D}=3 * T_{L D}
$$

$\mathrm{T}_{\mathrm{LD}}$ is the link delay and is defined as the sum of transmission delay, propagation delay and processing delay at each node.

Hence, 


$$
\begin{gathered}
T_{L D}=\mathrm{T}_{T R}+T_{P R}+T_{P R O C} \\
T_{L D}=\frac{S_{X *}}{B_{w l}}+L_{w l}+T_{P R O C} \\
\text { Note: } S_{x *} \text { can be SD or SC depending on the packet type }
\end{gathered}
$$

The switching delay is the time taken by each intermediate node to empty its buffer. This delay consists of 1) the delay of aware-LSR to empty its failure buffer which is directly dependent on the FIS 2) and the buffer emptying delay of other intermediate nodes which is dependent on the two way link delay experienced by the trigger packets.

The aware-LSR forwards the packets received by the next downstream node to the reverse path after generating the FIS. The time taken by the FIS to reach the next downstream LSR is $\mathrm{T}_{\mathrm{LD}}$. During this period, the downstream node is still forwarding the packets to aware-LSR. Considering packets are arriving at the rate of $\mathrm{P}_{\mathrm{CM}}$ and are forwarded on the reverse path equals to the LSP bandwidth $\mathrm{B}_{\mathrm{wl}}$,

$$
T_{A W A R E}=T_{L D} * \frac{P_{C M}}{B_{w l}}
$$

Every other intermediate LSR on the protected path upon receiving the FIS message, sends a trigger packet and stores the incoming packets until the trigger packet is received back on the reverse path. Hence, the storing period is $2 * \mathrm{~T}_{\mathrm{LD}}$ (two-way delay experienced by the trigger packet). As before, considering the packets are arriving at the rate of $\mathrm{B}_{\mathrm{CM}}$ and are forwarded on the reverse path equals to the LSP bandwidth $\mathrm{B}_{\mathrm{wl}}$,

$$
T_{L S R}=2 * T_{L D} * \frac{P_{C M}}{B_{w l}}
$$

The sum of delays in the intermediate LSRs is given as:

$$
T_{L S R \text { Com }}=\sum_{k=1}^{k=N-2} k T_{L S R}
$$

Hence,

$$
T_{S W}=T_{A W A R E}+T_{L S R \text { Com }}
$$

And,

$$
T_{R T}=T_{F D}+T_{S W}
$$

Hence,

$$
T_{R T(P R O P)}=3 * T_{L D}+T_{L D} * \frac{P_{C M}}{B_{w l}}+2 * T_{L D} * \frac{P_{C M}}{B_{w l}} \sum_{k=1}^{k=N-2} k
$$

On the other hand, RFR scheme experiences additional delay due to each node transmitting tagged packets to the next upstream node. Hence, 


$$
T_{R T(R F R)}=3 * T_{L D}+2 * T_{L D} * \frac{P_{C M}}{B_{w l}} \sum_{k=1}^{k=N-1} k+T_{L D} * \sum_{k=1}^{k=N-1} k
$$

The values of the parameters chosen for mathematical simulative analysis are listed in table 5.1.

\begin{tabular}{|c|c|}
\hline Parameters & Values \\
\hline$P_{C M}$ & $500 \mathrm{Mbps}$ \\
\hline$B_{w l}$ & $2.5 \mathrm{Gbps}$ \\
\hline $\mathrm{S}_{\mathrm{D}}$ & 1500 bytes \\
\hline $\mathrm{S}_{\mathrm{C}}$ & $20 \mathrm{bytes}$ \\
\hline $\mathrm{L}_{\mathrm{wl}}$ & $5 \mu \mathrm{s} / \mathrm{km}$ \\
\hline $\mathrm{d}$ & $5 \mathrm{~km}$ \\
\hline $\mathrm{T}_{\mathrm{PROc}}$ & $1 \mu \mathrm{s}$ \\
\hline
\end{tabular}

Table 5.1: The values of the parameters used for analysis

The comparison between analytical and simulative results for the proposed scheme and RFR scheme is shown in figure 5.11. The simulation models were developed in Optimized network engineering tool (OPNET), currently known as Riverbed Modular. Each simulation value is obtained by averaging 25 consecutive simulations with different seeds.

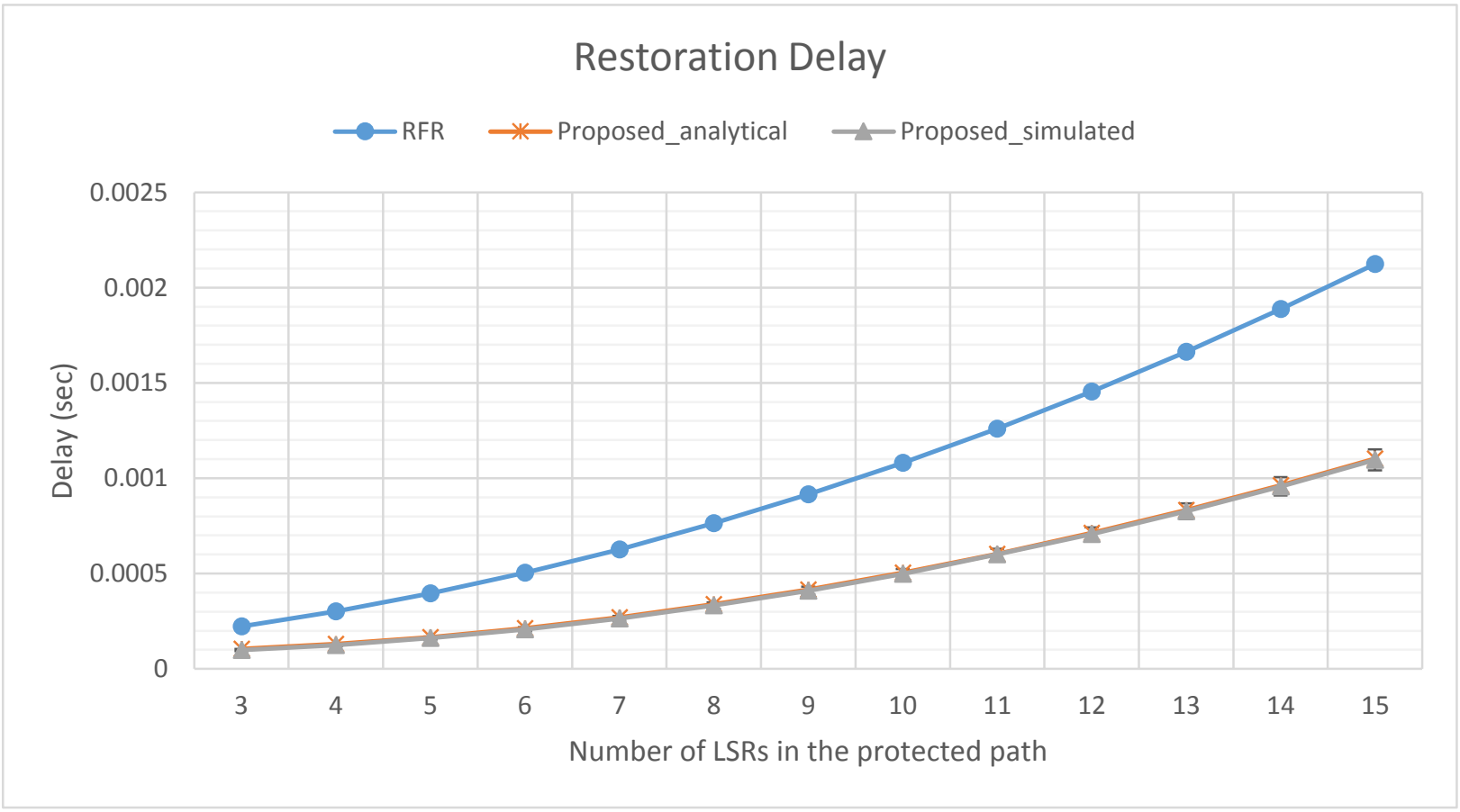

Figure 5.11 Total restoration delay compared to the number of LSRs in the protected path for the proposed scheme and RFR scheme 


\subsubsection{Buffer Size Analysis}

The buffering at PSL node starts when it receives the FIS packet and ends when it receives the trigger packet back. The time taken for the FIS packet to propagate to PSL node is:

$$
T_{\text {first }}=T_{L D} *(N-1)
$$

And the duration during which the packets are stored at the PSL node is equal to the difference between the restoration delay and the time PSL receives the first packet.

Hence,

$$
T_{P S L-S T}=T_{R T(P R O P)}-T_{\text {first }}
$$

At the arrival rate of $P_{C M}$, the buffer required at PSL is

$$
B u f f_{P S L}=T_{P S L-S T} * P_{C M}
$$

And the buffer size for each intermediate LSR is

$$
B u f f_{L S R}=2 * T_{L D} * P_{C M}
$$

Figure 5.12 provides the comparison between the minimum buffer size required at the PSL node for various number of LSRs in the protected path between the proposed and RFR scheme.

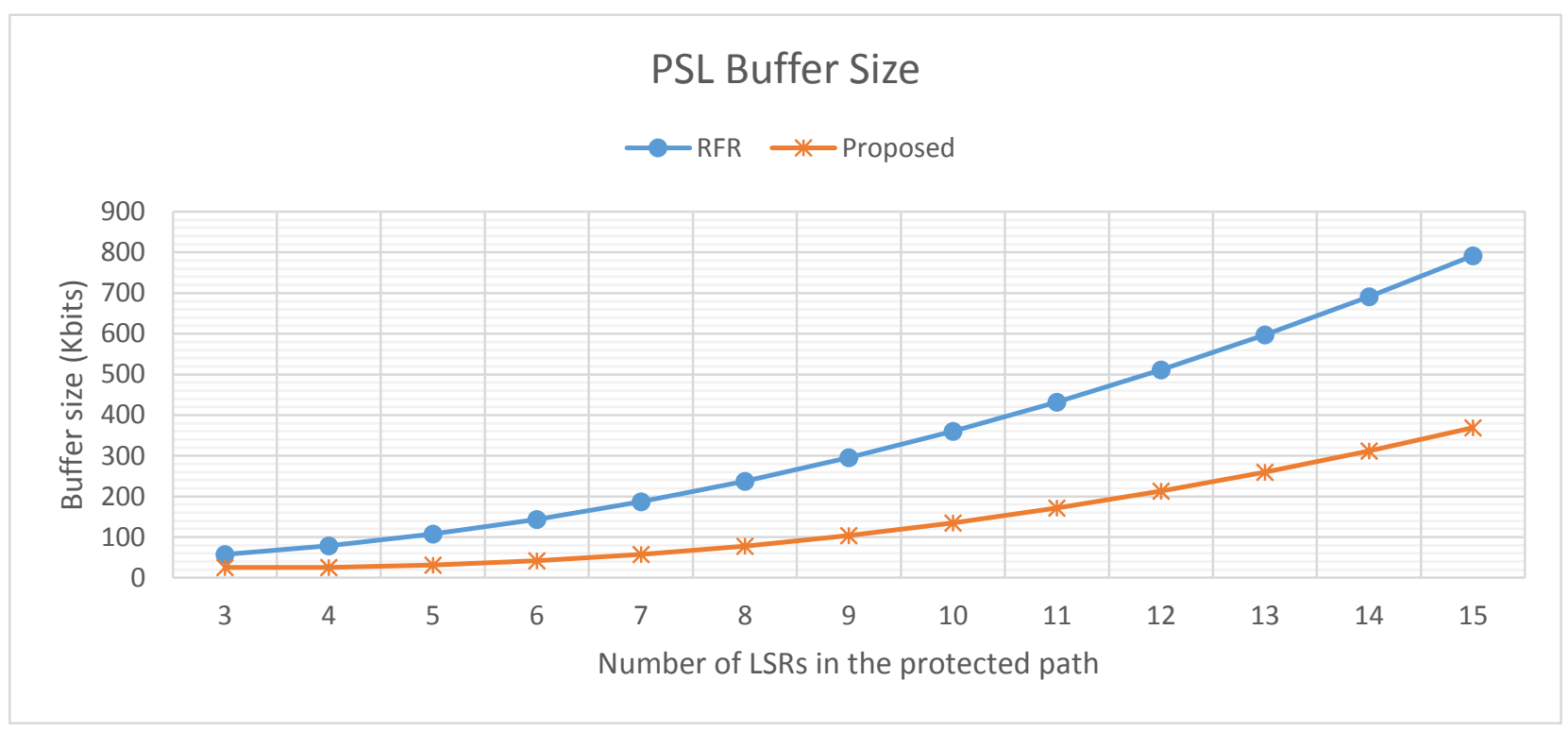

Figure 5.12 Buffer size required at PSL compared to the number of LSRs in the protected path

These results demonstrate that the proposed scheme has shorter path restoration time compared to RFR scheme. This is due to the fact that each node in RFR scheme generates and forwards the 
tagged packet to the next upstream node after it empties its buffer. The path restoration time of the proposed scheme is 50\%-60\% lesser than RFR scheme for 3-15 intermediate routers. Similarly, the minimum buffer size required at PSL node by the proposed scheme is $50 \%-70 \%$ smaller than RFR scheme as it has dependency on path restoration delay and the number of intermediate LSRs.

In addition to the improvement in path restoration delay, the proposed scheme addresses the ambiguities and limitations of the RFR scheme. RFR scheme uses packet tagging, which makes it unsuitable to be implemented in DiffServ aware MPLS architecture. The proposed scheme uses a special trigger packet for achieving preservation of packet sequence ordering. This approach makes the scheme suitable for both IntServ and DiffServ aware MPLS architectures. Secondly, the RFR scheme proposes to utilize the local buffers at each node for storing a copy of each outgoing packet. The scheme does not address the procedure adopted for discarding stored packets when there is no link failure. The proposed scheme, on the other hand, provides a threshold based limitation to the number of packets that would be stored in the local buffer and its periodic packet discard procedures. RFR scheme also proposes that the LSR has tagged the packet will take two different actions while forwarding it on the reverse path. In the case buffer is full, the tagged packet is forwarded "as is" on the reverse path after the buffer is emptied, to indicate to the next node that no more packets are expected on this link. However if the buffer is empty, the tagged packet is forwarded as normal packet after re-setting the EXP bit to 0. The purpose of this operation is unclear and raises serious questions on the validity of the scheme. Recall that purpose of the tagged packet is to act as the trigger initiating the buffer emptying operation at each LSR the packet passes through on its way to PSL node. If the tagged packet is forwarded as normal packet with unset bit, the upstream LSR will consider it as normal packet on the reverse path hence it will keep on buffering the incoming packets and will not initiate sending the already buffered packets back upstream using the reverse path. The proposed scheme, however, uses trigger packets that are generated by each node individually and do not employ forwarding of the same packet by all of LSRs. Hence, we conclude that the proposed scheme is more suitable for a MPLS based architecture that employs static LSPs for link failure protection and restoration. 


\section{Chapter 6: CONCLUSION \& FUTURE WORK}

\subsection{Conclusion}

The upcoming era of $5 \mathrm{G}$ has opened up new areas of research in the context of devices, systems and networks. The $5 \mathrm{G}$ of communication promises to support and serve communication needs of billions of devices with high data rate transfer, low latency and provide high bandwidth availability.

With these goals in mind, this work evaluated the feasibility of emerging technologies, VLC and mmWave, for use in $5 \mathrm{G}$ vehicular communication networks. To support seamless connectivity and continued QoS provision, a hierarchical MPLS based micro-mobility architecture was proposed that is also resilient to link failures. The contribution of this research is summarized as below:

- A detailed comparative performance analysis is carried out between the emerging technologies of VL and mmWave in the context of supporting ultra dense $5 \mathrm{G}$ vehicular communication networks. The analytical results address the behaviour VL and mmWave for various cell sizes. This analysis includes the effect of weather conditions. It was found that VL and mmWave exhibit similar behaviour in the outdoor LOS environment with VL experiencing more attenuation in heavy snow, whereas mmWave undergoes notable attenuation at certain frequencies in heavy rain. This result indicates that deployment decisions should be dependent on the snow versus rain weather history, the area of deployment has.

- A detailed comparative design analysis between VL and mmWave was carried out. A dual link VVLC system was evaluated for VVLC system, where the wide beam links supports low data rate communication and narrow beam links support high data rate communication. This system was also analyzed for path loss and the required increase in transmission power when experiencing adverse weather conditions. A LOS mmWave vehicular communication system based on the street canyon model was adopted for the link budget analysis. It was found that for the same cell size, implementation and maintenance of a mmWave vehicular system is considerably more costly compared to VVLC system. 
- This work proposes a hierarchical MPLS based micro-mobility architecture that is based on pre-established LSPs and provides QoS guarantees by integrating MPLS with IntServ architecture. The three-layer hierarchical architecture minimizes handoff latency and registration cost when the mobile user is changing subnets. This scheme also utilizes label stacking by LERs to minimize packet forwarding delay. The analytical and simulation results confirm the improvement in handoff latency and eradication of packet loss in the proposed architecture compared to existing solutions, hence making the proposed architecture an excellent candidate for 5G VVLC access networks.

- The proposed hierarchical MPLS scheme is based on static LSP configuration and hence may fail to fulfil performance guarantees in case of link failures. Since link failures in access networks may cause significant delays and packet loss, this would directly affect broadband and real-time applications and services. Hence to improve the survivability of the MPLS architecture, a fast and efficient rerouting scheme was proposed and integrated into the hierarchical MPLS architecture. This scheme is based on distributed recovery procedures with fast rerouting, utilizing pre-established LSPs. The proposed scheme reroutes traffic on the protected LSPs over pre-established back up LSPs in case of link failures while maintaining QoS guarantees. The scheme was compared against existing schemes and found to outperform the existing schemes in terms of restoration delay with no packet loss and eliminates packet disordering. In addition to link failures, this scheme may also be adopted to overcome network congestion issues, since it is based on notification signals. Similar to failure indication signals, LSR can also issue congestion notification signal to alert the network in case of congestion and protected flows can be rerouted on alternate LSPs to provide load balancing on the network. The proposed algorithm was evaluated for total restoration delay and buffer sizes required at the PSL and intermediate LSRs on the protected path. Both analytical and simulation results show an improvement on the restoration delay and buffer size requirement compared to existing schemes. The proposed algorithm also eliminated packet disordering and packet loss. 


\subsection{Future Work}

This work qualifies as an initial research in the direction of extensive research that is required for practical implementation of $5 \mathrm{G}$ vehicular communication networks with MPLS based micromobility. There are many research areas that can be explored further in-depth. Some of those areas are discussed henceforth.

- The evaluation of VVLC and mmWave outdoor system has been carried out based on existing empirical and software models. These models are mostly developed and simulated using sophisticated tools. However, extensive measurement campaigns are still missing from open literature. Hence measurement campaigns to affirm the behaviour of the outdoor communication channel is a potential area of research.

- This work evaluates the effect of adverse weather on the VL and mmWave signal in LOS outdoor environment. However, additional attenuation factors such as loss due to foliage, exterior building walls and road surface reflections in specific communication environments such as downtown, suburban areas and rural areas should be taken up for further study.

- The hierarchical MPLS based micro-mobility network architecture proposed in this work is based on static pre-established LSPs to reduce handoff latency. Another approach is to establish the micro-mobility architecture using dynamic LSPs. Dynamic LSPs have the advantage of utilizing optimum resources and better survivability at the expense of higher latency. A thorough study examining the trade-offs using different traffic characteristics and topologies is another potential area of research.

- For the fast restoration mechanism in case of link failures, the back-up links are also assumed to be pre-established. Dynamic re-configurability in the context of highly mobile and intermittent network is another research area that should be pursued. Furthermore, the implementation of the proposed hierarchical MPLS micro-mobility architecture along with fast failure rerouting in hardware-based platform would be useful to observe the real behavior, limitations and required modifications. Furthermore, the implementation of the proposed hierarchical MPLS micro-mobility architecture along with fast failure rerouting in hardware-based platform would be useful to observe the real behavior, limitations and required modifications. 
- Segment routing (SR) is an emerging source-routing architecture that aims to simplify and optimize packet forwarding in IP networks. In segment routing, the network nodes process and forward the packet based on specific routing instructions called "segments". Segments can represent network links, network nodes or services and are identified by a segment identifier (SID). The SIDs are learned across the network using IGP extensions for SR. In IPV4 / MPLS networks, the labels are used as SIDs. In IPV6 networks, segment routing version 6 (SRV6) uses the IPV6 destination header to encode the SID value. Multiple SIDs can be stacked utilizing the IPV6 destination header and SR extension header (if required) at the source to define a desired engineered path. Since SR leverages traditional routing protocols for control plane learning, it greatly simplifies the network architecture and eliminates the need to run separately path set up and label distribution protocols such as RSVP-TE and LDP in MPLS networks. The work presented in the thesis can be pursued with segment routing and results can be evaluated for scalability advantages and reduced complexity in the architecture. 


\section{REFERENCES}

[3GP-10] 3GPP, “Further Advancements for E-UTRA Physical Layer Aspects, ’ TR 36.814 (release 9), 2010.

[3GP-14] 3GPP TR 36.836; "Mobile Relay for Evolved Universal Terrestrial Radio Access". Website: http://www.tech-invite.com/3m36/tinv-3gpp-36-836.html. Last accessed $9^{\text {th }}$ November, 2019.

[3GP-17] 3GPP, “Architecture Enhancements for V2X Services (V14.3.0, Release 14),” 3GPP, Technical Report 23.285, June 2017.

[5GP-15] 5GPPP Automotive working group; "5G Automotive Vision"; White paper published on $20^{\text {th }}$ October 2015, Website: https://5g-ppp.eu/wp-content/uploads/2014/02/5G-PPP-WhitePaper-on-Automotive-Vertical-Sectors.pdf. Last accessed $13^{\text {th }}$ June 2019.

[ABB-11] M. K. Abbas, M.N. Karsiti, M. Napiah, B. B. Samir; “Traffic Light Control Using VANET System Architecture" IEEE National Postgraduate Conference (NPC), Kuala Lumpur, Malaysia, 19th -20th September 2011, pp 1-6.

[AGE-20] A.Gyasi-Ageyi; "Wireless Internet of Things: Principles and Practice" World Scientific Publishing, April 2020.

[AHM-08] I. Ahmed, D. Habibi "A Novel Mobile WiMAX Solution for Higher Throughput," Proceedings of 16th IEEE International Conference on Networks (ICON), New Delhi, India, $12^{\text {th }}-14^{\text {th }}$ December 2008, pg. 1-5.

[AKD-14] M. Akdeniz, Y.Liu, M.K.Samimi, S.Sun, S.Rangan, T.S.Rappaport, E.Erkip; "Millimeter Wave Channel Modeling and Cellular Capacity Evaluation," IEEE Journal in Selected Areas of Communication, Vol. 32, Issue. 6, June 2014, pp. 1164-1179.

[AKI-04] M. Akiba, K. Wakamori, S. Ito; "Measurement of Optical Propagation Characteristics for Free - Space Optical Communications During Rainfall", IEICE Transactions on Communications, Vol.E87 - B, Issue 7, 2004. pp.2053 - 2056.

[AKI-08] M. Akiba, K. Ogawa, K. Walkamori, K. Kodate, S. Ito, “ Measurement And Simulation of The Effect Of Snow Fall On Free Space Optical Propagation”, Applied Optics, Vol. 47, Issue 31, 2008. pp. 5736-5743.

[ALA-16] S.E.Alavi, M.R.K. Soltanian, I.S. Amiri, M.Khalily; "Towards 5G: A Photonic Based Millimeter Wave Signal Generation for Applying in 5 G Access Fronthaul" Scientific Reports, Vol.6, Article No. 19891. 2016. 
[ALT-84] E.E.Altshuler; “A Simple Expression for Estimating Attenuation by Fog at Millimeter Wavelengths"; IEEE Transactions on Antennas and Propagation, Vol.32, Issue.7, July 1984, pp.757-758.

[AMO-08] Aerodrome Meteorological Observation and Forecast Study Group (AMOFSG); "Snowfall Rate Thresholds for Light, Moderate and Heavy", $7^{\text {th }}$ Meeting, Montreal, Canada, September 2008. pp 1-3.

[AND-14] J.G.Andrews; “What will $5 G$ be?”, IEEE Journal on Selected Areas in Communication, Vol. 32, Issue 6, June 2014, pp 1065-1087.

[AND-17] I.Andrews; "The Environmental Benefits of Driverless Cars"; Published on $31^{\text {st }}$ August, 2017. Website: https://greenerideal.com/news/vehicles/driverless-cars-environmentalbenefits/\#Traffic_Congestion. Last accessed $4^{\text {th }}$ July, 2019.

[ANT-17] Antewrap.Manag.School; "New Alternative for Commuting. The 'Office On Wheels' That Turns Travel Time Into Work Time”; Published on $6^{\text {th }}$ June 2017. Website: https://www.antwerpmanagementschool.be/en/press-post/new-alternative-commuting-officewheels-turns-travel-time-work-time/. Last accessed 20 June 2019.

[ARA-08] S. Arai, S. Mase, T. Yamazato, T. Yendo, T. Fujii, M. Tanimoto, and Y. Kimura, "Feasible Study Of Road-To-Vehicle Communication System Using LED Array And High-Speed Camera," in Proceedings of $15^{\text {th }}$ World Congress on Intelligent Transportation Systems (WCITS'08), New York, NY, USA, $16^{\text {th }}-20^{\text {th }}$ November 2008, pp. 1-12.

[ARA-13] G. Araniti, C. Campolo, M. Condoluci, A. Iera, A. Molinaro; "LTE for Vehicular Networking: A Survey"; IEEE Communications Magazine, Vol.51, Issue. 5, May 2013.pp. 148157.

[ASE-17] P.Asel; “The Road to Transportation-As-A-Service”;Published on June 19 th $^{\text {th }} 2017$. Website: https://medium.com/reflections-by-ngp/the-road-to-transportation-as-a-serviceb72c3fe780ec. Last accessed $20^{\text {th }}$ June 2019.

[AUT-18] The Economist, “Autonomous-Vehicle Technology Is Advancing Ever Faster", Website: https://www.economist.com/news/special-report/21737420-making-vehicles-drive-themselveshard-getting-easier-autonomous-vehicle-technology. Last accessed 10 ${ }^{\text {th }}$ July, 2019.

[AWD-01] D. Awduche; "RSVP-TE: Extensions to RSVP for LSP Tunnels," IETF RFC3209, Dec. 2001. Website: https://tools.ietf.org/html/rfc3209. Last accessed $4^{\text {th }}$ November 2018.

[AXR-14]AXRTEK; "MOMO the World's Fastest VLC Device"; Website: www.axrtek.com/momo.html. Last accessed 22 ${ }^{\text {nd }}$ November 2019.

[AYA-14] O. El Ayach, S. Rajagopal, S. Abu-Surra, Z. Pi, R. Heath, "Spatially Sparse Precoding In Millimeter Wave MIMO Systems, " IEEE Transactions on Wireless Communication, Vol. 13, Issue 3, March 2014. pp. 1499-1513. 
[BAD-16] D.Bader; "Everything you need to know about 4G/LTE Frequency Band in Canada"; 2016. Website: https://www.androidcentral.com/everything-you-need-know-about-4g-lte-canada. Last accessed $27^{\text {th }}$ September 2019.

[BAL-10] A. Balasubramanian, R. Mahajan, and A. Venkataramani, "Augmenting Mobile $3 G$ Using Wifi," in Proceedings Of The 8th International Conference On Mobile Systems, Applications, And Services (MobiSys), June 2010, pp. 209-222.

[BAR-94] J. R. Barry, "Wireless Infrared Communications", Published by Kluwer Academic Publisher, 1994.

[BED-13] A. Bedekar and R. Agrawal, “Optimal Muting and Load Balancing for eICIC, ” in Proceedings of 11th International Symposium and Workshops on Modeling and Optimization in Mobile, Ad Hoc and Wireless Networks (WiOpt), Tsukuba Science City, Japan, $13^{\text {th }}-17^{\text {th }}$ May 2013, pp. 280-287.

[BEH-05] N.S. Behura; "The New ITE Light-Emitting Diode Traffic Signal Specifications—A Guide for Purchasers"; ITE Journal, November 2005. pp 38-40.

[BEN-11] A. Benslimane, T. Taleb, R. Sivaraj; "Dynamic Clustering-Based Adaptive Mobile Gateway Management In Integrated VANET-3G Heterogeneous Wireless Networks," IEEE Journal on Selected Areas in Communications, Vol. 29, No. 3, March 2011, pp. 559 -570.

[BLA-98] S.Black, K.Nichols, F.Baker, D.Black; "Definition of the Differentiated Services Field (DS Field) in the IPv4 and IPv6 Headers," IETF RFC 2474. Website:

https://tools.ietf.org/html/rfc2474. Last accessed $5^{\text {th }}$ February 2020.

[BLE-13] A. Bleicher; "Telecom Wireless News Millimeter Waves May Be the Future of $5 G$ Phones"; June 2013. Website: http://spectrum.ieee.org/telecom/wireless/millimeter-waves-maybethe-future-of-5g-phones. Last accessed $26^{\text {th }}$ September 2019.

[BUR-14] H. Burchardt, N. Serafimovski, D. Tsonev, S. Videv, H. Haas; "VLC: Beyond PointTo-Point Communication", IEEE Communications Magazine, Vol 52, Issue 7, July 2014. pp 98105.

[BUS-09] Business Insider; "Visible Light Communications Consortium (VLCC) Released Visible Light Communication Standard Based on IrDA Core Specification". Website: https://www.businesswire.com/news/home/20090312006284/en/Visible-Light-CommunicationsConsortium-VLCC-Released-Visible. Last accessed 3 ${ }^{\text {rd }}$ September 2019.

[CAI-16] AM. Cailean, M. Dimian, V. Popa, L.Chassagne, B.Cagneau; "Novel DSP Receiver Architecture for Multi-Channel Visible Light Communications In Automotive Applications"; IEEE Journal on Sensors, Vol. 16, Issue 10, Feb 2016, pp 3597-3604. 
[CAI-17] AM. Cailean, M. Dimian; "Impact of IEEE 802.15.7 Standard on Visible Light Communication Usage in Automotive Applications"; IEEE Communication Magazine, Vol 55, Issue 4, 2016, pp 169-175.

[CAM-00] A.T. Campbell, J. Gomez, S. Kim, A.G. Valko, Chieh-Yih Wan, Z.R. Turanyi; "Design, implementation, and evaluation of cellular IP"; IEEE Personal Communication, Volume 7, Issue 4, August 2000. pp 42-49.

[CAM-02] A.T.Campbell, J.Gomez, S.Kim, C.Y.Wan, Z.R.Turanyi, A.G.Valko; "Comparison of IP Micro-Mobility Protocols," IEEE Wireless Communications Magazine, Vol.9, No.1, Feb 2002. pp 72-82.

[CBC-17] CBC-NEWS; "Driverless Car Test in Ottawa a Canadian first"; Website: http://www.cbc.ca/news/canada/ottawa/driverless-car-test-ottawa-canadian-first-1.4351976. Last accessed $23^{\text {rd }}$ May 2019.

[CEA-04] CEATEC-04; “CEATEC JAPAN 2004”. Website: https://gizmodo.com/ceatec-japan2004-day-4-23076. Last accessed $3^{\text {rd }}$ September 2019.

[CET-11] R.Cetin; "RFC 6445 - Multiprotocol Label Switching Traffic Engineering"; 2011. Website: https://tools.ietf.org/html/rfc6445. Last accessed $4^{\text {th }}$ November 2019.

[CHE-04] H.Chen, J. Dai,Y.Liu; "Effect of Fog And Clouds On The Image Quality In Millimeter Communications"; International Journal of Infrared and Millimeter Waves, Vol.25, Issue.5,May 2004. pp. 749-757.

[CHE-13] C.Chen, Y.Zhu; "Augmenting Vehicular $3 G$ Users through Inter-vehicle Communications," IEEE Wireless Communications and Networking Conference (WCNC), Shanghai, China, $7^{\text {th }}-10^{\text {th }}$ April 2013, pp 1651-1656.

[CHE-14] X. Cheng, C. X. Wang, B. Ai, H. Aggoune; "Envelope Level Crossing Rate and Average Fade Duration of Non-Isotropic Vehicle-to-Vehicle Ricean Fading Channels" ; IEEE Transactions on Intelligent Transportation Systems, Volume 15, Issue 1, Feb. 2014. pp 62-72.

[CHI-02A] F. M. Chiussi, D. A. Khotimsky, S. Krishnan; "A Network Architecture for MPLSBased Micro-Mobility," IEEE Wireless Communications and Networking Conference (WCNC), Orlando, FL, USA, 17 ${ }^{\text {th }}-21$ st March 2002, Vol.2, pp 549-555.

[CHI-02B] F. M. Chiussi, D. A. Khotimsky, S. Krishnan; "Mobility Management in Third Generation All-IP Networks," IEEE Communications Magazine, Vol. 40, No. 9, September 2002, pp. 124-135.

[CLA-94] D.Clark; "RFC 1633-Integrated Services in the Internet Architecture: an Overview"; 1994. Website: https://tools.ietf.org/html/rfc1633. Last accessed $4^{\text {th }}$ November 2019. 
[CMR-11] CMRI, “C-RAN: The Road towards Green RAN”; October 2011, Website: http://www.labs.chinamobile.com/cran. Last accessed $9^{\text {th }}$ November 2019.

[COL-04] S. Collonge, G. Zaharia, and G. E. Zein; "Influence of the Human Activity on WideBand Characteristics of the $60 \mathrm{GHz}$ Indoor Radio Channel," IEEE Transactions on Wireless Communication, Vol. 3, Issue. 6, Nov.2004, pp. 2396-2406.

[COO-15] W.Coomans,R.B.Moraes, K. Hooghe, A.Duque, J.Calaro, M.Timmers; "XG-Fast: The $5^{\text {th }}$ Generation Broadband". IEEE Communications Magazine, Vol.53, No.12, 2015, pp 83-88.

[COR-14] G. Corbellini, K. Aks, S. Schmid, S. Mangold, T. R. Gross; "Connecting Networks Of Toys And Smartphones With Visible Light Communication", IEEE Communications Magazine, Vol.52, Issue 7, July 2014, pp 72-78.

[COS-11] G. Cossu, M. Presi, R. Corsini, P. Choudhury, A. M. Khalid, E. Ciaramella; "A Visible Light Localization Aided Optical Wireless System", IEEE 2nd Workshop on Optical Wireless Communications, 5th -9th December 2011, Houston, Texas, USA, pp 802-807.

[COW-10] COWA; "Center on Optical Wireless Applications (COWA)".

Website:http://www.iucrc.org/center/center-optical-wireless-applications. Last accessed $3^{\text {rd }}$ September 2019.

[DAN-10] R. C. Daniels, J. N. Murdock, T. S. Rappaport, and R. W. Heath; "60 Ghz Wireless: Up Close And Personal," IEEE Microwave Magazine, Vol. 11, Issue. 7, December 2010, pp 4450 .

[DAS-12] A.K. Das, A. Ghosh, A. M. Vibin, S. Prince; “ Underwater Communication System For Deep Sea Divers Using Visible Light”, IEEE Photonics Global Conference (PGC), 13th 16th December 2012, Singapore, pp 1-3.

[DAV-98] E.Davies; “RFC 2475 - An Architecture for Differentiated Services - IETF Tools”; 1998. Website: https://tools.ietf.org/html/rfc2475. Last accessed $4^{\text {th }}$ November 2019.

[DAV-02] B. Davie; “RFC 3246- Expedited Forwarding PHB”; 2002. Website: https://tools.ietf.org/html/rfc3246. Last accessed $4^{\text {th }}$ November 2019.

[DCS-18] Department of Computer Science, Standford University; "Chapter 5 Introducing SDN Control in MPLS Networks"; Online Resource. Website: http://yuba.stanford.edu/ sd2/Ch_5.pdf. Last accessed $4^{\text {th }}$ November 2019.

[DEB-14] S. Deb, P. Monogioudis, J. Miernik, J. P. Seymour; “Algorithms for Enhanced InterCell Interference Coordination (EICIC) In LTE Hetnets, ” IEEE/ACM Transactions on Networking, Vol. 22, Issue 1, Feb 2014, pp. 137-150. 
[DHI-12] H. S. Dhillon, R. K. Ganti, F. Baccelli, and J. G. Andrews; "Modeling and Analysis of K-Tier Downlink Heterogeneous Cellular Networks, ” IEEE Journal on Selected Areas of Communication, Vol. 30, Issue 3, April 2012, pp. 550-560.

[DIG-15] DIGIBYTE; "eCall in all new cars from April 2018”; Published on 28 ${ }^{\text {th }}$ April, 2015. Website: https://ec.europa.eu/digital-single-market/en/news/ecall-all-new-cars-april-2018. Last accessed 20th June 2019.

[DON-05] Y. Dong, D. Makrakis, N. D. Georganas; "Scalable and QoS Capable MPLS-based Micromobility Management"; 2nd Asia Pacific Conference on Mobile Technology, Applications and Systems, Guanghazou, China, $15^{\text {th }}-17^{\text {th }}$ November 2005.

[DON-17] P.Dong, T. Zheng, S. Yu, H. Zhang, X. Yan; “Enhancing Vehicular Communication Using 5G-Enabled Smart Collaborative Networking”; IEEE Wireless Communications, Vol.24, Issue 6, December 2017. pp 72-79.

[DOT-14] US Department of Transportation; “U.S. Department of Transportation Announces Decision to Move Forward with Vehicle-to-Vehicle Communication Technology for Light Vehicles". Published online on 3rd February 2014. Website: http://www.nhtsa.gov/About+NHTSA/Press+Releases/2014/USDOT+to+Move+Forward+with+ Vehicle-to-Vehicle+Communication+Technology+for+Light+Vehicles. Last accessed on 14th November 2019.

[DRE-14] F. Dressler, H. Hartenstein, O.Altintas, O. K. Tonguz; "Inter-Vehicle Communication: Quo Vadis", IEEE Communications Magazine, Volume 52, Issue 6, June 2014. pp 170-177.

[DRO-97] R.Droms; “RFC 2131- Dynamic Host Configuration Protocol-IETF Tools”; 1997.

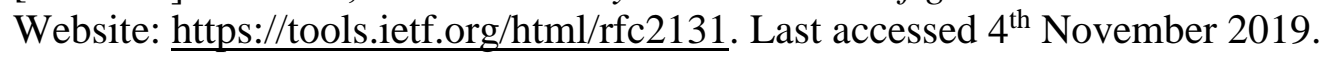

[DUB-03] R.Dube, M. Costa; “Bi-directional LSPs for classical MPLS,” IETF draft, July 2003. Website:https://tools.ietf.org/html/draft-dube-bidirectional-lsp-01. Last accessed $4^{\text {th }}$ February 2020 .

[ELG-09] H. Elgala, R. Mesleh, H. Haas, "Practical Considerations For Indoor Wireless Optical System Implementation Using OFDM," in Proceedings of $10^{\text {th }}$ International Conference on Telecommunications, Zagreb, Croatia, $8^{\text {th }}-10^{\text {th }}$ June 2009 , pp. 25-29.

[ENG-15] J.Fingas; “Audi’s Self- Driving Car Travelling to Las Vegas”. Website: https://www.engadget.com/2015/01/05/audi-self-driving-car-drives-to-ces/. Last accessed $23^{\text {rd }}$ May 2019.

[ENG-17] R.Baldwin; “Audi Takes Its Self-Driving Car Where Others Dare Not To Go", Website: https://www.engadget.com/audi-takes-its-self-driving-car-where-others-dare-not-go. Last accessed $23^{\text {rd }}$ May 2019. 
[ERI-19] Ericsson; "Massive MIMO brings high-speed mobile internet to football fans", Published online. Website: https://www.ericsson.com/en/networks/cases/massive-mimo-bringshigh-speed-mobile-internet-to-football-fans. Last accessed $6^{\text {th }}$ July, 2020.

[ETS-10] ETSI EN 302 665; "Intelligent Transport Systems (ITS); Communications Architecture"; September 2010.

[ETS-12] ETSI TR 102.962; “Intelligent Transportation Systems (ITS): Framework For Public Mobile Network”; February 2012.

[ETS-17] ETSI TR 138 901; "5G; Study On Channel Model for Frequencies from 0.5 to $100 \mathrm{Ghz}$ (3GPP TR 38.901 Version 14.0.0 Release 14)"; 2017.

Website:https://www.etsi.org/deliver/etsi_tr/138900_138999/138901/14.00.00_60/tr_138901v14 0000p.pdf. Last accessed $22^{\text {nd }}$ January 2019.

[FAN-16] P. Fan, J.Zhao, C.H.I; "5G High Mobility Wireless Communications: Challenges and Solutions"; China Communications, Supplement no. 2, 2016. pp 1-13.

[FCC-13] Federal Communication Commission; "ET-Docket No.13-49. Notice of Proposed Rulemaking". Published online on 20th February, 2013. Website: https://apps.fcc.gov/edocs_public/attachmatch/FCC-13-22A1.pdf. Last accessed 13th November 2019.

[FOO-13] D. Fooladivanda, C. Rosenberg; "Joint Resource Allocation and User Association For Heterogeneous Wireless Cellular Networks, " IEEE Transactions on Communication, Vol. 12, Issue 1, January 2013. pp. 248-257.

[FRI-46] H. T. Friis; "A Note on A Simple Transmission Formula," Proceedings of the IRE, Vol. 34, Issue. 5, May 1946. pp. 254-256.

[FUK-01] S.Fukushima, T.Ohno, Y.Doi; "Frequency stabilization of millimeter-wave subcarrier using laser heterodyne source and optical delay line", IEEE Photonics Technology Letters, Vol. 13, Issue 9, 2001. pp. 1002-1004.

[GAL-06] G.Galati, I.Dalmasso, G.Pavan, G. Brogi; "Fog Detection Using Airport Radar"; Proceedings of IRS 2006 International Radar Symposium, Krakow, Poland, May 24-26, 2006. pp 209-212.

[GAN-08] N.Gang; "A Micro-Mobility MPLS Scheme for QoS Supporting in Wireless Access Network"; 2008 ISECS International Colloquium on Computing, Communication, Control, and Management, $3^{\text {rd }}-4^{\text {th }}$ August 2008. pp 608-611.

[GAP-16] M. Gapeyenko, A. Samuylov, M. Gerasimenko, D.Moltchanov , S. Singh , E. Aryafar, S. Yeh, N. Himayat, S. Andreev, Y. Koucheryavy; "Analysis of Human-Body Blockage in Urban Millimeter-Wave Cellular Communications"; Proceedings of IEEE 
International Conference on Communications (ICC), Kuala Lampur, Malaysia, $22^{\text {nd }}-27^{\text {th }}$ May 2016, pp.1-7.

[GAR-10] A. P. Garcia, W. Kotterman, U. Trautwein, D. Bruckner, J. Kunisch, and R. S. Thoma; "60 GHz Time-Variant Shadowing Characterization within an Airbus 340"; in Proceedings of 4th EU Conference on Antenna And Propagation (EUCAP), Barcelona, Spain, $12^{\text {th }}-16^{\text {th }}$ April 2010, pp.1-5.

[GAR-16] J. P. Garzon, O. A.Hinojosa, P. Ameigeiras, J. J. R. Munoz, P. A. Maldonado, J. M. L.Soler; "Handover Implementation in a 5 G SDN-based Mobile Network Architecture"; IEEE 27th Annual IEEE International Symposium on Personal, Indoor and Mobile Radio Communications (PIMRC): Mobile and Wireless Networks, Valencia, Spain, $4^{\text {th }}-8^{\text {th }}$ September 2016, pp 1-6.

[GE-16] X.Ge, H.Cheng,G.Mao,Y.Yang, S.Tu; “Vehicular Communications for 5 G Cooperative Small-Cell Networks"; IEEE Transactions on Vehicular Technology, Vol. 65, Issue 10, October 2016. pp 7882-7894.

[GEO-95] J.B. Georges, D.M. Cutrer, O.Solgaard, K.Y.Lau, “Optical transmission of narrowband millimeter-wave signals". IEEE Transactions on Microwave Theory and Techniques, Vol. 43, 1995. pp 2229-2240.

[GHA-07] M. Ghavami, L. Michael, R.Kohno; "UWB: Ultra-Wideband Signals and Systems in Communication Engineering”, John Wiley \& Sons, Ltd., 2007.

[GHA-10] Z.Ghoomslay, W.O.Poopola; "Terrestrial Free-Space Optical Communication"; Open-access chapter, Mobile \& Wireless Communication, Published by intechopen, 2010. Website: https://www.intechopen.com/books/mobile-and-wireless-communications-networklayer-and-circuit-level-design/terrestrial-free-space-optical-communications. Last accessed September 24th, 2018.

[GHO-10] A. Ghosh, R. Ratasuk, B. Mondal, N. Mangalvedhe, T. Thomas; "LTE-Advanced: Next-Generation Wireless Broadband Technology," IEEE Wireless Communications, Vol 17, Issue 3, pg. 10-22, 2010.

[GHO-17] M. Gholibeig, N. Sarrionandia, M.Karimzadeh, M. Baratchi, H. V.D. Berg, G.Heijenk; "Reliable Vehicular Broadcast using 5 G Device-to-Device Communication"; Proceedings of 10th IFIP Wireless and Mobile Networking Conference (WMNC), Valencia, Spain, $25^{\text {th }}-27^{\text {th }}$ September 2017, pp 1-8.

[GIA-99] F. Giannetti, M. Luise, R. Reggiannini; "Mobile And Personal Communications In The $60 \mathrm{GHz}$ Band: A Survey"; Wireless Personal Communication, Vol. 10, Issue. 2, 1999. pp. 207243. 
[GIG-10] WiGig White Paper; "Defining the Future of Multi-Gigabit Wireless Communications"; July 2010. Website: http://wirelessgigabitalliance.org/specifications. Last accessed $26^{\text {th }}$ September 2019.

[GIG-14] Giga-IR “Multi- Gigabit Communications (Giga-IR) Using Infrared Technology" Website http://irdajp.info/gigair.html. Last accessed 1st October 2019.

[GIU-12] D. Giustiniano, N. O. Tippenhauer, S. Mangold; "Low-complexity visible light networking with LED-to-LED communication", IFIP Wireless Days (WD), Dublin, Ireland, $21^{\text {st }}$ $23^{\text {rd }}$ November 2012, pp 1-8.

[GON-03] L.H.Gonfa; "Enhanced Fast Rerouting Mechanisms for Protected Traffic In MPLS Networks” Ph.D thesis, Universitat Polit’ecnica de Catalunya, Barcelona, Spain. 2003.

[GOO-05] D. Goodman, R. A. Myers; "3G Cellular Standards and Patents"; International Conference on Wireless Networks, Communication and Mobile Computing (WIRELESSCOM), Maui, Hawaii, USA, 13th -16th June 2005, Vol 1, pp 415-420.

[GOO-17] W.Goodall, T.D.Fishman, J.Bornstein, B.Bonthron; "The Rise of Mobility As A Service”; Deloitte Review, Issue 20, 2017. Website: https://www2.deloitte.com/content/dam/Deloitte/nl/Documents/consumer-business/deloitte-nlcb-ths-rise-of-mobility-as-a-service.pdf. Last accessed 20 ${ }^{\text {th }}$ June 2016.

[GRI-01] J. Grimminger, H. P. Huth ;"Mobile MPLS - a MPLS based Micro-mobility Concept," The Wireless World Research Forum (WWRF), Stockholm, September 2001.

[GUO-15] C.Guojing; "Commercial Banks Eye Mobile Payment Innovations"; China Economic Net, June $28^{\text {th }} 2015$.

[GUS-04] E.Gustafsson, A.Jonsson, C. Perkins; "Mobile IPv4 Regional Registration". IETF RFC 4857. Webiste: https://tools.ietf.org/html/rfc4857. Last accessed $5^{\text {th }}$ February 2020.

[HAA-11] H.Haas, "Wireless Data from Every Light Bulb". TED Global. Published in July 2011.Website: https://www.ted.com/speakers/harald_haas. Last accessed 1st January 2020.

[HAA-19] H.Haas; "Opportunities and Challenges of Future LiFi”, IEEE Photonics Conference, San Antonio, Texas, U.S.A, $29^{\text {th }}$ September- $3^{\text {rd }}$ October 2019, pp 1-2.

[HAM-19] Hamamatsu; "Photodiodes-Hamamatsu Photonics". Website:

https://www.hamamatsu.com/resources/pdf/ssd/e02_handbook_si_photodiode.pdf. Last accessed $31^{\text {st }}$ October 2019.

[HAN-16] F. Han, S. Zhao, L.Zhang, J. Wu; "Survey of Strategies for Switching Off Base Stations in Heterogeneous Networks for Greener 5 G Systems"; IEEE Access, Vol 4, August 2016. pp 4959-4973. 
[HAR-10] Ed. H. Harteinstein, K.P. Labertaux; "VANET Vehicular Applications and InterNetworking Technologies", John Wiley \& Sons, Ltd., February 2010.

[HAS-00] D. Haskin , R. Krishnan; "A Method for Setting an Alternative Label Switched Paths to Handle Fast Reroute". IETF draft, November 2000.Website: https://tools.ietf.org/html/drafthaskin-mpls-fast-reroute-02. Last accessed $4^{\text {th }}$ February 2020.

[HEI-99] J. Heinanen; "RFC 2597- Assured Forwarding PHB"; 1999. Website: https://tools.ietf.org/html/rfc2597. Last accessed $4^{\text {th }}$ November 2019.

[HEM-18] I. A. Hemadeh, K. Satyanarayana, M.El-Hajjar, L. Hanzo; “Millimeter-Wave Communications: Physical Channel Models, Design Considerations, Antenna Constructions, and Link-Budget"; IEEE Communications Surveys \& Tutorials, Vol.20, Issue 2, 2018. pp 870913.

[HHI-13] Fraunhofer Heinrich Hertz Institute "High Speed Internet from the Ceiling Lamp" Website: http://www.hhi.fraunhofer.de/media/press/high-speed-internet-from-the-ceilinglamp.html. Last accessed 16th January, 2020.

[HHI-13A] Fraunhofer Heinrich Hertz Institute "Fraunhofer HHI Enables Visible Light Communication by LED at 3Gb/S". Published in November 2013. Website:

http://www.semiconductor-today.com/news_items/2013/NOV/HHI_151113.shtml. Last accessed 14th October 2019.

[HIE-16] S.Hietanen, S.Sahala; "Mobility as a Service"; March 2016. Website: https://www.itscanada.ca/files/MaaS\%20Canada\%20by\%20Sampo\%20Hietanen\%20and\%20Sa mi\%20Sahala.pdf. Last accessed $20^{\text {th }}$ June 2019.

[HOL-14] C. Holland, N. E. E. Alliance; "Are LEDs the Next CFL: A Diffusion of Innovation Analysis,” ACEEE Summer Study on Energy Efficiency in Buildings, 2014, pp. 9-184-9-196.

[HOR-18] J.Horwitz; " $5 G$ 's secret weapon will be low latency, empowering next-gen VR and gaming"; Venture beats Online Magazine. Published on 2nd March 2018. Website: https://venturebeat.com/2018/03/02/5gs-secret-weapon-will-be-low-latency-empowering-nextgen-vr-and-gaming/. Last accessed $9^{\text {th }}$ October 2019.

[HOU-11] X. Hou, P. Deshpande, S. Das; "Moving Bits from $3 G$ to Metroscale Wifi For Vehicular Network Access: An Integrated Transport Layer Solution," in Proceedings of IEEE International Conference on Network Protocol (ICNP), Vancouver, BC, Canada, $17^{\text {th }}-20^{\text {th }}$ October 2011, pp. 353-362.

[HOY-13] J. Hoydis, S. ten Brink, M. Debbah; "Massive MIMO in the UL/DL of Cellular Networks: How Many Antennas Do We Need?" IEEE Journal on. Selected Areas of Communication, Vol. 31, Issue 2, February 2013. pp. 160-171. 
[HUA-15] HUAWEI; "5G Vision: 100 Billion connections, 1 ms Latency, and 10 Gbps Throughput" Published 2015. Website: http://www.huawei.com/minisite/5g/en/defining-5g.html. Last accessed $20^{\text {th }}$ June 2019.

[HUA-19] J. Huang, Y. Liu, C.-X.Wang, J. Sun, H. Xiao; "5G Millimeter-Wave Channel Sounders, Measurements, and Models: Recent Developments and Future Challenges"; IEEE Communications Magazine, January 2019. pp 138-145.

[HUE-12] F. Hueger; "Platform Independent Applications for In-Vehicle Infotainment Systems Via Integration of CE Devices"; IEEE International Conference on Consumer Electronics (ICCC-Berlin), 3rd -5th September 2012, Berlin, Germany. pp 221-222.

[HUO-17] Y. Huo, X.Dong,W. Xu; "5G Cellular User Equipment: From Theory to Practical Hardware Design"; IEEE Access, 2017. Vol. 5, pp 13993-14010.

[IEE-09] IEEE 802.21-2008; “IEEE Standard for Local and Metropolitan Area Networks-Media Independent Handover Services", 2009, superseded by IEEE 802.21-2017.

[IET-02] A.Viswanathan; "RFC 3031 - Multiprotocol Label Switching Architecture - IETF Tools"; Published in 2001. Website: https://tools.ietf.org/html/rfc3031. Last accessed $19^{\text {th }}$ July 2019.

[INN-10] T.Innovations; "LTE in a Nutshell: The Physical Layer"; White paper published in 2010. Website: https://home.zhaw.ch/kunr/NTM1/literatur/LTE\%20in\%20a\%20Nutshell\%20\%20Physical\%20Layer.pdf. Last accessed $9^{\text {th }}$ October 2019.

[IrD-93] IrDA; “Infrared Data Association". Website: http://www.irda.org/. Last accessed $3^{\text {rd }}$ September 2019.

[ISH-12] H. Ishii, Y. Kishiyama, H. Takahashi; "Novel architecture for LTE-B: C-plane/U-plane split and phantom cell concept," in Proceedings of IEEE GLOBECOM Workshop, Anaheim, CA, USA, $3^{\text {rd }}-7^{\text {th }}$ December 2012, pp. 624-630.

[ISH-16] S.Ishii1, M. Kinugawa1, S.Wakiyama1, S. Sayama., T. Kamei; "Rain Attenuation in the Microwave-to-Terahertz Waveband"; Wireless Engineering and Technology, Vol. 7, 2016. pp 59-66.

[ISO-17] ISO2017; “ISO/TR 20529-1:2017 Intelligent Transport Systems - Framework for Green ITS (G-ITS) Standards - Part 1: General Information and Use Case Definitions". Website: https://www.iso.org/standard/73257.html. Last accessed $5^{\text {th }}$ February 2020.

[ITS-16] ITS-UK; "Mobility as a Service (MaaS) - the ITS topic of 2016"; Published on $16^{\text {th }}$ July 2016.Website: http://www.its-ukreview.org/mobility-as-a-service-maas-the-its-topic-of-2016/. Last accessed $20^{\text {th }}$ June 2016. 
[ITU-05] ITU-R P.838-3; "Specific attenuation model for rain for use in prediction methods"; 2005.

[ITU-07] ITU-R P.1814; "Prediction methods required for the design of terrestrial free-space optical links"; 2007.

[ITU-12] ITU-R P.1817-1; "Propagation data required for the design of terrestrial free-space optical links-P Series-Radio wave Propagation”; February 2012.

[ITU-14] D.Iturralde, C. Azurdia-Meza, N. Krommenacker, I.Soto, Z. Ghassemlooy, N.Becerra; "A New Location System For An Underground Mining Environment Using Visible Light Communications"; 9th International Symposium on Communication Systems, Networks and Digital Signal Processing (CSNDSP), 2014. pp 1165- 1169.

[ITU-16] ITU-R P.676-11; “Attenuation by Atmospheric Gases”; 2016.

[ITU-17] ITU-R P.840-7; "Attenuation due to clouds and fog-P Series-Radio Wave Propagation"; December 2017.

[IWA-08] S. Iwasaki, C. Premachandra, T. Endo, T. Fujii, M. Tanimoto, and Y. Kimura, "Visible Light Road-To-Vehicle Communication Using High-Speed Camera," in Proceedings of IEEE Intelligent Vehicles Symposium, Eindhoven, The Netherlands, June 2008, pp. 13-18.

[JAB-16] M. Jaber, M. A. Imran, R. Tafazolli , A.Tukmanov; "5G Backhaul Challenges and Emerging Research Directions: A Survey”; IEEE Access, Vol 4, April 2016. pp 1743-1766.

[JAC-98] D. Jackson, T. Buffaloe, and S. Leeb, "Fiat lux: a fluorescent lamp digital transceiver," IEEE Transactions on Industry Applications, Vol. 34, No. 3, 1998. pp. 625-630.

[JAM-15] T. Jamsa; "Deliverable D1.4 METIS Channel Models", document ICT-317669METIS/D1.4, METIS, New York, NY, USA, Feb. 2015. Webiste: https://www.metis2020.com/wpcontent/uploads/METIS_D1.4_v3.pdf. Last accessed 4th October 2019.

[JEI-07] JEITA Standards; “CP-1221-Visible Light Communication System, CP-1222-Visible Light Id System". Website:

https://www.jeita.or.jp/cgibin/standard_e/list.cgi?cateid=1\&subcateid=50. Last accessed $3^{\text {rd }}$ September 2019.

[JIA-06] Y.Jianjun, Z.Jia, L.Yi; “Optical millimeter-wave generation or up-conversion using external modulators", IEEE Photonics Technology Letters, Vol. 18, Issue 1, 2006. pp 265-267.

[JIN-10] L. Jingya, Z. Hui, X Xiaodong, T. Xiaofeng, T. Svensson, C. Botella, L. Baoling; “ $A$ Novel Frequency Reuse Scheme for Coordinated Multi-Point Transmission," Proceedings of IEEE Vehicular Technology Conference (VTC),Taipei, Taiwan, $16^{\text {th }}-19^{\text {th }}$ May 2010, pp. 1-5. 
[KAH-95] J. M. Kahn, W. J. Krause, J. B. Carruthers; "Experimental Characterization Of NonDirected Indoor Infrared Channels," IEEE Transactions on Communications, Vol. 43, 1995.pp. 1613-1623.

[KAH-97] J. Kahn, J. Barry; "Wireless Infrared Communications," in Proceedings of the IEEE, Vol.85, No.2, February 1997, pp. 265-298.

[KAR-11] S. V. Kartalopoulos; "Propagation of Light in Unguided Media"; Chapter 1, Free Space Optical Networks for Ultra Broadband Services, Published by Wiley \& Sons, 2011.

[KAR-88] S.Karp, R.M.Gagliardi,S.E.Moran, L.B.Sttots; "Optical Channel: Fibers, Clouds, Water and the Atmosphere"; Published 1988.

[KAU-12] A. Kaur, Dr. M. L Singh; "Comparing the Effect of Fog and Snow Induced Attenuation on Free Space Optics (FSO) and RF Links"; International Journal of Computer Science and Technology (IJCST), Vol. 3, Issue 1, Jan-Mar 2012. pp 554-556.

[KAU-17] H. Kaushal; "Free Space Optical Channel Models"; Chapter 2, Free Space Communication, Optical Networks, Published by Springer. pp 41-89. 2017.

[KAV-18] S. Kavanugh; " $5 G$ vs 4G: No Contest"; 5G Guides. Published on $27^{\text {th }}$ September 2018. Website: https://5g.co.uk/guides/4g-versus-5g-what-will-the-next-generation-bring/. Last accessed $9^{\text {th }}$ October 2019.

[KHA-11] F. Khan .Z. Pi; "mmWave mobile broadband (MMB): Unleashing the 3-300GHz Spectrum," in Proceedings of 34th IEEE Sarnoff Symposium, Princeton, NJ, USA, May 2011.pp. $1-6$.

[KIM-01A] I.I. Kim, E. Korevaar; “Availability of Free Space Optics (FSO) and Hybrid FSO/RF Systems"; Lightpointe technical report. Website: http://www.opticalaccess.com. Last accessed $25^{\text {th }}$ September 2019.

[KIM-01] H. Kim, K.S.D.Wong, W.Chen, C.L.Lau; "Mobility-Aware MPLS in IP-based Wireless Access Networks," IEEE Globecom, Vol 6, Issue 1, Nov. 2001, pp. 3444-3448.

[KIM-05] C.Kim, I.Kim, G.Li, M.R.Lange; “Optical microwave/millimeter-wave links using direct modulation of two-section gain-coupled DFB lasers"; IEEE Photonics Technology Letters, Vol.17, Issue 8, 2005.

[KIM-14] J. Kim, H.-W. Lee, S. Chong; "Virtual Cell Beamforming In Cooperative Networks," IEEE Journal on Selected Areas of Communication, Vol. 32, Issue 6, June 2014.pp 1126-1138.

[KIM-17] J. Kim, L. Xian, A. S. Sadri; "60 GHz Modular Antenna Array Link Budget Estimation with WiGig Baseband and Millimeter-Wave Specific Attenuation," International Journal of Antennas and Propagation, Vol. 2017, June 2017, pp 1-9. 
[KIN-18] A. Al-Kinani, J. Sun, C.X.Wang, W. Zhang, X.Ge, H.Haas. "A 2-D Non-Stationary GBSM for Vehicular Visible Light Communication Channels", IEEE Transactions on Wireless Communications, Vol. 17, No. 12, December 2018, pp 7981-7992.

[KIT-99] K. Kitayama , R. A. Grif; “Optical Downconversion from Millimeter-Wave to IF-Band Over 50-km-Long Optical Fiber Link Using an Electro absorption Modulator", IEEE Photonics Technology Letters, Vol. 11, No. 2, February 1999. pp. 287.289.

[KUM-12] N. Kumar, N. Lourenc, D. Terra, LN. Alves, RL. Aguiar; "Visible Light Communications In Intelligent Transportation Systems"; Proceedings of IEEE Symposium in Intelligent Vehicles (IV), Alcala de Henares, Spain, 2012, pp 748-753.

[KUM-15] S.Kumari, S.A.Rao, N.Kumar; "Characterization of mmWave Link for Outdoor Communications in $5 G$ Networks"; IEEE International Conference on Advances in Computing, Communications and Informatics (ICACCI), Kochi, India, September 2015, pp 44-49.

[KUO-17] P.H.Kuo, A.Mourad; "Millimeter Wave for 5 G Mobile Fronthaul and Backhaul"; Proceedings of IEEE European Conference on Networks and Communications (EuCNC), Oulu, Finland, $12^{\text {th }}-15^{\text {th }}$ June 2017. pp 1-5.

[KUT-16] S. Kutty, D.Sen; "Beamforming for Millimeter Wave Communications: An Inclusive Survey”; IEEE Communications Surveys \& Tutorials, Vol 18, Issue 2, 2016. pp 949-973.

[LAN-08] R. Langar, N. Bouabdallah, R. Boutaba; “A Comprehensive Analysis of Mobility Management in MPLS-Based Wireless Access Networks", IEEE/ACM Transactions on Networking, Vol. 16, Issue. 4, August 2008, pp 918-931.

[LAR-13] S. Larew, T. Thomas, and A. Ghosh; "Air Interface Design And Ray Tracing Study For $5 G$ Millimeter Wave Communications," in Proceedings of IEEE GLOBECOM Workshop, Atlanta, GA, USA, $9^{\text {th }}-13^{\text {th }}$ December 2013, pp. $117-122$.

[LEC-09] F. Lecoche, E.Tanguy, B. Charbonnier, H.Li, F. van Dijk, A. Enard, F. Blache, M. Goix, F. Mallécot; "Transmission Quality Measurement of Two Types of $60 \mathrm{GHz}$ MillimeterWave Generation and Distribution Systems"; Journal of Lightwave Technology, Vol.27, Issue 23, 2009. pp. 5469-5474.

[LEE-12] S. J. Lee, J. K. Kwon, S. Y. Jung, and Y. H. Kwon, "Simulation Modeling Of Visible Light Communication Channel For Automotive Applications," in Proceedings of IEEE Conference on Intelligent Transportation System (ITSC'12), Anchorage, AK, USA, $16^{\text {th }}-19^{\text {th }}$ September 2012, pp. 463-468.

[LEE-12A] S. J. Lee, J. K. Kwon, S. Y. Jung, and Y. H. Kwon, "Evaluation Of Visible Light Communication Channel Delay Profiles For Automotive Applications," EURASIP Journal on Wireless Communication and Networking, December 2012, pp. 1-8. 
[LEO-18] J.Leong; "Study Shows Autonomous Vehicles Can Help Improve Traffic Flow"; Published on $20^{\text {th }}$ February, 2018. Website: https://phys.org/news/2018-02-autonomous-vehiclestraffic.html. Last accessed $4^{\text {th }}$ July, 2019.

[LEU-05] K. Leung, S. Mukherjee, G. Rittenhouse; “Mobility Support for IEEE 802.1.6d Wireless Networks," IEEE Wireless Communications and Networking Conference (WCNC), Wuhan, China, $26^{\text {th }}$ September 2005, pg. 1446-1452.

[LI-05] Y.Li, M. Bystrom, D.Yoo, S.M. Goldwasser, P.R.Herczfeld; "Coherent optical vector modulation for fiber radio using electrooptic microchip lasers". IEEE Transactions on Microwave Theory and Techniques, Vol. 53, Issue. 10, 2005. pp 3121-3129.

[LI-10] J.Li, T.Ning, L.Pei, C.Qi; "60 GHz millimeter-wave generator based on a frequencyquadrupling feed-forward modulation technique", Optics Letters, Vol. 35, Issue 21, 2010. pp 3619-3621.

[LI-12] Yunxin (Jeff) Li; "An Overview of DSRC/WAVE Technology"; Quality, Reliability, Security and Robustness in Heterogeneous Networks, Lecture Notes of the Institute for Computer Sciences, Social Informatics and Telecommunications Engineering. Springer. Vol.74, 2012, pp 544-558.Website: http://link.springer.com/chapter/10.1007\%2F978-3-642-29222-4_38. Last accessed 30th September 2019.

[LiF-11] G.Povey; "Li-Fi Consortium Is Launched". Website: https://visiblelightcomm.com/li-ficonsortium-is-launched/. Last accessed $3^{\text {rd }}$ September 2019.

[LIN-09] C.T.Lin, P.T.Shih, W.Jiang; "A continuously tunable and filterless optical millimeterwave generation via frequency octupling”" Optics Express, Vol. 17, Issue 22, 2009. pp 1974919756

[LIN-16] C.-LIN. I, S. Han, Z. Xu, S. Wang, Q. Sun, Y. Chen; "New Paradigm of $5 G$ Wireless Internet"; IEEE Journal on Selected Areas in Communications, Vol 34, Issue 3, March 2016, pp 474-482.

[LIT-18] T.Litman; "Autonomous Vehicle Implementation Predictions Implications for Transport Planning"; Published on $24^{\text {th }}$ April 2018. Website: https://www.vtpi.org/avip.pdf. Last accessed $21^{\text {st }}$ June 2019.

[LIU-10] C.Liu, "The Case for Vehicular Visible Light Communication (V2 LC): Architecture, Services and Experiments"; M.S. Thesis, Rice University, Houston, TX, June 2010.

[LIU-12] J.Liu, PWC. Chan, Ng. DWK, Lo. ES, S.Shimamoto; "Hybrid Visible Light Communications in Intelligent Transportation Systems With Position Based Services"; IEEE GLOBECOM Workshop (GC Wkshps), Anaheim, CA, USA, 2012.pp 1254-1259. 
[LIU-16] D. Liu, L. Wang, Y. Chen, M.Elkashlan, K.K. Wong, R.Schober, L. Hanzo; "User Association in 5G Networks: A Survey and an Outlook"; IEEE Communications Surveys \& Tutorials, Vol 18, Issue 2, 2016. pp 1018-1044.

[LTE-14] LTE-3GPP; “LTE”. Website: http://www.3gpp.org/technologies/keywordsacronyms/98-lte. Last accessed 30th September 2019.

[LU-12] J. S. Lu, D. Steinbach, P. Cabrol, P. Pietraski; “Modeling Human Blockers In Millimeter Wave Radio Links," ZTE Communications, Vol. 4, Issue 4, December 2012.pp. 23-28. Website: http://wwwen.zte.com.cn/endata/magazine/ztecommunications/2012/4/ articles/201301/t20130106_380446.html. Last accessed 4th October 2019.

[LUO-15] P. Luo, Z. Ghassemlooy, H.- L. Minh, E. Bentley, A. Burton, and X. Tang, "Performance Analysis of a Car-To-Car Visible Light Communication System," Applied Optics, Vol. 54, No. 7, March 2015, pp. 1696-1706.

[LVX-10] LVX; "LVX Systems- First in Visible Light Communication”; Website: http://www.lvx-system.com/. Last accessed 22 ${ }^{\text {nd }}$ November 2019.

[MAC-16] G. R. MacCartney, S. Deng, S. Sun, T. S. Rappaport; "Millimeterwave human blockage at $73 \mathrm{GHz}$ with a simple double knife-edge diffraction model and extension for directional antennas," in Proceedings of IEEE 84th Vehicular Technology Conference (VTCFall), Montreal, Quebec, Canada, $18^{\text {th }}-21^{\text {st }}$ September 2016, pp. 1-6.

[MAD-14] U.Madhow; "Introduction to Communication Systems"; Cambridge University Press, 2014.

[MAG-17] K.Haneda, S.L.H. Nguyen, A.Karttunen; "Measurement Results and Final Mmmagic Channel Models, V1,” Technical Report, mmMAGIC, December 2017.

[MAL-00] K.E. Malki, H. Soliman; "Hierarchical Mobile IPv4/v6 and Fast Handoffs";2000. Website: https://tools.ietf.org/html/draft-elmalki-soliman-hmipv4v6-00. Last accessed $4^{\text {th }}$ November 2019.

[MAL-10] A. Maltsev ,R. Maslennikov , A. Sevastyanov , A. Lomayev , A. Khoryaev; "Statical Channel Models For 60 Ghz WLAN Systems in Conference Room Environment," Proceedings of the $4^{\text {th }}$ Eurpoean Conference on Antennas and Propagation, Barcelona, Spain, $12^{\text {th }}-16^{\text {th }}$ April 2010, pp. 1-5.

[MAL-14] A. Maltsev, A.Pudeyev, I. Karls, I. Bolotin, G. Morozov, R. Weiler, “QuasiDeterministic Approach To Mmwave Channel Modeling In A Non-Stationary Environment," in Proceedings of IEEE GLOBECOM Workshops, Austin, TX, USA, $8^{\text {th }}-12^{\text {th }}$ December 2014, pp. 966-971.

[MAL-14A] A. Maltsev, A.Pudeyev,I. Karls, I. Bolotin, G. Morozov, R. Weiler "Channel Modeling and Characterization", V1.0, document FP7-ICT-608637/D5.1, MiWEBA, Breitengüßbach, Germany, June 2014. 
[MAL-16] A. Maltsev, A.Pudeyev,I. Karls, I. Bolotin, G. Morozov, R. Weiler "Channel Modeling In The Next Generation Mmwave Wi-Fi: IEEE 802.1 lay Standard",22 ${ }^{\text {nd }}$ European Wireless Conference, Oulu, Finland, $18^{\text {th }}-20^{\text {th }}$ May 2016, pp.1-8.

[MAO-08] X. Mao, A. Maaref; K. Teo; "Adaptive Soft Frequency Reuse for Inter-cell Interference Coordination in SC-FDMA based 3GPP LTE Uplinks", IEEE Global Telecommunications Conference (GLOBECOM), New Orleans, LO, USA, $30^{\text {th }}$ November- $4^{\text {th }}$ December 2008, pp.1-6.

[MAR-05] M. Marcus, B. Pattan; "Millimeter Wave Propagation; Spectrum Management Implications,” IEEE Microwave Magazine, Vol. 6, Issue. 2, June 2005. pp. 54-62.

[MAR-14] E.P.Martin, T.Shao, V.Vujici, L.P.Barry; "25-Gb/s OFDM 60-GHz Radio Over Fiber System Based on a Gain Switched Laser", Journal of Lightwave Technology, Vol.33, Issue 8, 2014. pp 1635-1643.

[MAS-17] C.Masterson; "Massive MIMO and Beamforming: The Signal Processing Behind the 5 G Buzzwords"; Analog Dialogue, Vol 51, Issue 6, June 2017, pp. 1-5.

[MAS-17A] R.M.Mas, J.Gozalvez; "LTE-V for Sidelink 5G V2X Vehicular Communications: A New $5 G$ Technology for Short-Range Vehicle-to-Everything Communications"; IEEE Vehicular Technology Magazine, Vol.12, Issue 4, December 2017. pp 30-39.

[MEN-10] Y. S. Meng, Y. H. Lee; "Investigations ff Foliage Effect on Modern Wireless Communication Systems: A Review"; Progress in Electromagnetics Research, Vol. 105, 2010. pp 313-332.

[MER-19] Merriam-Webster "Angle of incidence". Website: https://www.merriamwebster.com/dictionary/angle\%20of\%20incidence. Last accessed $5^{\text {th }}$ February 2020.

[MES-14] A. Mesodiakaki, F. Adelantado, L. Alonso, C. Verikoukis; "Energy Efficient Context-Aware User Association For Outdoor Small Cell Heterogeneous Networks, " in Proceedings of IEEE International Conference on Communication (ICC), Sydney, Australia, $10^{\text {th }}-14^{\text {th }}$ June 2014, pp. 1614-1619.

[MET-14] METIS; "D1.1 - Scenarios, Requirements and KPIs for 5G Mobile and Wireless System", Published on $4^{\text {th }}$ April, 2014. Website https://cordis.europa.eu/docs/projects/cnect/9/317669/.../001-METISD11v1pdf.pdf. Last accessed $19^{\text {th }}$ July 2019.

[MFO-14] A.O. Mfoubat, F. Elbahhar, C.Tatkeu; "Novel Ultra-Wideband Multi-User Receiver for Transportation Systems Communication", IET Networks, Vol. 3, Issue 3, September 2014. pp. 169-175. 
[MIN-07] I.Minei; "RFC 5036- LDP Specification-IETF Tools"; 2007. Website: https://tools.ietf.org/html/rfc5036. Last accessed $4^{\text {th }}$ November 2019.

[MIT-08] 3GPP, R1-083615; "Combined Fixed and Adaptive Soft-Frequency Reuse for InterCell Interference Coordination," Mitsubishi Electric Report, September $28^{\text {th }}$-Oct. $3^{\text {rd }}, 2008$.

[MOH-08] M.Mohamed, X.Zhang, B.Hiramel; "Analysis of frequency quadrupling using a single Mach-Zehnder modulator for millimeter-wave generation and distribution over fiber systems", Optics Express, Vol.16, Issue 14, 2008. pp 10786-10802.

[MOR-10] Y.L.Morgan.; "Notes on DSRC \& WAVE Standards Suite: Its Architecture, Design, and Characteristics", IEEE Communications Survey and Tutorial, Vol 12, Issue 4, May 2010. pp 504-518.

[MOR-17] S.Morsalin, K.Mahmud, G.E.Town; "Scalability of Vehicular M2M Communications in a 4G Cellular Network", IEEE Transaction on Intelligent Transportation System, 2017. pp 18.

[MOR-97] A. J. C. Moreira, R. T. Valadas, A.M .O. Duarte; "Optical interference produced by artificial light," Wireless Networks, Vol. 3, No. 2, 1997. pp 131-140.

[MOY-98] J.Moy; "RFC 2328- OSPF Version. 2"; Website:https://tools.ietf.org/html/rfc2328. Last accessed $4^{\text {th }}$ November 2018.

[MUH-10] S.S.Muhammad, M.S. Awan, A. Rehman; "PDF Estimation and Liquid Water Content Based Attenuation Modeling for Fog in Terrestrial FSO Links"; Radio Engineering, Vol. 19, Issue. 2, June 2010. pp 228-236.

[MUM-15] S. Mumtaz, K.M.S. Huq, M. I. Ashraf, J. Rodriguez,V. Monteiro, C.Politis; "Cognitive Vehicular Communication for 5G"; IEEE Communications Magazine, Vol.53, Issue 7, July 2015. pp 109-117.

[MUR-13] J. Murdock and T. S. Rappaport; 'Consumption Factor And Power-Efficiency Factor: A Theory For Evaluating The Energy Efficiency Of Cascaded Communication Systems,", IEEE Journal on. Selected Areas of Communication, Vol 32, Issue 2, June 2013. pp 221-236.

[NAB-04] M.C. Al Naboulsi, H.Sizun, F. de Fornel; "Fog Attenuation Prediction for Optical and Infrared Waves"; Optical Engineering, Vol. 43, Issue 2. February 2004.

[NAD-08] F. Nadeem, B. Flecker, E. Leitgeb, M. S. Khan, M. S. Awan, T. Javornik;“Comparing The Fog Effects On Hybrid Network Using Optical Wireless And GHz Links", 6th International Symposium on Communication Systems, Networks and Digital Signal Processing, Graz, Austria, $25^{\text {th }}$ July 2008 , pp. 278-282.

[NAD-09] F. Nadeem, E. Leitgeb, M. S. Awan, G.Kandus; "Comparing The Snow Effects on Hybrid Network Using Optical Wireless and GHz Links"; Proceedings of International 
Workshop on Satellite and Space Communications, Siena-Tuscany, Italy, September $10^{\text {th }}$ September $11^{\text {th }} 2009$, pp. 171-175.

[NAK-09] B. Nakhkoob, M.Bilgi, M.M.Hella; "Multi-Transceiver Optical Wireless Spherical Structures for MANETs, ” IEEE Journal on Selected Areas of Communication, 2009, pp. 161222.

[NAT-13] National Instruments, "National Instruments and the University of Edinburgh Collaborate on Massive MIMO Visible Light Communication Networks to Advance 5G". Posted on 28th November 2013. Website:

http://www.cambridgewireless.co.uk/news/article/default.aspx?objid=45320. Last accessed 14th January 2020.

[NGU-13] T. Nguyen,M.Z.Chowdhury, Y.M.Jang; “A Novel Link Switching Scheme Using PreScanning and RSS Prediction in Visible Light Communication Networks"; EURASIP Journal of Wireless Communications and Networking, Vol. 2013 , Issue 1, 2013. pp 293-310.

[NHT-05] NHTSA; "Vehicle Safety Communications Project Task 3 Final Report”; Published in March 2005. Last accessed $7^{\text {th }}$ July, 2019.

[NOT-17] L.Notwell; "5G-Connection Density-Massive IoT and So Much More”, Brandpost Online Magazine. Published on 2nd November 2017. Website:

https://www.cio.com/article/3235971/internet-of-things/5g-connection-density-massive-iot-andso-much-more.html. Last accessed $9^{\text {th }}$ October 2019.

[OGU-83] T. Oguchi; "Electromagnetic Wave Propagation and Scattering in Rain and Other Hydrometeors", Proceedings of IEEE, Vol. 71, No.9, September 1983, pp 1029-1078.

[OME-11] OMEGA, “OMEGA, the Home Gigabit Access Project”. Website http://www.ictomega.eu/. Last accessed 16 January 2020.

[OOM-71] T. Oomori and S. Aoyagi; "A Presumptive Formula for Snowfall Attenuation of Radio Waves", Transactions of Industrial and Electronic Communication Engineering Japan (in Japanese), Vol. 58, pp. 451-458, 1971.

[OPF-15] Open Networking Foundation; "OpenFlow Switch Specification Version 1.5.1 (Protocol version 0x06 )"; March 26 ${ }^{\text {th }}$, 2015. Website: https://3vf60mmveq1g8vzn48q2o71awpengine.netdna-ssl.com/wp-content/uploads/2014/10/openflow-switch-v1.5.1.pdf. Last accessed $4^{\text {th }}$ November 2019.

[OWE-01] K. Owens, V. Sharma, S. Makam, and C. Huang; "A Path Protection/ Restoration Mechanism for MPLS Networks.” IETF draft, July 2001. Website: https://tools.ietf.org/html/draft-chang-mpls-path-protection-03. Last accessed 30 ${ }^{\text {th }}$ January 2020. 
[OXF-19] Oxford University Press; "Relaxation Frequency". Website:

https://www.encyclopedia.com/science/dictionaries-thesauruses-pictures-and-pressreleases/relaxation-frequency. Last accessed $5^{\text {th }}$ February 2020.

[PAR-07] C. S. Park, C. G. Lee, C-S Park; "Photonic Frequency Upconversion by SBS based Frequency Tripling"; Journal of Lightwave Technology, Vol.25, No. 7, July 2007. pp 17111718.

[PAN-02] G. Pang, T. Kwan, H. Liu, and C.-H Chan, “LED Wireless," IEEE Industry Applications Magazine, Vol. 8, No. 1, January 2002, pp. 21-28.

[PER-10] C. Perkins; "RFC 5944- IP Mobility Support for IPv4, Revised"; 2010. Website: https://tools.ietf.org/html/rfc5944. Last accessed $4^{\text {th }}$ November 2019.

[PER-11] R. Perez-Jimenez, J. Rufo, C. Quintana J. Rabadan, F.J. Lopez-Hernandez ; "Visible Light Communication Systems for Passenger In-Flight Data Networking", IEEE International Conference on Consumer Electronics (ICCE),9th -12th January 2011, Las Vegas, Nevada, USA, pp 445-446.

[PER-11A] C. Perkins, D.Johnson, J.Arkko; “Mobility Support in IPV6,” IETF RFC 6275. Website: https://tools.ietf.org/html/rfc6275. Last accessed $4^{\text {th }}$ February 2020.

[PHA-11] M.A.Phan, R. Rembarz, S.Sories; "A Capacity Analysis for the Transmission of Event and Cooperative Awareness Messages in LTE Networks"; Proceedings of $18^{\text {th }}$ World Congress on Intelligent Transportation System ITS World Congress, Orlando, USA. 16-20 October 2011.

[PHI-15] Philips; "Where Are the Discounts? Carrefour's LED Supermarket Lighting from Philips Will Guide You"; Press Release, Website:

https://www.philips.com/aw/about/news/archive/standard/news/press/2015/20150521-Where-are-thediscounts-Carrefours-LED-supermarket-lighting-from-Philips-will-guide-you.html\#.VgAF-t-qpBc. Last accessed $22^{\text {nd }}$ November 2019.

[PHO-95] K. P. Ho, J. M. Kahn; "Compound Parabolic Concentrators For Narrow-Band Wireless Infrared Receivers," Journal of Optical Engineering, Vol. 34, No. 5, May 1995, pp. 1385-1395.

[PI-11] Z. Pi, F. Khan; "System Design and Network Architecture For A Millimeter-Wave Mobile Broadband (MMB) System," in Proceedings of 34th IEEE Sarnoff Symposium, Princeton, NJ, USA, May 2011, pp. 1-6.

[PI-12] Z. Pi, F. Khan; “A Millimeter-Wave Massive MIMO System For Next Generation Mobile Broadband," in Proceedings of 2012 Conference Record of the Forty Sixth Asilomar Conference on Signals, Systems and Computers (ASILOMAR), Pacific Grove, CA, USA, Nov. 2012, pp. 693-698. 
[PIE-12] P.Pietraski, D.Britz, A.Roy, R. Pragada, G. Charlton; "Millimeter Wave and Terahertz Communications: Feasibility and Challenges"; ZTE Communications, Vol.10, Issue.4, December 2012. pp 3-12.

[POV-11] G.Povey; “D-Light Project Hits Target”. Website: https://visiblelightcomm.com/dlight-project-hits-target/. Last accessed $3^{\text {rd }}$ September 2019.

[PRI-12] G. B. Prince, T.D.C. Little; "A Two Phase Hybrid RSS/AOA Algorithm For Indoor Device Localization Using Visible Light", IEEE Global Communications Conference (GLOBECOM), 3rd -7th December 2012, Anaheim, California, USA, pp 3347-3352.

[QIA-18] J. Qiao, Y. He, X. Shen; “Improving Video Streaming Quality in 5 G Enabled Vehicular Networks”; IEEE Wireless Communications,Vol.25, Issue.2, April 2018. pp 133-139.

[QLI-13] Q. Li, H. Niu, G. Wu, R. Q. Hu; “Anchor-Booster Based Heterogeneous Networks With Mmwave Capable Booster Cells, " in Proceedings of IEEE GLOBECOM Workshop, Atlanta, GA,USA, $9^{\text {th }}-13^{\text {th }}$ December 2013, pp. 93-98.

[QUI-13] C. Quintana, V. Guerra, J. Rufo, J. Rabadan, R. Perez-Jimenez; "Reading Lamp-Based Visible Light Communication System For In-Flight Entertainment", IEEE Transactions on Consumer Electronics, Vol. 59, No. 1, February 2013, pp 31-37.

[QYE-13] Q. Ye, B. Rong, Y. Chen , M. Al-Shalash ,C. Caramanis ; J.G. Andrews; “User Association for Load Balancing in Heterogeneous Cellular Networks, ” IEEE Transactions on Wireless Communications, Vol. 12, Issue 6, June 2013. pp. 2706-2716.

[RAH-10] M. Rahman and H. Yanikomerouglu; "Enhancing Cell-Edge Performance: A Downlink Dynamic Intereference Avoidance Scheme With Inter-Cell Coordination," IEEE Transactions on Wireless Communications, Vol. 9, No. 4, 2010, pp. $1414-1425$.

[RAH-14] M.A.Rahman; "Design ff Wireless Sensor Network For Intra-Vehicular Communications"; International Conference on Wired/Wireless Internet Communication (WWIC 2014), Paris, France, $26^{\text {th }}-28^{\text {th }}$ May 2014, pp 29-40.

[RAM-02] R. Ramjee, K. Varadhan, L. Salgarelli, S. R. Thuel; "HAWAII: A Domain-Based Approach For Supporting Mobility In Wide-Area Wireless Networks," IEEE/ACM Transactions on Networking, Vol. 10, Issue 3, June 2002. pp. 396-410.

[RAN-14] S.Rangan, T.S.Rappaport, E.Erkip; "Millimeter-Wave Cellular Wireless Networks: Potentials and Challenges"; Proceedings of the IEEE, Vol. 102, Issue 3, March 2014. pp 366385.

[RAP-11] T. S. Rappaport, J. N. Murdock, and F. Gutierrez; "State of the art in $60 \mathrm{GHz}$ Integrated Circuits and Systems for Wireless Communications, ' Proceedings of IEEE, Vol. 99, Issue. 8, August 2011. pp 1390-1436. 
[RAP-13] T. S. Rappaport, S.Sun, H.Zhou, Y.Azar; "Millimeter Wave Mobile Communications for 5G Cellular: It Will Work!”, IEEE Access, Vol. 1, May 2013, pp. 335-349.

[RAZ-14] S. M. Razavizadeh, M. Ahn, I. Lee; “Three-Dimensional Beamforming-A New Enabling Technology For $5 G$ Wireless Networks”; IEEE Signal Processing Magazine, Vol 31, Issue 6, 2014. pp 94-101.

[REB-16] M. Rebato, M. Mezzavilla, S.Rangan, F. Boccardi, M. Zorzi; “Understanding Noise and Interference Regimes in 5 G Millimeter-Wave Cellular Networks "; Proceedings of $22^{\text {nd }}$ European Wireless Conference, Oulu, Finland, $18^{\text {th }}-20^{\text {th }}$ May 2016, pp 1-5.

[REB-17] M. Rebato, F. Boccardi, M. Mezzavilla, S. Rangan and M. Zorzi; "Hybrid Spectrum Sharing In mmWave Cellular Networks," in IEEE Transactions on Cognitive Communications and Networking, Vol 3, Issue 2, June 2017. pp. 155-168.

[REN-01] Z. Ren, C. Tham, C. Foo and C. Ko., "Integration of Mobile IP and Multi-Protocol Label Switching," Proceedings of IEEE International Conference on Communication (ICC2001), Helsinki, Finland, $11^{\text {th }}-14^{\text {th }}$ June 2001, Vol.7, pp 2123-2127.

[REN-01] Z. Ren, C. Tham, C. Foo, C. Ko; "Integration of Mobile IP and Multi-Protocol Label Switching," IEEE International Conference on Communication (ICC2001), Helsinki, Finland, $11^{\text {th }}-14^{\text {th }}$ June 2001, Vol.7, pp 2123-2127.

[ROB-09] R.Roberts, Z.Xu; “Update on VLC Link Budget Work”; IEEE Project: IEEE P802.15 Working Group for Wireless Personal Area Networks (WPANs).Website:

https://mentor.ieee.org> den > 15-09-0635-01-0007-update-on-vlc-link-budget. Last accessed 31 ${ }^{\text {st }}$ October, 2019.

[ROY-12] S. Corroy, L. Falconetti, R. Mathar; "Cell Association in Small Heterogeneous Networks: Downlink Sum Rate And Min Rate Maximization," in Proceedings of IEEE Wireless Network Communication Conference, Paris, France, $1^{\text {st }}-4^{\text {th }}$ April 2012, pp. 888-892.

[RSS-17] Remote Sensing System; “MSU/AMSU”; Website:

http://www.remss.com/missions/amsu/. Last accessed December 2019.

[RUS-13] F.Rusek, D.Persson,B.K.Lau,E.Larsson,T.Marzetta,O.Edfors, F.Tufvesson; "Scaling up MIMO-:Opportunities and Challenges With Very Large Arrays"; IEEE Signal Processing Magazine, Vol 30, Issue 1, January 2013.pp 40-60.

[SAE-14] Society of Automotive Engineers; "Automated Driving-Standard J3016", Website: https://www.sae.org/misc/pdfs/automated_driving.pdf. Last accessed 23 ${ }^{\text {rd }}$ May 2019.

[SAN-18] B.Santo; “5G:The Road to Low Latency”;Published on 23 ${ }^{\text {rd }}$ Febuary 2019. Website: https://www.edn.com/electronics-blogs/5g-waves/4460346/5G--The-road-to-low-latency. Last accessed $20^{\text {th }}$ June 2019. 
[SCH-06] T. Schneider, M. Junker, K.U.Lauterbach, "Theoretical and experimental investigation of Brillouin scattering for the generation of millimeter waves". JOSA B, Vol.23, Issue 6, 2006. pp. 1012-1019.

[SET-04] K. Sethom, H. Afifi, G. Pujolle; "Wireless MPLS: A New Layer 2.5 Micro-Mobility Scheme," ACM International Workshop on Mobility Management and Wireless Access (Mobimac'04), Philadelphia, PA, USA, October 2004, pp. 64-71.

[SHE-97] S.Shenker; "RFC 2212- Specification of Guaranteed Quality of Service-IETF Tools"; 1997. Website: https://tools.ietf.org/html/rfc2212. Last accessed $4^{\text {th }}$ November 2019.

[SHE-14] T. C. Shen, R. J. Drost, C.C. Davis, B. M. Sadler; "Design of Dual-link (Wide- and Narrow-beam) LED Communication Systems" Optics Express, Vol. 22, No. 9, May 2014, pp 11107-11118.

[SHI-10] P.T.Shih, J.Chen, C.T.Lin; "Optical millimeter-wave signal generation via frequency 12-tupling”, Journal of Lightwave Technology, Vol.28, Issue 1, 2010, pp 71-78.

[SIN-12] D.Singh, P.Singh; "Techniques of mmWave Signal Generation in ROF Systems: A Review"; International Journal of Computer Applications and Information Technology, Vol.1, Issue 2, September 2012. pp. 45-49.

[SLU-12] J. S. Lu, D. Steinbach, P. Cabrol, P. Pietraski; “'Modeling human blockers in millimeter wave radio links, ' ZTE Communication, Vol. 10, Issue 4, December 2012. pp. 23 28.

[SMI-98] G. H. Smith , D. Novak; "Broadband millimeter-wave fibre-radio network incorporating remote up/down conversion", Microwave Symposium Digest, IEEE MTT-S, vol. 3, June 1998. pp. 1509.1512.

[SOL-05] H. Soliman ; "Hierarchical Mobile IPv6 mobility management (HMIPv6)," Internet draft, IETF RFC4140. Website: https://tools.ietf.org/html/rfc4140. Last accessed 6th February 2020 .

[SON-14] J. Song, W. Ding, F.Yang, H. Yang, J. Wang, X.Wang, X. Zhang; "Indoor Hospital Communication Systems: An Integrated Solution Based On Power Line And Visible Light Communication", IEEE Faible Tension Faible Consommation (FTFC), $4^{\text {th }}-6^{\text {th }}$ May 2014, Monaco, pp 1-6.

[SON-98] P. P. Lawson. R. E. Stewart, L. J. Angus; "Observations and Numerical Simulations of Origin and Development ff Very Large Snowflakes", Journal of Atmospheric Science, Vol. 55, 1998. pp. 3209-3229.

[STD-10] IEEE Computer Society. IEEE Std 802.11p -2010. IEEE Standard for Information Technology -Telecommunications and Information Exchange between Systems- Local and 
Metropolitan Area Network- Specific Requirements: Part 11 Wireless LAN Medium Access Control and Physical Specifications, July 2010.

[STD-11] IEEE Standard for Local and Metropolitan Area Networks--Part 15.7: Short-Range Wireless Optical Communication Using Visible Light, September 2011.

[STD-18] IEEE Standard for Local and Metropolitan Area Networks--Part 15.7: Short-Range Wireless Optical Communication, December 2018.

[STE-12] M. Sternad ,M.Grieger, R.Apelfrojd, T.Syensson, D.Aronsson, A.Belen ; “Using 'Predictor Antennas' For Long-Range Prediction of Fast Fading for Moving Relays"; in Proceedings of IEEE Wireless Communications and Networking Conference Workshops (WCNC), Paris, France, April 2012, pp. 253-257.

[STI-14] L.Stinson; "IDEO Imagines the Wild Future of Self-Driving Cars"; 2014. Website: https://www.wired.com/2014/11/ideo-imagines-self-driving-cars/. Last accessed $6^{\text {th }}$ February 2020.

[SZA-15] K.G.Szarkowica, A.S.Monge; "MPLS in the SDN Era”; Published by O'Reilley Media, Juniper Networks.

[TAC-16] R. Tachet, P. Santi, S. Sobolevsky, L. I. Reyes-Castro, E.Frazzoli, D. Helbing, C. Ratti; "Revisiting Street Intersections Using Slot-Based Systems"; Published on $16^{\text {th }}$ March 2016. Website:

http://senseable.mit.edu/papers/pdf/20160316_Tachet_etal_RevisitingStreet_PLOS.pdf. Last accessed $4^{\text {th }}$ July, 2019.

[TAL-14] A.Talukdar, M.Cudak, A.Ghosh; "Handoff Rates for Millimeterwave 5 G Systems"; IEEE 79th Vehicular Technology Conference (VTC Spring),Seoul,South Korea, $18^{\text {th }}-21^{\text {st }}$ May 2014, pp 1-5.

[THO-18] Thorlabs; "Radiometric Vs Photometric Terms". Website: https://www.thorlabs.de/catalogPages/506.pdf. Last accessed 3rd October 2019.

[TOM-01] F.Tommasi, S.Molendini, A.Tricco; "Integrated Services across MPLS Domains Using CR-LDP Signalling," IETF draft, May 2001. Website: https://tools.ietf.org/html/drafttommasi-mpls-intserv-01. Last accessed $4^{\text {th }}$ February 2020.

[TRO-16] S.Trommer, V.Kolarova, E. Fraedrich, P. Phleps; "Autonomous Driving: The Impact of Vehicle Automation on Mobility Behaviour", Published in December 2016.

Website:https://www.researchgate.net/publication/312374304_Autonomous_DrivingThe_Impact _of_Vehicle_Automation_on_Mobility_Behaviour. Last accessed $21^{\text {st }}$ June 2019.

[TSA-07] H.Tsai, O.K.Tonguz, C.Saraydar, T.Talty, M.Ames,A.Macdonalds; “Zigbee-Based Intra-Car Wireless Sensor Networks: A Case Study"; IEEE Wireless Communications, Vol. 14, Issue 6, December 2007. pp 67-77. 
[TTA-12] TTA; “TS 808.220 Light Emitting Diode (LED) Traffic Signal Lamp Modules Material Specification". Published in 2012. Website: https://www.toronto.ca/wpcontent/uploads/2017/11/918e-ecs-specs-tcspecs-section-808-220-material-spec-for-ledmodules.pdf. Last accessed $5^{\text {th }}$ November 2019.

[UCL-10] California Lighting Technology Center; "Ubiquitous Communication by Light". Website: https://cltc.ucdavis.edu/uc-light. Last accessed $3^{\text {rd }}$ September 2019.

[UPV-15] UP-VLC;“UP-VLC: Ultra Parallel Visible Light Communication”. Website: https://www.eng.ed.ac.uk/research/projects/vlc-ultra-parallel-visible-light-communications. Last accessed $3^{\text {rd }}$ September 2019.

[URN-01] T. W. Urn, J. Choi, "A Study on Path Re-routing Algorithms at the MPLS based Hierarchical Mobile IP Network," IEEE TENCON 2001, IEEE Region 10 International Conference on Electrical and Electronic Technology, Singapore, $19^{\text {th }}-22^{\text {nd }}$ August 2001, Vol.2, pp.691-697.

[VAL-99] A. G. Valko; "Cellular IP: A New Approach to Internet Host Mobility," ACM SIGCOMM Computer Communication Review, Vol. 29, No. 1, January 1999, pp. 50-65.

[VAS-03] V. Vassiliou, H. L. Owen, D. Barlow, J. Sokol, H.-P. Huth, J. Grimminger; " $M$ MPLS: Micromobility-Enabled Multiprotocol Label Switching," IEEE International Conference on Communications, Anchorage, Alaska, USA, 11th -15th May 2003, Vol. 1, pp. 250-255.

[VDA-15] VDA; "Automation-From Drivers Assistance System to Automated Driving", Online Magazine, Website: https://www.vda.de/dam/vda/publications/2015/automation.pdf. Last accessed $23^{\text {rd }}$ May 2019.

[VEG-13] A. M. Vegni, M. Biagi, R. Cusani ;"Smart Vehicles, Technologies And Main Applications In Vehicular Ad Hoc Networks", Vehicular Technologies - Deployment and Applications, Dr. Lorenzo Galati Giordano (Ed.), Website:

http://www.intechopen.com/books/vehicular-technologies-deployment-and-applications/smartvehicles-technologies-and-main-applications-in-vehicular-ad-hoc-networks. Last accessed 30th September 2019.

[VIN-12] A. Vinel; “3GPP LTE Versus IEEE 802.11p/WAVE : Which Technology is able to Support Cooperative Vehicular Safety Applications"; IEEE Communications Letters, Vol. 1, No. 2, April 2012, pp. 125-128.

[VIN-13] J.Vincent, "Li-Fi Revolution: Internet Connections Using Light Bulbs Are 250 Times Faster Than Broadband", The Independent. Published on 28 October 2013. Website: http://www.independent.co.uk/news/science/lifi-revolution-internet-connections-using-lightbulbs-are-250-times-faster-than-broadband-8909320.html. Last accessed 28th April 2015. 
[VIR-13] W. Viriyasitavat, S.H. Yu , H.M. Tsai ; “ Short Paper: Channel Model for Visible Light Communications Using Off-the-shelf Scooter Taillight"; Proceedings of 2013 IEEE Vehicular Networking Conference (VNC-13), Boston, Ma, USA, 16 ${ }^{\text {th }}-18$ th December 2013, pp.170-173.

[VIS-01] A. Viswanathan; "RFC 3031 - Multiprotocol Label Switching Architecture - IETF Tools”; 2001. Website: https://tools.ietf.org/html/rfc3031. Last accessed $4^{\text {th }}$ November 2019.

[VIS-14] M.Viswanathan; "MIMO-Diversity and Spatial Multiplexing”; Published on $6^{\text {th }}$ August 2014. Website: https://www.gaussianwaves.com/2014/08/mimo-diversity-and-spatialmultiplexing/. Last accessed $3^{\text {rd }}$ July, 2019.

[VLC-03] VLCC; "Visible Light Communication Consortium". Website: http://www.vlcc.net/modules/xpage0/?ml_lang=en. Last accessed $3^{\text {rd }}$ September 2019.

[VNI-17] CISCO; "Cisco Visual Networking Index: Global Mobile Data Traffic Forecast Update, 2016-2021"; Published on 28 ${ }^{\text {th }}$ March, 2017. Website:

https://www.cisco.com/c/en/us/solutions/collateral/service-provider/visual-networking-indexvni/mobile-white-paper-c11-520862.html. Last accessed 20 ${ }^{\text {th }}$ June 2019.

[WAD-05] M. Wada, T. Yendo, T. Fujii, and M. Tanimoto, "Road-To-Vehicle Communication Using LED Traffic Light," in Proceedings of IEEE Intelligent Vehicles Symposium, Las Vegas, Nevada, USA, $6^{\text {th }}-8^{\text {th }}$ June 2005, pp. 601-606.

[WAL-88] J. Walfisch , H. L. Bertoni; "A Theoretical Model Of UHF Propagation In Urban Environments," IEEE Transactions on Antennas Propagation, Vol. 36, Issue. 12, December 1988. pp. 1788-1796.

[WAN-06] Q.Wang, H.Rideout, F. Zeng, J.Yao; "Millimeter-wave frequency tripling based on four-wave mixing in a semiconductor optical amplifier". IEEE Photonics Technology Letters, Vol. 18, 2006. pp. 2460-2462.

[WAN-17] L. Wang, T. Han, Q. Li, J.Yan, X. Liu, and D. Deng; "Cell-Less Communications in $5 G$ Vehicular Networks Based on Vehicle-Installed Access Points”; IEEE Wireless Communication, Vol.24, Issue 6, December 2017. pp 64-71.

[WAN-17A] H. Wang, S. Chen, M. Ai, H. Xu; "Localized Mobility Management for $5 G$ Ultra Dense Network"; IEEE Transactions On Vehicular Technology, Vol. 66, Issue. 9, September 2017. pp 8535-8552.

[WAN-18] C.X. Wang, J. Bian, J. Sun, W. Zhang, M. Zhang; "A Survey of 5G Channel Measurements and Models"; IEEE Communications Surveys \& Tutorials, Vol. 20, Issue. 4, fourth quarter 2018. pp 3142-3168.

[WEI-14] R. J. Weiler, M.Peter, W. Keusgen , E. Calvanese-Strinati , A. D. Domenico , I.Filippini , A.Capone , I.Siaud , A. Ulmer-Moll , A. Maltsev , T. Haustein, K.Sakaguchi; 
"Enabling 5G Backhaul and Access With Millimeter-Waves"; Proceedings of European Conference on Networks and Communications (EuCNC), Bologna, Italy, $23^{\text {rd }}-26^{\text {th }}$ June 2014, pp.1-5.

[WEI-82] M.A Weissberger; “An Initial Critical Summary Of Models For Predicting The Attenuation Of Radio Waves By Foliage"; ECAC-TR81-101, Electromagnetic Compatibility Analysis Center, Annapolis, MD, 1982. Website:

https://apps.dtic.mil/dtic/tr/fulltext/u2/a118343.pdf. Last accessed $6^{\text {th }}$ February 2020.

[WHO-18] WHO, ed. (2018); "Global Status Report on Road Safety 2018 " (PDF) (official report). Geneva, Switzerland: World Health Organisation (WHO). pp. vii, 1-8, 53ff (countries), 244-251 (table A2), 296-303 (table A10). Website:

http://www.who.int/violence_injury_prevention/road_safety_status/2018/en/. Last accessed 14th March 2019.

[WiM-10] WiMAx Forum, "WiMAX and the IEEE 802.16m Air Interface Standard- April 2010". Website:

http://www.wimaxforum.org/sites/wimaxforum.org/files/document_library/wimax_802.16m.pdf. Last accessed 28th November 2019.

[WLI-12] W. Li , C. Zhang, X. Duan, S. Jia, Y. Liu, L.Zhang ; "Performance Evaluation And Analysis On Group Mobility Of Mobile Relay For LTE Advanced System”; in Proceedings of IEEE Vehicular Technology Conference, Quebec City, QC, Canada, September 2012, pp. 1-5.

[WRO-97] J.Wroclawski; "RFC 2211- Specification of the Controlled Load Network ElementIETF Tools"; 1997. https://tools.ietf.org/html/rfc2211. Last accessed $4^{\text {th }}$ November 2019.

[WU-15] T. Wu, T. Rappaport, C. Collins; "The Human Body and Millimeterwave Wireless Communication Systems: Interactions and Implications," in Proceedings of IEEE International Conference on Communication (ICC), London, U.K., June 2015, pp. 2423-2429.

[XIE-02] K. Xie and V.C.M. Leung, "A MPLS Framework for Macro- And Micro-Mobility Management," 2nd IASTED International Conference Wireless and Optical Communications (WOC2002), Canada, July 2002, pp 1-6.

[XIE-03] K. Xie, V.Wong, V. Leung; "Support of Micro-Mobility in MPLS Based Wireless Access Networks," IEEE Wireless Communications and Networking (WCNC), New Orleans, Los Angeles, USA, 20 ${ }^{\text {th }}$ March 2003, Vol 2, pp 1242-1247.

[XIE-03] K. Xie, V.Wong, V. Leung; "Support of Micro-Mobility In MPLS Based Wireless Access Networks," IEEE Wireless Communications and Networking (WCNC), New Orleans, Los Angeles, USA, 20 ${ }^{\text {th }}$ March 2003, Vol 2, pp 1242-1247.

[XU-02] H. Xu, V. Kukshya, and T. S. Rappaport, "Spatial and temporal characteristics of 60$\mathrm{GHz}$ indoor channels," IEEE Journal on Selected Areas of Communication., Vol. 20, Issue. 3, April 2002. pp. 620-630. 
[YAN-01] T. Yang, D. Makrakis, "Hierarchical Mobile MPLS: Supporting Delay Sensitive Applications Over Wireless Internet”, IEEE International Conference on Info-tech and Info-net, 29th October-1st November 2001, Beijing, China. Vol 2, pp 453-458.

[YAN-02] T. Yang, Y. Dong, Y. Zhang, and D. Makrakis; "Practical Approaches For Supporting Micro Mobility With MPLS," International Conference of Telecommunications (ICT), Beijing, China, June 2002.

[YIN-16] X.Yin, X.Cheng; “Chapter 4-Geometry-based Stochastic Channel Modeling”;Wiley Online Library. Website: https://onlinelibrary.wiley.com/doi/10.1002/9781118188248.ch4. Last accessed $17^{\text {th }}$ January 2019.

[YU-13] S.H. Yu, O.Shih, H-M. Tsai; "Smart Automotive Lighting for Vehicle Safety", IEEE Communications Magazine, Volume 51, Issue 12, December 2013. pp 50-59.

[YUA-10] G. Yuan, X. Zhang, W. Wang, Y. Yang, ' Carrier Aggregation For LTE-Advanced Mobile Communication Systems, ” IEEE Communication Magazine, Vol. 48, Issue. 2, Febuary 2010. pp. 88-93.

[YUK-09] M. Yuksel ,J.Akela, P.Dutta, "Free-Space-Optical Mobile Ad Hoc Networks: AutoConfigurable Building Blocks, Wireless Networks, ”Wireless Networks, 2009, pp. 295-312.

[YUT-06] S. E. Yuter, D. E. Kingsmill, L. B. Nance, M. Loffler-Mang, “Observations Of Precipitation Size And Fall Speed Characteristics Within Coexisting Rain And Wet Snow", Journal of Applied Meteorology, Vol. 45, 2006. pp. 1450-1464.

[ZHA-07] L.Zhang; “RFC 2205 - Resource ReSerVation Protocol (RSVP - IETF Tools)”; 2007. Website: https://tools.ietf.org/html/rfc2205. Last accessed $4^{\text {th }}$ November 2019.

[ZHA-10] H. Zhang, S. Venkateswaran, U. Madhow, "Channel Modeling and MIMO Capacity for Outdoor Millimeter Wave Links," in Proceedings of IEEE Wireless Communication Network Conference (WCNC), Sydney, NSW, Australia, April 2010, pp. 1-6.

[ZHA-13] H. Zhao, R. Mayzus, S. Sun, M. Samimi,J. K. Schulz, Y. Azar, K. Wang, G. N. Wong,F. Gutierrez, T. S. Rappaport; "28 GHzmillimeter wave cellular communication measurements for reflection and penetrationloss in and around buildings in New York City, ' in Proceedings of IEEE International Conference on Communication., Budapest, Hungary, $9^{\text {th }}-13^{\text {th }}$ June 2013, pp. 5163-5167.

[ZHA-15] R.Zhang, J.Wang, Z.Wang, Z.Xu, C.Zhao, L.Hanzo; “Visible Light Communications in Heterogeneous Networks: Paving the Way for User-Centric Design”; IEEE Wireless Communications, April 2015. pp 8-16.

[ZHA-15A] Z.Zhang, J. Ryu, S.Subramanian, A.Sampath; "Coverage and Channel Characteristics of Millimeter Wave Band Using Ray Tracing"; IEEE International Conference on 
Communication Selected Areas in Communication - Millimeter-wave Communications, London, UK, $8^{\text {th }}-12^{\text {th }}$ June 2015. pp 1350-1385.

[ZHA-16] J.Zhang, X.Ge, Q. Li, M. Guizani, Y. Zhang; "5G Millimeter-Wave Antenna Array: Design and Challenges", IEEE Wireless Communication, Vol.24, No.2, April 201, pp. 106-112.

[ZHO-12] H. Zhu, S. Wang, D. Chen; "Energy-Efficient User Association For Heterogenous Cloud Cellular Networks, " in Proceedings of IEEE Globecom Workshops (GC Wkshps), Anaheim, CA, USA, $3^{\text {rd }}-7^{\text {th }}$ Dec. 2012, pp. 273-278.

[ZHO-17] H. Zhou, W. Xu, Y. Bi, J.Chen, Q. Yu, X. Shen; "Toward 5G Spectrum Sharing for Immersive-Experience-Driven Vehicular Communications"; IEEE Wireless Communication, Vol 24, Issue 6, December 2017. pp 30-37.

[ZHU-14] Na Zhu, Zhe Xu, Yuxiang Wang, Hendan Zhuge, Jibin Li; "Handover Method In Visible Light Communication Between the Moving Vehicle and Multiple LED Streetlights,"; Elsevier Optik, Vol.125, Issue 14, July 2014. pp 3540-3544. 